\title{
Enantioselective Construction of Spirooxindole-Fused Cyclopentanes
}

Vojtěch Dočekal, ${ }^{a)}$ Andrea Vopálenskáa, ${ }^{a)}$ Pavel Měrka, ${ }^{a)}$ Klára Konečná, ${ }^{b)}$ Ondřej Jand'ourek, ${ }^{b)}$ Milan Pour, ${ }^{c)}$ Ivana Císařová, ${ }^{d)}$ and Jan Veselý, ${ }^{a *}$

a) Department of Organic Chemistry, Faculty of Science, Charles University, Hlavova 2030/8, 12843 Prague 2, Czech Republic (email: jan.vesely@natur.cuni.cz)

b) Department of Biological and Medical Sciences, Faculty of Pharmacy in Hradec Králové, Charles University, Heyrovského 1203, 50005 Hradec Králové, Czech Republic

c) Department of Organic and Bioorganic Chemistry, Faculty of Pharmacy in Hradec Králové, Charles University, Heyrovského 1203, 50005 Hradec Králové, Czech Republic

d) Department of Inorganic Chemistry, Faculty of Science, Charles University, Hlavova 2030/8, 12843 Prague 2, Czech Republic

\section{Supporting information}

Unsuccessful attempts to prepare selected starting material .................................................................................. S2

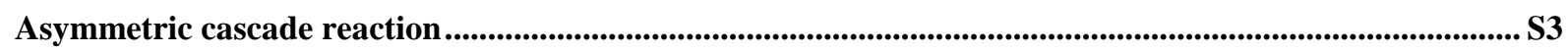

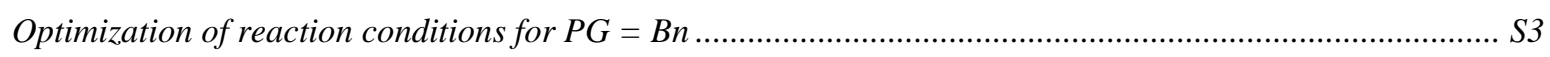

Example of determination of diastereomeric ratio for asymmetric cascade reaction ..................................... S10

Crystallographic data ................................................................................................................................................. S11

Computational methods..................................................................................................................................................... S15

Biological activity screening .......................................................................................................................................... S21

Antimycobacterial In Vitro Activity Screening Against Mycobacterium smegmatis, Mycobacterium aurum,

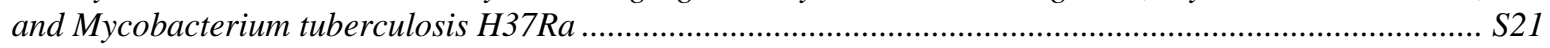

Basic screening of antibacterial activity ....................................................................................... S22

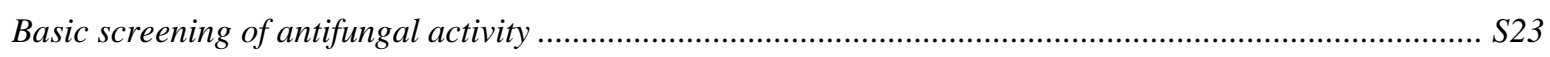

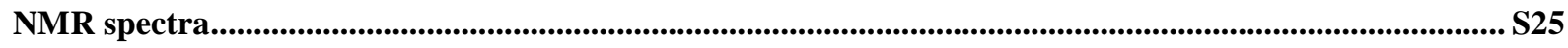

Chiral HPLC ............................................................................................................................................................. S111

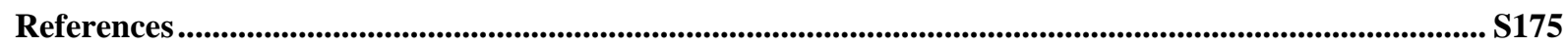




\section{Unsuccessful attempts to prepare selected starting material}

Following substrates (Figure S1) were not prepared by previously described procedures, including their optimization.<smiles>O=C1Nc2ccc([N+](=O)[O-])cc2C1CCBr</smiles><smiles>O=C1Nc2ccc(C(F)(F)F)cc2C1CCBr</smiles><smiles>O=C1C(CCBr)c2ccccc2N1Cc1cccc2ccccc12</smiles><smiles>O=C1C(CCBr)c2ccccc2N1S</smiles><smiles>CS(=O)(=O)N1C(=O)C(CCBr)c2ccccc21</smiles>

Figure S1. Not prepared starting material.

According to the previous methods, we tried to prepare oxindole bearing naphtalen-1-ylmethyl, and sulfonate protecting groups. We did not observe the formation of the corresponding oxindole derivative in the oxidation step $(\mathrm{DMSO} / \mathrm{HCl})$. We also tested other methods used previously for the synthesis of similar compounds without success. We successfully isolated only indole-based derivatives. We confirmed the indole-based structure of the isolated compound by X-ray.
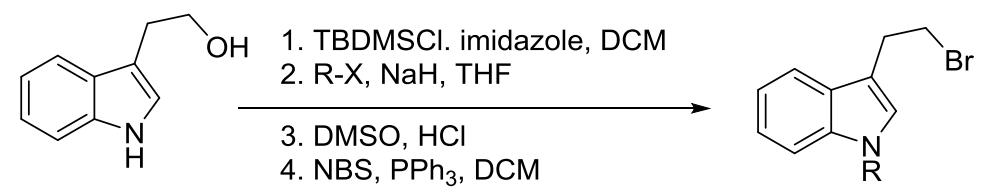
$\mathrm{R}=$ naphthalen-1-ylmethyl (29\%, over four steps) $\mathrm{R}=\mathrm{Ts}(33 \%$, over four steps $)$ $\mathrm{R}=4-\mathrm{Ns}$ (38\%, over four steps)
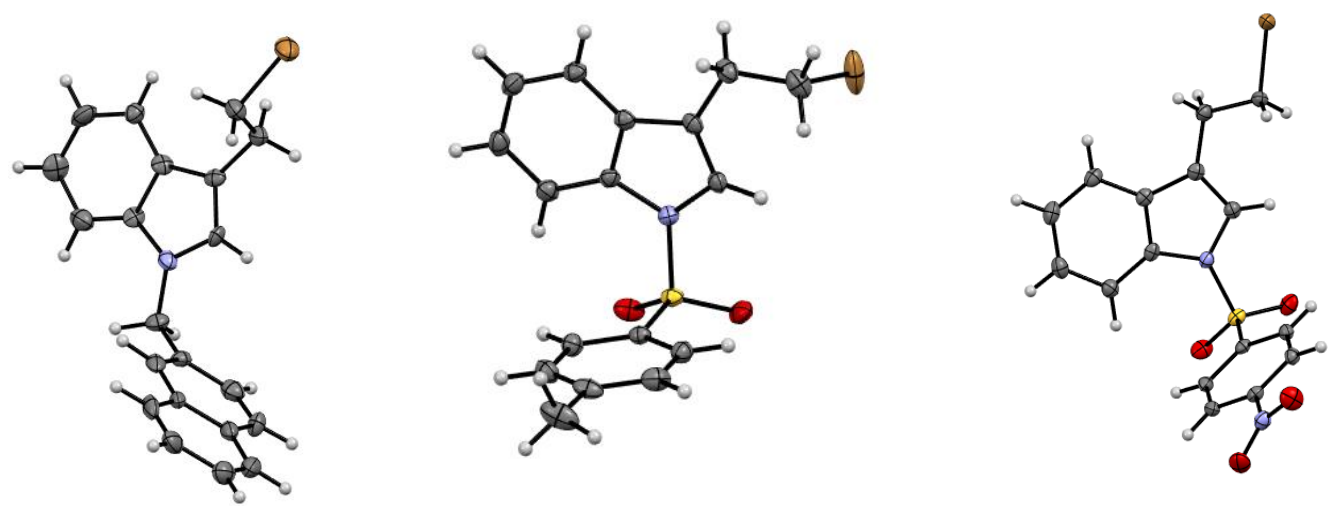

Additionally, we tried to prepare oxindole derivative bearing strong electron-withdrawing group $\left(-\mathrm{NO}_{2}\right.$, and $\left.-\mathrm{CF}_{3}\right)$ in position 5 of oxindole ring, but no success. 


\section{Asymmetric cascade reaction}

\section{Optimization of reaction conditions for $\mathbf{P G}=\mathbf{B n}$}

Figure S2. Screened organocatalysts.

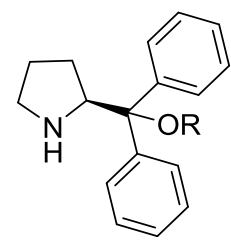

C1: $\mathrm{R}=\mathrm{TMS}$

C2: $R=$ TBDMS

C3: $R=$ MDPS

C4: $\mathrm{R}=$ TPS

C5: $\mathrm{R}=\mathrm{H}$

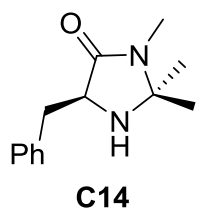

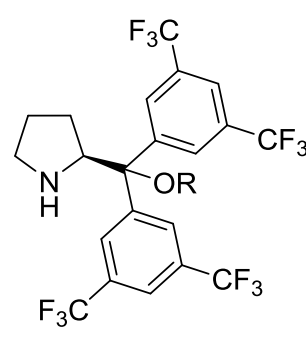

C6: $\mathrm{R}=\mathrm{TMS}$

C7: $R=$ TBDMS

C8: $R=$ MDPS

C9: $\mathrm{R}=\mathrm{H}$

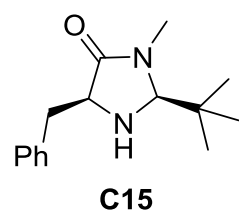

C15

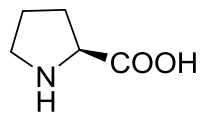

C16

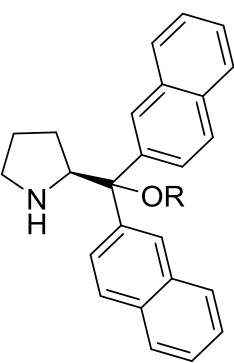

C10: $\mathrm{R}=\mathrm{TMS}$

C11: $R=$ TBDMS

C12: $R=$ MDPS

C13: $\mathrm{R}=\mathrm{H}$<smiles>[X]C(NC[C@@H]1CCCN1)Nc1cc(C(F)(F)F)cc(C(F)(F)F)c1</smiles>

C19: $X=O$

C20: $X=S$

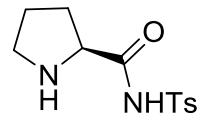

C21<smiles>C1CNC(CN2CCCC2)C1</smiles>

C17

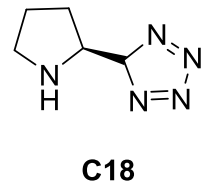


Table S1. Base screening.<smiles>[B]CCC1C(=O)Nc2ccccc21</smiles>

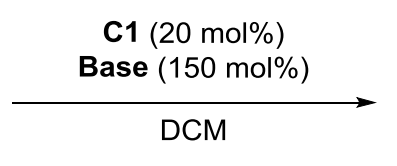

2a<smiles>O=CC=C[Pb]</smiles><smiles>O=C[C@H]1CC[C@@]2(C(=O)Nc3ccccc32)C1c1ccccc1</smiles>

$3 a$<smiles>O=C[C@H]1CC[C@]2(C(=O)Nc3ccccc32)[C@H]1c1ccccc1</smiles>

4a<smiles>O=C1Nc2ccccc2C12CC2</smiles>

\begin{tabular}{|c|c|c|c|c|c|c|}
\hline Entry & Base & Time (h) & $\operatorname{Dr}(3 a / 4 a)^{[a]}$ & Yield $(3 a / 4 a, \%)^{[b]}$ & Comb. yield (\%) ${ }^{[b]}$ & $e e(3 a / 4 a, \%)^{[c]}$ \\
\hline 1 & $\mathrm{NaHCO}_{3}$ & 120 & $1 / 1.2$ & $27 / 30$ & 57 & $93 / 94$ \\
\hline $2^{[\mathrm{d}]}$ & DABCO & 12 & $1 / 1.8$ & $17 / 38$ & 55 & $92 / 97$ \\
\hline $3^{[\mathrm{d}]}$ & $\mathrm{Et}_{3} \mathrm{~N}$ & 12 & $1 / 1.0$ & n.d. & n.d. & n.d. \\
\hline $4^{[\mathrm{d}]}$ & DIPEA & 12 & $1.6 / 1$ & n.d. & n.d. & n.d. \\
\hline 5 & 2,6-lutidine & 40 & $1 / 2.3$ & $30 / 68$ & 98 & $95 / 98$ \\
\hline 6 & pyridine & 120 & $1 / 1.8$ & $32 / 45$ & 77 & $94 / 95$ \\
\hline 7 & 2,4,6-collidine & 16 & $1 / 2.3$ & $26 / 72$ & 98 & $96 / 97$ \\
\hline 8 & 2,6-ditBu-4-Me-pyridine & 120 & $1 / 1.0$ & $25 / 24$ & 49 & $92 / 96$ \\
\hline 9 & 2,6-ditBu-pyridine & 120 & $1 / 1.0$ & $13 / 16$ & 29 & $93 / 95$ \\
\hline 10 & $\mathrm{AcONa}$ & 16 & $1.2 / 1$ & $55 / 43$ & 98 & $90 / 88$ \\
\hline 11 & $\mathrm{~K}_{2} \mathrm{CO}_{3}$ & 40 & $1 / 1.3$ & $39 / 57$ & 96 & $92 / 93$ \\
\hline
\end{tabular}

[a] Determined by $1 \mathrm{H}-\mathrm{NMR}$ analysis of the crude reaction mixture. ${ }^{[\mathrm{b}]}$ Isolated after column chromatography;

${ }^{[c]}$ Determined by chiral HPLC; ${ }^{[d]}$ Formation of cyclopropane by-product (5a) as major product was observed. 
Table S2. Catalyst screening.

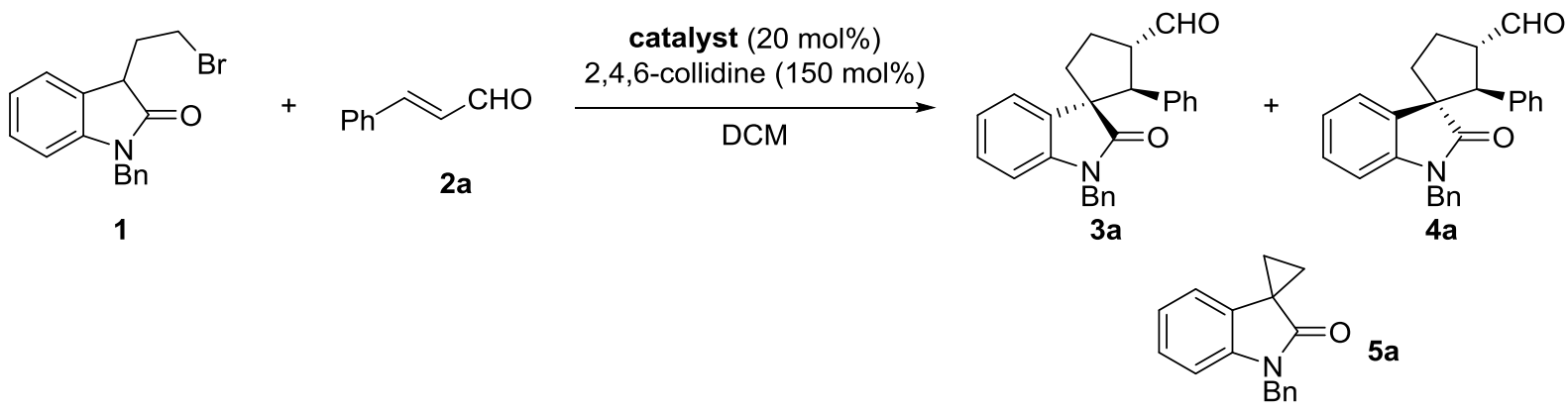

\begin{tabular}{|c|c|c|c|c|c|c|}
\hline Entry & Catalyst & Time (h) & $\operatorname{Dr}(3 a / 4 a)^{[a]}$ & Yield $(3 a / 4 a, \%)^{[b]}$ & Comb. yield $(\%)^{[b]}$ & $e e(3 a / 4 a, \%)^{[c]}$ \\
\hline 1 & C1 & 16 & $1 / 2.3$ & $30 / 68$ & 98 & $95 / 98$ \\
\hline $2^{[\mathrm{d}, \mathrm{e}]}$ & C5 & 120 & $1 / 1.1$ & n.d. & n.d. & n.d. \\
\hline 3 & $\mathrm{C2}$ & 12 & $1 / 2.3$ & $28 / 70$ & 98 & $99 / 98$ \\
\hline 4 & C3 & 12 & $1 / 1.8$ & $35 / 62$ & 97 & $98 / 98$ \\
\hline 5 & C4 & 12 & $1 / 1.7$ & $34 / 65$ & 99 & $98 / 99$ \\
\hline $6^{[\mathrm{e}]}$ & C9 & 120 & n.d. & n.d. & n.d. & n.d. \\
\hline 7 & C6 & 120 & $1 / 1.7$ & $27 / 52$ & 79 & $92 / 96$ \\
\hline 8 & C7 & 120 & $1 / 1.6$ & $34 / 45$ & 79 & $96 / 99$ \\
\hline 9 & $\mathrm{C8}$ & 96 & $1 / 1.6$ & $32 / 55$ & 87 & $97 / 98$ \\
\hline $10^{[\mathrm{d}, \mathrm{e}]}$ & $\mathrm{C} 13$ & 120 & $1.1 / 1$ & n.d. & n.d. & n.d. \\
\hline 11 & C10 & 16 & $1 / 2.3$ & $26 / 71$ & 97 & $94 / 96$ \\
\hline 12 & C11 & 16 & $1 / 2.3$ & $28 / 71$ & 99 & $97 / 98$ \\
\hline 13 & C12 & 16 & $1 / 2.0$ & $30 / 67$ & 97 & $98 / 99$ \\
\hline $14^{[\mathrm{e}]}$ & C14 & 120 & $1.2 / 1$ & n.d. & n.d. & n.d. \\
\hline $15^{[\mathrm{d}, \mathrm{e}]}$ & C15 & 120 & $1.6 / 1$ & n.d. & n.d. & n.d. \\
\hline $16^{[\mathrm{d}, \mathrm{e}]}$ & C16 & 120 & $1 / 1.3 / 1.2^{[f]}$ & n.d. & n.d. & n.d. \\
\hline 17 & C17 & 12 & $1 / 1.2$ & $30 / 42$ & 72 & $-8 /-9$ \\
\hline 18 & C18 & 12 & $1 / 1.3$ & $23 / 41$ & 64 & $60 /-16$ \\
\hline 19 & C19 & 120 & $1.9 / 1$ & $43 / 25$ & 68 & $5 / 4$ \\
\hline 20 & $\mathrm{C} 20$ & 120 & $3.0 / 1$ & $39 / 8$ & 47 & $-11 /-12$ \\
\hline $21^{[\mathrm{e}]}$ & C21 & 120 & n.d. & n.d. & n.d. & n.d. \\
\hline
\end{tabular}

${ }^{[a]}$ Determined by $1 \mathrm{H}-\mathrm{NMR}$ analysis of the crude reaction mixture. ${ }^{[\mathrm{b}]}$ Isolated after column chromatography;

${ }^{[c]}$ Determined by chiral HPLC; ${ }^{[d]}$ Formation of cyclopropane by-product (5a) as major product was observed.

${ }^{[\mathrm{e}]}$ Almost no conversion of starting cinnamldehyde was observed. ${ }^{[\mathrm{ff}}$ Formation of uncyclized Micheal product was observed. 
Table S3. Solvent screening.<smiles>O=C1Nc2ccccc2C1CCBr</smiles>

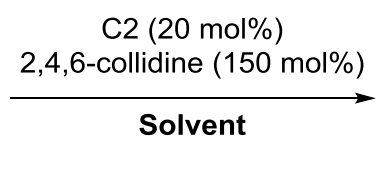

2a<smiles>O=C[C@H]1CC[C@]2(C(=O)Nc3ccccc32)[C@H]1c1ccccc1</smiles><smiles>O=C1Nc2ccccc2C12CC2</smiles>

\begin{tabular}{|c|c|c|c|c|c|c|}
\hline Entry & Solvent & Time (h) & $\operatorname{Dr}(3 a / 4 a)^{[a]}$ & Yield $(3 a / 4 a, \%)^{[b]}$ & Comb. yield $(\%)^{[b]}$ & $e e(3 a / 4 a, \%)^{[c]}$ \\
\hline 1 & $\mathrm{DCM}$ & 12 & $1 / 2.3$ & $28 / 70$ & 98 & $99 / 98$ \\
\hline 2 & $\mathrm{CHCl}_{3}$ & 36 & $1 / 1.7$ & $30 / 53$ & 83 & $95 / 99$ \\
\hline 3 & $\mathrm{CCl}_{4}$ & 96 & $1.7 / 1$ & $34 / 16$ & 50 & $97 / 95$ \\
\hline 4 & 1,2-DCE & 14 & $1 / 1.1$ & $45 / 52$ & 97 & $97 / 99$ \\
\hline 5 & toluene & 36 & $1.7 / 1$ & $54 / 31$ & 85 & $99 / 95$ \\
\hline 6 & benzene & 40 & $1.5 / 1$ & $60 / 38$ & 98 & $96 / 93$ \\
\hline 7 & $\mathrm{MeCN}$ & 10 & $1 / 1.3$ & $40 / 55$ & 95 & $97 / 98$ \\
\hline 8 & EtOAc & 40 & $1.1 / 1$ & $50 / 42$ & 92 & $98 / 94$ \\
\hline 9 & $\mathrm{Et}_{2} \mathrm{O}$ & 24 & $1.9 / 1$ & $56 / 35$ & 91 & $94 / 93$ \\
\hline 10 & THF & 40 & $1 / 1.1$ & $35 / 42$ & 77 & $95 / 97$ \\
\hline 11 & $\mathrm{MeOH}$ & 24 & $2.8 / 1$ & $63 / 24$ & 87 & $88 / 93$ \\
\hline 12 & iPrOH & 24 & $1.4 / 1$ & $42 / 30$ & 72 & $95 / 95$ \\
\hline $13^{[\mathrm{e}]}$ & DMSO & 120 & n.d. & n.d. & n.d. & n.d. \\
\hline 14 & heptane & 120 & $1.3 / 1$ & $45 / 34$ & 79 & $96 / 94$ \\
\hline
\end{tabular}

[a] Determined by $1 \mathrm{H}-\mathrm{NMR}$ analysis of the crude reaction mixture. ${ }^{[b]}$ Isolated after column chromatography;

${ }^{[c]}$ Determined by chiral HPLC; ${ }^{[d]}$ Formation of cyclopropane by-product (5a) as major product was observed.

${ }^{[\mathrm{e}]}$ Almost no conversion of starting cinnamaldehyde was observed. 
Table S4. Reagent ratio, temperature, concentration screening.

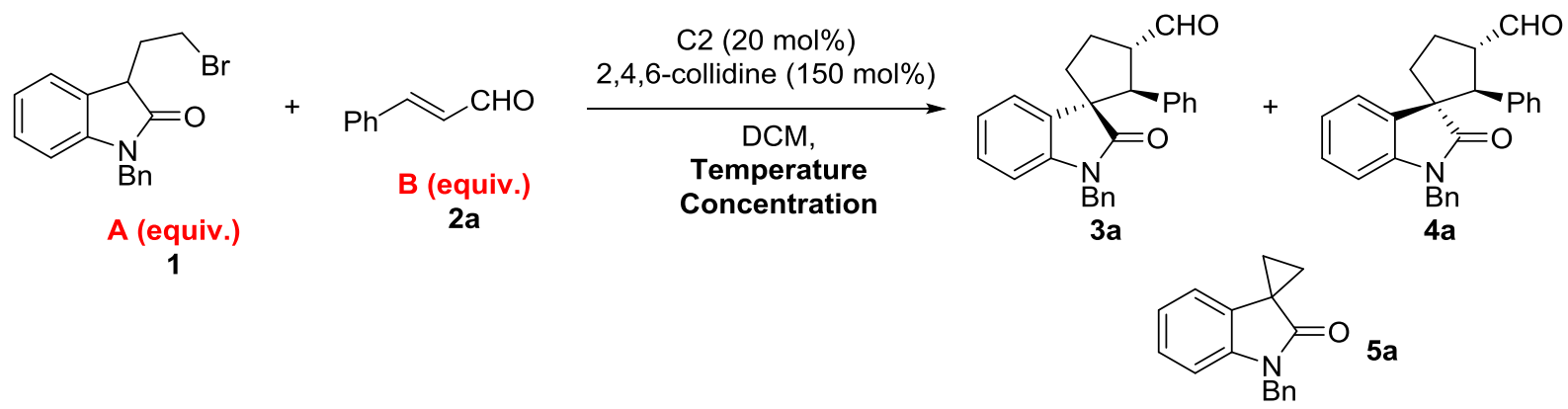

\begin{tabular}{|c|c|c|c|c|c|c|c|c|c|}
\hline Entry & & B & Temp. $\left({ }^{\circ} \mathrm{C}\right)$ & Conc. (mol/l) & Time (h) & $\operatorname{Dr}(3 \mathbf{a} / 4 a)^{[a]}$ & Yield $(3 a / 4 a, \%)^{[b]}$ & Com. yield $(\%)^{[b]}$ & $(3 \mathrm{a} / 4 \mathrm{a}, \%)^{[c]}$ \\
\hline 1 & 1.05 & 1.0 & r.t. & 0.2 & 12 & $1 / 2.3$ & $28 / 70$ & 98 & 99/98 \\
\hline 2 & 1.05 & 1.0 & 0 & 0.2 & 24 & $1 / 1.8$ & $33 / 57$ & 90 & $99 / 99$ \\
\hline 3 & 1.05 & 1.0 & 40 & 0.2 & 3 & $1 / 1.5$ & $32 / 60$ & 82 & $98 / 99$ \\
\hline 4 & 1.05 & 1.0 & r.t. & 0.1 & 24 & $1 / 1.8$ & $32 / 65$ & 97 & $99 / 99$ \\
\hline 5 & 1.05 & 1.0 & r.t. & 0.4 & 10 & $1 / 1.4$ & $34 / 57$ & 91 & $97 / 99$ \\
\hline 6 & 1.5 & 1.0 & r.t. & 0.2 & 12 & $1 / 2.1$ & $29 / 59$ & 88 & $98 / 99$ \\
\hline $7^{[\mathrm{e}]}$ & 1.0 & 1.0 & r.t. & 0.2 & 12 & $1 / 2.3$ & $28 / 61$ & 89 & $98 / 99$ \\
\hline 8 & 1.0 & 1.2 & r.t. & 0.2 & 12 & $1 / 2.2$ & $21 / 62$ & 83 & 99/99 \\
\hline
\end{tabular}

[a] Determined by $1 \mathrm{H}-\mathrm{NMR}$ analysis of the crude reaction mixture. ${ }^{[\mathrm{b}]}$ Isolated after column chromatography;

${ }^{[c]}$ Determined by chiral HPLC; ${ }^{[d]}$ Formation of cyclopropane by-product (5a) as major product was observed.

[e] Starting oxindole was consumed.

Table S5. Catalyst and base ratio screening.

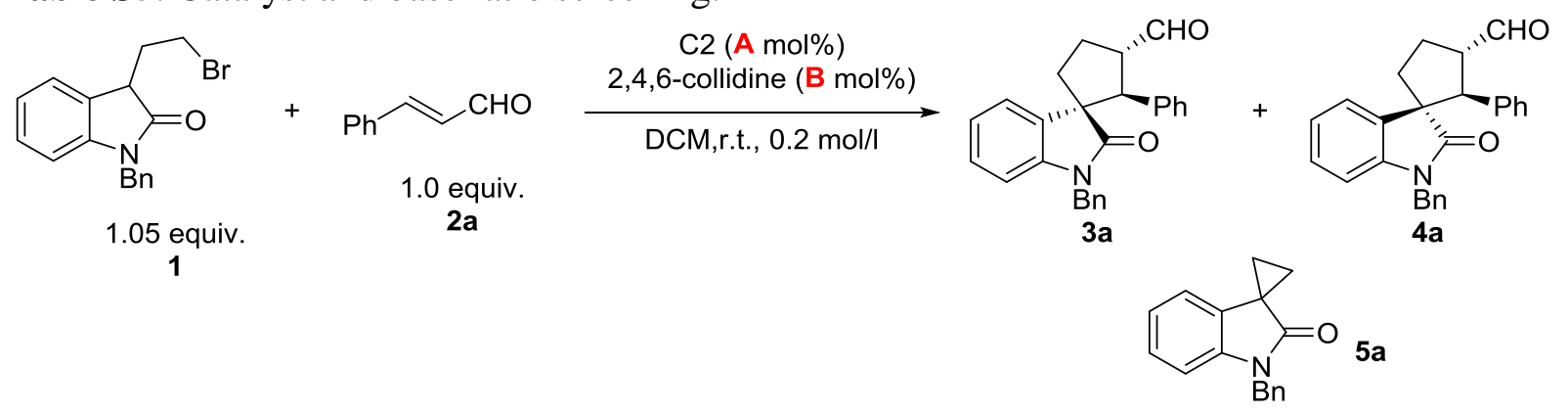

\begin{tabular}{|c|c|c|c|c|c|c|c|}
\hline Entry & $\mathbf{A}$ & B & Time (h) & $\operatorname{Dr}(3 a / 4 a)^{[a]}$ & Yield $(3 a / 4 a, \%)^{[b]}$ & Com. yield (\%) ${ }^{[\mathrm{b}]}$ & ee $(3 a / 4 a, \%)^{[c]}$ \\
\hline 1 & 20 & 150 & 12 & $1 / 2.3$ & $28 / 70$ & 98 & $99 / 98$ \\
\hline 2 & 15 & 150 & 36 & $1 / 2.1$ & $32 / 65$ & 98 & $98 / 99$ \\
\hline 3 & 10 & 150 & 120 & $1 / 2.0$ & $30 / 58$ & 88 & $97 / 99$ \\
\hline $4^{[\mathrm{e}]}$ & 20 & 200 & 36 & $1 / 2.2$ & $28 / 63$ & 91 & $98 / 99$ \\
\hline 5 & 20 & 100 & 36 & $1 / 2.1$ & $30 / 62$ & 92 & $98 / 99$ \\
\hline
\end{tabular}

[a] Determined by $1 \mathrm{H}-\mathrm{NMR}$ analysis of the crude reaction mixture. ${ }^{[\mathrm{b}]}$ Isolated after column chromatography;

${ }^{[c]}$ Determined by chiral HPLC; ${ }^{[d]}$ Formation of cyclopropane by-product (5a) as major product was observed.

[e] Starting oxindole was consumed. 


\section{Optimization of reaction conditions for $\mathbf{P G}=\mathbf{H}$}

Table S6. Solvent screening.<smiles>[B]CCC1C(=O)Nc2ccccc21</smiles>

1d
C2 $(20 \mathrm{~mol} \%)$ 2,4,6-collidine (150 mol\%)

Solvent

2a<smiles>O=C[C@H]1CC[C@]2(C(=O)Nc3ccccc32)[C@H]1c1ccccc1</smiles><smiles>O=C[C@H]1CCC2(C(=O)Nc3ccccc32)[C@H]1c1ccccc1</smiles><smiles>O=C1Nc2ccccc2C12CC2</smiles>

\begin{tabular}{|c|c|c|c|c|c|c|}
\hline Entry & Solvent & Time (h) & $\operatorname{Dr}(3 d / 4 d)^{[a]}$ & Yield (3d/4d, \%) $)^{[b]}$ & Comb. yield (\%) ${ }^{[\mathrm{b}]}$ & ee $(3 d / 4 d, \%)^{[c]}$ \\
\hline 1 & $\mathrm{DCM}$ & 15 & $3.4 / 1$ & $60 / 17$ & 77 & $90 / 96$ \\
\hline 2 & $\mathrm{CCl}_{4}$ & 15 & $4.8 / 1$ & $77 / 16$ & 93 & $67 / 80$ \\
\hline 3 & toluene & 15 & $3.9 / 1$ & $70 / 18$ & 88 & $60 / 73$ \\
\hline 4 & EtOAc & 15 & $2.0 / 1$ & $55 / 32$ & 87 & $60 / 90$ \\
\hline 5 & $\mathrm{Et}_{2} \mathrm{O}$ & 15 & $3.8 / 1$ & $50 / 14$ & 64 & $72 / 83$ \\
\hline 6 & $\mathrm{MeOH}$ & 15 & $2.1 / 1$ & $52 / 25$ & 77 & $61 / 72$ \\
\hline 7 & $\mathrm{DCM}$ & 15 & $4.1 / 1$ & 72/n.d. & 72 & $92 / 95$ \\
\hline 8 & $\mathrm{DCM}^{[\mathrm{e}]}$ & 15 & $3.3 / 1$ & $68 / 20$ & 88 & $92 / 88$ \\
\hline 9 & EtOAc & 15 & $2.1 / 1$ & $52 / 25$ & 77 & $62 / 95$ \\
\hline
\end{tabular}

[a] Determined by $1 \mathrm{H}-\mathrm{NMR}$ analysis of the crude reaction mixture. ${ }^{[\mathrm{b}]}$ Isolated after column chromatography.

${ }^{[c]}$ Determined by chiral HPLC. ${ }^{[d]}$ Formation of cyclopropane by-product (5d) as major product was observed.

${ }^{[\mathrm{e}]}$ Acidic work-up. 
Table S7. Base screening.<smiles>O=C1Nc2ccccc2C1CCBr</smiles>

1<smiles>O=CC=C[Pb]</smiles>

$2 a$

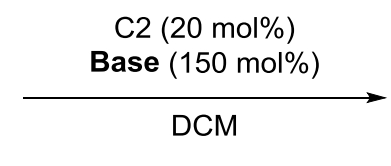<smiles>O=C[C@H]1CC[C@]2(C(=O)Nc3ccccc32)[C@H]1c1ccccc1</smiles>

3d<smiles>O=C[C@H]1CC[C@]2(C(=O)Nc3ccccc32)[C@H]1c1ccccc1</smiles>

4d

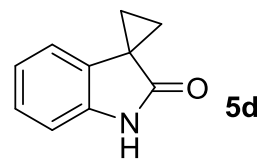

\begin{tabular}{|c|c|c|c|c|c|c|}
\hline Entry & Base & Time (h) & $\operatorname{Dr}(3 b / 4 b)^{[a]}$ & Yield $(3 b / 4 b, \%)^{[b]}$ & Comb. yield $(\%)^{[b]}$ & $e e(3 b / 4 b, \%)^{[c]}$ \\
\hline 1 & $2,4,6$-collidine ${ }^{[f]}$ & 15 & $3.4 / 1$ & $60 / 17$ & 77 & $90 / 96$ \\
\hline 2 & $\mathrm{NaHCO}_{3}$ & 120 & $4.0 / 1$ & $77 / 19$ & 96 & $46 / 81$ \\
\hline 3 & $\mathrm{DABCO}{ }^{[\mathrm{d}, \mathrm{e}]}$ & 120 & $1.8 / 1$ & n.d. & n.d. & n.d. \\
\hline 4 & $\mathrm{Et}_{3} \mathrm{~N}^{[\mathrm{d}, \mathrm{e}]}$ & 120 & $3.0 / 1$ & n.d. & n.d. & n.d. \\
\hline 5 & DIPEA $^{[\mathrm{e}]}$ & 120 & $1.7 / 1$ & n.d. & n.d. & n.d. \\
\hline 6 & 2,6-lutidine & 96 & $4.0 / 1$ & $72 / 17$ & 89 & $69 / 85$ \\
\hline 7 & $\mathrm{AcONa}$ & 15 & $4.0 / 1$ & $58 / 15$ & 73 & $42 / 64$ \\
\hline 8 & $\mathrm{~K}_{2} \mathrm{CO}_{3}$ & 96 & $3.6 / 1$ & 47/n.d. & 47 & 62/n.d. \\
\hline
\end{tabular}

${ }^{[\mathrm{a}]}$ Determined by $1 \mathrm{H}-\mathrm{NMR}$ analysis of the crude reaction mixture. ${ }^{[\mathrm{b}]}$ Isolated after column chromatography.

${ }^{[c]}$ Determined by chiral HPLC. ${ }^{[d]}$ Formation of cyclopropane by-product (5d) as major product was observed.

${ }^{[\mathrm{e}]}$ Almost no conversion of starting cinnamaldehyde was observed. ${ }^{[f]}$ Acidic work-up. 


\section{Example of determination of diastereomeric ratio for asymmetric cascade reaction}<smiles>O=C1Nc2ccccc2C1CCBr</smiles>

$1 \mathrm{a}$<smiles>O=C/C=C/c1ccccc1</smiles>

$2 \mathbf{a}$

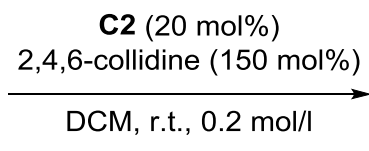

ill<smiles>O=C1Nc2ccccc2[C@]12CC[C@H](C=Cc1ccccc1)C2c1ccccc1</smiles>

3a<smiles>O=C[C@H]1CC[C@]2(C(=O)Nc3ccccc32)[C@H]1c1ccccc1</smiles>

4a

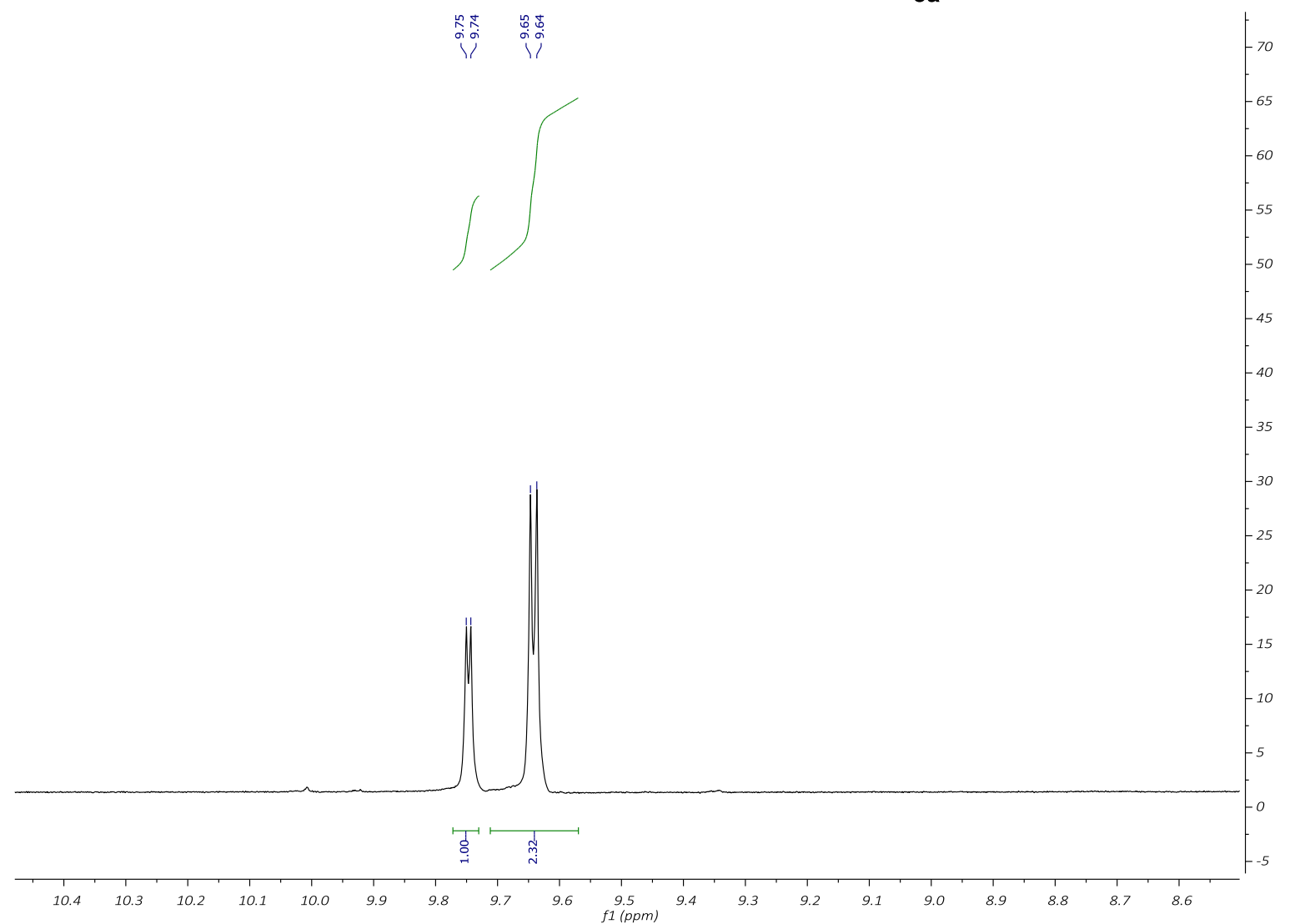

Figure S3. Aldehydic part of ${ }^{1} \mathrm{H}$ NMR of the crude reaction mixture.

The diastereomeric ratio between spirocycles $\mathbf{3}$ and $\mathbf{4}$ was determined from the crude reaction mixture (after full composition of enal, the solvent was evaporated, and the crude product was directly analyzed by ${ }^{1} \mathrm{H}$ NMR).

Aldehydic doublet of diastereomers 3 was located close to $9.8 \mathrm{ppm}$ with a coupling constant near to $2 \mathrm{~Hz}$ (based on substitution).

Aldehydic doublet of diastereomers 4 was located close to $9.65 \mathrm{ppm}$ with a coupling constant near to $3 \mathrm{~Hz}$ (based on substitution).

The absolute configuration of all prepared spirocompounds $3, \mathbf{4}$ was determined by chemical correlation with spirocycles with defined stereochemistry from X-ray $(\mathbf{3 v}, \mathbf{4 b}, \mathbf{4 p})$. Both diastereomers can be identified by ${ }^{1} \mathrm{H}-\mathrm{NMR}$ spectra - based on chemical shifts on typical values of $J$ constant for aldehyde and cyclopentane hydrogens. Additionally, specific rotation showed a positive value for spirocycles $\mathbf{4}$ and a negative value for spirocycles $\mathbf{3}$. 


\section{Crystallographic data}

The diffraction experiments for 3v, $\mathbf{4 b}, \mathbf{4 p}$ were performed on Bruker D8 VENTURE Kappa Duo PHOTONIII with $\mathrm{I} \mu \mathrm{S}$ micro-focus sealed tube $\mathrm{CuK} \alpha(\lambda=1.54178 \AA)$ radiation at a temperature $120(2) \mathrm{K}$. The structures were solved by direct methods (XT)1 and refined by full matrix least squares based on $F^{2}$ (SHELXL2018) ${ }^{2}$. The hydrogen atoms on carbon were fixed into idealized positions (riding model) and assigned temperature factors either $\mathrm{H}_{\text {iso }}(\mathrm{H})=1.2$ $\mathrm{U}_{\mathrm{eq}}\left(\right.$ pivot atom) or $\mathrm{H}_{\mathrm{iso}}(\mathrm{H})=1.5 \mathrm{U}_{\mathrm{eq}}$ (pivot atom) for methyl moiety. The hydrogen atoms in $\mathrm{O}-\mathrm{H}$ moieties were found on difference Fourier maps and refined under assumption of rigid body movement with temperature factors $\mathrm{H}_{\text {iso }}(\mathrm{H})=1.2 \mathrm{U}_{\text {eq }}$ (pivot $\mathrm{O}$ ), The determination of absolute structures were based on anomalous scattering of the heaviest atoms in the crystals (either $\mathrm{Br}$, or $\mathrm{O}, \mathrm{N}$ ). The isopropyl moiety in $\mathbf{4 p}$ crystal is disorder over two positions, however the precision of the results including absolute structure determination were not diminished. 
Table S8. Summary of crystallographic data for $3 \mathbf{v}, \mathbf{4 b}, \mathbf{4 p}$ and structure refinement parameters.

\begin{tabular}{|c|c|c|c|}
\hline Compound & $3 \mathbf{v}$ & $4 b$ & $4 p$ \\
\hline Formula & $\mathrm{C}_{26} \mathrm{H}_{22} \mathrm{BrNO}_{2}$ & $\mathrm{C}_{22} \mathrm{H}_{21} \mathrm{NO}_{2}$ & $\mathrm{C}_{29} \mathrm{H}_{30} \mathrm{~N}_{2} \mathrm{O}_{5}$ \\
\hline$M$ & 460.35 & 331.40 & 486.55 \\
\hline Crystal system & orthorhombic & orthorhombic & monoclinic \\
\hline Space group & $P 2{ }_{1}{ }_{1} 2_{1}$ & $P 2{ }_{1}{ }_{1} 2_{1}$ & $P 2_{1}$ \\
\hline$T / \mathrm{K}$ & $120(2)$ & $120(2)$ & $120(2)$ \\
\hline$a / \AA$ & $8.4943(5)$ & $8.3891(8)$ & $8.3133(6)$ \\
\hline$b / \AA$ & $13.3083(7)$ & $13.3775(13)$ & $17.1241(12)$ \\
\hline$c / \AA$ & $19.1273(11)$ & $15.4488(15)$ & $9.6093(7)$ \\
\hline$\alpha /^{\circ}$ & 90 & 90 & 90 \\
\hline$\beta /{ }^{\circ}$ & 90 & 90 & $112.331(2)$ \\
\hline$\gamma /{ }^{\circ}$ & 90 & 90 & 90 \\
\hline$V / \AA^{3}$ & $2162.2(2)$ & $1733.7(3)$ & $1265.37(16)$ \\
\hline$Z$ & 4 & 4 & 2 \\
\hline$\mu(\mathrm{Mo} \mathrm{K} \alpha) / \mathrm{mm}^{-1}$ & 2.762 & 0.640 & 0.710 \\
\hline Diffrns collected & 49082 & 28831 & 49490 \\
\hline Independent diffrns & 4277 & 3607 & 4461 \\
\hline Observed $^{b}$ diffrns & 4233 & 3598 & 4457 \\
\hline$R_{\text {int }}^{c} / \%$ & 0.0281 & 0.0233 & 0.0216 \\
\hline No. of parameters & 271 & 226 & 336 \\
\hline$R^{c}$ obsd diffrns $/ \%$ & 0.0174 & 0.0265 & 0.0238 \\
\hline$R, w R^{c}$ all data $/ \%$ & $0.0177,0.0465$ & $0.0266,0.0687$ & $0.0238,0.0650$ \\
\hline$\Delta \rho / \mathrm{e} \AA^{-3}$ & $0.467,-0.289$ & $0.191,-0.150$ & $0.208,-0.114$ \\
\hline CCDC entry & 2053815 & 2053813 & 2053814 \\
\hline
\end{tabular}

${ }^{a}$ The range of transmission factors. ${ }^{b}$ Diffractions with $I>2 \sigma(I) .{ }^{c}$ Definitions: $R_{\text {int }}=\Sigma \mid F_{\mathrm{o}}{ }^{2}-F_{\mathrm{o}}{ }^{2}($ mean $) \mid / \Sigma F_{\mathrm{o}}{ }^{2}$, where $F_{\mathrm{o}}^{2}\left(\right.$ mean) is the average intensity of symmetry-equivalent diffractions. $R=\Sigma|| F_{\mathrm{o}}|-| F_{\mathrm{c}}|| / \Sigma\left|F_{\mathrm{o}}\right|$, $\mathrm{w} R=\left[\Sigma\left\{\mathrm{w}\left(F_{\mathrm{o}}^{2}-F_{\mathrm{c}}^{2}\right)^{2}\right\} / \Sigma \mathrm{w}\left(F_{\mathrm{o}}^{2}\right)^{2}\right]^{1 / 2}$. 


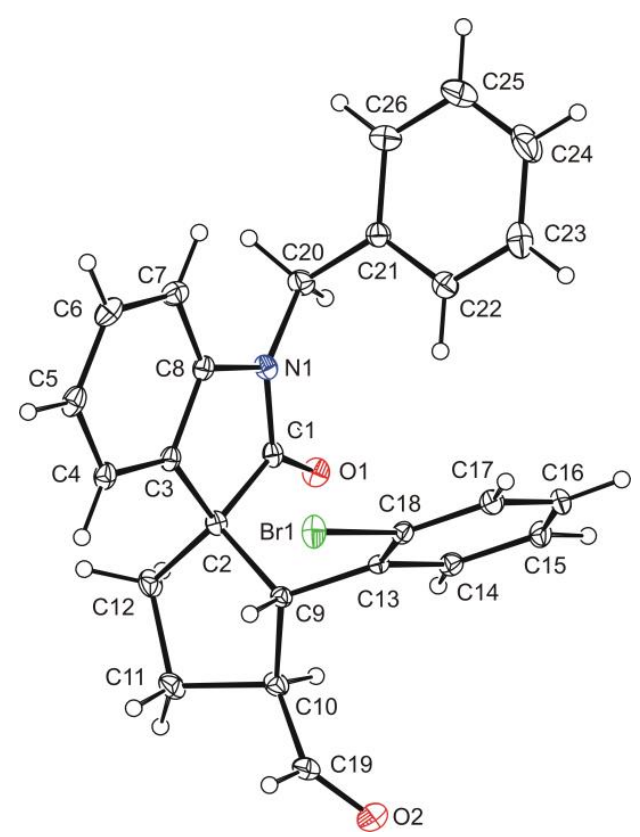

Figure S4. View on molecule of $\mathbf{3 v}$, the displacement ellipsoids at $30 \%$ probability level.

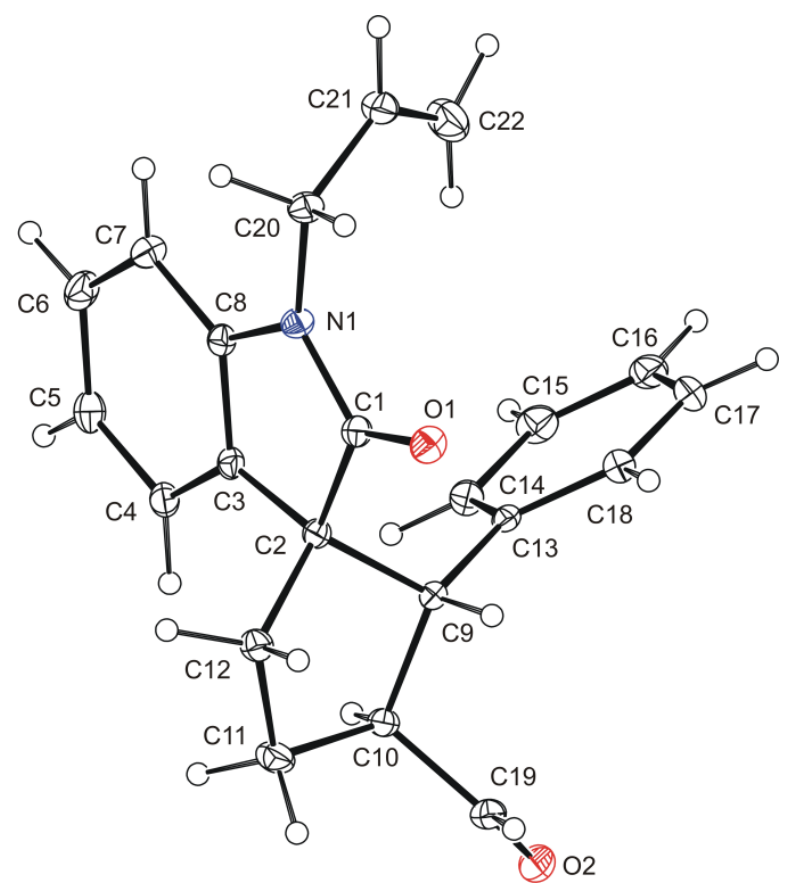

Figure S5. View on molecule of $\mathbf{4 b}$, the displacement ellipsoids at $30 \%$ probability level. 


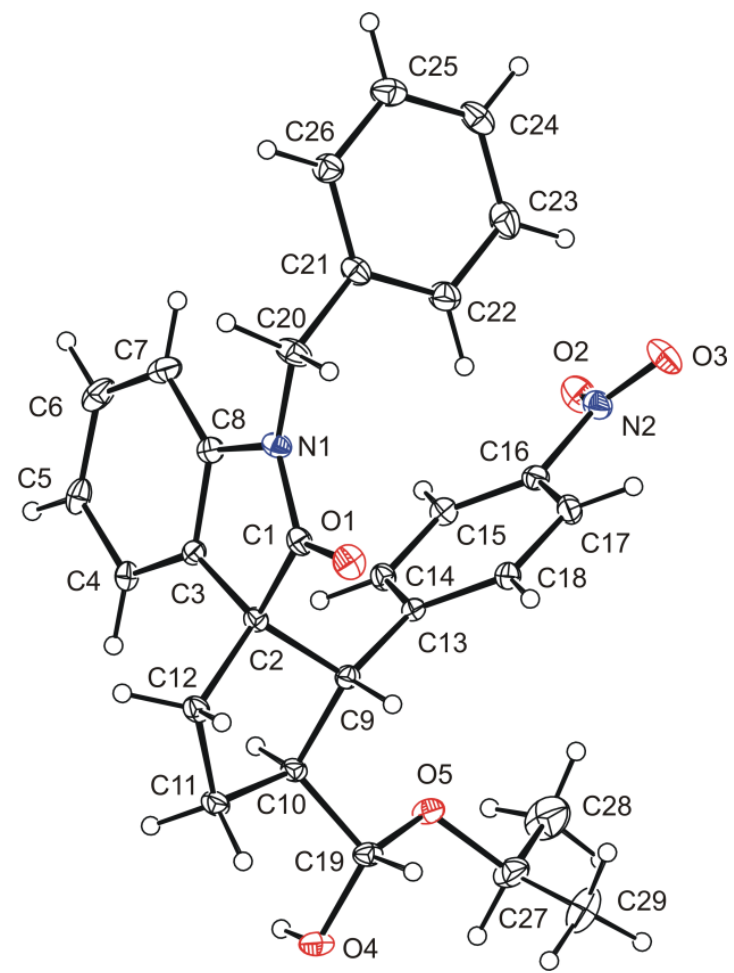

Figure S6. View on molecule of $\mathbf{4 p}$, the displacement ellipsoids at $30 \%$ probability level.

CCDC 2053813 (4b), 2053814 (4p), 2053815 (3v) contain the supplementary crystallographic data of this paper. These data can be obtained free of charge via www.ccdc.cam.ac.uk/data_request/cif, or by emailing data_request@ccdc.cam.ac.uk, or by contacting The Cambridge Crystallographic Data Centre, 12 Union Road, Cambridge CB2 1EZ, UK; fax: +44 1223336033. 


\section{Computational methods}

The calculations of activation energy of the ring-closing step were performed using Gaussian $16 .^{3}$ Density functional theory (DFT) was applied using the M06-2X functional. ${ }^{4}$ Doble zeta basis set def $2 \mathrm{SV}$ was used for the geometry optimization, polarized triple zeta basis set def2TZVP was used for the single point calculations. ${ }^{5}$ The solvent effect (dichloromethane) was included by the polarizable continuum model (PCM) for all calculations. ${ }^{6}$ The stationary points were characterized as local minima or transition states by the vibrational frequency calculations. The Gibbs free energies were computed as a sum of the electronic energy (def2TZVP) and thermal corrections to Gibbs free energy at $298 \mathrm{~K}$ (def2SV). All calculations were performed for a singlet state of the molecules. 
Table S9. The summary of electronic, thermal and activation energies for 5 and 6-exo-tet ring closing reactions. Thermal correction to Gibbs free energy was calculated only with the smaller basis set (def2SV) and used for calculation of sum of electronic and thermal free energies in both cases (def2TZVP, def2SV). Activation energy was calculated as the difference between sum of electronic and thermal free energies for the local minima and transition state.

\section{5-exo-tet}

The local minima

\begin{tabular}{lccc}
\hline Basis set & $\begin{array}{c}\text { Electronic energy } \\
(\mathrm{kJ} / \mathrm{mol})\end{array}$ & $\begin{array}{c}\text { Thermal correction to Gibbs } \\
\text { Free Energy }(\mathrm{kJ} / \mathrm{mol})\end{array}$ & $\begin{array}{c}\text { Sum of electronic and thermal } \\
\text { Free Energies }(\mathrm{kJ} / \mathrm{mol})\end{array}$ \\
\hline def2TZVP & $-12755808,2$ & - & $-12753833,4$ \\
def2SV & $-12748651,8$ & 1974,8 & $-12746677,0$ \\
\hline
\end{tabular}

Transition state

\begin{tabular}{|c|c|c|c|}
\hline Basis set & $\begin{array}{l}\text { Electronic energy } \\
(\mathrm{kJ} / \mathrm{mol})\end{array}$ & $\begin{array}{c}\text { Thermal correction to Gibbs } \\
\text { Free Energy }(\mathrm{kJ} / \mathrm{mol})\end{array}$ & $\begin{array}{c}\text { Sum of electronic and thermal } \\
\text { Free Energies }(\mathrm{kJ} / \mathrm{mol})\end{array}$ \\
\hline def2TZVP & $-12755728,9$ & - & $-12753754,3$ \\
\hline def2SV & $-12748563,8$ & 1974,5 & $-12746589,3$ \\
\hline
\end{tabular}

\section{Activation energy}

\begin{tabular}{cc}
\hline Basis set & ${ } G^{298}(\mathrm{~kJ} / \mathrm{mol})$ \\
\hline def2TZVP & 79,0 \\
def2SV & 87,7 \\
\hline
\end{tabular}

\section{6-exo-tet}

The local minima

\begin{tabular}{lccc}
\hline Basis set & $\begin{array}{c}\text { Electronic energy } \\
(\mathrm{kJ} / \mathrm{mol})\end{array}$ & $\begin{array}{c}\text { Thermal correction to Gibbs } \\
\text { Free Energy }(\mathrm{kJ} / \mathrm{mol})\end{array}$ & $\begin{array}{c}\text { Sum of electronic and thermal } \\
\text { Free Energies }(\mathrm{kJ} / \mathrm{mol})\end{array}$ \\
\hline def2TZVP & $-12858008,5$ & - & $-12855965,0$ \\
def2SV & $-12850729,2$ & 2043,4 & $-12848685,7$ \\
\hline
\end{tabular}

Transition state

\begin{tabular}{lccc}
\hline Basis set & $\begin{array}{c}\text { Electronic energy } \\
(\mathrm{kJ} / \mathrm{mol})\end{array}$ & $\begin{array}{c}\text { Thermal correction to Gibbs } \\
\text { Free Energy }(\mathrm{kJ} / \mathrm{mol})\end{array}$ & $\begin{array}{c}\text { Sum of electronic and thermal } \\
\text { Free Energies }(\mathrm{kJ} / \mathrm{mol})\end{array}$ \\
\hline def2TZVP & $-12857918,2$ & - & $-12855865,2$ \\
def2SV & $-12850632,3$ & 2053,0 & $-12848579,3$ \\
\hline
\end{tabular}

\section{Activation energy}

\begin{tabular}{cc}
\hline Basis set & ${ } G^{298}(\mathrm{~kJ} / \mathrm{mol})$ \\
\hline def2TZVP & 99,8 \\
def2SV & 106,5 \\
\hline
\end{tabular}


Table S10. Geometry of stationary points for local minima of 5-exo-tet ring closing reaction using M062x/def2SV basis set including PMC model for the solvent (DCM).

\begin{tabular}{|c|c|c|c|}
\hline \multicolumn{4}{|c|}{0 imaginary frequency } \\
\hline \multicolumn{4}{|c|}{ Charge $=0$, Multiplicity $=1$} \\
\hline $\mathrm{H}$ & -4.61386 & -2.78877 & -4.76800 \\
\hline $\mathrm{H}$ & -6.58254 & -1.57001 & -3.83896 \\
\hline $\mathrm{C}$ & -4.54283 & -2.28496 & -3.80058 \\
\hline $\mathrm{H}$ & -2.48365 & -2.85734 & -3.46948 \\
\hline $\mathrm{H}$ & 2.91156 & -3.39039 & -2.35067 \\
\hline $\mathrm{C}$ & -5.64336 & -1.60344 & -3.28070 \\
\hline $\mathrm{C}$ & -3.35145 & -2.32365 & -3.07294 \\
\hline $\mathrm{H}$ & -3.85345 & 0.80527 & -3.25582 \\
\hline $\mathrm{H}$ & -3.38112 & 2.50634 & -3.47405 \\
\hline $\mathrm{H}$ & -1.54221 & 0.46765 & -2.89447 \\
\hline $\mathrm{H}$ & 3.55261 & -4.80942 & -0.40105 \\
\hline $\mathrm{C}$ & 2.75921 & -3.02459 & -1.33178 \\
\hline $\mathrm{H}$ & 8.39046 & -3.83810 & -1.12948 \\
\hline $\mathrm{H}$ & 6.06036 & -3.71383 & -0.24585 \\
\hline $\mathrm{H}$ & 1.91721 & -1.15807 & -1.99408 \\
\hline $\mathrm{C}$ & -3.38165 & 1.66828 & -2.76130 \\
\hline $\mathrm{C}$ & -5.55108 & -0.95901 & -2.04523 \\
\hline $\mathrm{C}$ & 7.86017 & -2.88263 & -1.10845 \\
\hline $\mathrm{C}$ & 6.55749 & -2.81283 & -0.61518 \\
\hline $\mathrm{C}$ & -3.26188 & -1.68047 & -1.83859 \\
\hline $\mathrm{C}$ & 3.12216 & -3.81719 & -0.24185 \\
\hline $\mathrm{H}$ & 5.43800 & 1.15259 & -2.06606 \\
\hline $\mathrm{C}$ & -1.96497 & 1.30333 & -2.30659 \\
\hline $\mathrm{H}$ & -6.42022 & -0.42188 & -1.65745 \\
\hline $\mathrm{C}$ & 2.19770 & -1.76286 & -1.12900 \\
\hline $\mathrm{H}$ & 9.50781 & -1.76503 & -1.95499 \\
\hline $\mathrm{C}$ & 8.48553 & -1.72195 & -1.57021 \\
\hline $\mathrm{H}$ & -1.26222 & 2.15678 & -2.39550 \\
\hline $\mathrm{C}$ & 5.87850 & -1.59223 & -0.58506 \\
\hline $\mathrm{H}$ & 4.85776 & -1.54699 & -0.19122 \\
\hline $\mathrm{O}$ & 3.05130 & 1.32471 & -1.83643 \\
\hline $\mathrm{C}$ & -4.35940 & -0.98663 & -1.31085 \\
\hline $\mathrm{H}$ & -2.32255 & -1.70424 & -1.27911 \\
\hline $\mathrm{C}$ & 7.80937 & -0.50344 & -1.53453 \\
\hline $\mathrm{C}$ & 6.49869 & -0.42860 & -1.04718 \\
\hline $\mathrm{H}$ & -5.20506 & 1.83192 & -1.53030 \\
\hline $\mathrm{H}$ & 8.30466 & 0.40584 & -1.88933 \\
\hline $\mathrm{C}$ & -4.12176 & 2.01697 & -1.45954 \\
\hline $\mathrm{C}$ & 5.79143 & 0.91320 & -1.04989 \\
\hline $\mathrm{N}$ & -2.11645 & 0.94068 & -0.91107 \\
\hline $\mathrm{C}$ & 2.93050 & -3.33247 & 1.05289 \\
\hline $\mathrm{H}$ & 6.50118 & 1.70443 & -0.75019 \\
\hline $\mathrm{H}$ & -3.97594 & 3.08179 & -1.21394 \\
\hline $\mathrm{C}$ & 3.35009 & 1.19613 & -0.66843 \\
\hline $\mathrm{C}$ & 1.98361 & -1.26950 & 0.16645 \\
\hline $\mathrm{C}$ & 0.21832 & 0.38468 & -0.56396 \\
\hline $\mathrm{H}$ & 3.21007 & -3.94283 & 1.91571 \\
\hline $\mathrm{C}$ & -3.44568 & 1.16317 & -0.37418 \\
\hline $\mathrm{N}$ & 4.62658 & 0.97191 & -0.20053 \\
\hline $\mathrm{C}$ & -4.17386 & -0.17978 & -0.02511 \\
\hline
\end{tabular}

\begin{tabular}{|c|c|c|c|}
\hline $\mathrm{C}$ & -1.04269 & 0.61821 & -0.13422 \\
\hline $\mathrm{H}$ & -6.01847 & -2.03501 & 0.64819 \\
\hline $\mathrm{C}$ & 2.36586 & -2.07105 & 1.25160 \\
\hline $\mathrm{C}$ & 1.32503 & 0.08447 & 0.41082 \\
\hline $\mathrm{C}$ & -6.31357 & -1.04247 & 1.00129 \\
\hline $\mathrm{O}$ & -3.27933 & -0.82932 & 0.84567 \\
\hline $\mathrm{C}$ & -5.50144 & 0.06337 & 0.70859 \\
\hline $\mathrm{C}$ & 2.39291 & 1.23775 & 0.53311 \\
\hline $\mathrm{H}$ & -3.59852 & -4.67549 & 1.35640 \\
\hline $\mathrm{H}$ & -3.35469 & 1.70666 & 0.58064 \\
\hline $\mathrm{C}$ & 1.69662 & 2.61739 & 0.68896 \\
\hline $\mathrm{H}$ & 2.19837 & -1.70748 & 2.27007 \\
\hline $\mathrm{C}$ & 4.64154 & 0.86663 & 1.19552 \\
\hline $\mathrm{H}$ & -8.10970 & -1.78383 & 1.93407 \\
\hline $\mathrm{C}$ & -7.49823 & -0.90336 & 1.72020 \\
\hline $\mathrm{C}$ & -5.92047 & 1.32155 & 1.15563 \\
\hline $\mathrm{H}$ & -5.33169 & 2.21646 & 0.94789 \\
\hline $\mathrm{H}$ & 0.86963 & 0.04294 & 1.41627 \\
\hline $\mathrm{H}$ & -0.71261 & -2.30966 & 1.05914 \\
\hline $\mathrm{H}$ & 6.74278 & 0.55555 & 1.63994 \\
\hline $\mathrm{H}$ & -4.66377 & -3.73085 & 0.28318 \\
\hline $\mathrm{C}$ & -3.63877 & -3.79528 & 0.68743 \\
\hline $\mathrm{C}$ & 3.33724 & 1.00051 & 1.68899 \\
\hline $\mathrm{Si}$ & -3.11906 & -2.28279 & 1.67601 \\
\hline $\mathrm{C}$ & 5.73063 & 0.66249 & 2.03556 \\
\hline $\mathrm{C}$ & 1.35759 & 3.32752 & -0.61270 \\
\hline $\mathrm{C}$ & -7.90246 & 0.35845 & 2.16259 \\
\hline $\mathrm{C}$ & -7.11102 & 1.46769 & 1.87468 \\
\hline $\mathrm{C}$ & -1.27483 & -2.35874 & 2.00967 \\
\hline $\mathrm{H}$ & -2.95206 & -3.98180 & -0.15571 \\
\hline $\mathrm{H}$ & -8.83238 & 0.47447 & 2.72498 \\
\hline $\mathrm{H}$ & -0.95186 & -1.51510 & 2.64626 \\
\hline $\mathrm{H}$ & -7.41650 & 2.46211 & 2.21010 \\
\hline $\mathrm{C}$ & 3.10274 & 0.93715 & 3.05360 \\
\hline $\mathrm{Br}$ & 0.14330 & 4.82960 & -0.26589 \\
\hline $\mathrm{H}$ & -0.99808 & -3.29729 & 2.52327 \\
\hline $\mathrm{H}$ & -5.14521 & -2.38070 & 3.17172 \\
\hline $\mathrm{C}$ & 5.47949 & 0.59324 & 3.41222 \\
\hline $\mathrm{C}$ & -4.06183 & -2.21743 & 3.29970 \\
\hline $\mathrm{H}$ & 2.08894 & 1.04659 & 3.45053 \\
\hline $\mathrm{H}$ & -3.91970 & -1.23932 & 3.79324 \\
\hline $\mathrm{C}$ & 4.18632 & 0.72776 & 3.91982 \\
\hline $\mathrm{H}$ & 6.31539 & 0.43087 & 4.09741 \\
\hline $\mathrm{H}$ & -3.67987 & -2.99995 & 3.98101 \\
\hline $\mathrm{H}$ & 4.01693 & 0.67118 & 4.99757 \\
\hline $\mathrm{H}$ & 2.24136 & 3.76578 & -1.09210 \\
\hline $\mathrm{H}$ & 0.83563 & 2.68565 & -1.32774 \\
\hline $\mathrm{H}$ & 2.33783 & 3.28213 & 1.28983 \\
\hline $\mathrm{H}$ & 0.77080 & 2.44673 & 1.26439 \\
\hline $\mathrm{H}$ & -1.27278 & 0.54682 & 0.93436 \\
\hline $\mathrm{H}$ & 0.46265 & 0.43667 & -1.62913 \\
\hline
\end{tabular}


Table S11. Geometry of stationary points for transtiton state of 5-exo-tet ring closing reaction using M06-2x/def2SV basis set including PMC model for the solvent (DCM).

\begin{tabular}{|lrrl|}
\hline 1 imaginary frequency & $(-538.39$ & $\mathrm{cm}$ \\
\hline
\end{tabular}

\begin{tabular}{|c|c|c|c|}
\hline $\mathrm{C}$ & -1.10936 & 0.92516 & 0.03570 \\
\hline $\mathrm{H}$ & -6.05645 & -1.98277 & -0.57068 \\
\hline $\mathrm{C}$ & 1.97465 & -2.08056 & 1.36084 \\
\hline $\mathrm{C}$ & 1.21046 & 0.21348 & 0.62276 \\
\hline $\mathrm{C}$ & -6.41185 & -1.14341 & 0.03066 \\
\hline $\mathrm{O}$ & -3.32115 & -0.61812 & 0.91404 \\
\hline $\mathrm{C}$ & -5.56663 & -0.05326 & 0.27959 \\
\hline $\mathrm{C}$ & 2.42952 & 1.17389 & 0.73228 \\
\hline $\mathrm{H}$ & -3.97743 & -4.31158 & 1.92992 \\
\hline $\mathrm{H}$ & -3.54271 & 1.82100 & 0.65215 \\
\hline $\mathrm{C}$ & 1.85944 & 2.59741 & 0.88521 \\
\hline $\mathrm{H}$ & 1.94877 & -1.70893 & 2.38963 \\
\hline $\mathrm{C}$ & 4.65322 & 0.54875 & 1.25254 \\
\hline $\mathrm{H}$ & -8.34545 & -2.04682 & 0.33408 \\
\hline $\mathrm{C}$ & -7.70944 & -1.18278 & 0.54277 \\
\hline $\mathrm{C}$ & -6.06453 & 1.00103 & 1.05827 \\
\hline $\mathrm{H}$ & -5.44397 & 1.86942 & 1.28790 \\
\hline $\mathrm{H}$ & 0.75274 & 0.21737 & 1.62808 \\
\hline $\mathrm{H}$ & -1.05560 & -2.15304 & 2.10957 \\
\hline $\mathrm{H}$ & 6.72328 & -0.02371 & 1.56495 \\
\hline $\mathrm{H}$ & -4.80875 & -3.51433 & 0.57045 \\
\hline $\mathrm{C}$ & -3.87584 & -3.52548 & 1.15858 \\
\hline $\mathrm{C}$ & 3.40587 & 0.82903 & 1.82688 \\
\hline $\mathrm{Si}$ & -3.52761 & -1.88964 & 2.00944 \\
\hline $\mathrm{C}$ & 5.75681 & 0.19840 & 2.02200 \\
\hline $\mathrm{C}$ & 0.82940 & 2.93047 & -0.16873 \\
\hline $\mathrm{C}$ & -8.19082 & -0.12660 & 1.31591 \\
\hline $\mathrm{C}$ & -7.36266 & 0.96700 & 1.56874 \\
\hline $\mathrm{C}$ & -1.85328 & -1.95334 & 2.84831 \\
\hline $\mathrm{H}$ & -3.04753 & -3.81512 & 0.48899 \\
\hline $\mathrm{H}$ & -9.20676 & -0.15401 & 1.71784 \\
\hline $\mathrm{H}$ & -1.62564 & -0.99920 & 3.35649 \\
\hline $\mathrm{H}$ & -7.72584 & 1.80309 & 2.17176 \\
\hline $\mathrm{C}$ & 3.24711 & 0.77100 & 3.20259 \\
\hline $\mathrm{Br}$ & 1.25606 & 5.41491 & -0.32516 \\
\hline $\mathrm{H}$ & -1.82062 & -2.75652 & 3.60622 \\
\hline $\mathrm{H}$ & -5.87939 & -1.50210 & 2.81585 \\
\hline $\mathrm{C}$ & 5.58134 & 0.13757 & 3.41073 \\
\hline $\mathrm{C}$ & -4.86821 & -1.48821 & 3.25928 \\
\hline $\mathrm{H}$ & 2.27937 & 0.99182 & 3.66298 \\
\hline $\mathrm{H}$ & -4.70015 & -0.49256 & 3.70721 \\
\hline $\mathrm{C}$ & 4.34723 & 0.41904 & 3.99864 \\
\hline $\mathrm{H}$ & 6.43102 & -0.13566 & 4.04161 \\
\hline $\mathrm{H}$ & -4.84452 & -2.23367 & 4.07563 \\
\hline $\mathrm{H}$ & 4.23761 & 0.36626 & 5.08419 \\
\hline $\mathrm{H}$ & 1.10652 & 2.89563 & -1.21903 \\
\hline $\mathrm{H}$ & -0.16964 & 3.26356 & 0.09147 \\
\hline $\mathrm{H}$ & 2.68388 & 3.32297 & 0.83241 \\
\hline $\mathrm{H}$ & 1.40574 & 2.68205 & 1.88671 \\
\hline $\mathrm{H}$ & -1.39256 & 0.75657 & 1.08014 \\
\hline $\mathrm{H}$ & 0.46095 & 0.78191 & -1.40486 \\
\hline
\end{tabular}


Table S12. Geometry of stationary points for local minima of 6-exo-tet ring closing reaction using M062x/def2SV basis set including PMC model for the solvent (DCM).

\begin{tabular}{|c|c|c|c|}
\hline \multicolumn{4}{|c|}{0 imaginary frequency } \\
\hline \multicolumn{4}{|c|}{ Charge $=0$, Multiplicity $=1$} \\
\hline $\mathrm{H}$ & -4.53536 & -3.71566 & -4.29542 \\
\hline $\mathrm{H}$ & -6.52175 & -2.36968 & -3.61108 \\
\hline $\mathrm{C}$ & -4.47840 & -3.05179 & -3.42890 \\
\hline $\mathrm{H}$ & -2.41918 & -3.54133 & -2.98362 \\
\hline $\mathrm{H}$ & 3.02460 & -3.82004 & -1.99535 \\
\hline $\mathrm{C}$ & -5.58881 & -2.29906 & -3.04585 \\
\hline $\mathrm{C}$ & -3.29498 & -2.95467 & -2.69387 \\
\hline $\mathrm{H}$ & -3.86306 & 0.12520 & -3.42143 \\
\hline $\mathrm{H}$ & -3.38463 & 1.76789 & -3.90514 \\
\hline $\mathrm{H}$ & -1.56723 & -0.17956 & -2.99383 \\
\hline $\mathrm{H}$ & 3.65207 & -4.97439 & 0.12585 \\
\hline $\mathrm{C}$ & 2.84282 & -3.33550 & -1.03234 \\
\hline $\mathrm{H}$ & 8.48114 & -4.00531 & -0.59224 \\
\hline $\mathrm{H}$ & 6.12709 & -3.80944 & 0.20996 \\
\hline $\mathrm{H}$ & 1.98206 & -1.58132 & -1.93484 \\
\hline $\mathrm{C}$ & -3.38742 & 1.05312 & -3.06880 \\
\hline $\mathrm{C}$ & -5.51456 & -1.45051 & -1.93934 \\
\hline $\mathrm{C}$ & 7.93904 & -3.06268 & -0.70270 \\
\hline $\mathrm{C}$ & 6.62276 & -2.95295 & -0.25492 \\
\hline $\mathrm{C}$ & -3.22357 & -2.10781 & -1.58794 \\
\hline $\mathrm{C}$ & 3.19821 & -3.98020 & 0.15361 \\
\hline $\mathrm{H}$ & 5.49816 & 0.78276 & -2.21790 \\
\hline $\mathrm{C}$ & -1.97113 & 0.75584 & -2.56384 \\
\hline $\mathrm{H}$ & -6.39108 & -0.86111 & -1.65884 \\
\hline $\mathrm{C}$ & 2.25248 & -2.07128 & -0.99690 \\
\hline $\mathrm{H}$ & 9.59506 & -2.03222 & -1.63905 \\
\hline $\mathrm{C}$ & 8.56238 & -1.95803 & -1.28824 \\
\hline $\mathrm{H}$ & -1.25282 & 1.56497 & -2.80956 \\
\hline $\mathrm{C}$ & 5.92773 & -1.74873 & -0.39300 \\
\hline $\mathrm{H}$ & 4.89537 & -1.67239 & -0.03538 \\
\hline $\mathrm{O}$ & 3.10115 & 0.85267 & -2.08048 \\
\hline $\mathrm{C}$ & -4.33126 & -1.34294 & -1.19844 \\
\hline $\mathrm{H}$ & -2.29024 & -2.02692 & -1.02351 \\
\hline $\mathrm{C}$ & 7.87019 & -0.75526 & -1.42023 \\
\hline $\mathrm{C}$ & 6.54587 & -0.64132 & -0.97961 \\
\hline $\mathrm{H}$ & -5.21005 & 1.40558 & -1.87725 \\
\hline $\mathrm{H}$ & 8.36285 & 0.11123 & -1.87252 \\
\hline $\mathrm{C}$ & -4.12779 & 1.60574 & -1.83927 \\
\hline $\mathrm{C}$ & 5.82224 & 0.67834 & -1.16969 \\
\hline $\mathrm{N}$ & -2.11464 & 0.65387 & -1.12480 \\
\hline $\mathrm{C}$ & 2.96900 & -3.34514 & 1.37502 \\
\hline $\mathrm{H}$ & 6.51287 & 1.51179 & -0.95081 \\
\hline $\mathrm{H}$ & -3.99025 & 2.69760 & -1.77025 \\
\hline $\mathrm{C}$ & 3.36101 & 0.92272 & -0.89839 \\
\hline $\mathrm{C}$ & 2.00151 & -1.42742 & 0.22391 \\
\hline $\mathrm{C}$ & 0.20550 & 0.08296 & -0.71102 \\
\hline $\mathrm{H}$ & 3.24232 & -3.83897 & 2.31132 \\
\hline $\mathrm{C}$ & -3.44710 & 0.94463 & -0.62940 \\
\hline $\mathrm{N}$ & 4.63148 & 0.82431 & -0.36936 \\
\hline $\mathrm{C}$ & -4.16507 & -0.32924 & -0.06552 \\
\hline $\mathrm{C}$ & -1.04154 & 0.42082 & -0.31282 \\
\hline
\end{tabular}

\begin{tabular}{|c|c|c|c|}
\hline $\mathrm{H}$ & -6.00697 & -2.06883 & 0.86735 \\
\hline $\mathrm{C}$ & 2.37546 & -2.08155 & 1.40641 \\
\hline $\mathrm{C}$ & 1.32058 & -0.06523 & 0.28807 \\
\hline $\mathrm{C}$ & -6.31108 & -1.03596 & 1.06113 \\
\hline $\mathrm{O}$ & -3.26931 & -0.81296 & 0.90612 \\
\hline $\mathrm{C}$ & -5.50063 & 0.01649 & 0.61006 \\
\hline $\mathrm{C}$ & 2.36802 & 1.11316 & 0.25936 \\
\hline $\mathrm{H}$ & -3.54798 & -4.50799 & 2.09665 \\
\hline $\mathrm{H}$ & -3.36292 & 1.63596 & 0.22548 \\
\hline $\mathrm{C}$ & 1.65091 & 2.48675 & 0.19888 \\
\hline $\mathrm{H}$ & 2.17920 & -1.59914 & 2.36896 \\
\hline $\mathrm{C}$ & 4.60588 & 0.90673 & 1.02713 \\
\hline $\mathrm{H}$ & -8.11388 & -1.63573 & 2.07909 \\
\hline $\mathrm{C}$ & -7.50339 & -0.79505 & 1.73936 \\
\hline $\mathrm{C}$ & -5.92741 & 1.32599 & 0.85816 \\
\hline $\mathrm{H}$ & -5.33945 & 2.18235 & 0.52389 \\
\hline $\mathrm{H}$ & 0.86608 & 0.01857 & 1.29129 \\
\hline $\mathrm{H}$ & -0.70020 & -2.19095 & 1.42676 \\
\hline $\mathrm{H}$ & 6.69987 & 0.72528 & 1.56758 \\
\hline $\mathrm{H}$ & -4.63645 & -3.78670 & 0.88349 \\
\hline $\mathrm{C}$ & -3.60868 & -3.75880 & 1.28492 \\
\hline $\mathrm{C}$ & 3.28274 & 1.05971 & 1.46143 \\
\hline $\mathrm{Si}$ & -3.11701 & -2.08806 & 1.99205 \\
\hline $\mathrm{C}$ & 5.67289 & 0.85045 & 1.91707 \\
\hline $\mathrm{C}$ & -0.02453 & 3.81156 & -1.18919 \\
\hline $\mathrm{C}$ & -7.91631 & 0.51730 & 1.98076 \\
\hline $\mathrm{C}$ & -7.12498 & 1.57382 & 1.53640 \\
\hline $\mathrm{C}$ & -1.27808 & -2.08451 & 2.36311 \\
\hline $\mathrm{H}$ & -2.92055 & -4.07575 & 0.48279 \\
\hline $\mathrm{H}$ & -8.85218 & 0.71309 & 2.51023 \\
\hline $\mathrm{H}$ & -0.97364 & -1.14167 & 2.85266 \\
\hline $\mathrm{H}$ & -7.43640 & 2.60614 & 1.71518 \\
\hline $\mathrm{C}$ & 3.00716 & 1.16856 & 2.81546 \\
\hline $\mathrm{Br}$ & 0.10461 & 5.41365 & -0.05458 \\
\hline $\mathrm{H}$ & -1.00289 & -2.91919 & 3.03300 \\
\hline $\mathrm{H}$ & -5.16793 & -1.92468 & 3.44802 \\
\hline $\mathrm{C}$ & 5.38022 & 0.95497 & 3.28337 \\
\hline $\mathrm{C}$ & -4.08714 & -1.73625 & 3.56169 \\
\hline $\mathrm{H}$ & 1.97837 & 1.29423 & 3.16665 \\
\hline $\mathrm{H}$ & -3.95450 & -0.68462 & 3.87335 \\
\hline $\mathrm{C}$ & 4.06806 & 1.11207 & 3.73212 \\
\hline $\mathrm{H}$ & 6.19831 & 0.91178 & 4.00693 \\
\hline $\mathrm{H}$ & -3.71501 & -2.38140 & 4.37886 \\
\hline $\mathrm{H}$ & 3.86580 & 1.19205 & 4.80274 \\
\hline $\mathrm{C}$ & 1.24886 & 2.99470 & -1.19256 \\
\hline $\mathrm{H}$ & -0.27569 & 4.18667 & -2.18990 \\
\hline $\mathrm{H}$ & -0.87302 & 3.23622 & -0.78857 \\
\hline $\mathrm{H}$ & 1.07033 & 2.15182 & -1.87617 \\
\hline $\mathrm{H}$ & 2.07131 & 3.58133 & -1.63571 \\
\hline $\mathrm{H}$ & 2.29318 & 3.24158 & 0.68036 \\
\hline $\mathrm{H}$ & 0.75132 & 2.39667 & 0.83567 \\
\hline $\mathrm{H}$ & -1.25810 & 0.53359 & 0.75498 \\
\hline $\mathrm{H}$ & 0.43433 & -0.04655 & -1.77286 \\
\hline
\end{tabular}


Table S13. Geometry of stationary points for transition state of 6-exo-tet ring closing reaction using M06-2x/def2SV basis set including PMC model for the solvent (DCM).

\begin{tabular}{|c|c|c|c|}
\hline \multicolumn{4}{|c|}{1 imaginary frequency $\left(-533.14 \mathrm{~cm}^{-1}\right)$} \\
\hline \multicolumn{4}{|c|}{ Charge $=0$, Multiplicity $=1$} \\
\hline $\mathrm{H}$ & -2.77401 & -3.84393 & -4.29816 \\
\hline $\mathrm{H}$ & -5.02162 & -2.77126 & -4.13215 \\
\hline $\mathrm{C}$ & -3.00616 & -3.17887 & -3.46245 \\
\hline $\mathrm{H}$ & -1.06093 & -3.38642 & -2.54284 \\
\hline $\mathrm{H}$ & 1.27634 & -3.63566 & -0.55911 \\
\hline $\mathrm{C}$ & -4.26336 & -2.57969 & -3.36863 \\
\hline $\mathrm{C}$ & -2.04962 & -2.92392 & -2.47922 \\
\hline $\mathrm{H}$ & -3.46082 & 0.21478 & -3.54469 \\
\hline $\mathrm{H}$ & -3.24673 & 1.94582 & -3.87368 \\
\hline $\mathrm{H}$ & -1.20549 & 0.23327 & -2.85912 \\
\hline $\mathrm{H}$ & 2.06380 & -4.32390 & 1.70601 \\
\hline $\mathrm{C}$ & 1.50573 & -2.87862 & 0.19583 \\
\hline $\mathrm{H}$ & 6.46506 & -5.49427 & -0.40044 \\
\hline $\mathrm{H}$ & 4.58372 & -4.28559 & 0.70590 \\
\hline $\mathrm{H}$ & 0.99972 & -1.23889 & -1.10782 \\
\hline $\mathrm{C}$ & -3.19002 & 1.17701 & -3.08951 \\
\hline $\mathrm{C}$ & -4.55837 & -1.72933 & -2.30273 \\
\hline $\mathrm{C}$ & 6.26144 & -4.44926 & -0.64762 \\
\hline $\mathrm{C}$ & 5.20951 & -3.77338 & -0.02998 \\
\hline $\mathrm{C}$ & -2.34999 & -2.07755 & -1.41036 \\
\hline $\mathrm{C}$ & 1.95253 & -3.26450 & 1.46032 \\
\hline $\mathrm{H}$ & 4.99033 & -0.27583 & -2.65107 \\
\hline $\mathrm{C}$ & -1.78217 & 1.09807 & -2.48621 \\
\hline $\mathrm{H}$ & -5.54410 & -1.25942 & -2.25423 \\
\hline $\mathrm{C}$ & 1.35412 & -1.52487 & -0.11415 \\
\hline $\mathrm{H}$ & 7.88680 & -4.29929 & -2.06777 \\
\hline $\mathrm{C}$ & 7.05729 & -3.78010 & -1.58065 \\
\hline $\mathrm{H}$ & -1.18704 & 2.00497 & -2.69449 \\
\hline $\mathrm{C}$ & 4.94783 & -2.43692 & -0.34275 \\
\hline $\mathrm{H}$ & 4.12116 & -1.91567 & 0.15156 \\
\hline $\mathrm{O}$ & 2.87113 & 0.75806 & -2.20779 \\
\hline $\mathrm{C}$ & -3.60683 & -1.46886 & -1.30730 \\
\hline $\mathrm{H}$ & -1.59572 & -1.87631 & -0.64451 \\
\hline $\mathrm{C}$ & 6.79895 & -2.44544 & -1.88834 \\
\hline $\mathrm{C}$ & 5.73912 & -1.76380 & -1.27729 \\
\hline $\mathrm{H}$ & -5.12558 & 1.08589 & -2.02452 \\
\hline $\mathrm{H}$ & 7.42802 & -1.92141 & -2.61476 \\
\hline $\mathrm{C}$ & -4.11550 & 1.50722 & -1.90430 \\
\hline $\mathrm{C}$ & 5.47933 & -0.32017 & -1.66450 \\
\hline $\mathrm{N}$ & -1.99455 & 0.98439 & -1.04579 \\
\hline $\mathrm{C}$ & 2.25338 & -2.28587 & 2.40919 \\
\hline $\mathrm{H}$ & 6.43949 & 0.21936 & -1.74519 \\
\hline $\mathrm{H}$ & -4.22029 & 2.59860 & -1.79773 \\
\hline $\mathrm{C}$ & 3.37575 & 0.88519 & -1.11174 \\
\hline $\mathrm{C}$ & 1.63380 & -0.53194 & 0.83540 \\
\hline $\mathrm{C}$ & 0.34610 & 1.18070 & -0.51192 \\
\hline $\mathrm{H}$ & 2.59863 & -2.57400 & 3.40560 \\
\hline $\mathrm{C}$ & -3.40445 & 0.96037 & -0.65980 \\
\hline $\mathrm{N}$ & 4.61040 & 0.38839 & -0.75660 \\
\hline $\mathrm{C}$ & -3.85385 & -0.46067 & -0.18109 \\
\hline $\mathrm{C}$ & -0.98232 & 1.01635 & -0.17407 \\
\hline $\mathrm{H}$ & -5.66365 & -2.54049 & -0.11175 \\
\hline $\mathrm{C}$ & 2.08925 & -0.93517 & 2.09896 \\
\hline $\mathrm{C}$ & 1.43427 & 0.95298 & 0.52025 \\
\hline
\end{tabular}

\begin{tabular}{|c|c|c|c|}
\hline $\mathrm{C}$ & -6.06421 & -1.63074 & 0.33934 \\
\hline $\mathrm{O}$ & -2.99692 & -0.73052 & 0.90548 \\
\hline $\mathrm{C}$ & -5.30856 & -0.44952 & 0.32183 \\
\hline $\mathrm{C}$ & 2.78278 & 1.62541 & 0.10204 \\
\hline $\mathrm{H}$ & -3.26612 & -4.28505 & 2.45929 \\
\hline $\mathrm{H}$ & -3.52114 & 1.62531 & 0.20624 \\
\hline $\mathrm{C}$ & 2.65198 & 3.13459 & -0.21245 \\
\hline $\mathrm{H}$ & 2.29434 & -0.17976 & 2.86205 \\
\hline $\mathrm{C}$ & 4.93197 & 0.72597 & 0.56313 \\
\hline $\mathrm{H}$ & -7.89144 & -2.61078 & 0.92770 \\
\hline $\mathrm{C}$ & -7.32775 & -1.67437 & 0.92903 \\
\hline $\mathrm{C}$ & -5.86367 & 0.69098 & 0.91933 \\
\hline $\mathrm{H}$ & -5.31567 & 1.63434 & 0.94526 \\
\hline $\mathrm{H}$ & 1.13326 & 1.44563 & 1.46376 \\
\hline $\mathrm{H}$ & -0.56836 & -1.91815 & 2.10135 \\
\hline $\mathrm{H}$ & 6.90639 & -0.13848 & 0.82003 \\
\hline $\mathrm{H}$ & -4.24007 & -3.77527 & 1.05750 \\
\hline $\mathrm{C}$ & -3.28267 & -3.61412 & 1.57999 \\
\hline $\mathrm{C}$ & 3.87739 & 1.45039 & 1.13129 \\
\hline $\mathrm{Si}$ & -3.03598 & -1.84966 & 2.17333 \\
\hline $\mathrm{C}$ & 6.09248 & 0.43281 & 1.27131 \\
\hline $\mathrm{C}$ & 0.17544 & 3.42225 & -0.50086 \\
\hline $\mathrm{C}$ & -7.86660 & -0.53075 & 1.51808 \\
\hline $\mathrm{C}$ & -7.12853 & 0.65225 & 1.50850 \\
\hline $\mathrm{C}$ & -1.31355 & -1.68078 & 2.88264 \\
\hline $\mathrm{H}$ & -2.46358 & -3.92247 & 0.90674 \\
\hline $\mathrm{H}$ & -8.85614 & -0.56062 & 1.98105 \\
\hline $\mathrm{H}$ & -1.12151 & -0.65400 & 3.24253 \\
\hline $\mathrm{H}$ & -7.53594 & 1.55748 & 1.96578 \\
\hline $\mathrm{C}$ & 3.98165 & 1.91895 & 2.43157 \\
\hline $\mathrm{Br}$ & -0.35710 & 5.82380 & -0.08669 \\
\hline $\mathrm{H}$ & -1.15350 & -2.37425 & 3.72787 \\
\hline $\mathrm{H}$ & -5.36409 & -1.51116 & 3.07113 \\
\hline $\mathrm{C}$ & 6.17782 & 0.89706 & 2.59006 \\
\hline $\mathrm{C}$ & -4.33359 & -1.37049 & 3.44150 \\
\hline $\mathrm{H}$ & 3.16950 & 2.49708 & 2.88275 \\
\hline $\mathrm{H}$ & -4.21402 & -0.31253 & 3.73620 \\
\hline $\mathrm{C}$ & 5.14088 & 1.63216 & 3.16695 \\
\hline $\mathrm{H}$ & 7.07678 & 0.68012 & 3.17264 \\
\hline $\mathrm{H}$ & -4.21000 & -1.98948 & 4.34931 \\
\hline $\mathrm{H}$ & 5.23360 & 1.98809 & 4.19555 \\
\hline $\mathrm{C}$ & 1.53000 & 3.54782 & -1.15755 \\
\hline $\mathrm{H}$ & -0.73904 & 3.39442 & -1.08842 \\
\hline $\mathrm{H}$ & 0.09280 & 3.34776 & 0.58001 \\
\hline $\mathrm{H}$ & 1.56266 & 2.96430 & -2.09061 \\
\hline $\mathrm{H}$ & 1.68263 & 4.60136 & -1.43381 \\
\hline $\mathrm{H}$ & 3.62065 & 3.47329 & -0.61773 \\
\hline $\mathrm{H}$ & 2.51029 & 3.66764 & 0.74495 \\
\hline $\mathrm{H}$ & -1.28174 & 0.97925 & 0.87944 \\
\hline $\mathrm{H}$ & 0.63552 & 1.05506 & -1.557 \\
\hline
\end{tabular}




\section{Biological activity screening}

Antimycobacterial In Vitro Activity Screening Against Mycobacterium smegmatis, Mycobacterium aurum, and Mycobacterium tuberculosis H37Ra

Antimycobacterial assay was performed with fast growing Mycobacterium smegmatis DSM 43465 (ATCC 607), Mycobacterium aurum DSM 43999 (ATCC 23366) from German Collection of Microorganisms and Cell Cultures (Braunschweig, Germany) and with avirulent strain of Mycobacterium tuberculosis H37Ra ITM-M006710 (ATCC 9431) from Belgian Coordinated Collections of Micro-organisms (Antwerpen, Belgium). The technique used for activity determination was microdilution broth panel method using 96-well microtitration plates. Culturing medium was Middlebrook 7H9 broth (Sigma-Aldrich, Steinheim, Germany) enriched with $0.4 \%$ of glycerol (Sigma-Aldrich, Steinheim, Germany) and $10 \%$ of Middlebrook OADC growth supplement (Himedia, Mumbai, India).

Mycobacterial strains were cultured on Middlebrook 7H9 agar (MB) and suspensions were prepared in Middlebrook $7 \mathrm{H} 9$ broth. Final density was adjusted to value 1.0 according to McFarland scale and diluted in ratio 1:20 (for fast growing mycobacteria) or 1:10 (for $M$. tuberculosis) with broth.

Tested compounds were dissolved in DMSO (Sigma-Aldrich, Steinheim, Germany) then MB broth was added to obtain concentration $2000 \mu \mathrm{g} / \mathrm{mL}$. Standards used for activity determination were isoniazid (INH), rifampicin (RIF) and ciprofloxacin (CPX) (Sigma-Aldrich, Steinheim, Germany). Final concentrations were reached by binary dilution and addition of mycobacterial suspension and were set as 500, 250, 125, 62.5, 31.25, 15.625, 7.81 and $3.91 \mu \mathrm{g} / \mathrm{mL}$. Isoniazid was diluted in range $500-3.91 \mu \mathrm{g} / \mathrm{mL}$ for screening against fast growing mycobacteria and in range $1-0.0078 \mu \mathrm{g} / \mathrm{mL}$ for screening against $M$. tuberculosis. Rifampicin final concentrations ranged from 50 to $0.39 \mu \mathrm{g} / \mathrm{mL}$ for fast growing mycobacteria and from 0.1 to $0.00078 \mu \mathrm{g} / \mathrm{mL}$ for M. tuberculosis. Ciprofloxacin was used for screening antimycobacterial activity with the final concentrations $1,0.5,0.25,0.125,0.0625,0.0313,0.0156,0.0078 \mu \mathrm{g} / \mathrm{mL}$. The final concentration of DMSO did not exceed $2.5 \%(\mathrm{v} / \mathrm{v})$ and did not affect the growth of $M$. smegmatis, M. aurum nor M. tuberculosis. Positive (broth, DMSO, bacteria) and negative (broth, DMSO) growth controls were included.

Plates were sealed with polyester adhesive film and incubated in dark at $37^{\circ} \mathrm{C}$ without agitation. The addition of $0.01 \%$ solution of resazurin sodium salt followed after 48 hours of incubation for M. smegmatis, after 72 hours of incubation for M. aurum and after 120 hours of incubation for M. tuberculosis, respectively. Microtitration panels were then incubated for further 2.5 hours for determination of activity against $M$. smegmatis, 4 hours for $M$. aurum and 24 hours for $M$. tuberculosis, respectively. Antimycobacterial activity was expressed as minimal inhibition concentration (MIC) and the value was read on the basis of stain color change (blue color - active compound; pink color not active compound).. All experiments were conducted in duplicate. 
Table S14. MIC of standards in $\mu \mathrm{g} / \mathrm{mL}$.

\begin{tabular}{|l|l|l|l|}
\hline & M. smegmatis & M. aurum & M. tuberculosis $\mathrm{H} 37 \mathrm{Ra}$ \\
\hline INH & $15.625-31.25$ & 3.91 & $0.125-0.25$ \\
\hline RFM & $12.5-25$ & $0.39-0.78$ & $0.0015625-0.003125$ \\
\hline CPX & $0.0625-0.125$ & $0.0156-0.03125$ & $0.125-0.25$ \\
\hline
\end{tabular}

Table S15. Antimycobacterial activities expressed as MIC (minimal inhibition concentration) of compounds 10-14 against following strains: M. smeg.: Mycobacterium smegmatis DSM 43465 (ATCC 607), M. aurum: Mycobacterium aurum DSM 43999 (ATCC 23366), M. tbc H37Ra: Mycobacterium tuberculosis H37Ra ITM-M006710 (ATCC 9431).

\begin{tabular}{|c|c|c|c|c|c|c|c|c|c|c|}
\hline \multirow{2}{*}{$\begin{array}{c}\text { Bacterial } \\
\text { strain }\end{array}$} & \multicolumn{10}{|c|}{ Compound [MIC, $\mu \mathrm{g} / \mathbf{m L}]$} \\
\hline & $\mathbf{1 0 a}$ & $\mathbf{1 0 d}$ & $\mathbf{1 1 a}$ & $\mathbf{1 1 d ^ { \prime }}$ & $\mathbf{1 2 a}$ & $\mathbf{1 2 d}^{\prime}$ & $\mathbf{1 3 a}$ & $\mathbf{1 3 a ^ { \prime }}$ & $\mathbf{1 3 d}^{\prime}$ & $\mathbf{1 4 a}$ \\
\hline M. smeg. & 125 & 250 & $>500$ & 125 & $>500$ & 250 & 7.81 & 7.81 & $\mathbf{6 2 . 5}$ & $>500$ \\
\hline $\begin{array}{c}\text { M. } \\
\text { aurum }\end{array}$ & $\mathbf{6 2 . 5}$ & 250 & $>500$ & $\mathbf{6 2 . 5}$ & $\mathbf{3 1 . 2 5}$ & 125 & $\mathbf{3 . 9 1}$ & $\mathbf{3 . 9 1}$ & $\mathbf{3 1 . 2 5}$ & $>500$ \\
\hline $\begin{array}{c}\text { M. tbc } \\
\text { H37Ra }\end{array}$ & $\mathbf{6 2 . 5}$ & 250 & $>500$ & $\mathbf{3 1 . 2 5}$ & $\mathbf{1 5 . 6 2 5}$ & $\mathbf{6 2 . 5}$ & $\mathbf{1 . 9 8}$ & $\mathbf{1 . 9 8}$ & $\mathbf{1 5 . 6 2 5}$ & $>500$ \\
\hline
\end{tabular}

\section{Basic screening of antibacterial activity}

The microdilution broth method was performed according to EUCAST (The European Committee on Antimicrobial Susceptibility Testing) instructions, ${ }^{7}$ with slight modification. Eight tested bacterial strains, purchased from the Czech Collection of Microorganisms (CCM, Brno, Czech Republic) or the German Collection of Microorganisms and Cell Cultures (DSM, Braunschweig, Germany), were employed in the study. Namely, four Gram-positive reference strains, Staphylococcus aureus subsp. aureus CCM 4223 (ATCC 29213), Staphylococcus aureus subsp. aureus methicillin-resistant (MRSA) CCM 4750 (ATCC 43300), Staphylococcus epidermidis CCM 4418 (ATCC 12228), Enterococcus faecalis CCM 4224 (ATCC 29212), and four Gram-negative reference strains, Escherichia coli CCM 3954 (ATCC 25922), Klebsiella pneumoniae CCM 4415 (ATCC 10031), Acinetobacter baumannii DSM 30007 (ATCC 19606), and Pseudomonas aeruginosa CCM 3955 (ATCC 27853) were included in activity screening.

Briefly, the cultivation was done in Cation-adjusted Mueller-Hinton broth (CAMHB, M-H 2 Broth, Sigma-Aldrich) at $35 \pm 2{ }^{\circ} \mathrm{C}$. Tested compounds were dissolved in DMSO (SigmaAldrich, USA) to produce stock solutions. The final concentration of DMSO in the testing medium did not exceed $1 \%(\mathrm{v} / \mathrm{v})$ of the total solution composition. Positive growth controls (microbe solely), negative growth controls (cultivation medium and DMSO) and internal quality standards (ciprofloxacin, gentamicin, and vancomycin (Sigma-Aldrich, USA)) were involved in assays. Antibacterial activity, expressed as minimum inhibitory concentration (MIC, reported in $\mu \mathrm{M}$ ), was evaluated after 24 and $48 \mathrm{~h}$ of static incubation in the dark and humidified atmosphere at $35 \pm 2{ }^{\circ} \mathrm{C}$. Visual inspection and spectrophotometric measurement (530 nm, Synergy HTX Multi-Mode Microplate reader, BioTek, USA) were used for MIC endpoint evaluation. 
Table S16. Antibacterial activities expressed as MIC (minimal inhibition concentration) of compounds 11-14 against following strains, SA: Staphylococcus aureus subsp. aureus CCM 4223 (ATCC 29213), MRSA: Staphylococcus aureus subsp. aureus methicillin-resistant CCM 4750 (ATCC 43300), SE: Staphylococcus epidermidis CCM 4418 (ATCC 12228), EF: Enterococcus faecalis CCM 4224 (ATCC 29212), EC: Escherichia coli CCM 3954 (ATCC 25922), KP Klebsiella pneumoniae CCM 4415 (ATCC 10031), ACI: Acinetobacter baumannii DSM 30007 (ATCC 19606) ; and PA: Pseudomonas aeruginosa CCM 3955 (ATCC 27853).

\begin{tabular}{|c|c|c|c|c|c|c|c|c|c|c|c|}
\hline \multirow{2}{*}{\multicolumn{2}{|c|}{$\begin{array}{c}\text { Bacterial } \\
\text { strain } \\
\text { (incubation } \\
\text { period) }\end{array}$}} & \multicolumn{10}{|c|}{ Compound $\left[\mathrm{MIC}, \mu \mathrm{mol} \cdot \mathrm{I}^{-1}\right]$} \\
\hline & & $11 a$ & 11d & $12 a$ & $12 d^{\prime}$ & $10 a$ & $10 d^{\prime}$ & $13 a$ & $13 a^{\prime}$ & $13 d^{\prime}$ & $14 a$ \\
\hline \multirow[t]{2}{*}{ SA } & $24 \mathrm{~h}$ & $>125$ & $>125$ & $>125$ & 250 & $>500$ & $>500$ & 31.25 & 15.62 & 250 & $>500$ \\
\hline & $48 \mathrm{~h}$ & $>125$ & $>125$ & $>125$ & 250 & $>500$ & $>500$ & 31.25 & 31.25 & 250 & $>500$ \\
\hline \multirow[t]{2}{*}{ MRSA } & $24 \mathrm{~h}$ & $>125$ & $>125$ & $>125$ & 500 & $>500$ & $>500$ & 15.62 & 15.62 & 250 & $>500$ \\
\hline & $48 \mathrm{~h}$ & $>125$ & $>125$ & $>125$ & $>500$ & $>500$ & $>500$ & 31.25 & 15.62 & 250 & $>500$ \\
\hline \multirow[t]{2}{*}{ SE } & $24 \mathrm{~h}$ & $>125$ & $>125$ & 125 & 500 & $>500$ & 500 & 7.81 & 7.81 & 250 & $>500$ \\
\hline & $48 \mathrm{~h}$ & $>125$ & $>125$ & 125 & 500 & $>500$ & 500 & 15.62 & 7.81 & 250 & $>500$ \\
\hline \multirow[t]{2}{*}{ EF } & $24 \mathrm{~h}$ & $>125$ & $>125$ & $>125$ & $>500$ & $>500$ & $>500$ & 125 & 15.62 & $>250$ & $>500$ \\
\hline & $48 \mathrm{~h}$ & $>125$ & $>125$ & $>125$ & $>500$ & $>500$ & $>500$ & 500 & 62.5 & $>250$ & $>500$ \\
\hline \multirow[t]{2}{*}{ EC } & $24 \mathrm{~h}$ & $>125$ & $>125$ & $>125$ & $>500$ & $>500$ & $>500$ & $>500$ & 31.25 & $>250$ & $>500$ \\
\hline & $48 \mathrm{~h}$ & $>125$ & $>125$ & $>125$ & $>500$ & $>500$ & $>500$ & $>500$ & 31.25 & $>250$ & $>500$ \\
\hline \multirow[t]{2}{*}{ KP } & $24 \mathrm{~h}$ & $>125$ & $>125$ & $>125$ & $>500$ & $>500$ & $>500$ & $>500$ & 15.62 & $>250$ & $>500$ \\
\hline & $48 \mathrm{~h}$ & $>125$ & $>125$ & $>125$ & $>500$ & $>500$ & $>500$ & $>500$ & 15.62 & $>250$ & $>500$ \\
\hline \multirow[t]{2}{*}{ ACI } & $24 \mathrm{~h}$ & $>125$ & $>125$ & $>125$ & $>500$ & $>500$ & $>500$ & $>500$ & $>500$ & $>250$ & $>500$ \\
\hline & $48 \mathrm{~h}$ & $>125$ & $>125$ & $>125$ & $>500$ & $>500$ & $>500$ & $>500$ & $>500$ & $>250$ & $>500$ \\
\hline \multirow[t]{2}{*}{ PA } & $24 \mathrm{~h}$ & $>125$ & $>125$ & $>125$ & $>500$ & $>500$ & $>500$ & $>500$ & $>500$ & $>250$ & $>500$ \\
\hline & $48 \mathrm{~h}$ & $>125$ & $>125$ & $>125$ & $>500$ & $>500$ & $>500$ & $>500$ & $>500$ & $>250$ & $>500$ \\
\hline
\end{tabular}

\section{Basic screening of antifungal activity}

A microdilution broth method was performed according to EUCAST instructions with slight modifications. ${ }^{8}$ Eight fungal strains, purchased from the Czech Collection of Microorganisms (CCM, Czech Republic) or American Type Culture Collection (ATCC, USA), were employed in the study. Namely, four yeast reference strains Candida albicans ATCC 24433 (CCM 8320), Candida krusei ATCC 6258 (CCM 8271), Candida parapsilosis ATCC 22019 (CCM 8260), Candida tropicalis ATCC 750 (CCM 8264) and four filamentous fungi Aspergillus fumigatus ATCC 204305, Aspergillus flavus CCM 8363, Lichtheimia corymbifera CCM 8077, Trichophyton interdigitale ATCC 9533, CCM 8377 we included in activity testing.

Briefly, the cultivation was done in RPMI-1640 medium with L-glutamine, supplemented with $2 \%$ glucose (w/v), and buffered to $\mathrm{pH} 7.0$ with 3 morpholinopropane-1sulfonic acid (all components were purchased from Sigma-Aldrich, USA). Tested compounds were dissolved in DMSO (Sigma-Aldrich, USA) to produce stock solutions. The final concentration of DMSO in the testing medium did not exceed $1 \%(\mathrm{v} / \mathrm{v})$ of the total solution composition. Positive growth controls (microbe solely), negative growth controls (cultivation medium and DMSO) and internal quality standards (amphotericin, voriconazole (SigmaAldrich, USA)) were involved in assays. Static incubation was performed in the dark and humidified atmosphere at $35 \pm 2{ }^{\circ} \mathrm{C}$ for 24 and $48 \mathrm{~h}(72$ and $120 \mathrm{~h}$ for Trichophyton interdigitale). Antifungal activity was expressed as minimum inhibitory concentration (MIC, reported in $\mu \mathrm{M}$ ) after visual inspection and spectrophotometric measurement (530 nm, Synergy HTX Multi-Mode Microplate reader, BioTek, USA). 
Table S17. Antifungal activities expressed as MIC (minimal inhibition concentration) of compounds 11-14 against following strains, CA: Candida albicans ATCC 24433 (CCM 8320), CK: Candida krusei ATCC 6258 (CCM 8271), CP: Candida parapsilosis ATCC 22019 (CCM 8260), CT: Candida tropicalis ATCC 750 (CCM 8264), AF: Aspergillus fumigatus ATCC 204305, AFla: Aspergillus flavus CCM 8363, AC: Lichtheimia corymbifera CCM 8077, TI: Trichophyton interdigitale ATCC 9533, CCM 8377.

\begin{tabular}{|c|c|c|c|c|c|c|c|c|c|c|c|}
\hline \multirow{2}{*}{\multicolumn{2}{|c|}{$\begin{array}{c}\text { Fungal } \\
\text { strain } \\
\text { (incubation } \\
\text { period) } \\
\end{array}$}} & \multicolumn{10}{|c|}{ Compound [MIC, $\mu \mathrm{mol.l^{-1 }}$ ] } \\
\hline & & \multirow{2}{*}{$\begin{array}{c}11 a \\
>125\end{array}$} & \multirow{2}{*}{$\begin{array}{c}\text { 11d } \\
>125\end{array}$} & \multirow{2}{*}{$\begin{array}{c}12 a \\
>500\end{array}$} & \multirow{2}{*}{$\begin{array}{c}12 d \\
>500\end{array}$} & \multirow{2}{*}{$\frac{10 a^{\prime}}{>>500}$} & \multirow{2}{*}{$\frac{10 d^{\prime}}{=500}$} & \multirow{2}{*}{$\frac{13 a}{}$} & \multirow{2}{*}{$\frac{13 a^{\prime}}{125}$} & \multirow{2}{*}{$\frac{13 d^{\prime}}{>250}$} & \multirow{2}{*}{$\begin{array}{r}14 a \\
>125\end{array}$} \\
\hline CA & $24 \mathrm{~h}$ & & & & & & & & & & \\
\hline & $48 \mathrm{~h}$ & $>125$ & $>125$ & $>500$ & $>500$ & $>500$ & $>500$ & $>500$ & 125 & $>250$ & $>125$ \\
\hline \multirow[t]{2}{*}{ CK } & $24 \mathrm{~h}$ & $>125$ & $>125$ & $>500$ & $>500$ & 500 & $>500$ & 125 & 62.5 & $>250$ & $>125$ \\
\hline & $48 \mathrm{~h}$ & $>125$ & $>125$ & $>500$ & $>500$ & $>500$ & $>500$ & 125 & 62.5 & $>250$ & $>125$ \\
\hline \multirow[t]{2}{*}{ CP } & $24 \mathrm{~h}$ & $>125$ & $>125$ & $>500$ & $>500$ & 500 & $>500$ & 125 & 125 & 250 & $>125$ \\
\hline & $48 \mathrm{~h}$ & $>125$ & $>125$ & $>500$ & $>500$ & $>500$ & $>500$ & 125 & 125 & 250 & $>125$ \\
\hline \multirow[t]{2}{*}{ CT } & $24 h$ & $>125$ & $>125$ & $>500$ & $>500$ & $>500$ & $>500$ & 125 & 125 & $>250$ & $>125$ \\
\hline & $48 \mathrm{~h}$ & $>125$ & $>125$ & $>500$ & $>500$ & $>500$ & $>500$ & 500 & 250 & $>250$ & $>125$ \\
\hline \multirow[t]{2}{*}{ AF } & $24 h$ & $>125$ & $>125$ & $>500$ & $>500$ & $>500$ & $>500$ & $>500$ & $>500$ & $>250$ & $>125$ \\
\hline & $48 \mathrm{~h}$ & $>125$ & $>125$ & $>500$ & $>500$ & $>500$ & $>500$ & $>500$ & $>500$ & $>250$ & $>125$ \\
\hline \multirow[t]{2}{*}{ AFla } & $24 \mathrm{~h}$ & $>125$ & $>125$ & $>500$ & $>500$ & $>500$ & $>500$ & $>500$ & $>500$ & $>250$ & $>125$ \\
\hline & $48 \mathrm{~h}$ & $>125$ & $>125$ & $>500$ & $>500$ & $>500$ & $>500$ & $>500$ & $>500$ & $>250$ & $>125$ \\
\hline \multirow[t]{2}{*}{ AC } & $24 \mathrm{~h}$ & $>125$ & $>125$ & $>500$ & $>500$ & $>500$ & $>500$ & $>500$ & 125 & $>250$ & $>125$ \\
\hline & $48 \mathrm{~h}$ & $>125$ & $>125$ & $>500$ & $>500$ & $>500$ & $>500$ & $>500$ & 125 & $>250$ & $>125$ \\
\hline \multirow[t]{2}{*}{ TI } & $72 \mathrm{~h}$ & $>125$ & $>125$ & $>500$ & $>500$ & $>500$ & 250 & 62,5 & 62.5 & $>250$ & $>125$ \\
\hline & $120 \mathrm{~h}$ & $>125$ & $>125$ & $>500$ & $>500$ & $>500$ & 250 & 125 & 62.5 & $>250$ & $>125$ \\
\hline
\end{tabular}




\section{NMR spectra}

(R)-2-((tert-butyldimethylsilyl)oxy)diphenylmethyl)pyrrolidine (ent-C2)

${ }^{1} \mathrm{H}$ NMR of ent-C2 $\left(400 \mathrm{MHz}, \mathrm{CDCl}_{3}\right)$

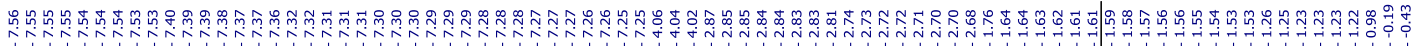

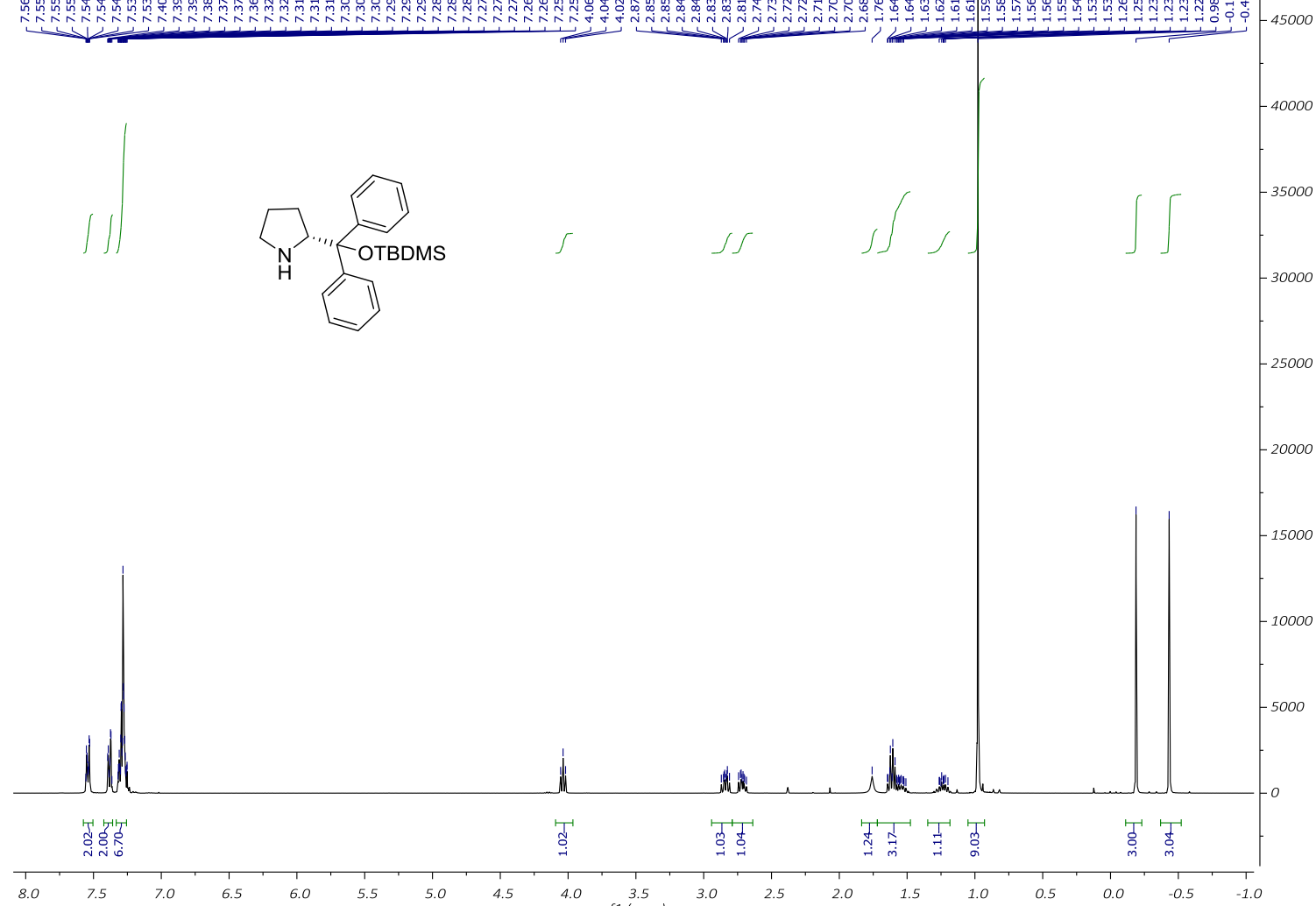

${ }^{13} \mathrm{C}\left\{{ }^{1} \mathrm{H}\right\}$ NMR of ent-C2 $\left(101 \mathrm{MHz}, \mathrm{CDCl}_{3}\right)$

虽勇

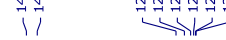

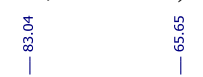

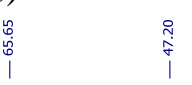

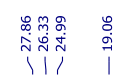

5000
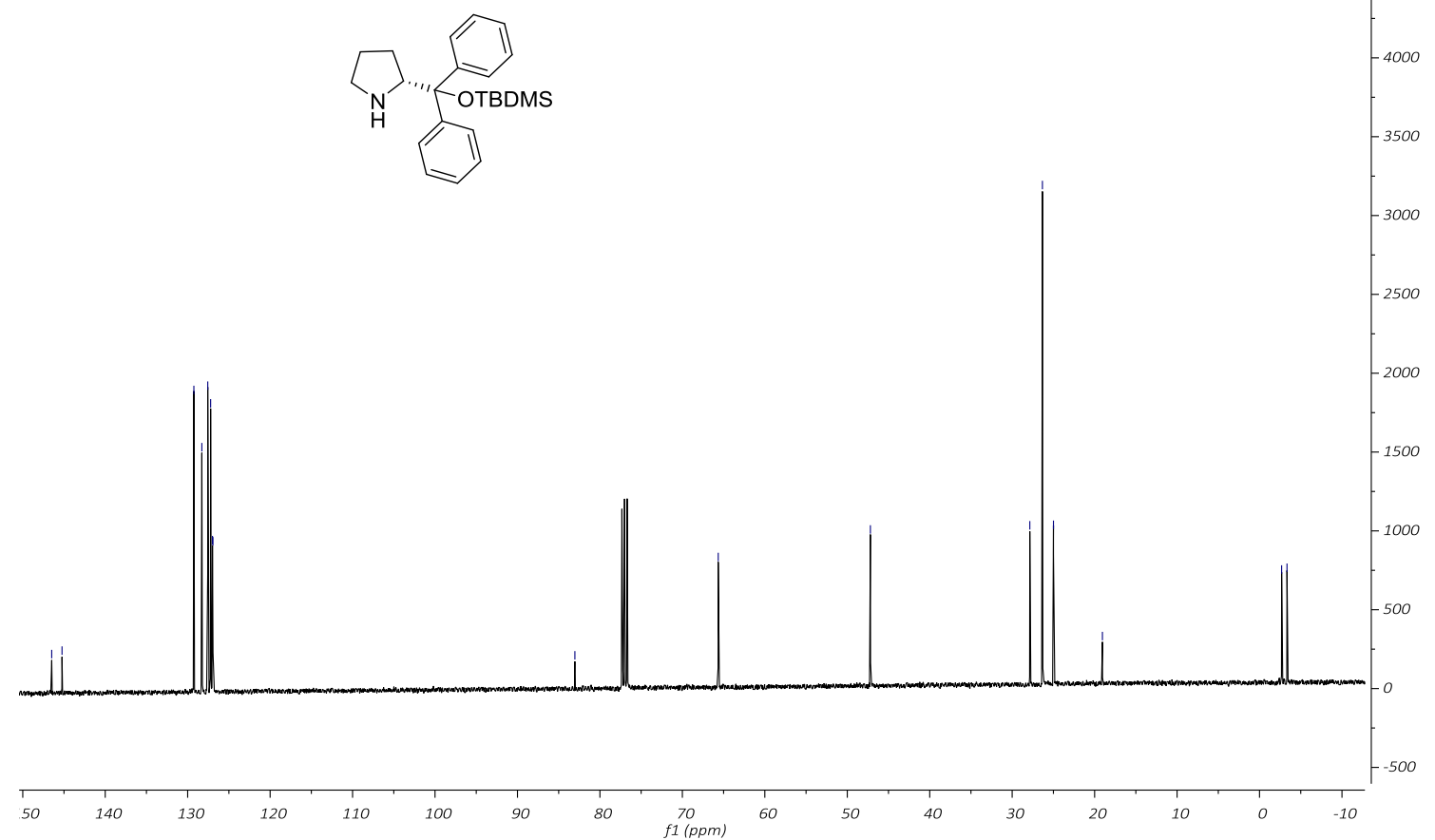
1-Benzyl-3-(2-bromoethyl)-5-methylindolin-2-one (1e)

${ }^{1} \mathrm{H}$ NMR of 1 e $\left(400 \mathrm{MHz}, \mathrm{CDCl}_{3}\right)$

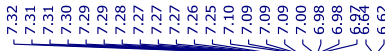

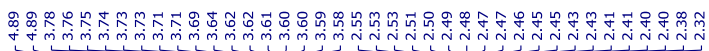
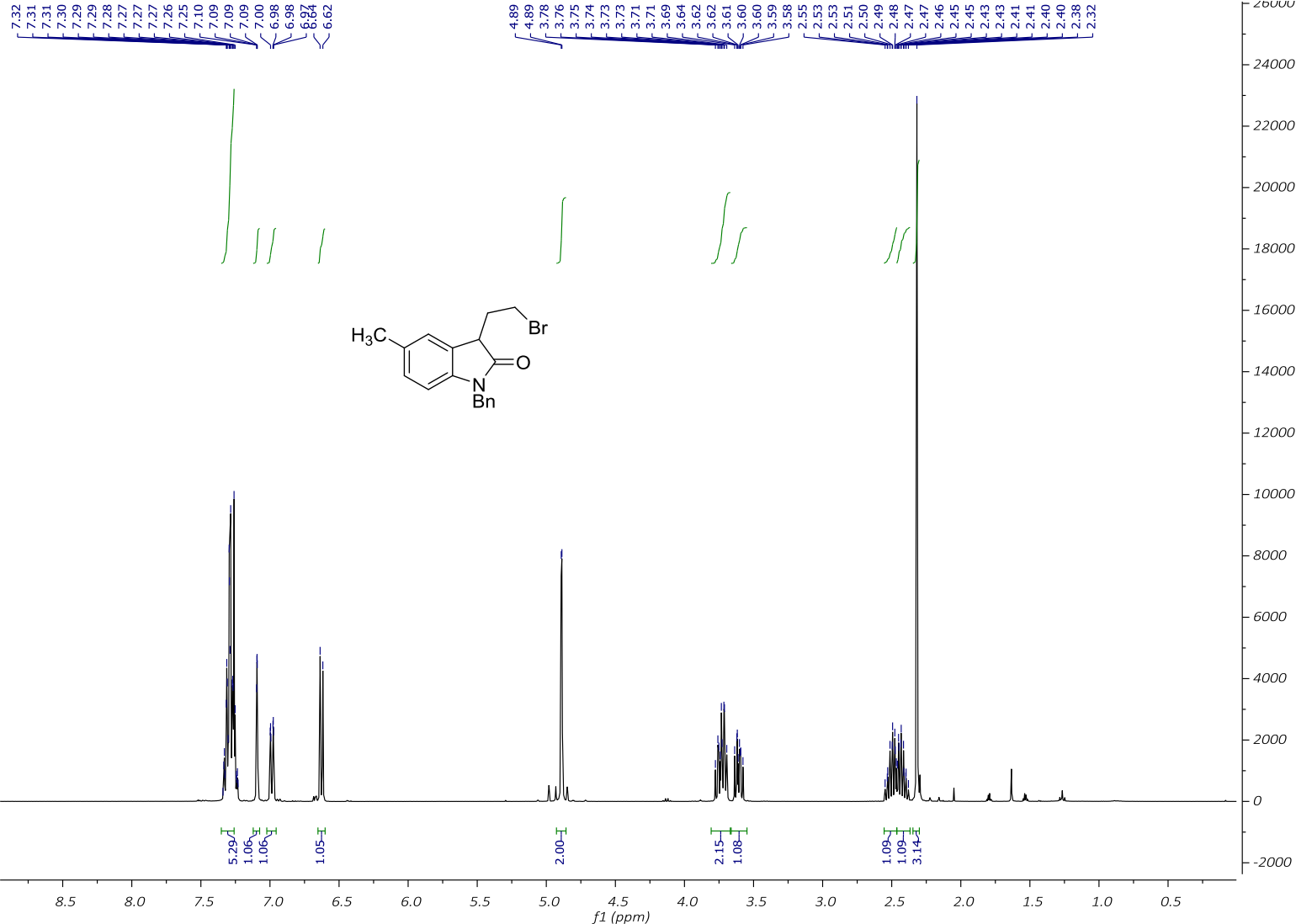

${ }^{13} \mathrm{C}\left\{{ }^{1} \mathrm{H}\right\}$ NMR of $\mathbf{1 e}\left(101 \mathrm{MHz}, \mathrm{CDCl}_{3}\right)$
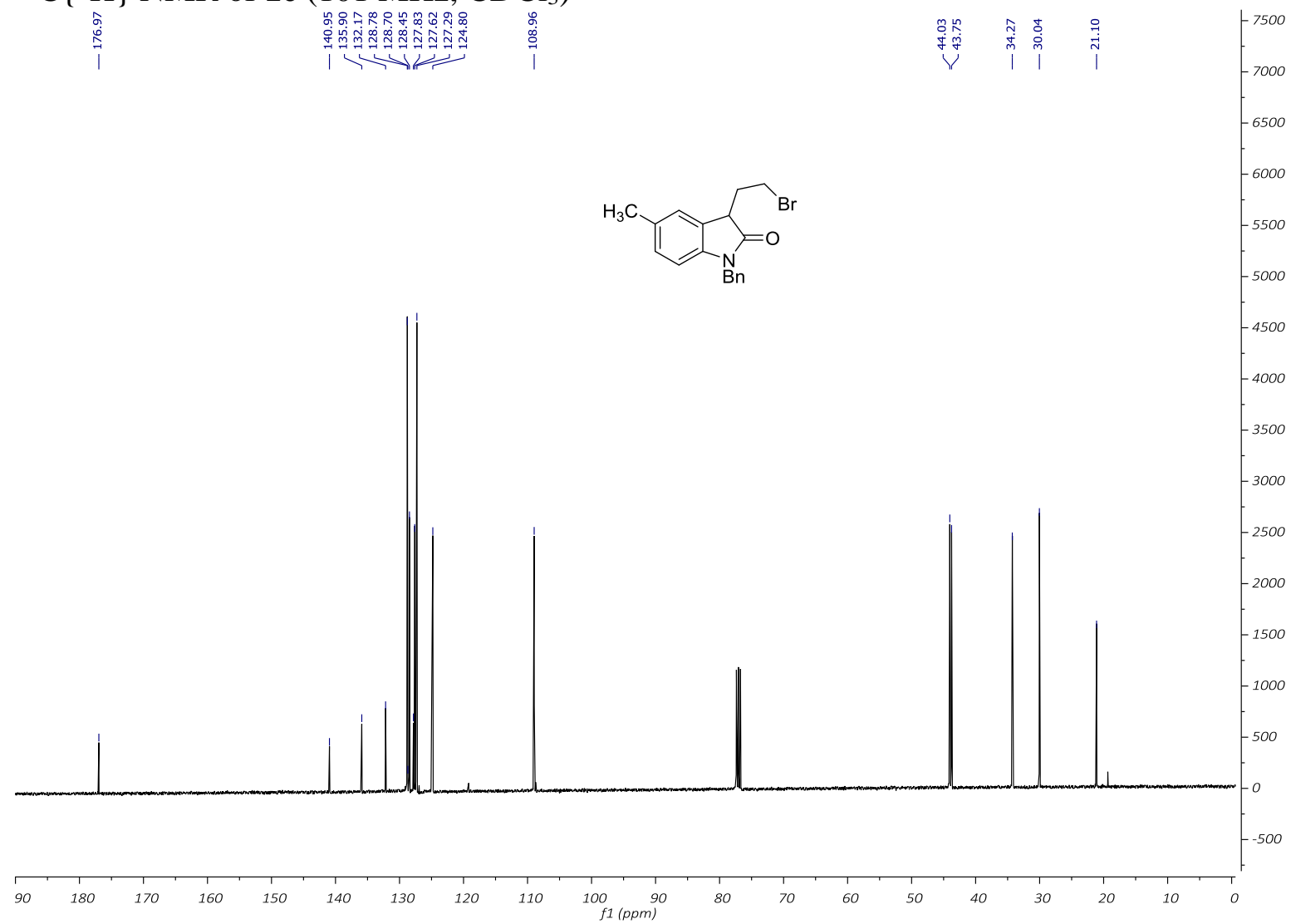
1-Benzyl-3-(2-bromoethyl)-5-methoxyindolin-2-one (1f)

${ }^{1} \mathrm{H}$ NMR of $1 \mathbf{f}\left(400 \mathrm{MHz}, \mathrm{CDCl}_{3}\right)$

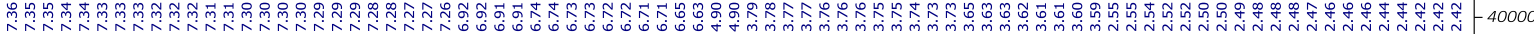

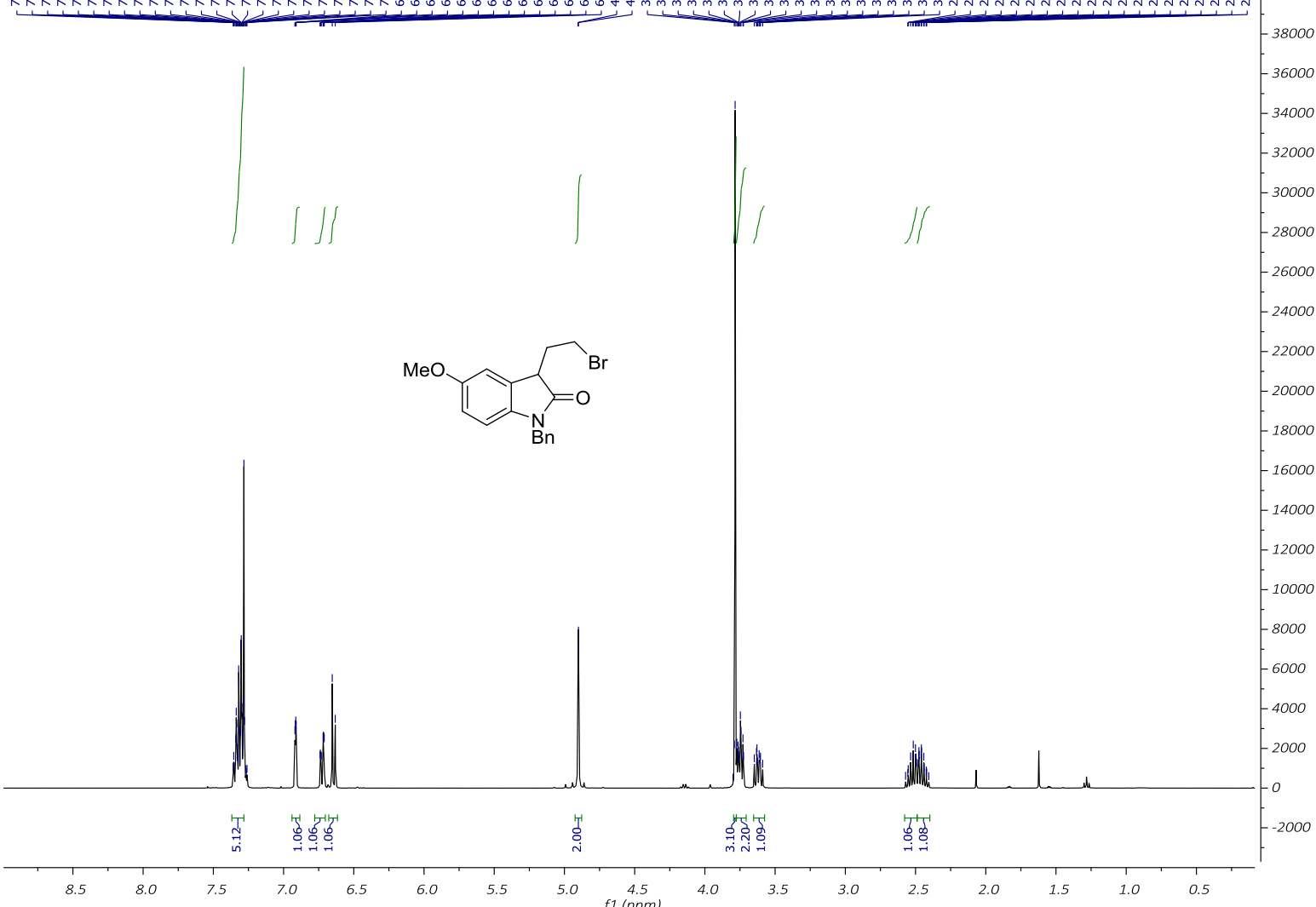

${ }^{13} \mathrm{C}\left\{{ }^{1} \mathrm{H}\right\}$ NMR of $\mathbf{1 f}\left(101 \mathrm{MHz}, \mathrm{CDCl}_{3}\right)$

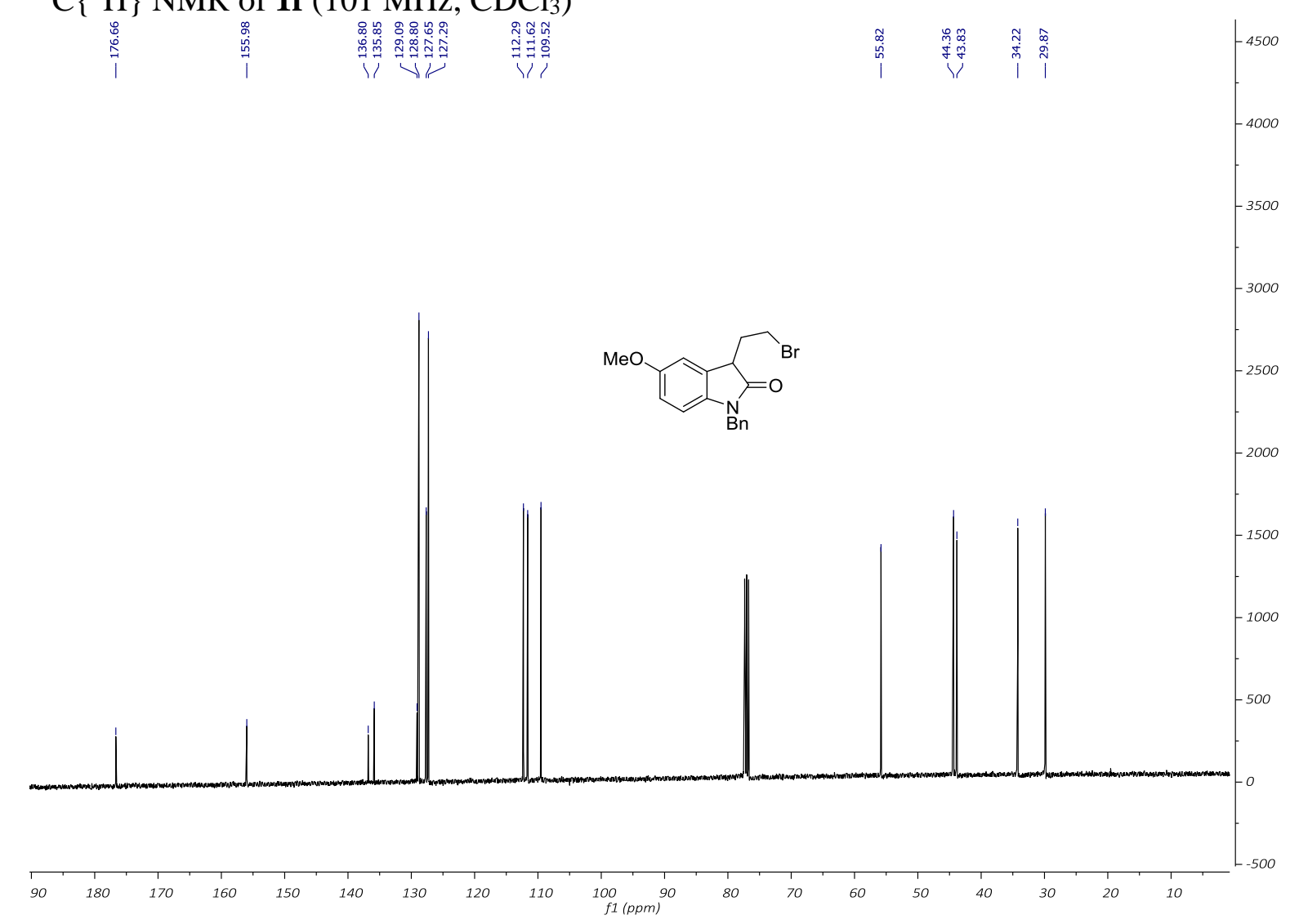


1-Benzyl-3-(2-bromoethyl)-5-fluoroindolin-2-one (1g)

${ }^{1} \mathrm{H}$ NMR of $1 \mathrm{~g}\left(400 \mathrm{MHz}, \mathrm{CDCl}_{3}\right)$

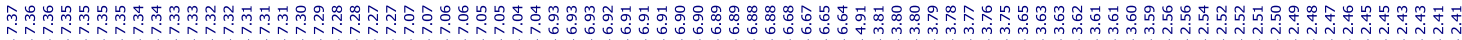

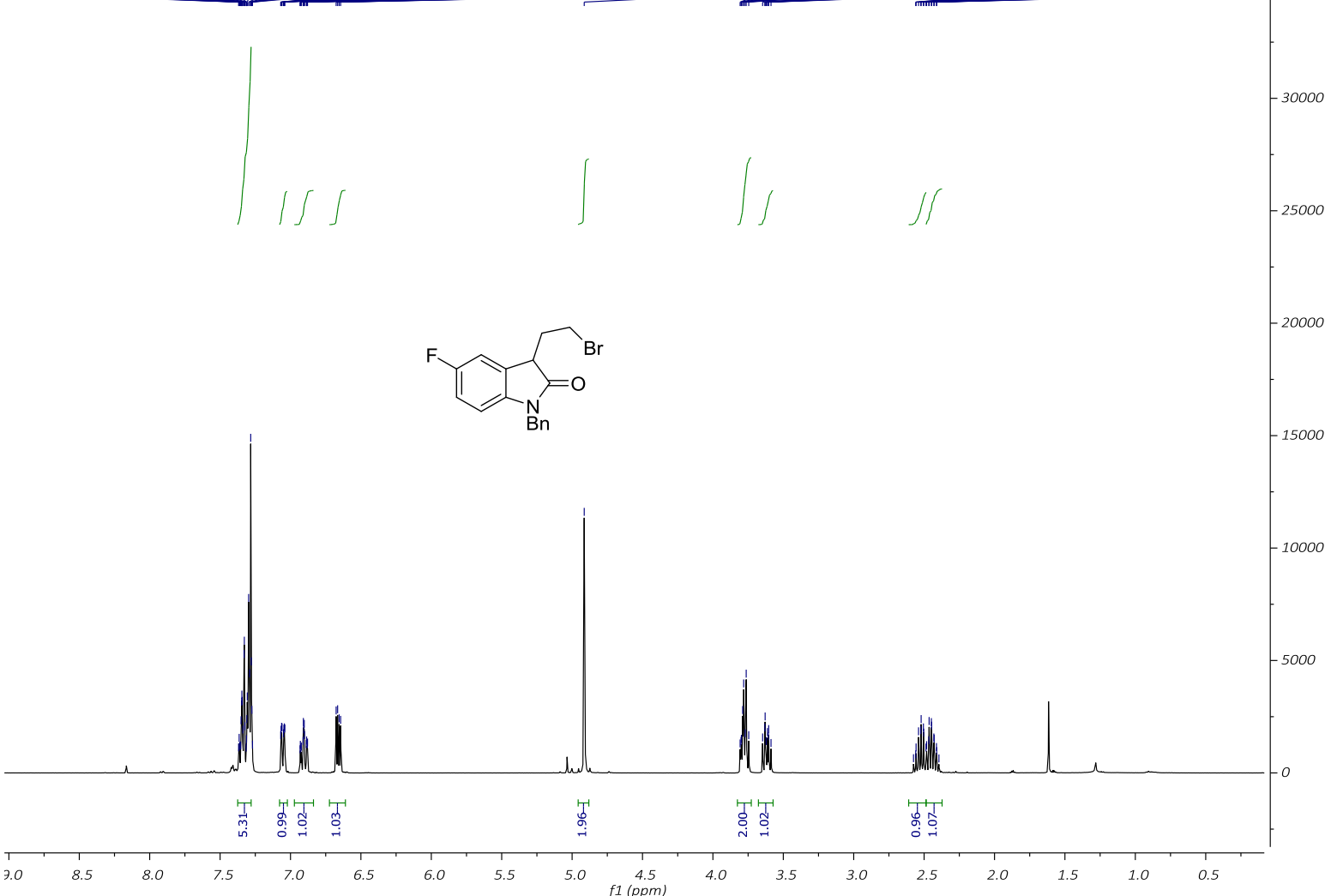

${ }^{13} \mathrm{C}\left\{{ }^{1} \mathrm{H}\right\} \mathrm{NMR}$ of $\mathbf{1 g}\left(101 \mathrm{MHz}, \mathrm{CDCl}_{3}\right)$

$\begin{array}{ll}0 & \\ 0 & 0\end{array}$
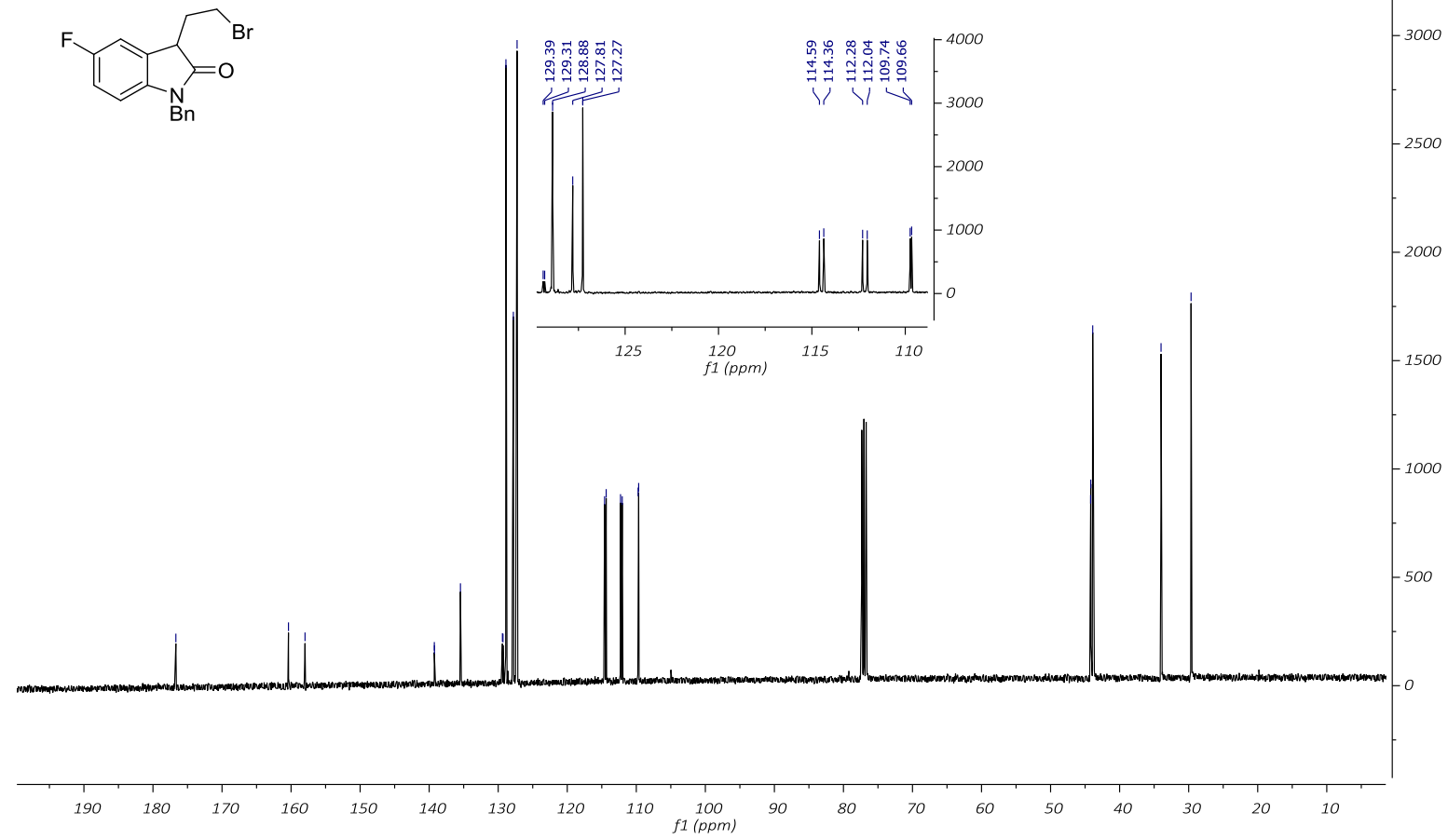

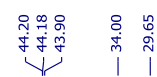

$-4000$ 
${ }^{19} \mathrm{~F}$ NMR of $1 \mathrm{~g}\left(376 \mathrm{MHz} \mathrm{MHz}, \mathrm{CDCl}_{3}\right)$

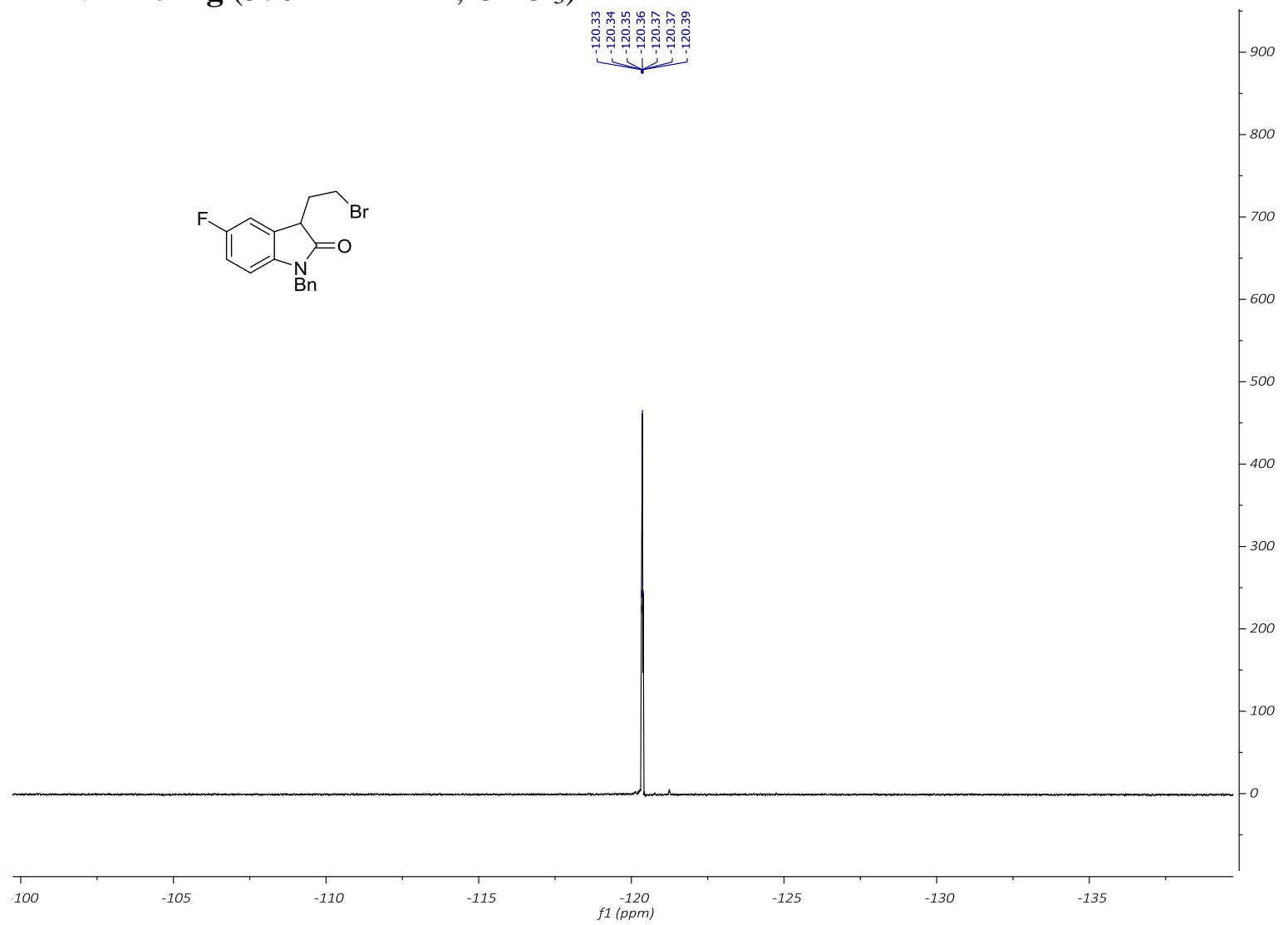


1-Benzyl-3-(2-bromoethyl)-5-chloroindolin-2-one (1h)

${ }^{1} \mathrm{H} \mathrm{NMR}$ of $\mathbf{1 h}\left(400 \mathrm{MHz}, \mathrm{CDCl}_{3}\right)$

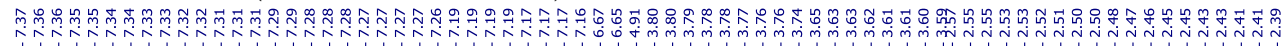

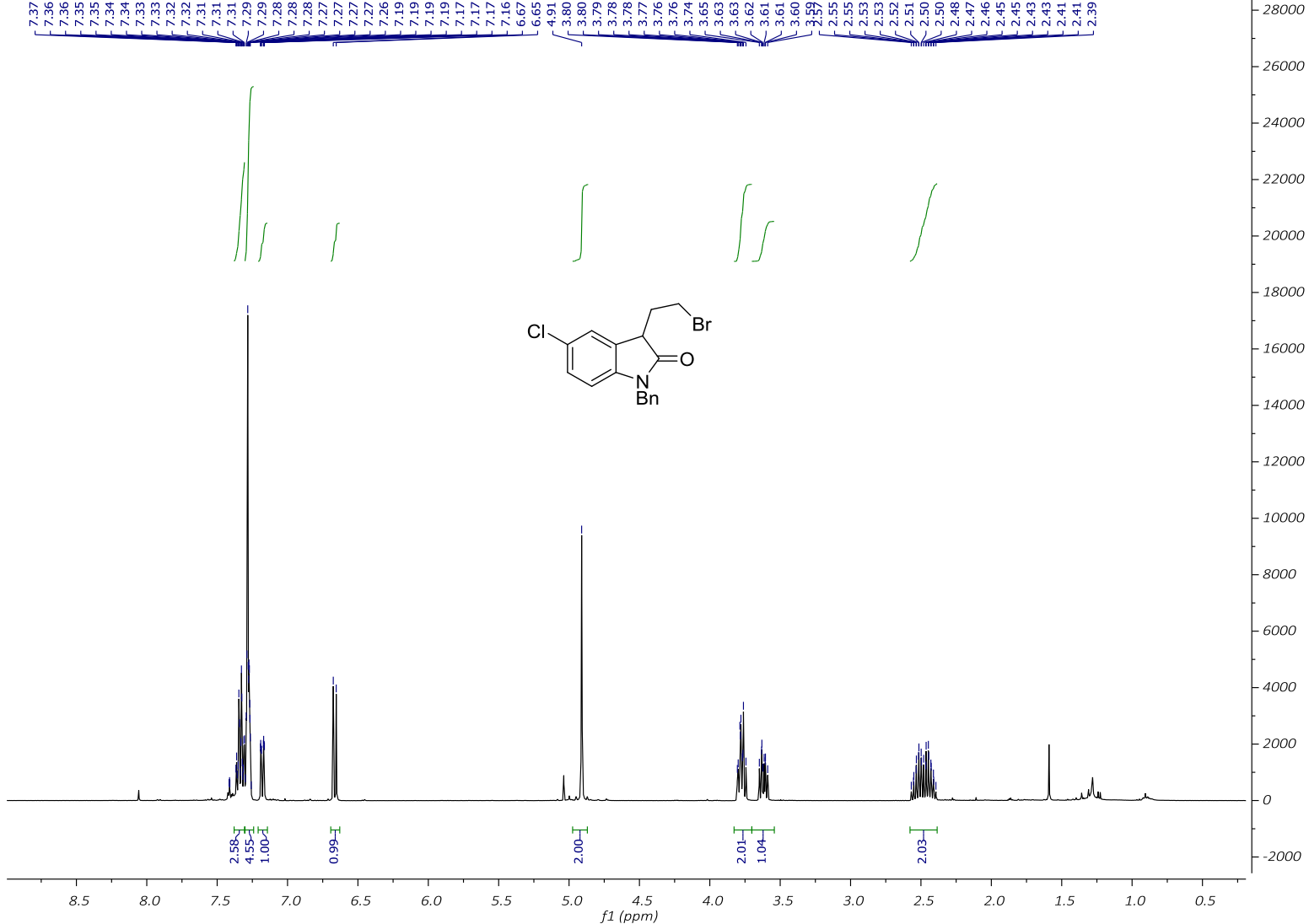

${ }^{13} \mathrm{C}\left\{{ }^{1} \mathrm{H}\right\} \mathrm{NMR}$ of $\mathbf{1 h}\left(101 \mathrm{MHz}, \mathrm{CDCl}_{3}\right)$

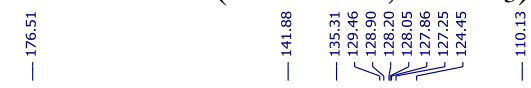

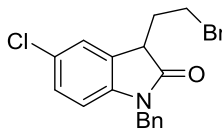

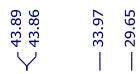

4000

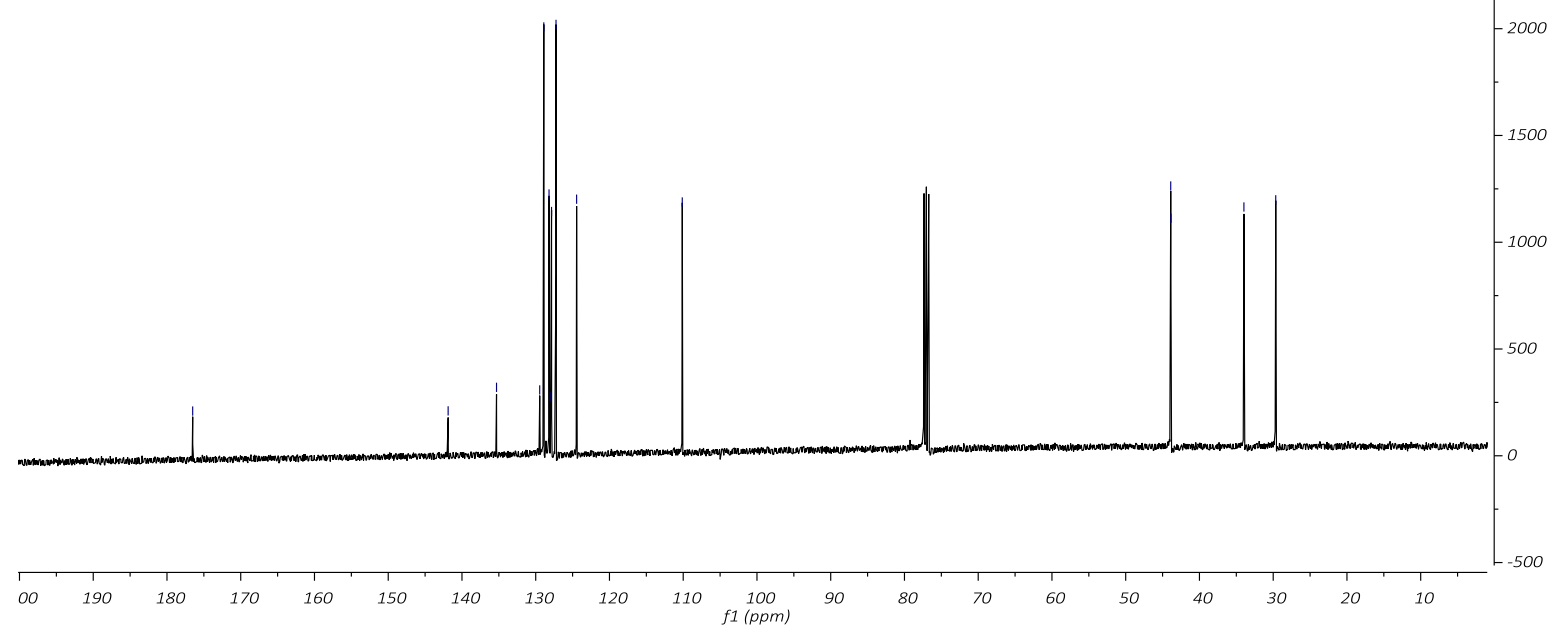


1-Benzyl-5-bromo-3-(2-bromoethyl)indolin-2-one (1i)

${ }^{1} \mathrm{H} \mathrm{NMR}$ of $1 \mathbf{i}\left(400 \mathrm{MHz}, \mathrm{CDCl}_{3}\right)$

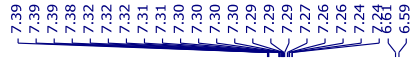

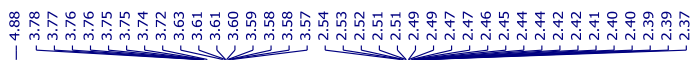

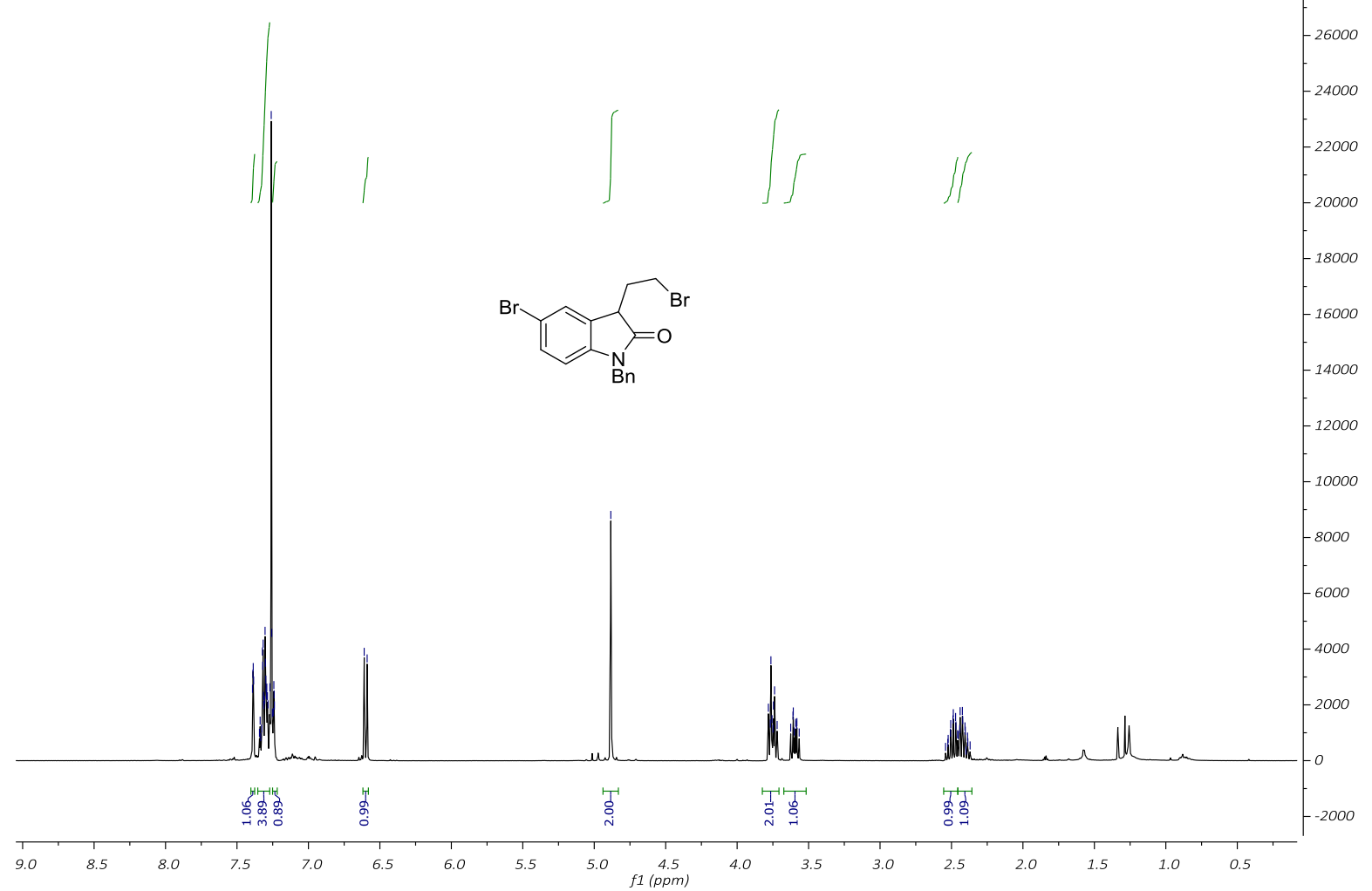

${ }^{13} \mathrm{C}\left\{{ }^{1} \mathrm{H}\right\}$ NMR of $\mathbf{1 i}\left(101 \mathrm{MHz}, \mathrm{CDCl}_{3}\right)$

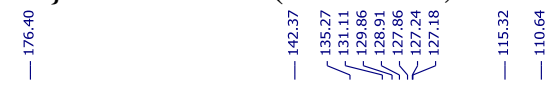

$\overbrace{\mathrm{Bn}}^{\mathrm{Br}}$

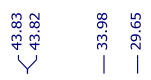

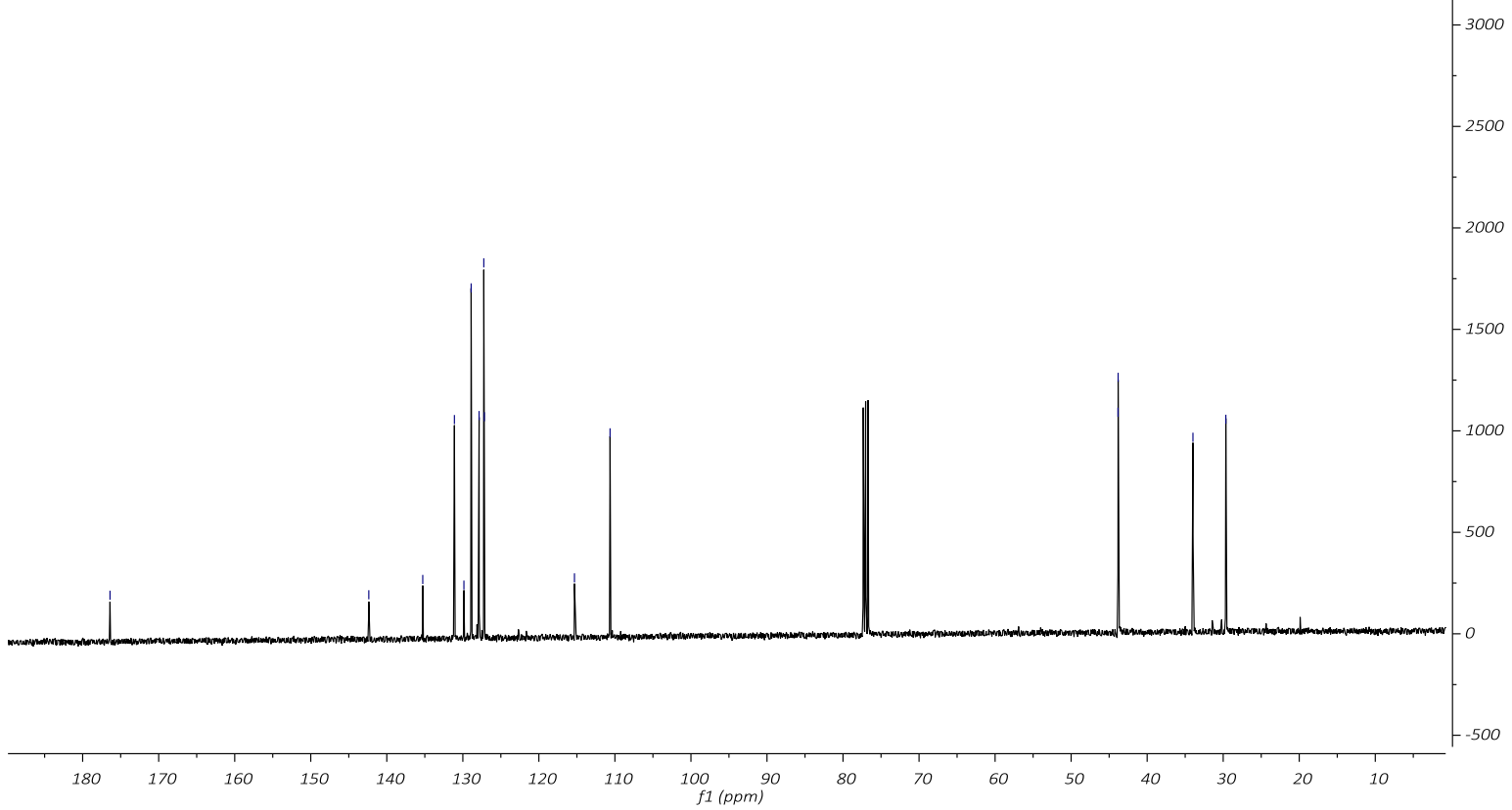


1-Benzyl-3-(2-bromoethyl)-4-methylindolin-2-one (1j)

${ }^{1} \mathrm{H} \mathrm{NMR}$ of $\mathbf{1 j}\left(400 \mathrm{MHz}, \mathrm{CDCl}_{3}\right)$

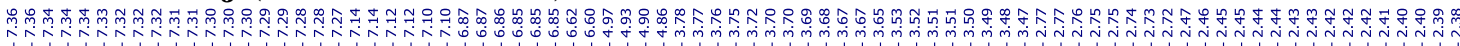

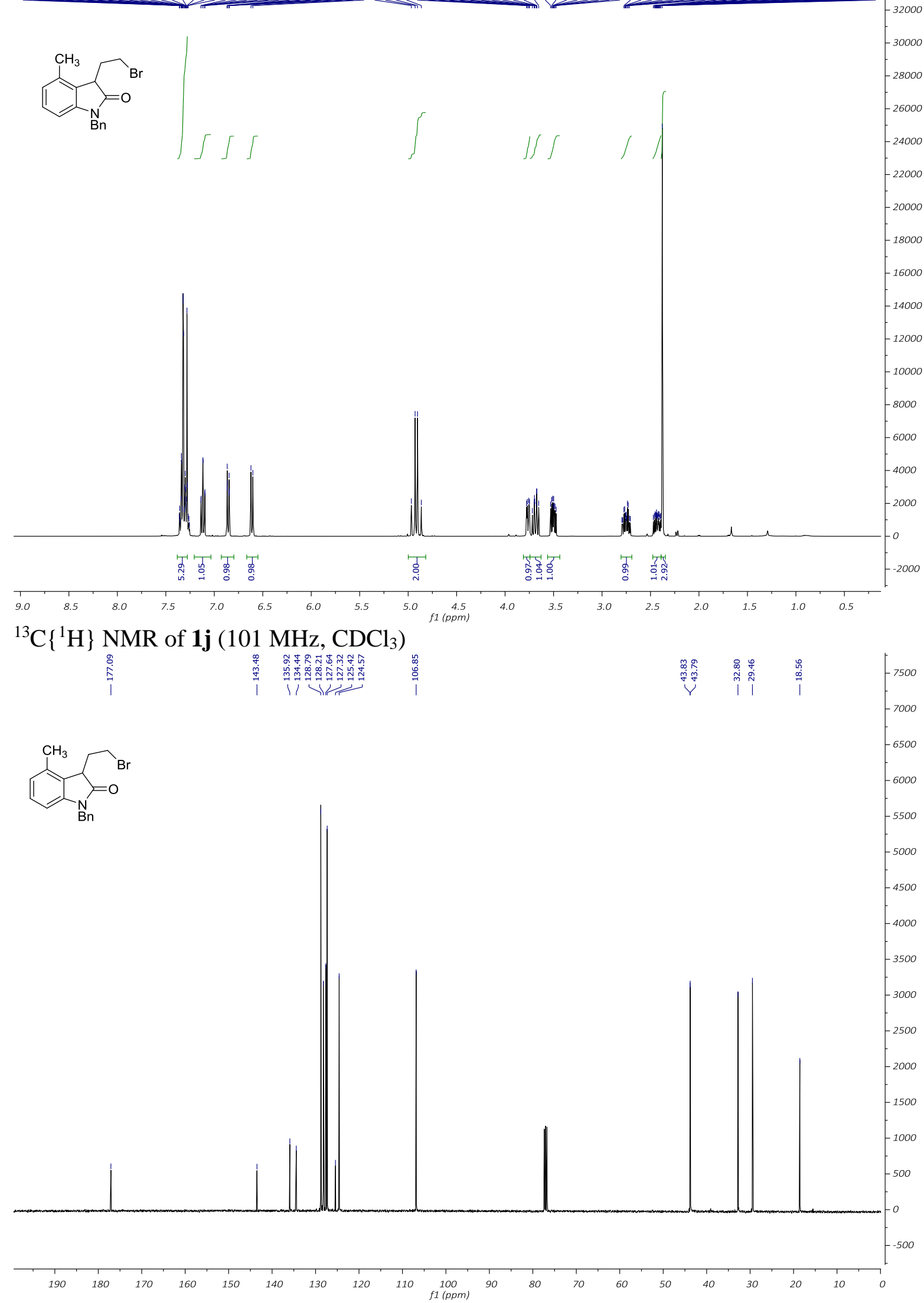


1-Benzyl-3-(2-bromoethyl)-6-methylindolin-2-one (1k)

${ }^{1} \mathrm{H}$ NMR of $1 \mathbf{k}\left(400 \mathrm{MHz}, \mathrm{CDCl}_{3}\right)$

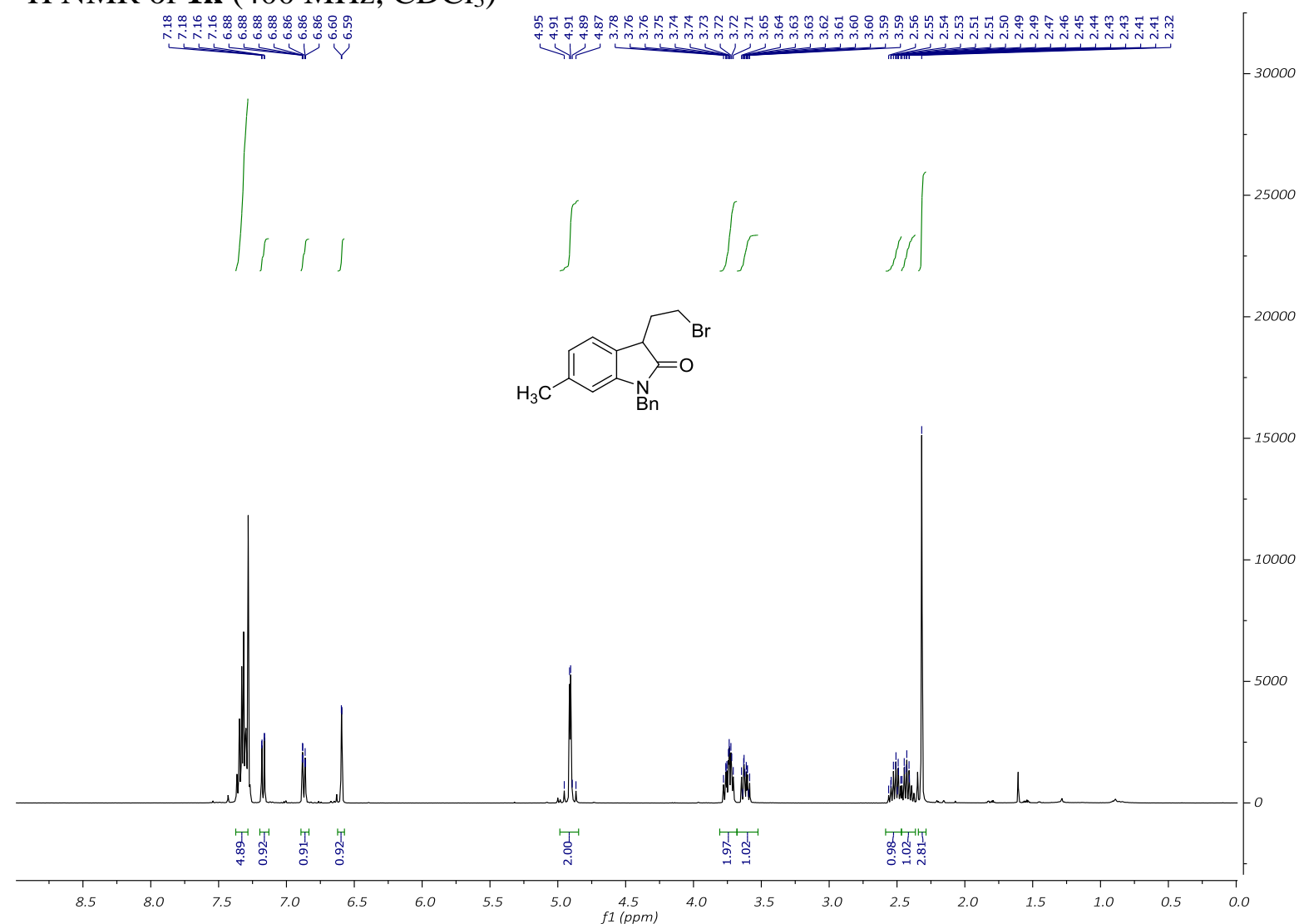

${ }^{13} \mathrm{C}\left\{{ }^{1} \mathrm{H}\right\}$ NMR of $\mathbf{1 k}\left(101 \mathrm{MHz}, \mathrm{CDCl}_{3}\right)$

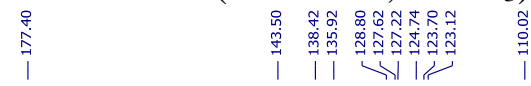

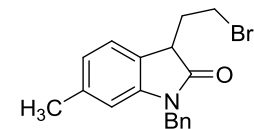

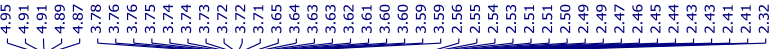

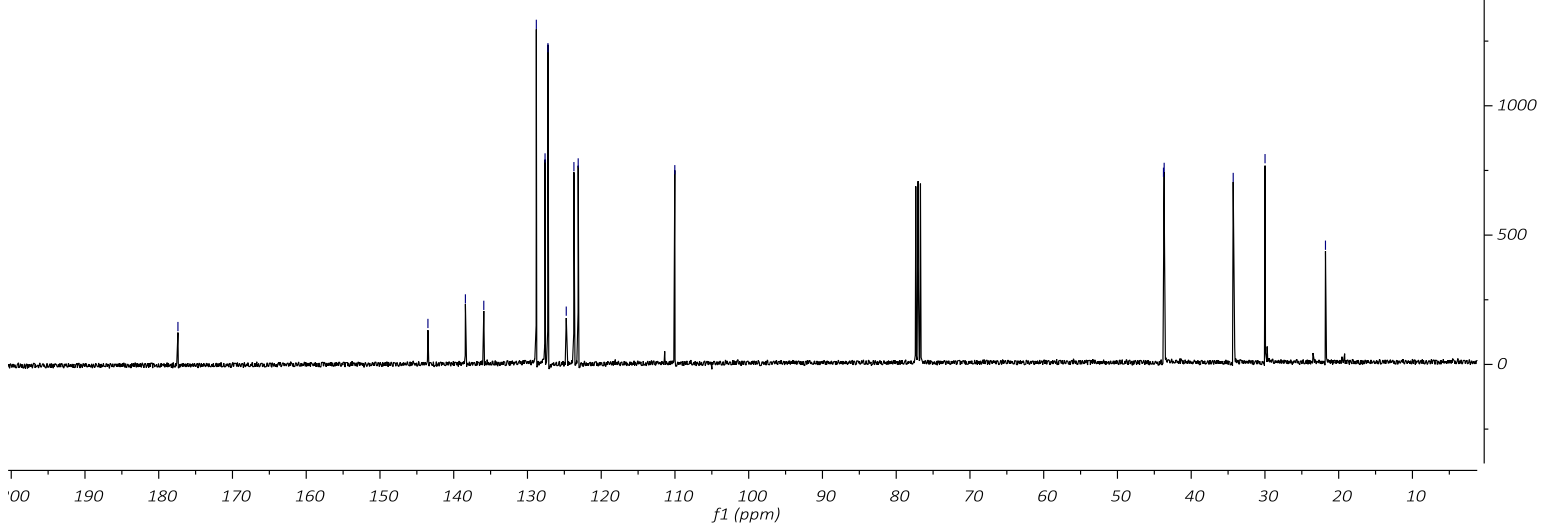


1-Benzyl-3-(2-bromoethyl)-7-methylindolin-2-one (11)

${ }^{1} \mathrm{H} \mathrm{NMR}$ of $11\left(400 \mathrm{MHz}, \mathrm{CDCl}_{3}\right)$

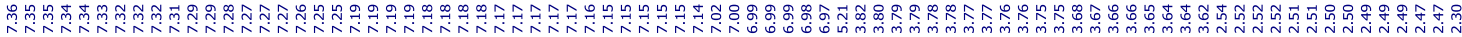

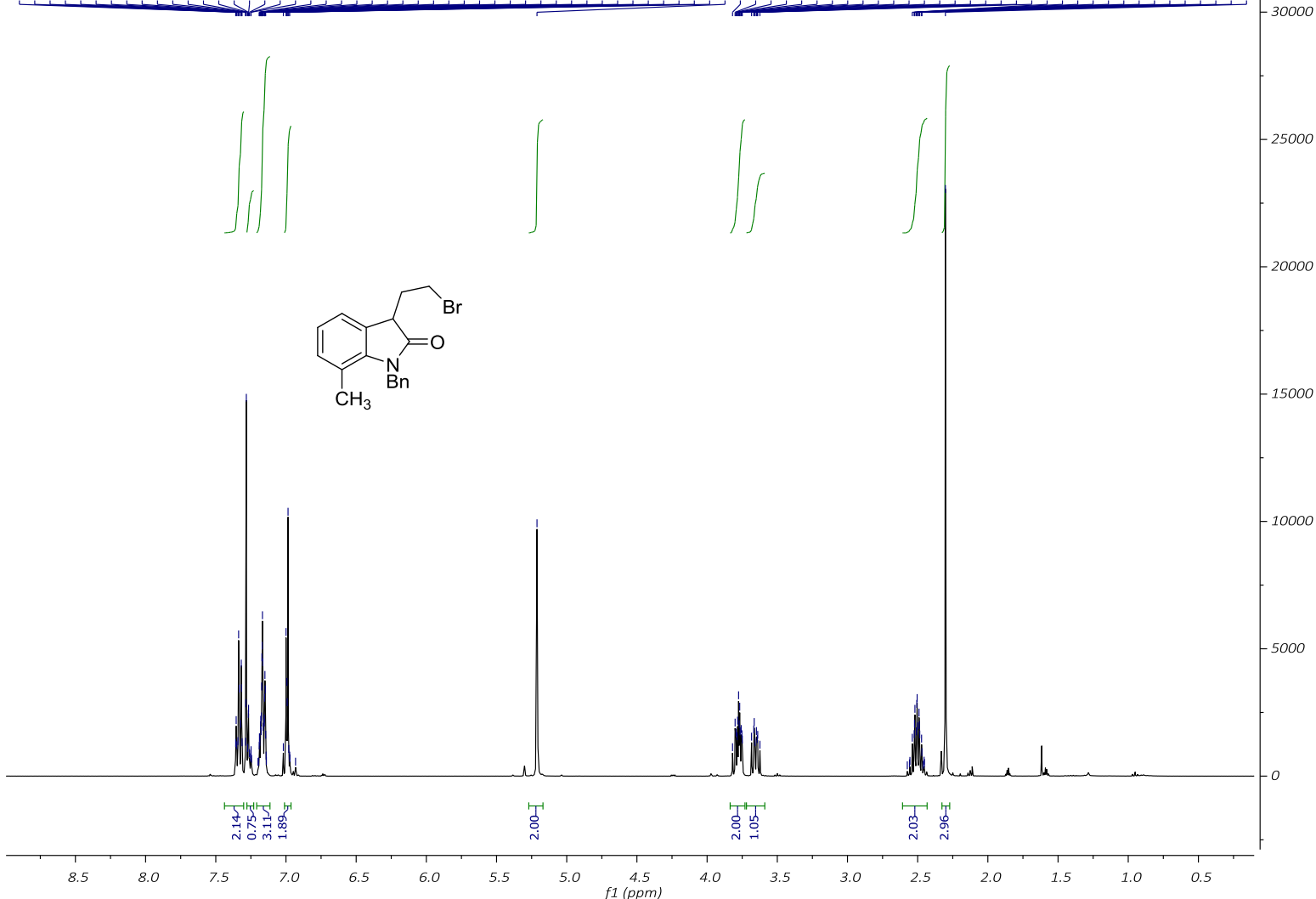

${ }^{13} \mathrm{C}\left\{{ }^{1} \mathrm{H}\right\}$ NMR of $11\left(101 \mathrm{MHz}, \mathrm{CDCl}_{3}\right)$

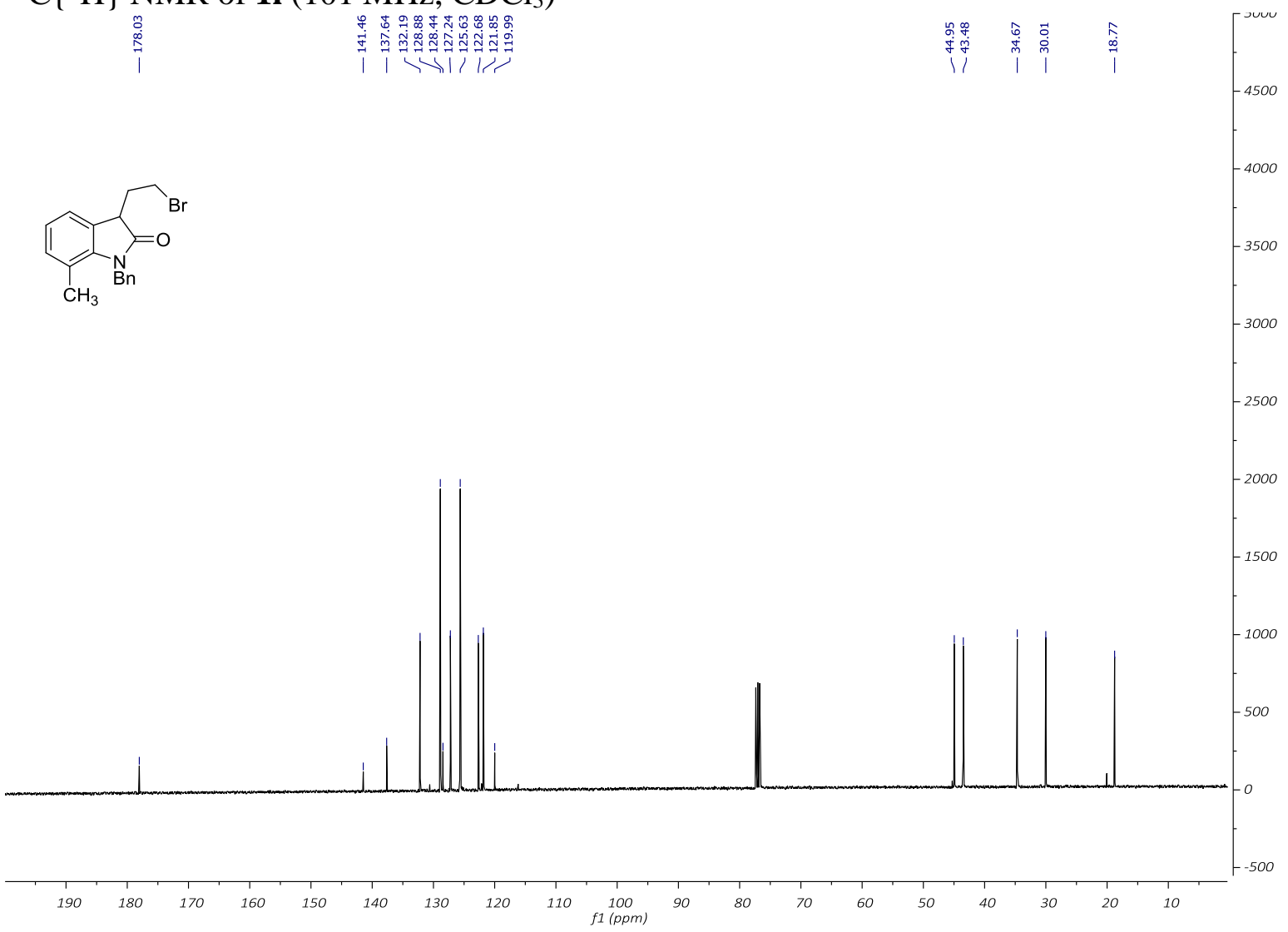


1-Benzyl-3-(4-bromopropyl)indolin-2-one (1n)

${ }^{1} \mathrm{H}$ NMR of 1 n $\left(400 \mathrm{MHz}, \mathrm{CDCl}_{3}\right)$

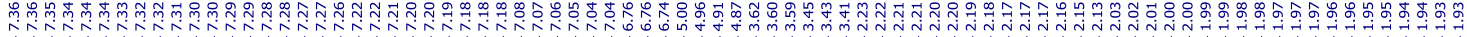

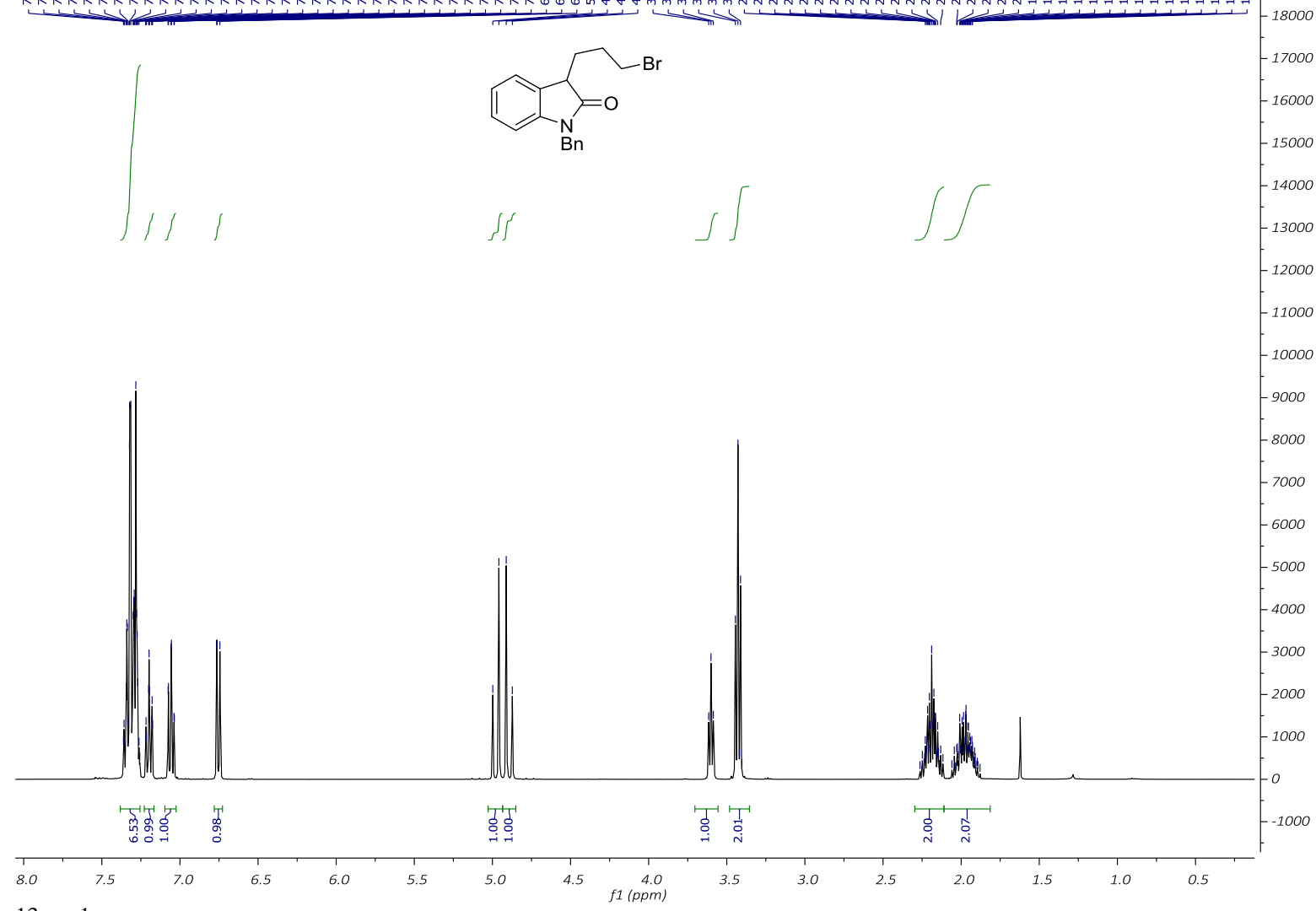

${ }^{13} \mathrm{C}\left\{{ }^{1} \mathrm{H}\right\}$ NMR of 1 n $\left(101 \mathrm{MHz}, \mathrm{CDCl}_{3}\right)$

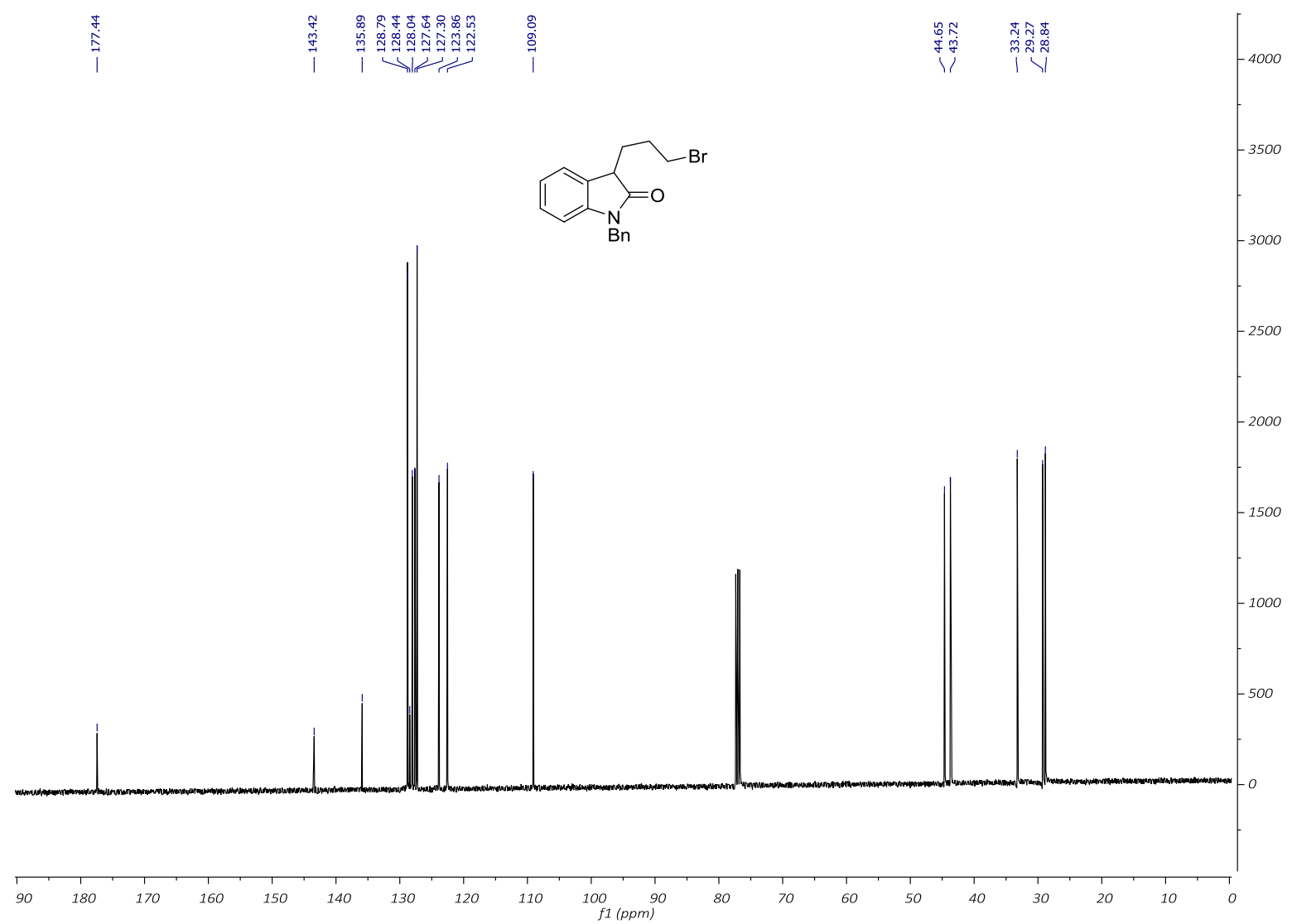


1-Benzyl-3-(4-bromobutyl)indolin-2-one (10)

${ }^{1} \mathrm{H}$ NMR of $10\left(400 \mathrm{MHz}, \mathrm{CDCl}_{3}\right)$

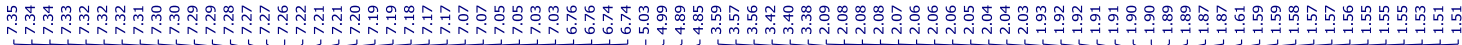

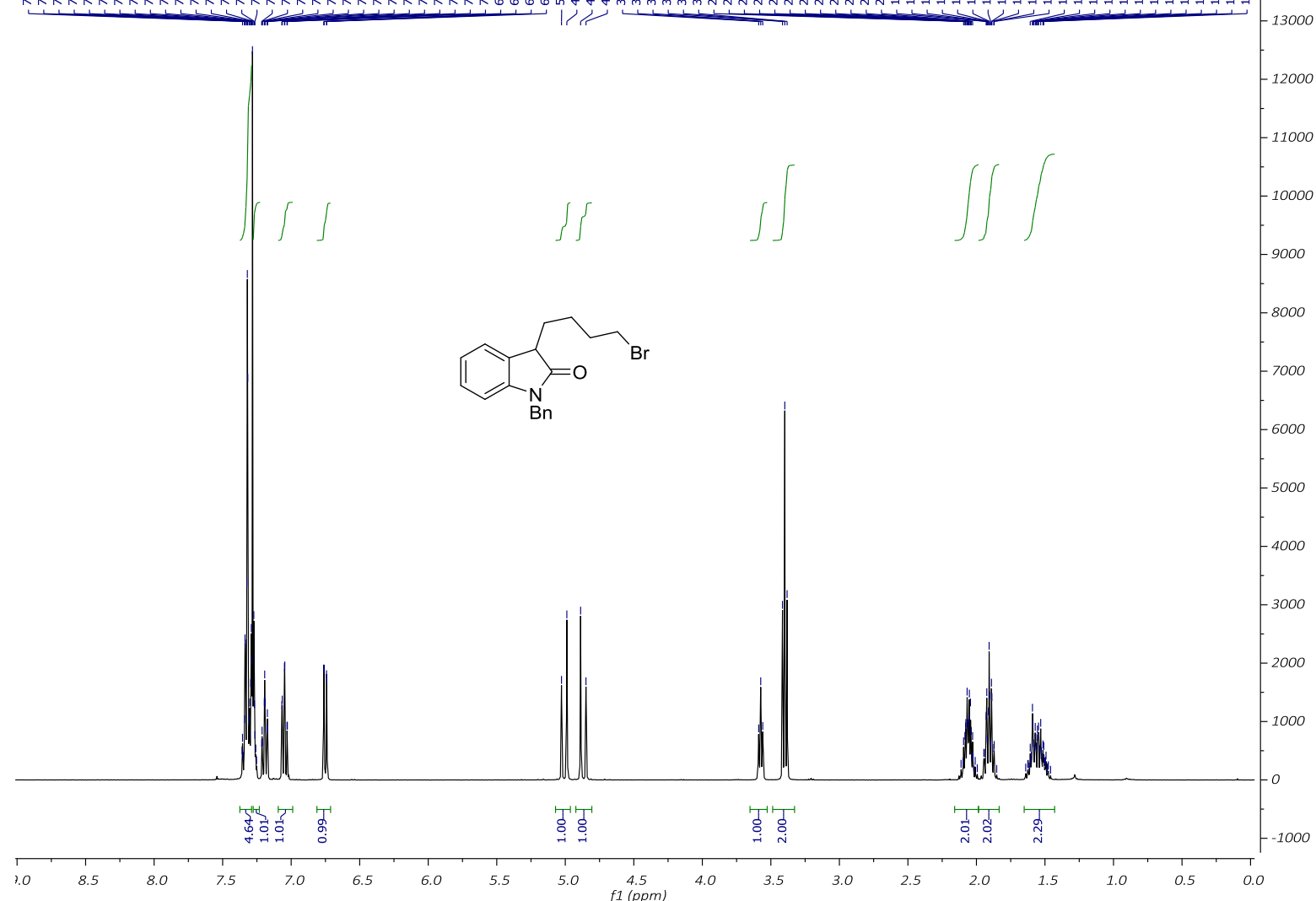

${ }^{13} \mathrm{C}\left\{{ }^{1} \mathrm{H}\right\}$ NMR of $10\left(101 \mathrm{MHz}, \mathrm{CDCl}_{3}\right)$
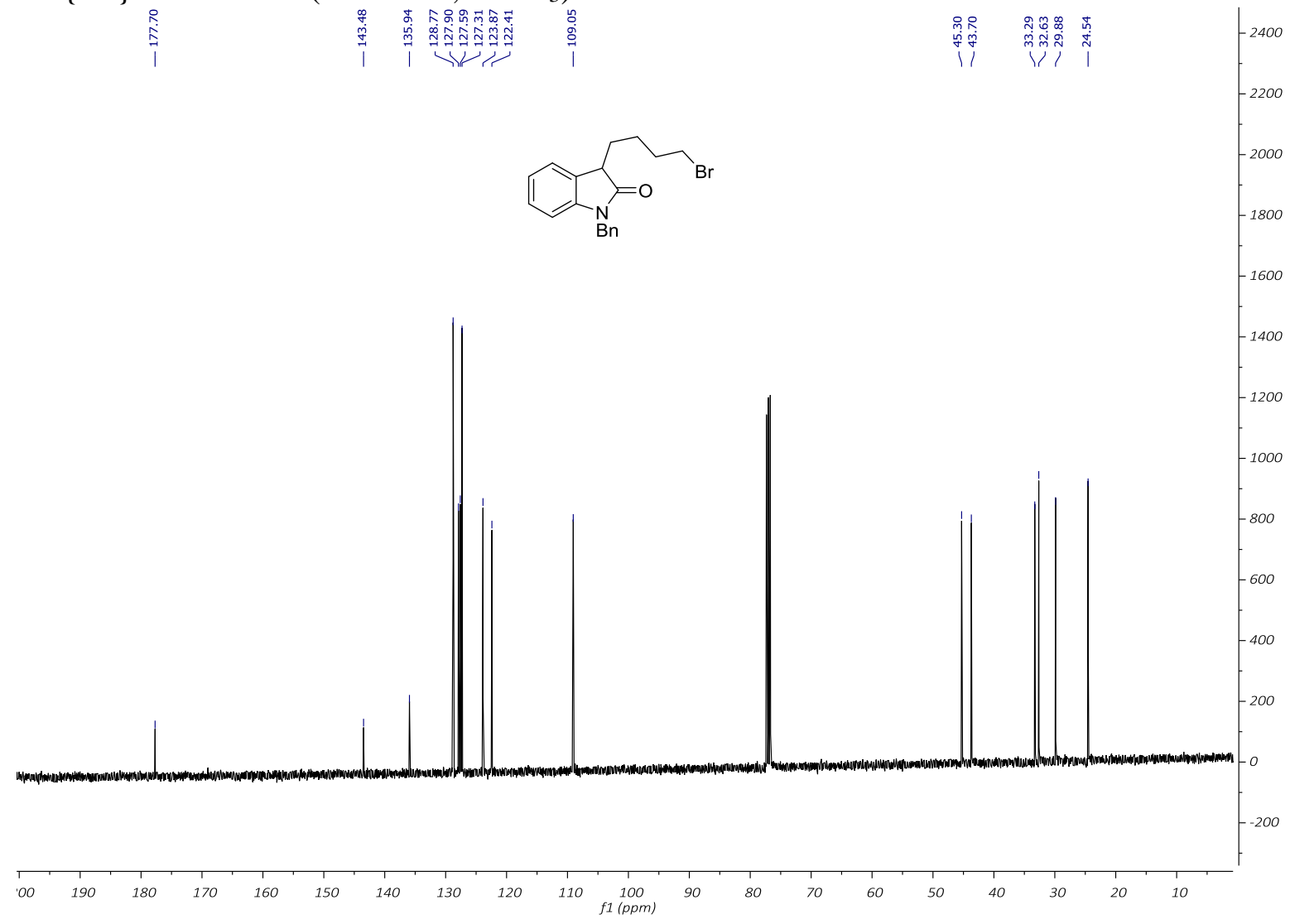
(1S,2S,3S)-1'-Benzyl-2'-oxo-2-phenylspiro[cyclopentane-1,3'-indoline]-3-carbaldehyde (3a)

${ }^{1} \mathrm{H} \mathrm{NMR}$ of $\mathbf{3 a}\left(400 \mathrm{MHz}, \mathrm{CDCl}_{3}\right)$

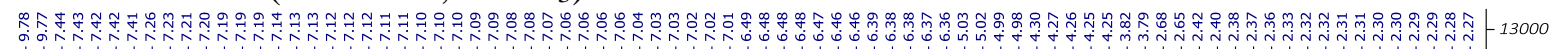

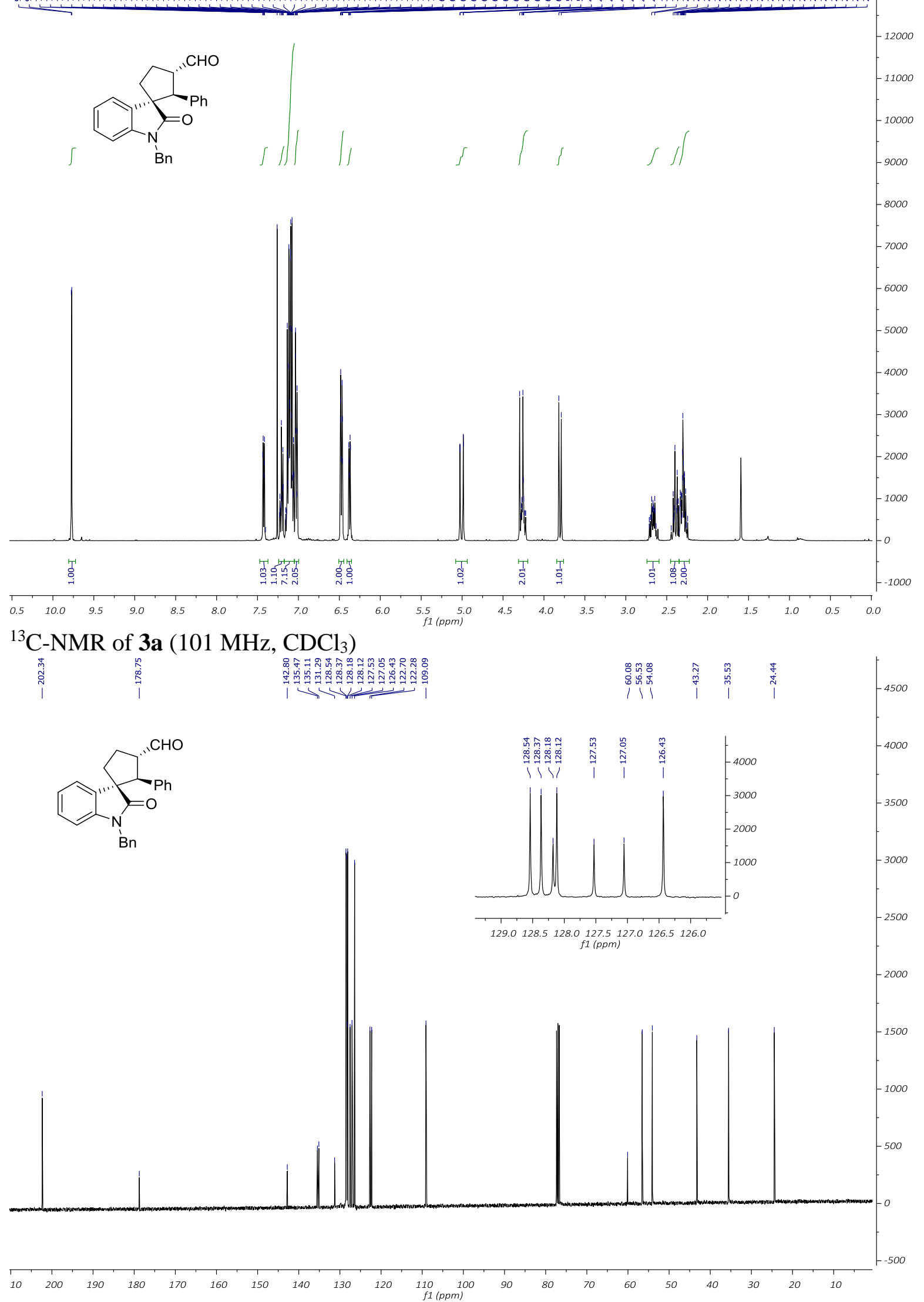


(1R,2S,3S)-1'-Benzyl-2'-oxo-2-phenylspiro[cyclopentane-1,3'-indoline]-3-carbaldehyde (4a)

${ }^{1} \mathrm{H} \mathrm{NMR}$ of $4 \mathbf{a}\left(400 \mathrm{MHz}, \mathrm{CDCl}_{3}\right)$

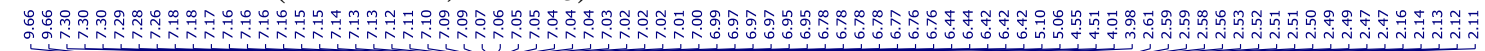

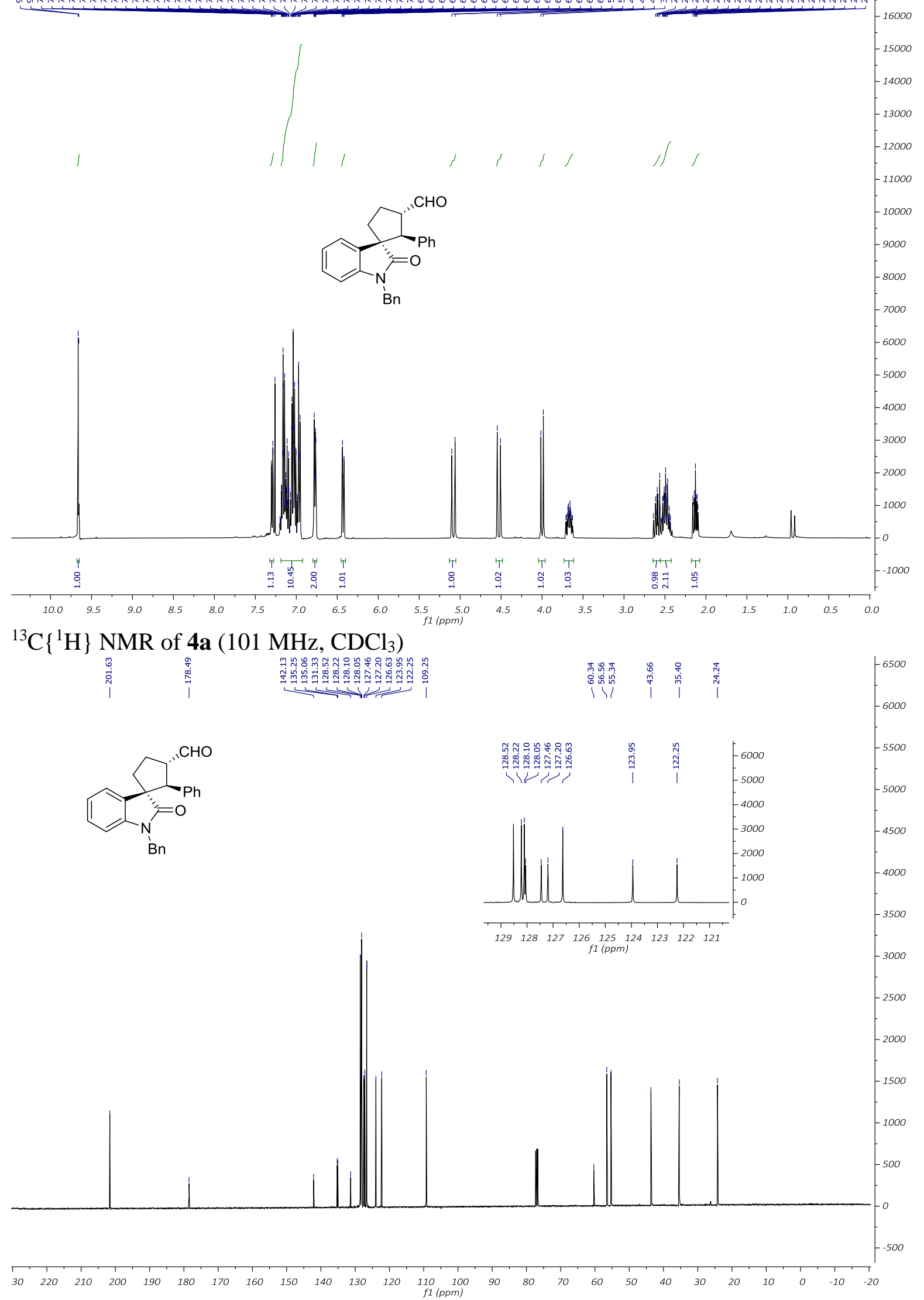


(1S,2S,3S)-1'-Allyl-2'-oxo-2-phenylspiro[cyclopentane-1,3'-indoline]-3-carbaldehyde (3b) ${ }^{1} \mathrm{H} \mathrm{NMR}$ of $\mathbf{3 b}\left(400 \mathrm{MHz}, \mathrm{CDCl}_{3}\right)$

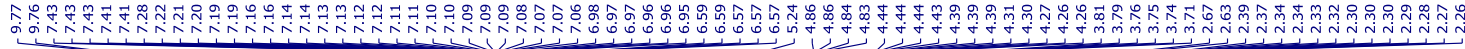

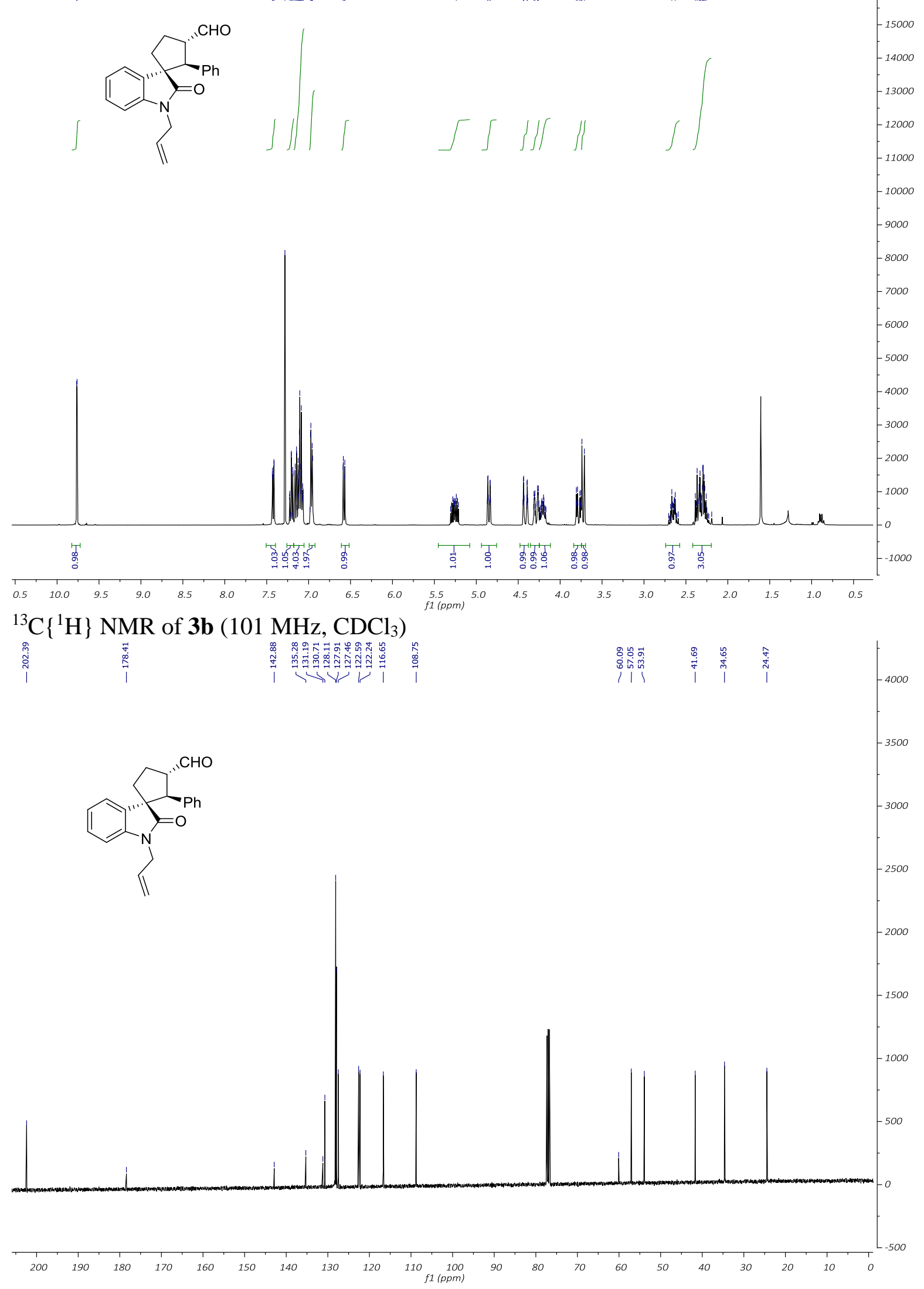


$(1 R, 2 S, 3 S)-1$ '-Allyl-2'-oxo-2-phenylspiro[cyclopentane-1,3'-indoline]-3-carbaldehyde (4b)

${ }^{1} \mathrm{H}$ NMR of $\mathbf{4 b}\left(400 \mathrm{MHz}, \mathrm{CDCl}_{3}\right)$

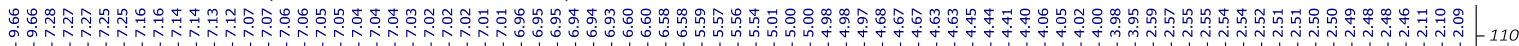

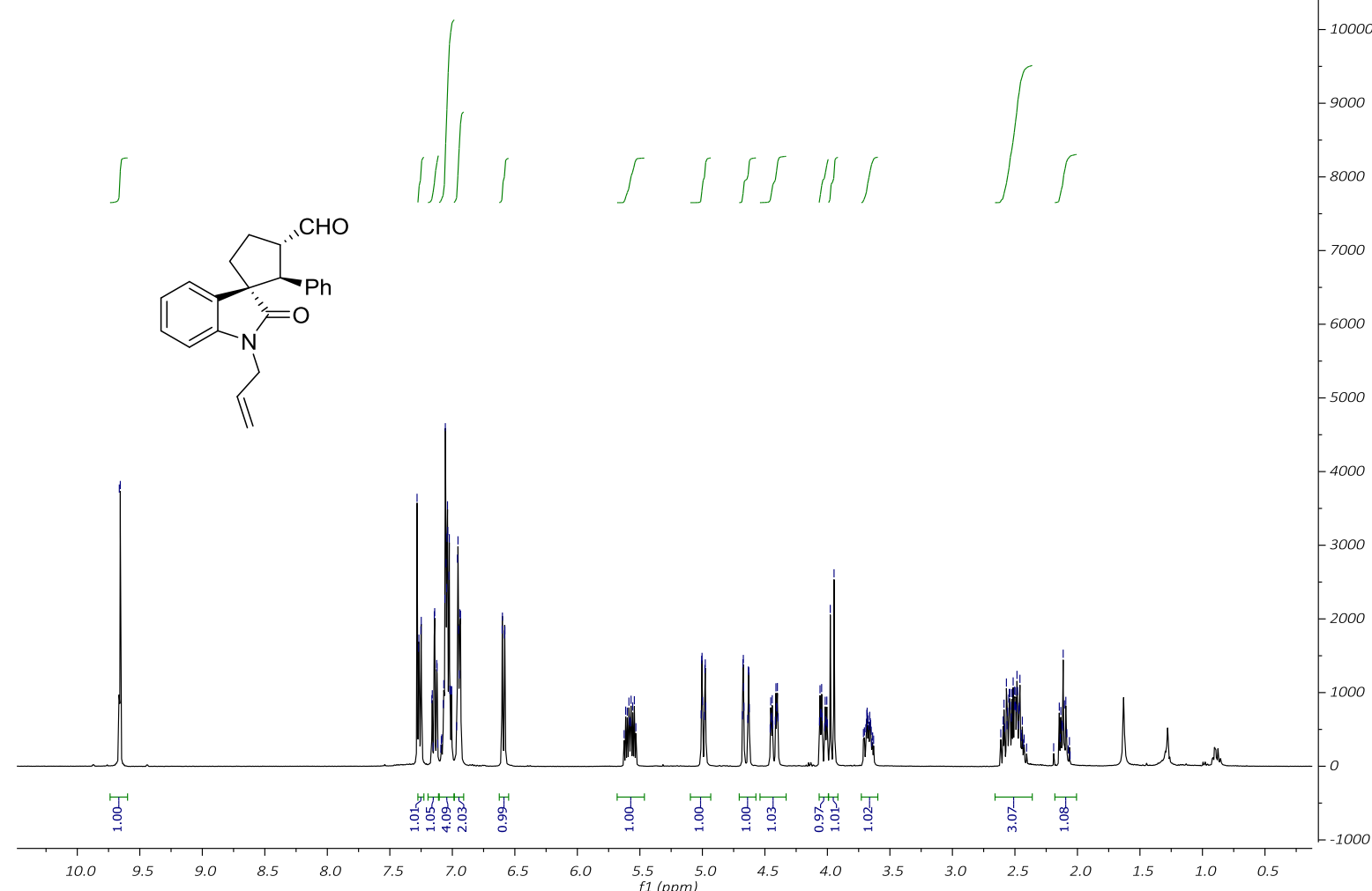

${ }^{13} \mathrm{C}\left\{{ }^{1} \mathrm{H}\right\} \mathrm{NMR}$ of $\mathbf{4 b}\left(101 \mathrm{MHz}, \mathrm{CDCl}_{3}\right)$

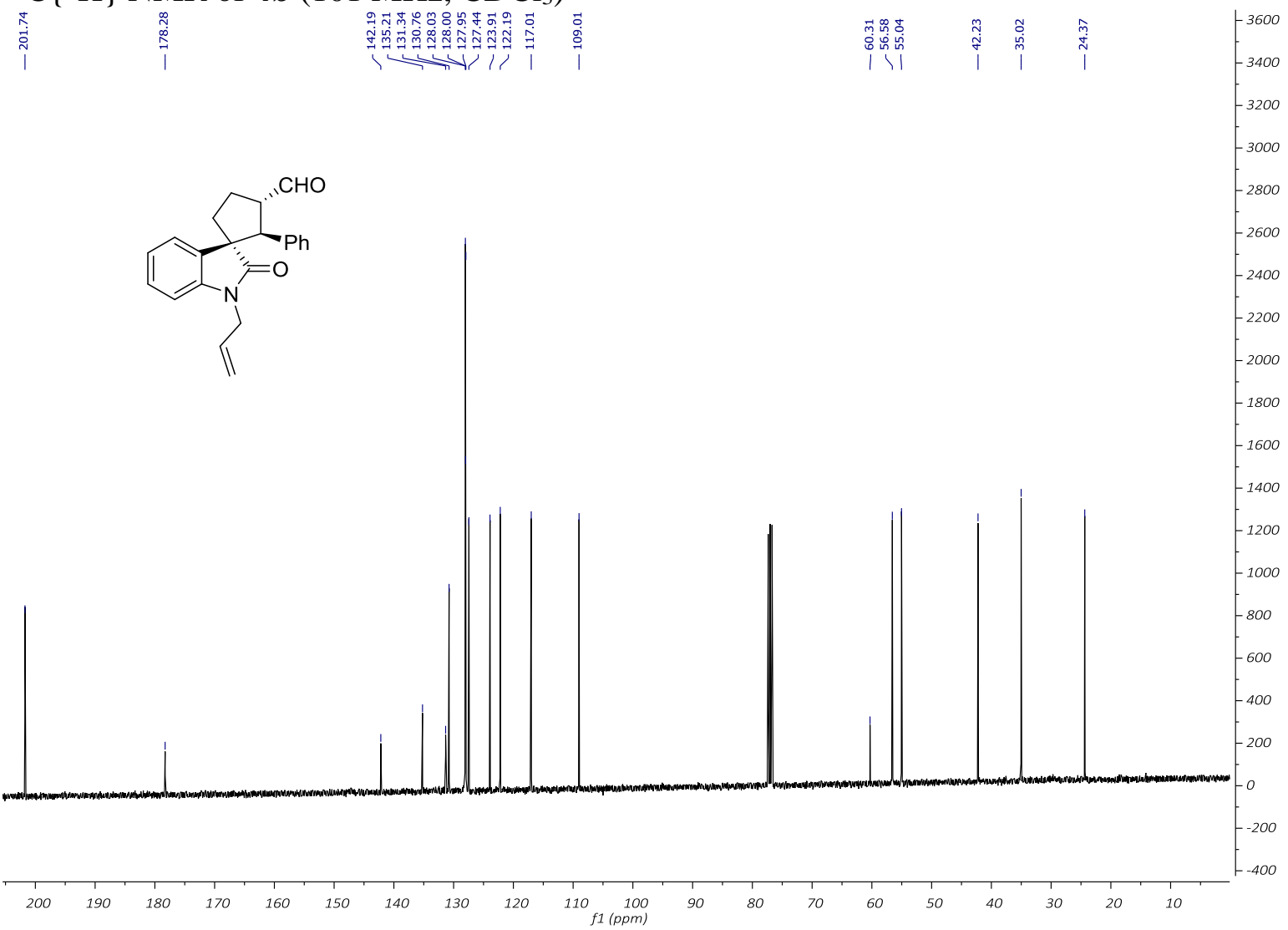


(1S,2S,3S)-1'-Methyl-2'-oxo-2-phenylspiro[cyclopentane-1,3'-indoline]-3-carbaldehyde (3c)

${ }^{1} \mathrm{H} \mathrm{NMR}$ of $3 \mathbf{c}\left(400 \mathrm{MHz}, \mathrm{CDCl}_{3}\right)$

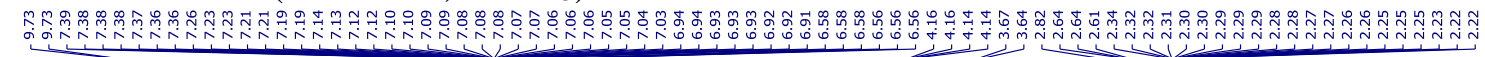

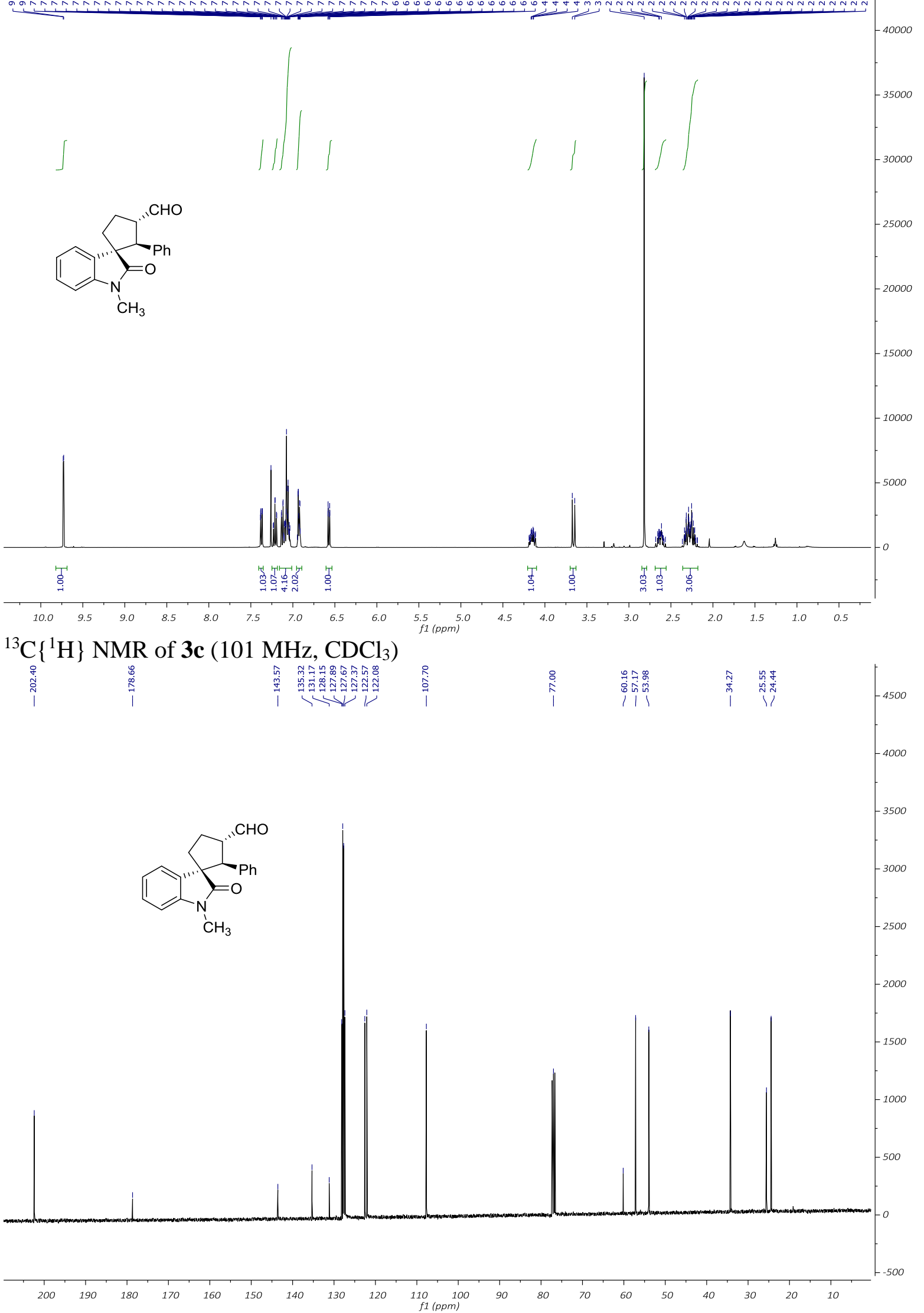


$(1 R, 2 S, 3 S)$-1'-Methyl-2'-oxo-2-phenylspiro[cyclopentane-1,3'-indoline]-3-carbaldehyde (4c)

${ }^{1} \mathrm{H}$ NMR of $4 \mathbf{c}\left(400 \mathrm{MHz}, \mathrm{CDCl}_{3}\right)$

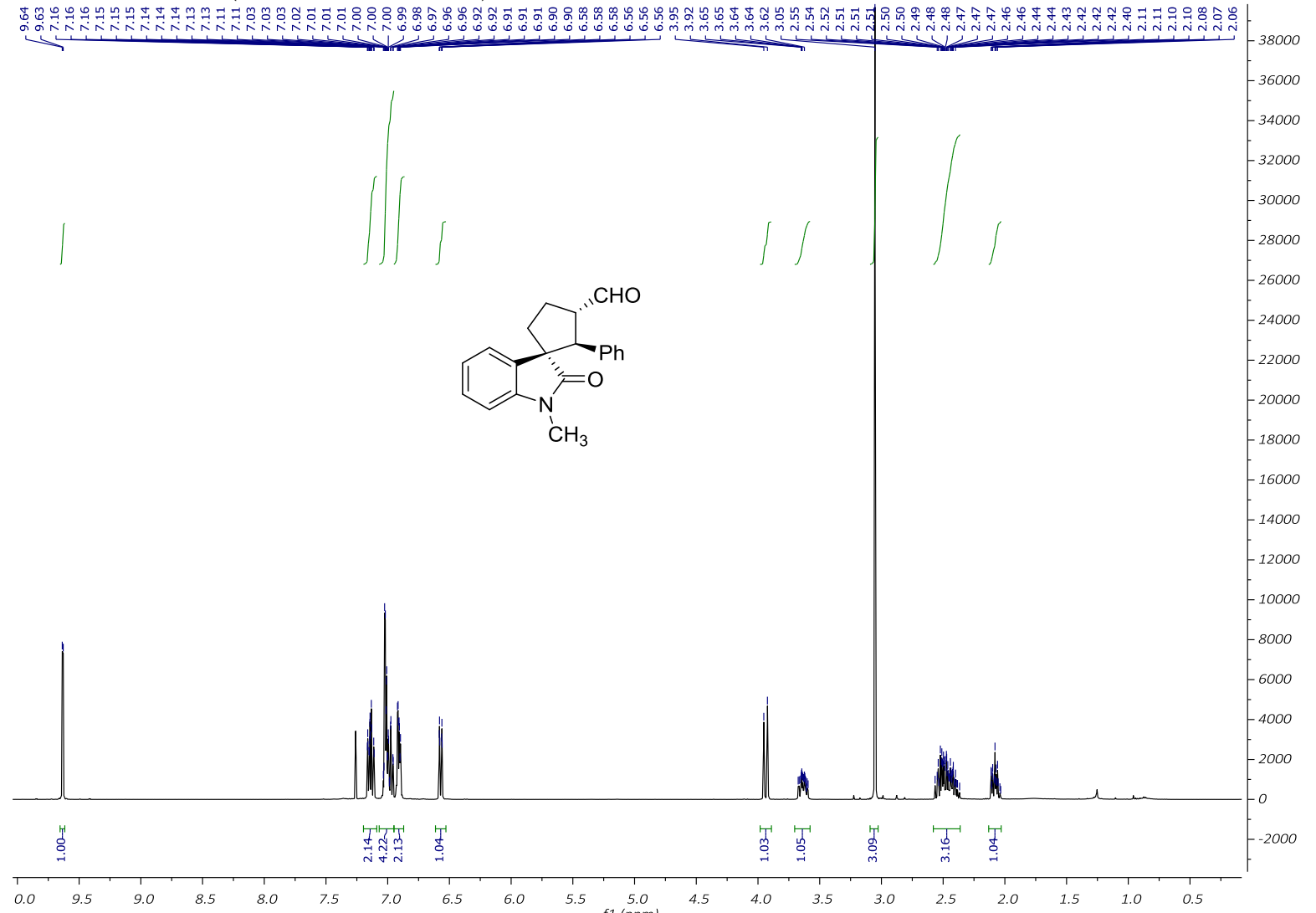

${ }^{13} \mathrm{C}\left\{{ }^{1} \mathrm{H}\right\}$ NMR of $4 \mathbf{c}\left(101 \mathrm{MHz}, \mathrm{CDCl}_{3}\right)$

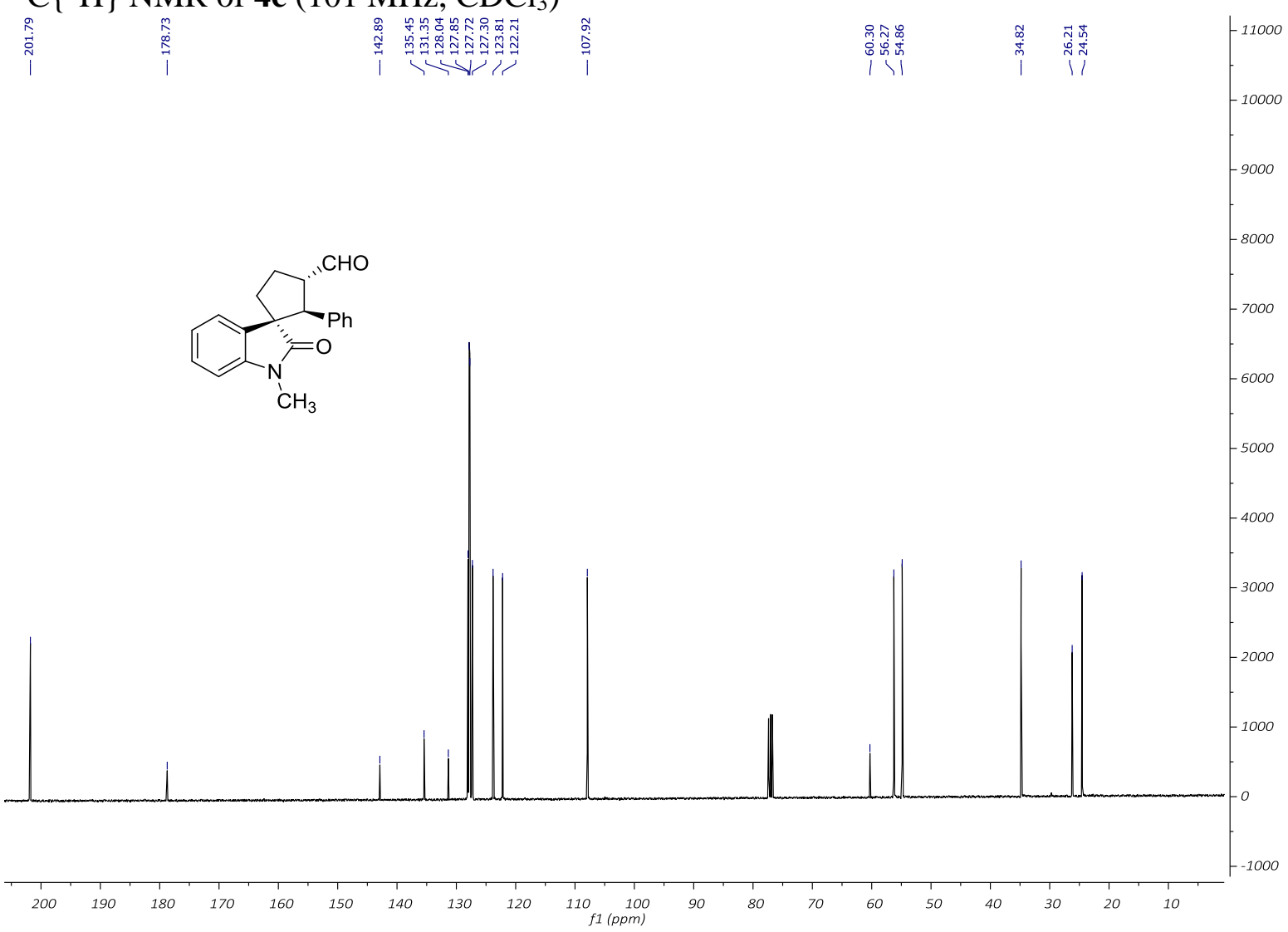




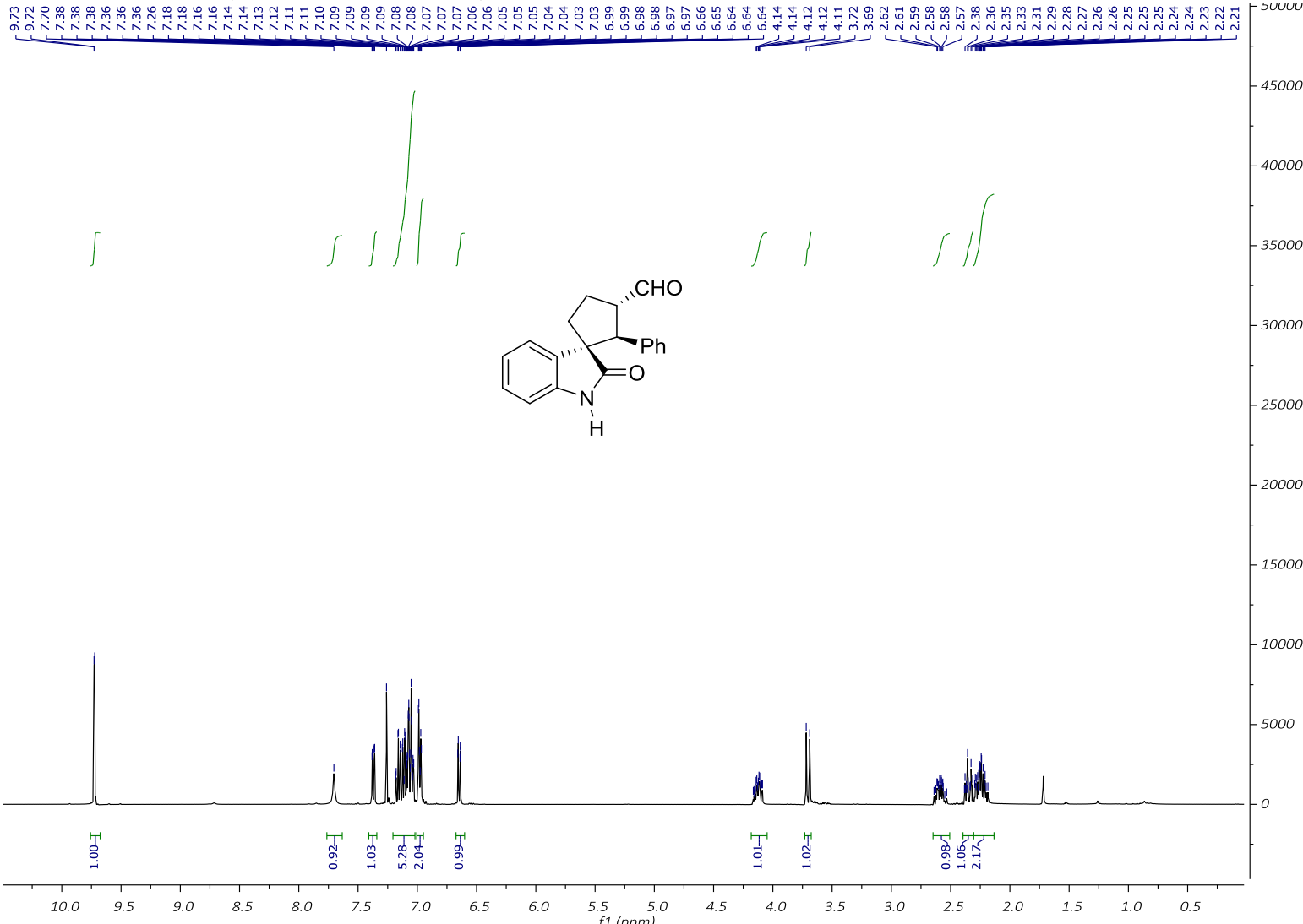

${ }^{13} \mathrm{C}\left\{{ }^{1} \mathrm{H}\right\}$ NMR of $3 d\left(101 \mathrm{MHz}, \mathrm{CDCl}_{3}\right)$

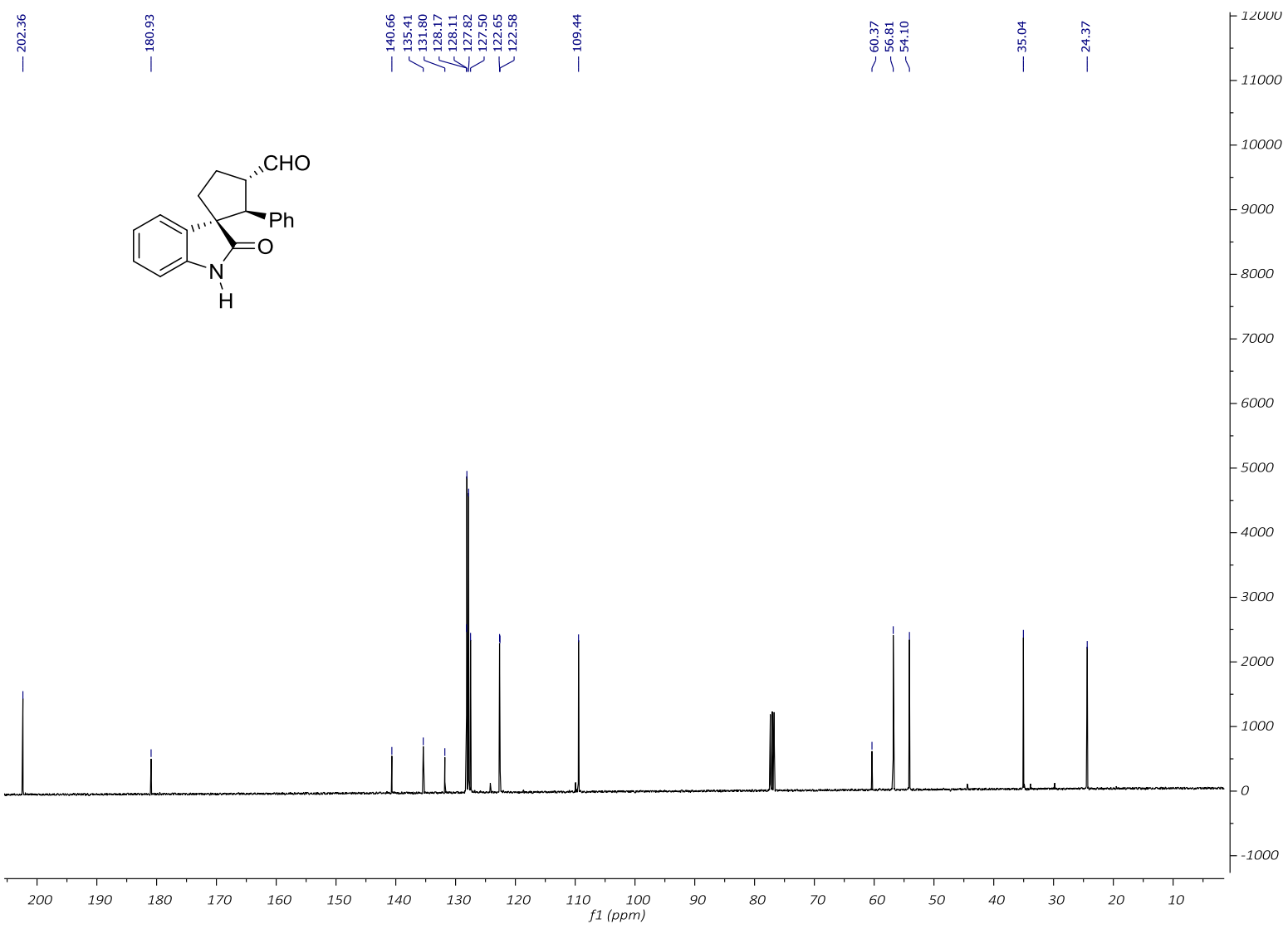




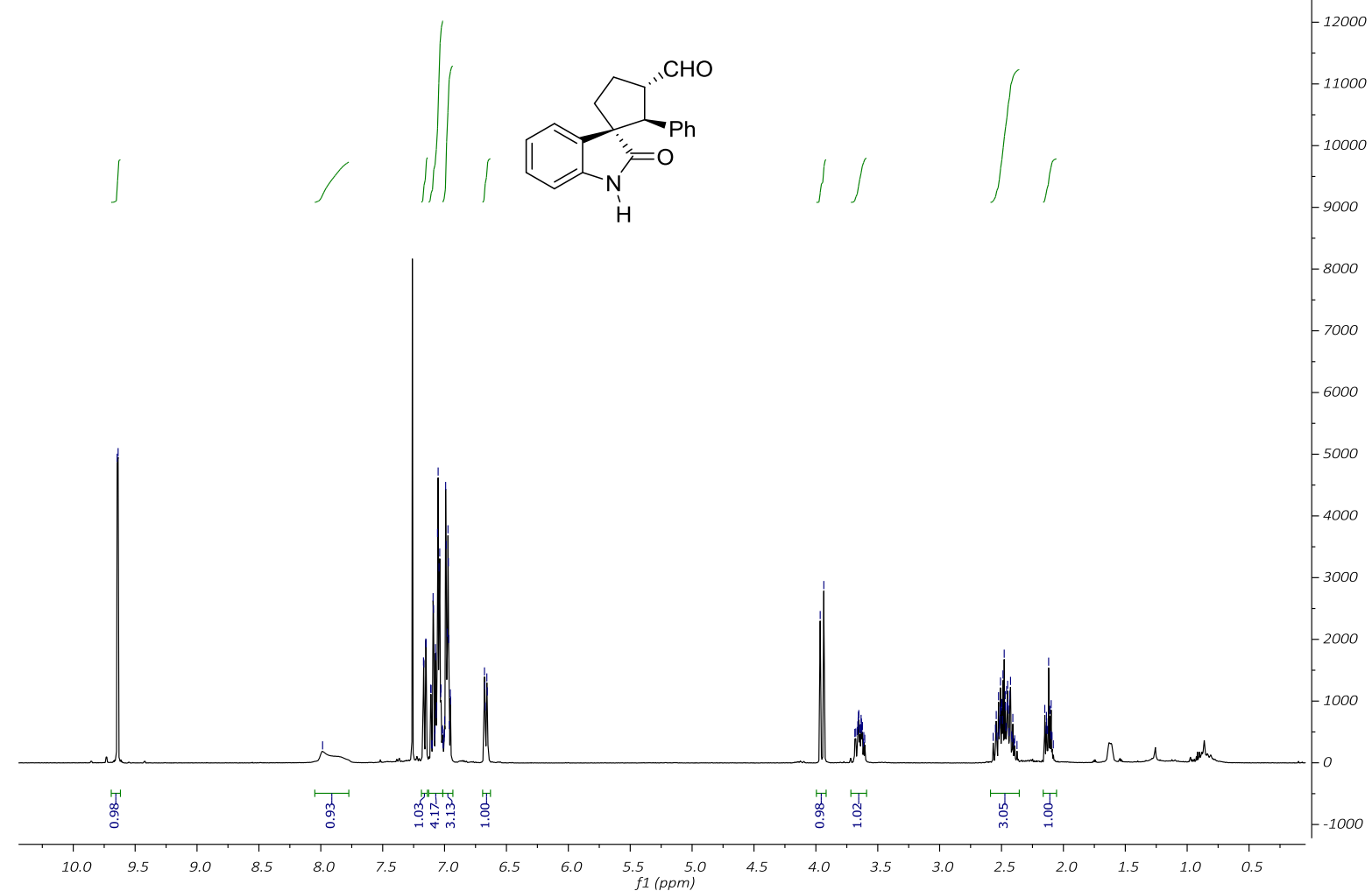

${ }^{13} \mathrm{C}\left\{{ }^{1} \mathrm{H}\right\}$ NMR of $\mathbf{4 d}\left(101 \mathrm{MHz}, \mathrm{CDCl}_{3}\right)$

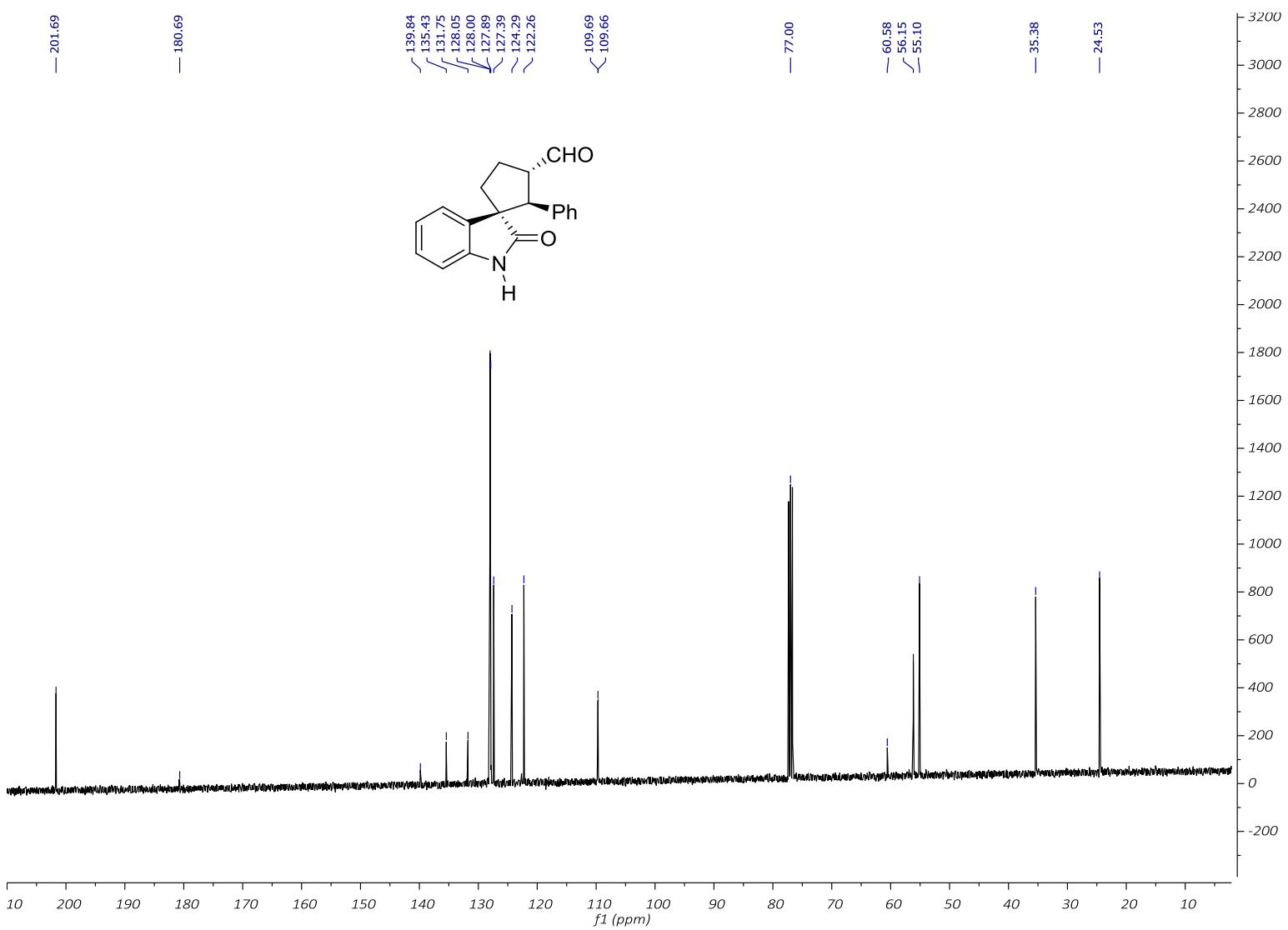


(1S,2S,3S)-1'-Benzyl-5'-methyl-2'-oxo-2-phenylspiro[cyclopentane-1,3'-indoline]-3carbaldehyde (3e)

${ }^{1} \mathrm{H} \mathrm{NMR}$ of $3 \mathbf{e}\left(400 \mathrm{MHz}, \mathrm{CDCl}_{3}\right)$

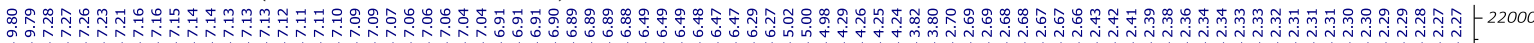
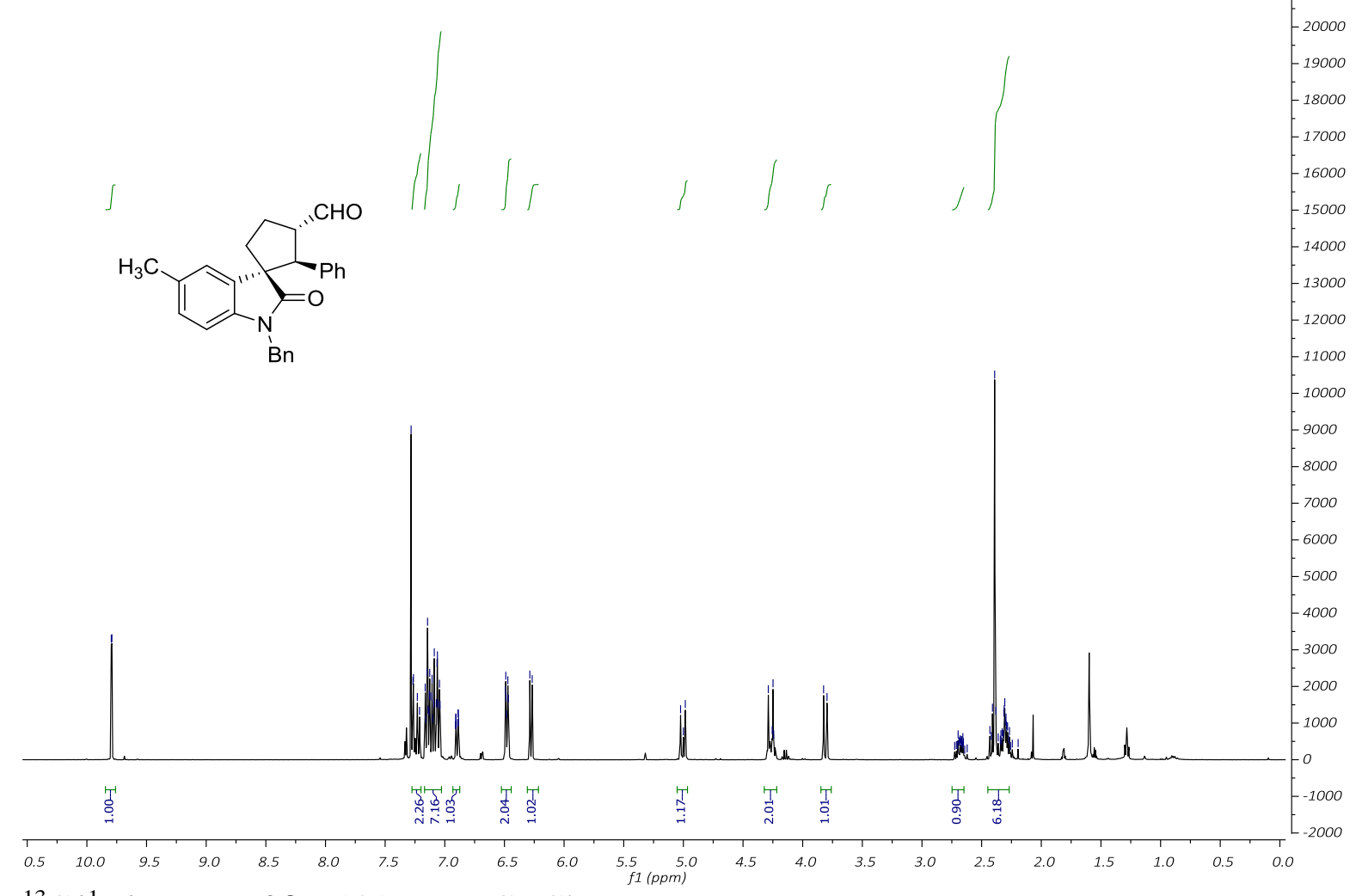

${ }^{13} \mathrm{C}\left\{{ }^{1} \mathrm{H}\right\}$ NMR of $\mathbf{3 e}\left(101 \mathrm{MHz}, \mathrm{CDCl}_{3}\right)$

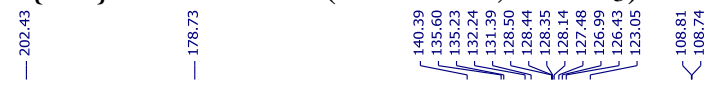
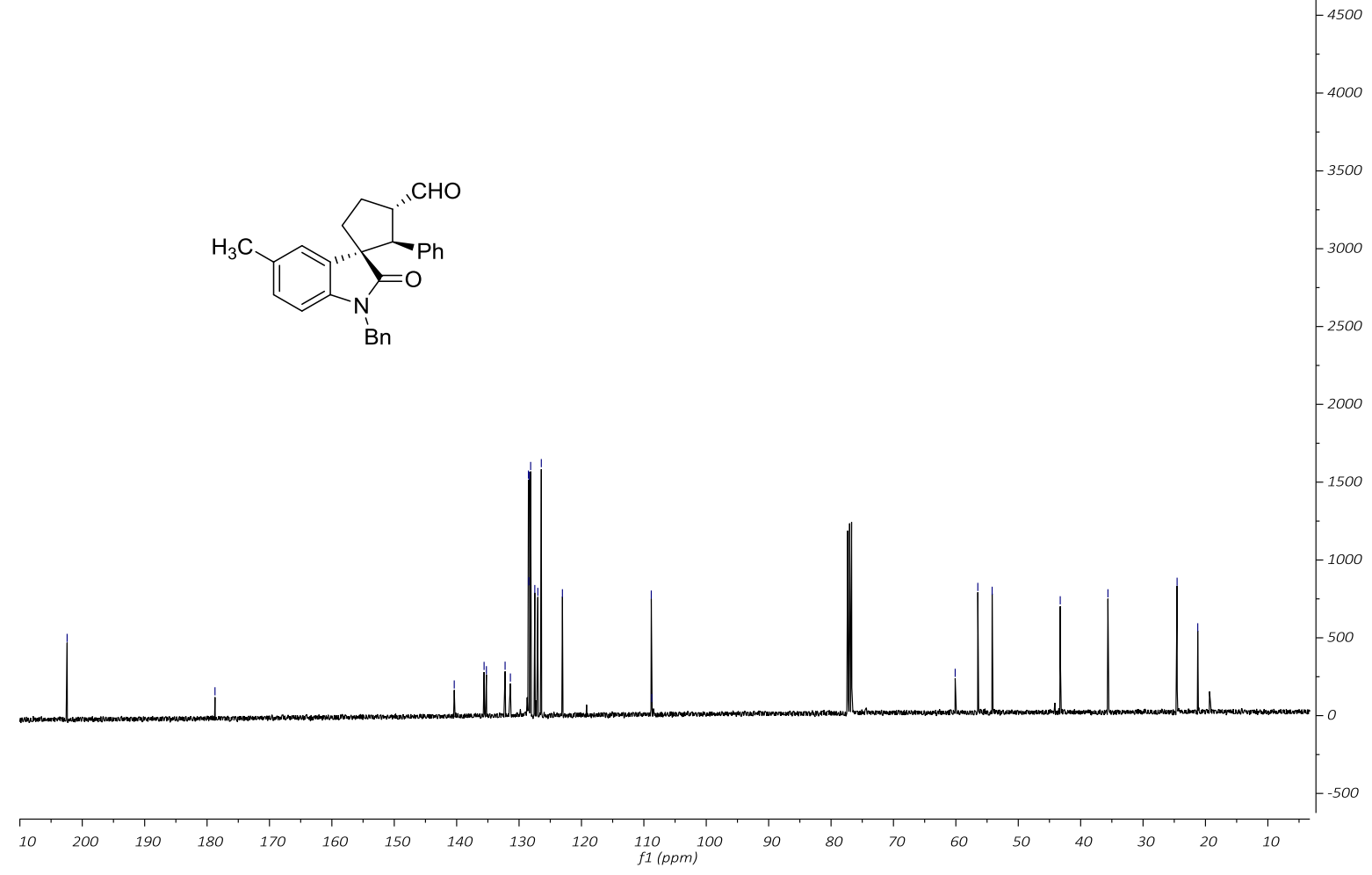
$(1 R, 2 S, 3 S)-1$ '-Benzyl-5'-methyl-2' -oxo-2-phenylspiro[cyclopentane-1,3'-indoline]-3carbaldehyde (4e)

${ }^{1} \mathrm{H} \mathrm{NMR}$ of $4 \mathbf{e}\left(400 \mathrm{MHz}, \mathrm{CDCl}_{3}\right)$

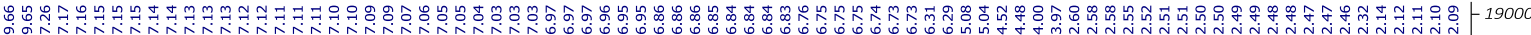

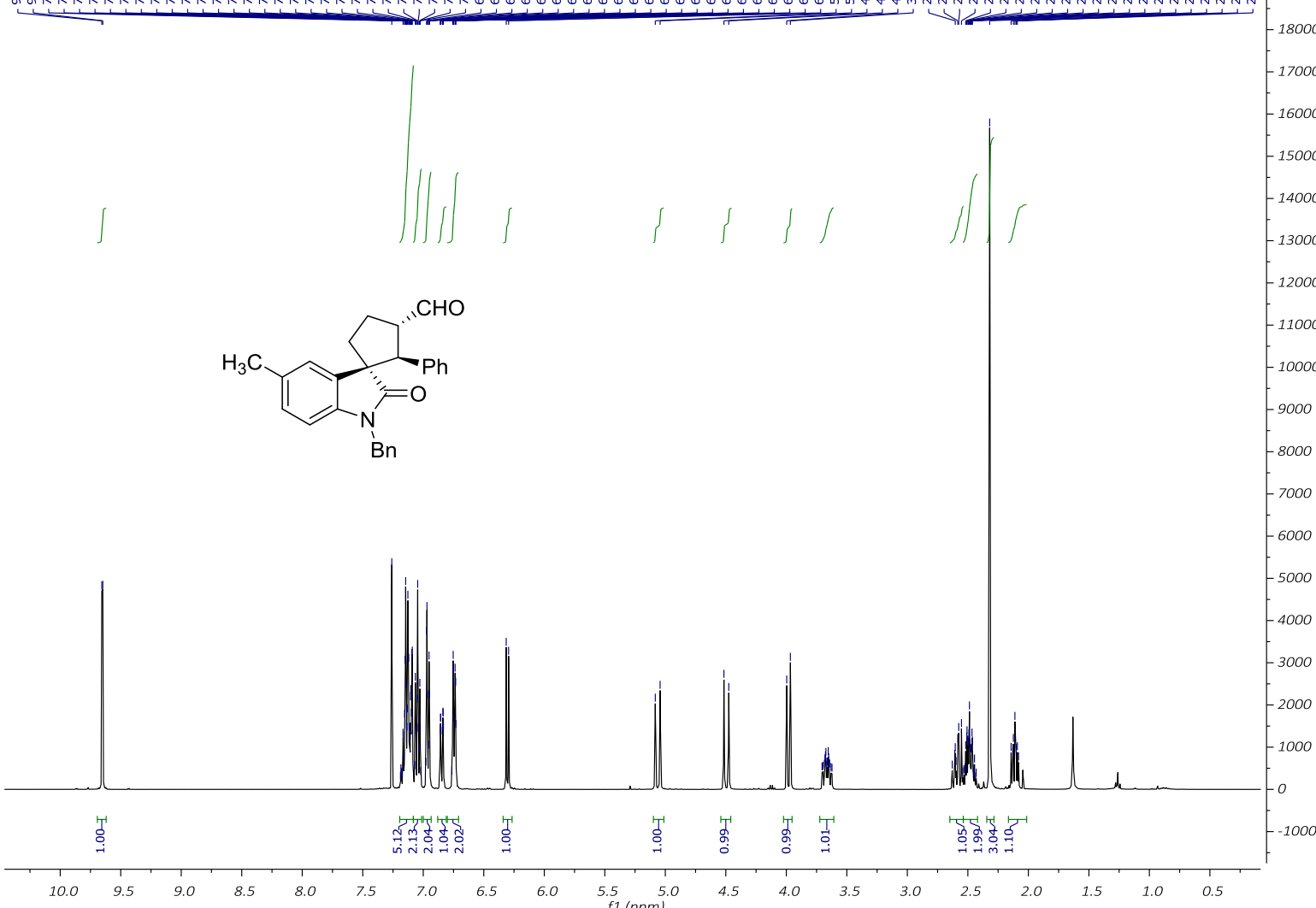

${ }^{13} \mathrm{C}\left\{{ }^{1} \mathrm{H}\right\}$ NMR of $4 \mathbf{e}\left(101 \mathrm{MHz}, \mathrm{CDCl}_{3}\right)$
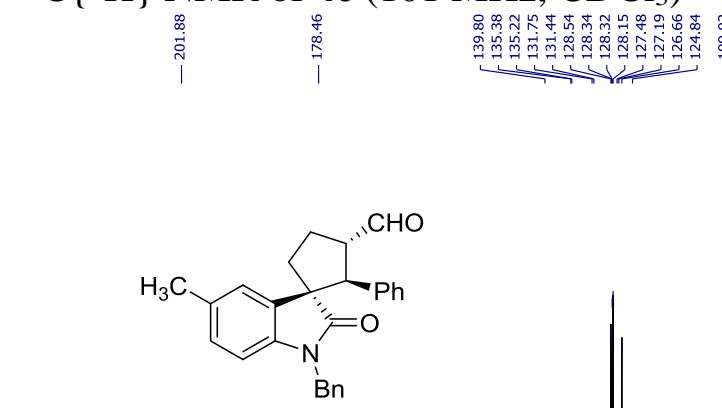

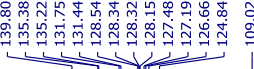

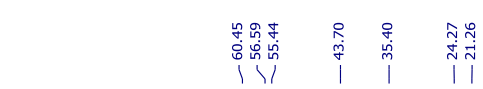

6500

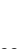

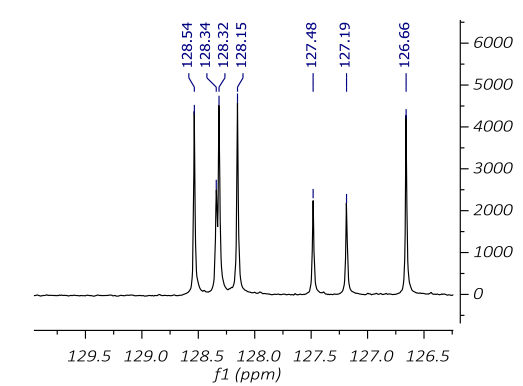

000

00

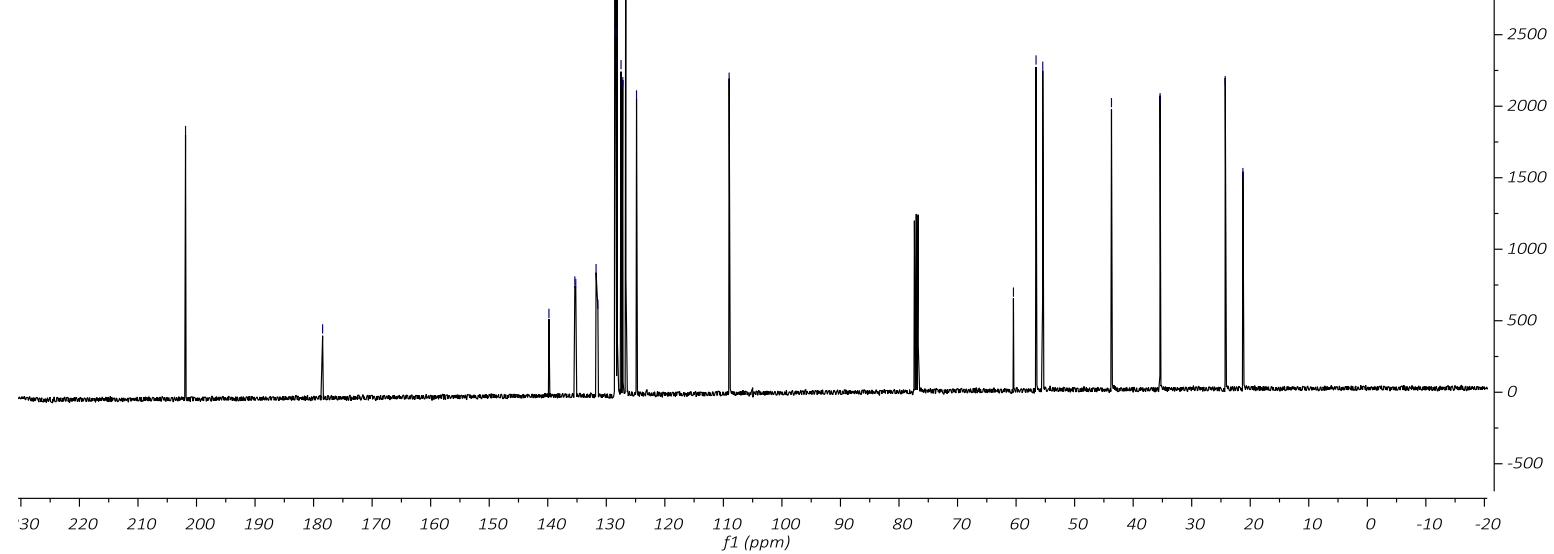


(1S,2S,3S)-1'-Benzyl-5'-methoxy-2' -oxo-2-phenylspiro[cyclopentane-1,3'-indoline]-3carbaldehyde (3f)

${ }^{1} \mathrm{H}$ NMR of $\mathbf{3 f}\left(400 \mathrm{MHz}, \mathrm{CDCl}_{3}\right)$

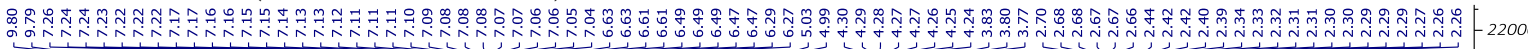<smiles>[2H]C1C(C=O)CCC12Cc1cc(OC)ccc1N(Cc1ccccc1)C2=O</smiles>
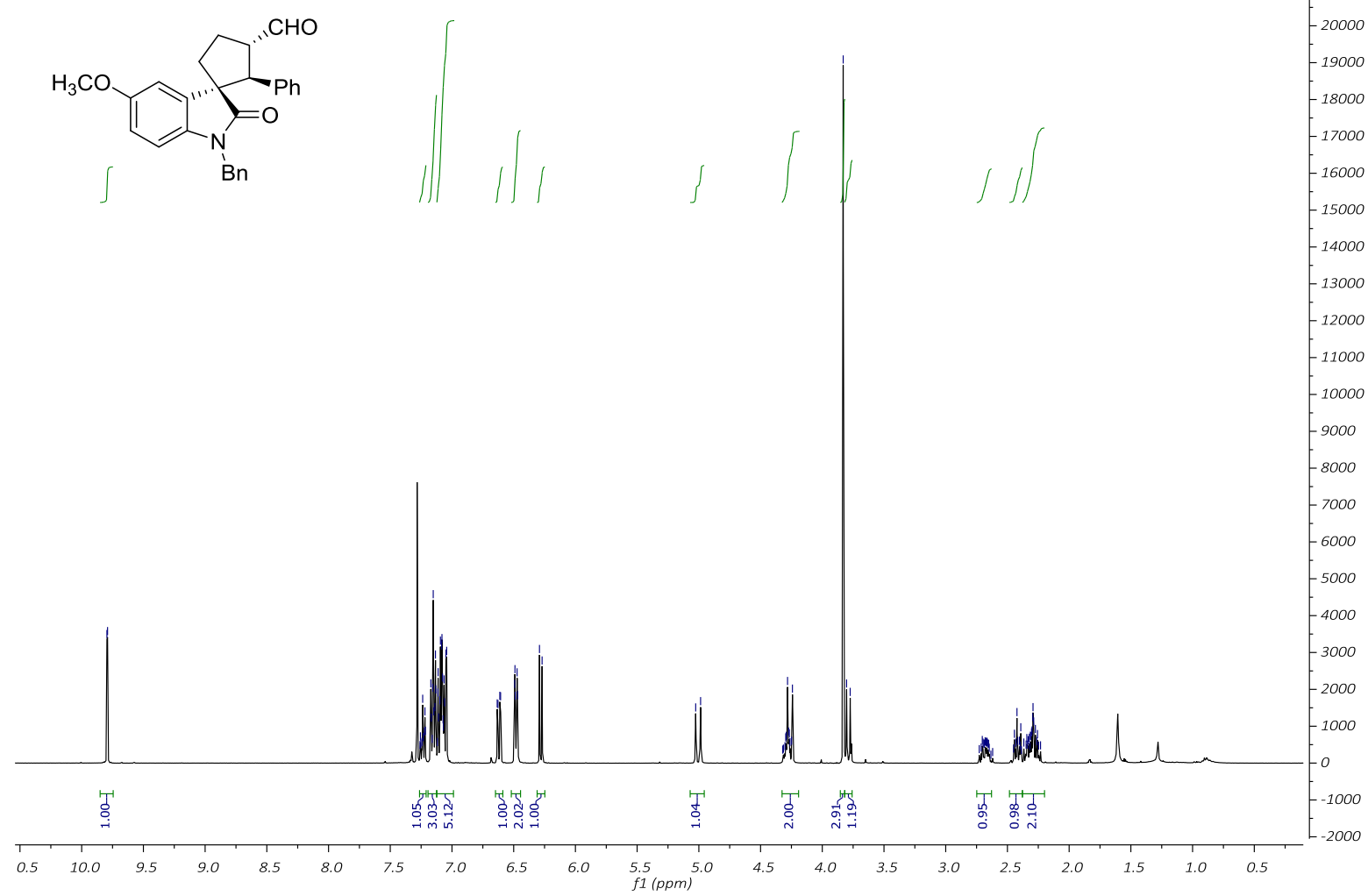

${ }^{13} \mathrm{C}\left\{{ }^{1} \mathrm{H}\right\}$ NMR of $\mathbf{3 f}\left(101 \mathrm{MHz}, \mathrm{CDCl}_{3}\right)$
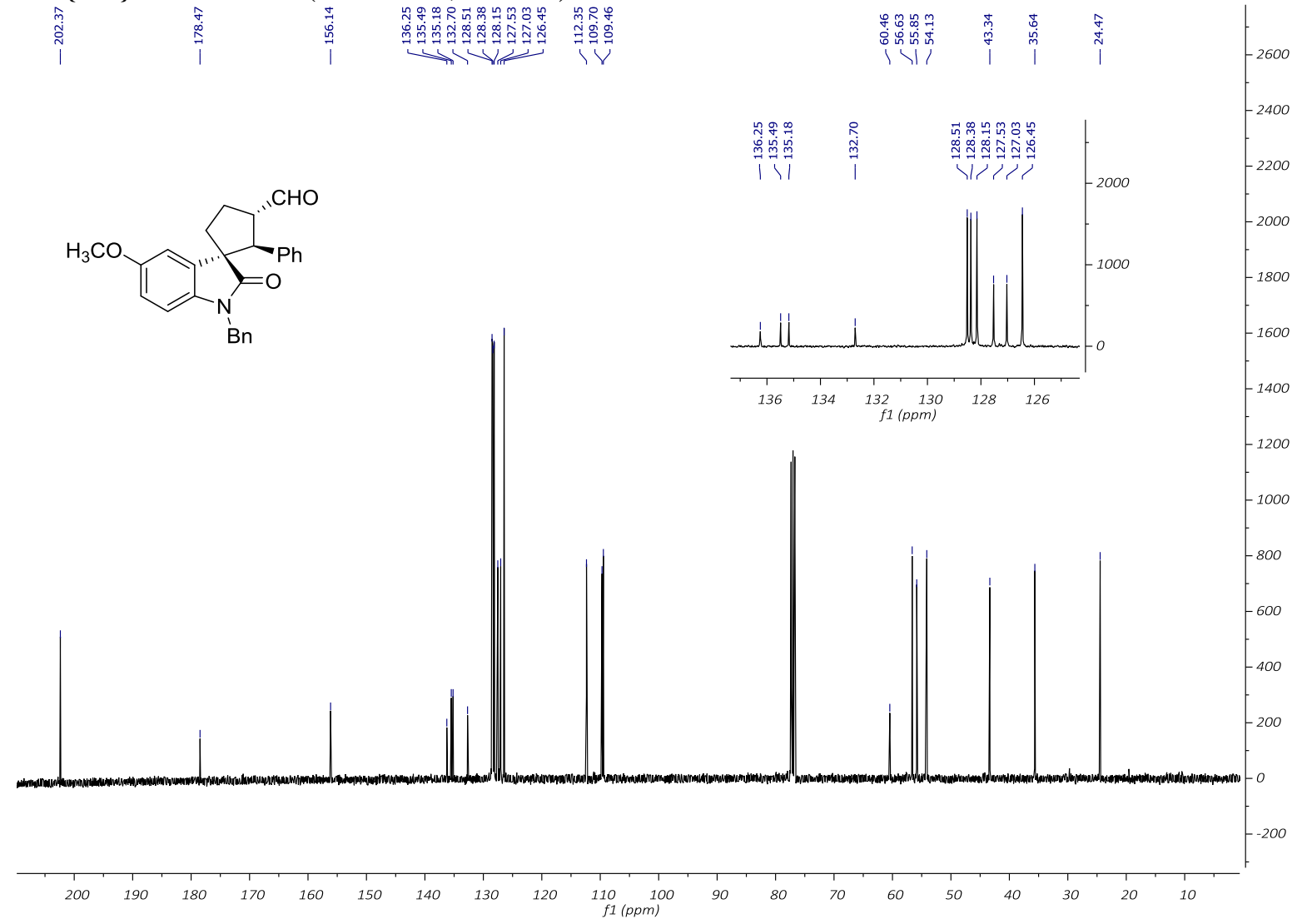
(1R,2S,3S)-1'-Benzyl-5'-methoxy-2'-oxo-2-phenylspiro[cyclopentane-1,3'-indoline]-3carbaldehyde (4f)

${ }^{1} \mathrm{H} \mathrm{NMR}$ of $\mathbf{4 f}\left(400 \mathrm{MHz}, \mathrm{CDCl}_{3}\right)$
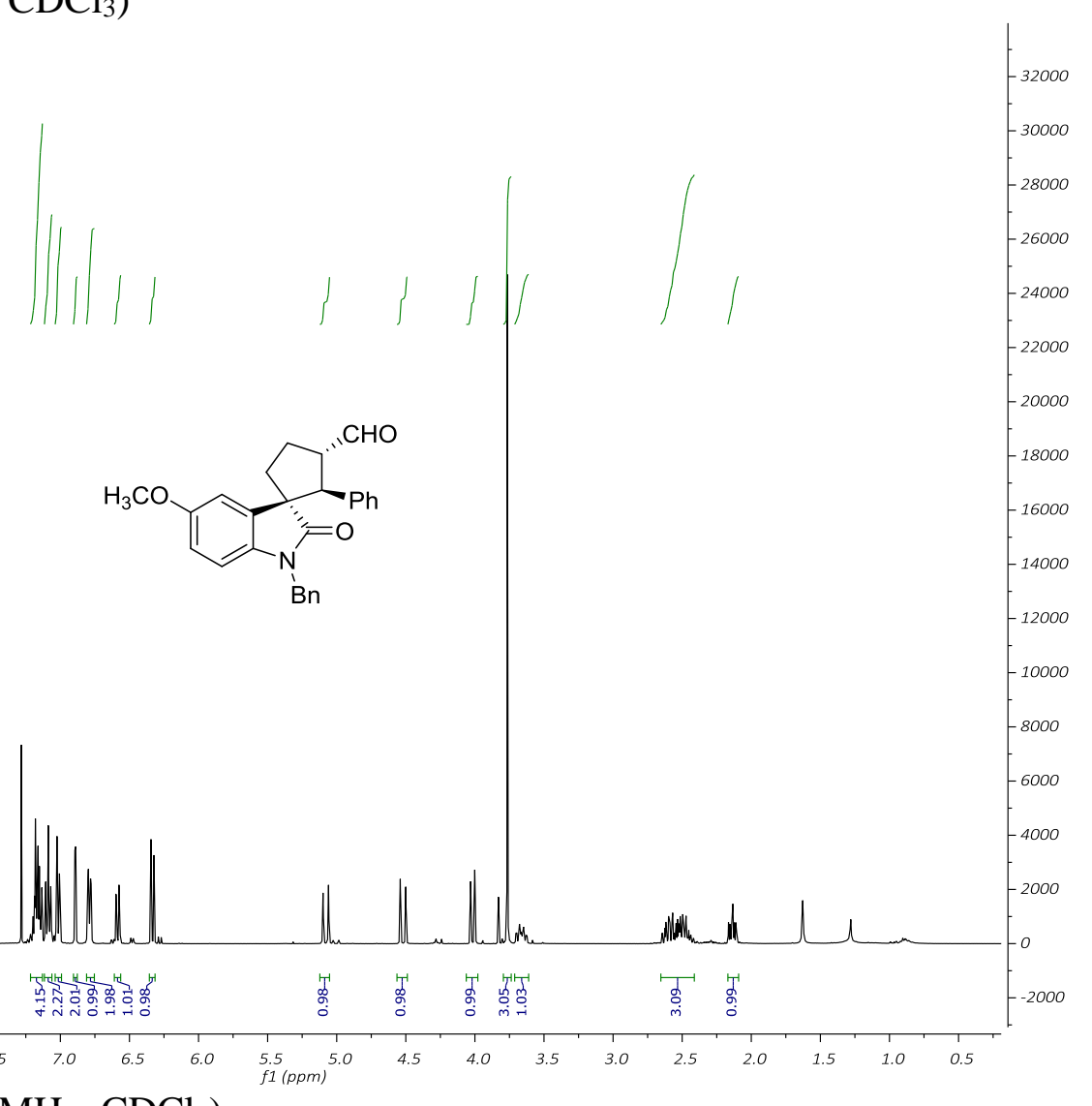

${ }^{13} \mathrm{C}\left\{{ }^{1} \mathrm{H}\right\} \mathrm{NMR}$ of $\mathbf{4 f}\left(101 \mathrm{MHz}, \mathrm{CDCl}_{3}\right)$
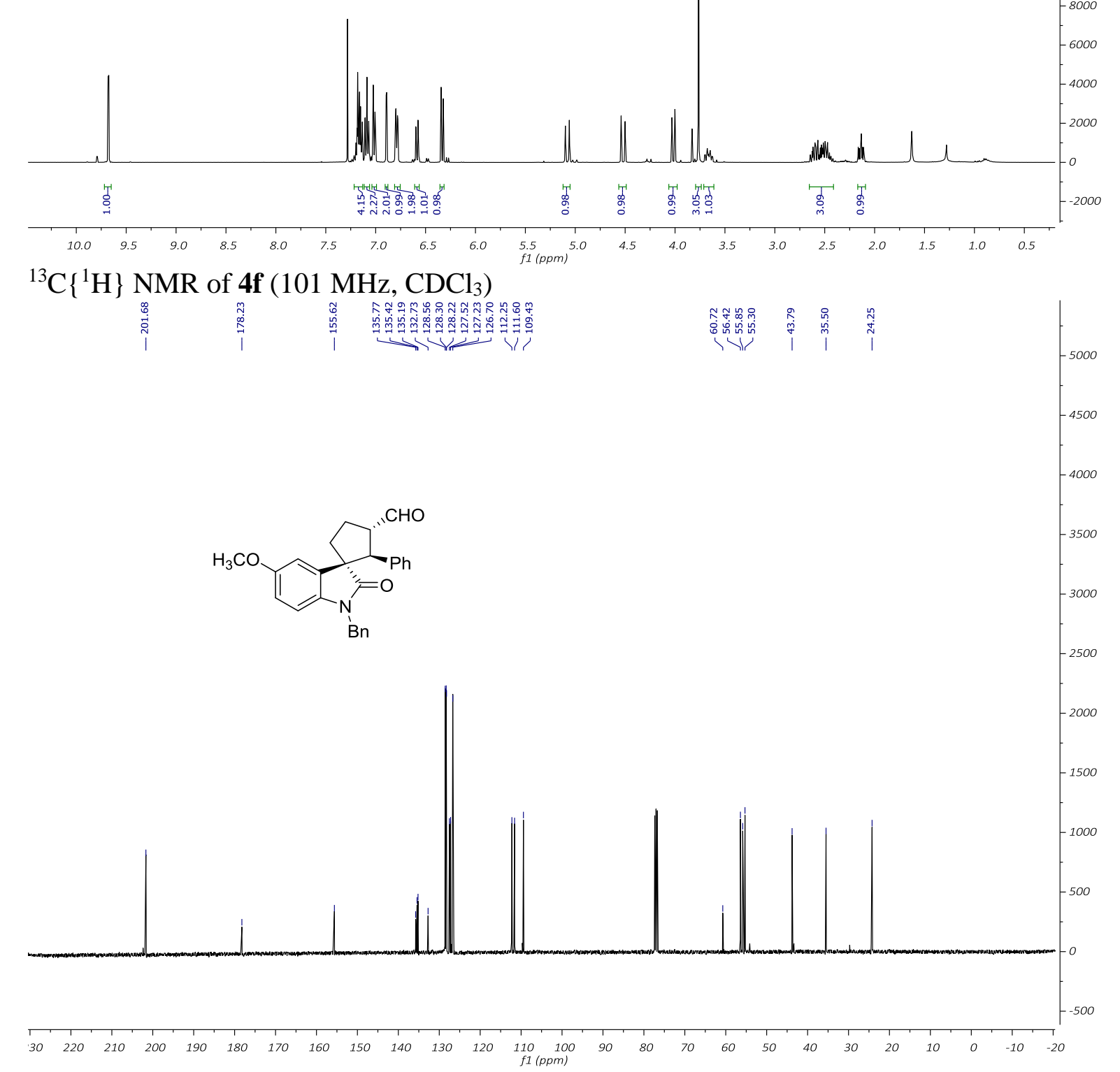
(1S,2S,3S)-1'-Benzyl-5'-fluoro-2'-oxo-2-phenylspiro[cyclopentane-1,3'-indoline]-3carbaldehyde (3g)

${ }^{1} \mathrm{H}$ NMR of $\mathbf{3 g}\left(400 \mathrm{MHz}, \mathrm{CDCl}_{3}\right)$

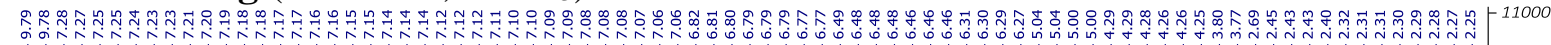

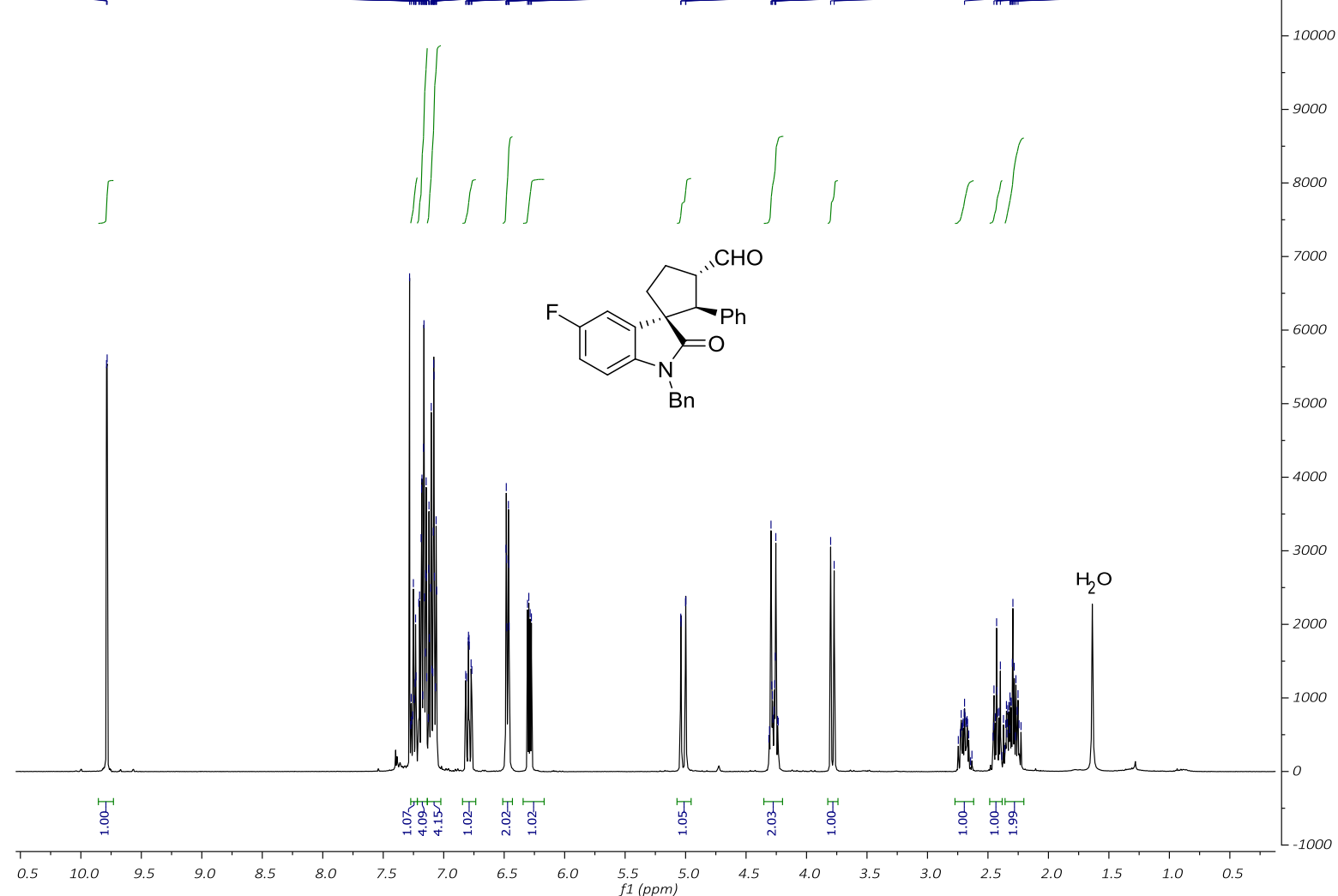

${ }^{13} \mathrm{C}\left\{{ }^{1} \mathrm{H}\right\}$ NMR of $\mathbf{3 g}\left(101 \mathrm{MHz}, \mathrm{CDCl}_{3}\right)$

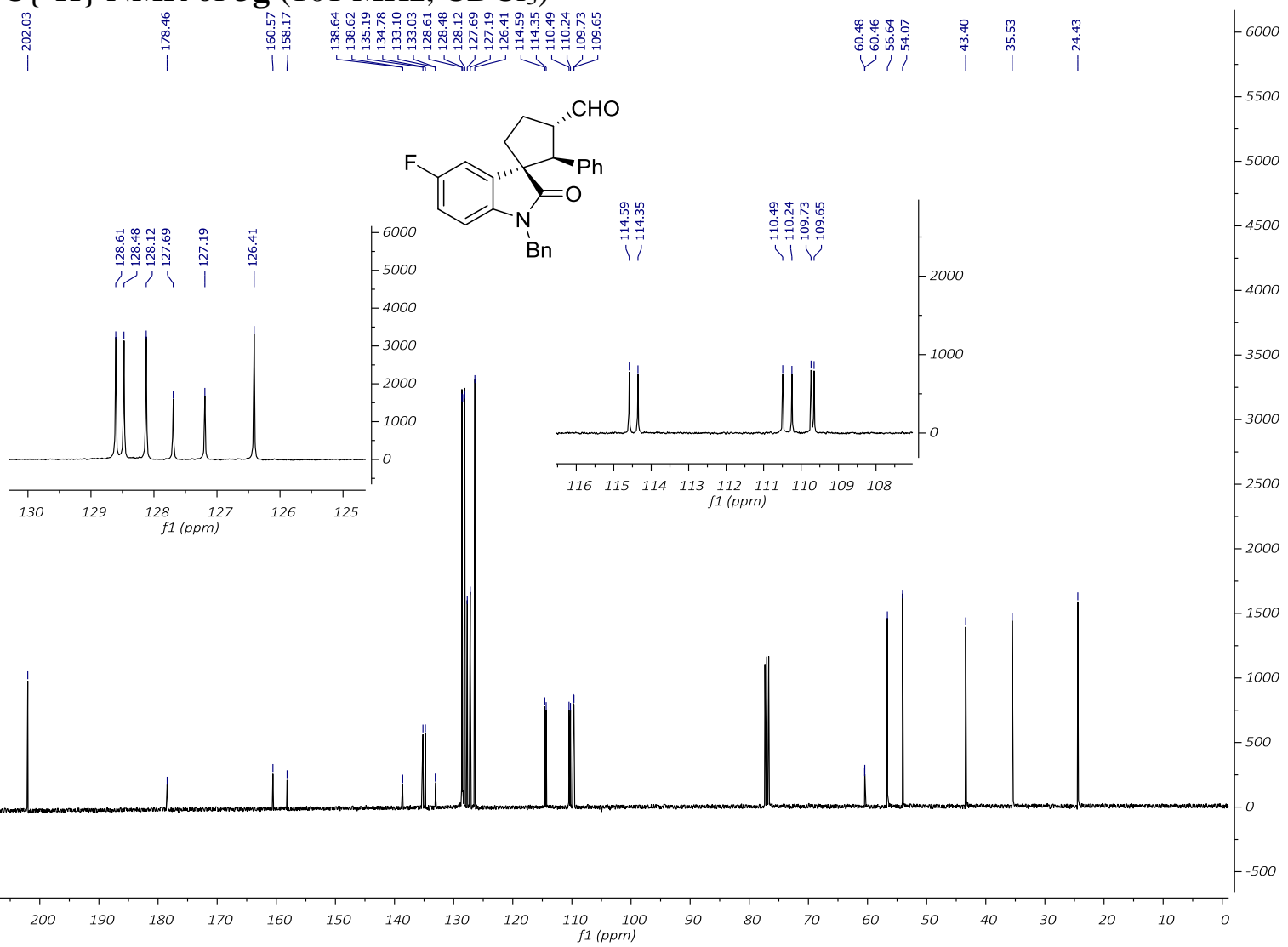




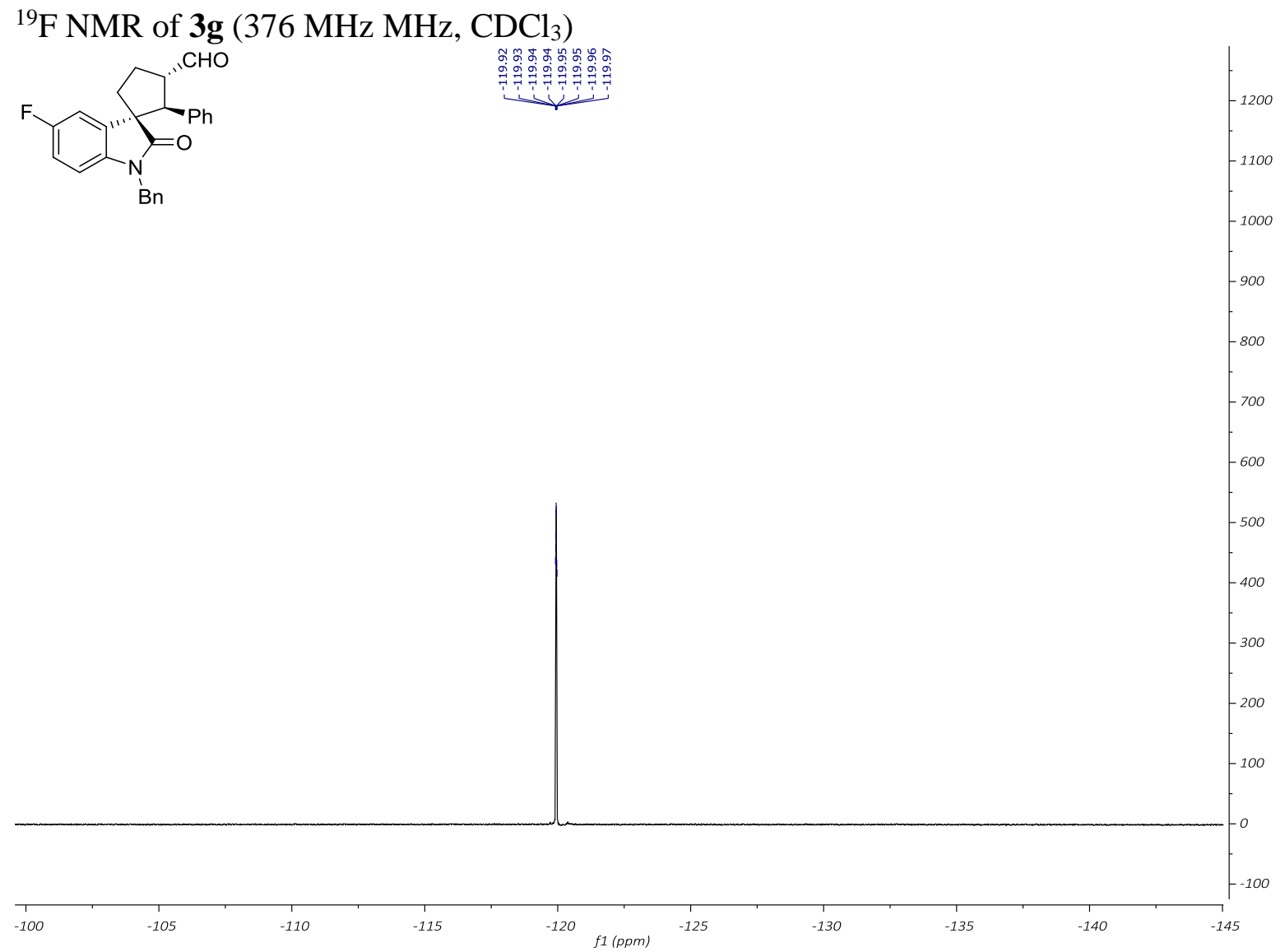


(1R,2S,3S)-1'-Benzyl-5'-fluoro-2'-oxo-2-phenylspiro[cyclopentane-1,3'-indoline]-3carbaldehyde (4g)

${ }^{1} \mathrm{H} \mathrm{NMR}$ of $4 \mathbf{g}\left(400 \mathrm{MHz}, \mathrm{CDCl}_{3}\right)$

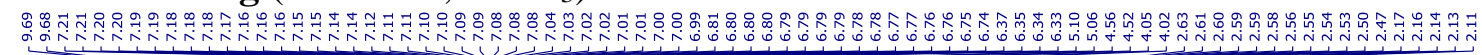

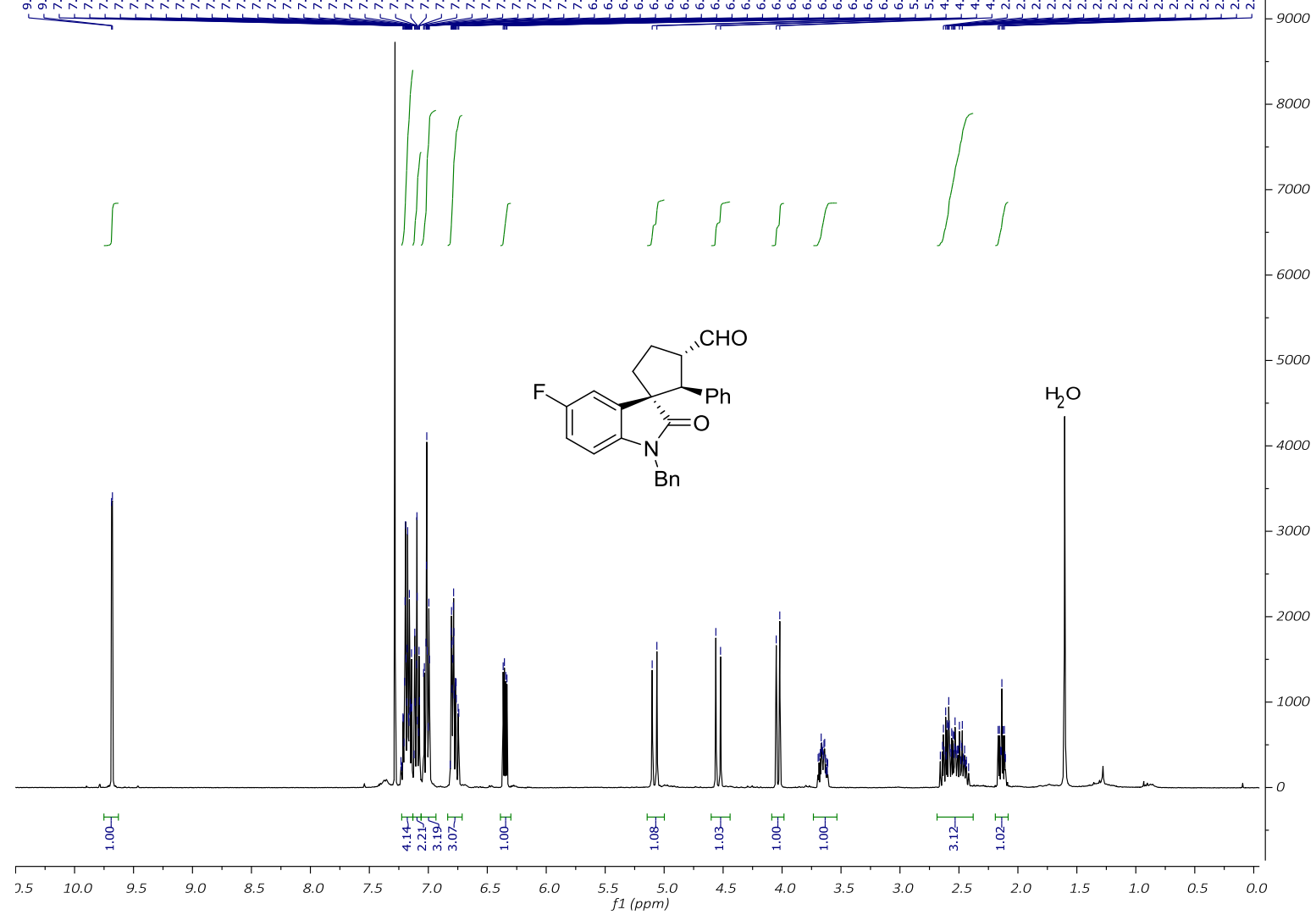

${ }^{13} \mathrm{C}\left\{{ }^{1} \mathrm{H}\right\} \mathrm{NMR}$ of $\mathbf{4 g}\left(101 \mathrm{MHz}, \mathrm{CDCl}_{3}\right)$

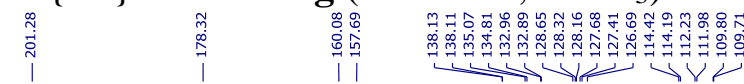
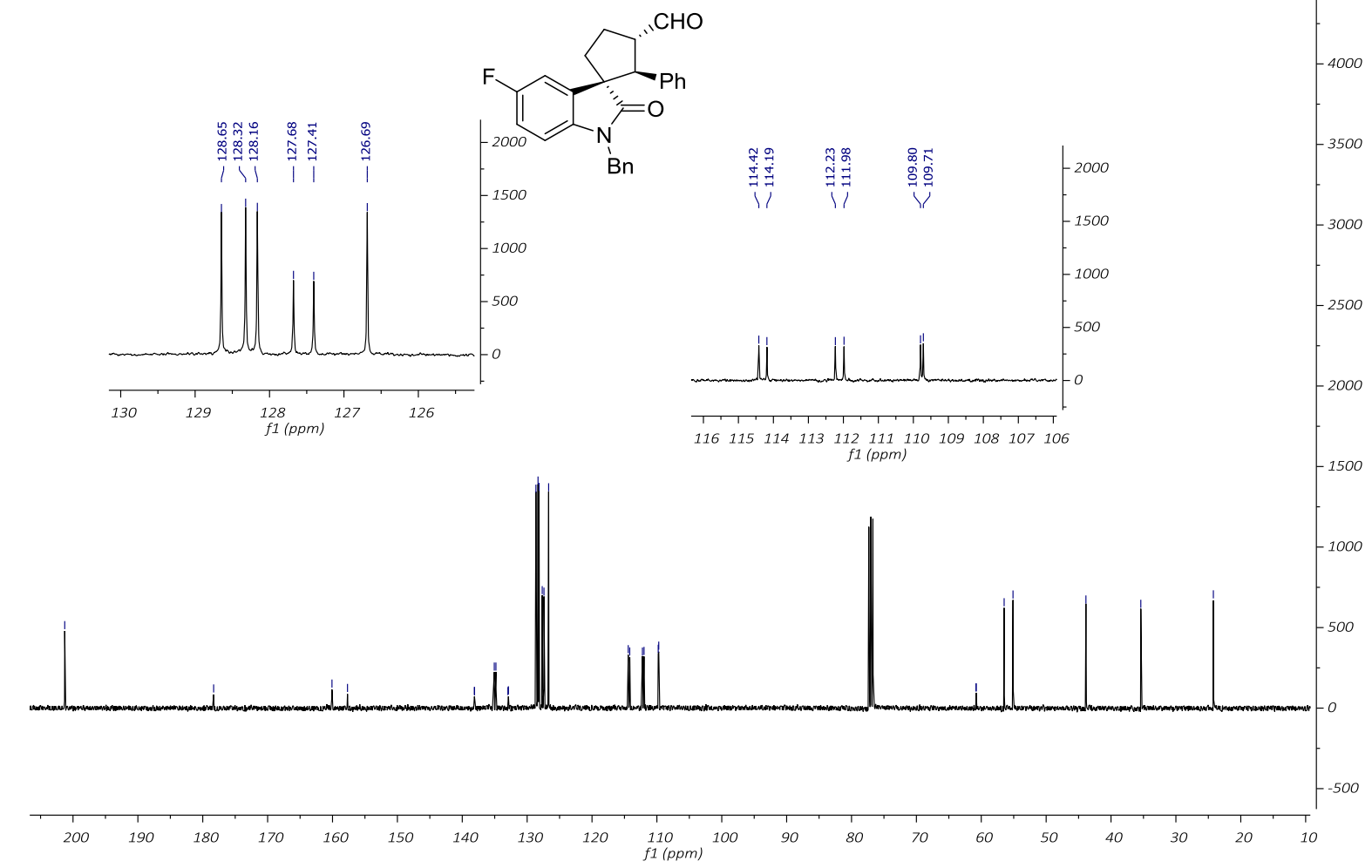


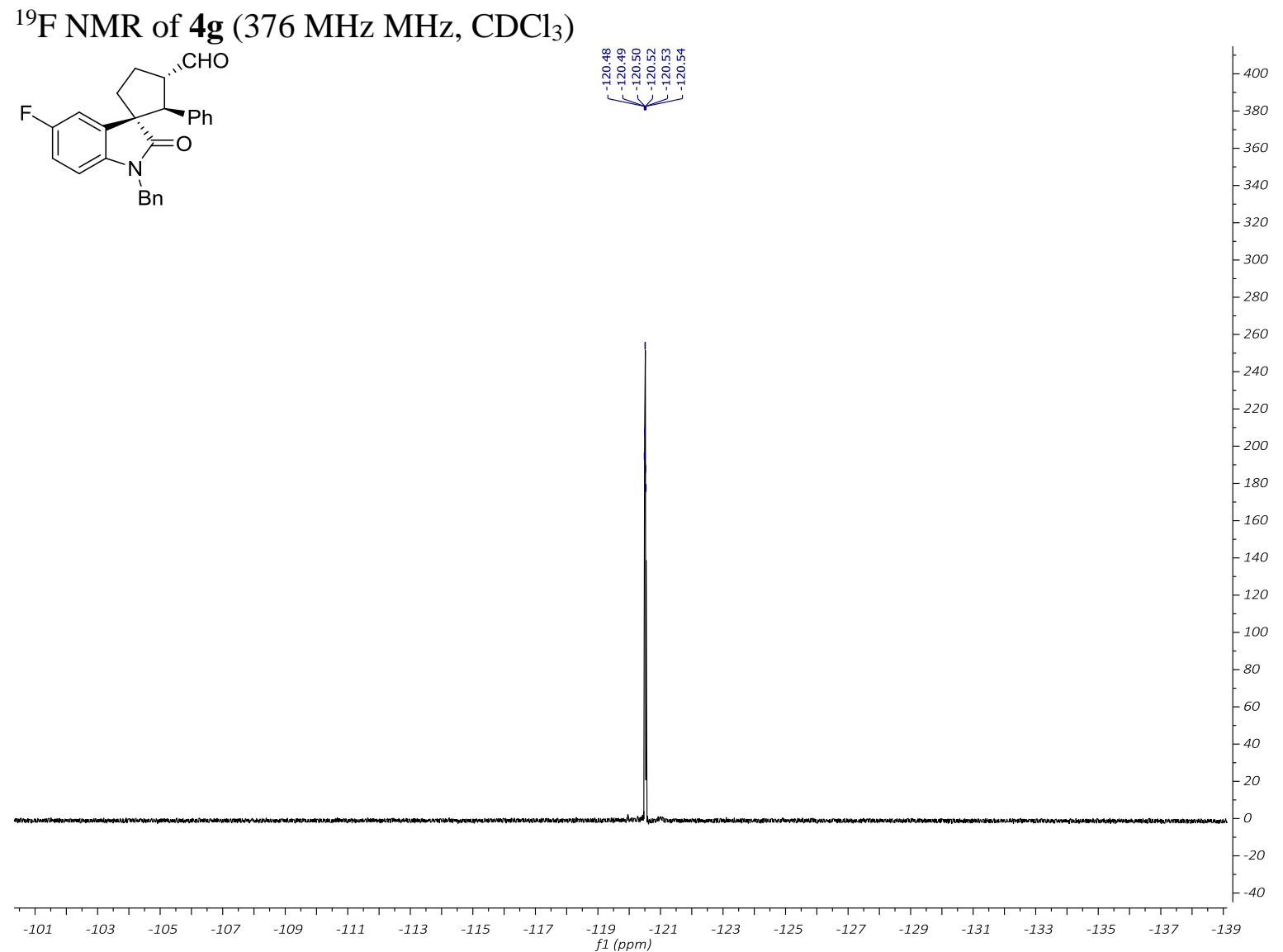


(1S,2S,3S)-1'-Benzyl-5' -chloro-2'-oxo-2-phenylspiro[cyclopentane-1,3'-indoline]-3carbaldehyde (3h)

${ }^{1} \mathrm{H}$ NMR of $\mathbf{3 h}\left(400 \mathrm{MHz}, \mathrm{CDCl}_{3}\right)$

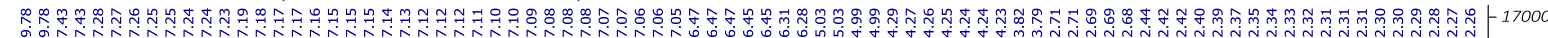

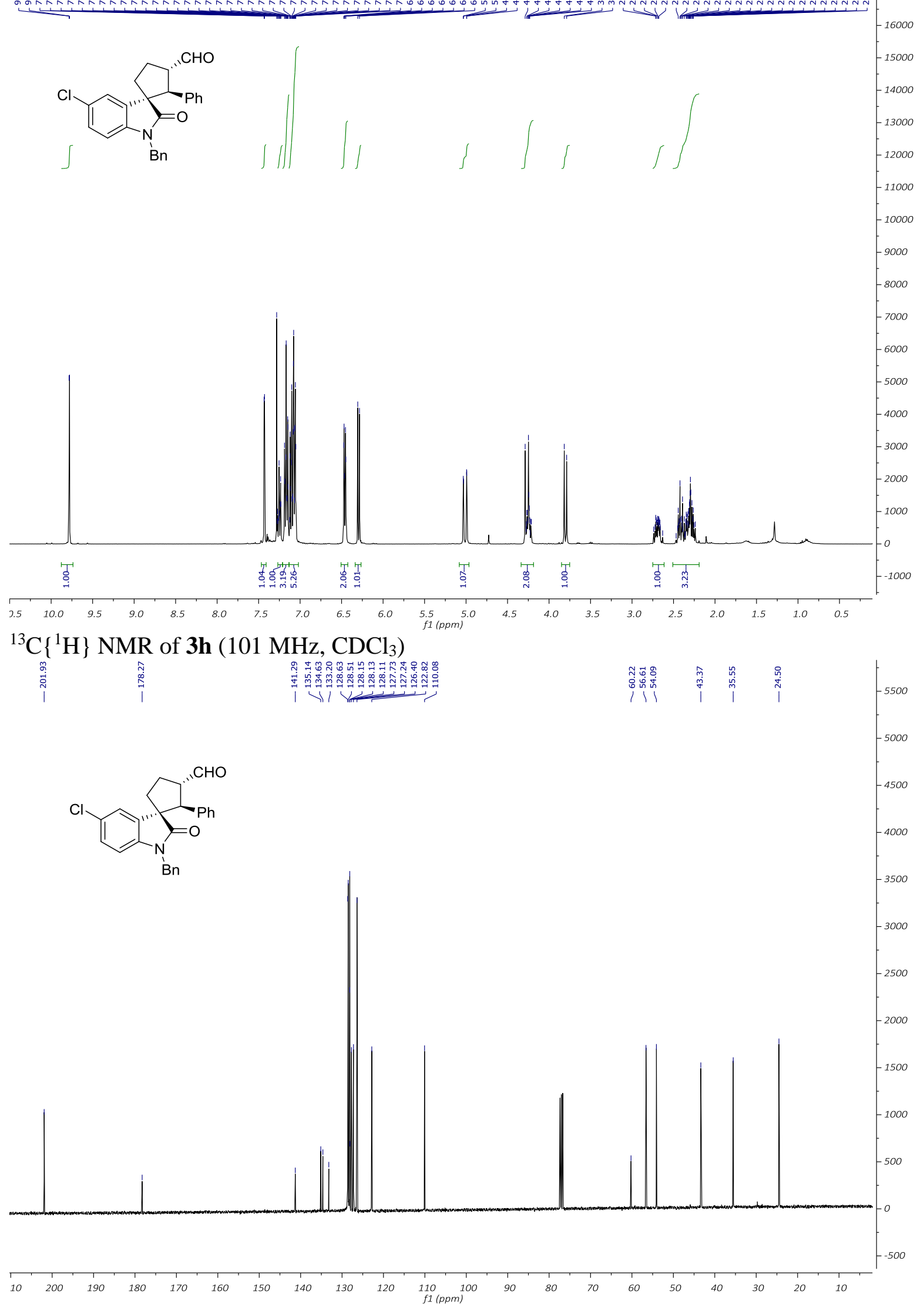


(1R,2S,3S)-1'-Benzyl-5' -chloro-2'-oxo-2-phenylspiro[cyclopentane-1,3'-indoline]-3carbaldehyde (4h)

${ }^{1} \mathrm{H}$ NMR of $4 \mathbf{h}\left(400 \mathrm{MHz}, \mathrm{CDCl}_{3}\right)$

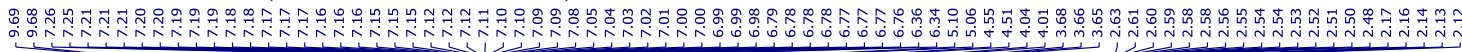

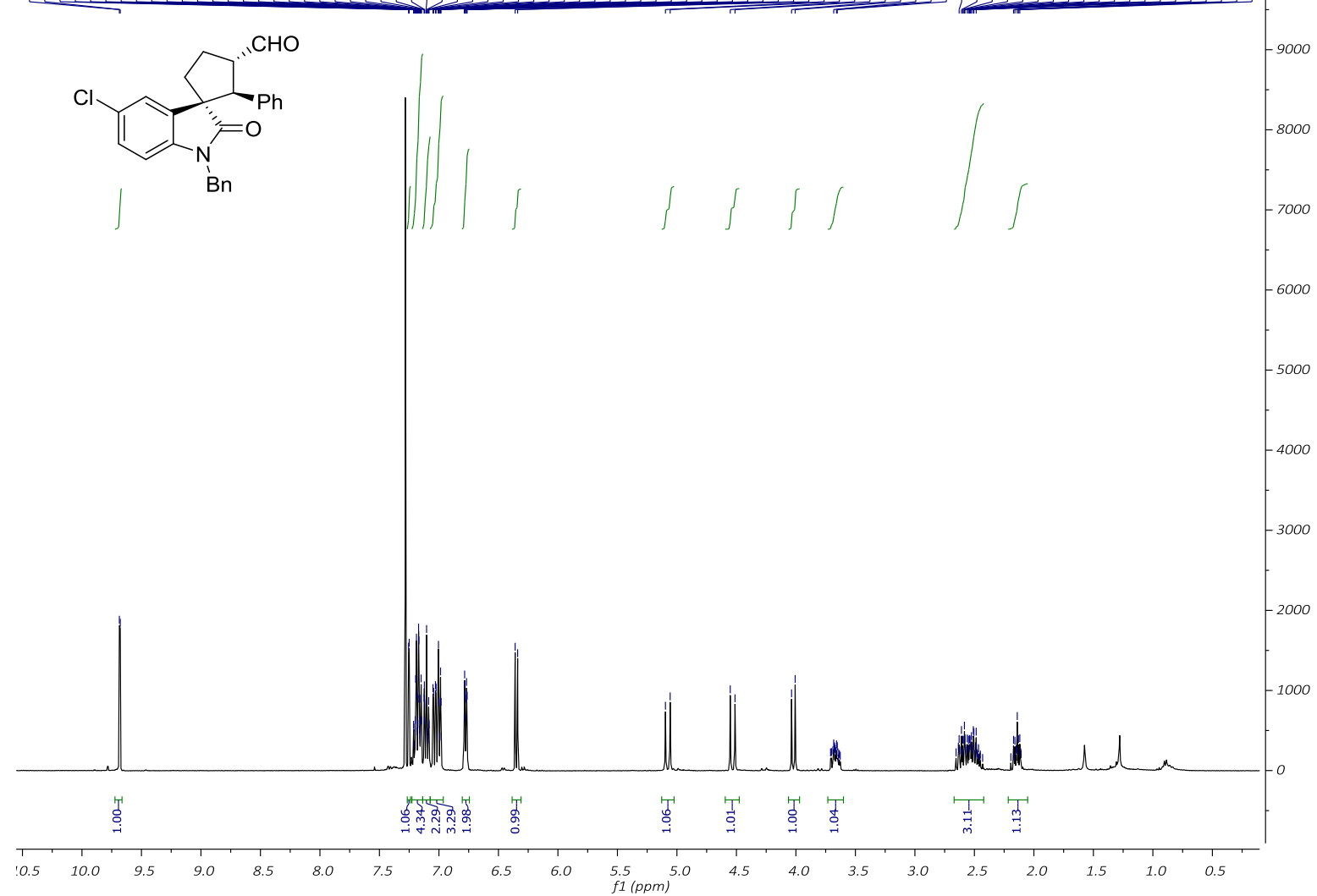

${ }^{13} \mathrm{C}\left\{{ }^{1} \mathrm{H}\right\}$ NMR of $4 \mathbf{h}\left(101 \mathrm{MHz}, \mathrm{CDCl}_{3}\right)$

i

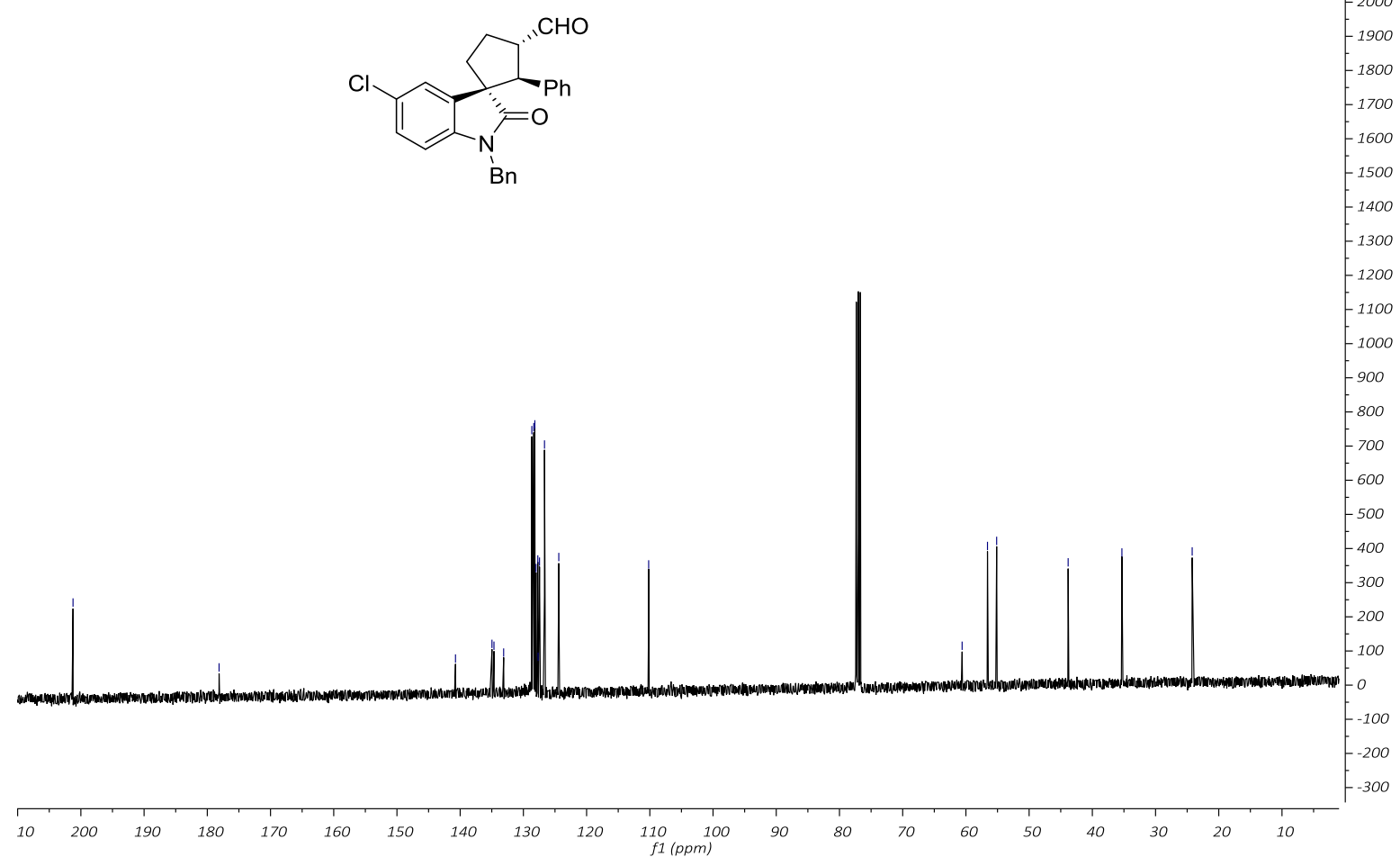

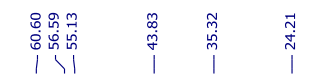

$-2300$ 
(1S,2S,3S)-1'-Benzyl-5'-bromo-2'-oxo-2-phenylspiro[cyclopentane-1,3'-indoline]-3carbaldehyde (3i)

${ }^{1} \mathrm{H}$ NMR of $3 \mathbf{i}\left(400 \mathrm{MHz}, \mathrm{CDCl}_{3}\right)$

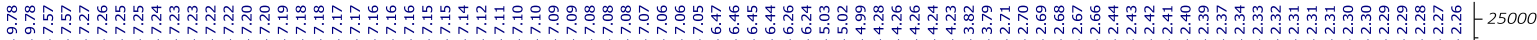

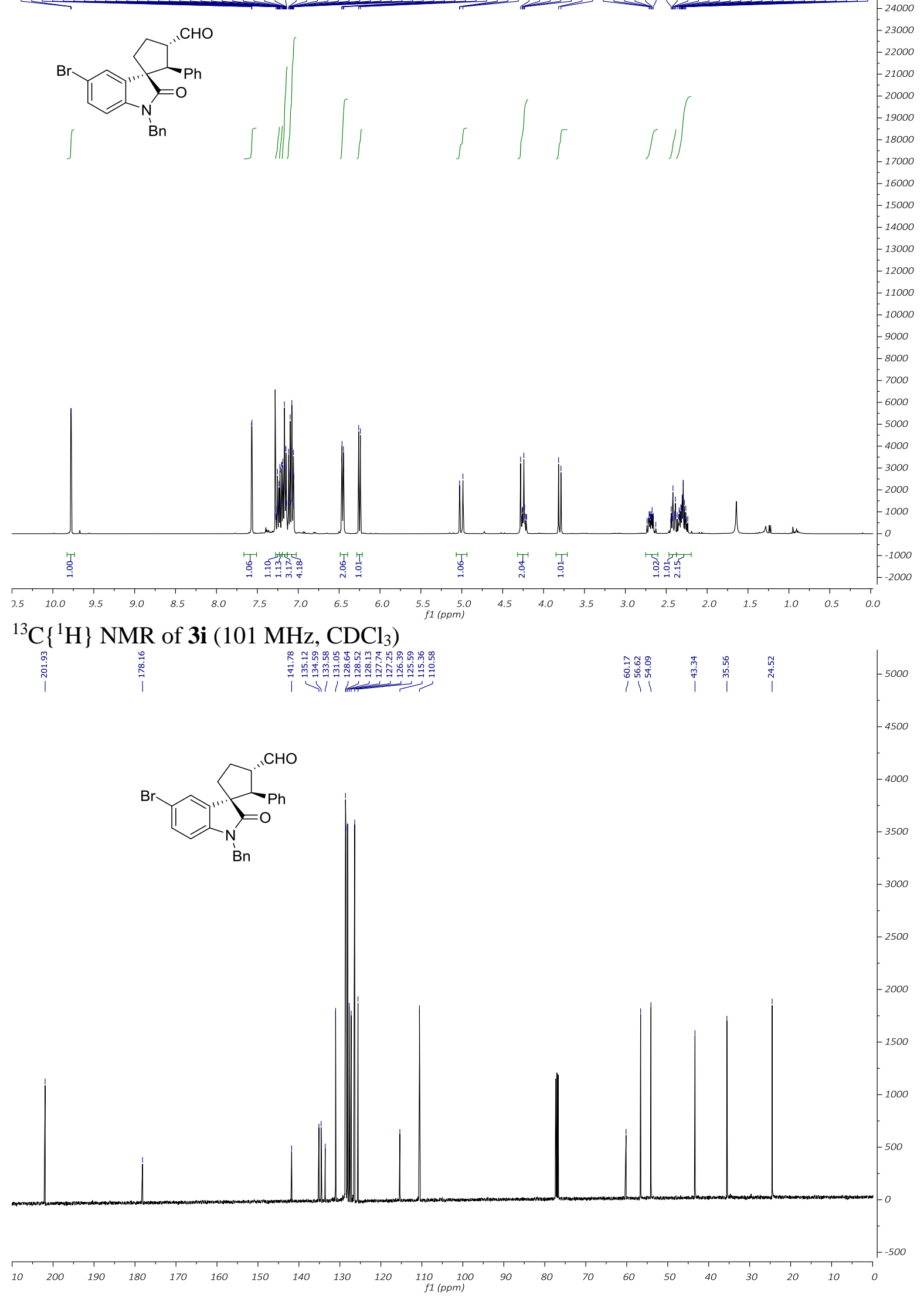


$(1 R, 2 S, 3 S)-1$ '-Benzyl-5' -bromo-2'-oxo-2-phenylspiro[cyclopentane-1,3'-indoline]-3carbaldehyde (4i)

${ }^{1} \mathrm{H}$ NMR of $4 \mathbf{i}\left(400 \mathrm{MHz}, \mathrm{CDCl}_{3}\right)$

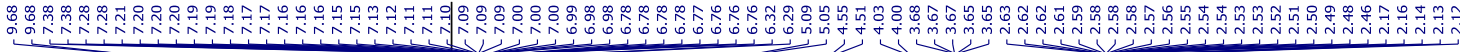

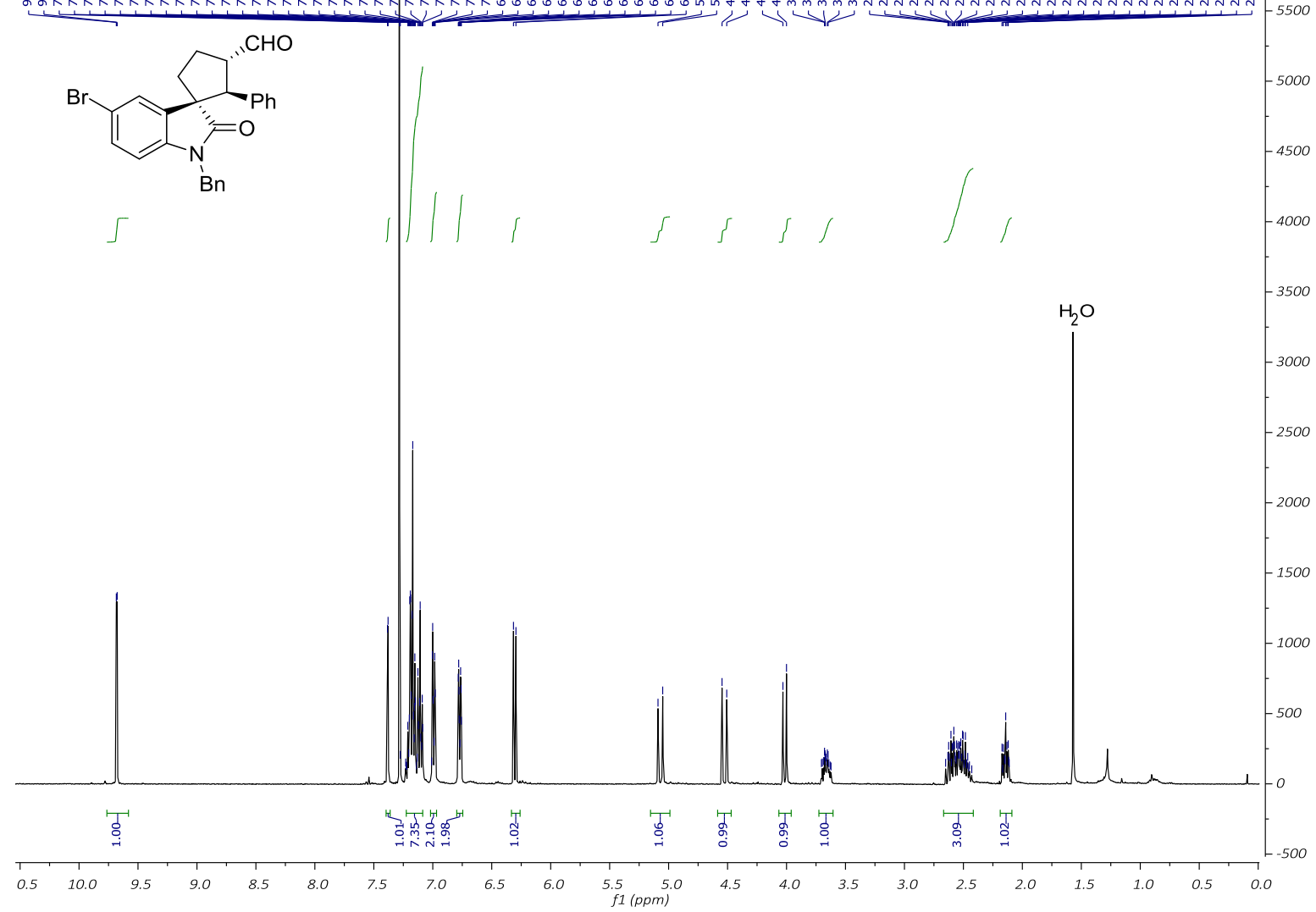

${ }^{13} \mathrm{C}\left\{{ }^{1} \mathrm{H}\right\} \mathrm{NMR}$ of $4 \mathbf{i}\left(101 \mathrm{MHz}, \mathrm{CDCl}_{3}\right)$

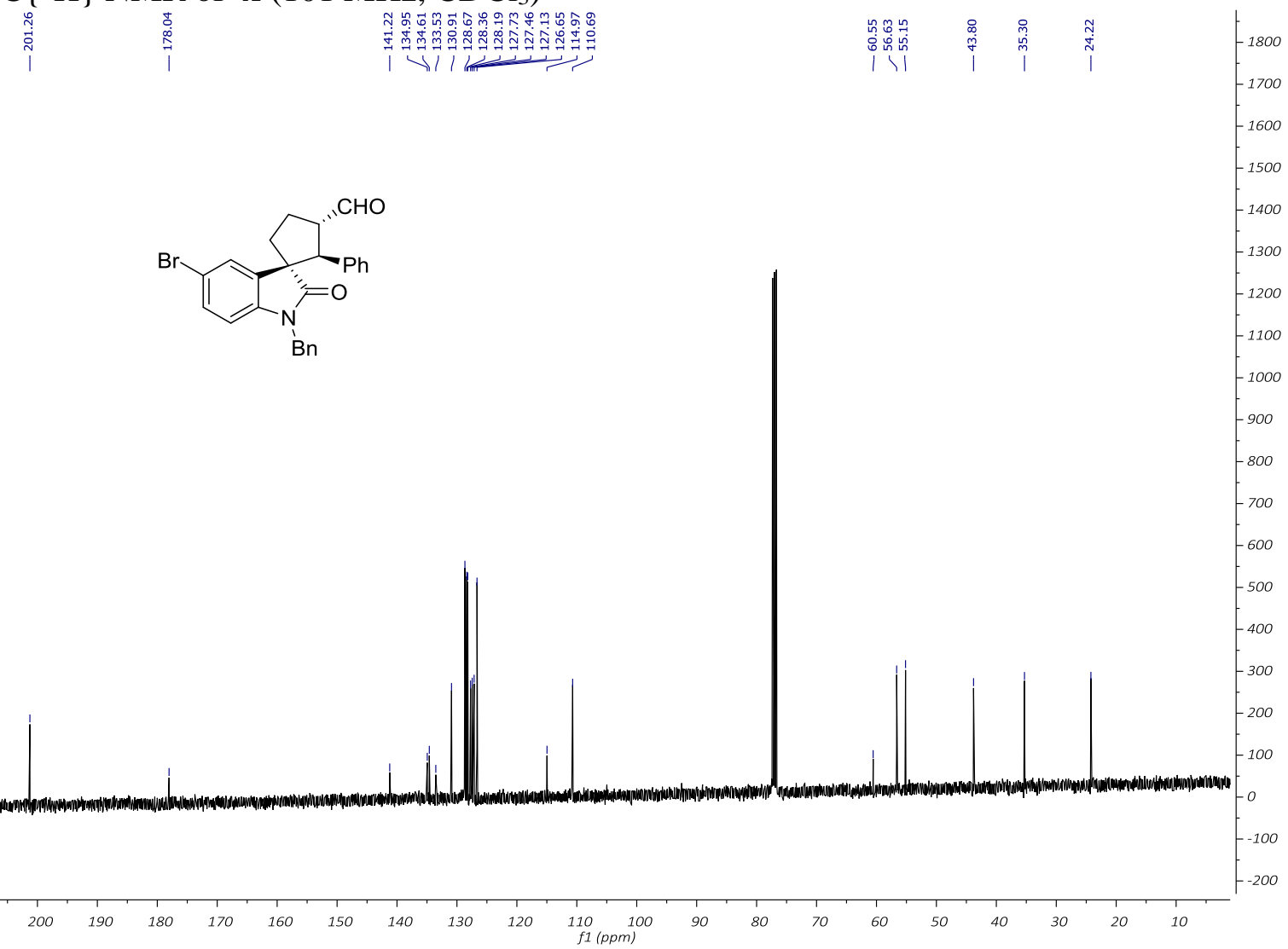


(1S,2S,3S)-1'-Benzyl-4' -methyl-2'-oxo-2-phenylspiro[cyclopentane-1,3'-indoline]-3carbaldehyde (3j)

${ }^{1} \mathrm{H}$ NMR of $\mathbf{3 j}\left(400 \mathrm{MHz}, \mathrm{CDCl}_{3}\right)$

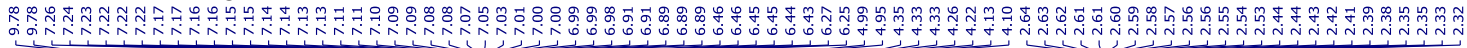
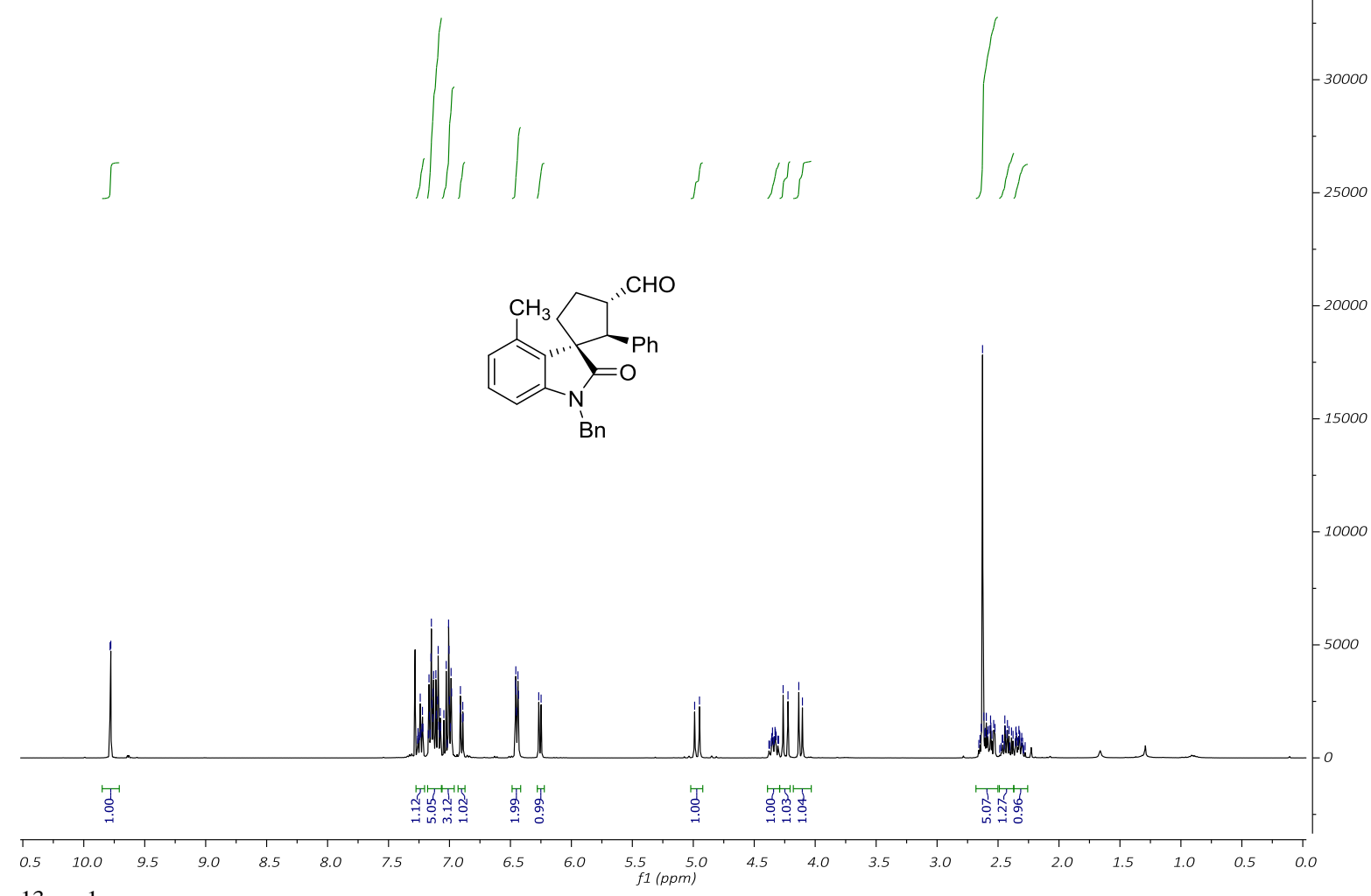

30000

25000

${ }^{13} \mathrm{C}\left\{{ }^{1} \mathrm{H}\right\}$ NMR of $\mathbf{3 j}\left(101 \mathrm{MHz}, \mathrm{CDCl}_{3}\right)$

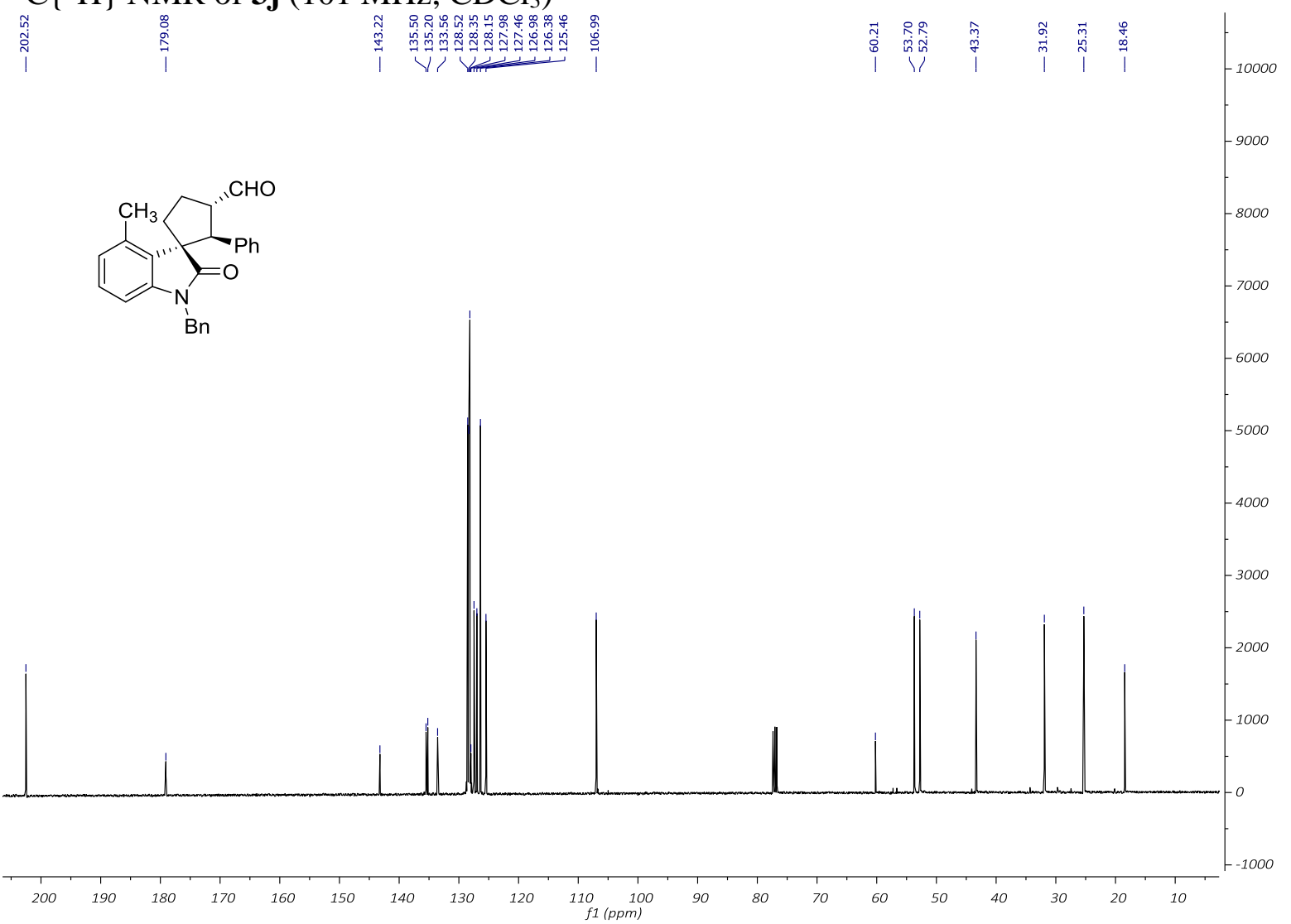


(1S,2S,3S)-1'-Benzyl-6'-methyl-2'-oxo-2-phenylspiro[cyclopentane-1,3'-indoline]-3carbaldehyde (3k)

${ }^{1} \mathrm{H}$ NMR of $3 \mathbf{k}\left(400 \mathrm{MHz}, \mathrm{CDCl}_{3}\right)$

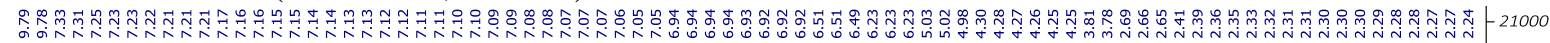

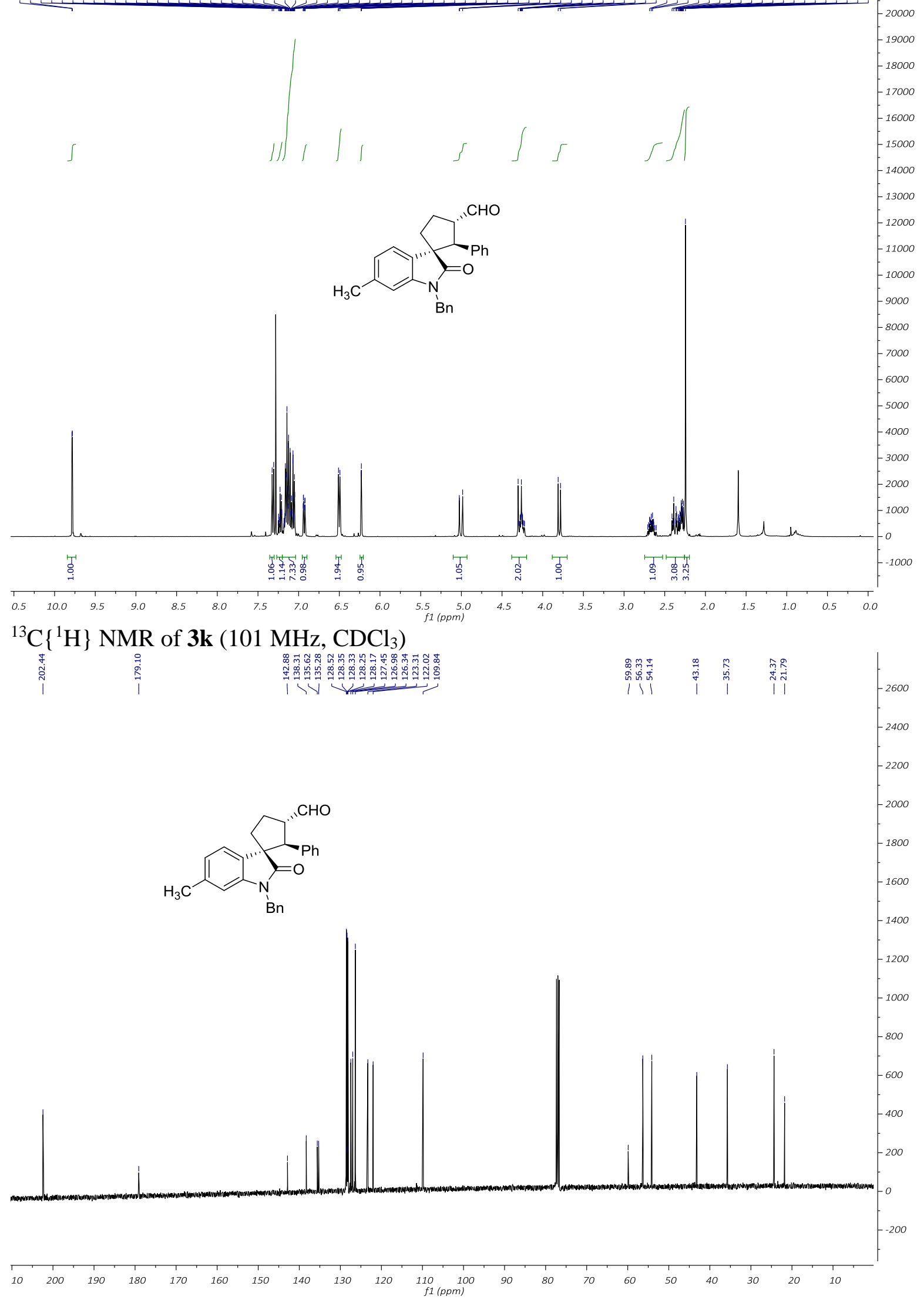


$(1 R, 2 S, 3 S)-1 '$-Benzyl-6' -methyl-2' -oxo-2-phenylspiro[cyclopentane-1,3'-indoline]-3carbaldehyde (4k)

${ }^{1} \mathrm{H}$ NMR of $4 \mathbf{k}\left(400 \mathrm{MHz}, \mathrm{CDCl}_{3}\right)$

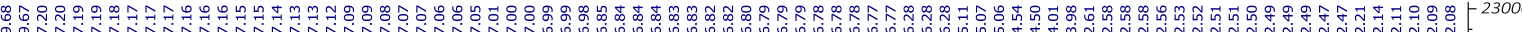

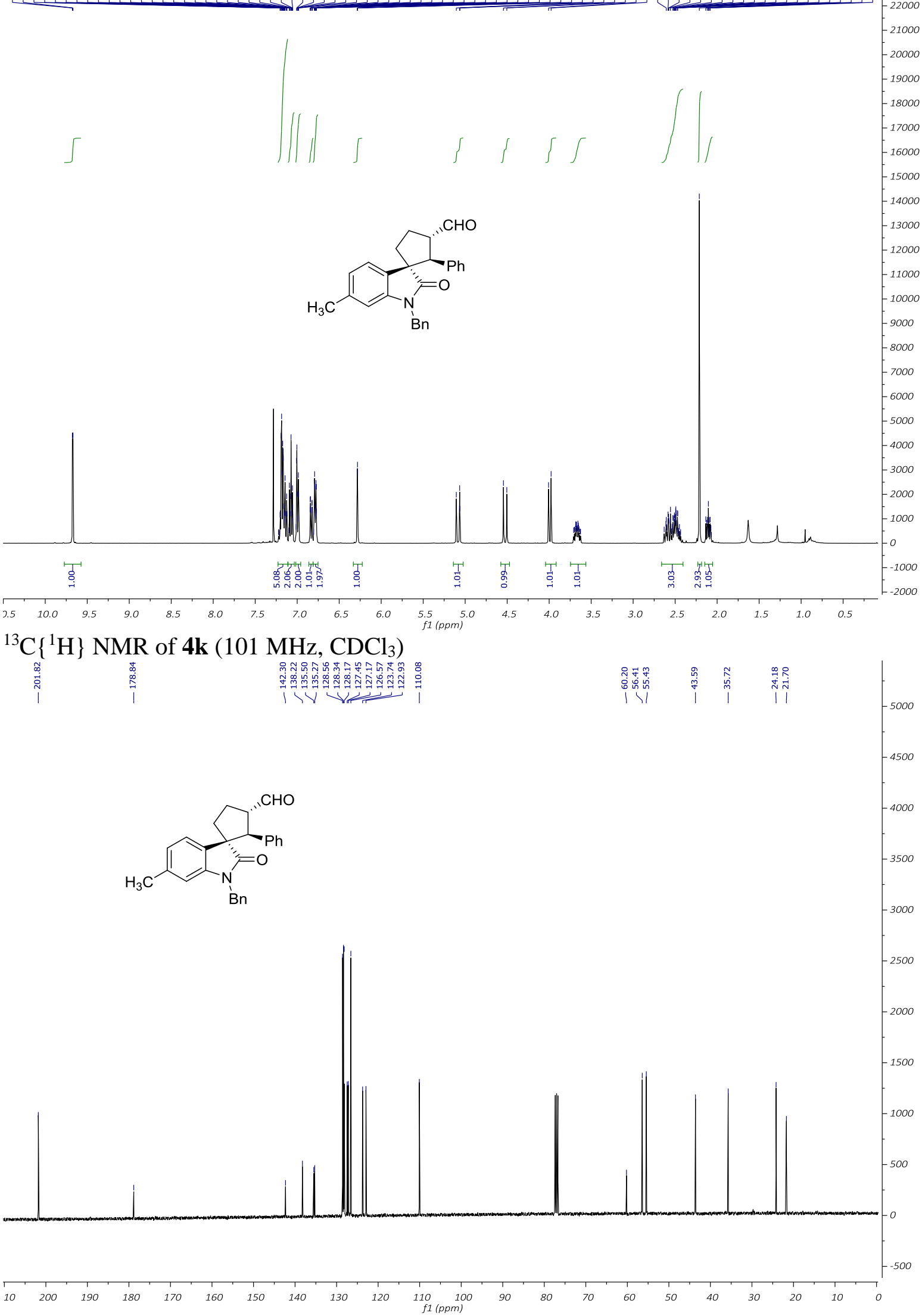


(1S,2S,3S)-1'-Benzyl-7'-methyl-2'-oxo-2-phenylspiro[cyclopentane-1,3'-indoline]-3carbaldehyde (3I)

${ }^{1} \mathrm{H}$ NMR of 31 (400 MHz, $\left.\mathrm{CDCl}_{3}\right)$

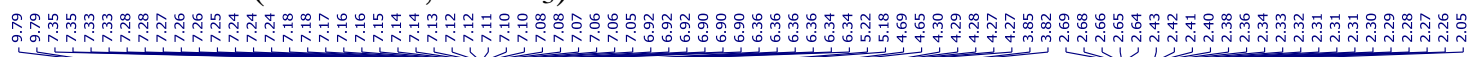

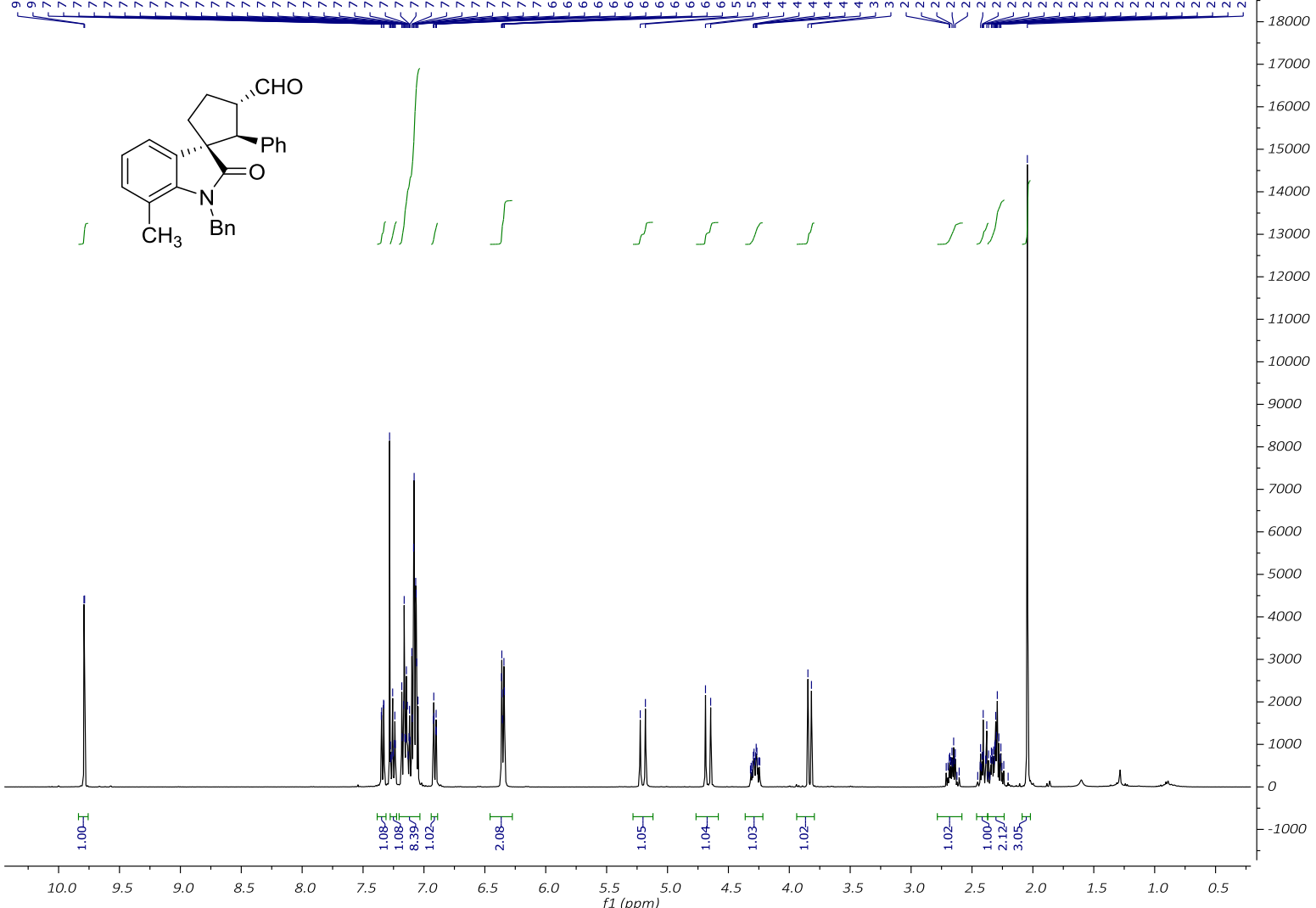

${ }^{13} \mathrm{C}\left\{{ }^{1} \mathrm{H}\right\} \mathrm{NMR}$ of $3 \mathbf{l}\left(101 \mathrm{MHz}, \mathrm{CDCl}_{3}\right)$

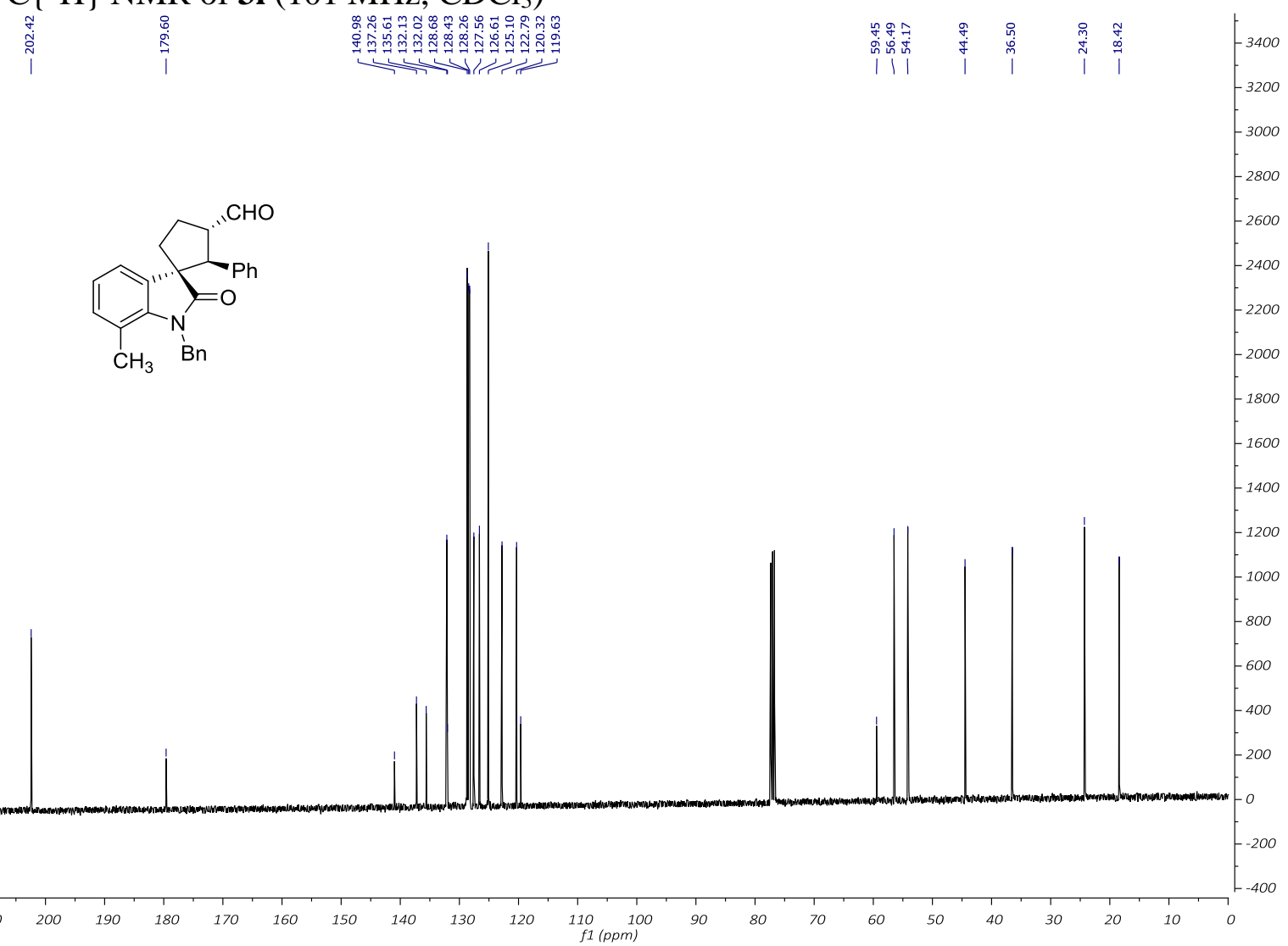


$(1 R, 2 S, 3 S)-1$ '-Benzyl-7'-methyl-2' -oxo-2-phenylspiro[cyclopentane-1,3'-indoline]-3carbaldehyde (4I)

${ }^{1} \mathrm{H} \mathrm{NMR}$ of $4 \mathbf{l}\left(400 \mathrm{MHz}, \mathrm{CDCl}_{3}\right)$

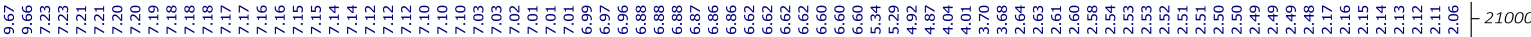

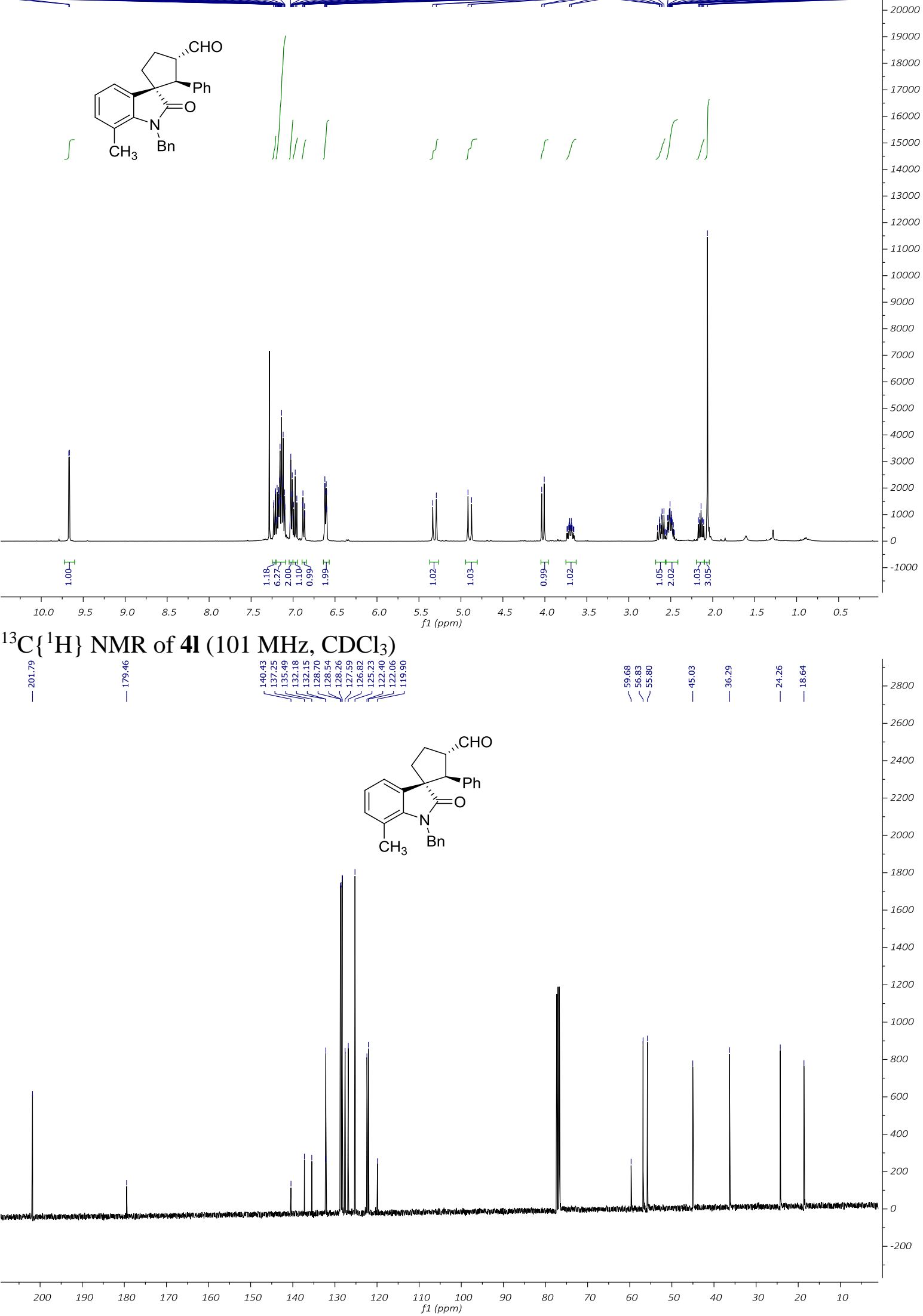


(1S,2S,3S)-1'-Benzyl-2-(naphthalen-2-yl)-2'-oxospiro[cyclopentane-1,3'-indoline]-3carbaldehyde (3m)

${ }^{1} \mathrm{H} \mathrm{NMR}$ of $\mathbf{3 m}\left(400 \mathrm{MHz}, \mathrm{CDCl}_{3}\right)$

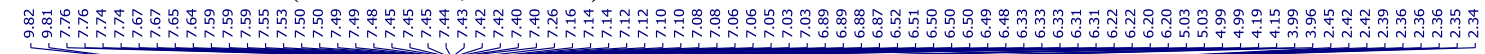

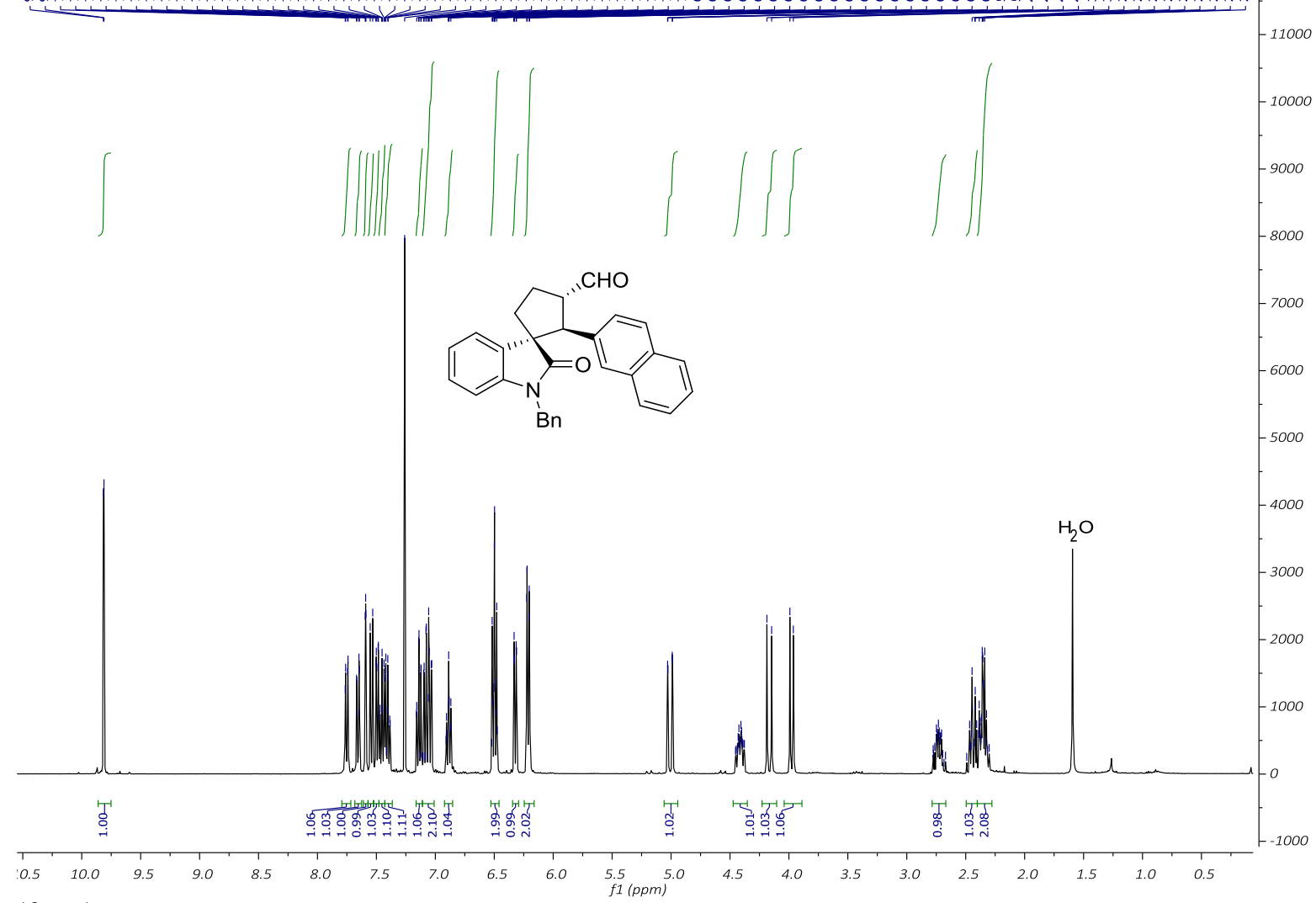

${ }^{13} \mathrm{C}\left\{{ }^{1} \mathrm{H}\right\} \mathrm{NMR}$ of $\mathbf{3 m}\left(101 \mathrm{MHz}, \mathrm{CDCl}_{3}\right)$
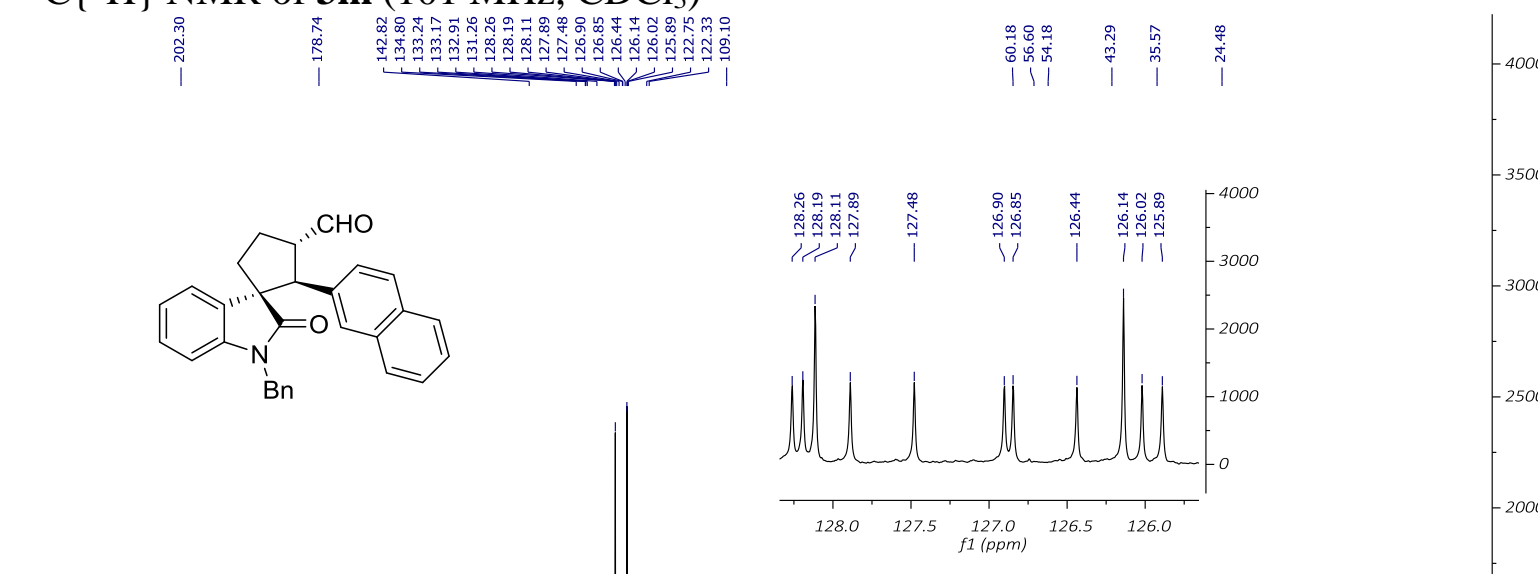

$-3500$

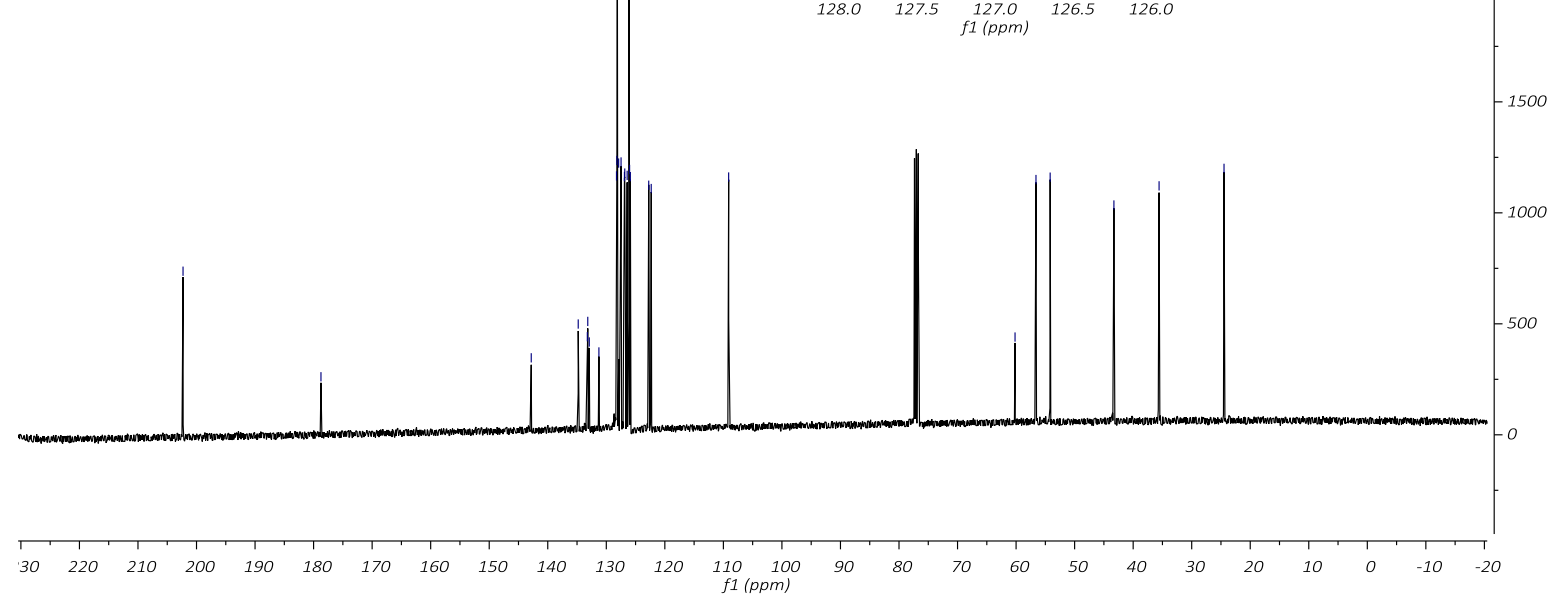


(1R,2S,3S)-1'-Benzyl-2-(naphthalen-2-yl)-2'-oxospiro[cyclopentane-1,3'-indoline]-3carbaldehyde $(\mathbf{4 m})$

${ }^{1} \mathrm{H} \mathrm{NMR}$ of $\mathbf{4 m}\left(400 \mathrm{MHz}, \mathrm{CDCl}_{3}\right)$

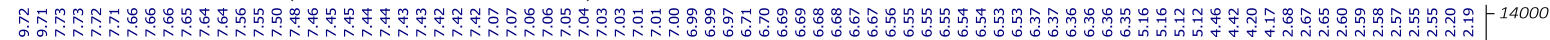

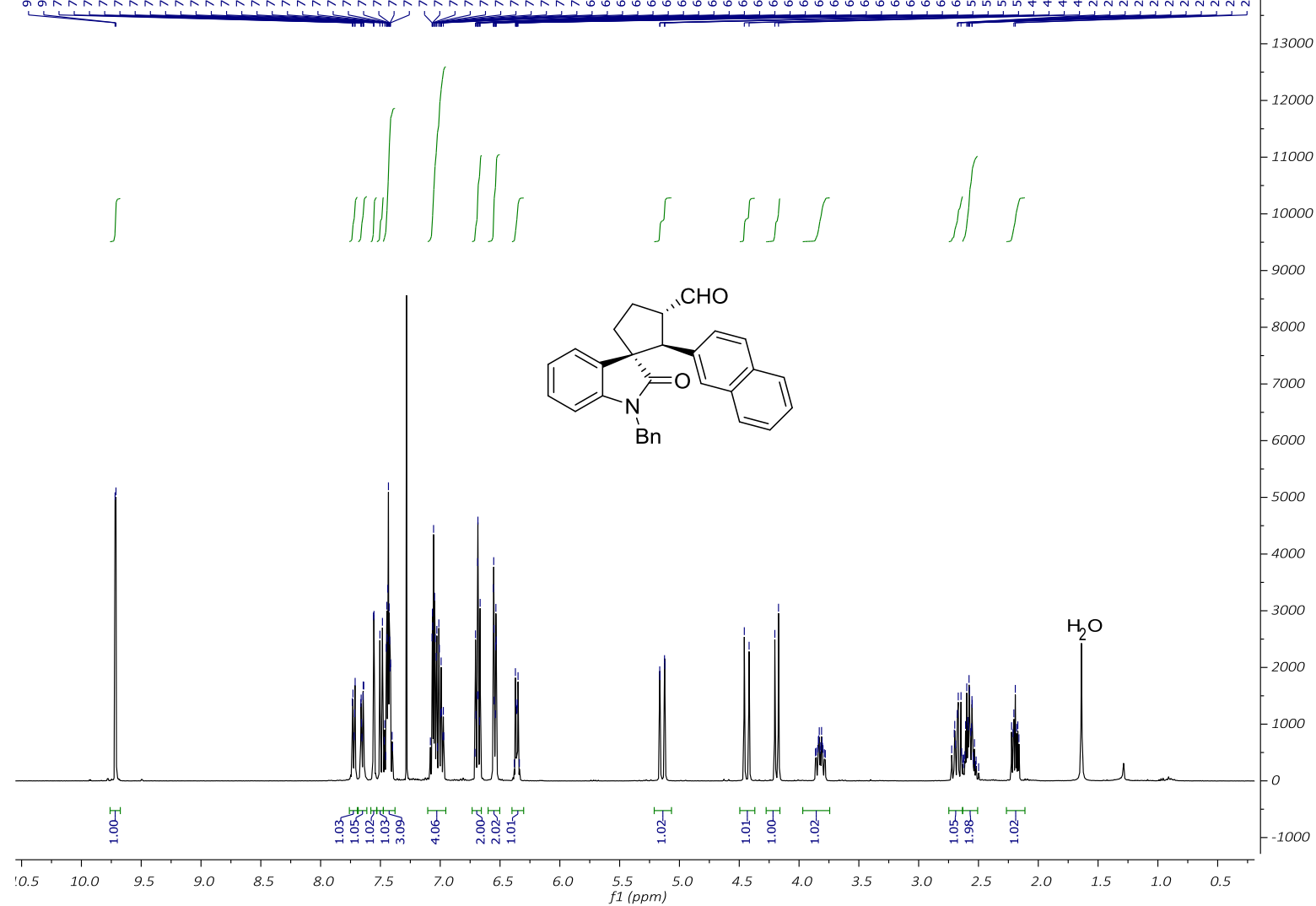

${ }^{13} \mathrm{C}\left\{{ }^{1} \mathrm{H}\right\} \mathrm{NMR}$ of $\mathbf{4 m}\left(101 \mathrm{MHz}, \mathrm{CDCl}_{3}\right)$

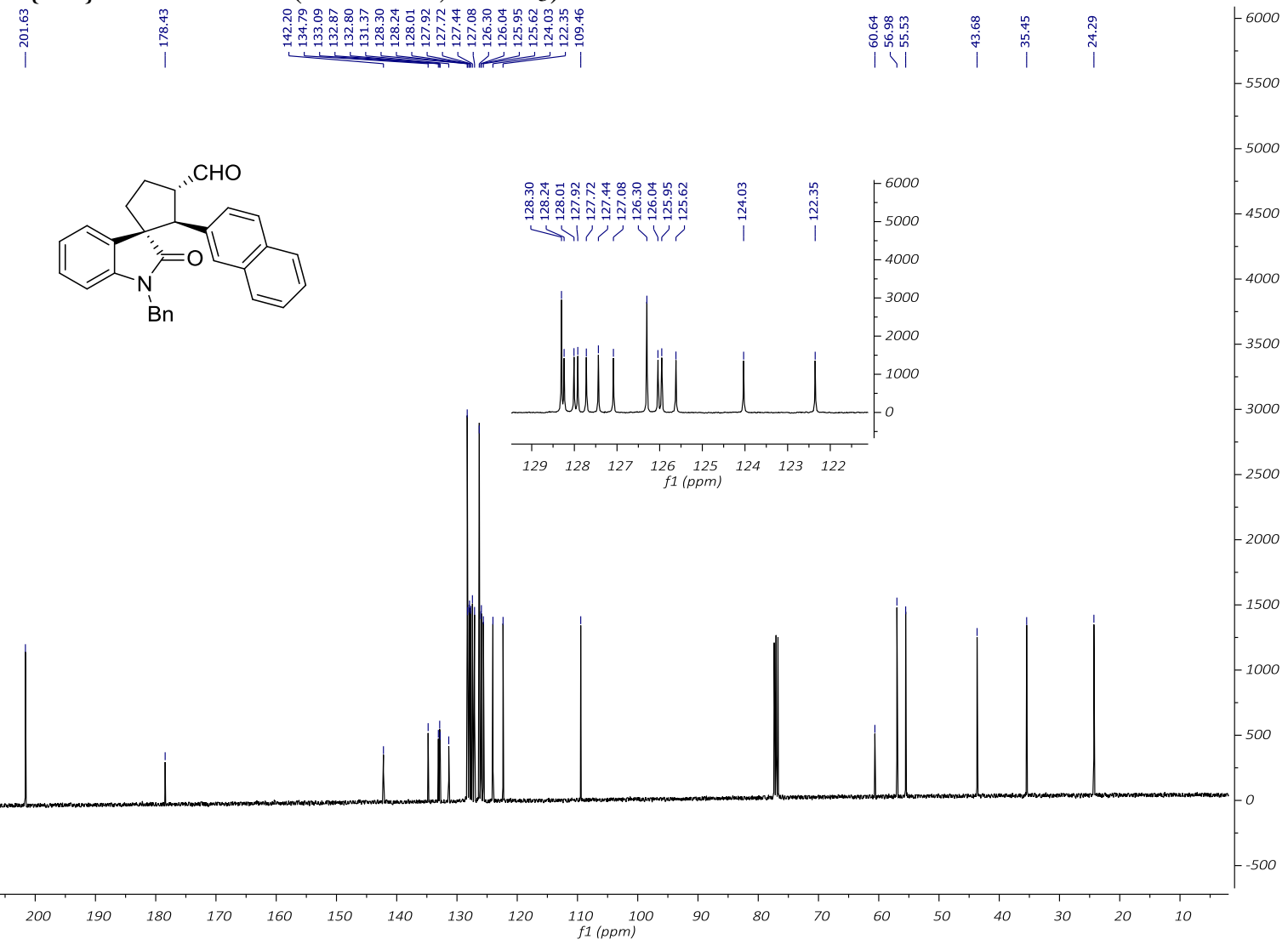


(1S,2S,3S)-1'-Benzyl-2' -oxo-2-(p-tolyl)spiro[cyclopentane-1,3'-indoline]-3-carbaldehyde (3n)

${ }^{1} \mathrm{H}$ NMR of $3 \mathbf{n}\left(400 \mathrm{MHz}, \mathrm{CDCl}_{3}\right)$

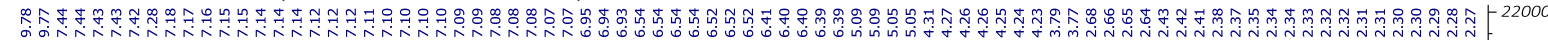

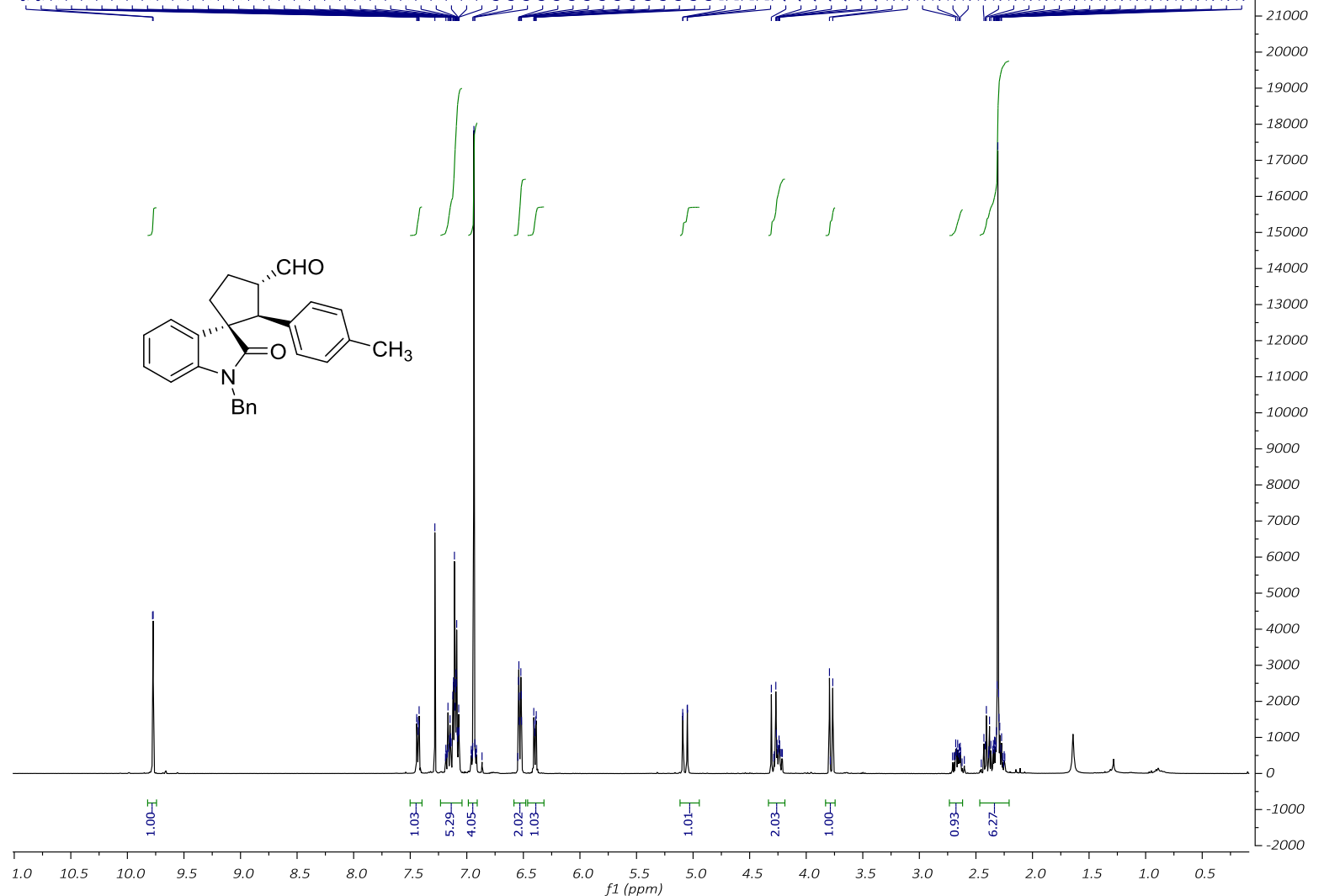

${ }^{13} \mathrm{C}\left\{{ }^{1} \mathrm{H}\right\}$ NMR of 3n $\left(101 \mathrm{MHz}, \mathrm{CDCl}_{3}\right)$

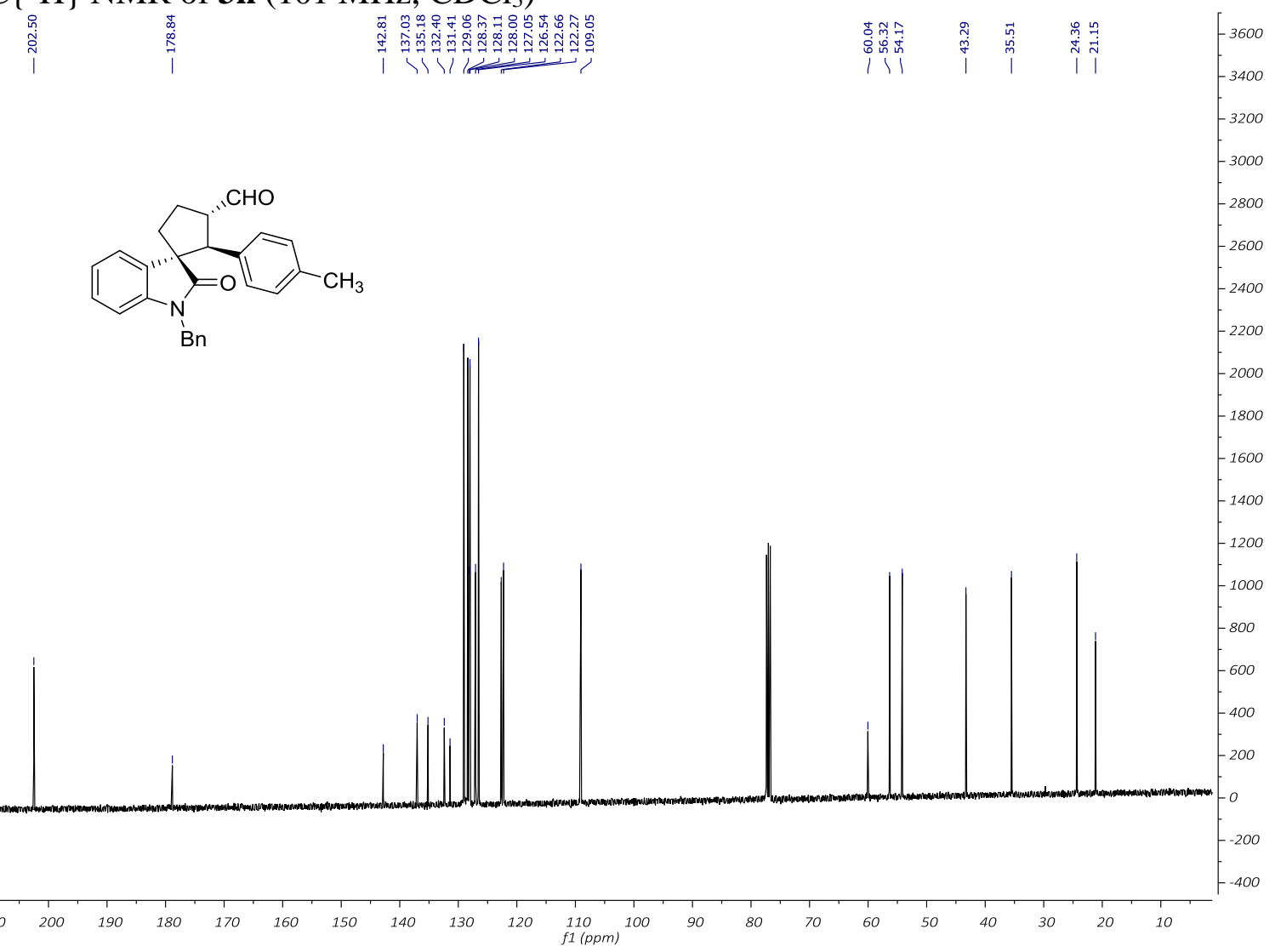


$(1 R, 2 S, 3 S)-1 '$-Benzyl-2'-oxo-2-(p-tolyl)spiro[cyclopentane-1,3'-indoline]-3-carbaldehyde (4n)

${ }^{1} \mathrm{H}$ NMR of $4 \mathbf{n}\left(400 \mathrm{MHz}, \mathrm{CDCl}_{3}\right)$

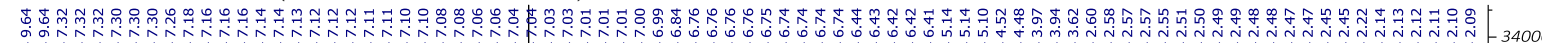

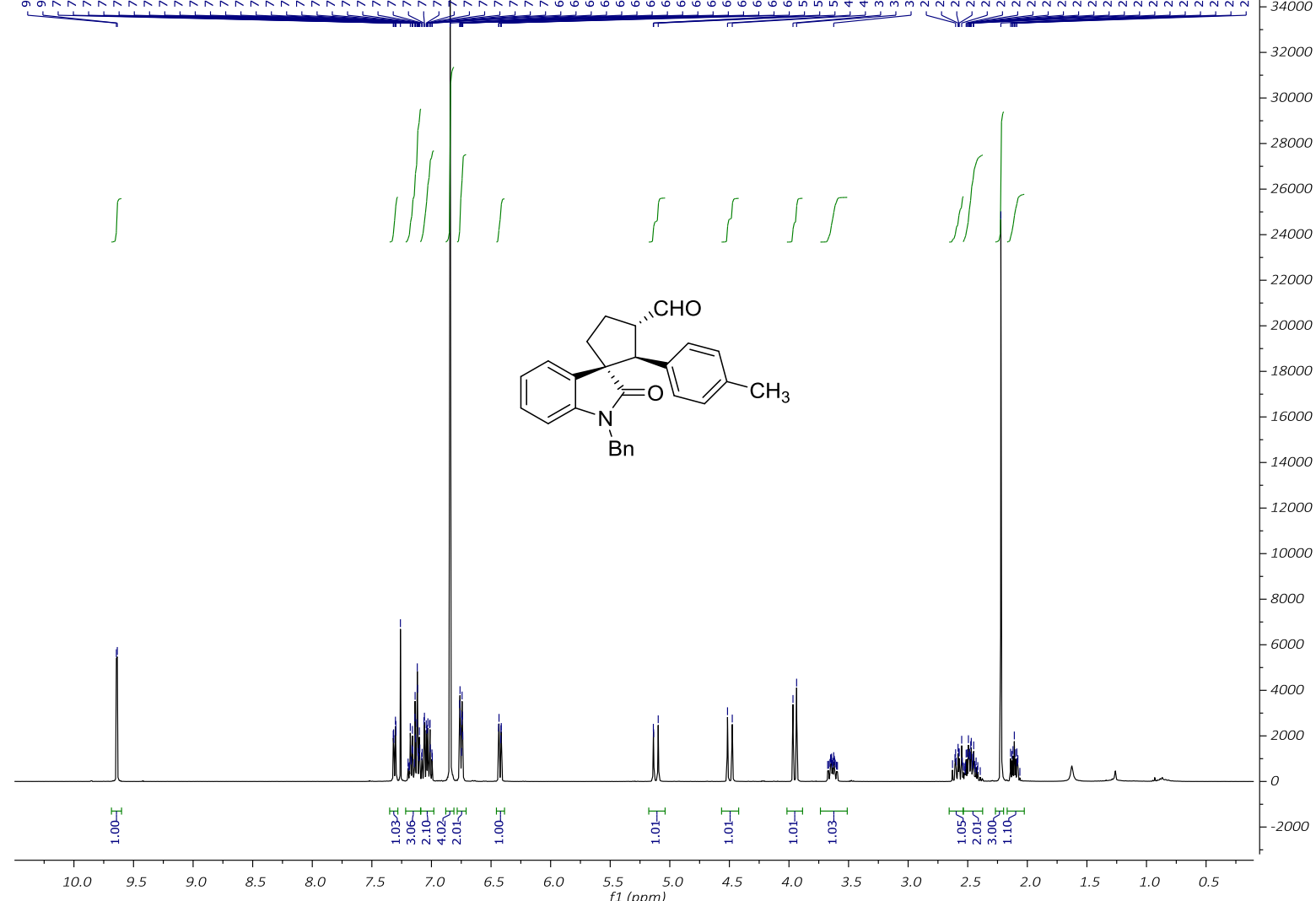

${ }^{13} \mathrm{C}\left\{{ }^{1} \mathrm{H}\right\}$ NMR of $4 \mathbf{n}\left(101 \mathrm{MHz}, \mathrm{CDCl}_{3}\right)$

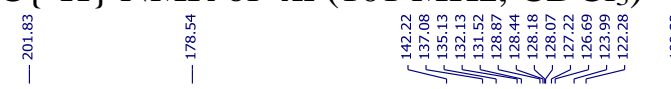
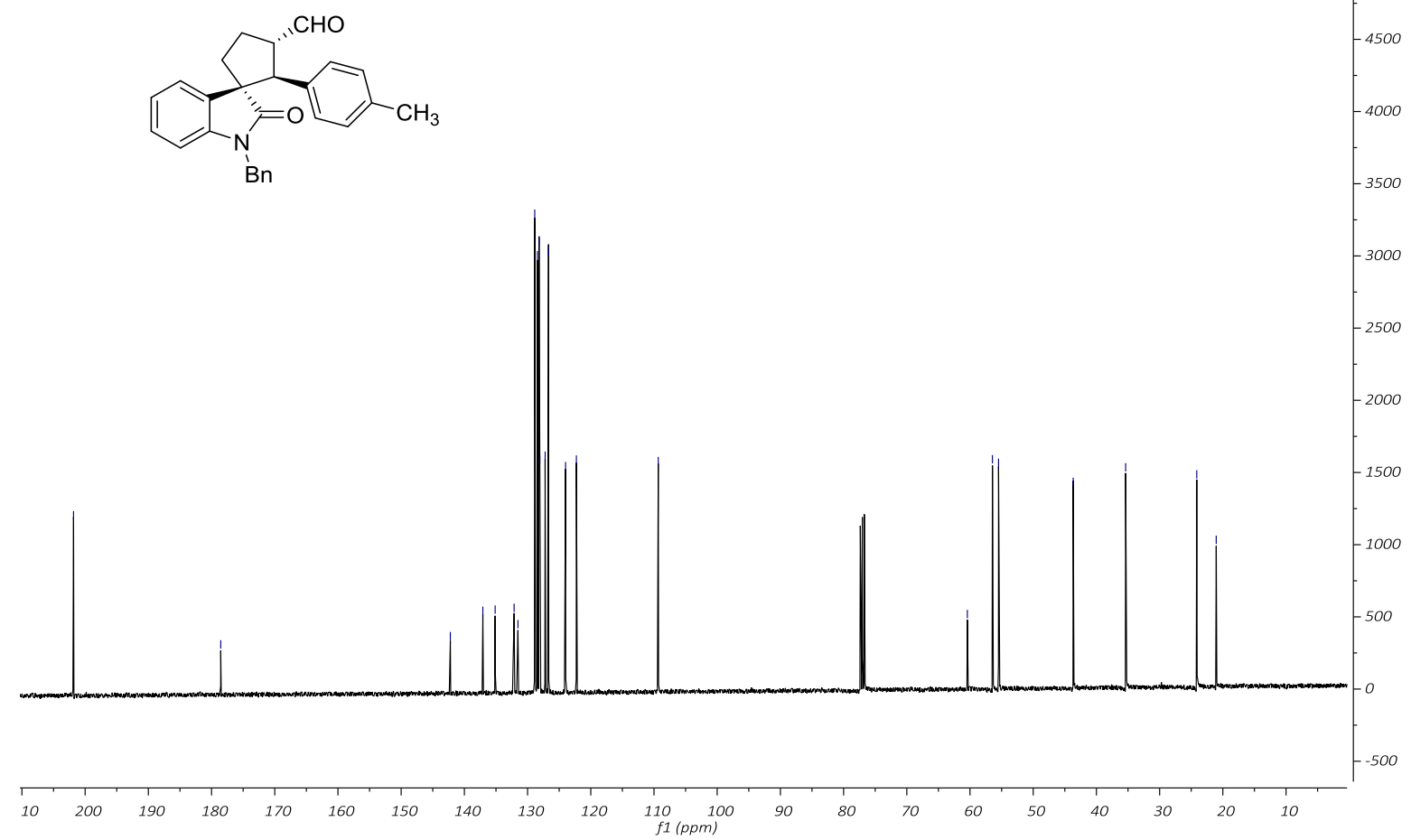
(1S,2S,3S)-1'-Benzyl-2-(4-methoxyphenyl)-2'-oxospiro[cyclopentane-1,3'-indoline]-3carbaldehyde (3o)

${ }^{1} \mathrm{H}$ NMR of $30\left(400 \mathrm{MHz}, \mathrm{CDCl}_{3}\right)$

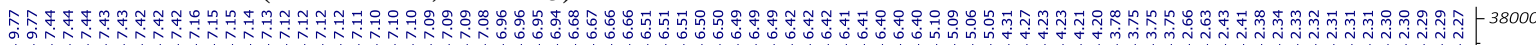

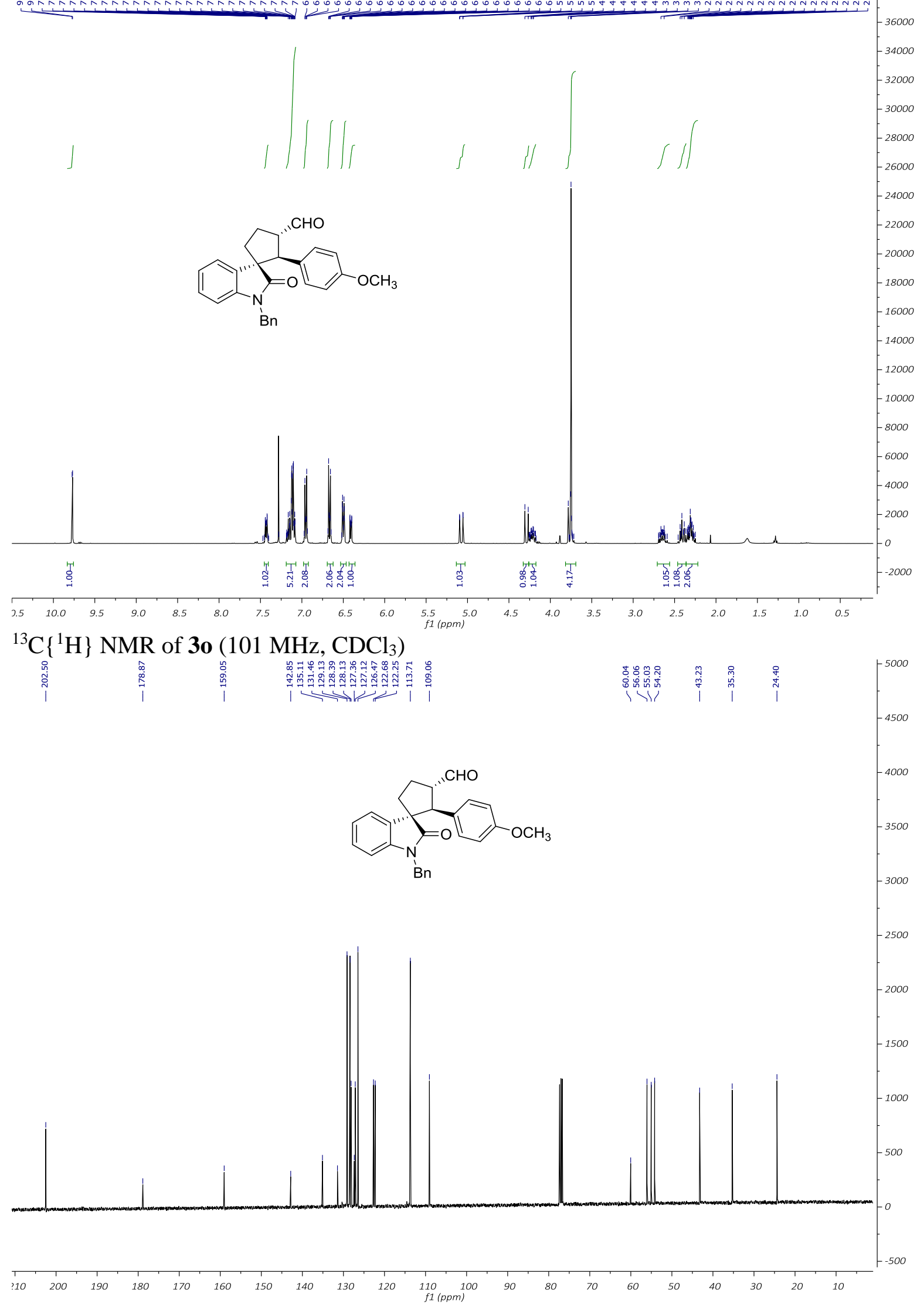


$(1 R, 2 S, 3 S)-1$ '-benzyl-2-(4-methoxyphenyl)-2' -oxospiro[cyclopentane-1,3'-indoline]-3carbaldehyde (4o)

${ }^{1} \mathrm{H} \mathrm{NMR}$ of $4 \mathbf{o}\left(400 \mathrm{MHz}, \mathrm{CDCl}_{3}\right)$

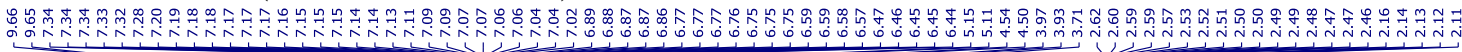

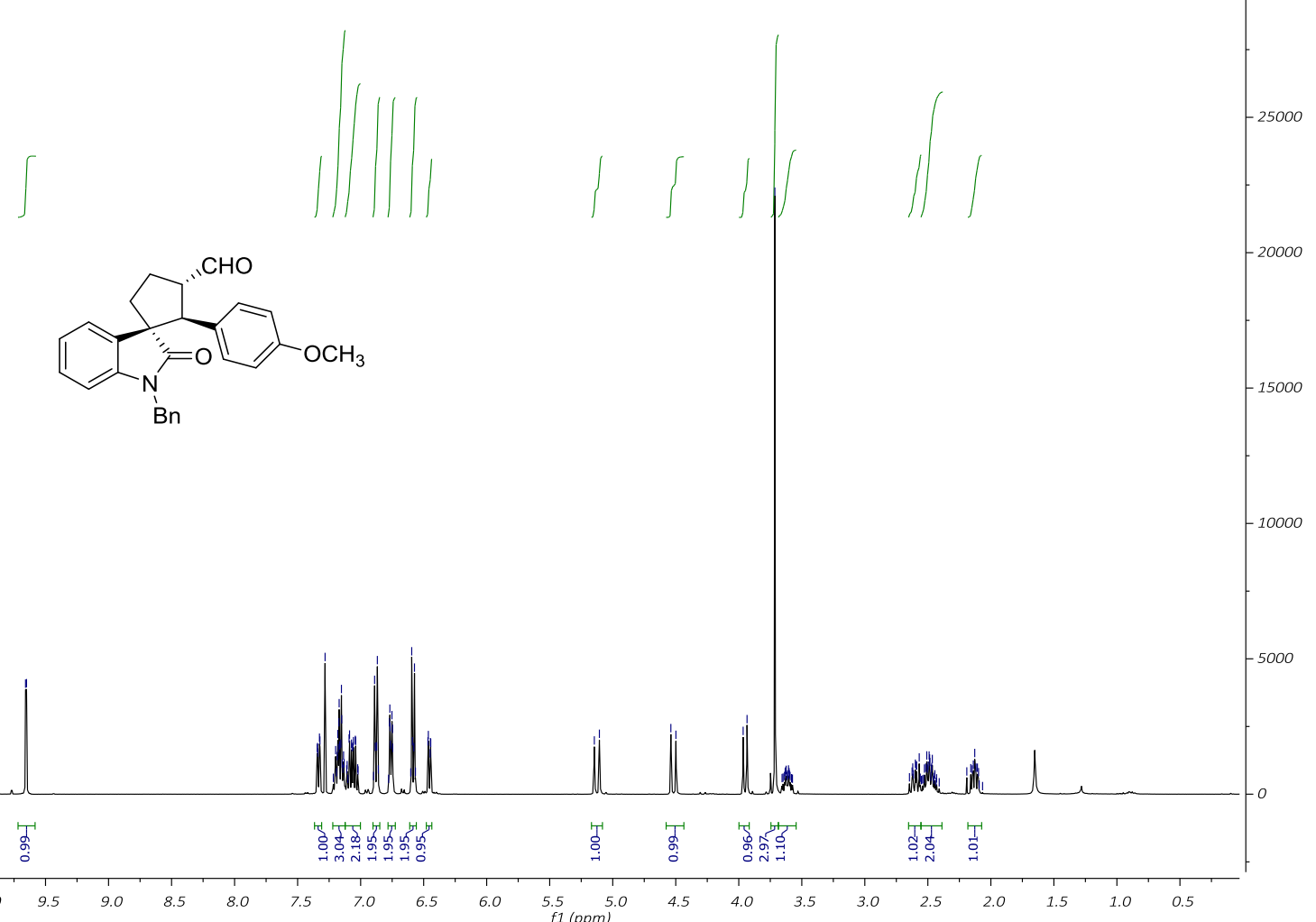

${ }^{13} \mathrm{C}\left\{{ }^{1} \mathrm{H}\right\} \mathrm{NMR}$ of $4 \mathrm{o}\left(101 \mathrm{MHz}, \mathrm{CDCl}_{3}\right)$

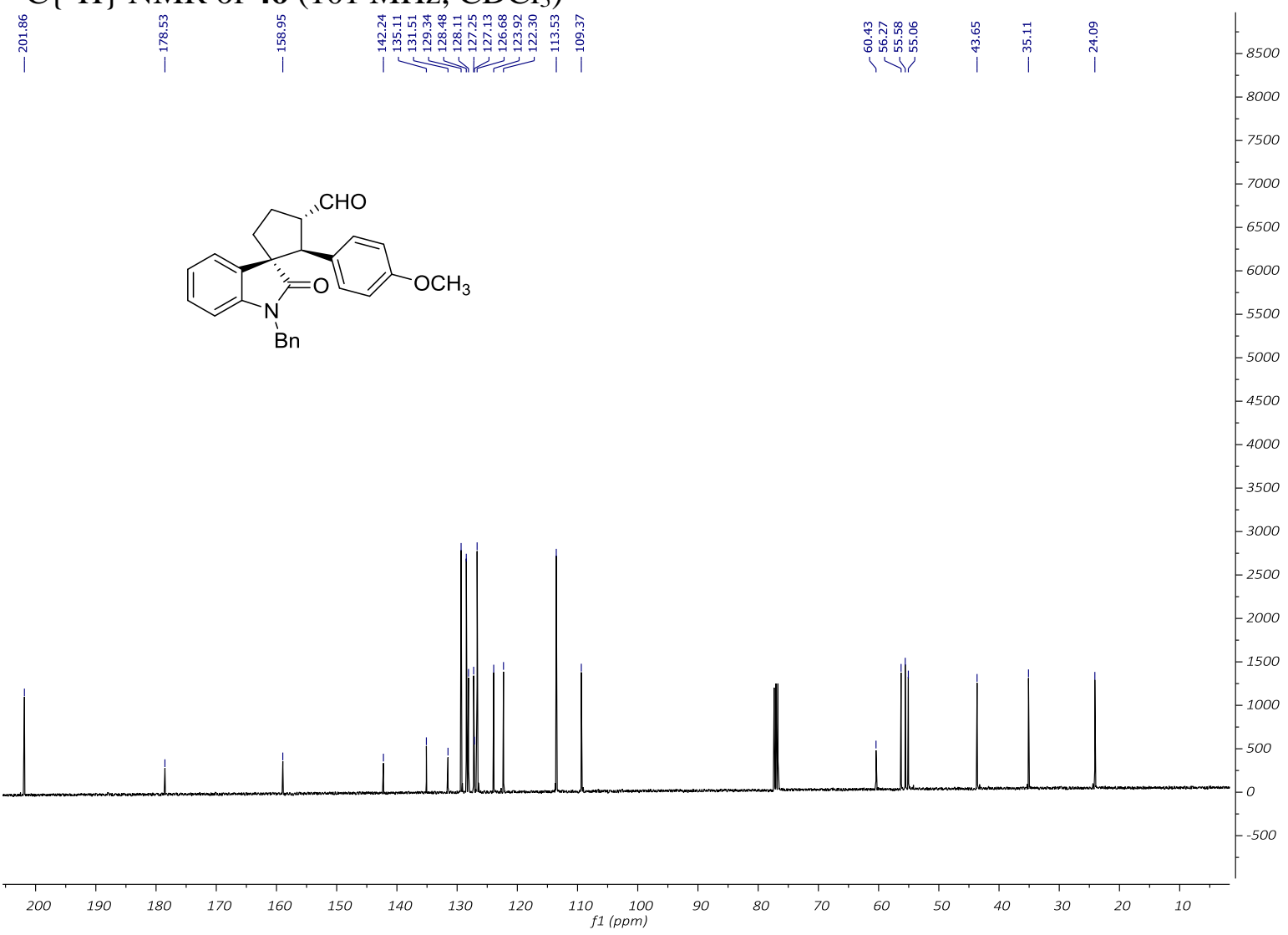


(1S,2S,3S)-1'-Benzyl-2-(4-nitrophenyl)-2'-oxospiro[cyclopentane-1,3'-indoline]-3carbaldehyde (3p)

${ }^{1} \mathrm{H} \mathrm{NMR}$ of $\mathbf{3 p}\left(400 \mathrm{MHz}, \mathrm{CDCl}_{3}\right)$

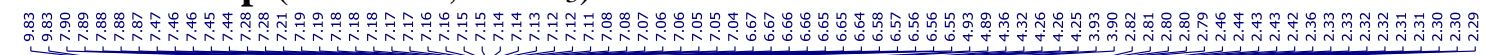

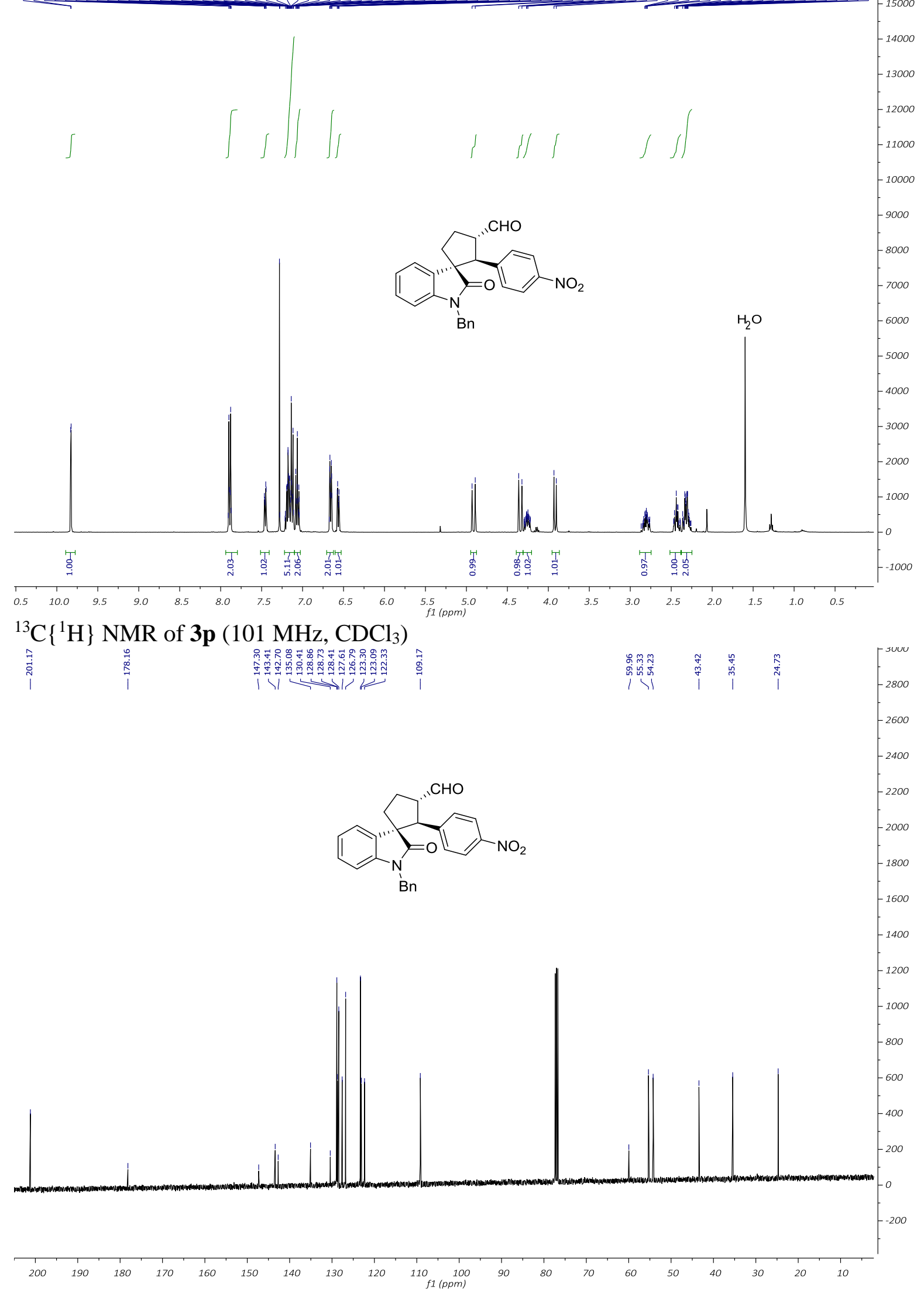




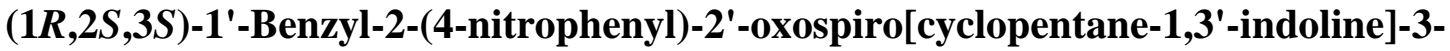
carbaldehyde (4p)

${ }^{1} \mathrm{H} \mathrm{NMR}$ of $\mathbf{4 p}\left(400 \mathrm{MHz}, \mathrm{CDCl}_{3}\right)$

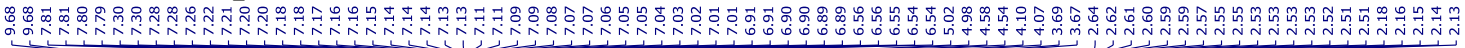

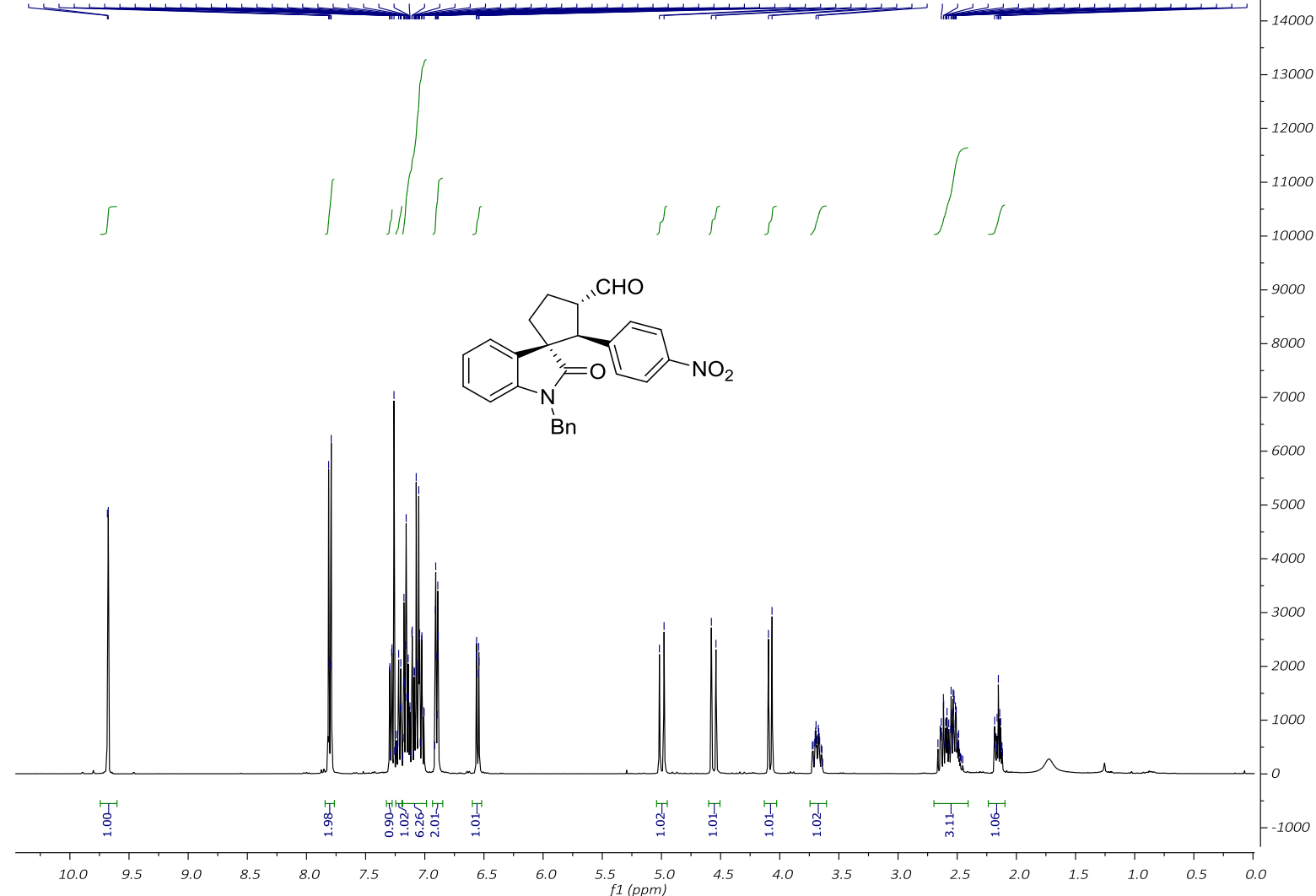

${ }^{13} \mathrm{C}\left\{{ }^{1} \mathrm{H}\right\}$ NMR of $\mathbf{4 p}\left(101 \mathrm{MHz}, \mathrm{CDCl}_{3}\right)$

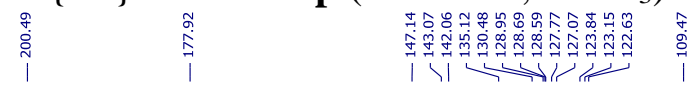
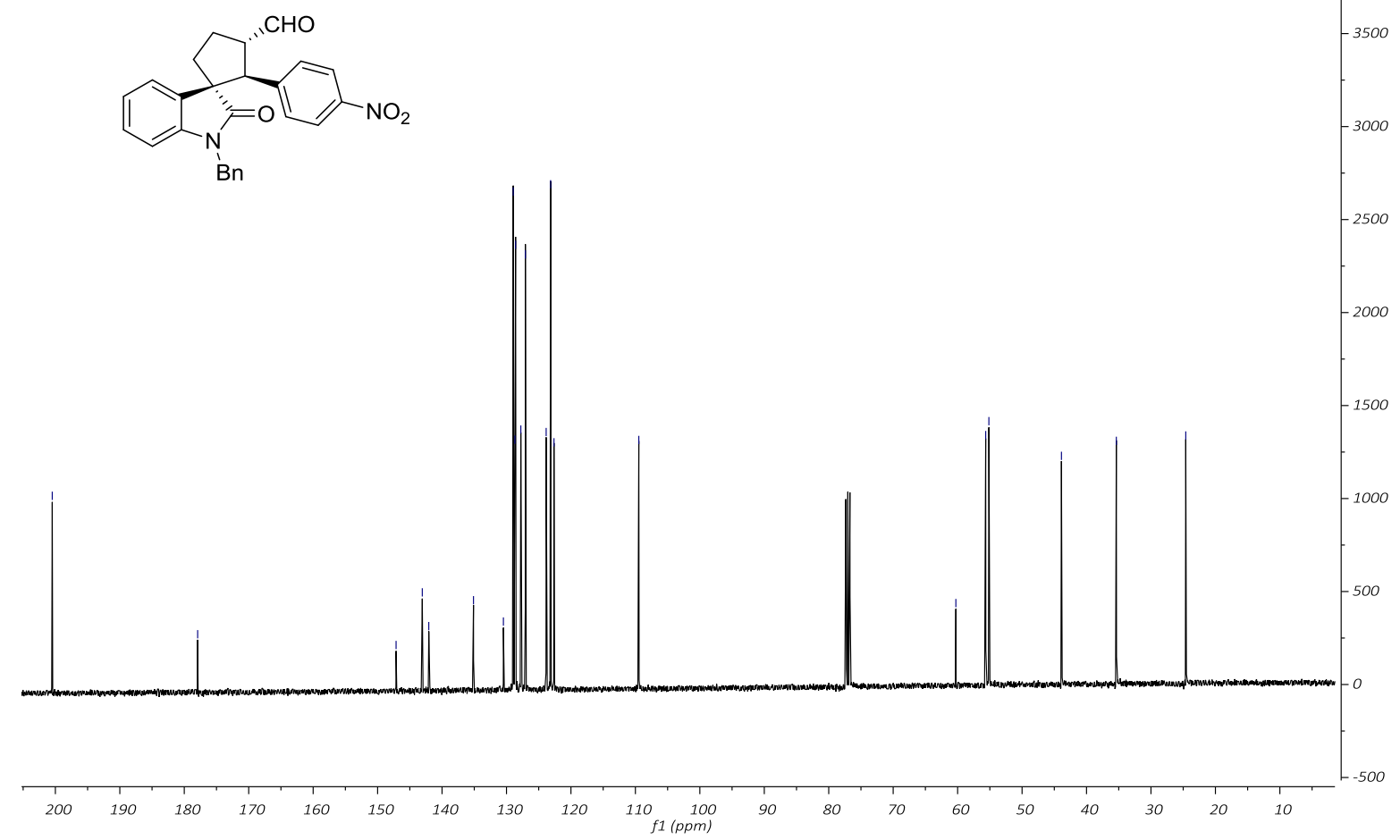
(1S,2S,3S)-1'-Benzyl-2'-oxo-2-(4-(trifluoromethyl)phenyl)spiro[cyclopentane-1,3'indoline]-3-carbaldehyde (3q)

${ }^{1} \mathrm{H}$ NMR of $\mathbf{3 q}\left(400 \mathrm{MHz}, \mathrm{CDCl}_{3}\right)$

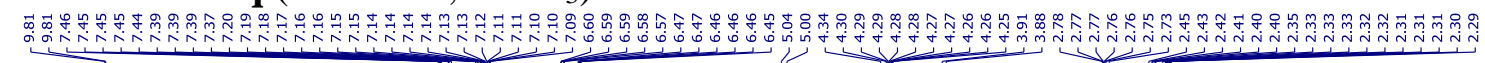

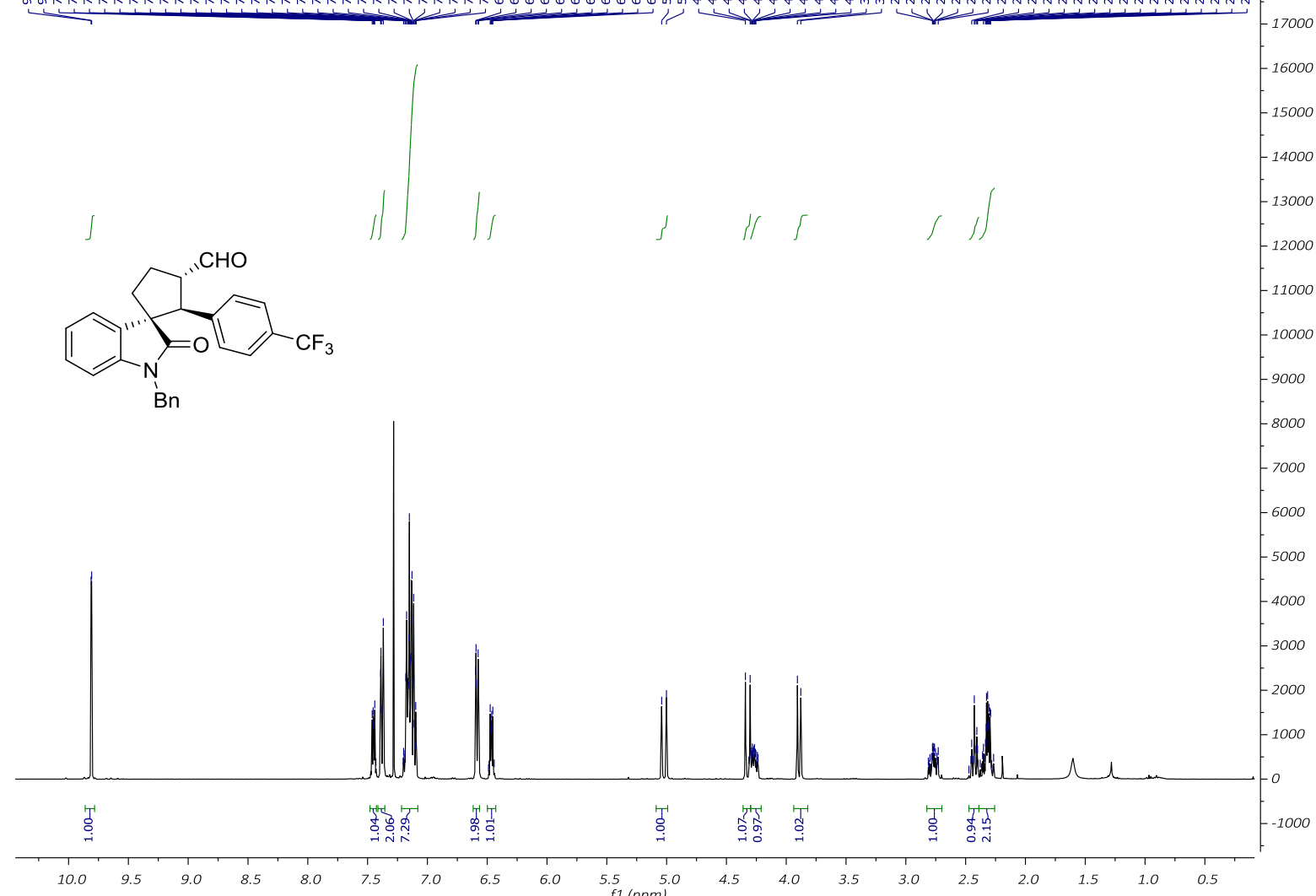

${ }^{13} \mathrm{C}\left\{{ }^{1} \mathrm{H}\right\}$ NMR of $\mathbf{3 q}\left(101 \mathrm{MHz}, \mathrm{CDCl}_{3}\right)$

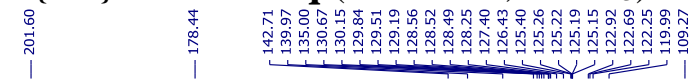
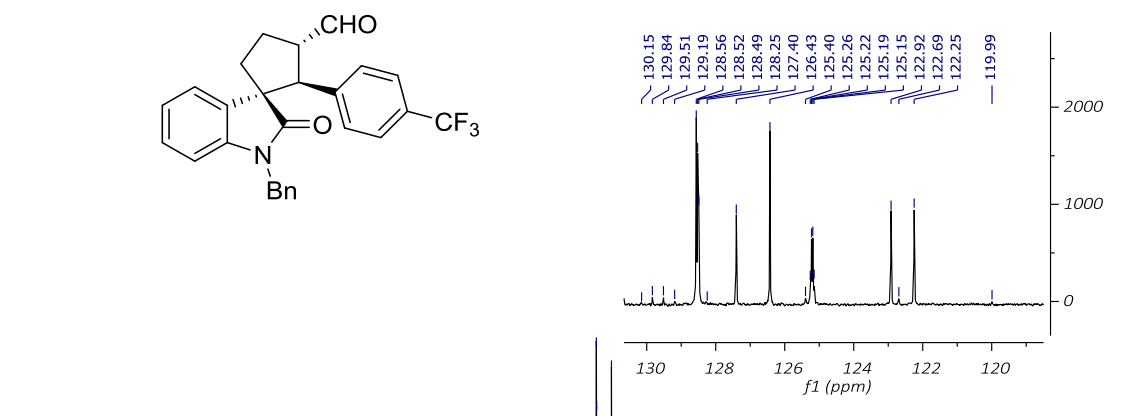

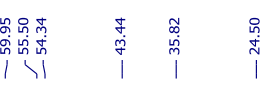

$-4500$

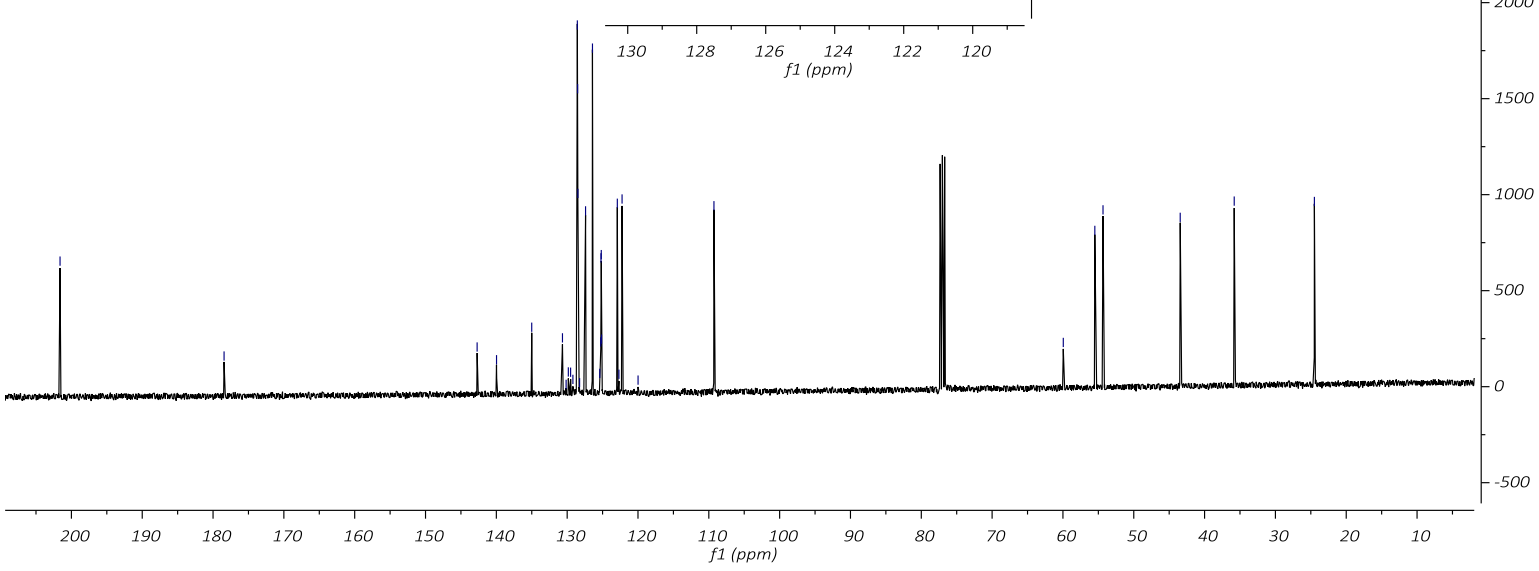


${ }^{19} \mathrm{~F} \mathrm{NMR}$ of 3q $\left(376 \mathrm{MHz} \mathrm{MHz}, \mathrm{CDCl}_{3}\right)$

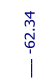<smiles></smiles>

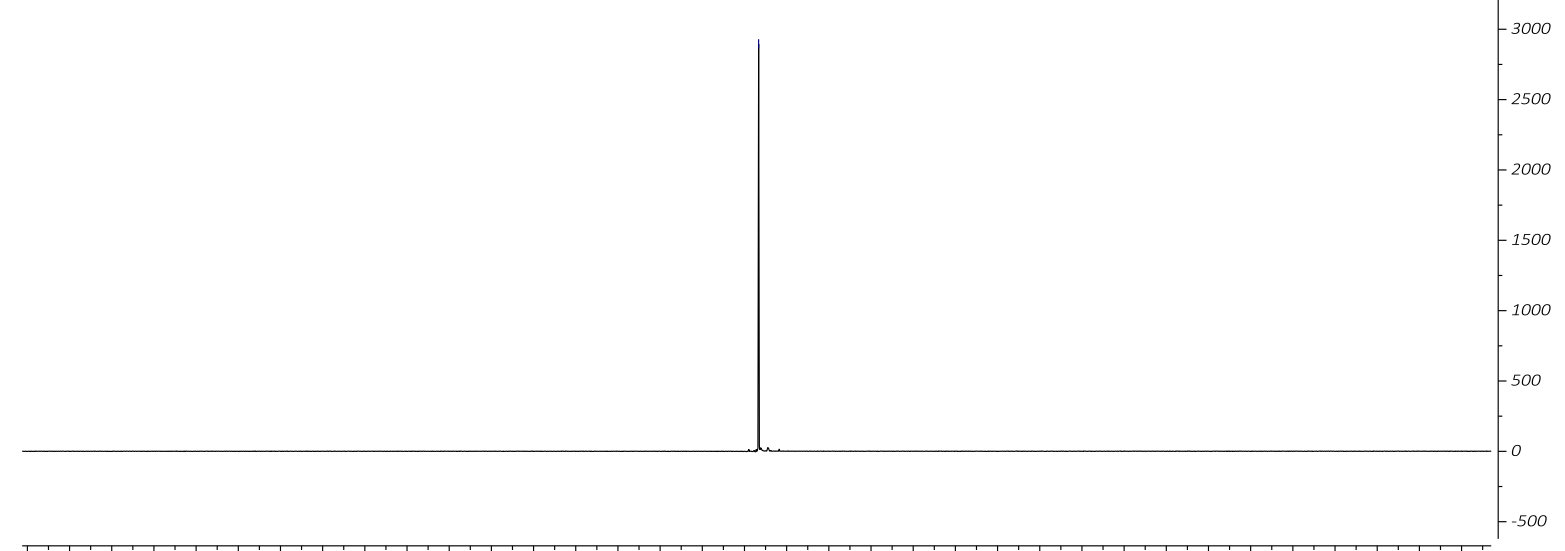

$\begin{array}{llllllllllllllllllllllllllllllllllllllllllllll}45 & -46 & -47 & -48 & -49 & -50 & -51 & -52 & -53 & -54 & -55 & -56 & -57 & -58 & -59 & -60 & -61 & -62 & -63 & -64 & -65 & -66 & -67 & -68 & -69 & -70 & -71 & -72 & -73 & -74 & -75 & -76 & -77 & -78 & -79\end{array}$ 


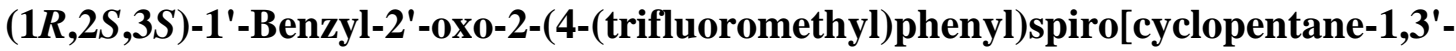
indoline]-3-carbaldehyde (4q)

${ }^{1} \mathrm{H}$ NMR of $\mathbf{4 q}\left(400 \mathrm{MHz}, \mathrm{CDCl}_{3}\right)$

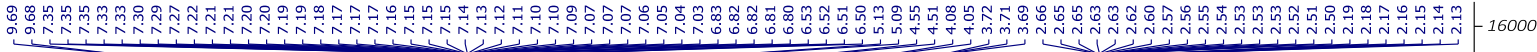

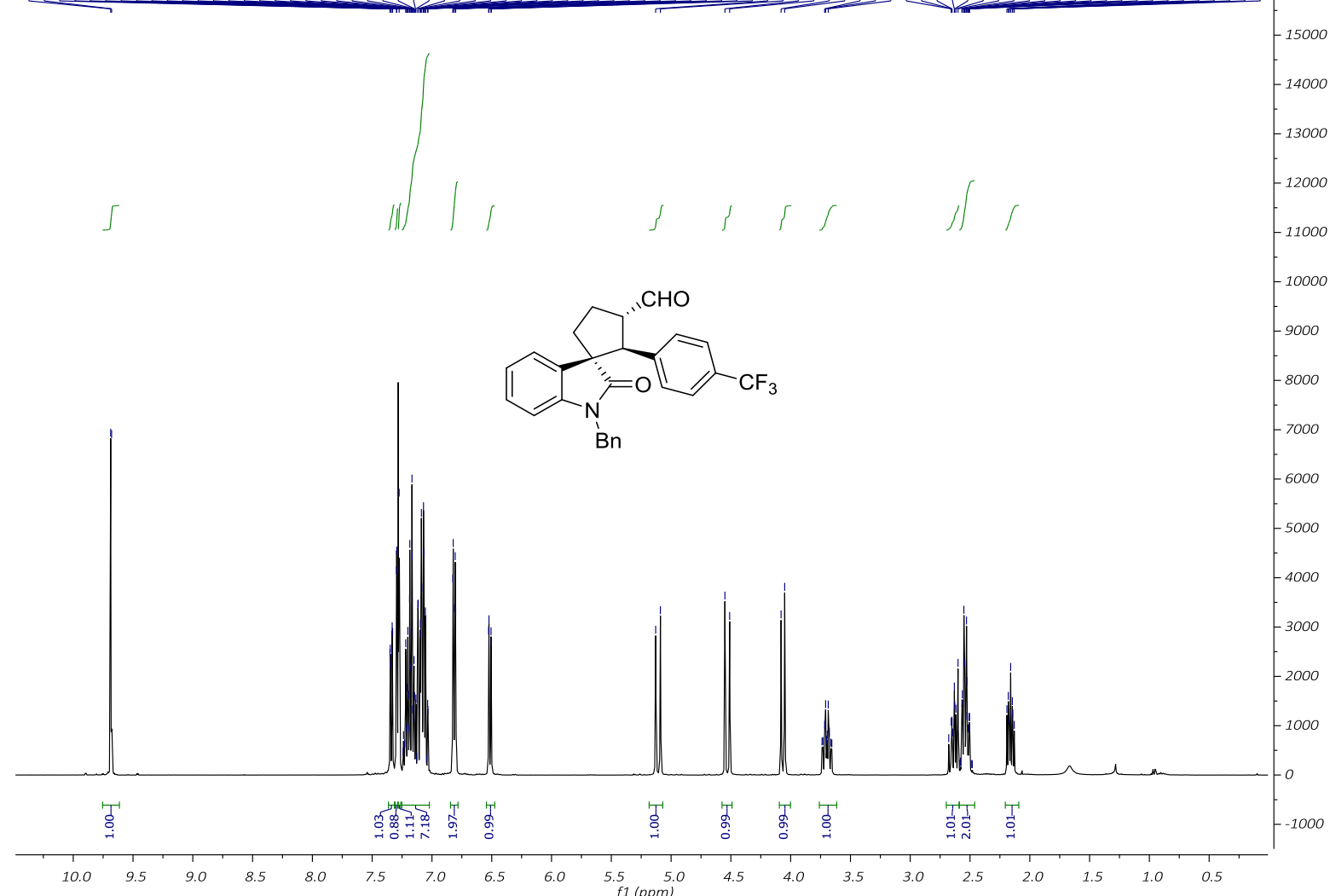

${ }^{13} \mathrm{C}\left\{{ }^{1} \mathrm{H}\right\}$ NMR of $\mathbf{4 q}\left(101 \mathrm{MHz}, \mathrm{CDCl}_{3}\right)$

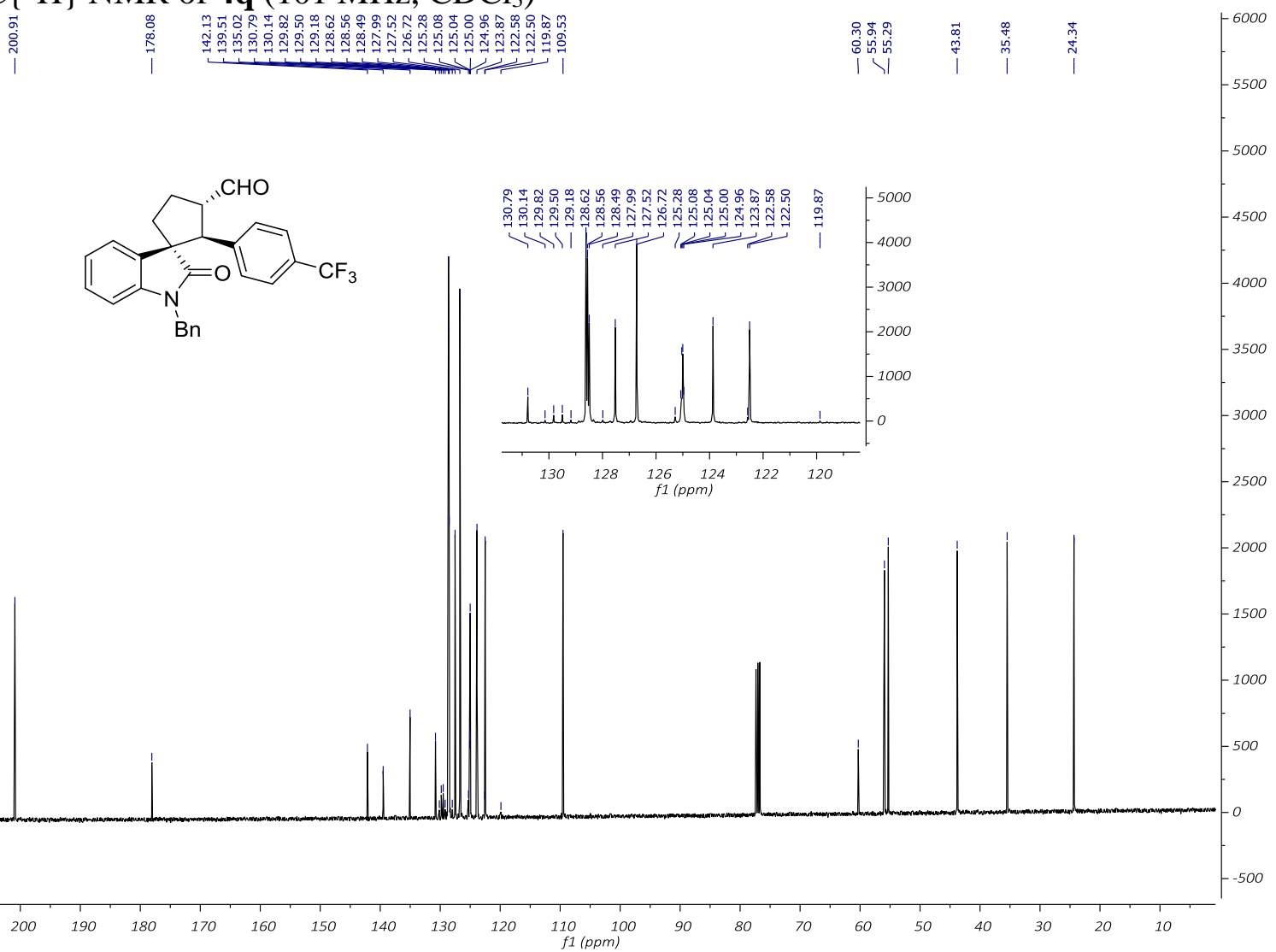


${ }^{19}$ F NMR of 4q (376 MHz MHz, $\mathrm{CDCl}_{3}$ )

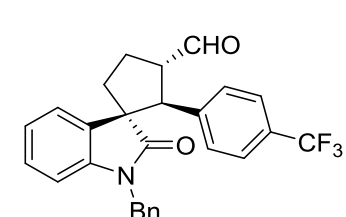

$\mathrm{Bn}$ 
(1S,2S,3S)-1'-Benzyl-2-(4-fluorophenyl)-2'-oxospiro[cyclopentane-1,3'-indoline]-3carbaldehyde (3r)

${ }^{1} \mathrm{H} \mathrm{NMR}$ of $3 \mathbf{r}\left(400 \mathrm{MHz}, \mathrm{CDCl}_{3}\right)$

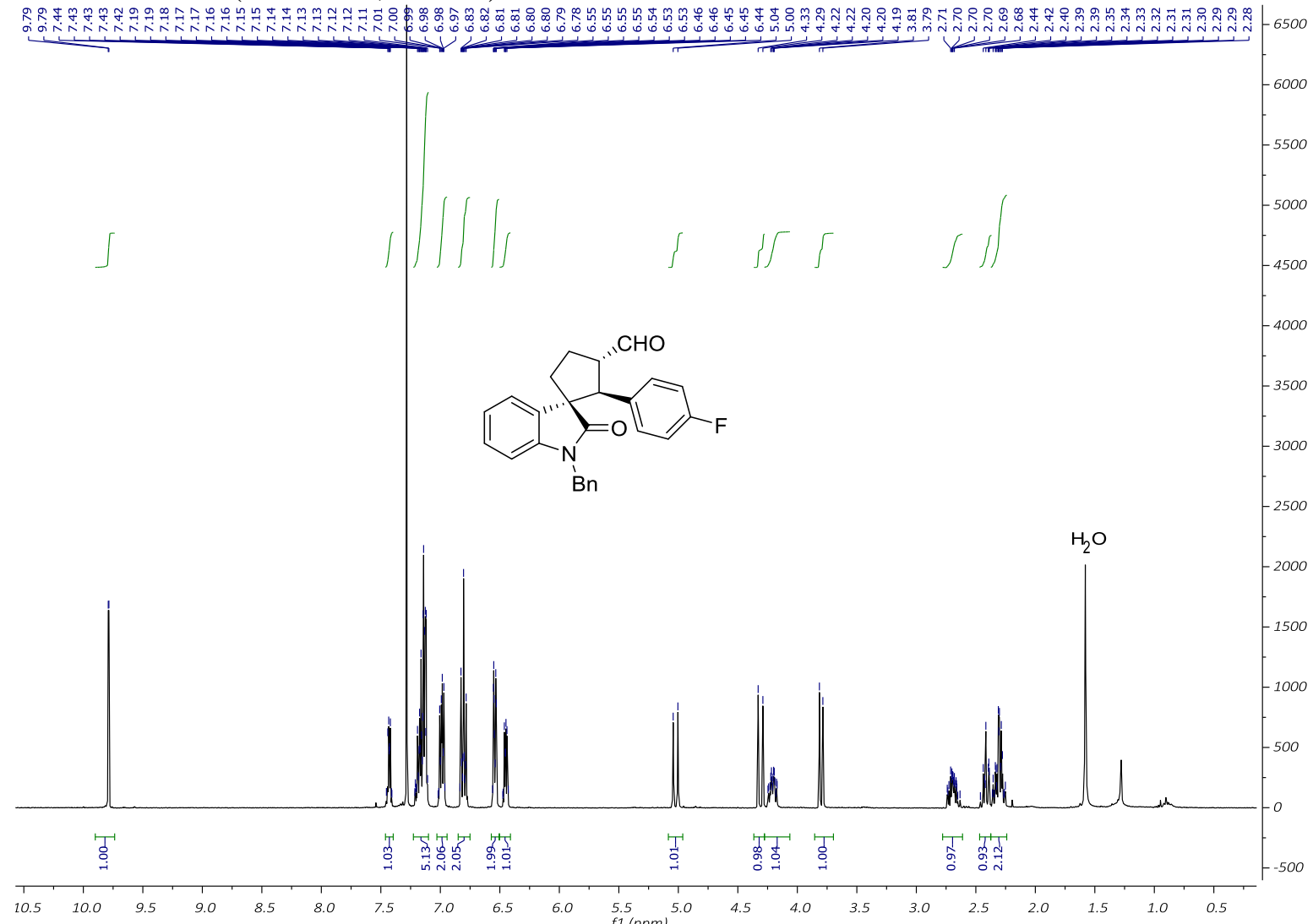

${ }^{13} \mathrm{C}\left\{{ }^{1} \mathrm{H}\right\}$ NMR of $3 \mathbf{r}\left(101 \mathrm{MHz}, \mathrm{CDCl}_{3}\right)$

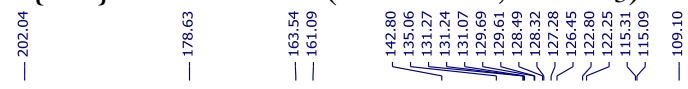
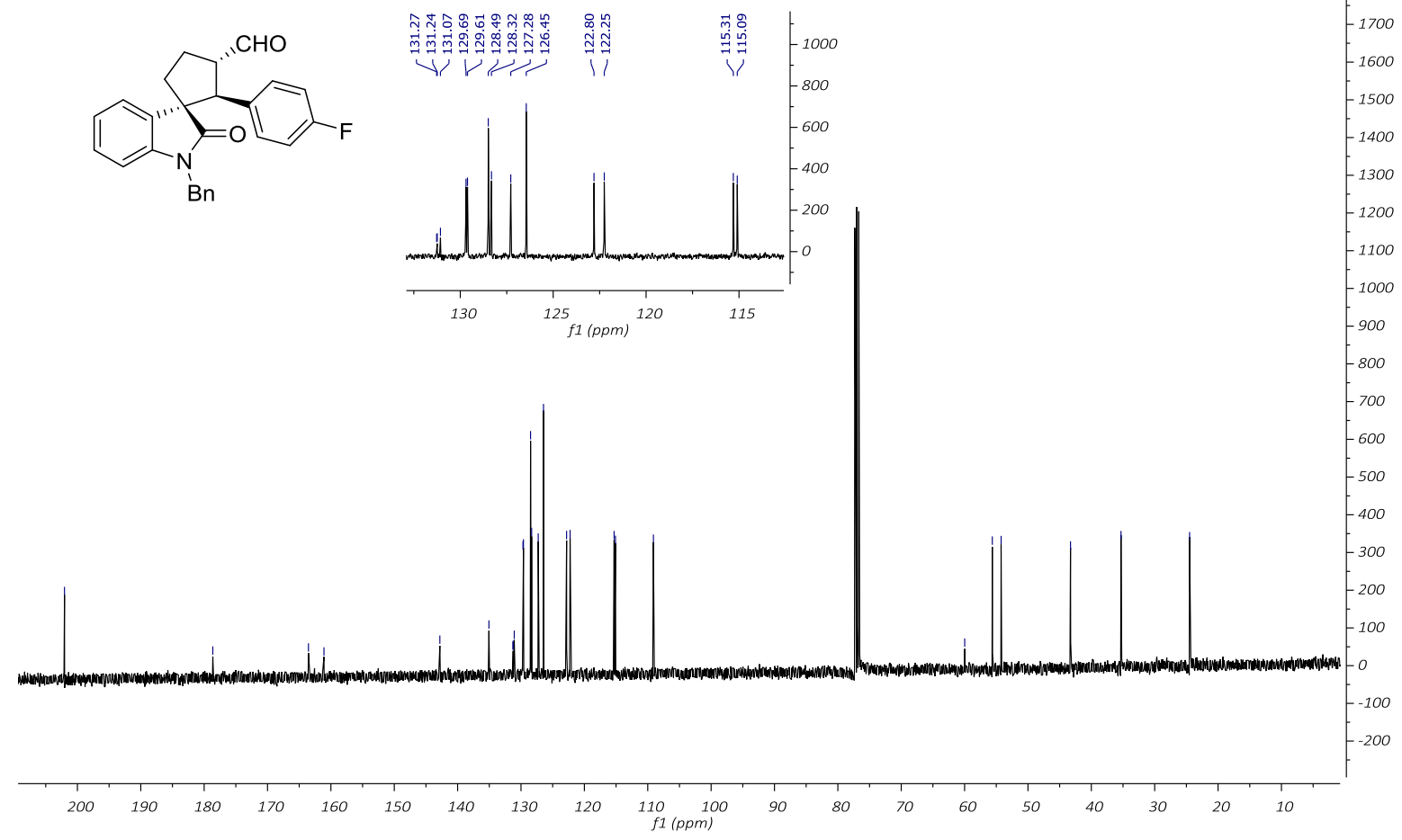

I

$-2100$ 
${ }^{19} \mathrm{~F} \mathrm{NMR}$ of $3 r\left(376 \mathrm{MHz} \mathrm{MHz}, \mathrm{CDCl}_{3}\right)$

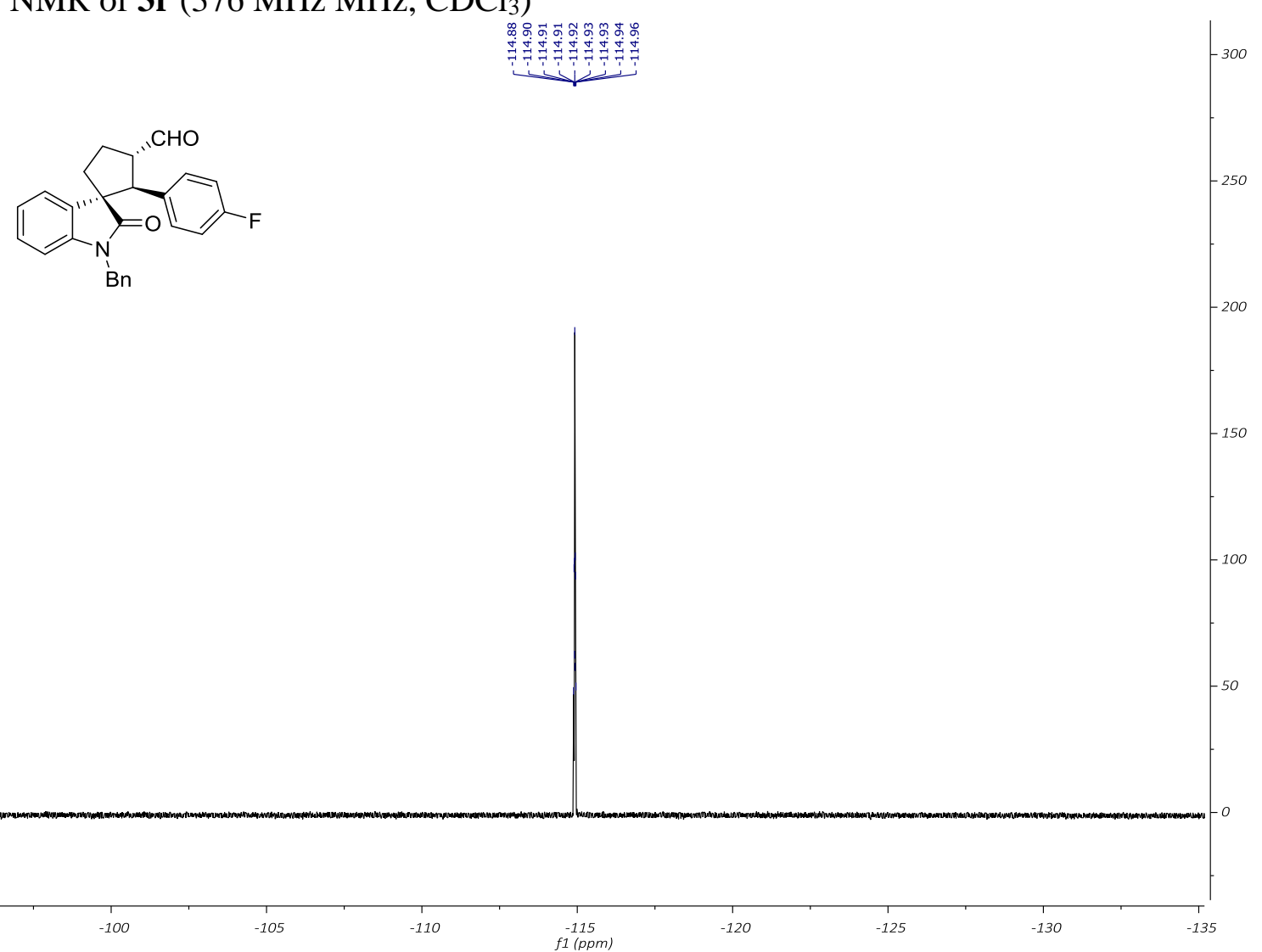


(1R,2S,3S)-1'-Benzyl-2-(4-fluorophenyl)-2'-oxospiro[cyclopentane-1,3'-indoline]-3carbaldehyde (4r)

${ }^{1} \mathrm{H} \mathrm{NMR}$ of $4 \mathbf{r}\left(400 \mathrm{MHz}, \mathrm{CDCl}_{3}\right)$

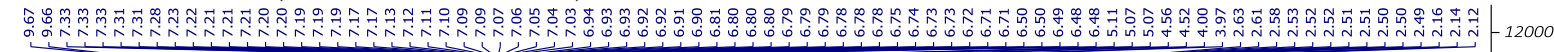

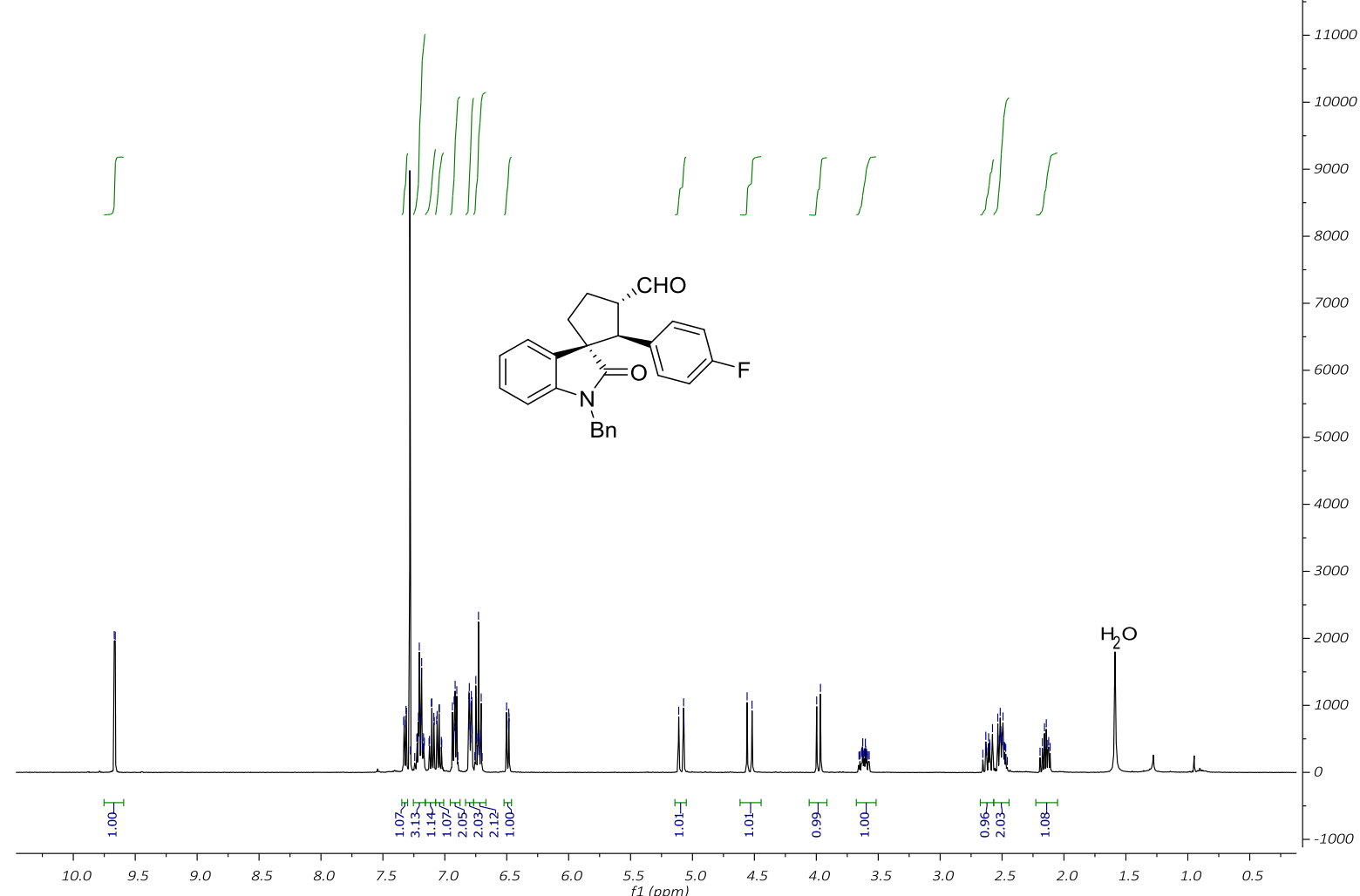

${ }^{13} \mathrm{C}\left\{{ }^{1} \mathrm{H}\right\}$ NMR of $4 \mathbf{r}\left(101 \mathrm{MHz}, \mathrm{CDCl}_{3}\right)$

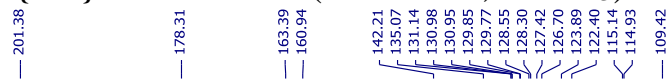
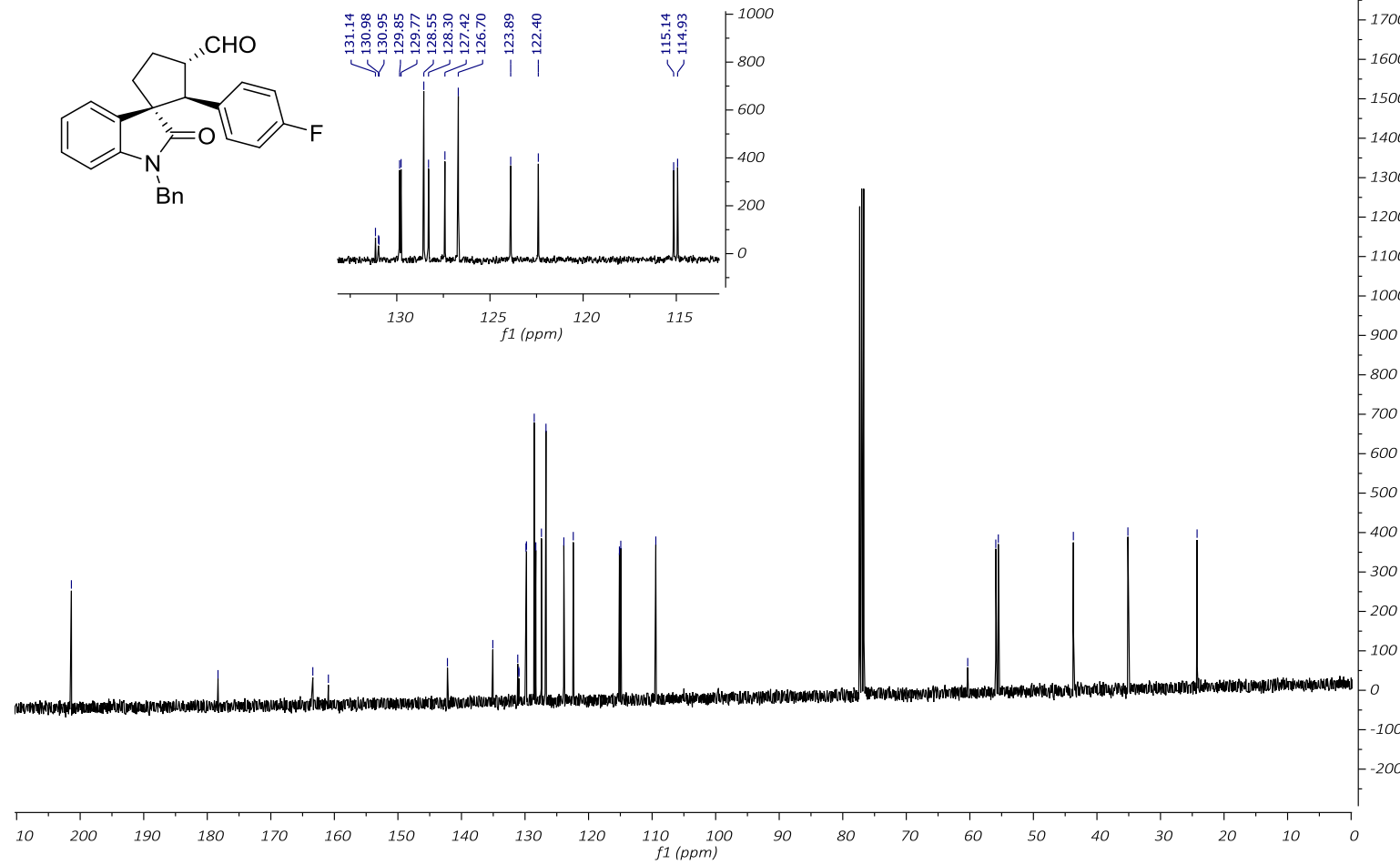
${ }^{19} \mathrm{~F}$ NMR of $4 \mathbf{r}\left(376 \mathrm{MHz} \mathrm{MHz}, \mathrm{CDCl}_{3}\right)$

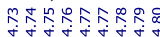

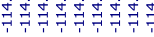
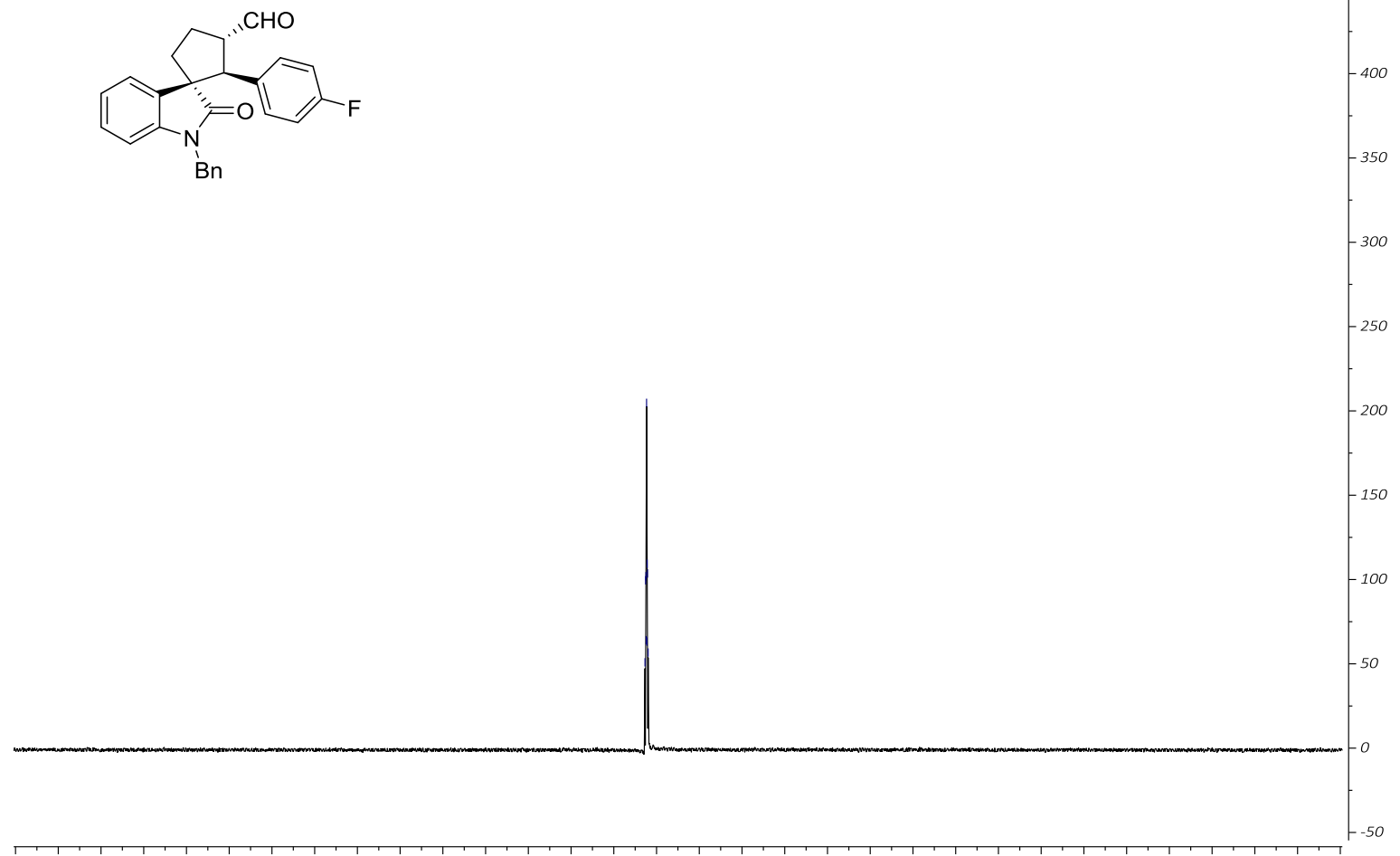

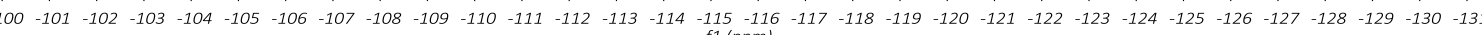


(1S,2S,3S)-1'-Benzyl-2-(4-chlorophenyl)-2'-oxospiro[cyclopentane-1,3'-indoline]-3carbaldehyde (3s)

${ }^{1} \mathrm{H}$ NMR of $3 \mathbf{s}\left(400 \mathrm{MHz}, \mathrm{CDCl}_{3}\right)$

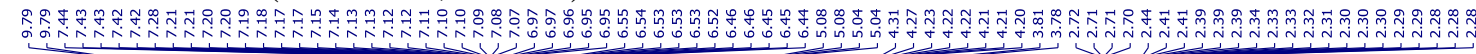

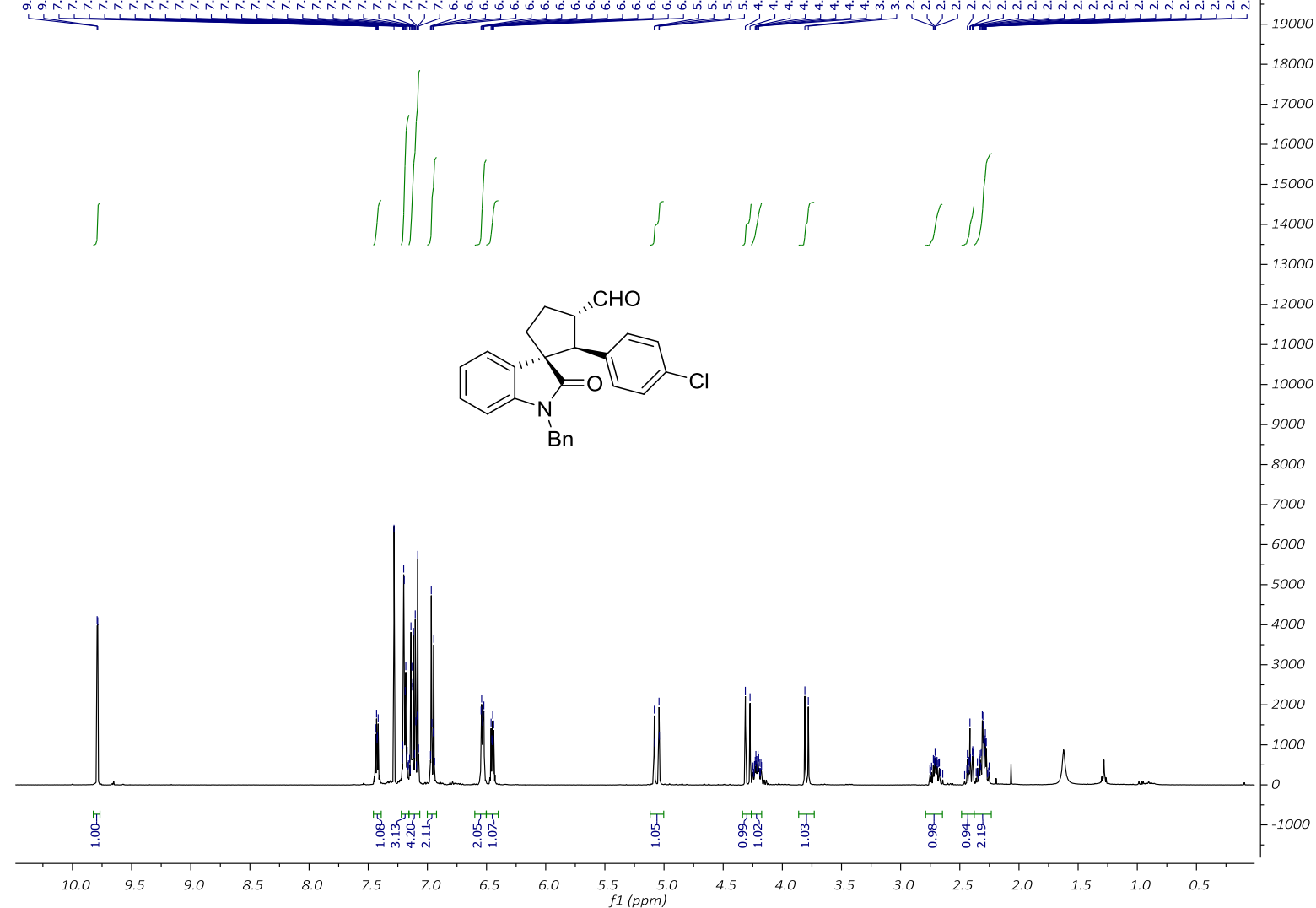

${ }^{13} \mathrm{C}\left\{{ }^{1} \mathrm{H}\right\}$ NMR of $3 \mathbf{s}\left(101 \mathrm{MHz}, \mathrm{CDCl}_{3}\right)$

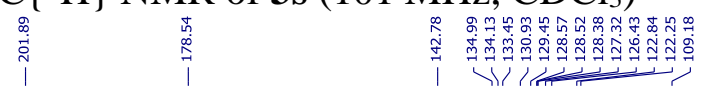

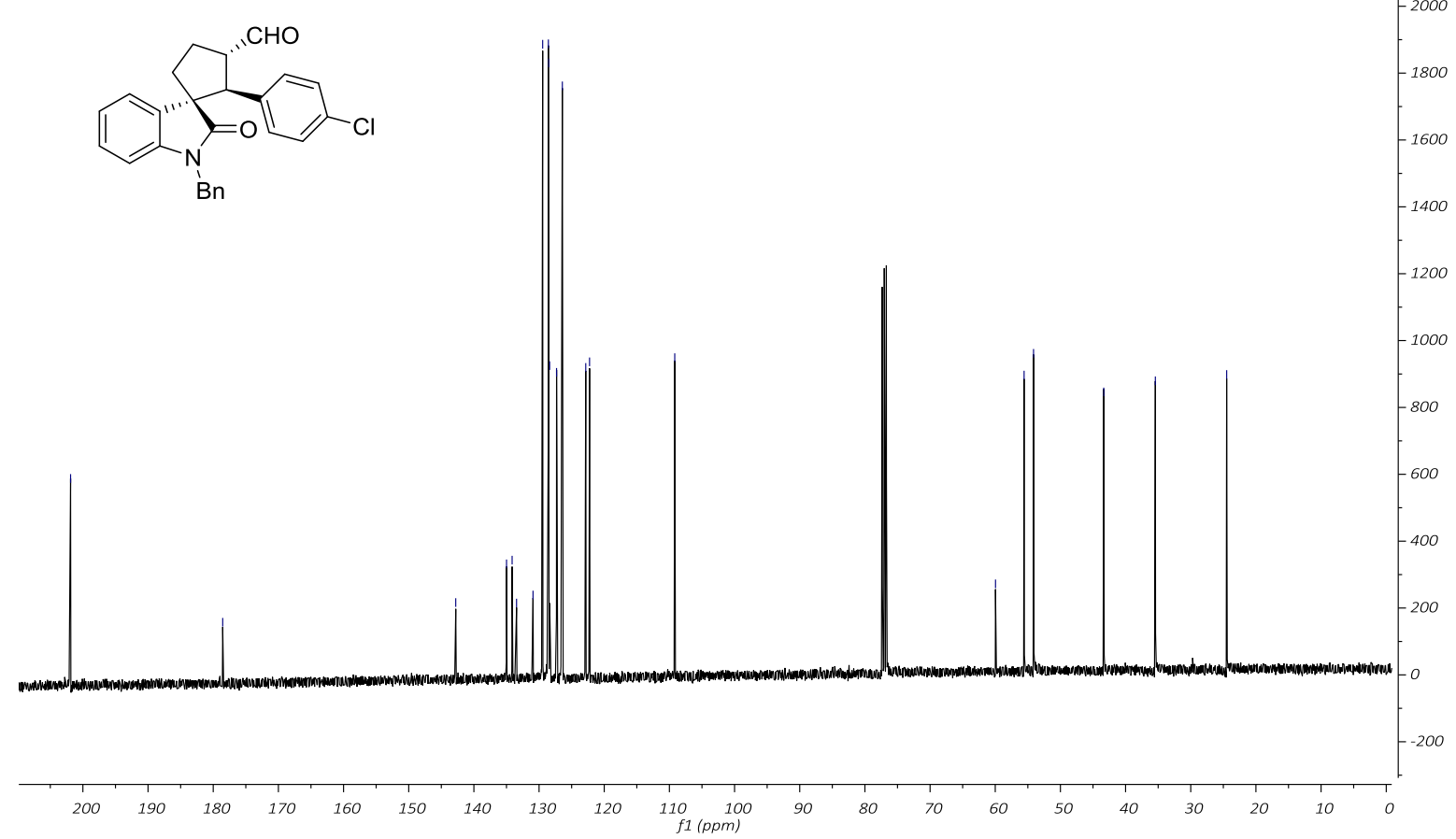

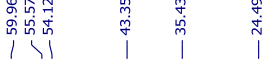

2400 
(1R,2S,3S)-1'-Benzyl-2-(4-chlorophenyl)-2'-oxospiro[cyclopentane-1,3'-indoline]-3carbaldehyde (4s)

${ }^{1} \mathrm{H} \mathrm{NMR}$ of $4 \mathbf{s}\left(400 \mathrm{MHz}, \mathrm{CDCl}_{3}\right)$

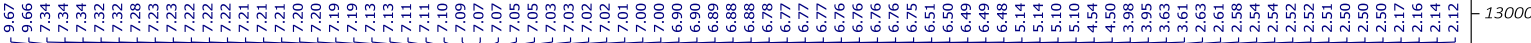

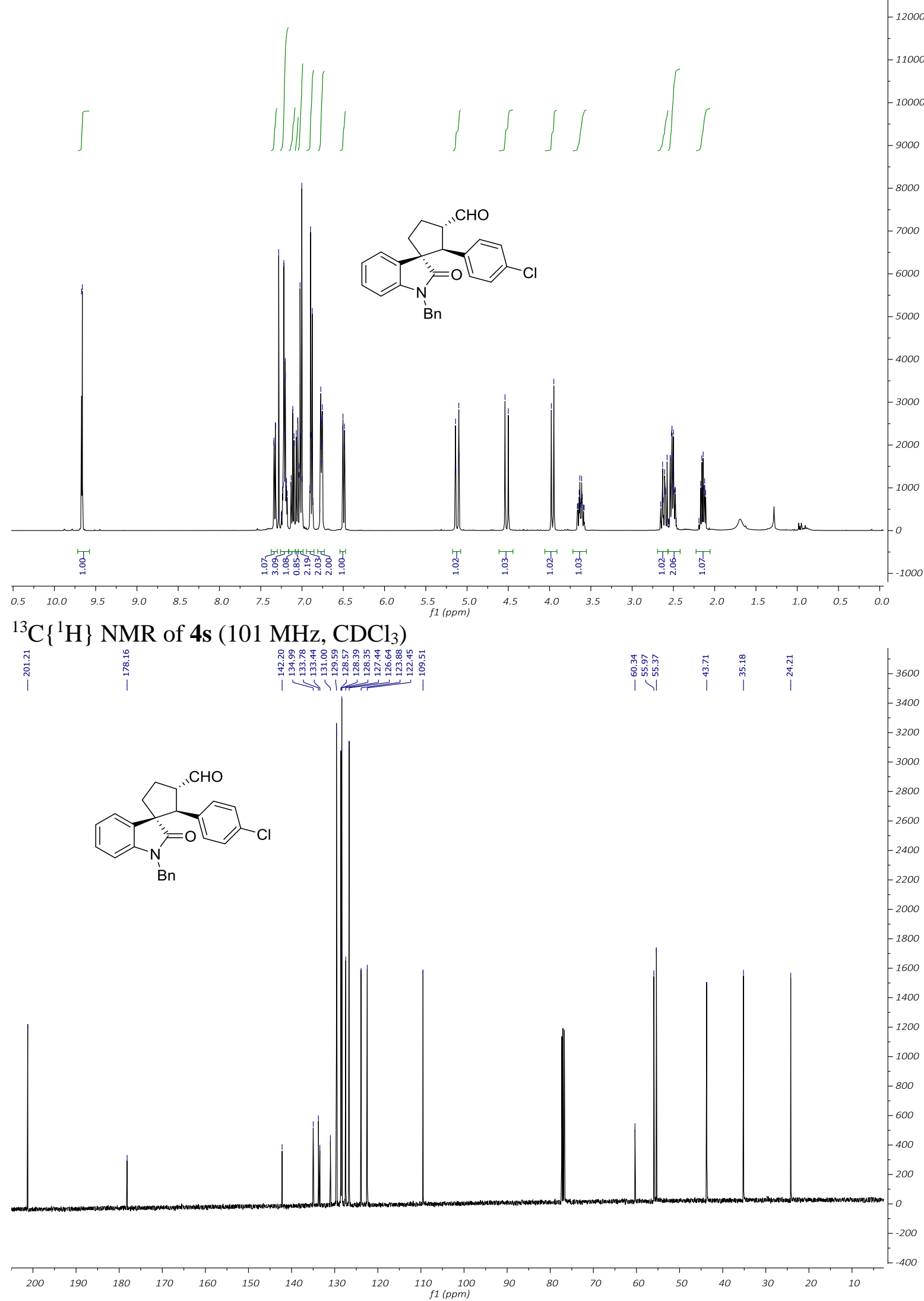


(1S,2S,3S)-1'-Benzyl-2-(4-bromophenyl)-2'-oxospiro[cyclopentane-1,3'-indoline]-3carbaldehyde (3t)

${ }^{1} \mathrm{H} \mathrm{NMR}$ of $\mathbf{3 t}\left(400 \mathrm{MHz}, \mathrm{CDCl}_{3}\right)$

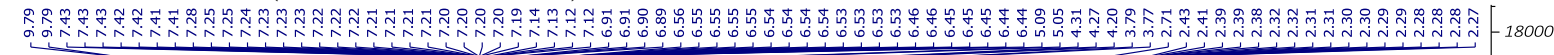<smiles>O=CC1CCCC1C(=O)Nc1ccccc1</smiles>
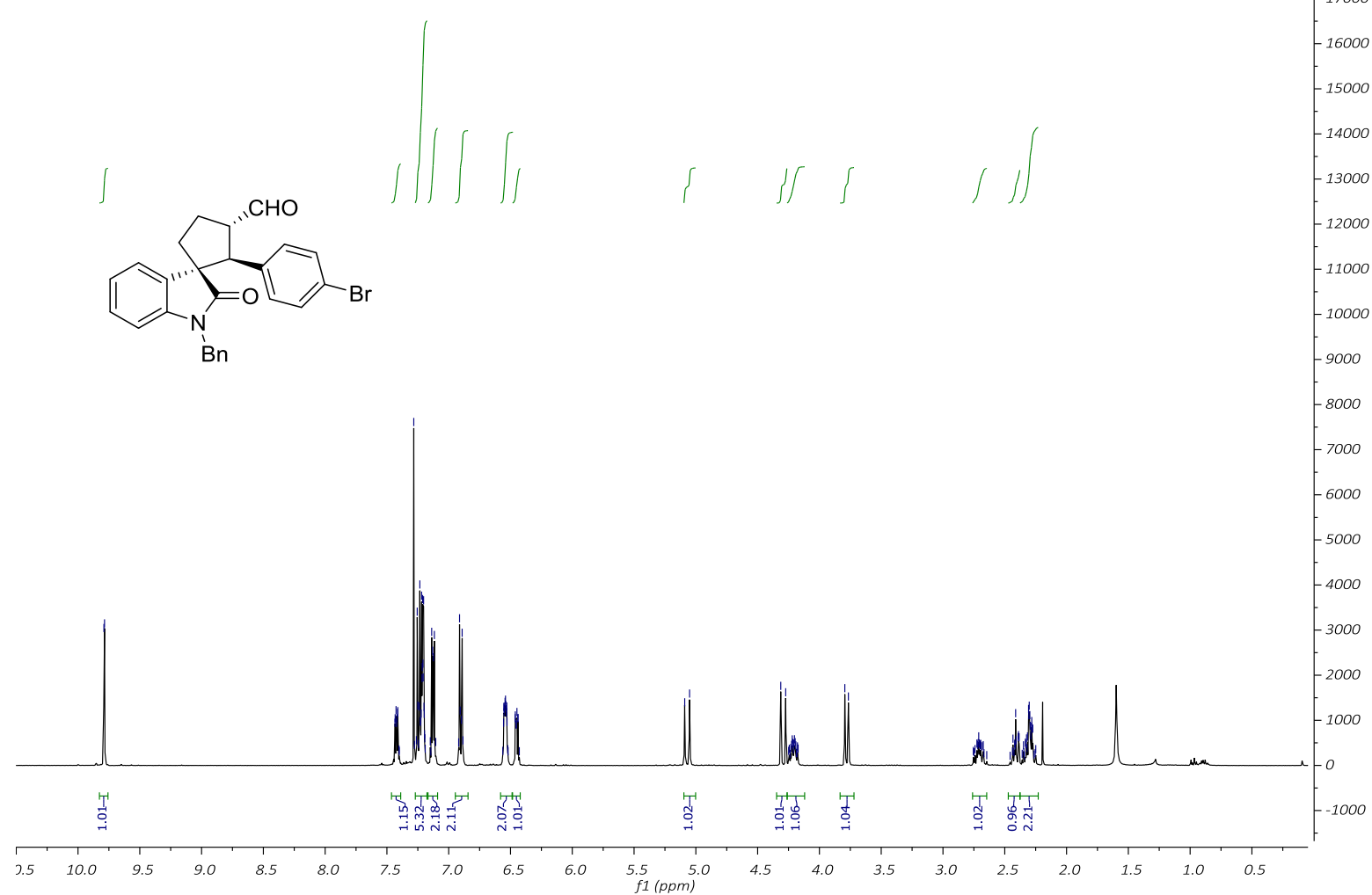

${ }^{13} \mathrm{C}\left\{{ }^{1} \mathrm{H}\right\} \mathrm{NMR}$ of $3 \mathbf{t}\left(101 \mathrm{MHz}, \mathrm{CDCl}_{3}\right)$

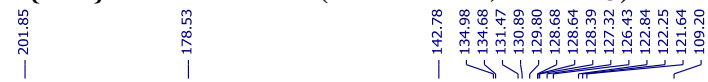
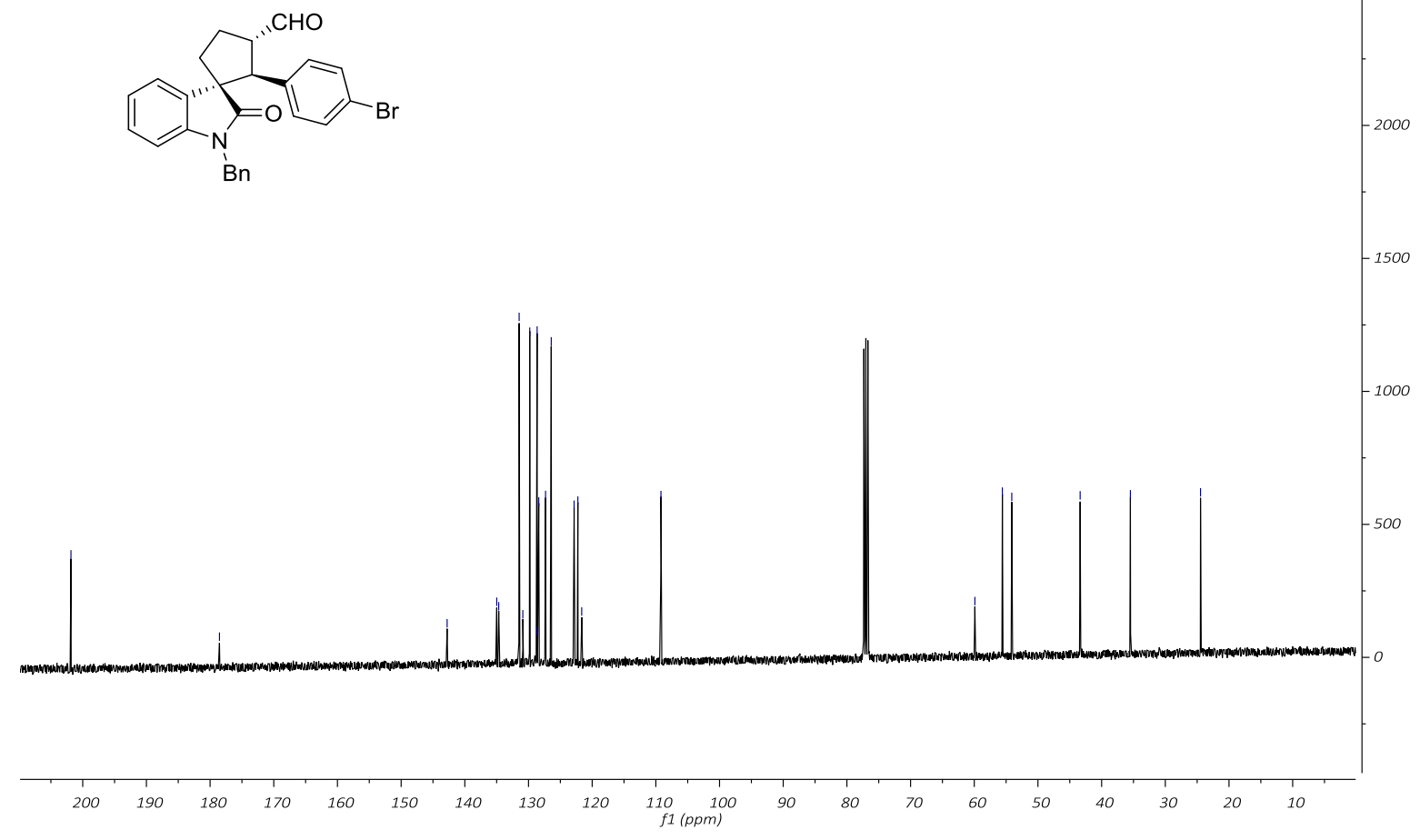
(1R,2S,3S)-1'-Benzyl-2-(4-bromophenyl)-2'-oxospiro[cyclopentane-1,3'-indoline]-3carbaldehyde (4t)

${ }^{1} \mathrm{H} \mathrm{NMR}$ of $\mathbf{4 t}\left(400 \mathrm{MHz}, \mathrm{CDCl}_{3}\right)$

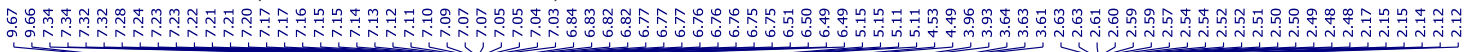

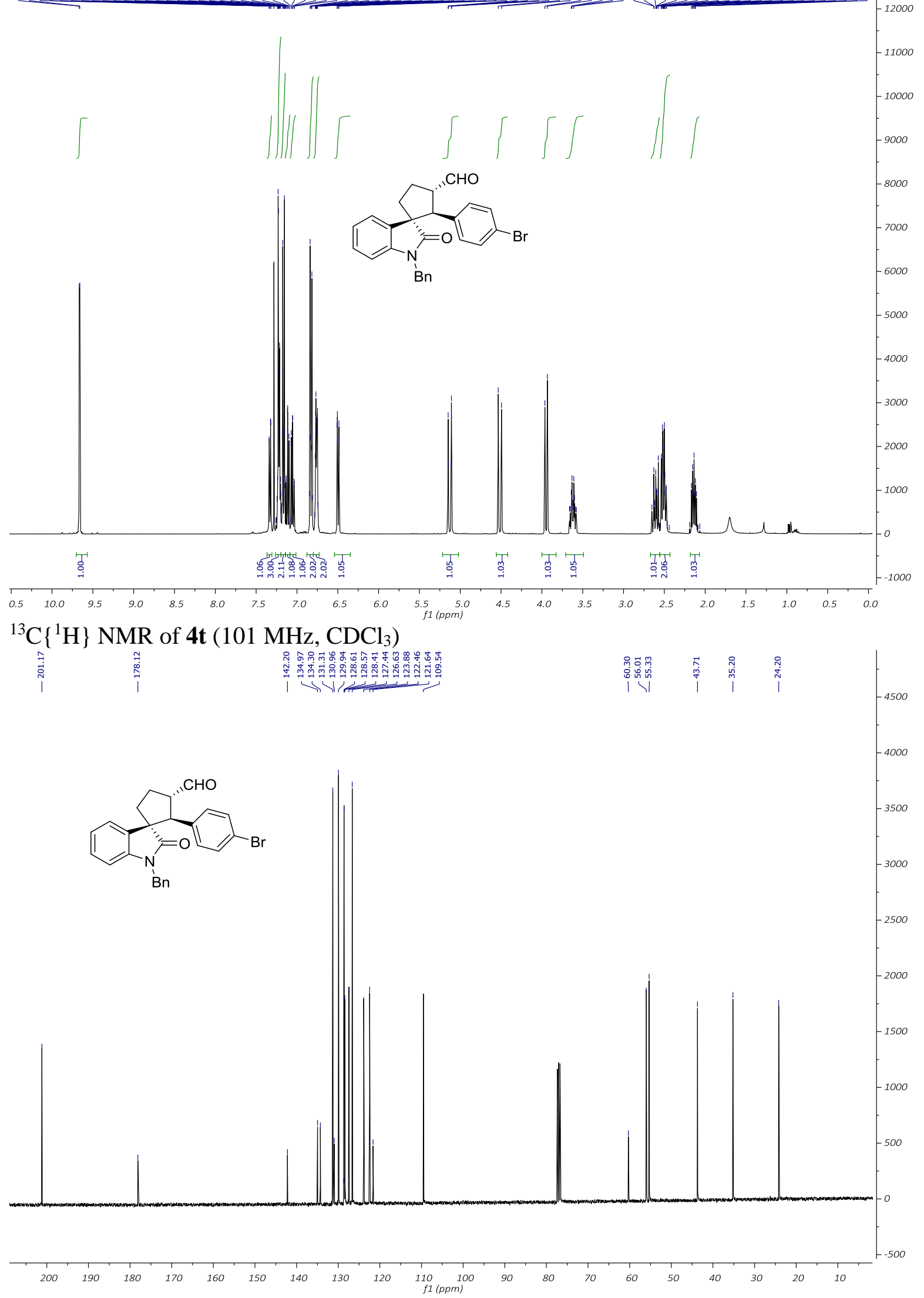


(1S,2S,3S)-1'-Benzyl-2-(3-bromophenyl)-2'-oxospiro[cyclopentane-1,3'-indoline]-3carbaldehyde (3u)

${ }^{1} \mathrm{H}$ NMR of $3 \mathbf{u}\left(400 \mathrm{MHz}, \mathrm{CDCl}_{3}\right)$

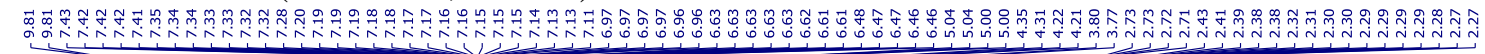

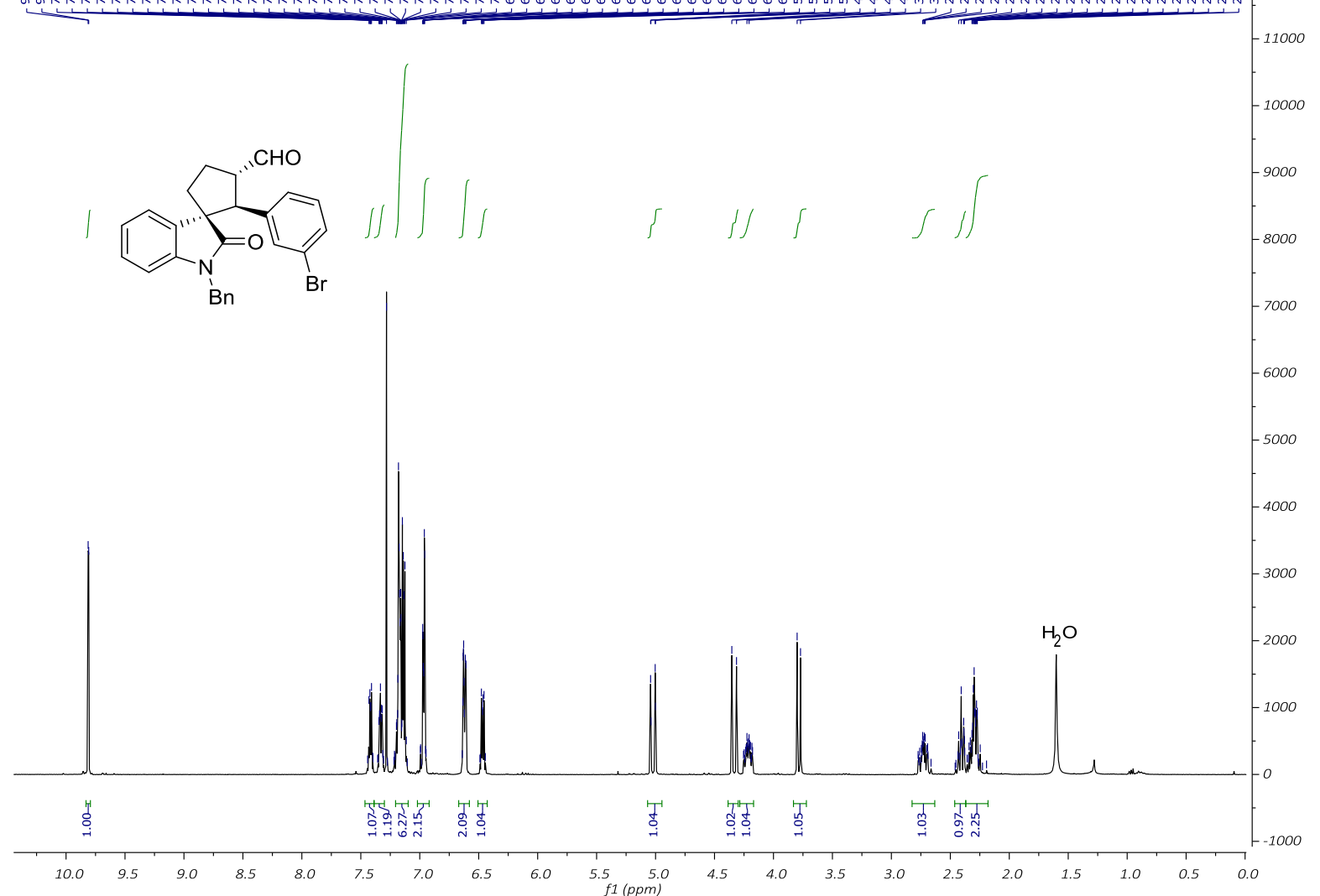

${ }^{13} \mathrm{C}\left\{{ }^{1} \mathrm{H}\right\}$ NMR of $3 \mathbf{u}\left(101 \mathrm{MHz}, \mathrm{CDCl}_{3}\right)$

|
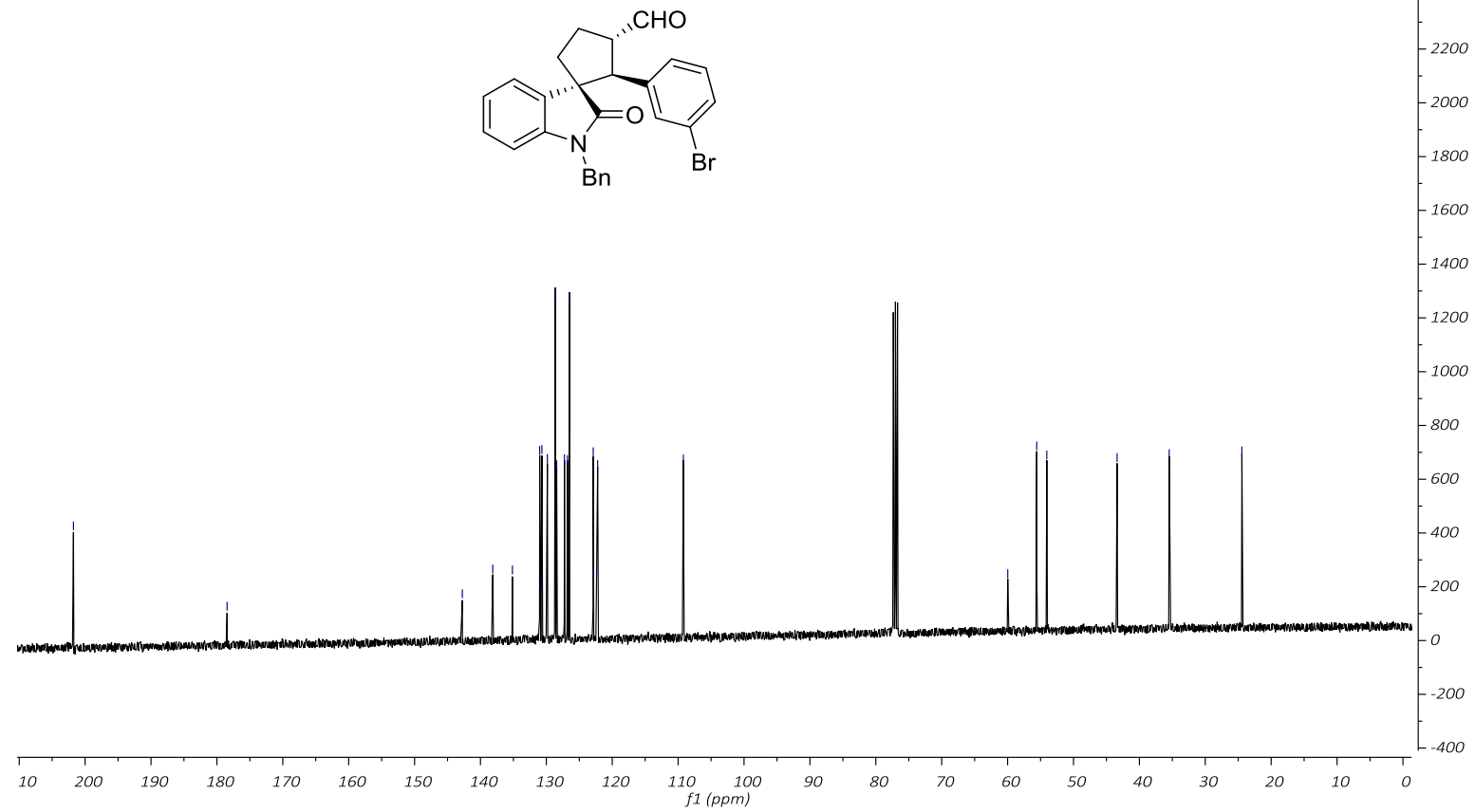
(1R,2S,3S)-1'-benzyl-2-(3-bromophenyl)-2'-oxospiro[cyclopentane-1,3'-indoline]-3carbaldehyde (4u)

${ }^{1} \mathrm{H} \mathrm{NMR}$ of $4 \mathbf{u}\left(400 \mathrm{MHz}, \mathrm{CDCl}_{3}\right)$

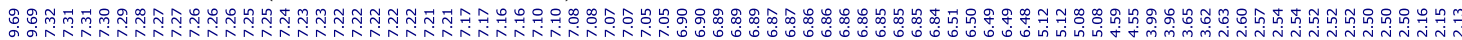

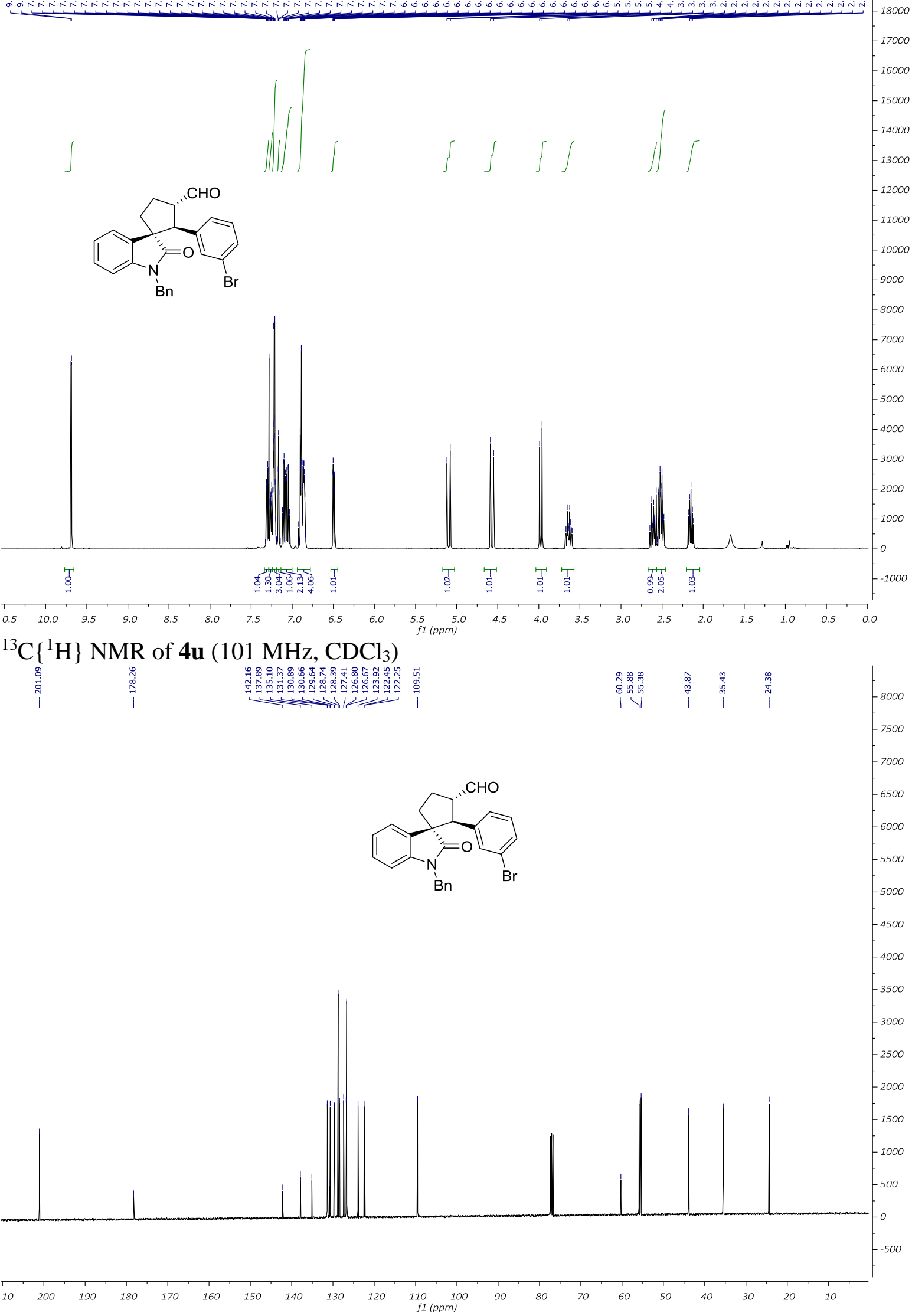


(1S,2R,3S)-1'-Benzyl-2-(2-bromophenyl)-2'-oxospiro[cyclopentane-1,3'-indoline]-3carbaldehyde (3v)

${ }^{1} \mathrm{H}$ NMR of $3 \mathbf{v}\left(400 \mathrm{MHz}, \mathrm{CDCl}_{3}\right)$

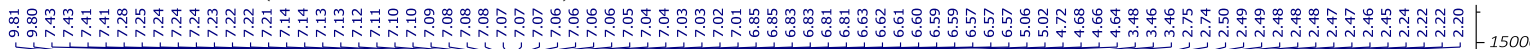

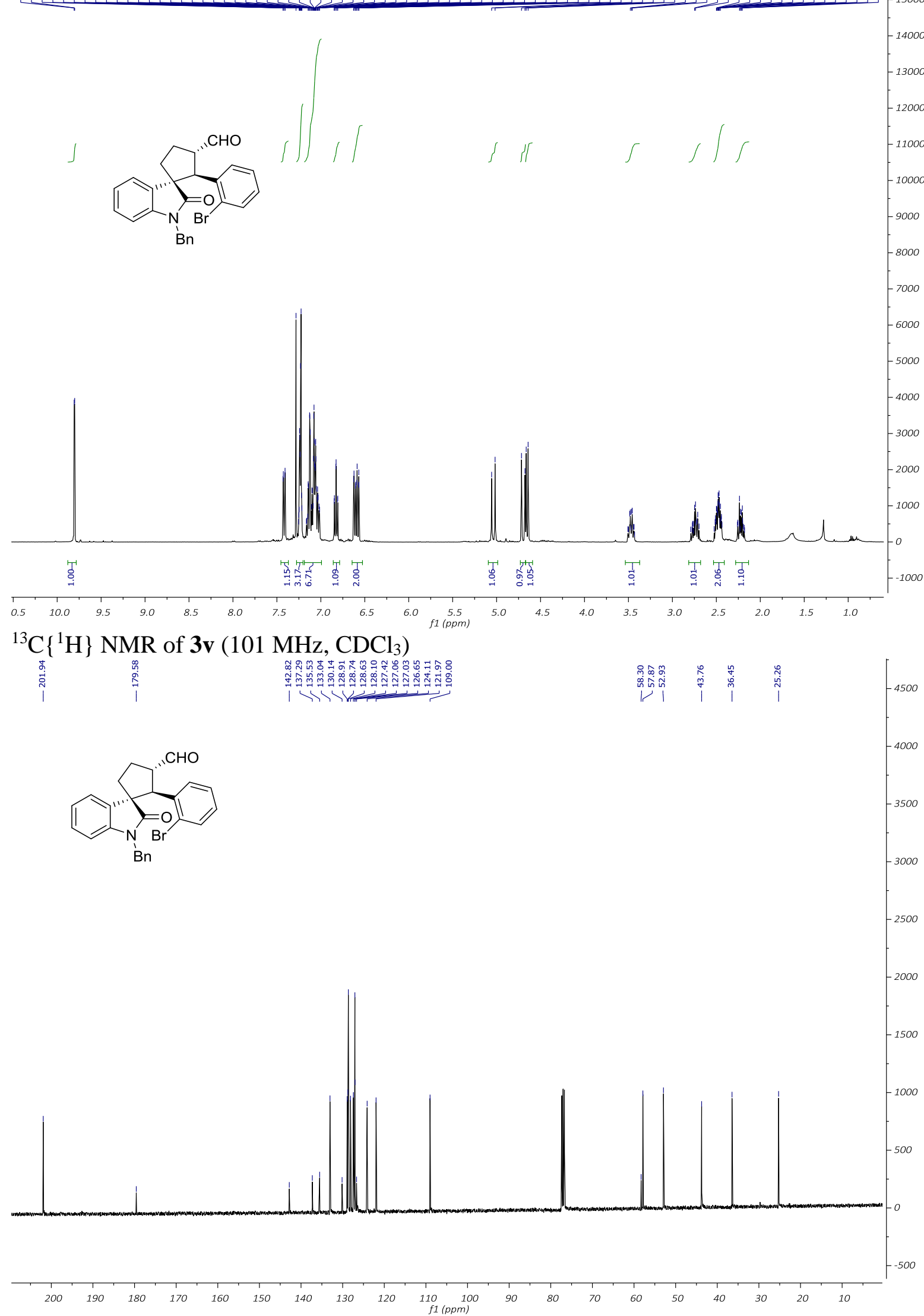


$(1 R, 2 R, 3 S)-1$ '-Benzyl-2-(2-bromophenyl)-2'-oxospiro[cyclopentane-1,3'-indoline]-3carbaldehyde (4v)

${ }^{1} \mathrm{H}$ NMR of $4 \mathbf{v}\left(400 \mathrm{MHz}, \mathrm{CDCl}_{3}\right)$

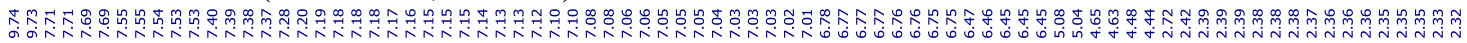

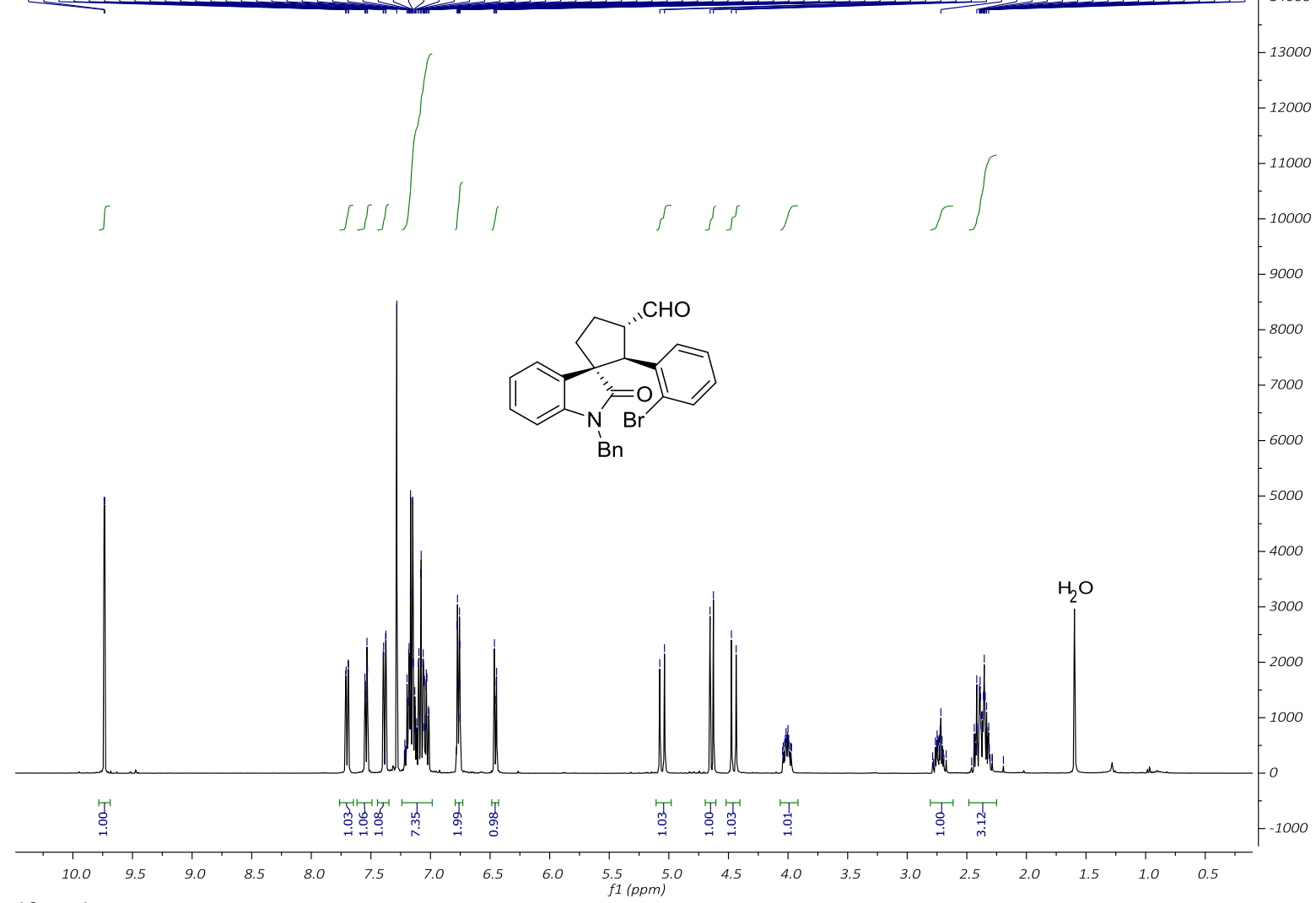

${ }^{13} \mathrm{C}\left\{{ }^{1} \mathrm{H}\right\}$ NMR of $4 \mathbf{v}\left(101 \mathrm{MHz}, \mathrm{CDCl}_{3}\right)$

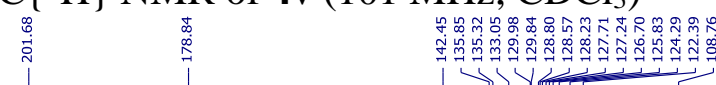
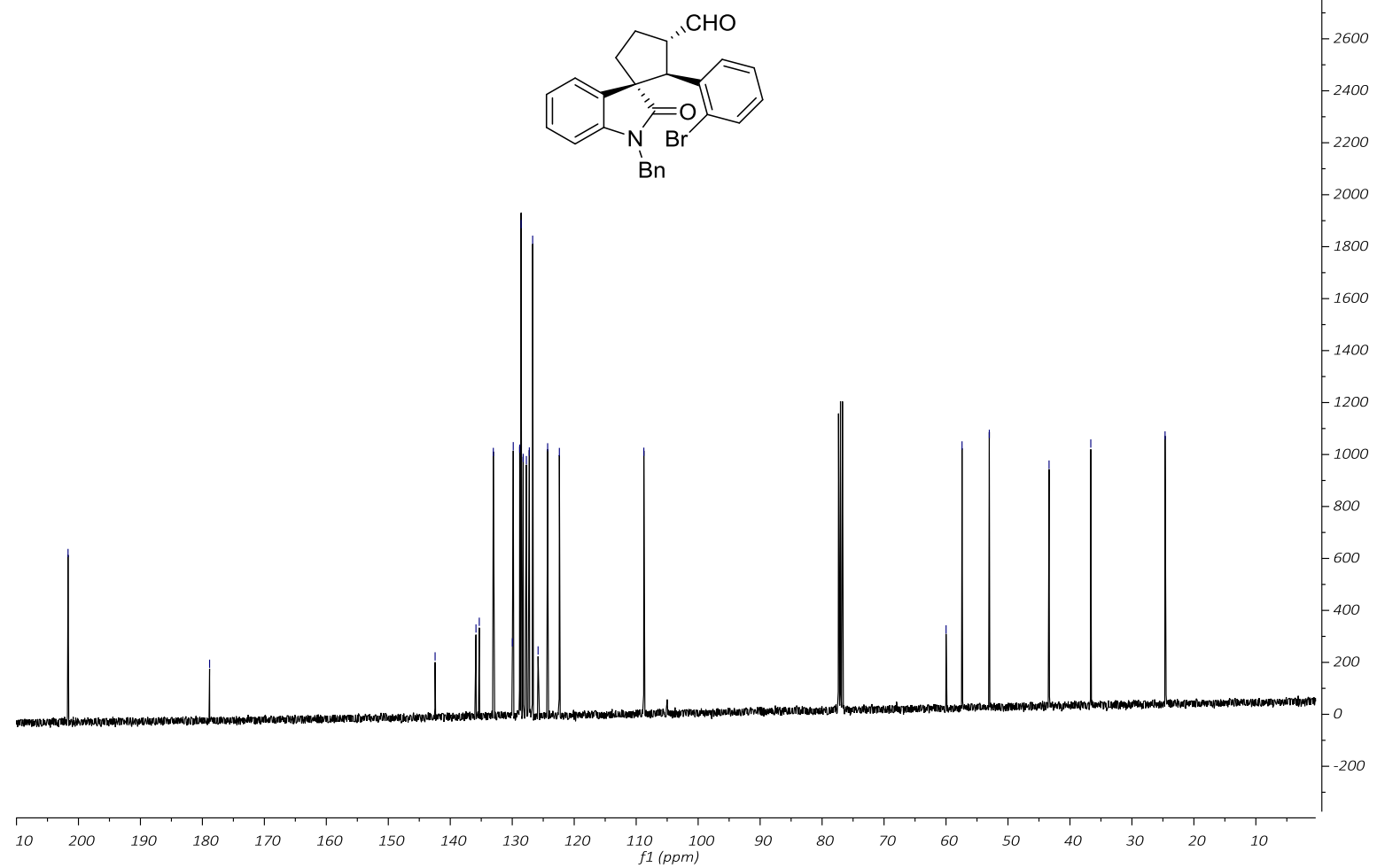
(1S,2R,3S)-1'-Benzyl-2-(furan-2-yl)-2'-oxospiro[cyclopentane-1,3'-indoline]-3carbaldehyde (3w)

${ }^{1} \mathrm{H}$ NMR of $3 \mathbf{w}\left(400 \mathrm{MHz}, \mathrm{CDCl}_{3}\right)$

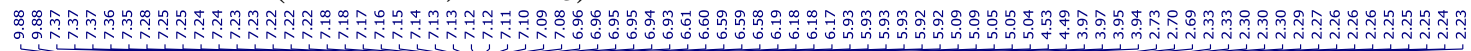<smiles>O=C[C@H]1CC[C@@]2(C(=O)N(Cc3ccccc3)c3ccccc32)[C@H]1c1ccco1</smiles>
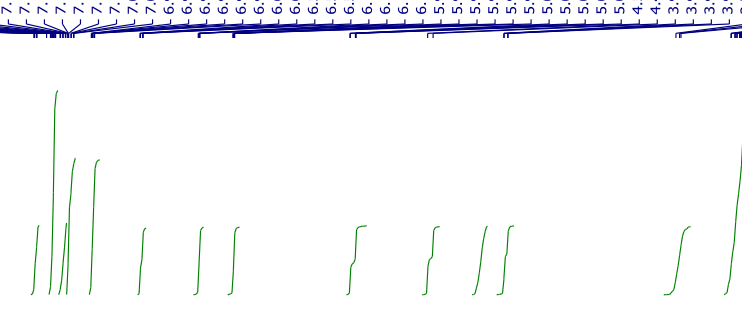

15000

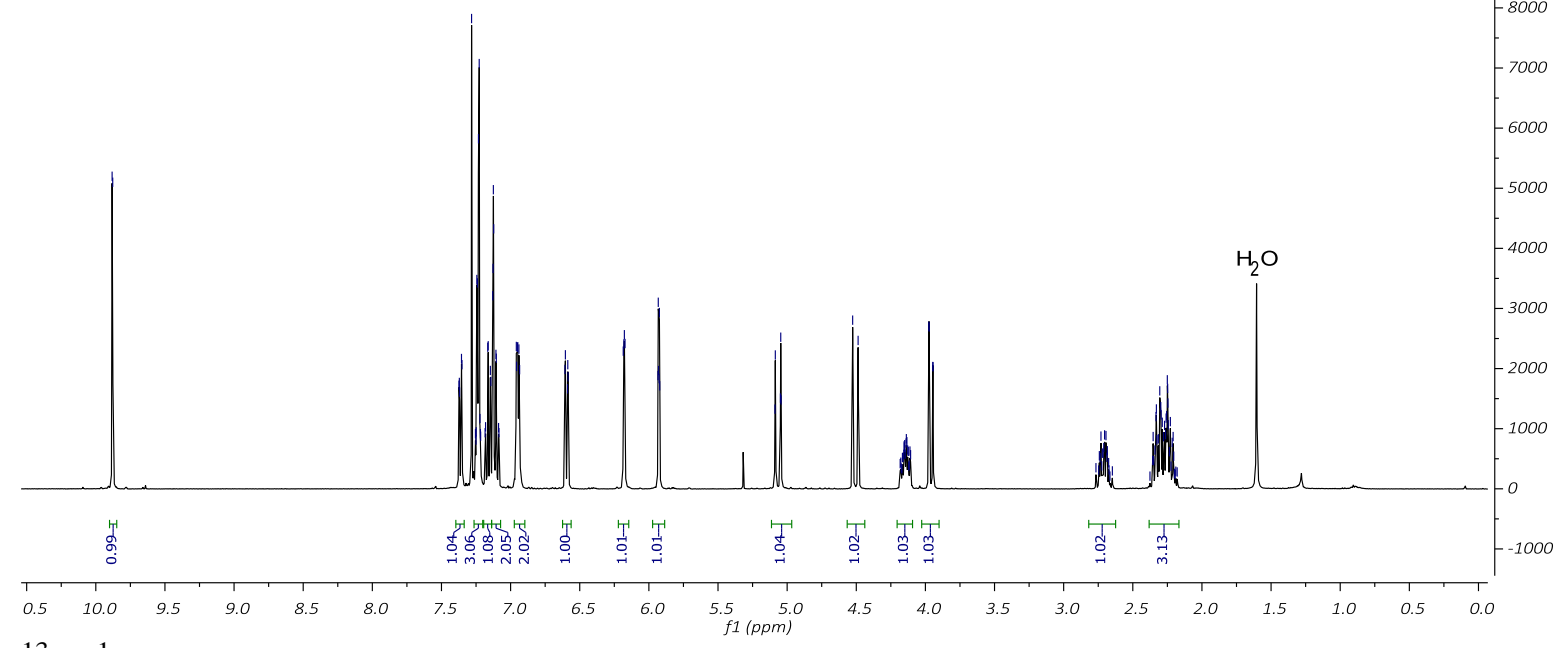

${ }^{13} \mathrm{C}\left\{{ }^{1} \mathrm{H}\right\}$ NMR of $\mathbf{3 w}\left(101 \mathrm{MHz}, \mathrm{CDCl}_{3}\right)$

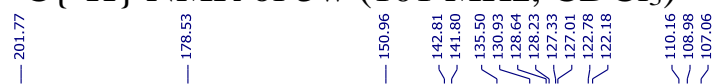

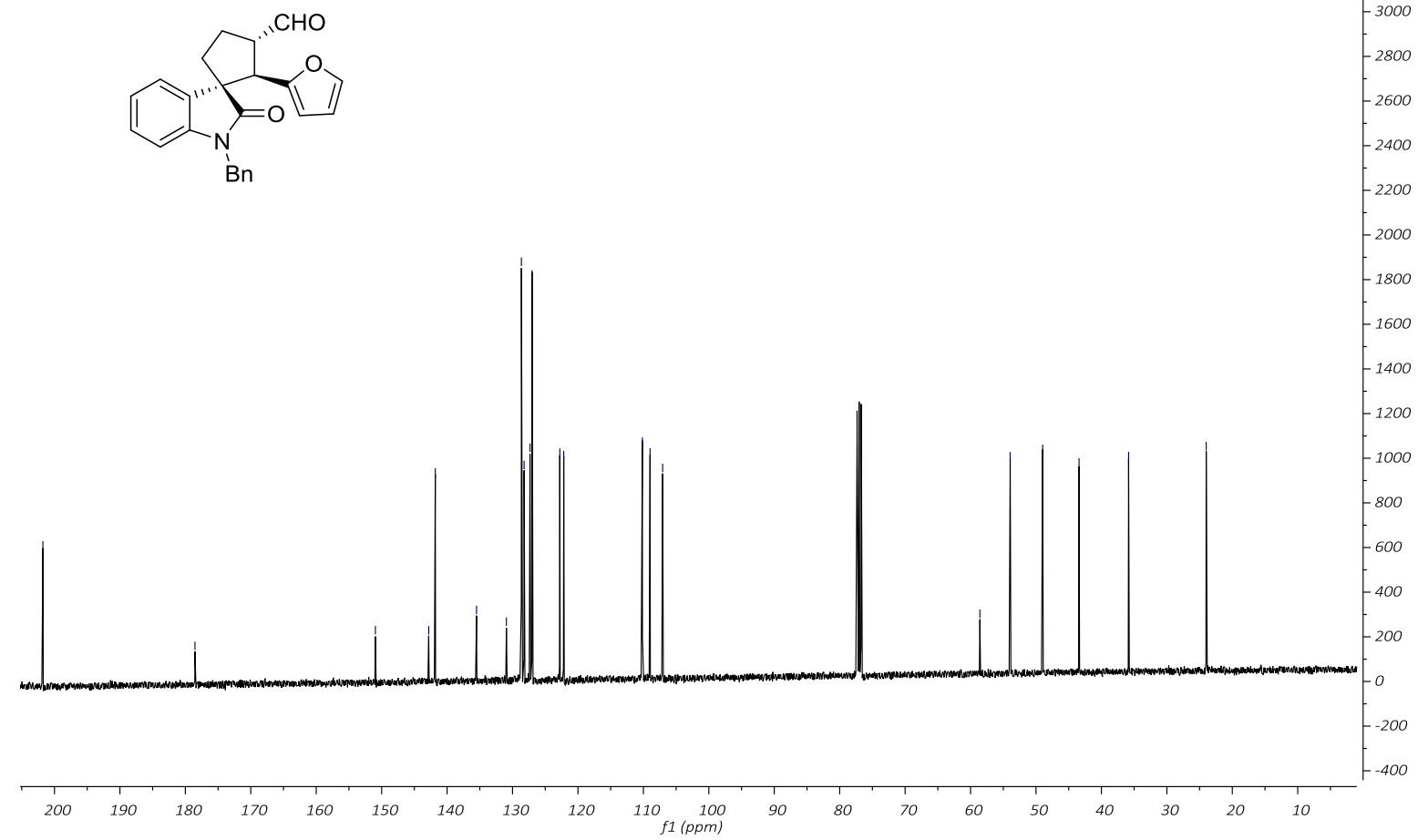


$(1 R, 2 R, 3 S)-1$ '-Benzyl-2-(furan-2-yl)-2'-oxospiro[cyclopentane-1,3'-indoline]-3carbaldehyde (4w)

${ }^{1} \mathrm{H}$ NMR of $4 \mathbf{w}\left(400 \mathrm{MHz}, \mathrm{CDCl}_{3}\right)$

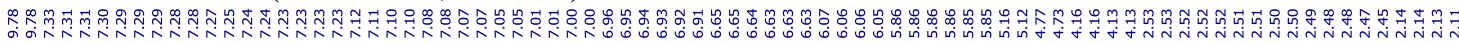
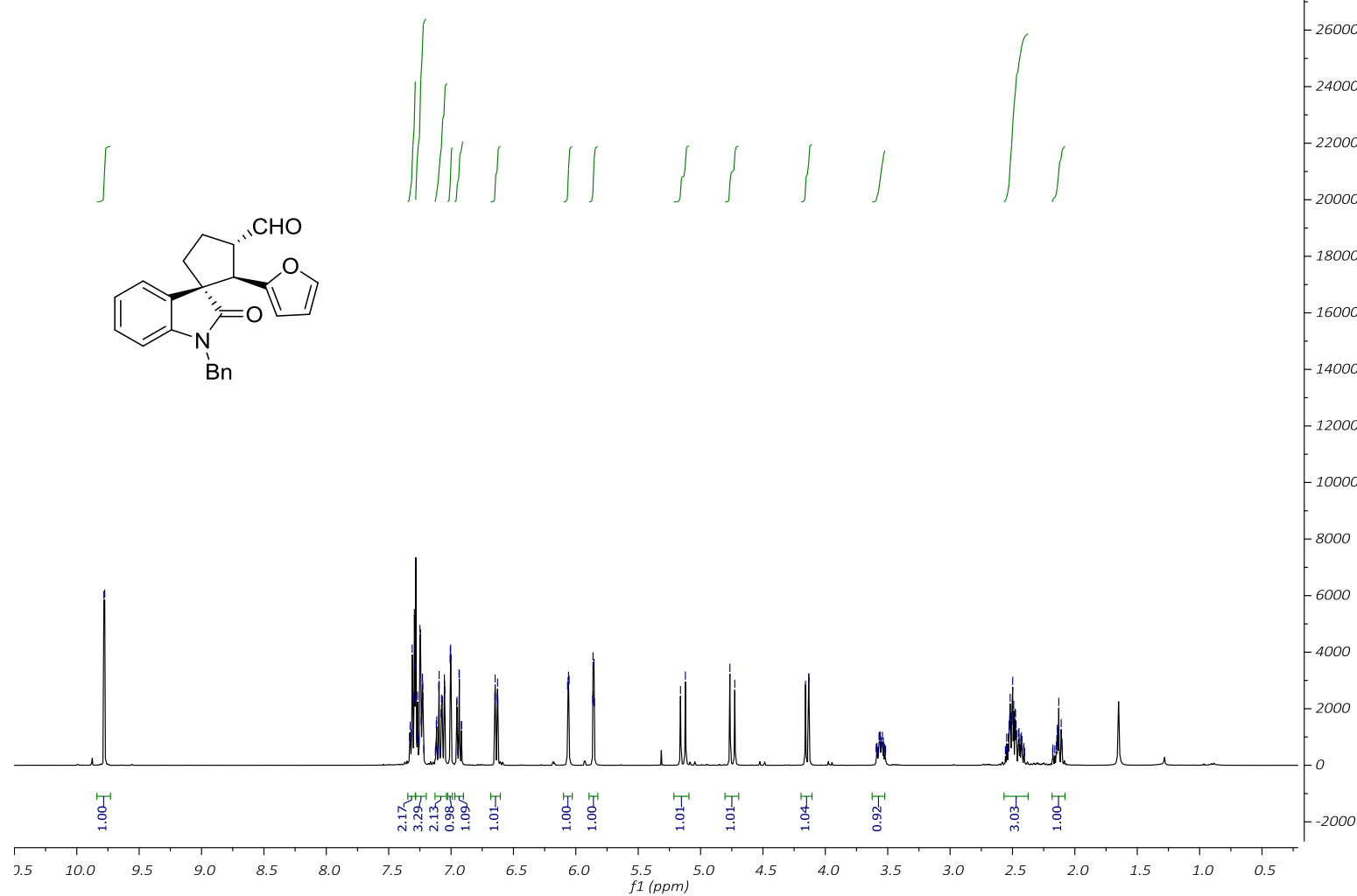

${ }^{13} \mathrm{C}\left\{{ }^{1} \mathrm{H}\right\} \mathrm{NMR}$ of $\mathbf{4 w}\left(101 \mathrm{MHz}, \mathrm{CDCl}_{3}\right)$
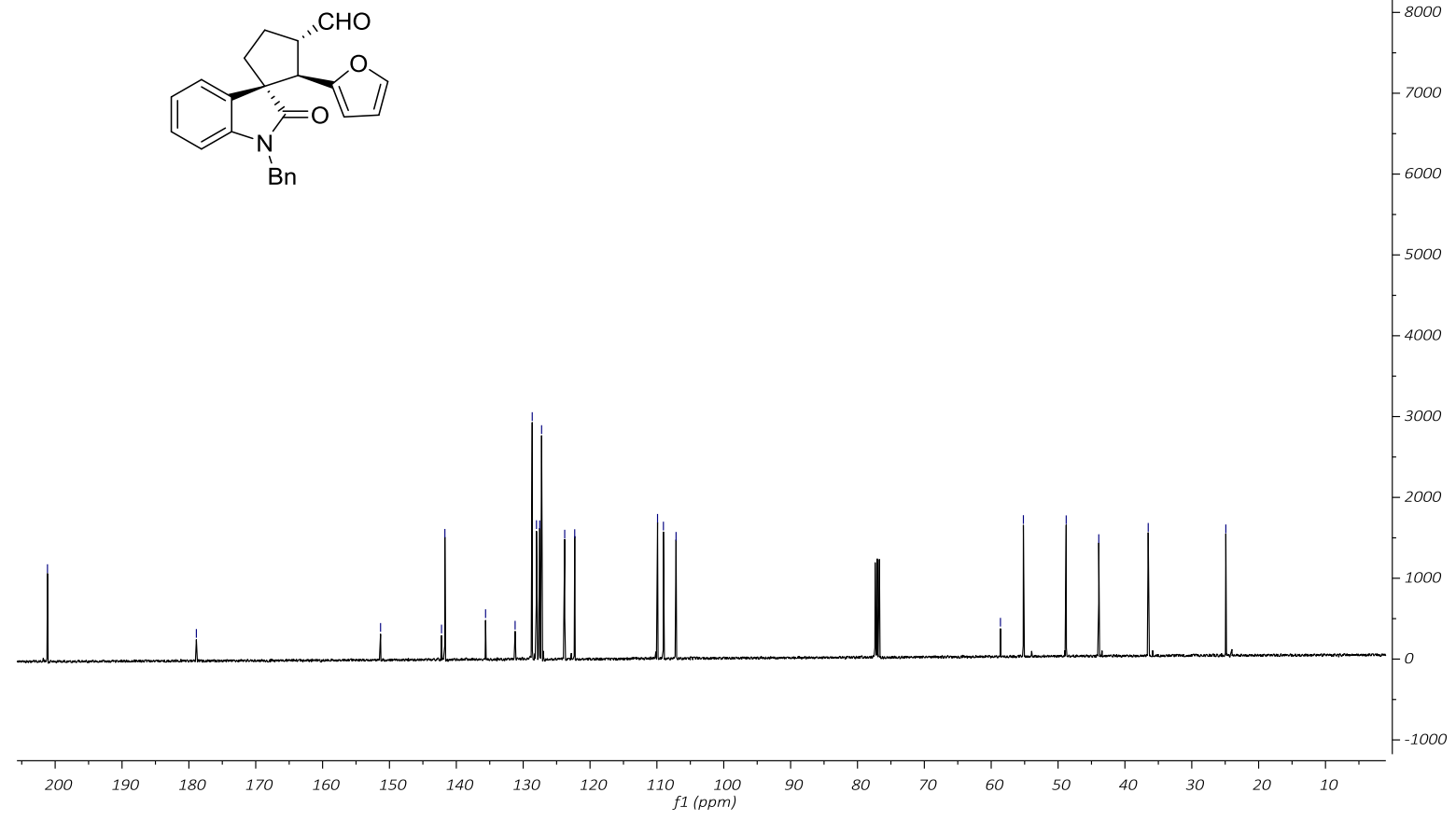
(1S,2R,3S)-1'-Benzyl-2'-oxo-2-(thiophen-2-yl)spiro[cyclopentane-1,3'-indoline]-3carbaldehyde (3x)

${ }^{1} \mathrm{H} \mathrm{NMR}$ of $\mathbf{3 x}\left(400 \mathrm{MHz}, \mathrm{CDCl}_{3}\right)$

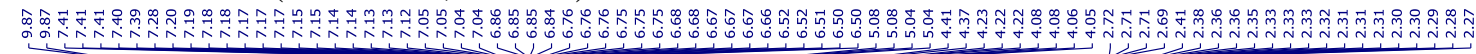

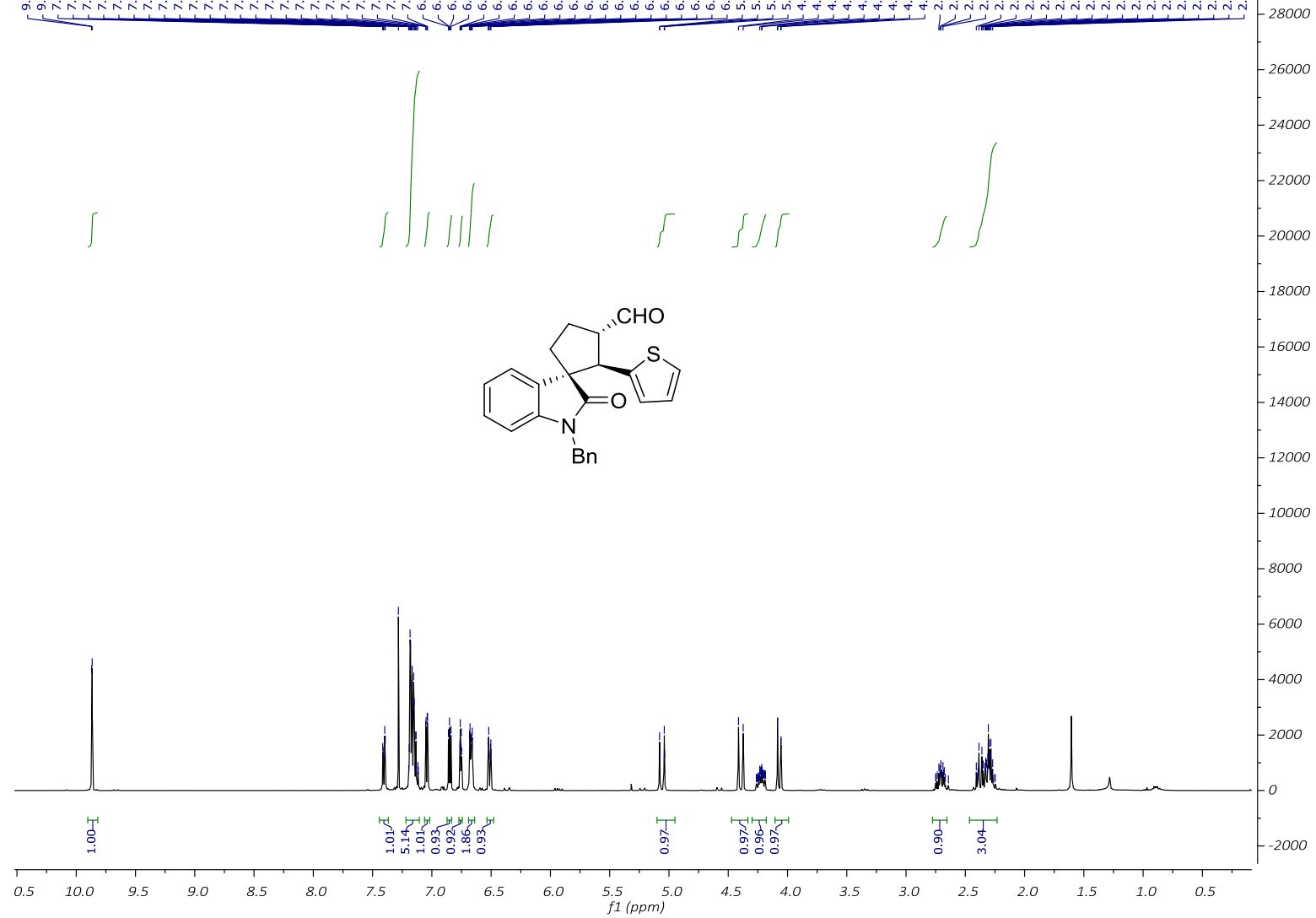

${ }^{13} \mathrm{C}\left\{{ }^{1} \mathrm{H}\right\} \mathrm{NMR}$ of $\mathbf{3 x}\left(101 \mathrm{MHz}, \mathrm{CDCl}_{3}\right)$

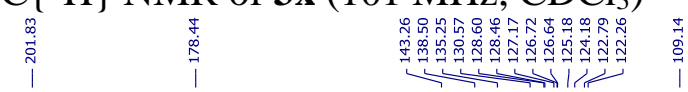<smiles>O=CC1CCC(C(=O)Nc2ccccc2)C1c1ccco1</smiles>

(1)

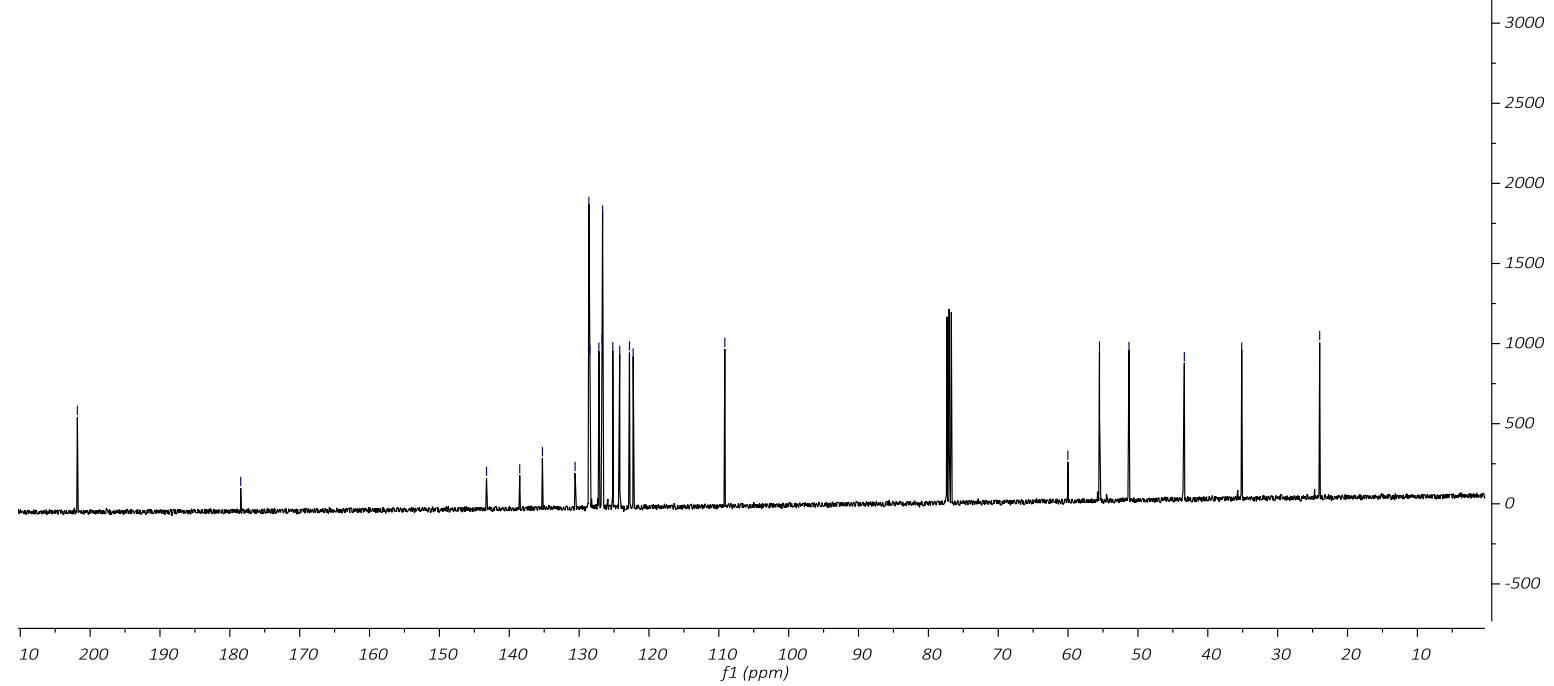


$(1 R, 2 R, 3 S)-1 '$-Benzyl-2'-oxo-2-(thiophen-2-yl)spiro[cyclopentane-1,3'-indoline]-3carbaldehyde (4x)

${ }^{1} \mathrm{H} \mathrm{NMR}$ of $\mathbf{4 x}\left(400 \mathrm{MHz}, \mathrm{CDCl}_{3}\right)$

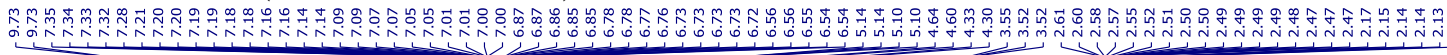

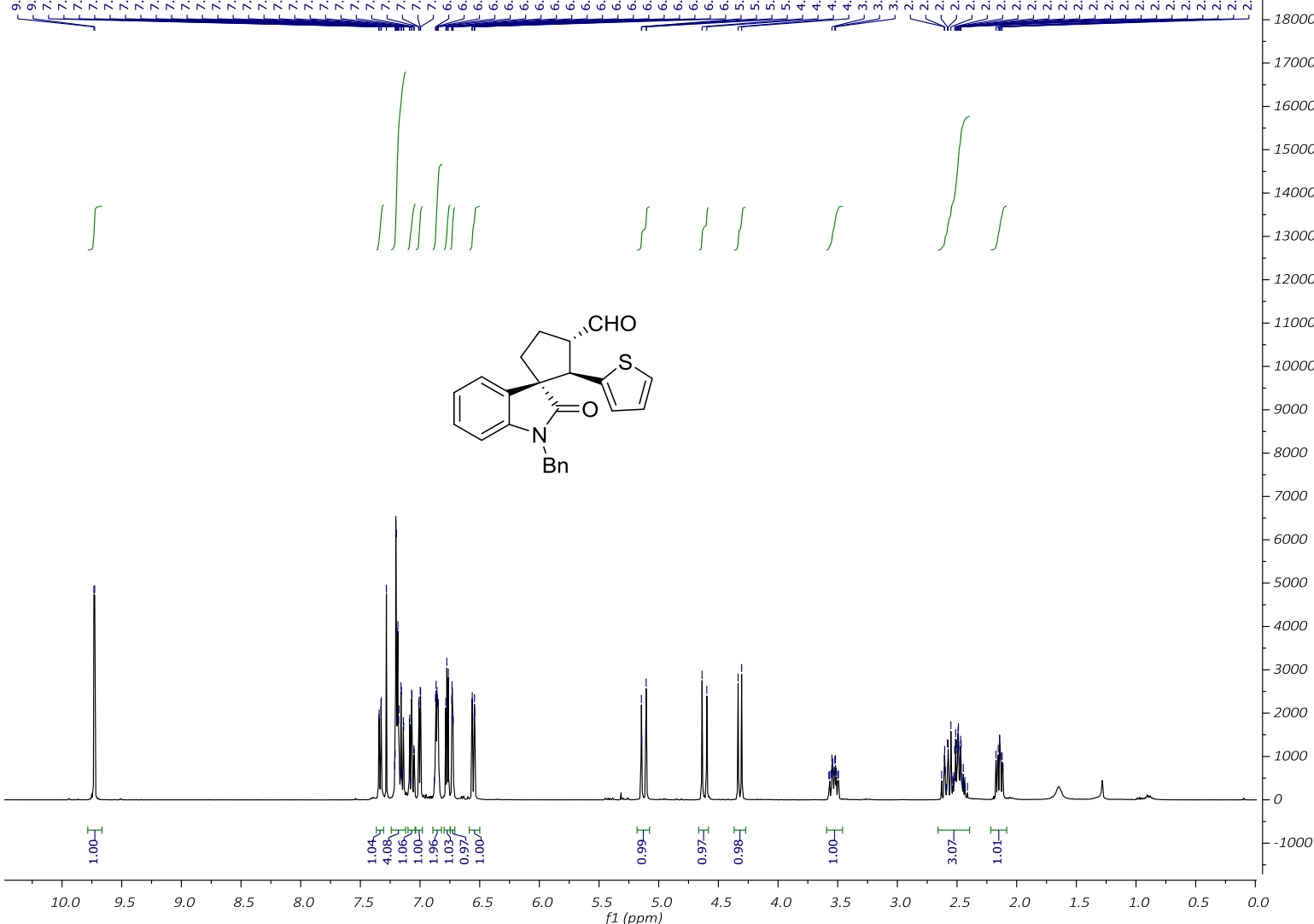

${ }^{13} \mathrm{C}\left\{{ }^{1} \mathrm{H}\right\} \mathrm{NMR}$ of $\mathbf{4 x}\left(101 \mathrm{MHz}, \mathrm{CDCl}_{3}\right)$

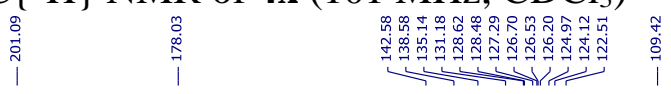

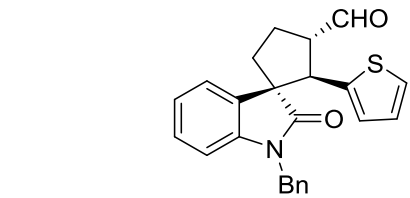

₹

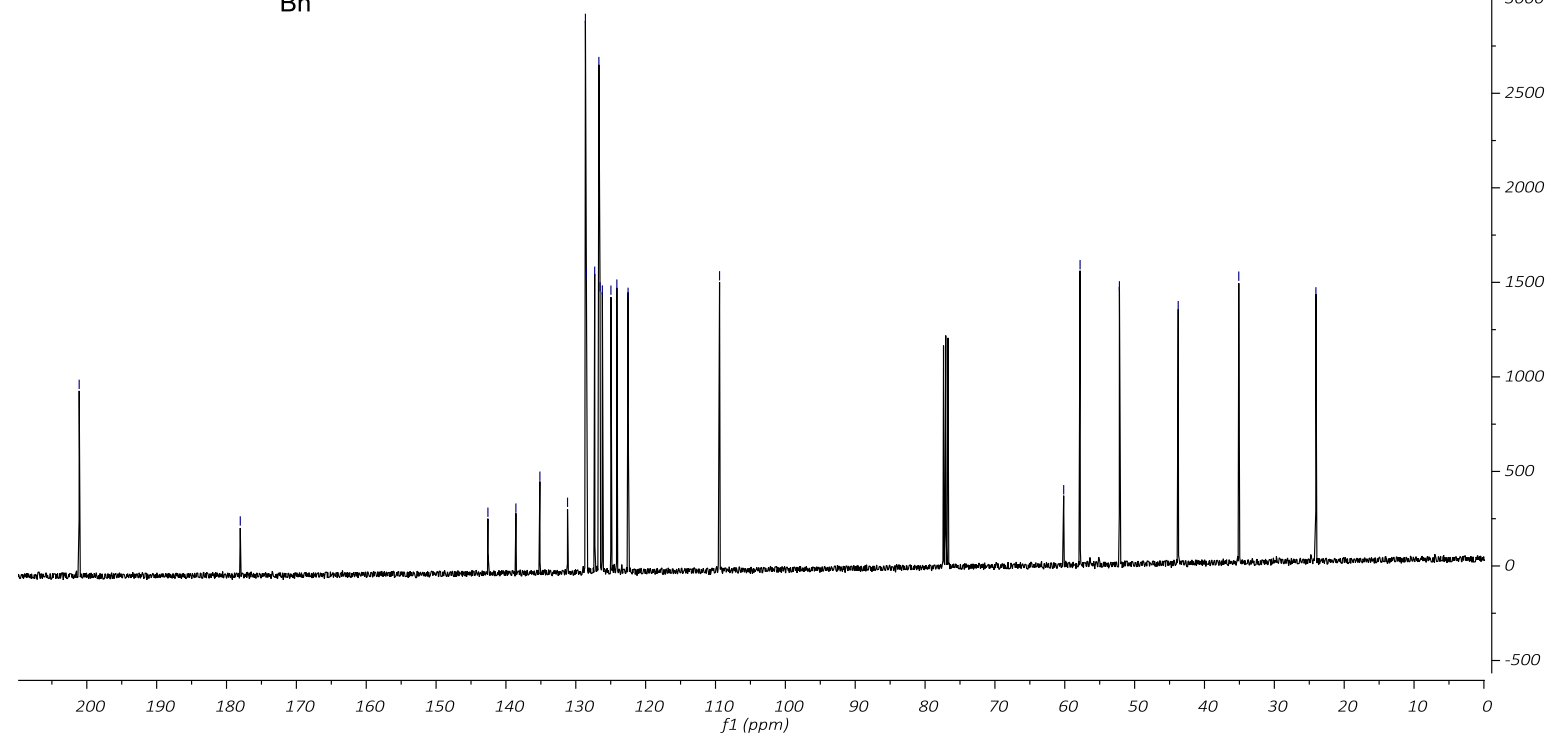


Ethyl $(1 S, 2 R, 3 S)-1$ '-benzyl-3-formyl-2'-oxospiro[cyclopentane-1,3'-indoline]-2carboxylate (3y)

${ }^{1} \mathrm{H}$ NMR of $\mathbf{3 y}\left(400 \mathrm{MHz}, \mathrm{CDCl}_{3}\right)$

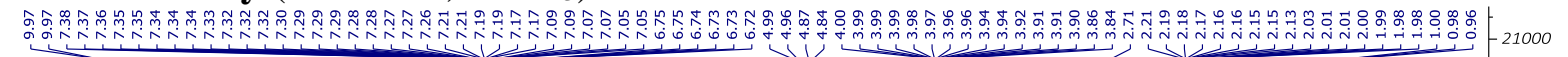

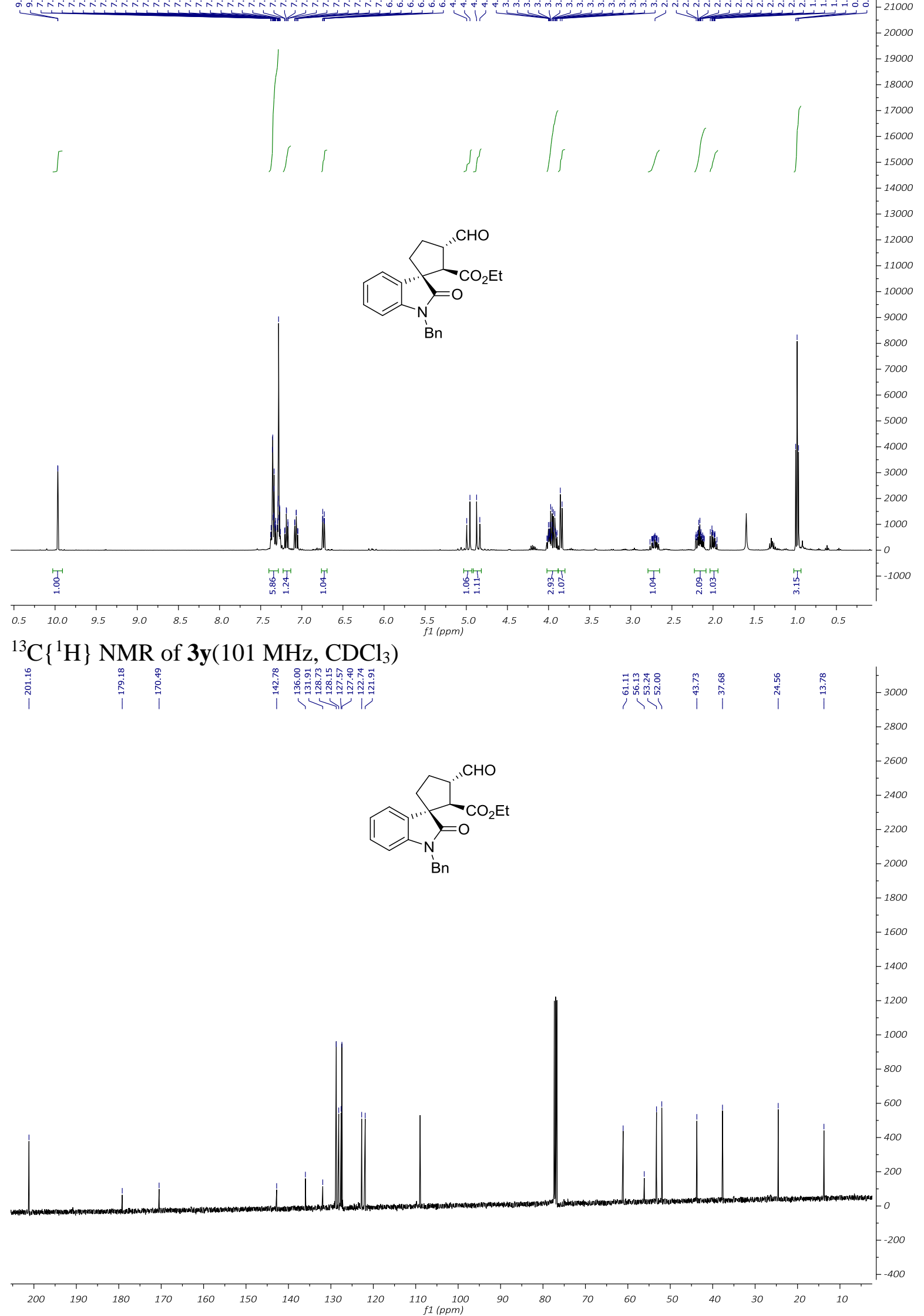


Ethyl (1R,2R,3S)-1'-benzyl-3-formyl-2' -oxospiro[cyclopentane-1,3'-indoline]-2carboxylate (4y)

${ }^{1} \mathrm{H}$ NMR of $4 \mathbf{y}\left(400 \mathrm{MHz}, \mathrm{CDCl}_{3}\right)$

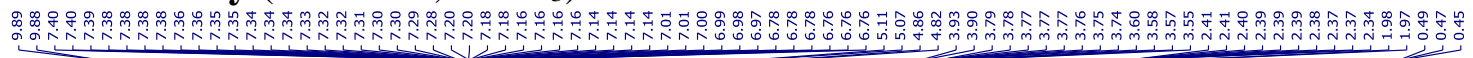

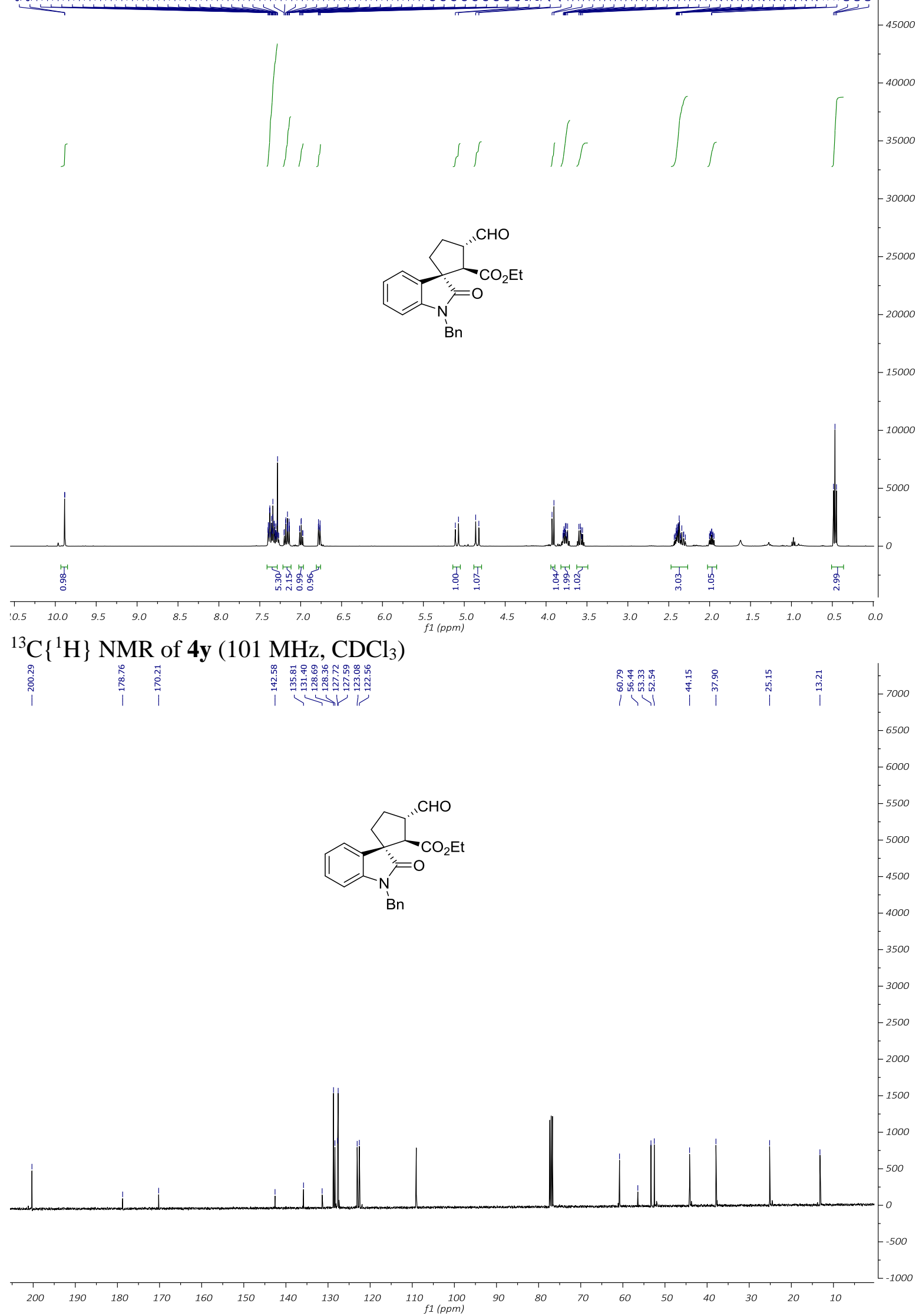


Methyl (E)-3-(1'-benzyl-2'-oxo-2-phenylspiro[cyclopropane-1,3'-indolin]-3-yl)acrylate

(5)

${ }^{1} \mathrm{H}$ NMR of $5\left(400 \mathrm{MHz}, \mathrm{CDCl}_{3}\right)$

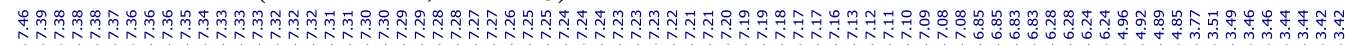

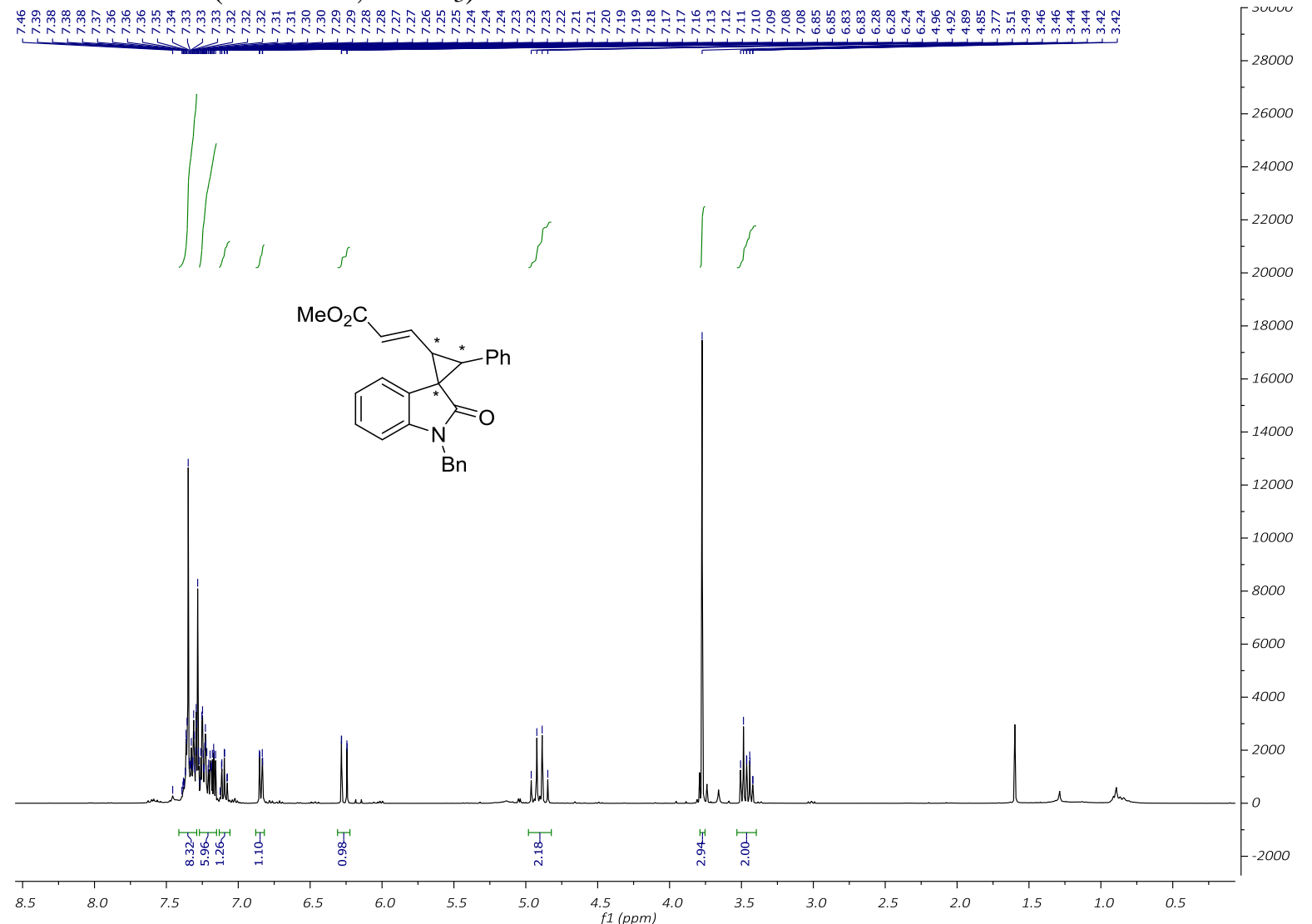

${ }^{13} \mathrm{C}\left\{{ }^{1} \mathrm{H}\right\}$ NMR of $5\left(101 \mathrm{MHz}, \mathrm{CDCl}_{3}\right)$

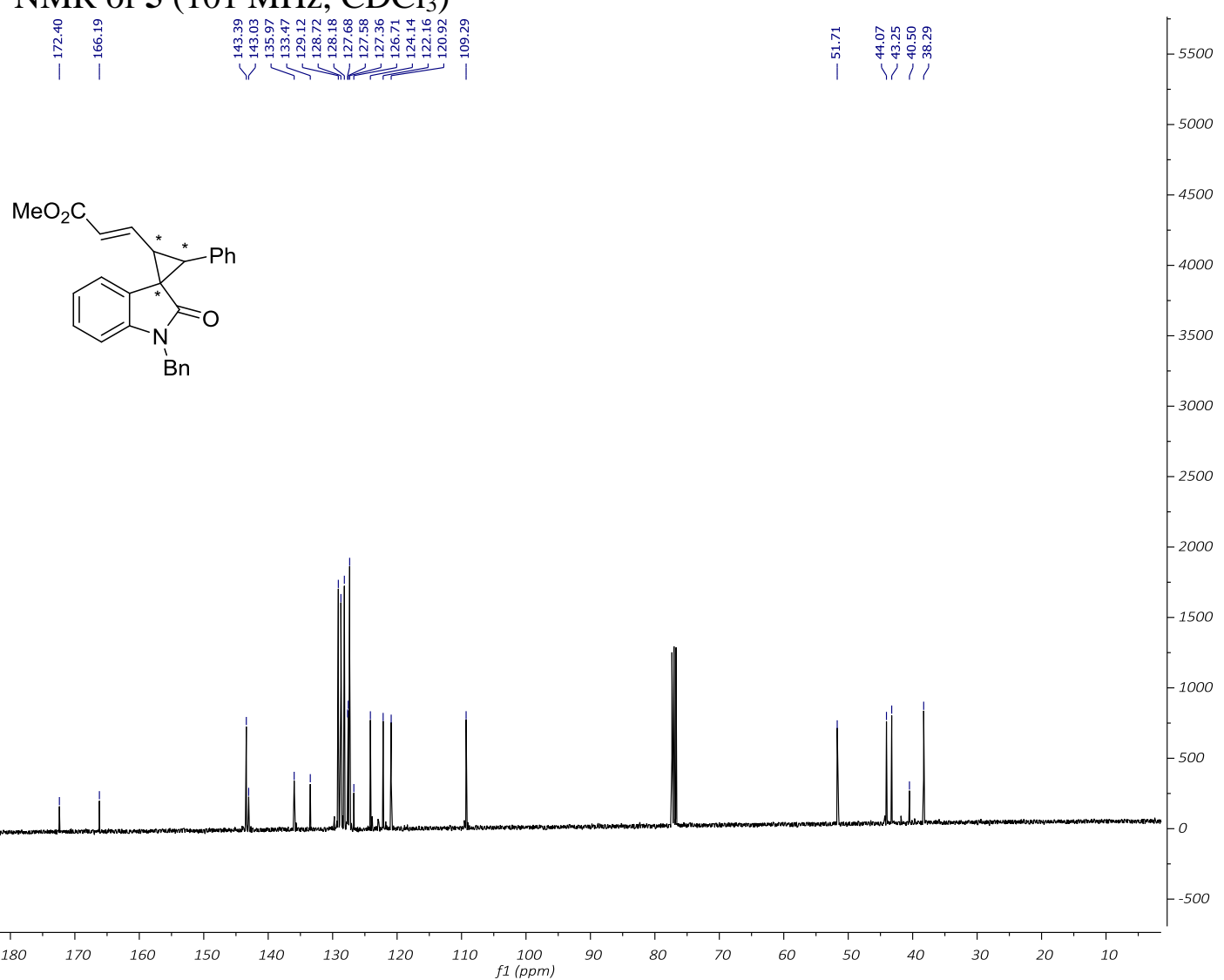


3-(1-Benzyl-3-(3-bromopropyl)-2-oxoindolin-3-yl)-3-phenylpropanal (6) ${ }^{1} \mathrm{H} \mathrm{NMR}$ of $6\left(400 \mathrm{MHz}, \mathrm{CDCl}_{3}\right)$

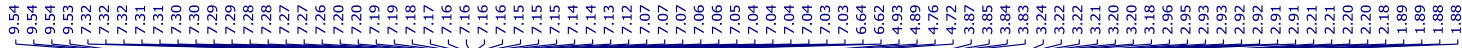

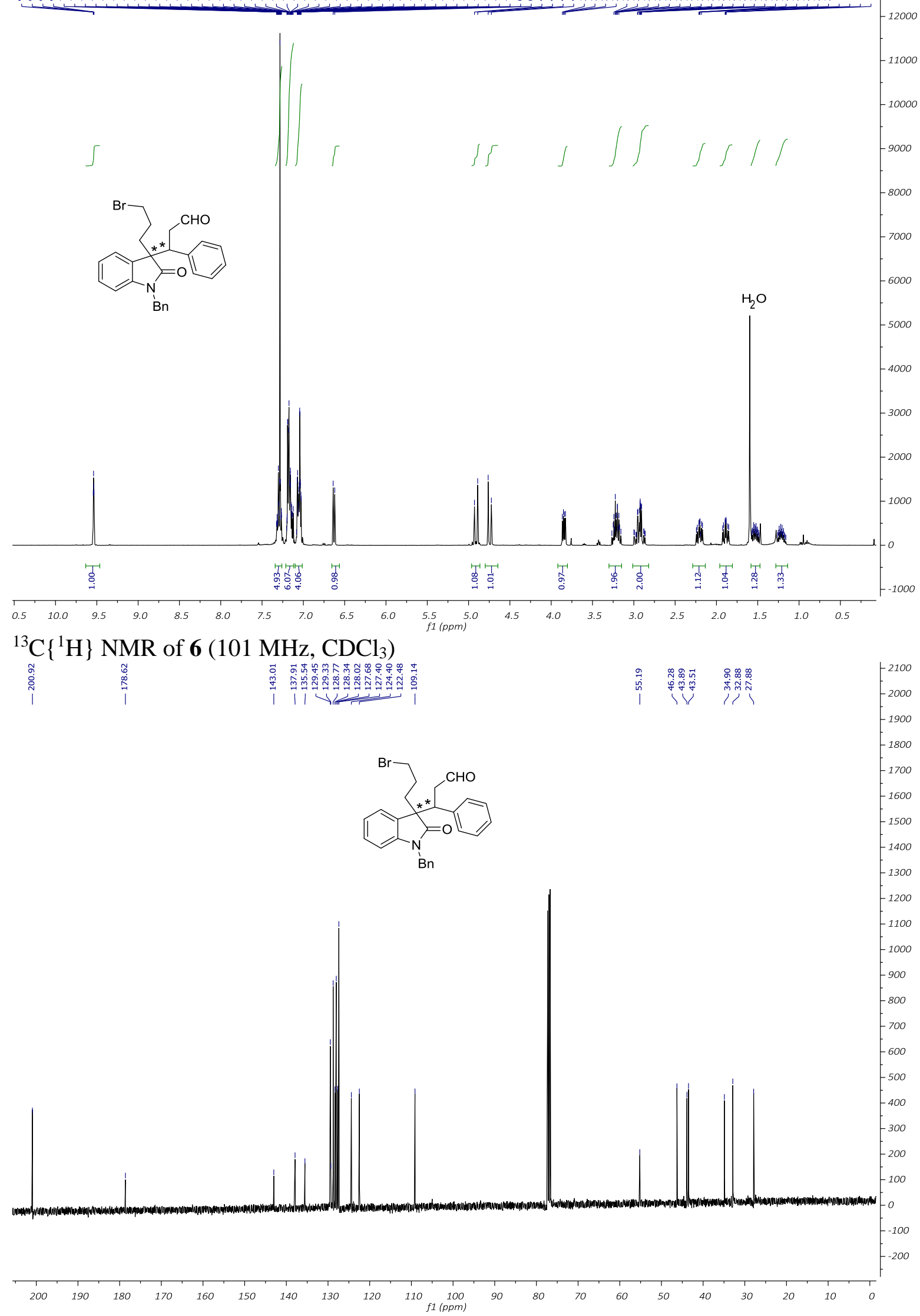


3-(1-Benzyl-3-(3-bromopropyl)-2-oxoindolin-3-yl)-3-phenylpropanal (7)

${ }^{1} \mathrm{H} \mathrm{NMR}$ of $7\left(400 \mathrm{MHz}, \mathrm{CDCl}_{3}\right)$

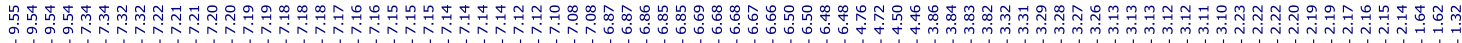

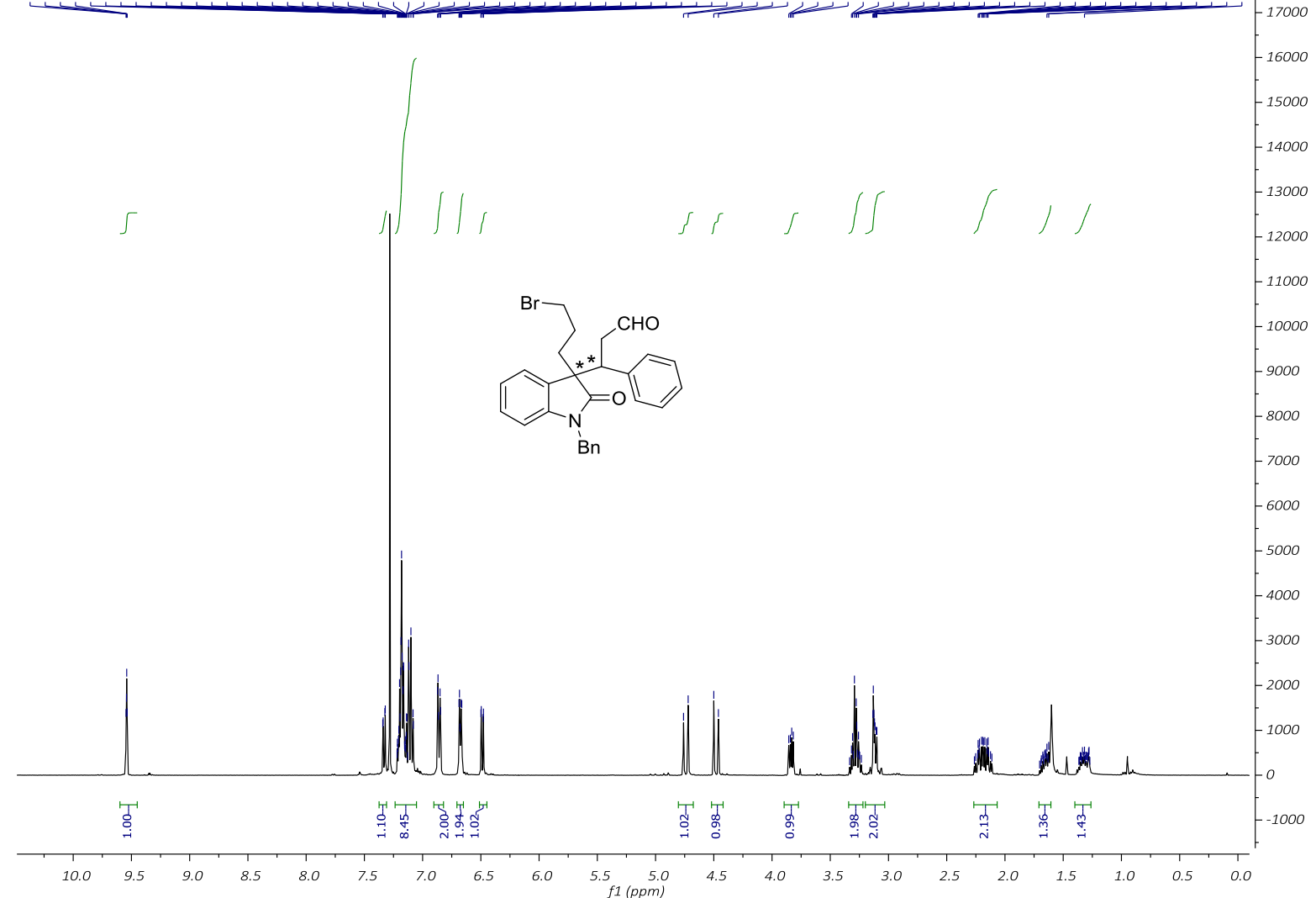

${ }^{13} \mathrm{C}\left\{{ }^{1} \mathrm{H}\right\}$ NMR of $7\left(101 \mathrm{MHz}, \mathrm{CDCl}_{3}\right)$

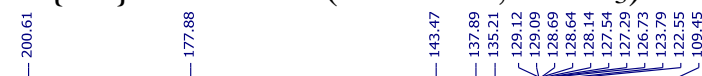

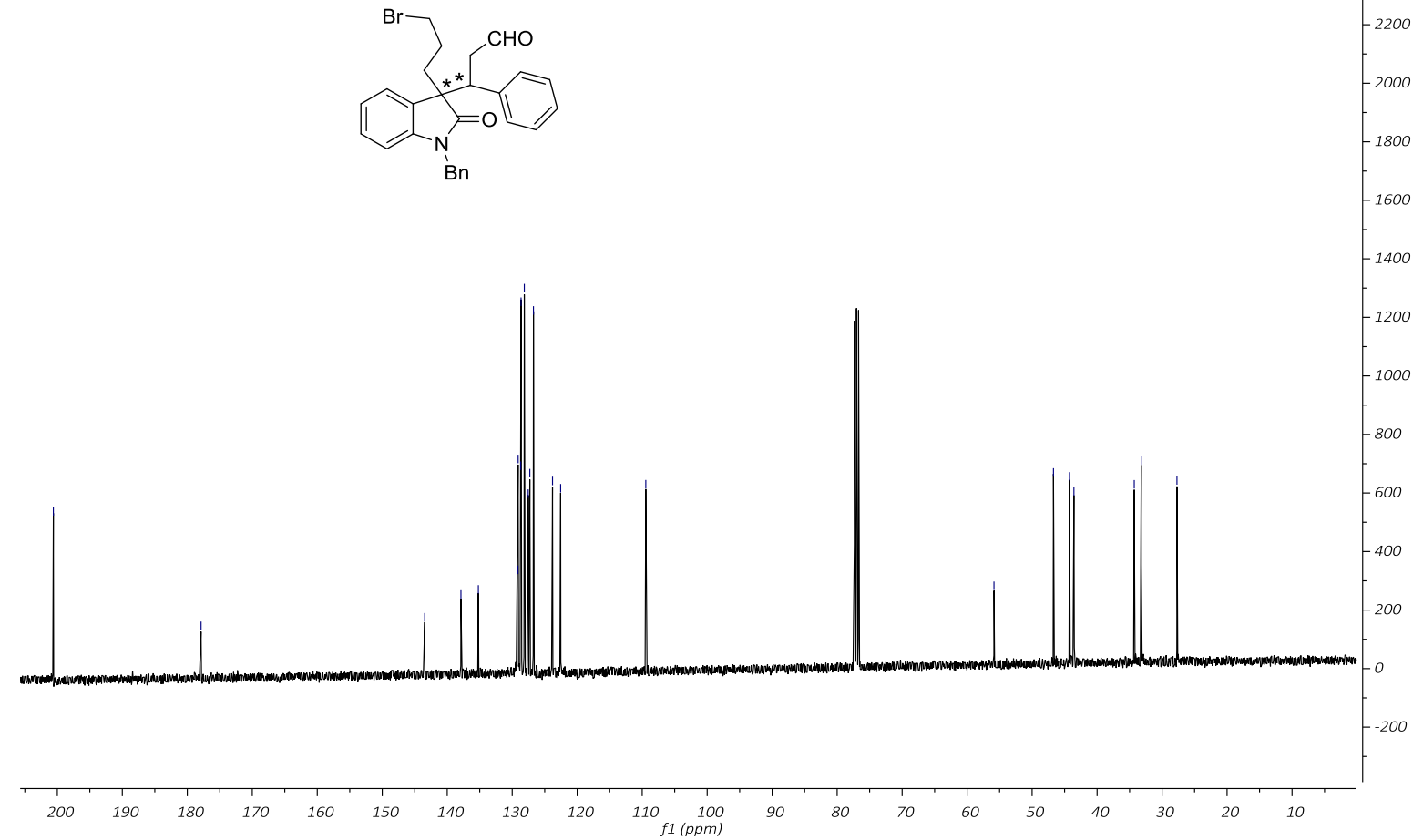

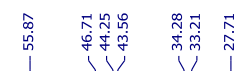

2800

2600

2400 
3-(1-Benzyl-3-(4-bromobutyl)-2-oxoindolin-3-yl)-3-phenylpropanal (8)

${ }^{1} \mathrm{H} \mathrm{NMR}$ of $8\left(400 \mathrm{MHz}, \mathrm{CDCl}_{3}\right)$

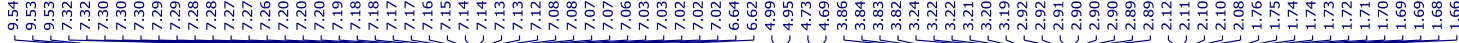

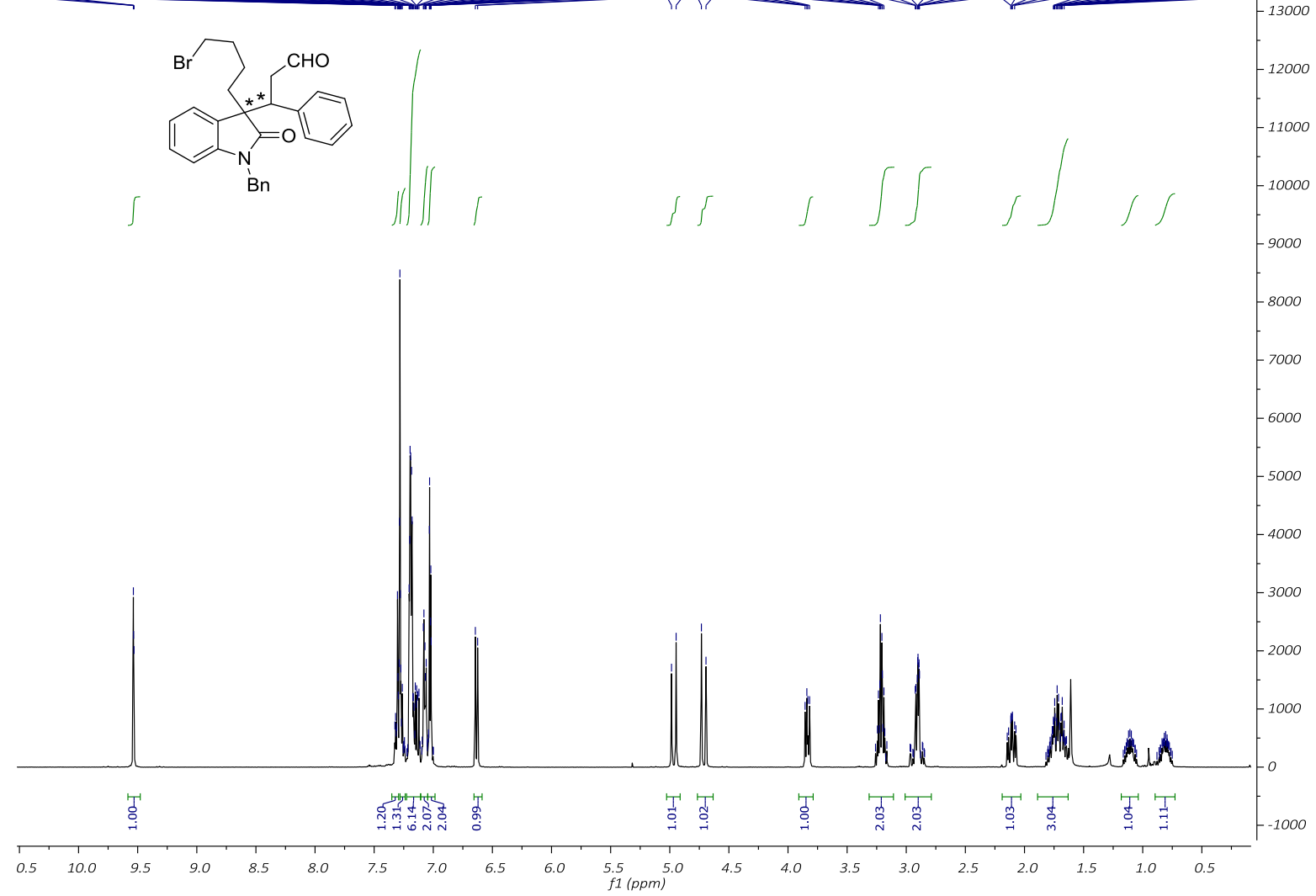

${ }^{13} \mathrm{C}\left\{{ }^{1} \mathrm{H}\right\} \mathrm{NMR}$ of $8\left(101 \mathrm{MHz}, \mathrm{CDCl}_{3}\right)$

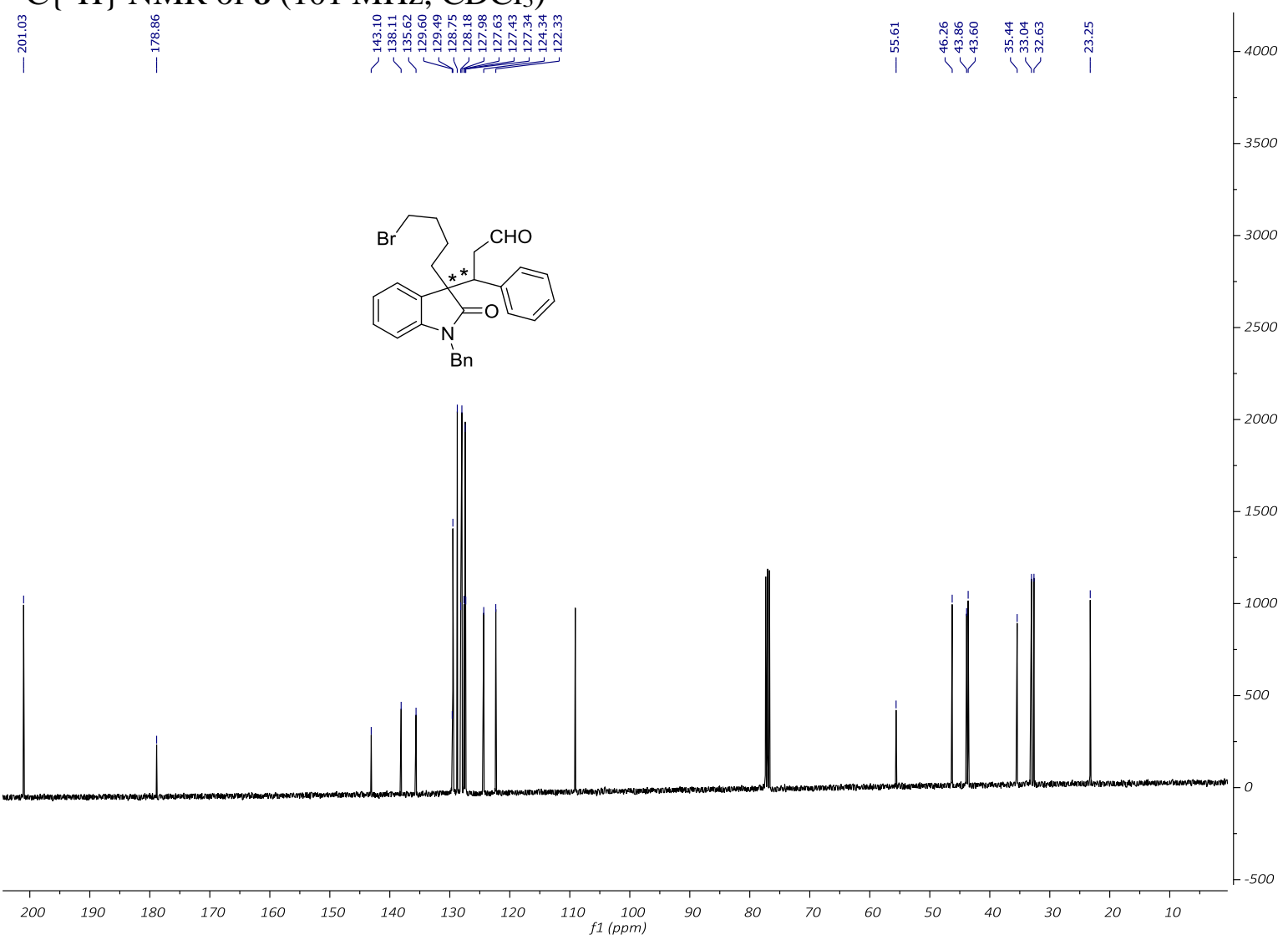


3-(1-Benzyl-3-(4-bromobutyl)-2-oxoindolin-3-yl)-3-phenylpropanal (9)

${ }^{1} \mathrm{H} \mathrm{NMR}$ of $9\left(400 \mathrm{MHz}, \mathrm{CDCl}_{3}\right)$

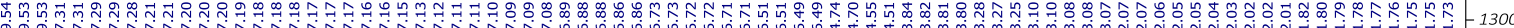

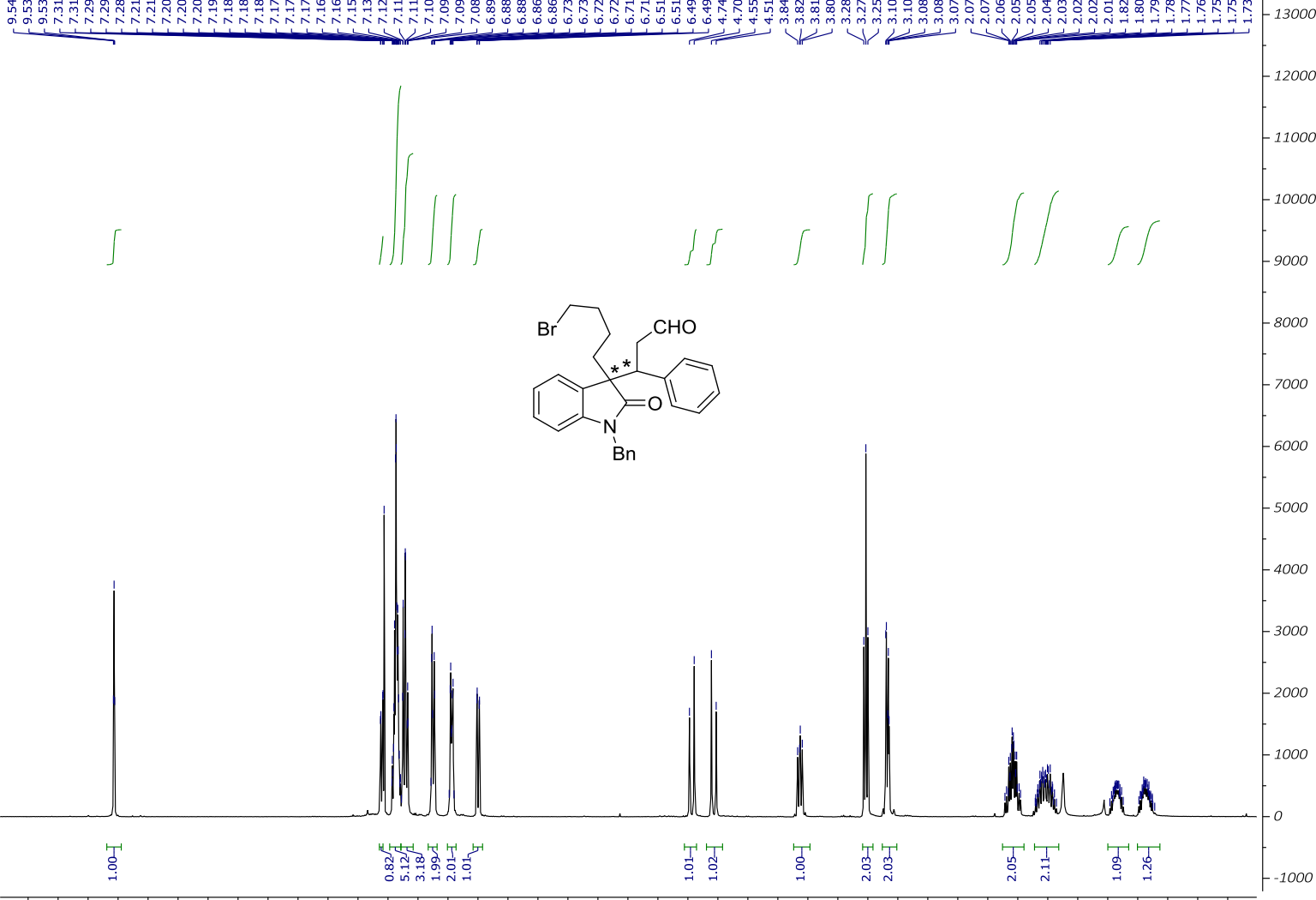

${ }^{13} \mathrm{C}\left\{{ }^{1} \mathrm{H}\right\} \mathrm{NMR}$ of $9\left(101 \mathrm{MHz}, \mathrm{CDCl}_{3}\right)$

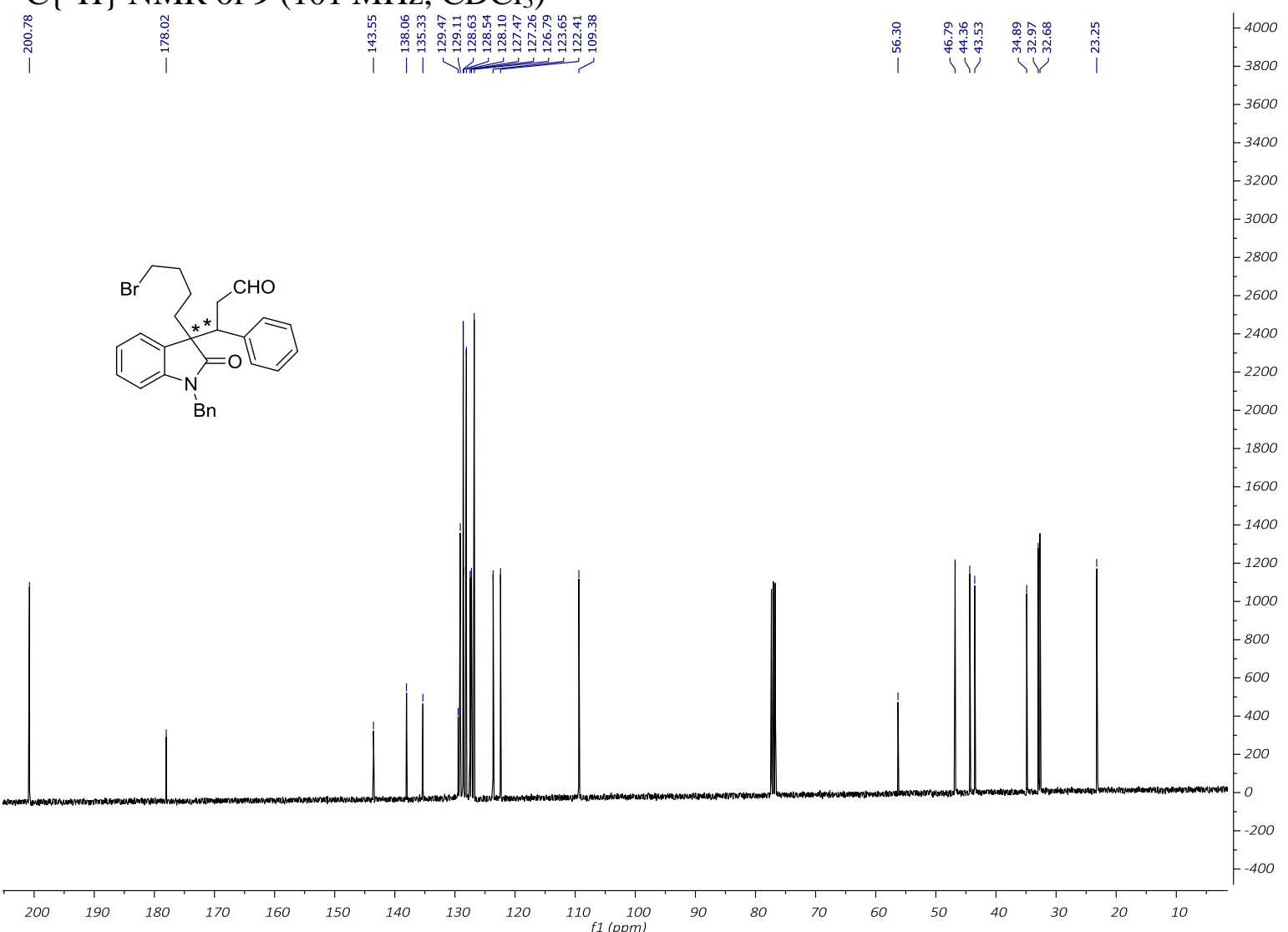


$(1 R, 2 S, 3 S)-1 '$-Benzyl-2' -oxo-2-phenylspiro[cyclopentane-1,3'-indoline]-3-carboxylic acid (10a)

${ }^{1} \mathrm{H}$ NMR of $\mathbf{1 0 a}\left(400 \mathrm{MHz}, \mathrm{CDCl}_{3}\right)$

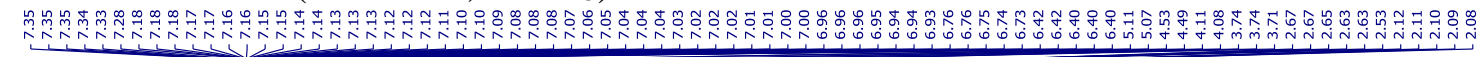

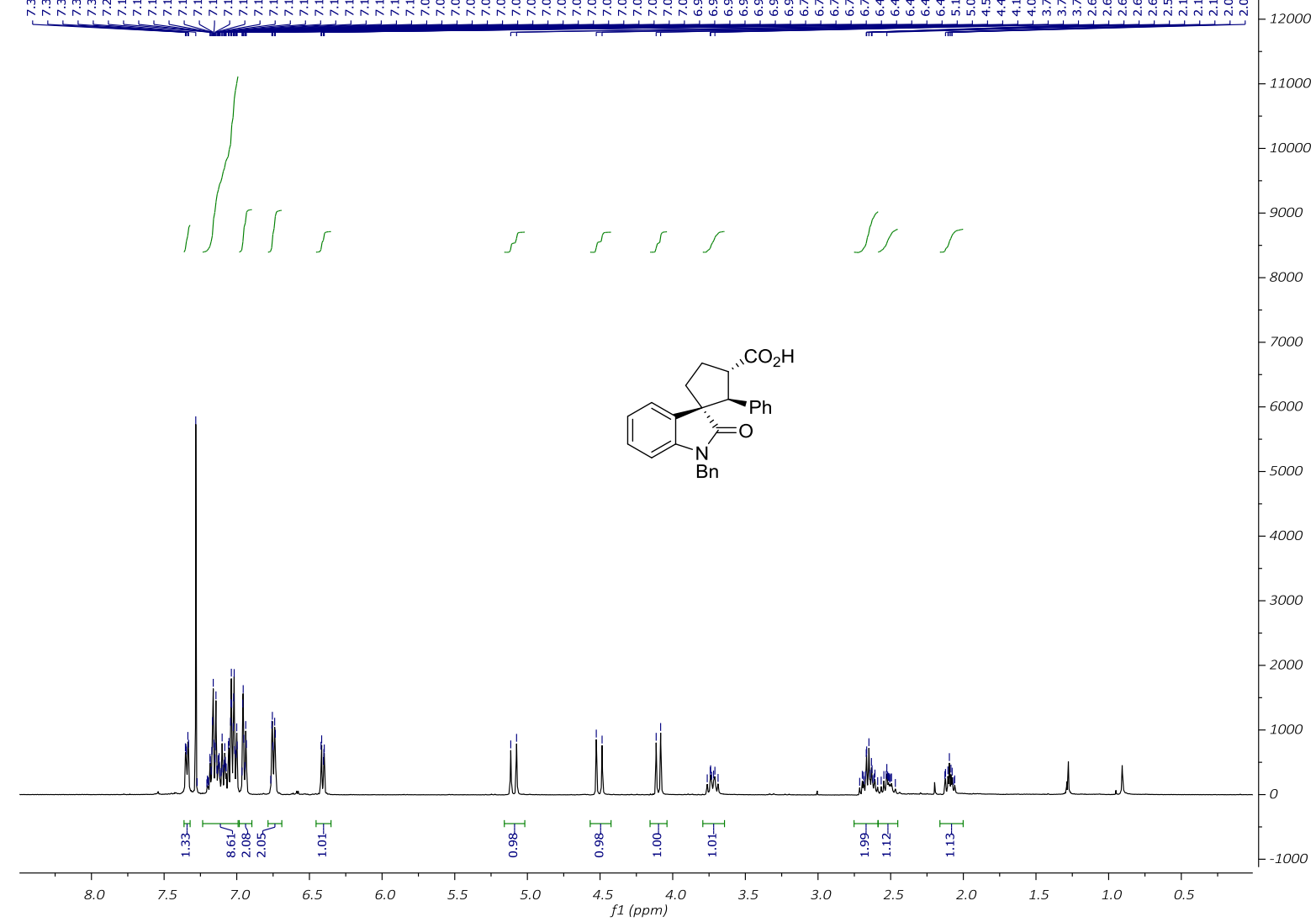

${ }^{13} \mathrm{C}\left\{{ }^{1} \mathrm{H}\right\} \mathrm{NMR}$ of $\mathbf{1 0 a}\left(101 \mathrm{MHz}, \mathrm{CDCl}_{3}\right)$

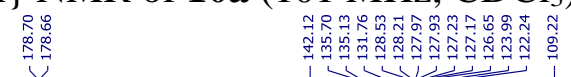

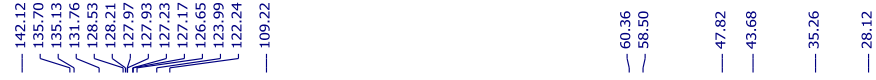
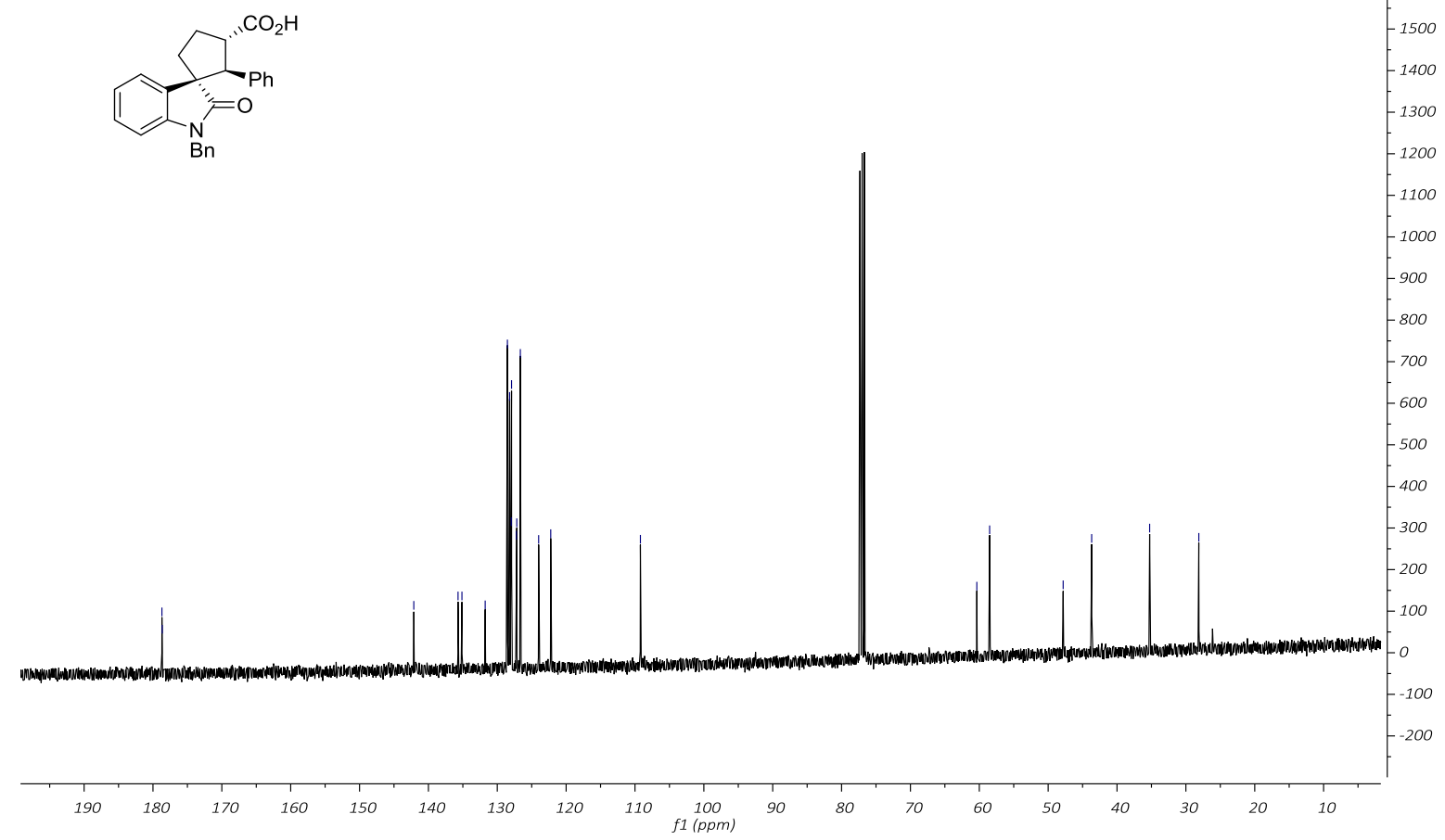


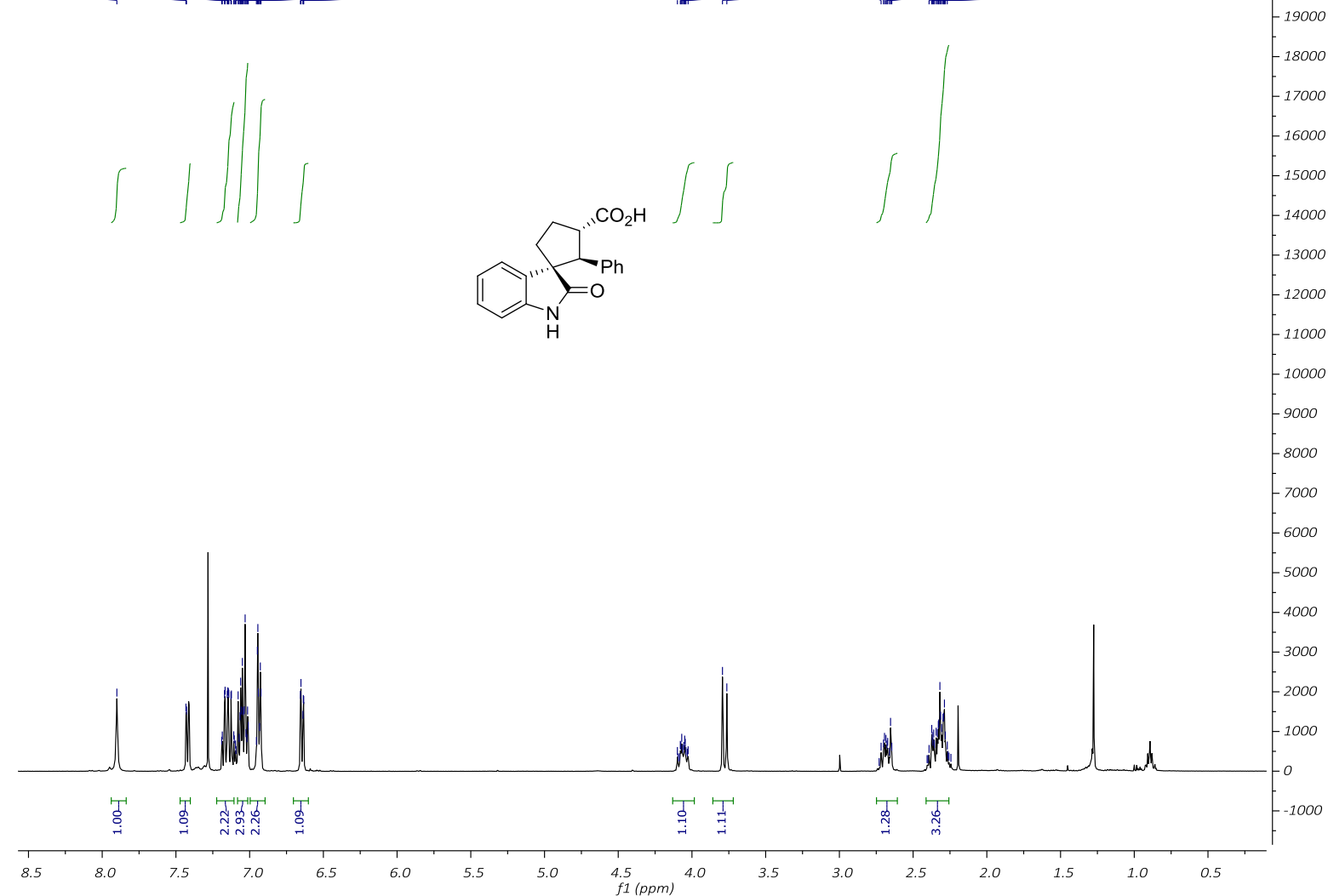

${ }^{13} \mathrm{C}\left\{{ }^{1} \mathrm{H}\right\} \mathrm{NMR}$ of $\mathbf{1 0 d}{ }^{\prime}\left(101 \mathrm{MHz}, \mathrm{CDCl}_{3}\right)$
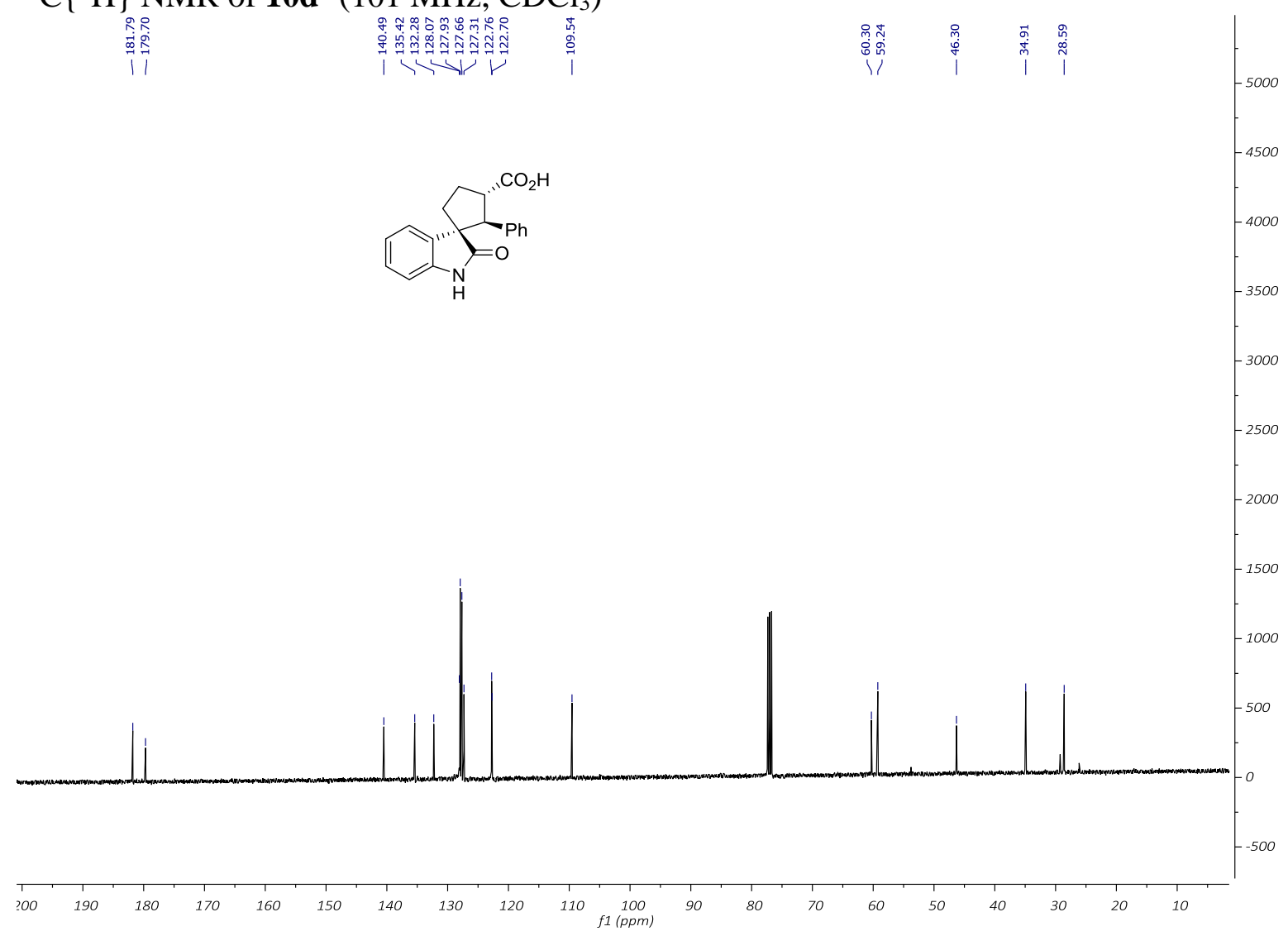

\section{)}

1 रा । 
carboxylate (11a)

${ }^{1} \mathrm{H} \mathrm{NMR}$ of $11 \mathrm{a}\left(400 \mathrm{MHz}, \mathrm{CDCl}_{3}\right)$

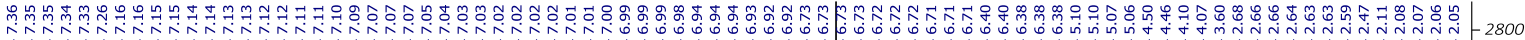

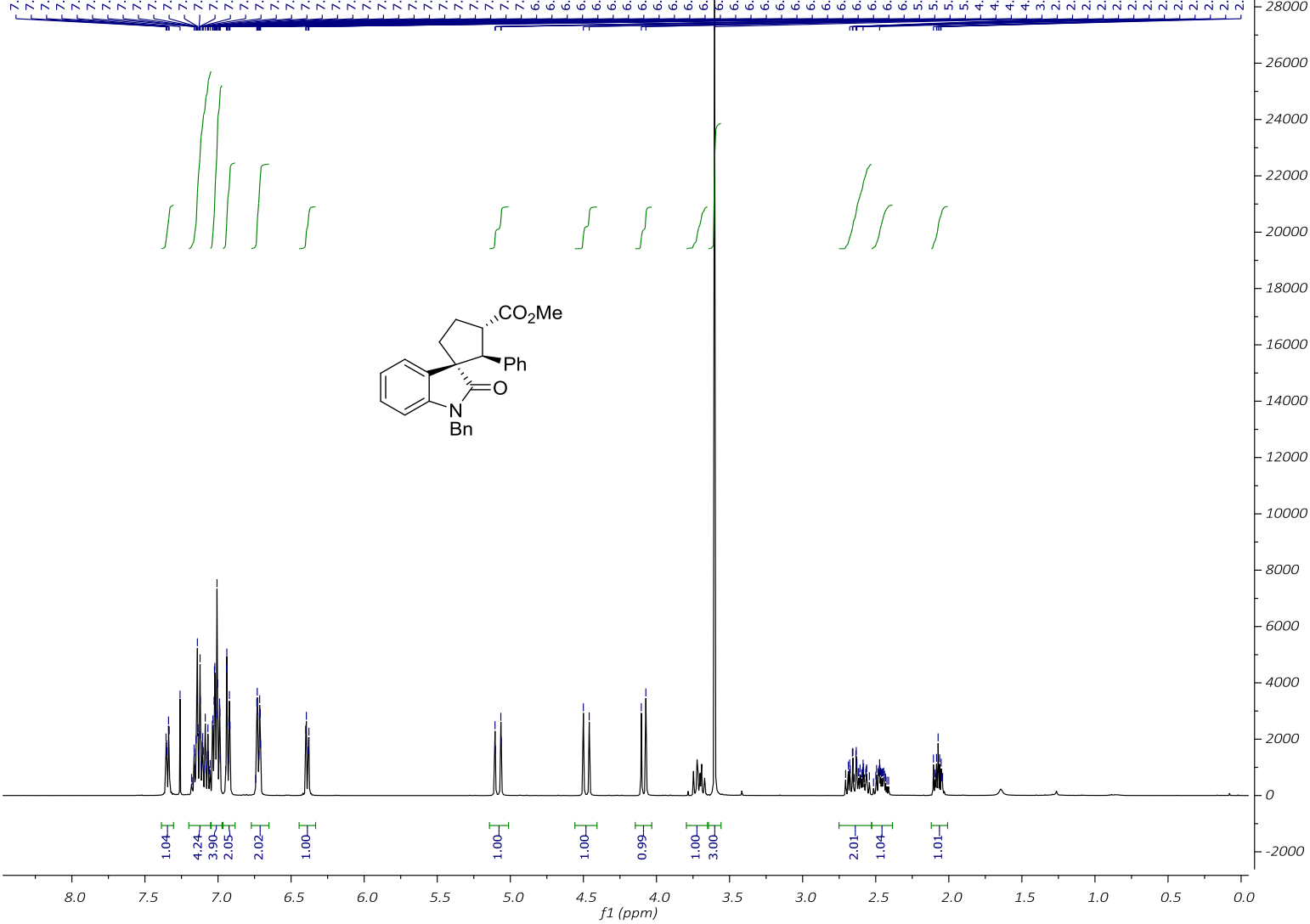

${ }^{13} \mathrm{C}\left\{{ }^{1} \mathrm{H}\right\}$ NMR of $11 \mathbf{a}\left(101 \mathrm{MHz}, \mathrm{CDCl}_{3}\right)$

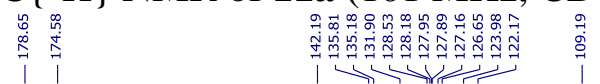

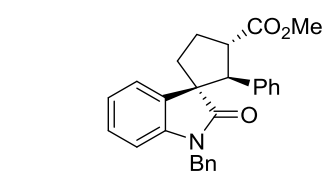

|

6000

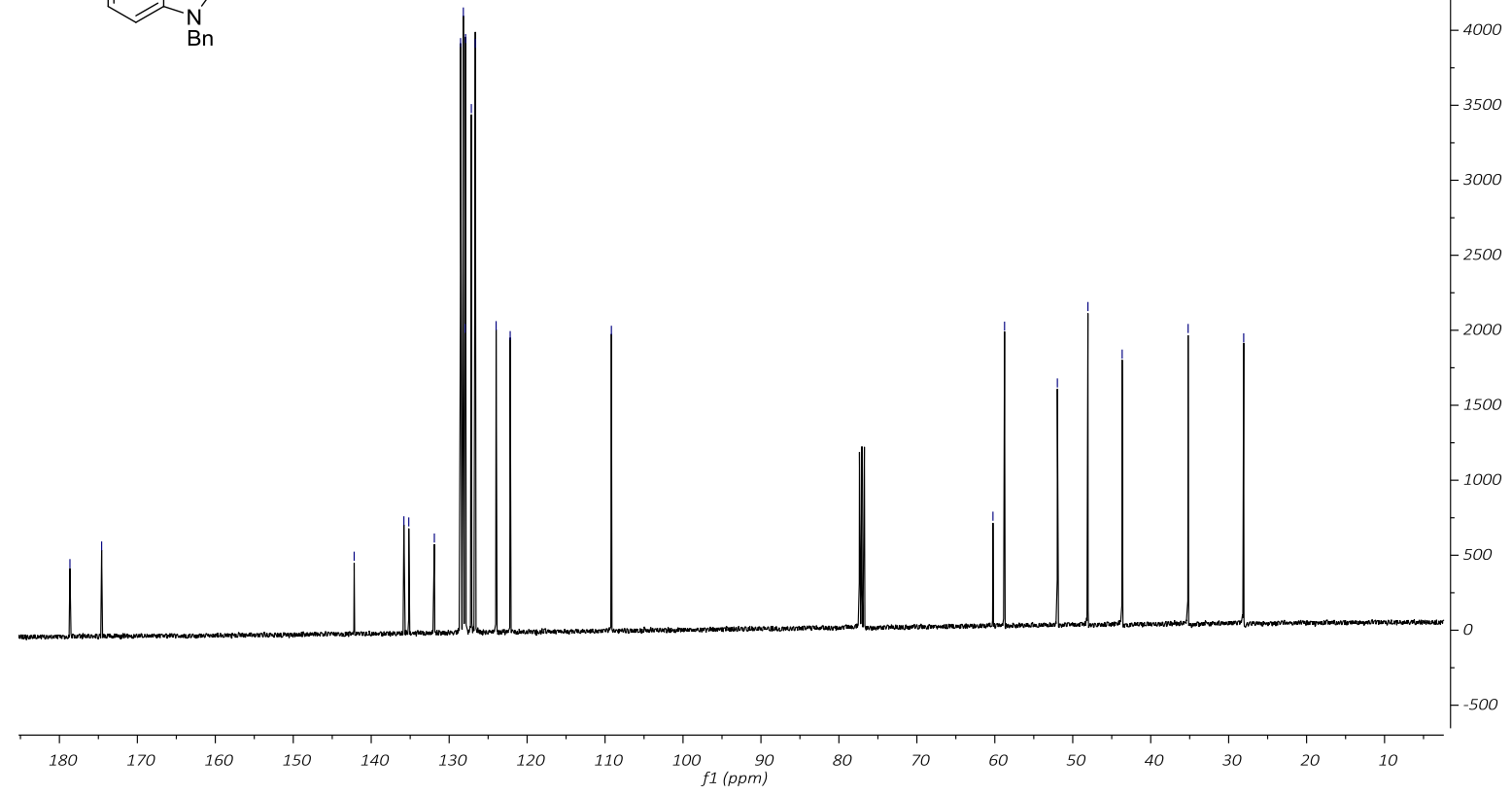


Methyl (1S,2S,3S)-2'-oxo-2-phenylspiro[cyclopentane-1,3'-indoline]-3-carboxylate (11d') ${ }^{1} \mathrm{H}$ NMR of 11d' $\left(400 \mathrm{MHz}, \mathrm{CDCl}_{3}\right)$

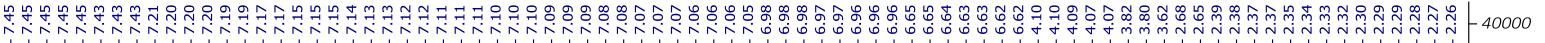

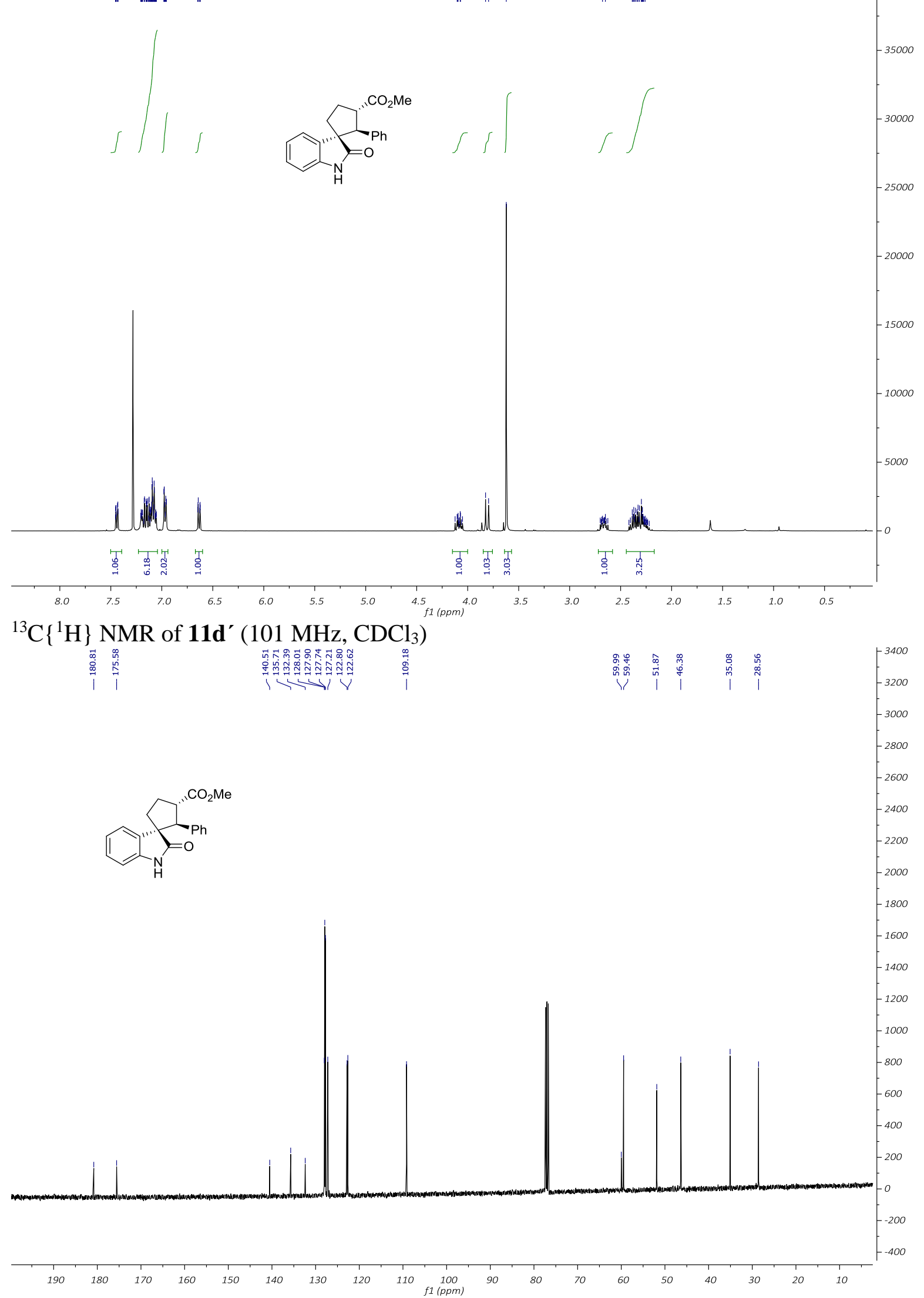


$(1 R, 2 S, 3 S)-1 '$-Benzyl-3-(hydroxymethyl)-2-phenylspiro[cyclopentane-1,3'-indolin]-2' -one (12a)

${ }^{1} \mathrm{H} \mathrm{NMR}$ of $\mathbf{1 2 a}\left(400 \mathrm{MHz}, \mathrm{CDCl}_{3}\right)$

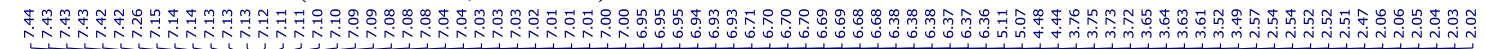

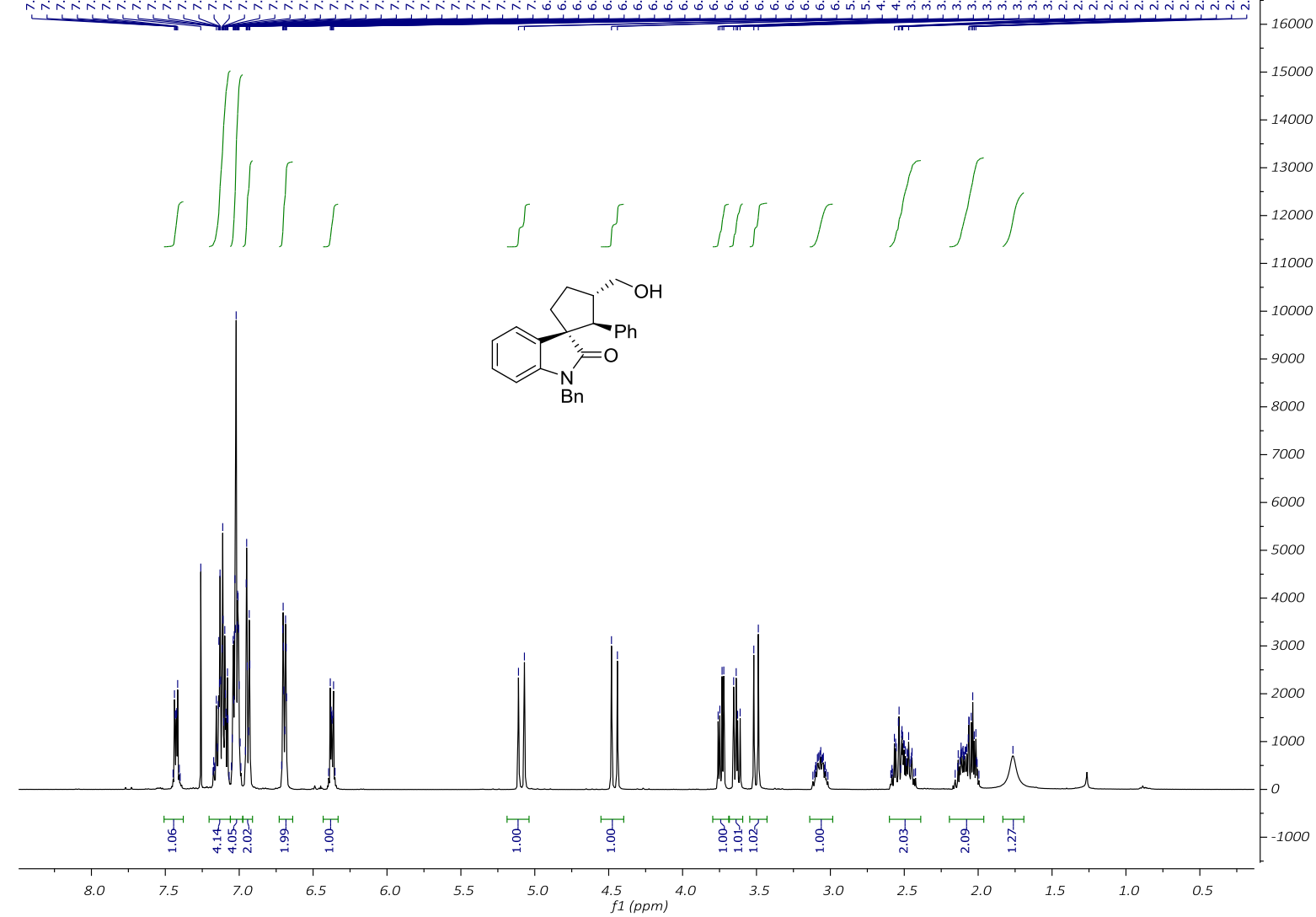

${ }^{13} \mathrm{C}\left\{{ }^{1} \mathrm{H}\right\} \mathrm{NMR}$ of $\mathbf{1 2 a}\left(101 \mathrm{MHz}, \mathrm{CDCl}_{3}\right)$

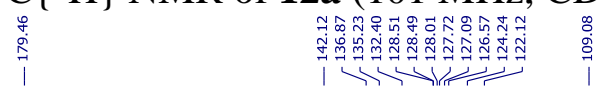

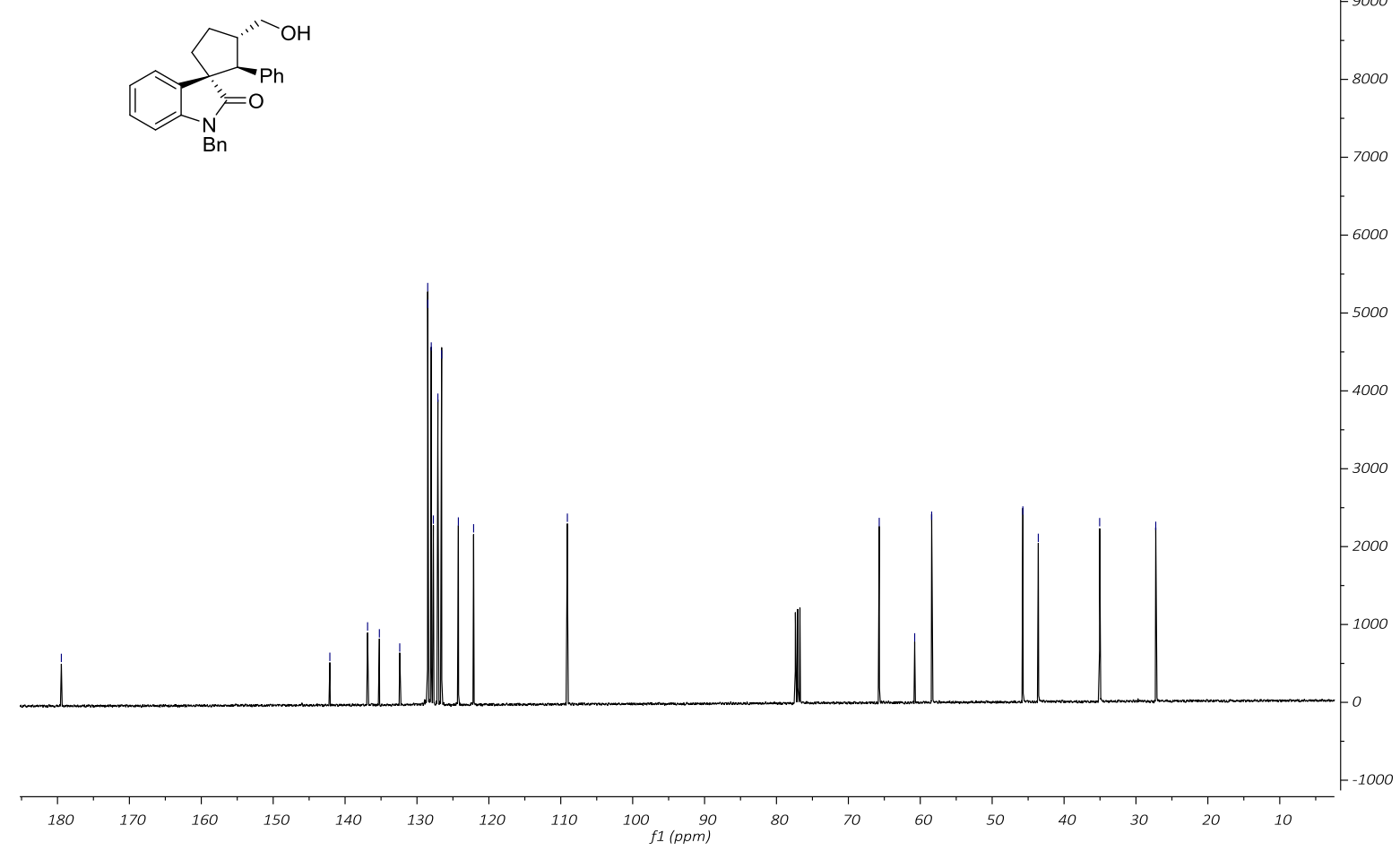

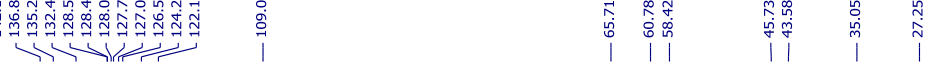


(1S,2S,3S)-3-(Hydroxymethyl)-2-phenylspiro[cyclopentane-1,3'-indolin]-2'-one (12d') ${ }^{1} \mathrm{H} \mathrm{NMR}$ of $12 \mathbf{d}^{\prime}\left(400 \mathrm{MHz}, \mathrm{CDCl}_{3}\right)$

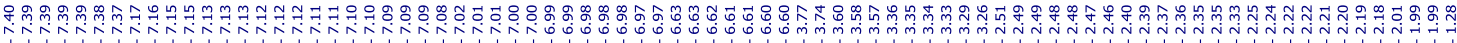

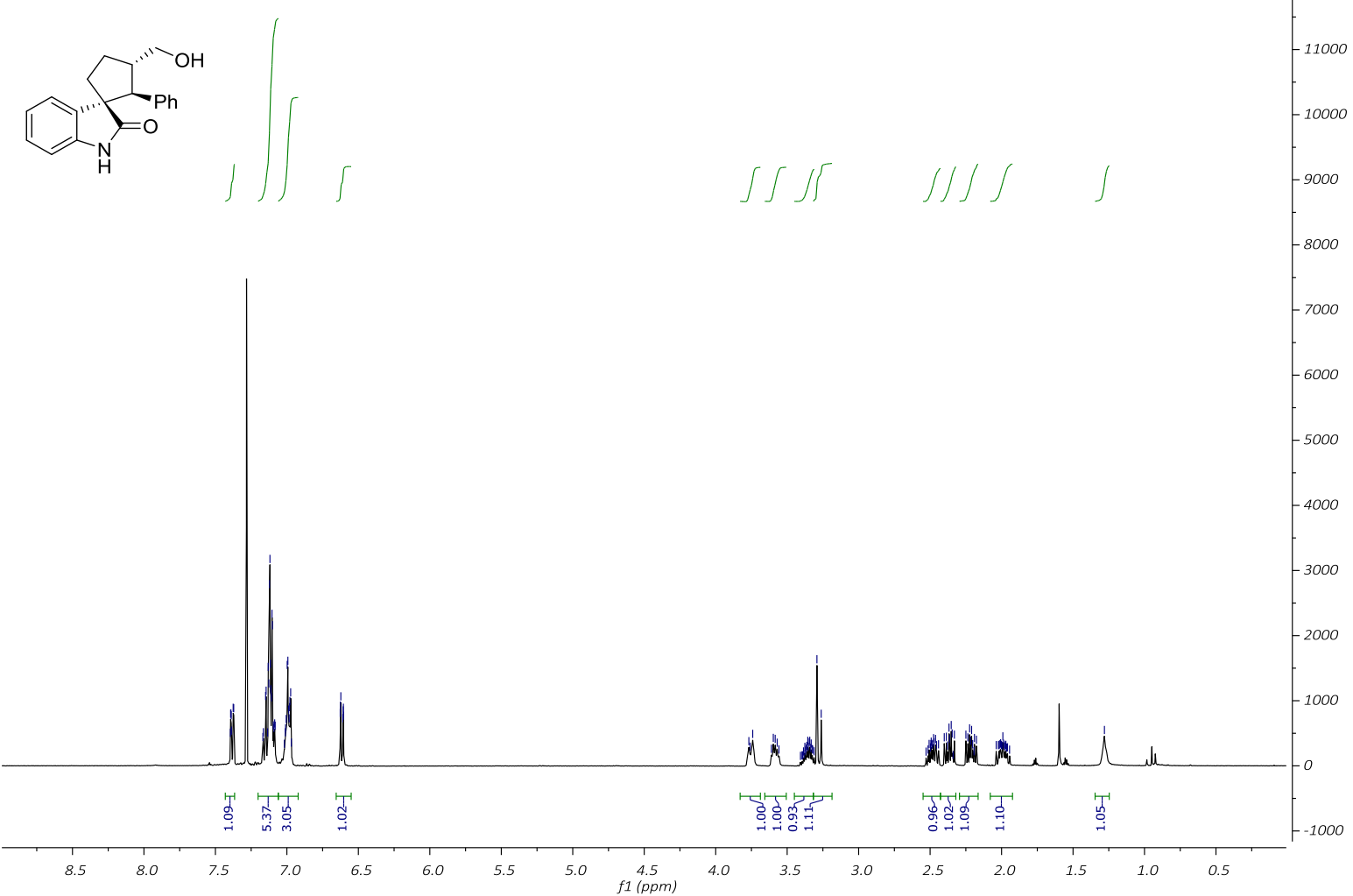

${ }^{13} \mathrm{C}\left\{{ }^{1} \mathrm{H}\right\} \mathrm{NMR}$ of $\mathbf{1 2 d}^{\prime}\left(101 \mathrm{MHz}, \mathrm{CDCl}_{3}\right)$

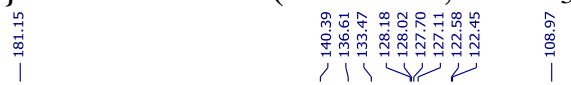

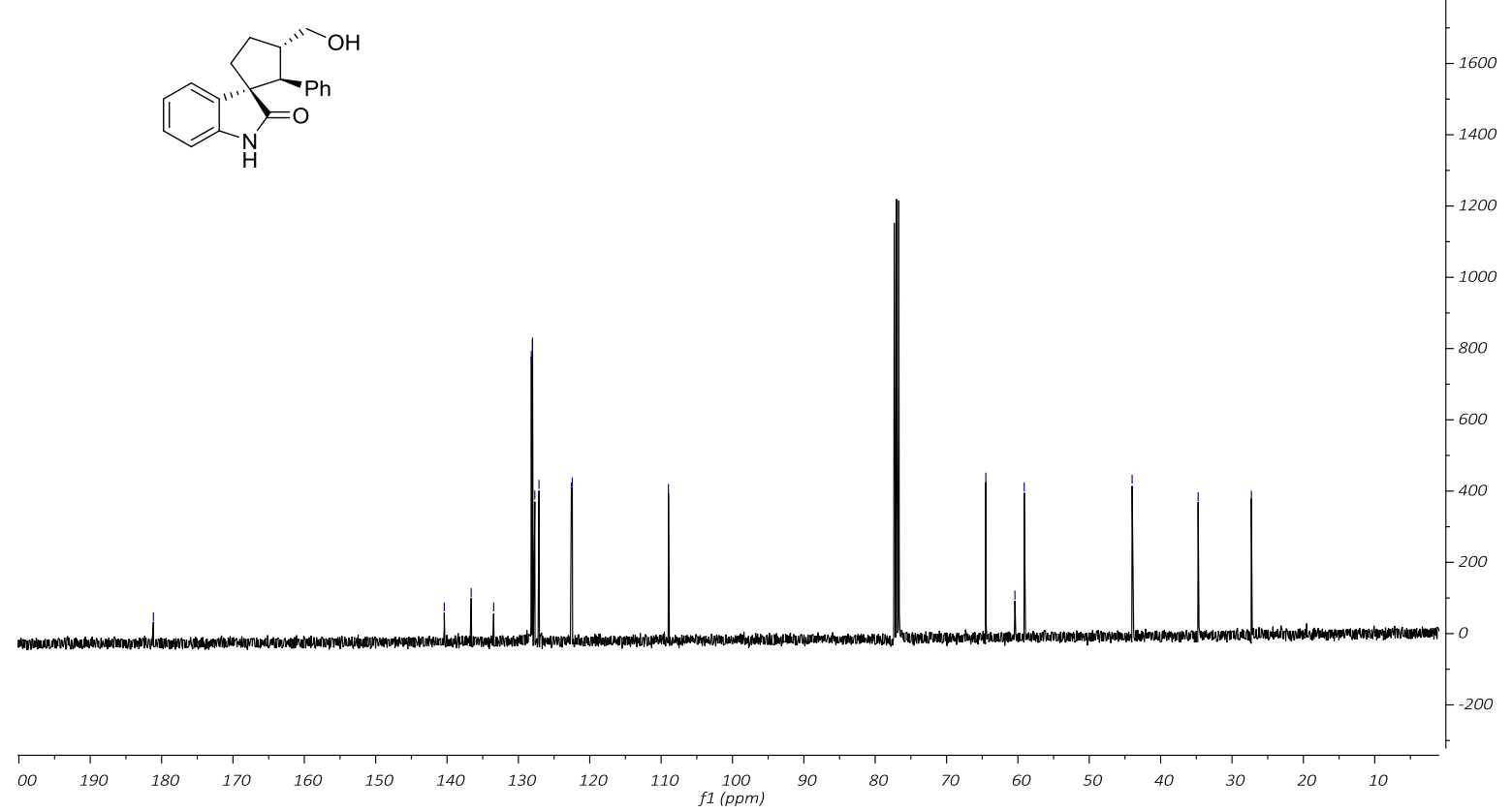
3) 
$(1 R, 2 S, 3 S)-1 '$-Benzyl-3-((benzylamino)methyl)-2-phenylspiro[cyclopentane-1,3'-indolin]2'-one (13a)

${ }^{1} \mathrm{H} \mathrm{NMR}$ of $13 \mathbf{a}\left(400 \mathrm{MHz}, \mathrm{CDCl}_{3}\right)$

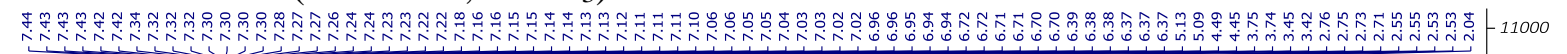

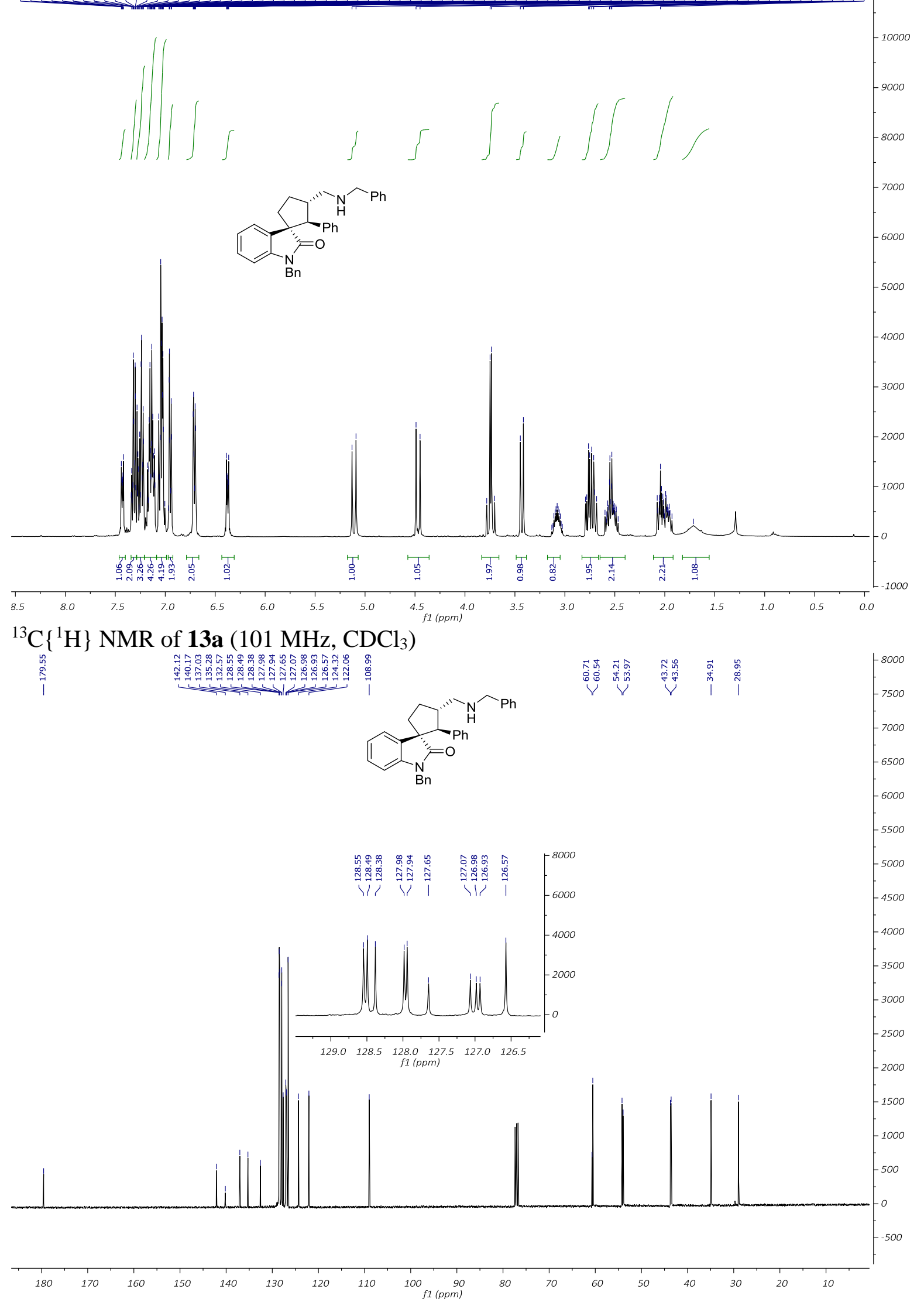


$(1 S, 2 S, 3 S)-1$ '-Benzyl-3-((benzylamino)methyl)-2-phenylspiro[cyclopentane-1,3'-indolin]2 '-one (13a')

${ }^{1} \mathrm{H}$ NMR of 13a' $\left(400 \mathrm{MHz}, \mathrm{CDCl}_{3}\right)$

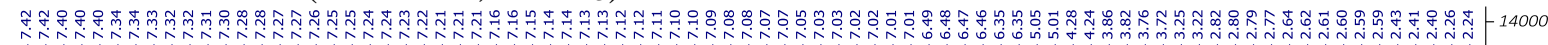

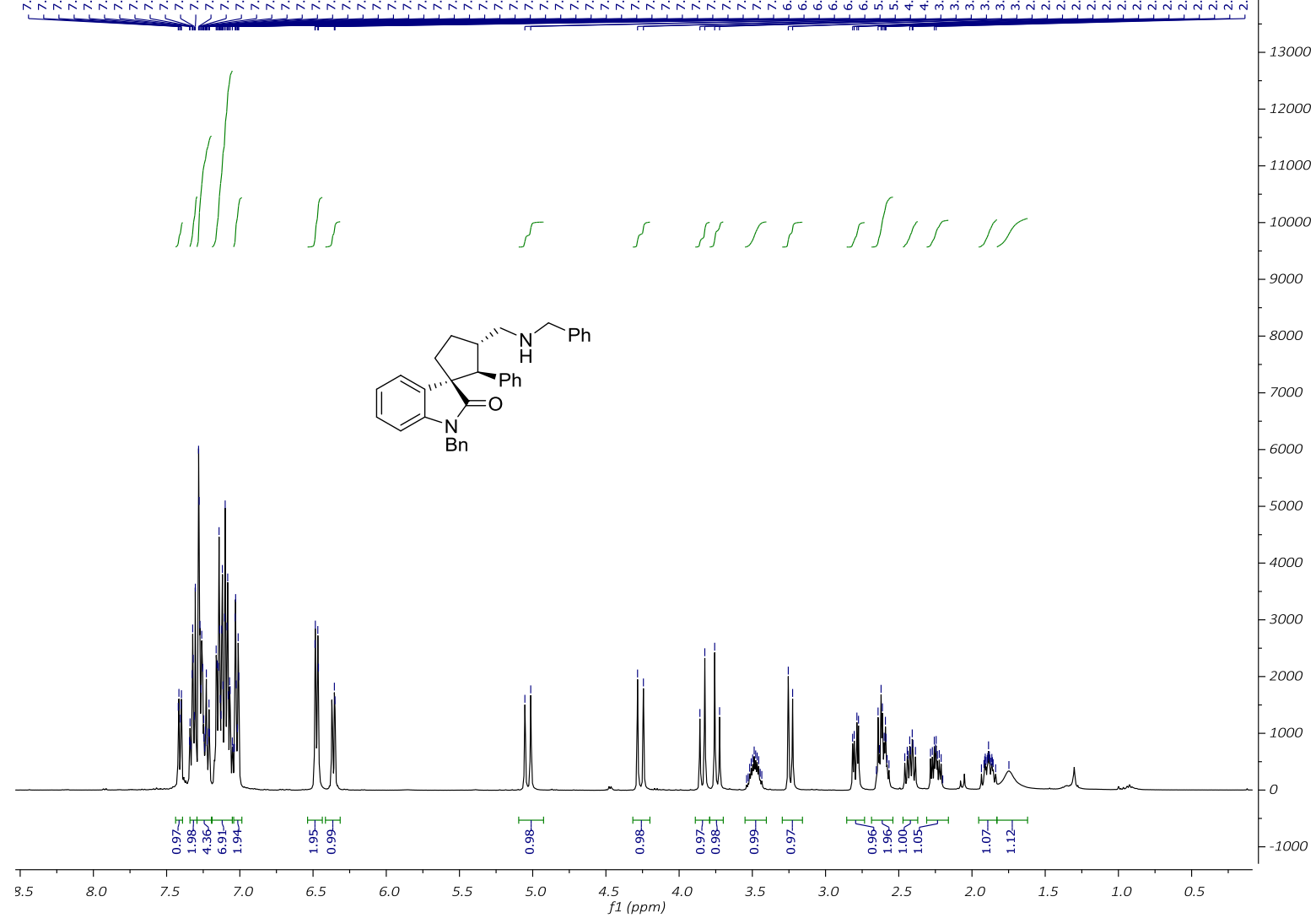

${ }^{13} \mathrm{C}\left\{{ }^{1} \mathrm{H}\right\}$ NMR of 13a' (101 MHz, $\left.\mathrm{CDCl}_{3}\right)$

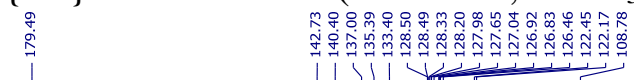

|

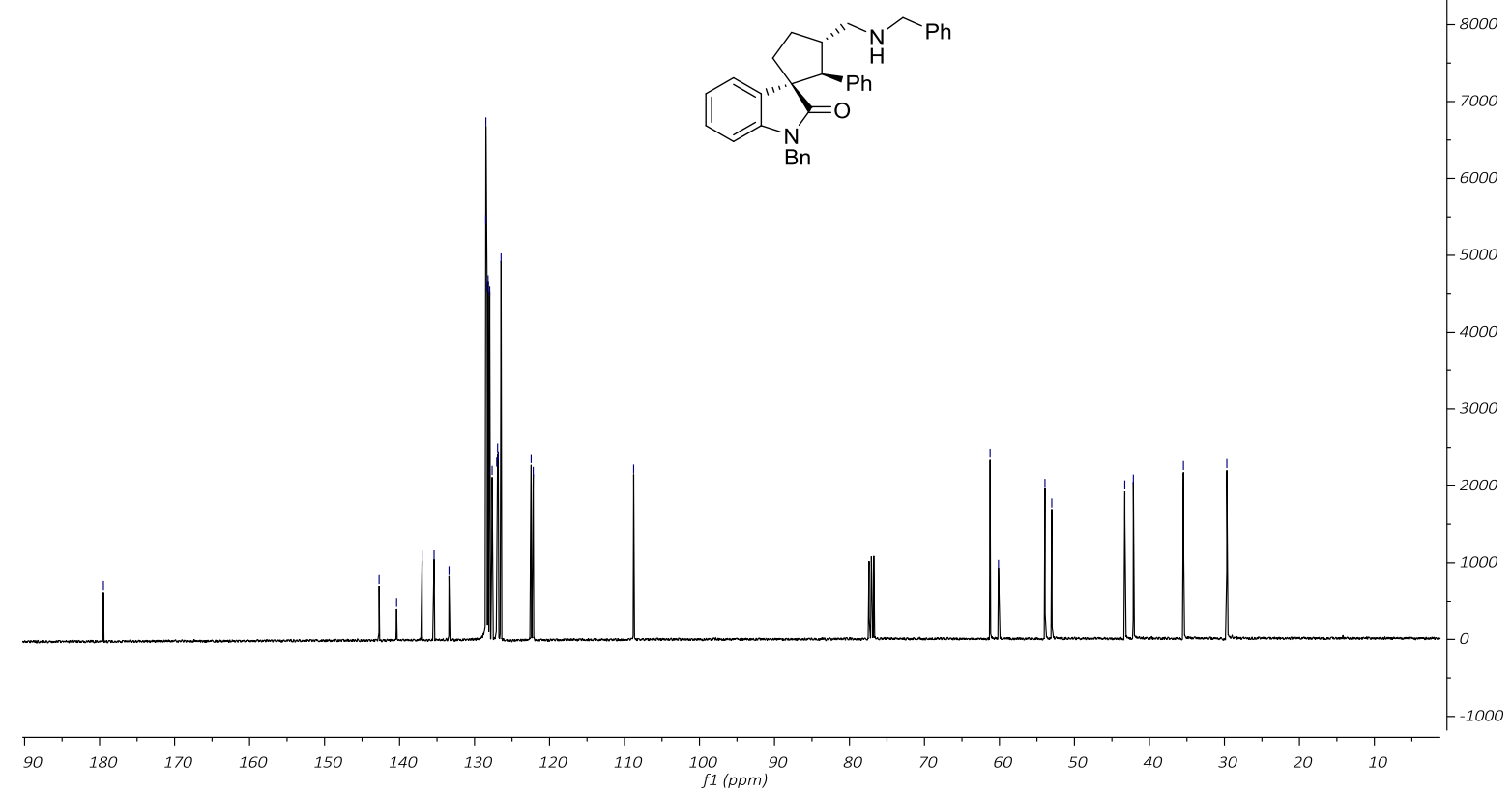


(1S,2S,3S)-3-((Benzylamino)methyl)-2-phenylspiro[cyclopentane-1,3'-indolin]-2'-one $\left(13 d^{\prime}\right)$

${ }^{1} \mathrm{H}$ NMR of 13d' (400 MHz, $\mathrm{CDCl}_{3}$ )

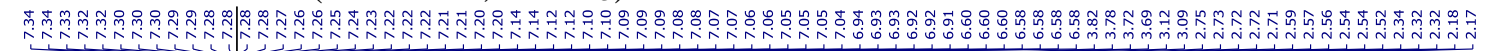

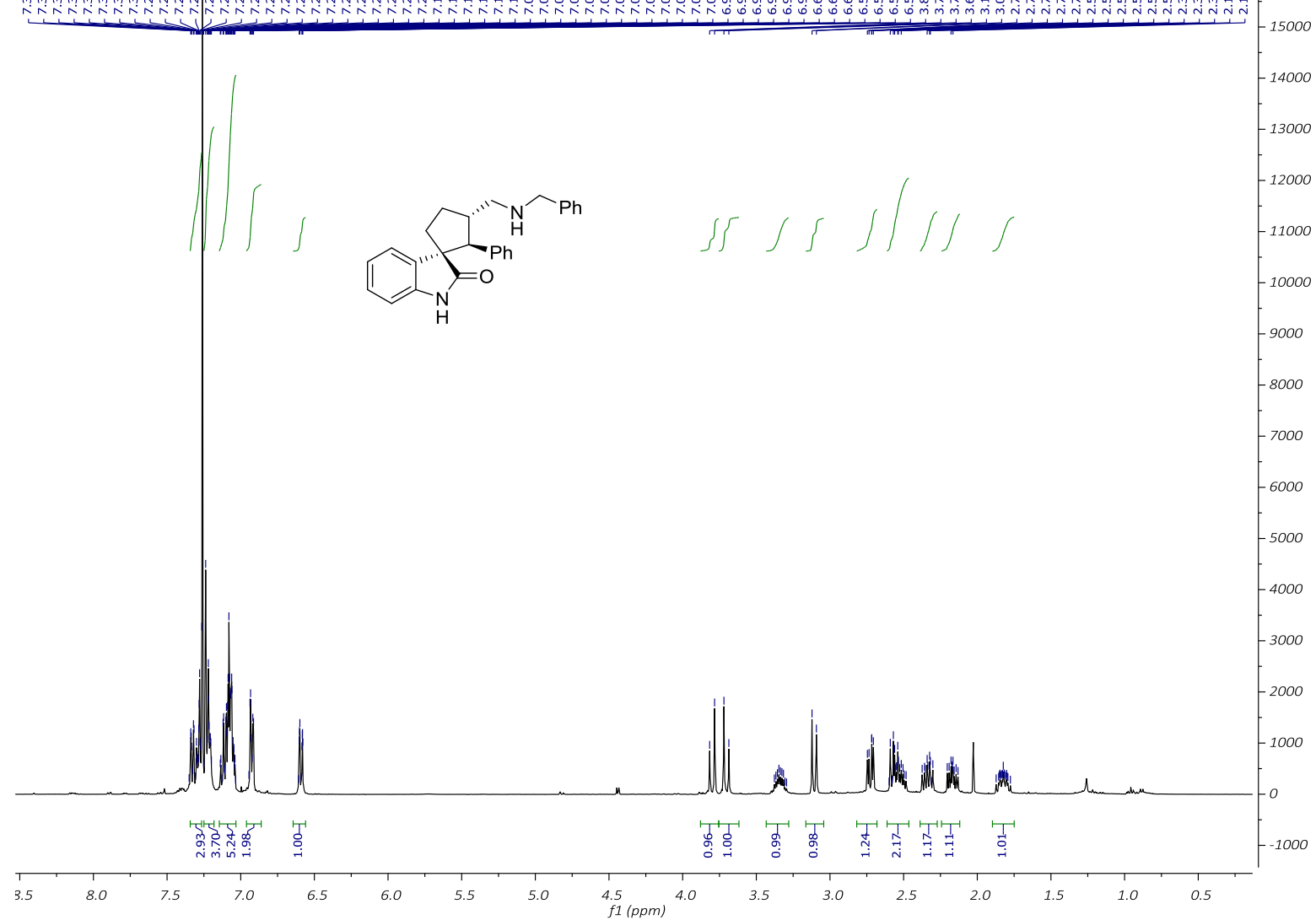

${ }^{13} \mathrm{C}\left\{{ }^{1} \mathrm{H}\right\}$ NMR of $\mathbf{1 3 d}^{\prime}\left(101 \mathrm{MHz}, \mathrm{CDCl}_{3}\right)$

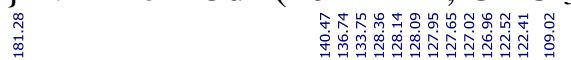

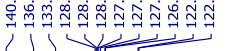

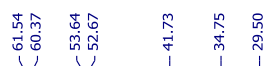

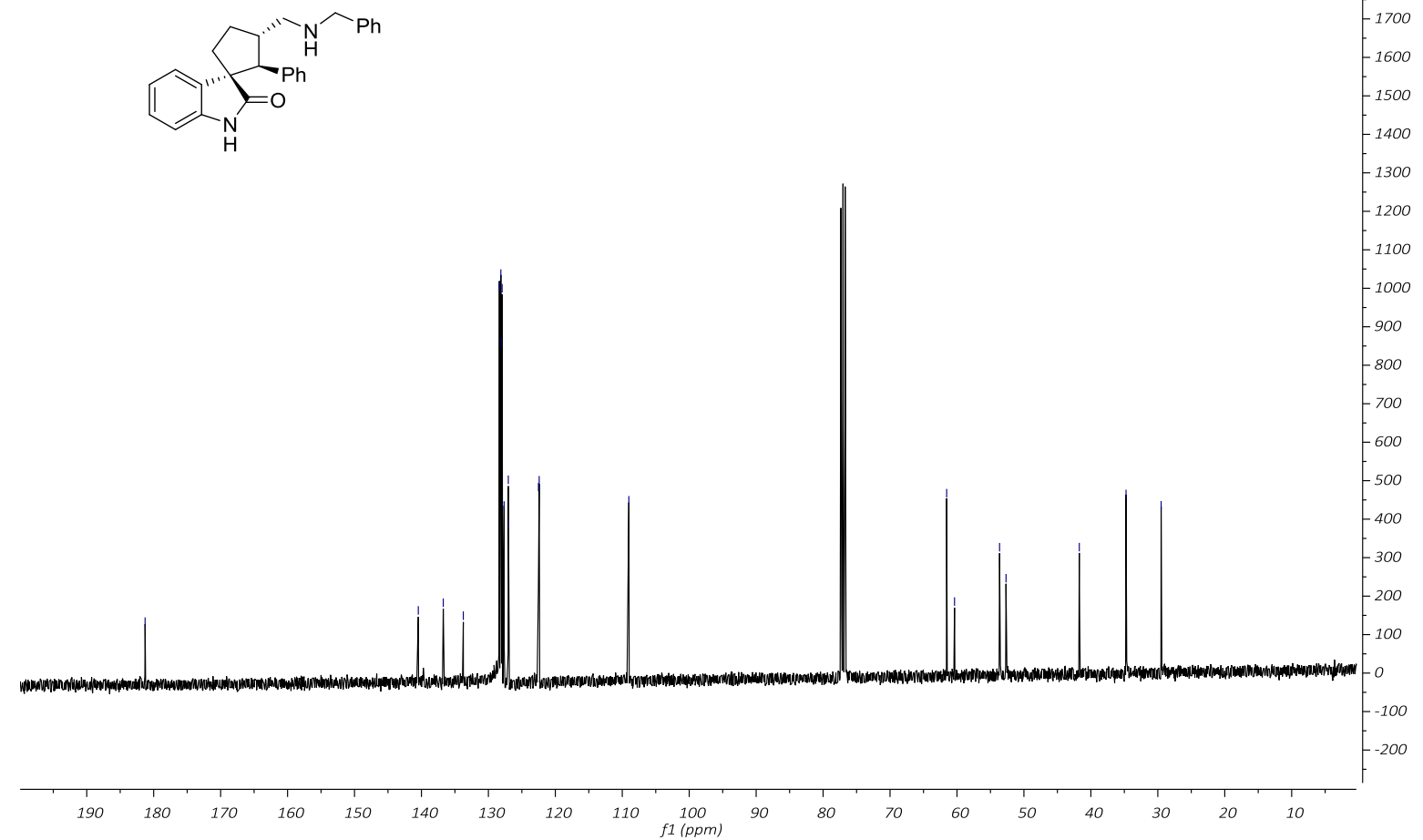


Methyl $(E)-3-((1 R, 2 S, 3 R)-1 '$-benzyl-2'-oxo-2-phenylspiro[cyclopentane-1,3'-indolin]-3yl)acrylate (14a)

${ }^{1} \mathrm{H} \mathrm{NMR}$ of $\mathbf{1 4 a}\left(400 \mathrm{MHz}, \mathrm{CDCl}_{3}\right)$

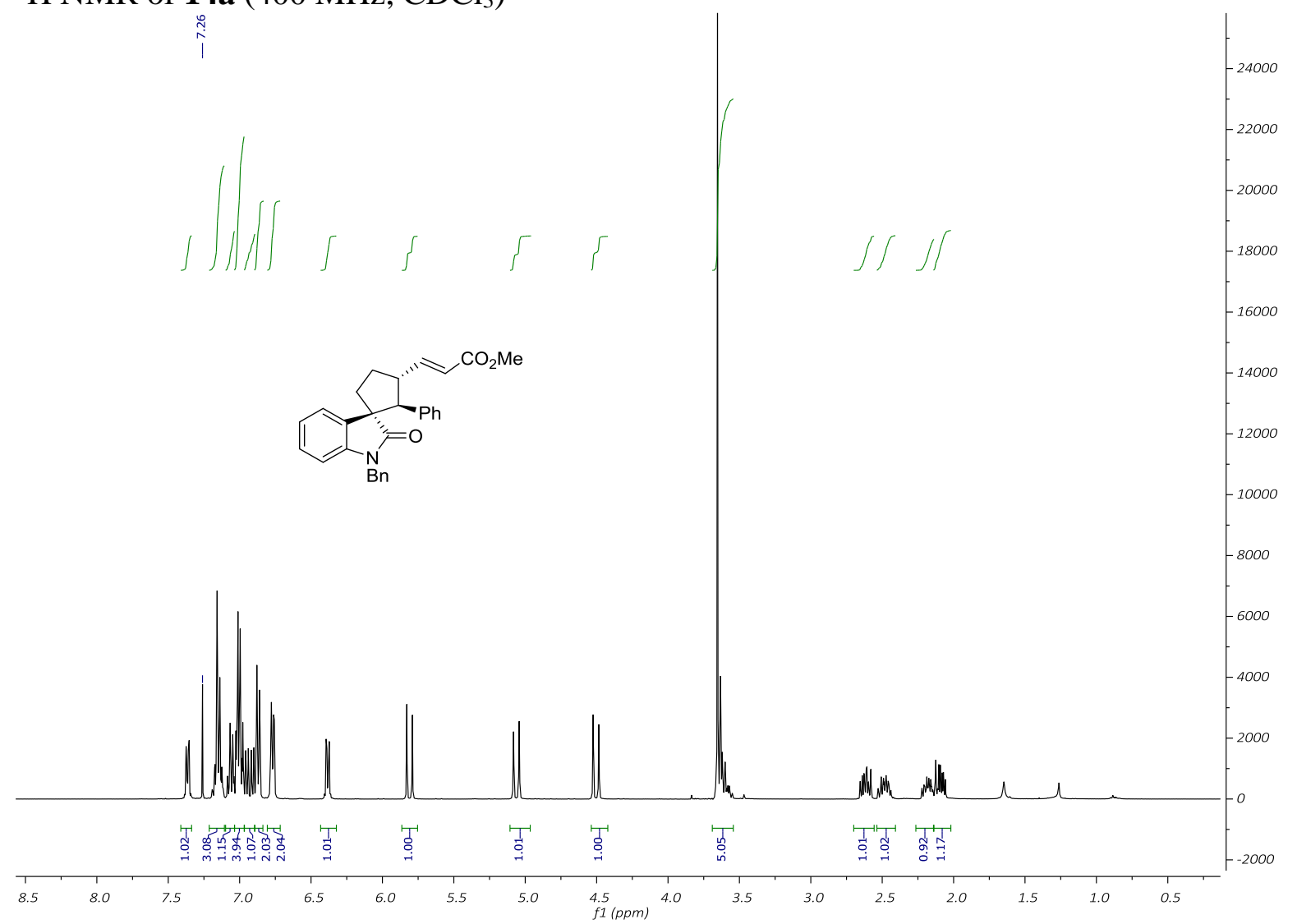

${ }^{13} \mathrm{C}\left\{{ }^{1} \mathrm{H}\right\}$ NMR of $\mathbf{1 4 a}\left(101 \mathrm{MHz}, \mathrm{CDCl}_{3}\right)$

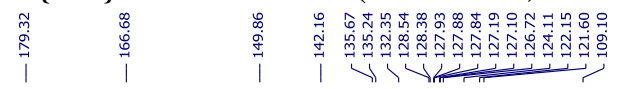

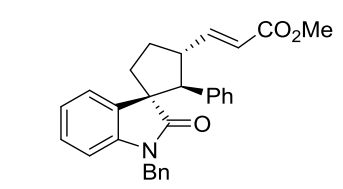

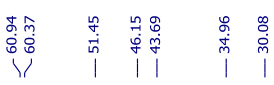
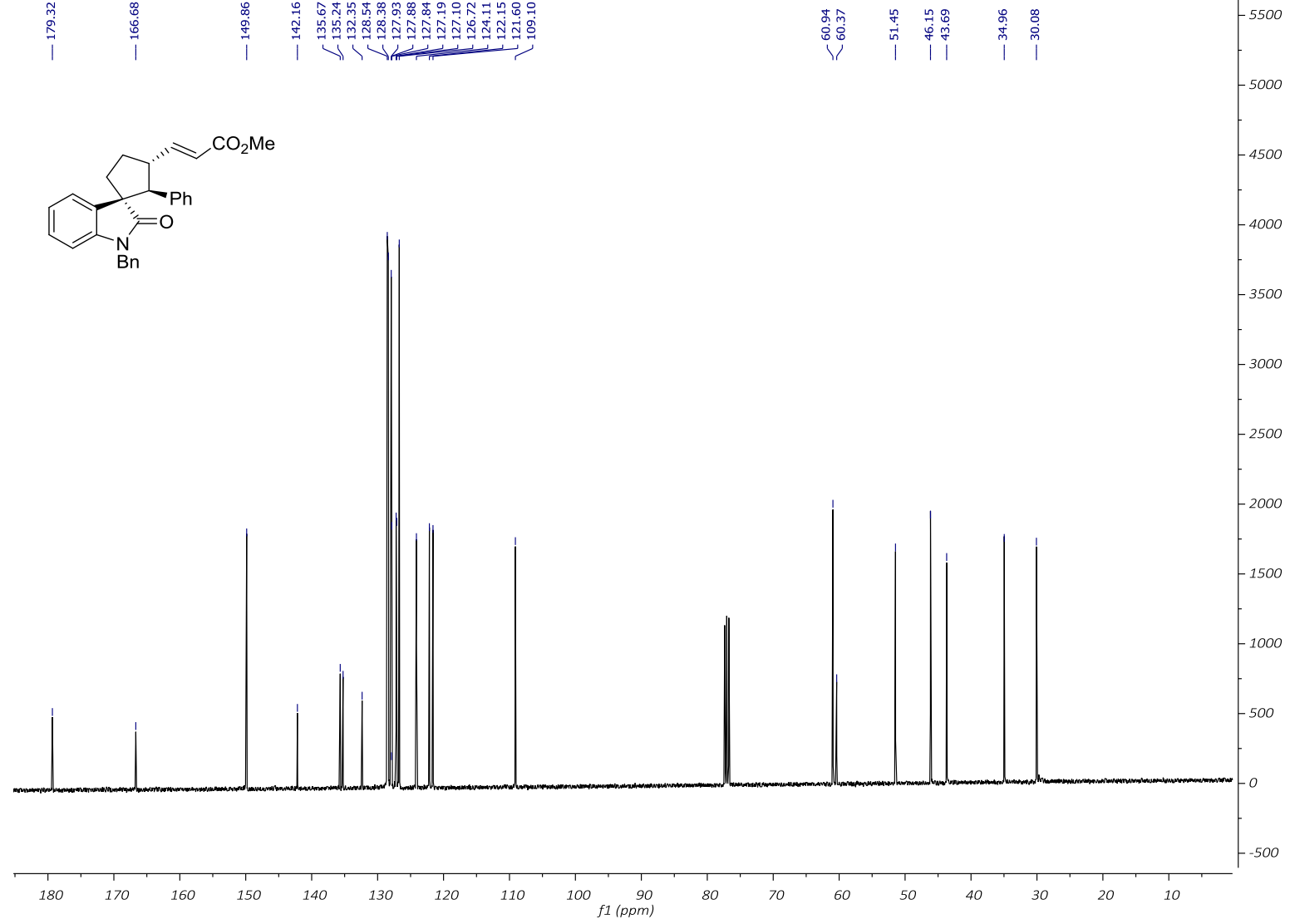
Methyl $\quad(E)-3-((1 R, 2 S, 3 R)-1$ '-allyl-2'-oxo-2-phenylspiro[cyclopentane-1,3'-indolin]-3yl)acrylate (14b)

${ }^{1} \mathrm{H} \mathrm{NMR}$ of $\mathbf{1 4 b}\left(400 \mathrm{MHz}, \mathrm{CDCl}_{3}\right)$

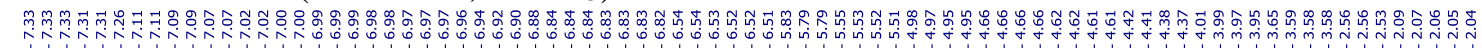

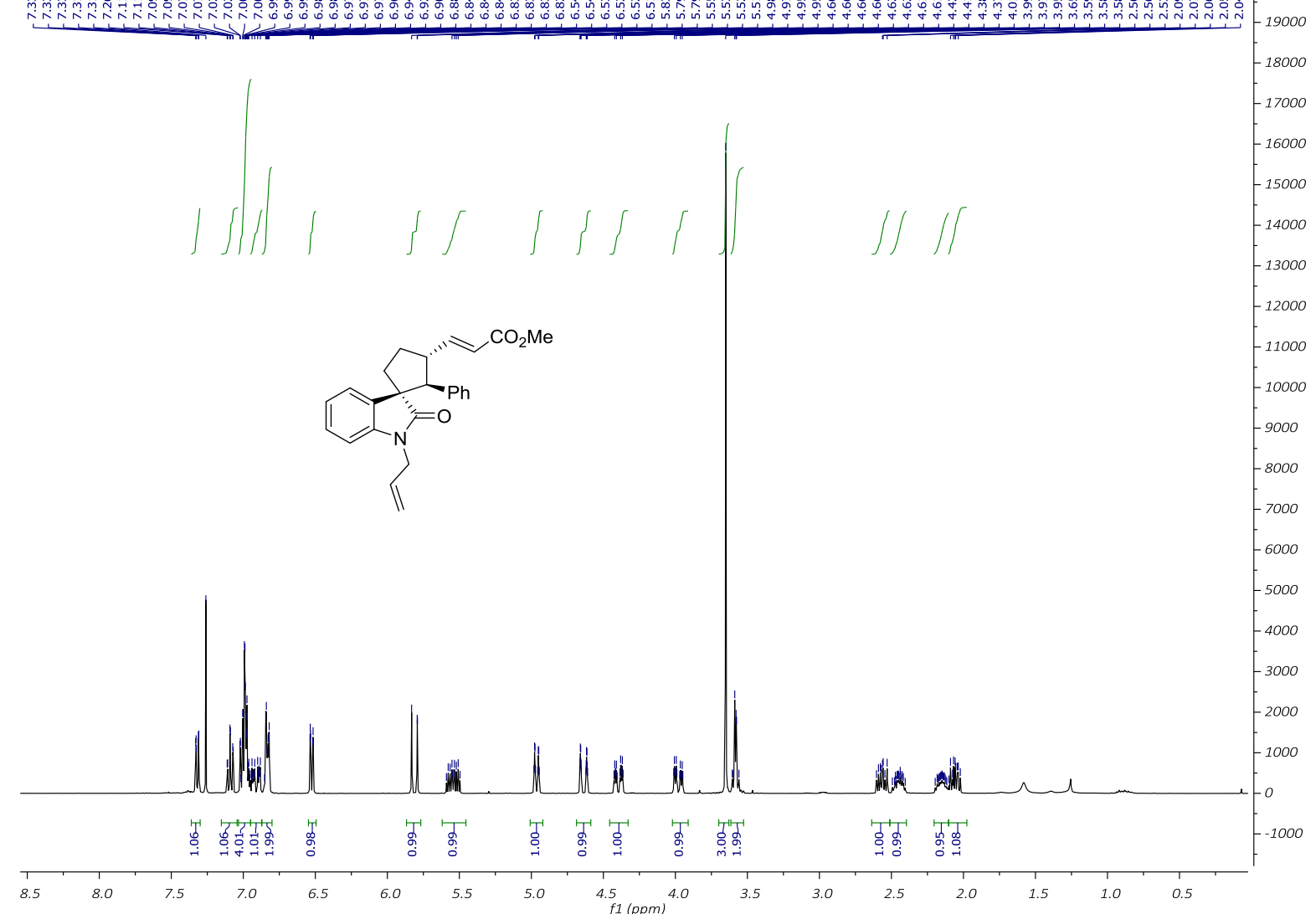

${ }^{13} \mathrm{C}\left\{{ }^{1} \mathrm{H}\right\}$ NMR of $\mathbf{1 4 b}\left(101 \mathrm{MHz}, \mathrm{CDCl}_{3}\right)$

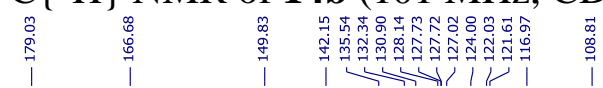

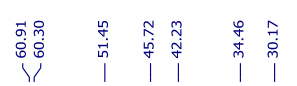

$-2100$

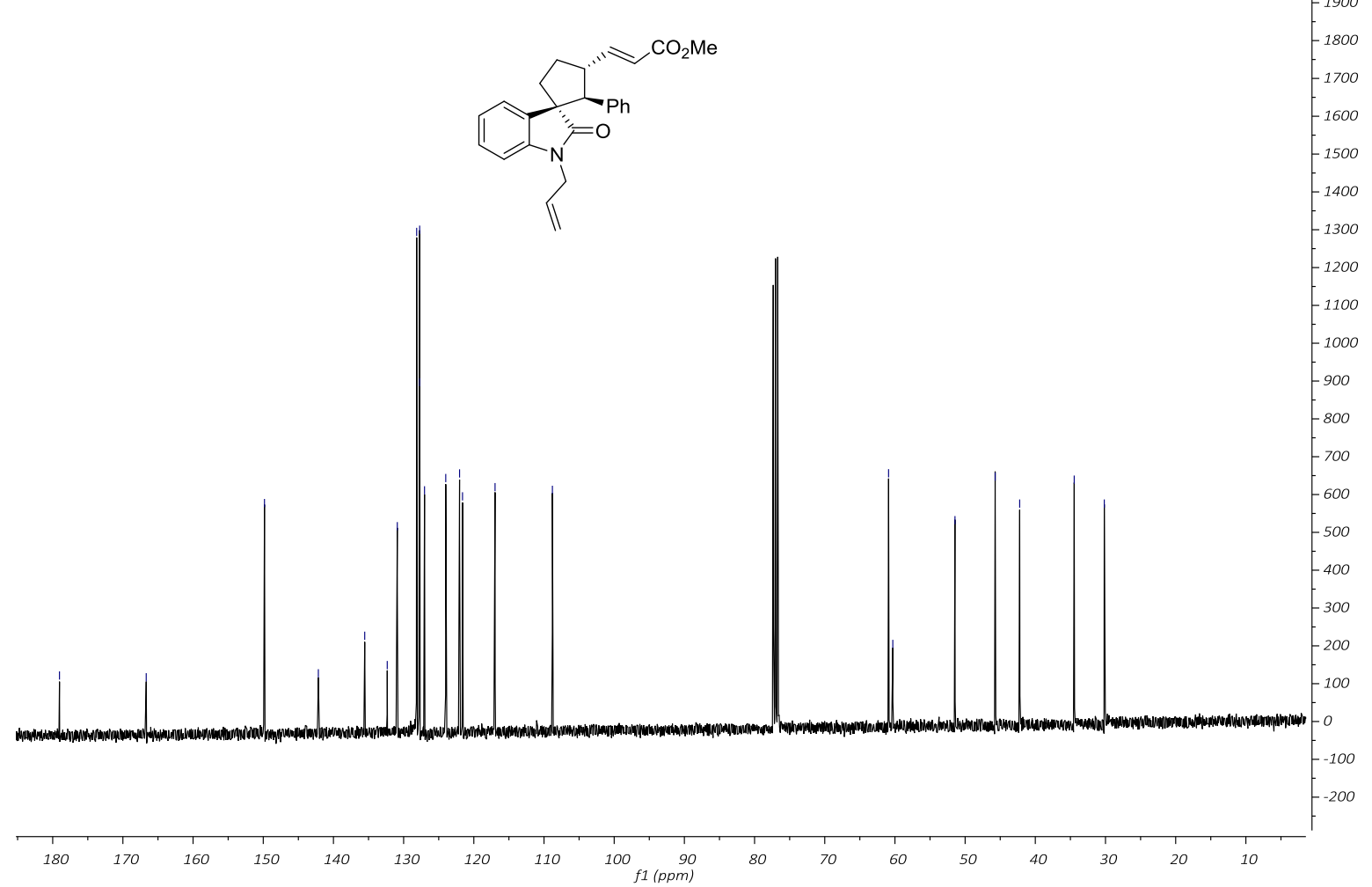


Methyl $(E)-3-((1 S, 2 S, 3 R)-1 '$-allyl-2'-oxo-2-phenylspiro[cyclopentane-1,3'-indolin]-3yl)acrylate (14b')

${ }^{1} \mathrm{H}$ NMR of 14b' $\left(400 \mathrm{MHz}, \mathrm{CDCl}_{3}\right)$

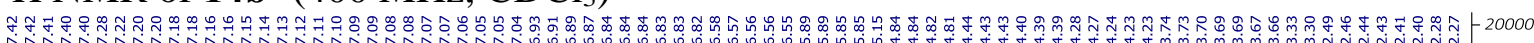

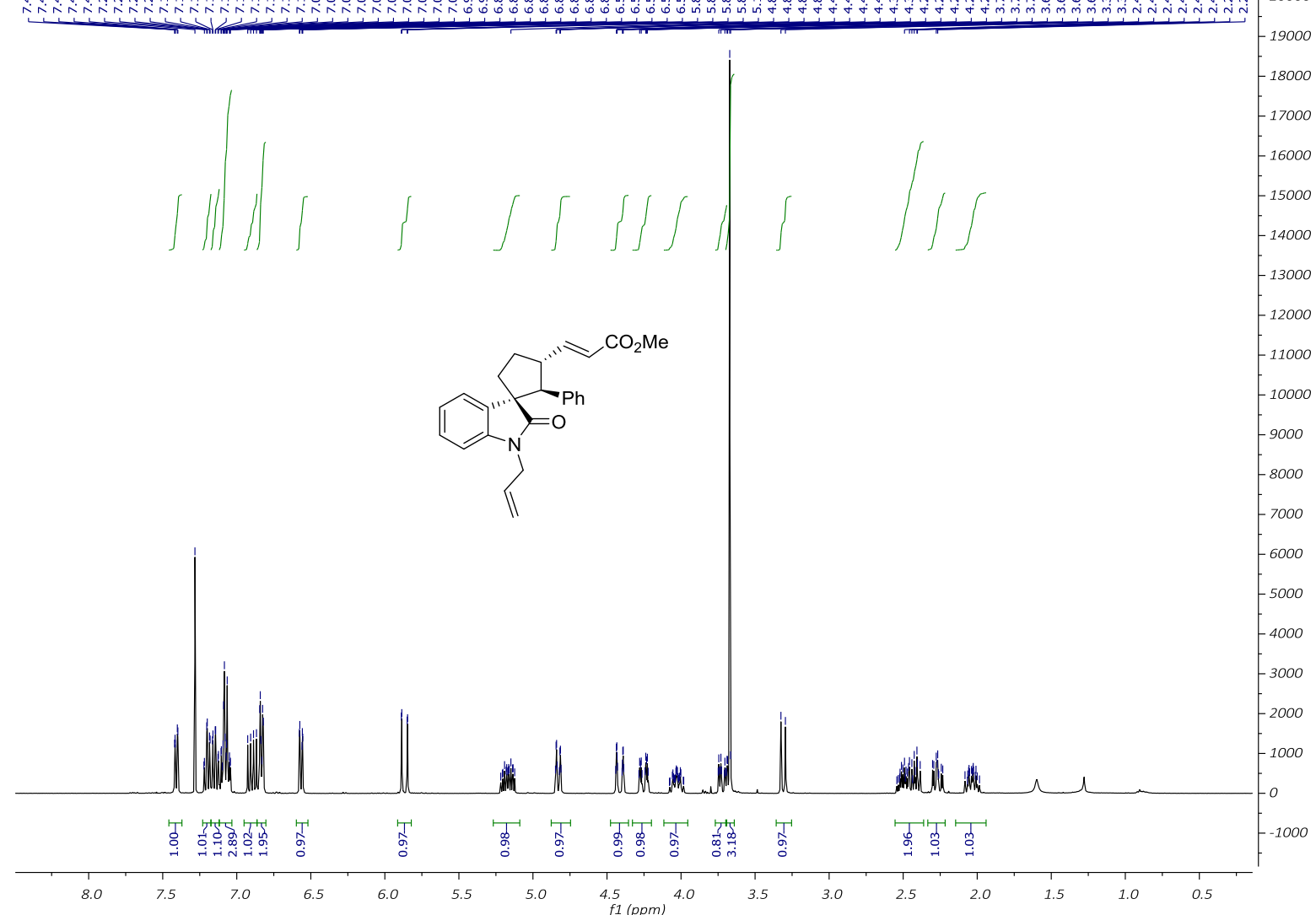

${ }^{13} \mathrm{C}\left\{{ }^{1} \mathrm{H}\right\}$ NMR of $\mathbf{1 4} \mathbf{b}^{\prime}\left(101 \mathrm{MHz}, \mathrm{CDCl}_{3}\right)$

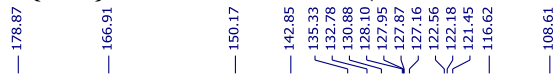
$f_{1}($

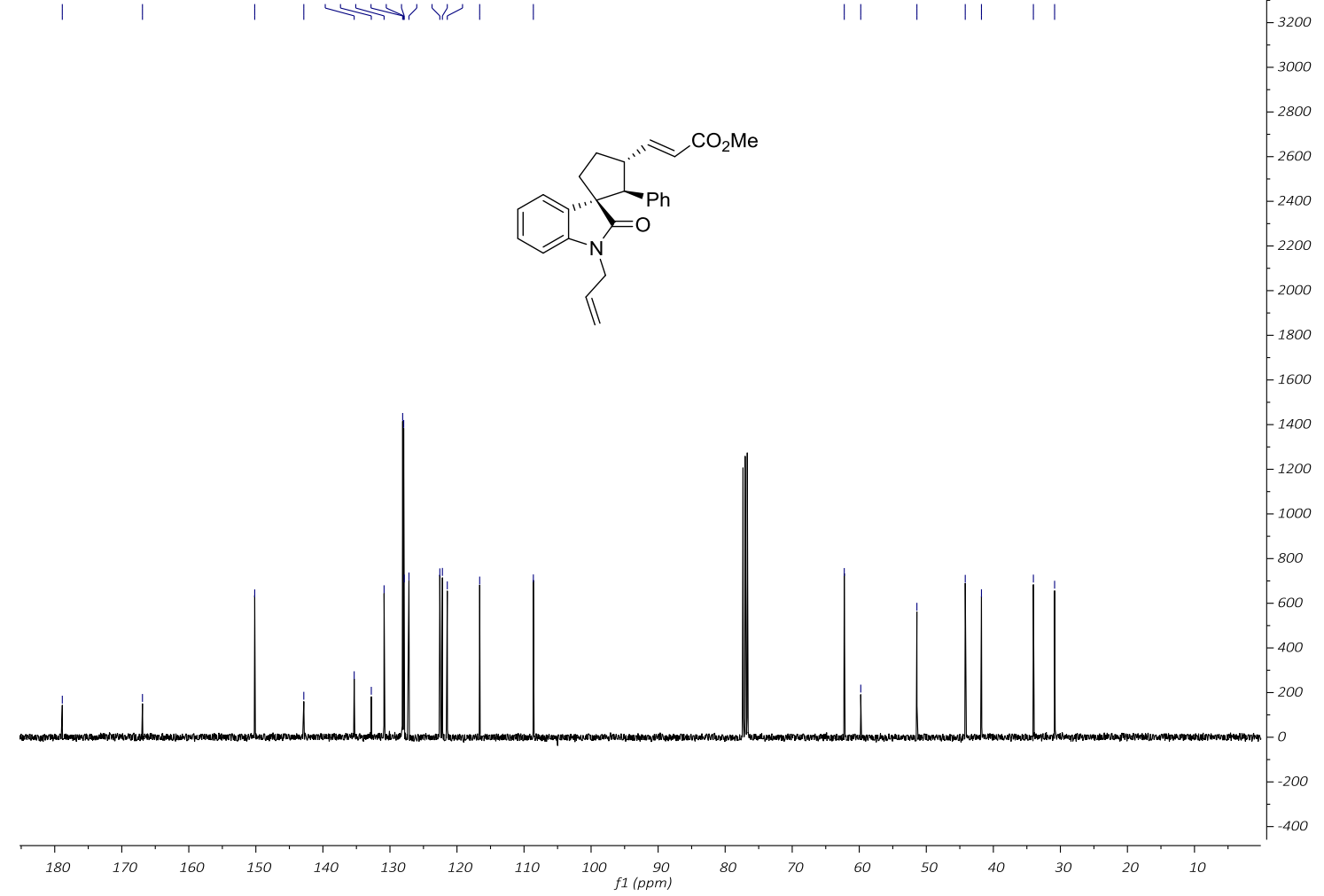


Methyl $(E)-3-((1 R, 2 S, 3 R)-1$ '-methyl-2'-oxo-2-phenylspiro[cyclopentane-1,3'-indolin]-3yl)acrylate (14c)

${ }^{1} \mathrm{H} \mathrm{NMR}$ of $14 \mathbf{c}\left(400 \mathrm{MHz}, \mathrm{CDCl}_{3}\right)$

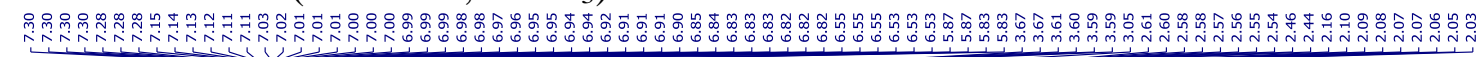

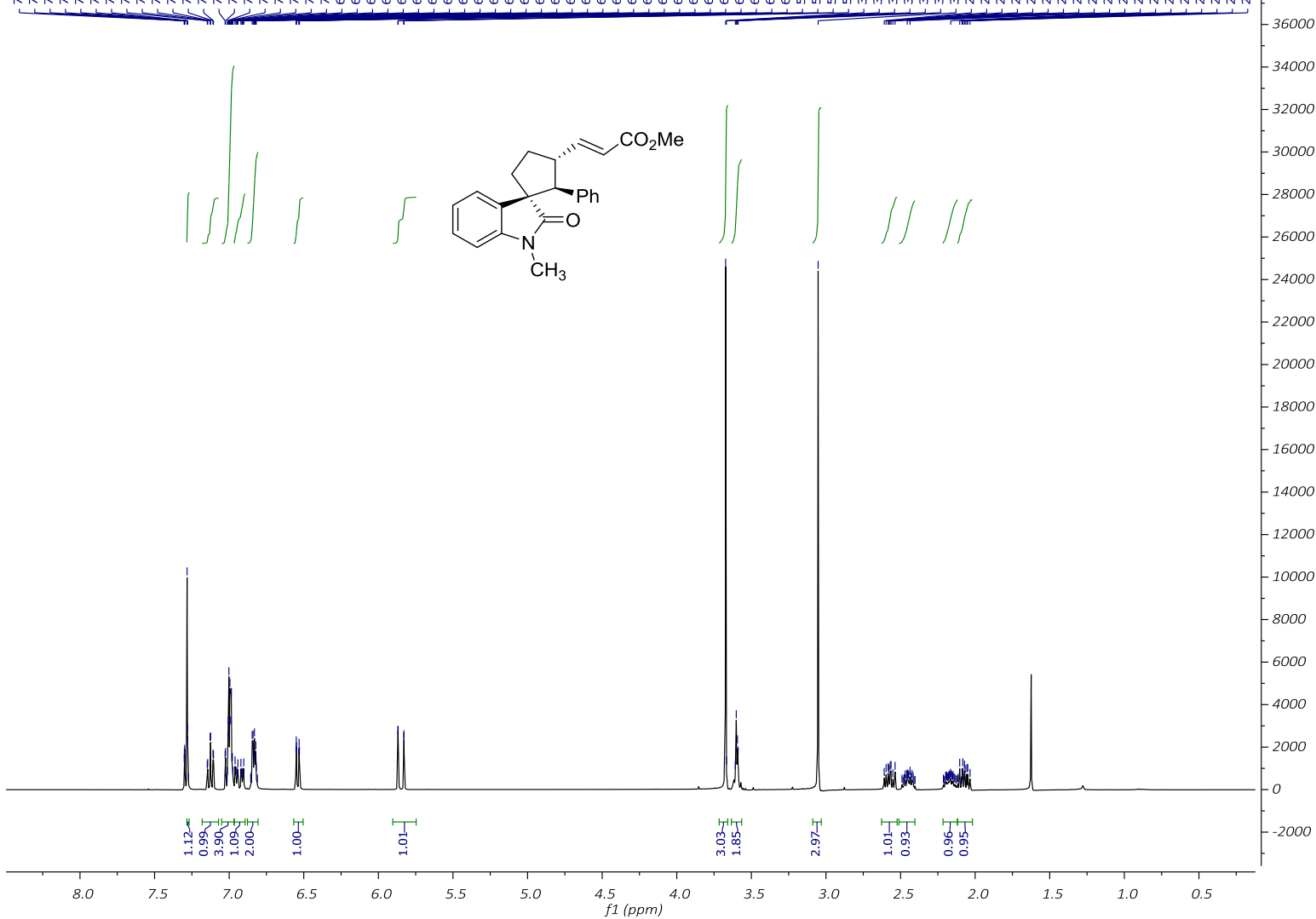

${ }^{13} \mathrm{C}\left\{{ }^{1} \mathrm{H}\right\} \mathrm{NMR}$ of $\mathbf{1 4 c}\left(101 \mathrm{MHz}, \mathrm{CDCl}_{3}\right)$

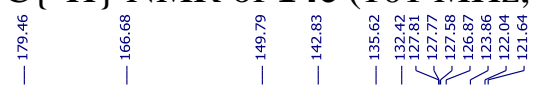
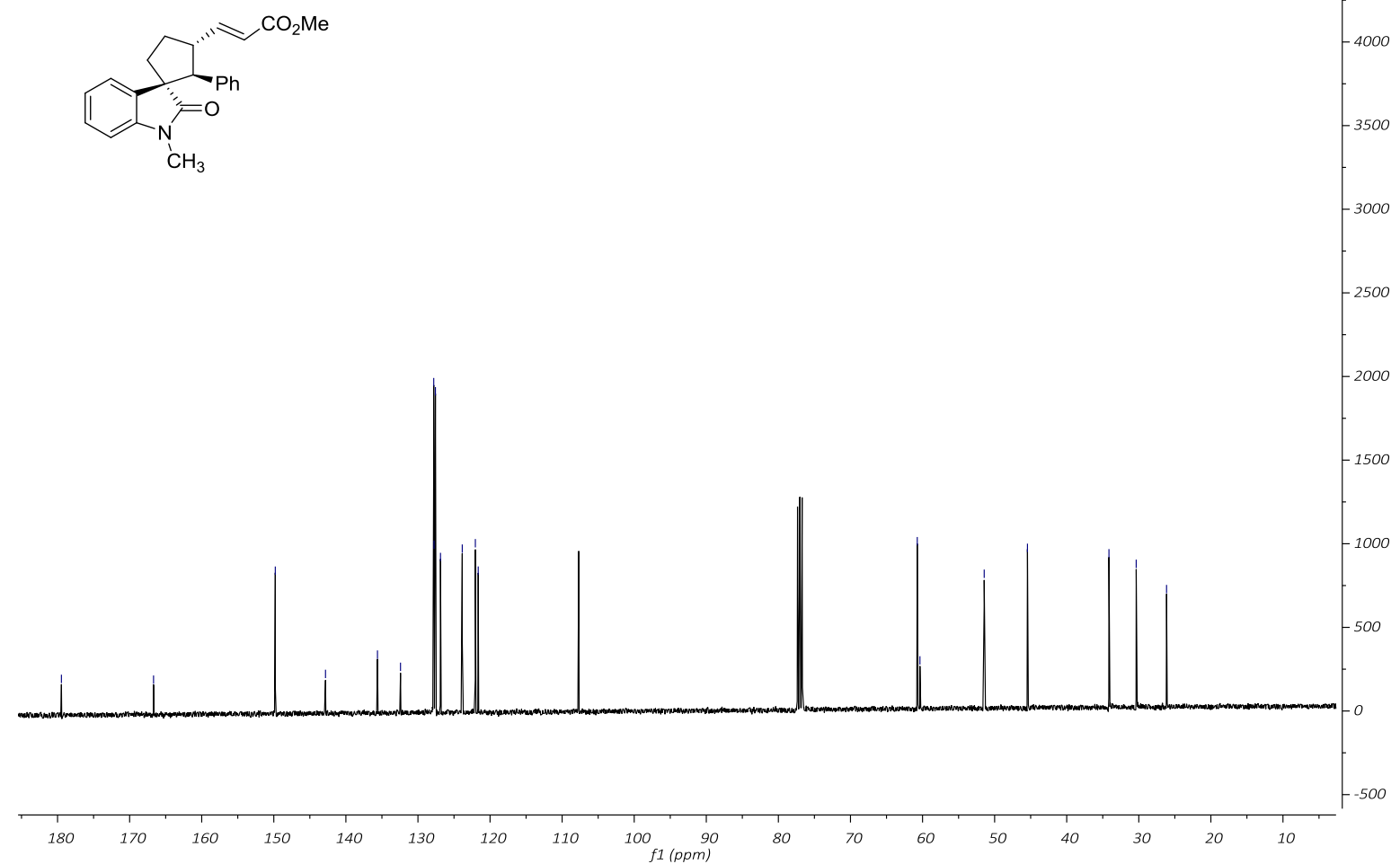
Methyl (E)-3-((1S,2S,3R)-1'-methyl-2'-oxo-2-phenylspiro[cyclopentane-1,3'-indolin]-3yl)acrylate $\left(14 c^{\prime}\right)$

${ }^{1} \mathrm{H}$ NMR of $14 \mathbf{c}^{\prime}\left(400 \mathrm{MHz}, \mathrm{CDCl}_{3}\right)$

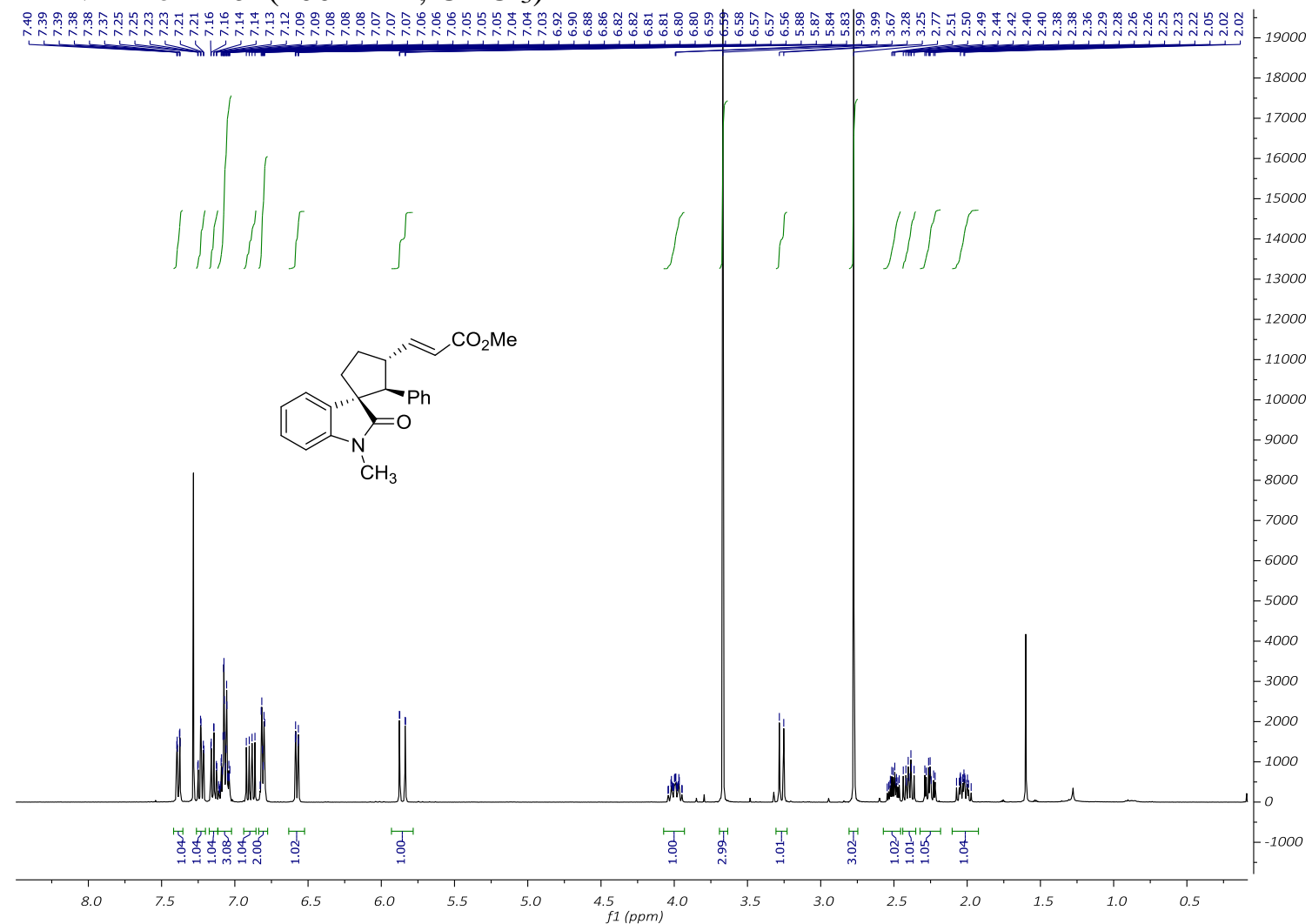

${ }^{13} \mathrm{C}\left\{{ }^{1} \mathrm{H}\right\} \mathrm{NMR}$ of $\mathbf{1 4 c}^{\prime}\left(101 \mathrm{MHz}, \mathrm{CDCl}_{3}\right)$

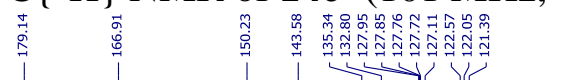

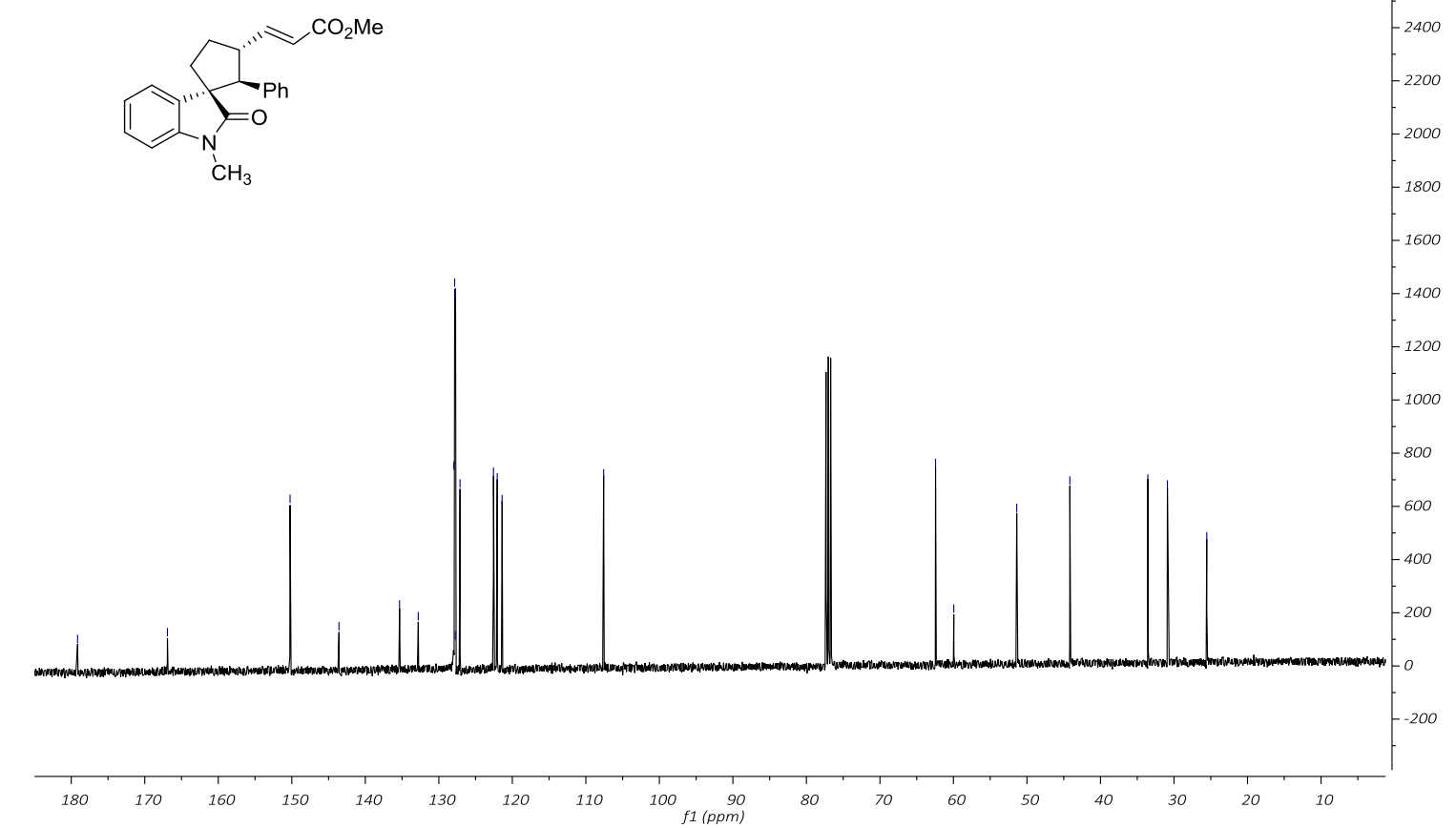




\section{Chiral HPLC}<smiles>C[C@@H]1CCC2(C(=O)Nc3ccccc32)[C@H]1c1ccccc1</smiles>

Bn

Conditions: IA column

mobile phase: heptane / $i$-PrOH $-80: 20$

$\lambda=214 \mathrm{~nm}, V=1.0 \mathrm{ml} / \mathrm{min}, t=25^{\circ} \mathrm{C}$

for 3a: $t_{\mathrm{R}}=9.6 \mathrm{~min}$ (minor), $t_{\mathrm{R}}=25.7 \mathrm{~min}$ (major).

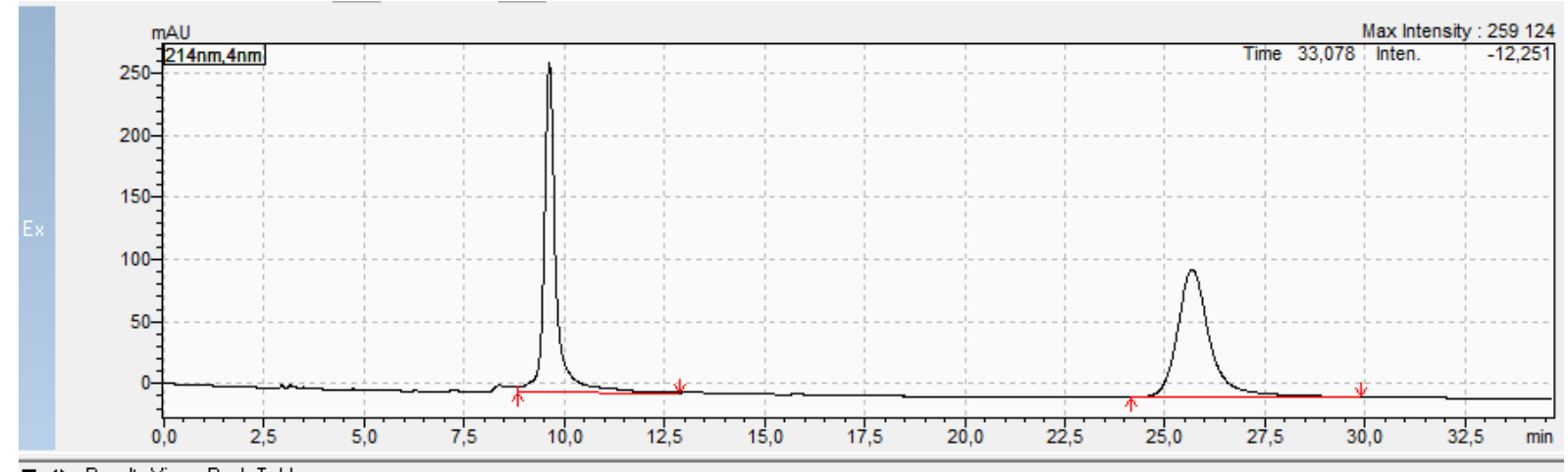

口〈 Results View - Peak Table

\begin{tabular}{l|l|l|l}
\hline Peak Table Compound & Group & Calibration Curve \\
\hline
\end{tabular}

\begin{tabular}{|c|c|c|c|c|c|c|c|c|c|}
\hline Peak\# & Ret. Time & Conc. & Area & Height & Similarity Index & Mark & Peak Start & Peak End & Area\% \\
\hline 1 & 9,622 & 48,746 & 5547936 & 266286 & 0,000000 & & 8,843 & 12,885 & 48,746 \\
\hline Total & & 100,000 & 11381381 & 369340 & & & & & 100,000 \\
\hline
\end{tabular}

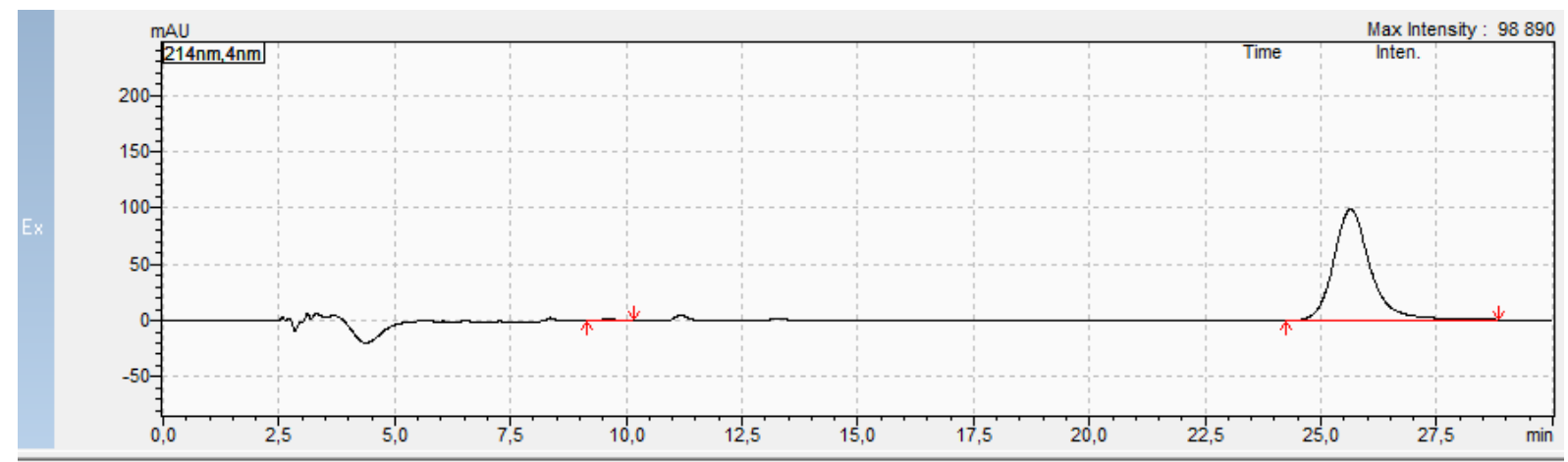

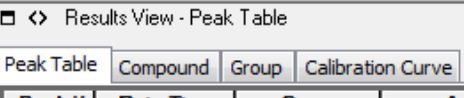

\begin{tabular}{|l|r|r|r|r|r|r|r|r|r|r|}
\hline Peak\# & Ret. Time & \multicolumn{1}{|c|}{ Conc. } & \multicolumn{1}{c|}{ Area } & \multicolumn{1}{c|}{ Height } & Similarity Index & Mark & \multicolumn{1}{c|}{ Peak Start } & Peak End & Area\% \\
\hline 1 & 9,624 & 0,893 & 48983 & 2473 & 0,000000 & & 9,163 & 10,165 & 0,893 \\
\hline 2 & 25,638 & 99,107 & 5436086 & 98624 & 0,000000 & & 24,256 & 28,832 & 99,107 \\
\hline Total & & 100,000 & 5485068 & 101097 & & & & & \\
\hline
\end{tabular}

for 3a: $e e=98 \%$ 
<smiles>O=CC1CCC2(C(=O)N(Cc3ccccc3)c3ccccc32)[C@H]1c1ccccc1</smiles>

Conditions: IA column

mobile phase: heptane / $i$-PrOH $-80: 20$

$\lambda=214 \mathrm{~nm}, V=1.0 \mathrm{ml} / \mathrm{min}, t=25^{\circ} \mathrm{C}$

for ent $-3 \mathrm{a}: t_{\mathrm{R}}=9.6 \mathrm{~min}$ (minor), $t_{\mathrm{R}}=25.7 \mathrm{~min}$ (major).

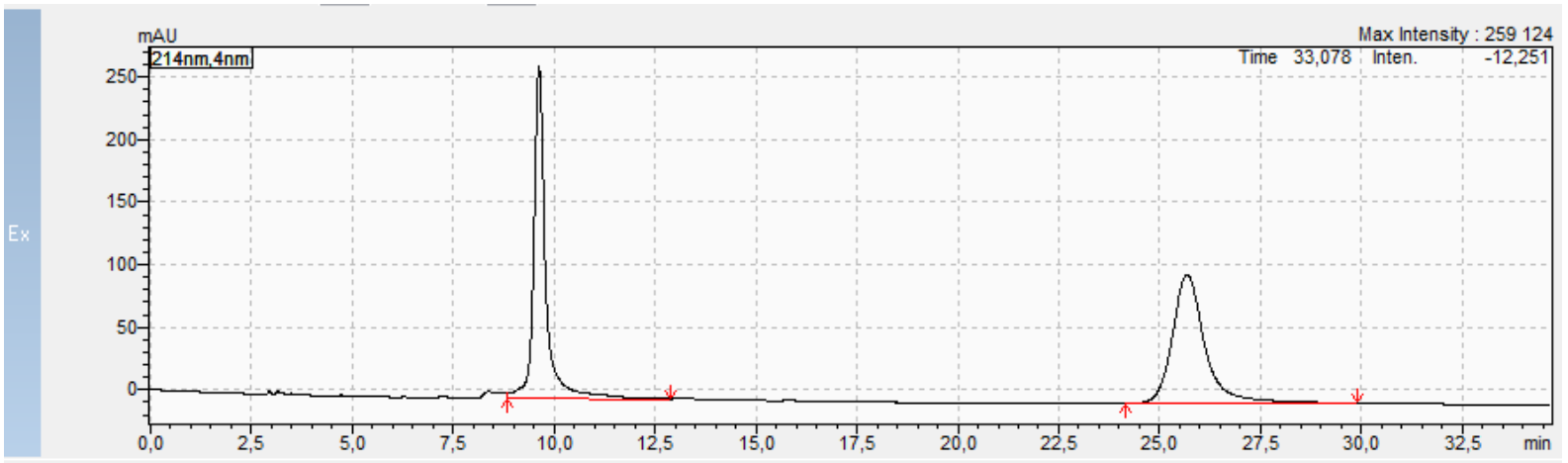

- $〉$ Results View - Peak Table

\begin{tabular}{l|l|l|l|}
\hline Peak Table & Compound & Group & Calibration Curve \\
\hline
\end{tabular}

\begin{tabular}{|l|r|r|r|r|r|r|r|r|r|r|}
\hline Peak\# & Ret. Time & \multicolumn{1}{|c|}{ Conc. } & \multicolumn{1}{c|}{ Area } & Height & Similarity Index & Mark & \multicolumn{1}{|c|}{ Peak Start } & Peak End & Area\% \\
\hline 1 & 9,622 & 48,746 & 5547936 & 266286 & 0,000000 & & 8,843 & 12,885 & 48,746 \\
\hline 2 & 25,682 & 51,254 & 5833445 & 103054 & 0,000000 & & 24,171 & 29,888 \\
\hline Total & & 100,000 & 11381381 & 369340 & & & & 51,254 \\
\hline
\end{tabular}

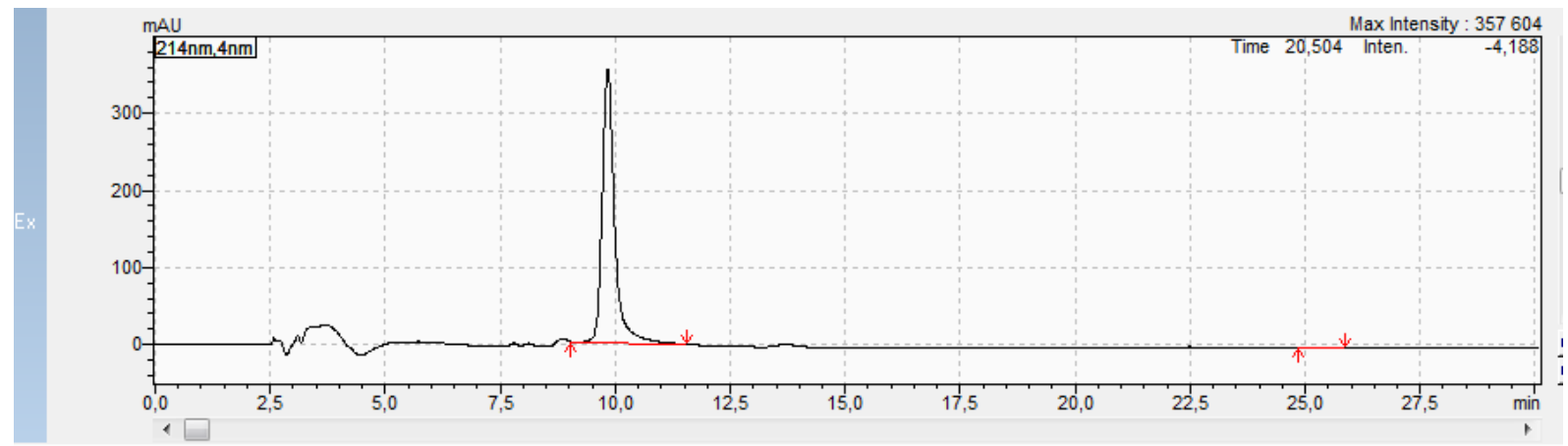

(

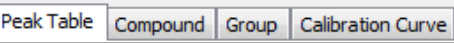

\begin{tabular}{|l|r|r|r|r|r|r|r|r|r|r|}
\hline Peak\# & Ret. Time & \multicolumn{1}{|c|}{ Conc. } & \multicolumn{1}{c|}{ Area } & \multicolumn{1}{c|}{ Height } & Similarity Index & Mark & \multicolumn{1}{c|}{ Peak Start } & Peak End & Area\% \\
\hline 1 & 9,839 & 99,984 & 6620361 & 355274 & 0,000000 & M & 9,035 & 11,573 \\
\hline 2 & 25,460 & 0,016 & 1034 & 65 & 0,000000 & M & 24,843 & 25,856 \\
\hline Total & & 100,000 & 6621395 & 355339 & & & & & \\
\hline
\end{tabular}

for $e n t-3 \mathbf{a}: e e=99 \%$ 


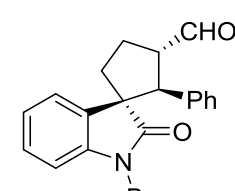

$\mathrm{Bn}$

Conditions: IA column

mobile phase: heptane / $i$-PrOH $-80: 20$

$\lambda=208 \mathrm{~nm}, V=1.0 \mathrm{ml} / \mathrm{min}, t=25^{\circ} \mathrm{C}$

for $4 \mathrm{a}: t_{\mathrm{R}}=11.2 \mathrm{~min}$ (major), $t_{\mathrm{R}}=14.9 \mathrm{~min}$ (minor).

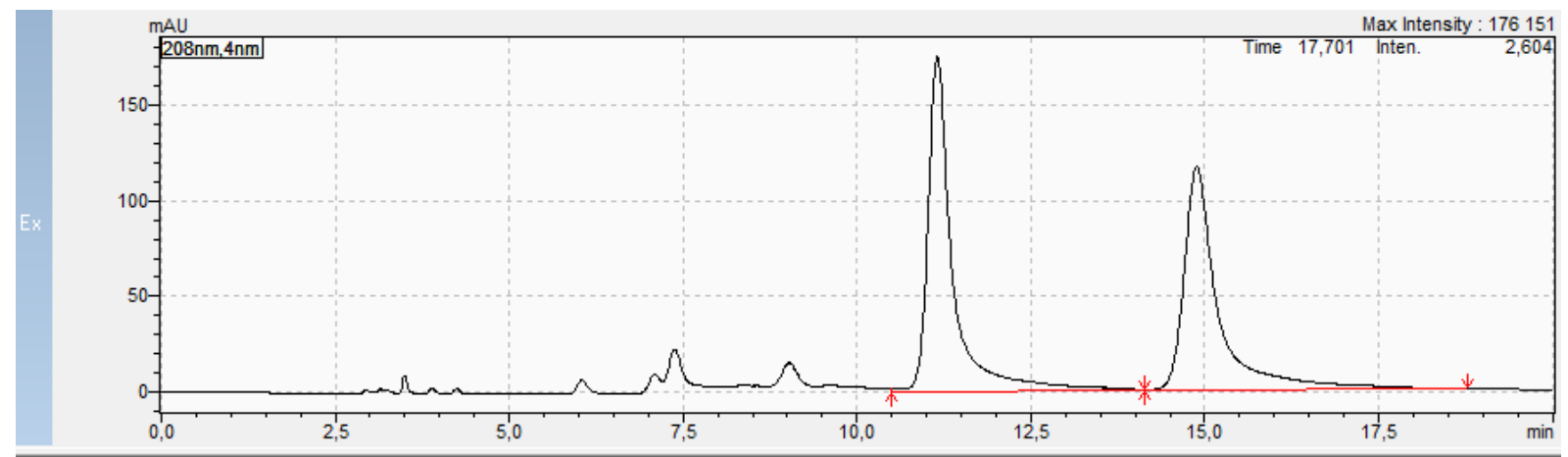

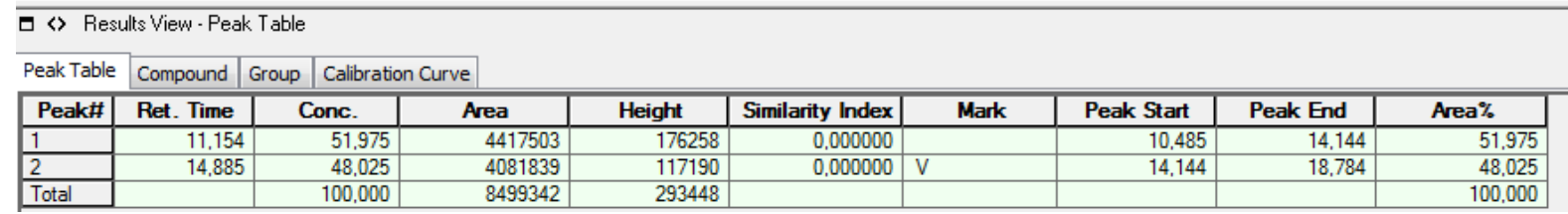

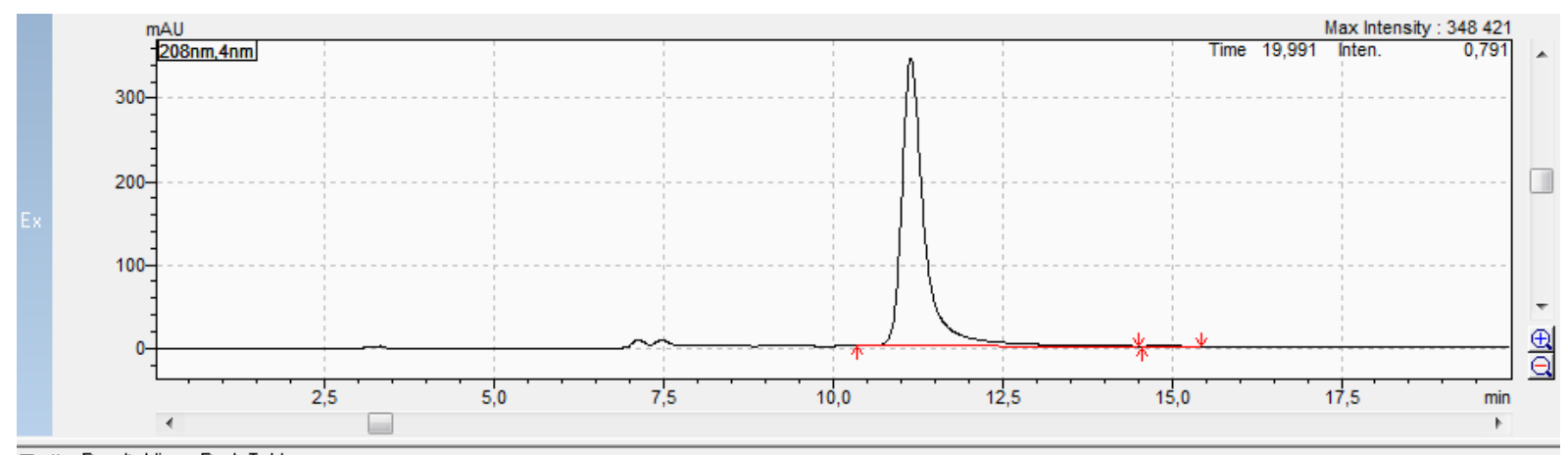

口 〈 Results View - Peak Table

\begin{tabular}{|l|l|l|l|}
\hline Peak Table & Compound & Group & Calibration Curve \\
\hline
\end{tabular}

\begin{tabular}{|c|c|c|c|c|c|c|c|c|c|}
\hline Peak\# & Ret. Time & Conc. & Area & Height & Similarity Index & Mark & Peak Start & Peak End & Area\% \\
\hline 1 & 11,147 & 99,693 & 7968895 & 345798 & 0,000000 & S & 10,357 & 14,496 & 99,693 \\
\hline 2 & 14,880 & 0,307 & 24536 & 1037 & 0,000000 & & 14,560 & 15,424 & 0,307 \\
\hline Total & & 100,000 & 7993431 & 346834 & & & & & 100,000 \\
\hline
\end{tabular}

for 4a: $e e=99 \%$ 
(N)

$\mathrm{Bn}$

Conditions: IA column

mobile phase: heptane / $i$-PrOH $-80: 20$

$\lambda=208 \mathrm{~nm}, V=1.0 \mathrm{ml} / \mathrm{min}, t=25^{\circ} \mathrm{C}$

for ent $-4 \mathrm{a}: t_{\mathrm{R}}=11.2 \mathrm{~min}$ (minor), $t_{\mathrm{R}}=14.9 \mathrm{~min}$ (major).

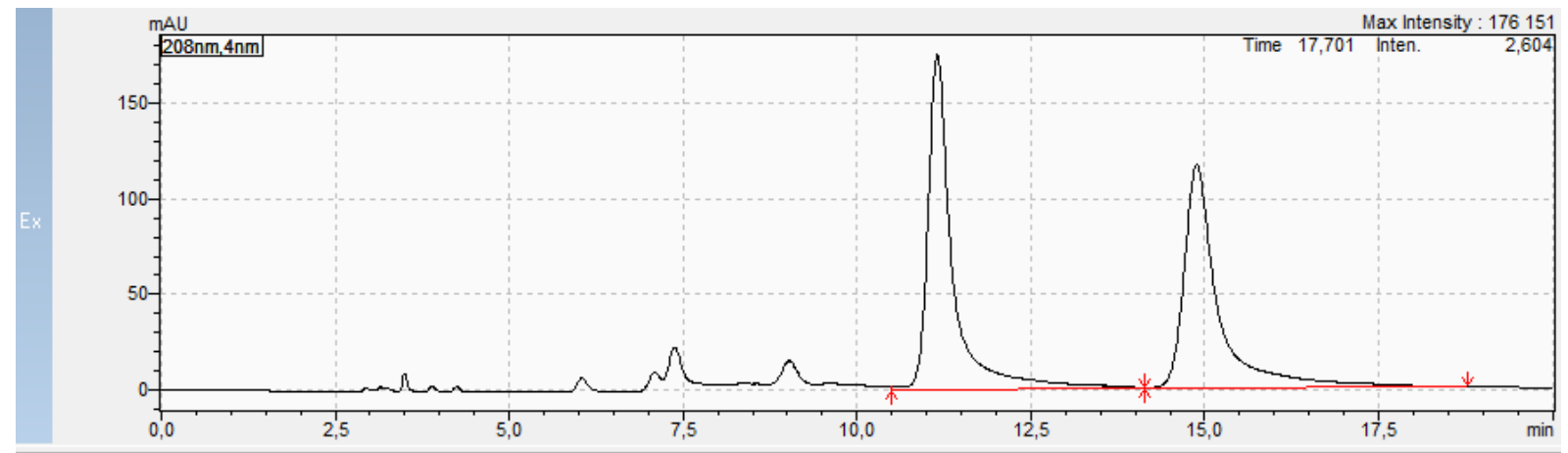

\begin{tabular}{|c|c|c|c|c|c|c|c|c|c|}
\hline \multirow{2}{*}{$\begin{array}{l}\text { Peak Table } \\
\text { Peak\# }\end{array}$} & \multirow{2}{*}{\begin{tabular}{l|} 
Compound \\
Ret. Time
\end{tabular}} & \multicolumn{8}{|c|}{ Calibration Curve } \\
\hline & & Conc. & Area & Height & Similarity Index & Mark & Peak Start & Peak End & Area\% \\
\hline 1 & 11,154 & 51,975 & 4417503 & 176258 & 0,000000 & & 10,485 & 14,144 & 51,975 \\
\hline 2 & 14,885 & 48,025 & 4081839 & 117190 & 0,000000 & V & 14,144 & 18,784 & 48,025 \\
\hline Total & & 100,000 & 8499342 & 293448 & & & & & 100,000 \\
\hline
\end{tabular}

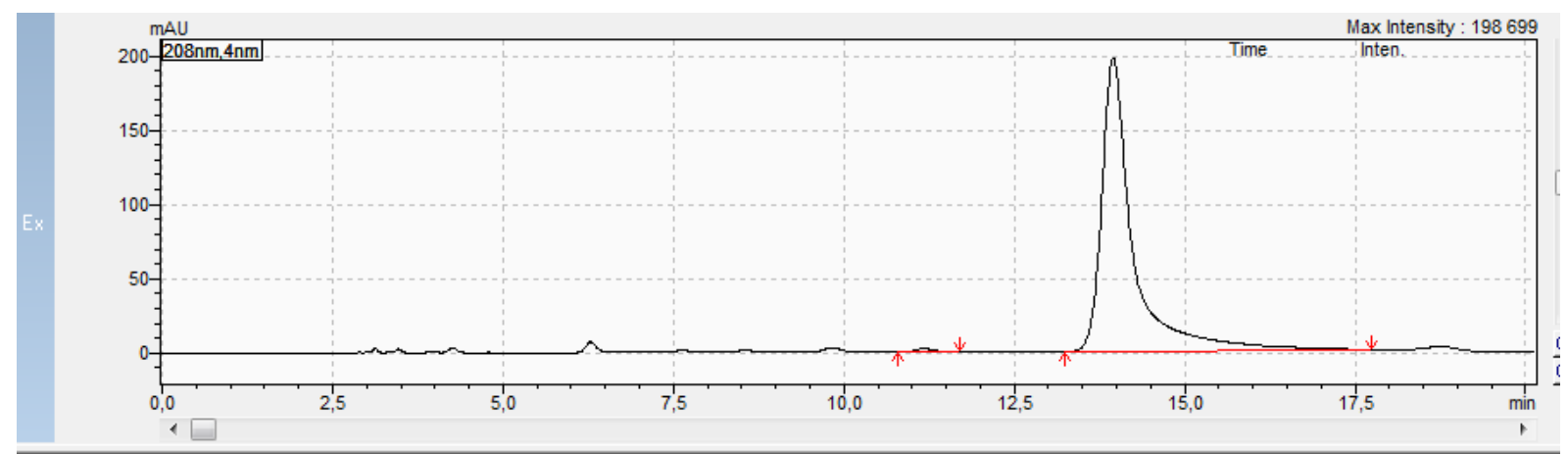

口〈 Results View - Peak Table

\begin{tabular}{l|l|l|l|}
\hline Peak Table & Compound & Group & Calibration Curve \\
\hline
\end{tabular}

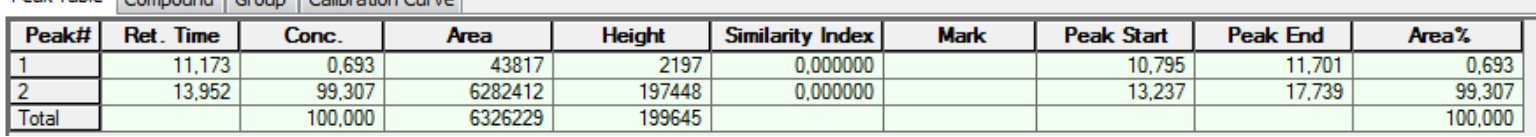

for $e n t-\mathbf{4 a}: e e=99 \%$ 


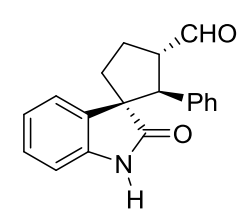

Conditions: IA column

mobile phase: heptane / $i$-PrOH $-90: 10$

$\lambda=254 \mathrm{~nm}, V=1.0 \mathrm{ml} / \mathrm{min}, t=25^{\circ} \mathrm{C}$

for 3d: $t_{\mathrm{R}}=12.2 \mathrm{~min}$ (minor), $t_{\mathrm{R}}=13.8 \mathrm{~min}$ (major).
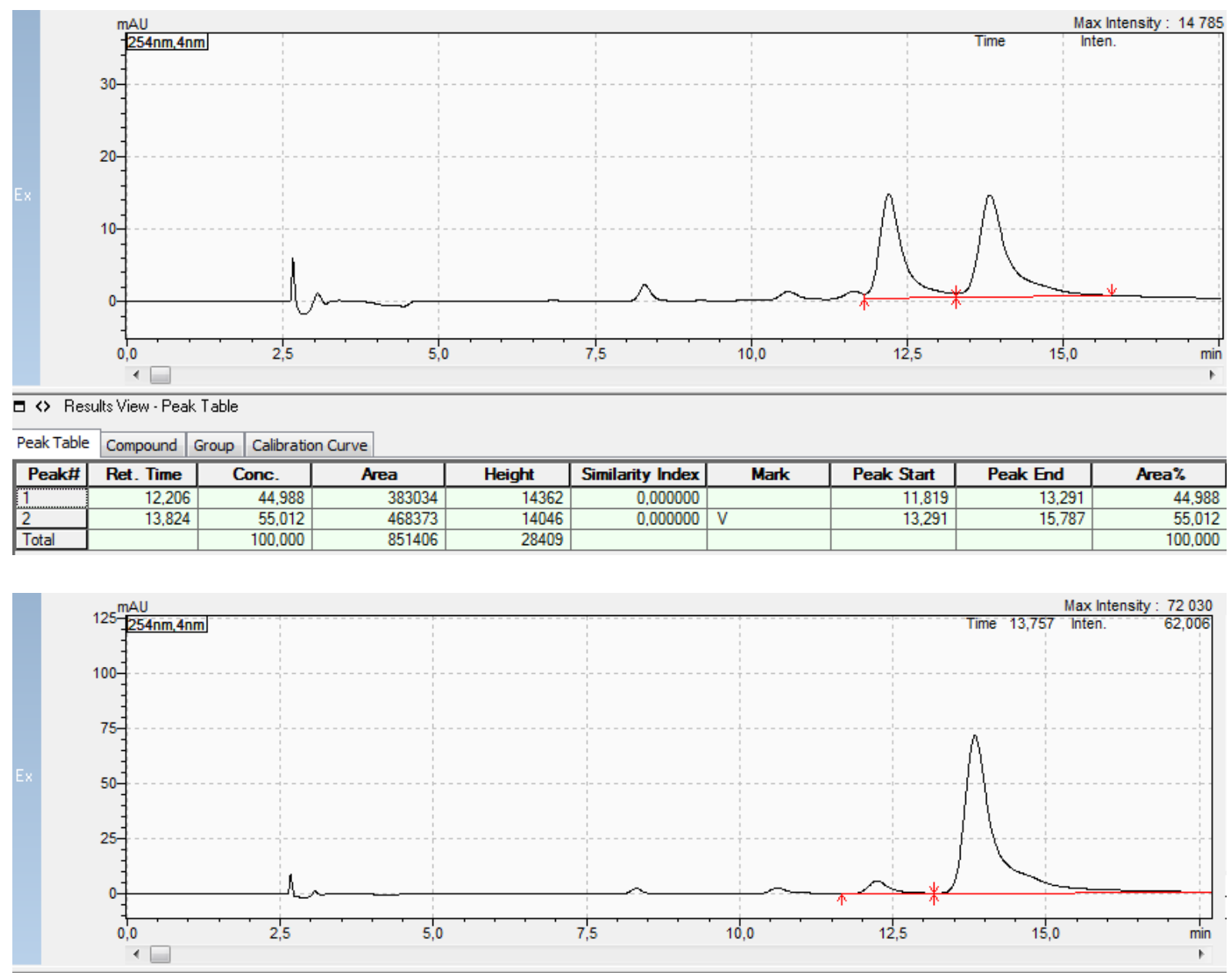

口

\begin{tabular}{|l|l|l|l|}
\hline Peak Table & Compound & Group & Calibration Curve \\
\hline
\end{tabular}

\begin{tabular}{|c|c|c|c|c|c|c|c|c|c|}
\hline Peak\# & Ret. Time & Conc. & Area & Height & Similarity Index & Mark & Peak Start & Peak End & Area\% \\
\hline $1 \ldots$ & 12,244 & 5,105 & 130573 & 5568 & 0,000000 & $M$ & 11,669 & 13,173 & 5.105 \\
\hline 2 & 13,840 & 94,895 & 2427325 & 71522 & 0,000000 & M & 13,173 & 17,813 & 94,895 \\
\hline Total & & 100,000 & 2557898 & 77090 & & & & & 100,000 \\
\hline
\end{tabular}

for 3d: $e e=90 \%$ 


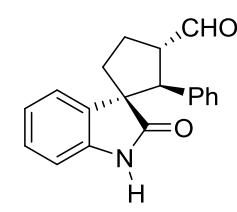

Conditions: IA column

mobile phase: heptane / $i$-PrOH $-90: 10$

$\lambda=254 \mathrm{~nm}, V=1.0 \mathrm{ml} / \mathrm{min}, t=25{ }^{\circ} \mathrm{C}$

for $4 \mathbf{d}$ : $t_{\mathrm{R}}=10.6 \mathrm{~min}$ (major), $t_{\mathrm{R}}=11.6 \mathrm{~min}$ (minor).
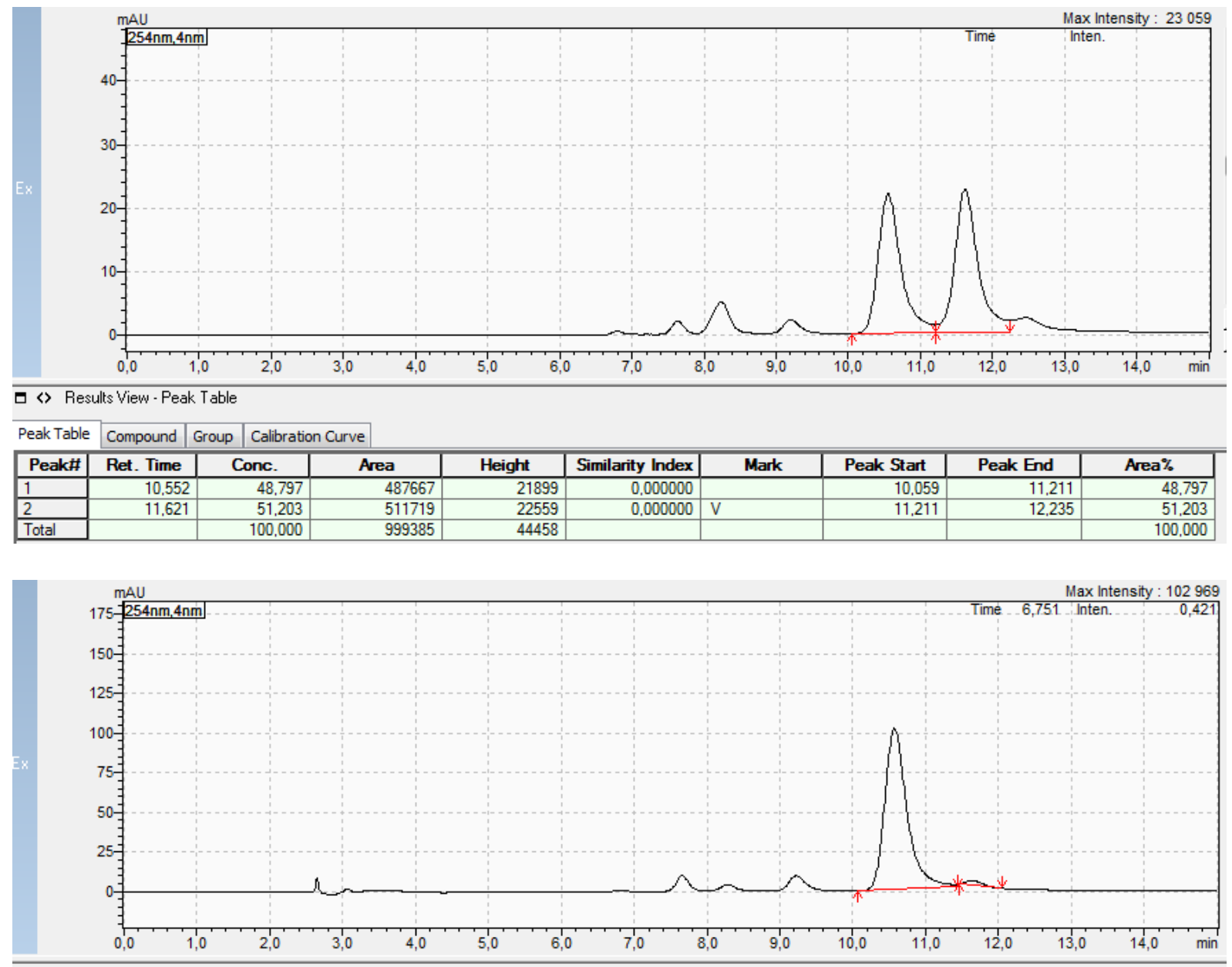

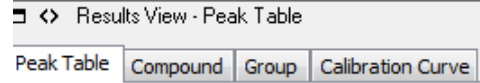

\begin{tabular}{|l|r|r|r|r|r|r|r|r|r|}
\hline Peak\# & Ret. Time & \multicolumn{1}{|c|}{ Conc. } & \multicolumn{1}{|c|}{ Area } & \multicolumn{1}{c|}{ Height } & Similarity Index & Mark & Peak Start & \multicolumn{1}{c|}{ Peak End } & \multicolumn{1}{c|}{ Area\% } \\
\hline 1 & 10,572 & 97,933 & 2158449 & 101289 & 0,000000 & M & 10,069 & 11,445 & 97,933 \\
\hline 2 & 11,641 & 2,067 & 45550 & 2948 & 0,000000 & M & 11.456 & 12,053 \\
\hline Total & & 100,000 & 2203999 & 104237 & & & 2,067 \\
\hline
\end{tabular}

for 4d: $e e=96 \%$ 
<smiles>Cc1ccc(N(Cc2ccccc2)C(=O)c2ccccc2)c(C=O)c1</smiles>

Conditions: IA column

mobile phase: heptane / $i$-PrOH $-80: 20$

$\lambda=217 \mathrm{~nm}, V=1.0 \mathrm{ml} / \mathrm{min}, t=25^{\circ} \mathrm{C}$

for $3 \mathrm{e}: t_{\mathrm{R}}=8.2 \min$ (minor), $t_{\mathrm{R}}=18.2 \mathrm{~min}$ (major).

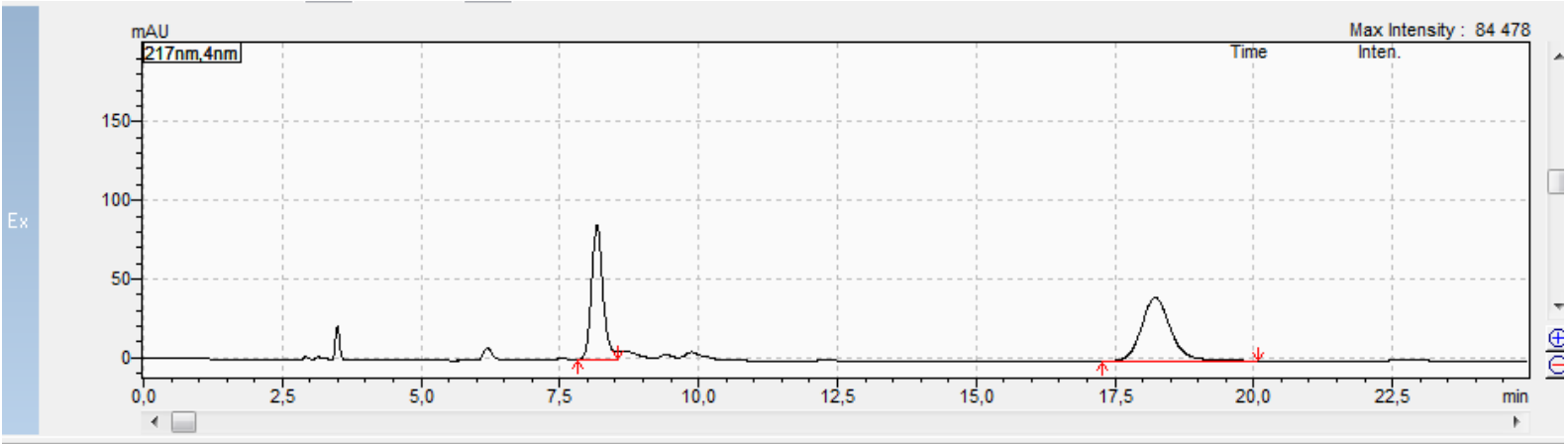

口〈R Results View - Peak Table

\begin{tabular}{|l|l|l|l|}
\hline Peak Table Compound & Group & Calibration Curve \\
\hline
\end{tabular}

\begin{tabular}{|c|c|c|c|c|c|c|c|c|c|}
\hline Peak\# & Ret. Time & Conc. & Area & Height & Similarity Index & Mark & Peak Start & Peak End & Area\% \\
\hline 1 & 8,170 & 45,826 & 1248266 & 85917 & 0,000000 & $\bar{M}$ & 7,819 & 8.544 & 45,826 \\
\hline 2 & 18,225 & 54,174 & 1475648 & 40408 & 0,000000 & & 17,259 & 20,075 & 54,174 \\
\hline Total & & 100,000 & 2723914 & 126325 & & & & & 100,000 \\
\hline
\end{tabular}

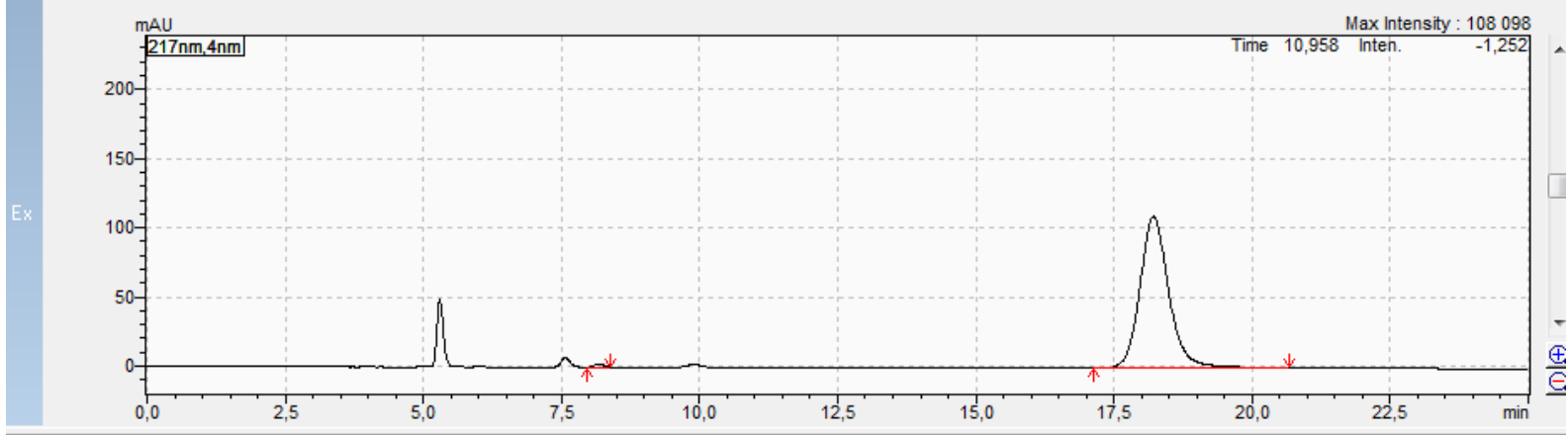

口〈 Results View - Peak Table

\begin{tabular}{|l|l|l|l|} 
Peak Table & Compound & Group & Calibration Curve \\
\hline
\end{tabular}

\begin{tabular}{|l|r|r|r|r|r|r|r|r|r|}
\hline Peak\# & Ret. Time & \multicolumn{1}{|c|}{ Conc. } & \multicolumn{1}{c|}{ Area } & Height & Similarity Index & Mark & Peak Start & Peak End & \multicolumn{1}{|c|}{ Area\% } \\
\hline 1 & 8,180 & 0,671 & 26964 & 2237 & 0,000000 & M & 7,957 & 0,384 & 0,671 \\
\hline 2 & 18,218 & 99,329 & 3992280 & 109472 & 0,000000 & & 17,141 & 20,661 & \\
\hline Total & & 100,000 & 4019244 & 111709 & & & & 99,329 \\
\hline
\end{tabular}

for 3e: $e e=99 \%$ 


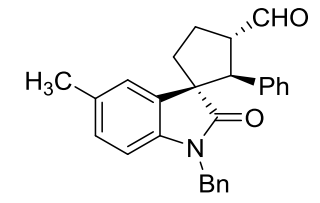

Conditions: IA column

mobile phase: heptane / $i$-PrOH $-80: 20$

$\lambda=208 \mathrm{~nm}, V=1.0 \mathrm{ml} / \mathrm{min}, t=25^{\circ} \mathrm{C}$

for $4 \mathrm{e}: t_{\mathrm{R}}=9.0 \mathrm{~min}$ (major), $t_{\mathrm{R}}=12.3 \mathrm{~min}$ (minor).
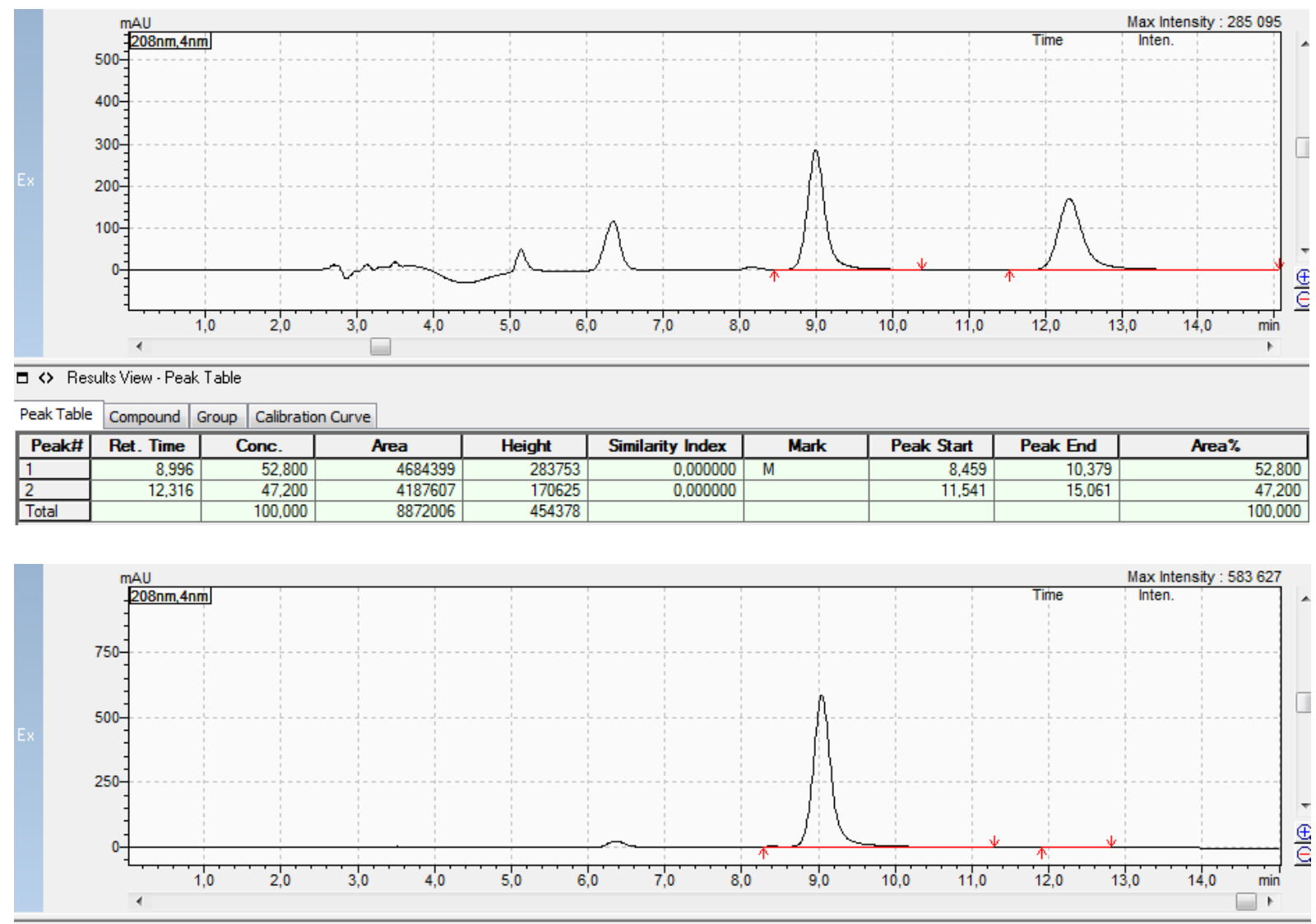

口〈 Results View - Peak Table

\begin{tabular}{|l|l|l|l} 
Peak Table & Compound & Group & Calibration Curve \\
\hline
\end{tabular}

\begin{tabular}{|c|c|c|c|c|c|c|c|c|c|}
\hline Peak\# & Ret. Time & Conc. & Area & Height & Similarity Index & Mark & Peak Start & Peak End & Area\% \\
\hline 1 & 9.043 & 99,523 & 9658402 & 581824 & 0.000000 & $\bar{M}$ & 8.288 & 11.285 & 99.523 \\
\hline 2 & 12,342 & 0,477 & 46269 & 2247 & 0,000000 & & 11,915 & 12,811 & 0.477 \\
\hline Total & & 100,000 & 9704671 & 584071 & & & & & 100,000 \\
\hline
\end{tabular}

for $4 \mathrm{e}: e e=99 \%$ 


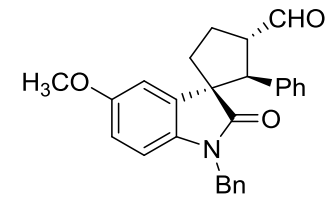

Conditions: IA column

mobile phase: heptane / $i$-PrOH $-80: 20$

$\lambda=208 \mathrm{~nm}, V=1.0 \mathrm{ml} / \mathrm{min}, t=25^{\circ} \mathrm{C}$

for 3f: $t_{\mathrm{R}}=15.2 \mathrm{~min}$ (minor), $t_{\mathrm{R}}=41.6 \mathrm{~min}$ (major).

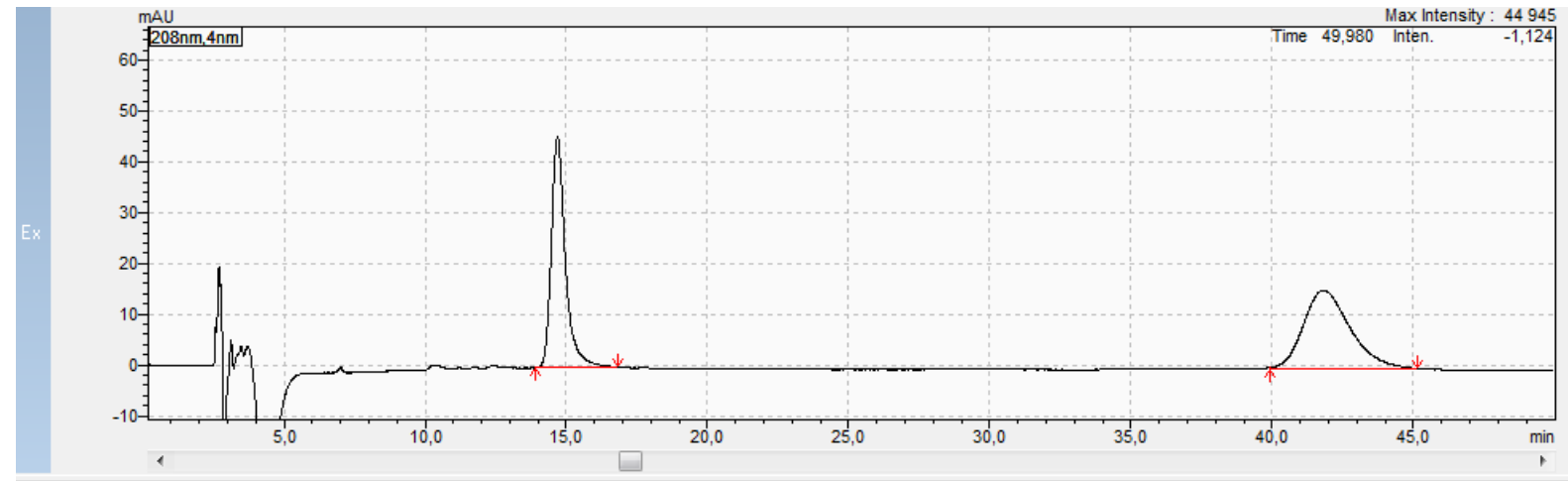

口〈 Results View - Peak Table

\begin{tabular}{|l|l|l|l|}
\hline Peak Table & Compound & Group & Calibration Curve \\
\hline
\end{tabular}

\begin{tabular}{|c|c|c|c|c|c|c|c|c|c|}
\hline Peak\# & Ret. Time & Conc. & Area & Height & Similarity Index & Mark & Peak Start & Peak End & Area\% \\
\hline $1 \cdots \cdots$ & 14,688 & 47,216 & 1580047 & 45509 & 0,000000 & S & 13,888 & 16,853 & 47,216 \\
\hline 2 & 41,832 & 52,784 & 1766390 & 15455 & 0,000000 & S & 39,904 & 45,163 & 52,784 \\
\hline Total & & 100,000 & 3346436 & 60963 & & & & & 100,000 \\
\hline
\end{tabular}

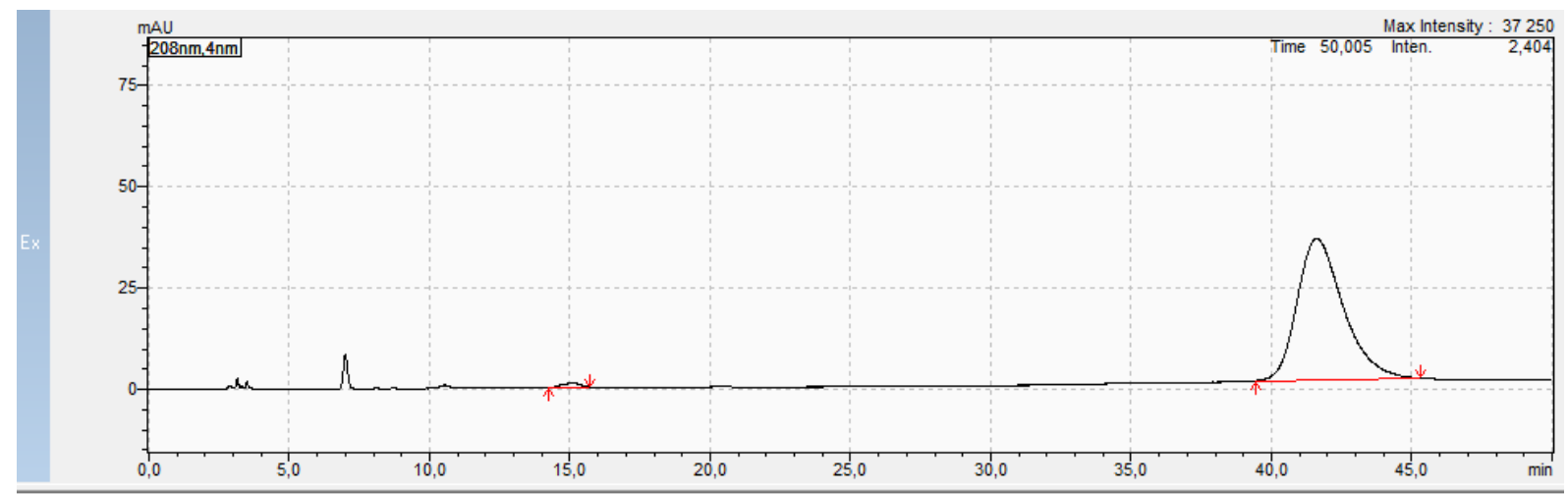

Peak Table Compound Group Calibration Curve

\begin{tabular}{|c|c|c|c|c|c|c|c|c|c|}
\hline Peak\# & Ret. Time & Conc. & Area & Height & Similarity Index & Mark & Peak Start & Peak End & Area\% \\
\hline 1 & 15,110 & 1,220 & 49472 & 1117 & 0,000000 & & 14,229 & 15,701 & 1,220 \\
\hline 2 & 41,609 & 98.780 & 4006466 & 34944 & 0,000000 & SV & 39,413 & 45,291 & 98,780 \\
\hline Total & & 100,000 & 4055938 & 36062 & & & & & 100,000 \\
\hline
\end{tabular}

for 3f: $e e=98 \%$ 


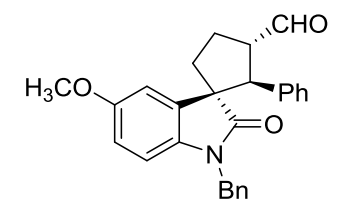

Conditions: IA column

mobile phase: heptane / $i$-PrOH $-80: 20$

$\lambda=208 \mathrm{~nm}, V=1.0 \mathrm{ml} / \mathrm{min}, t=25^{\circ} \mathrm{C}$

for 4f: $t_{\mathrm{R}}=15.2 \min$ (major), $t_{\mathrm{R}}=19.0 \mathrm{~min}$ (minor).

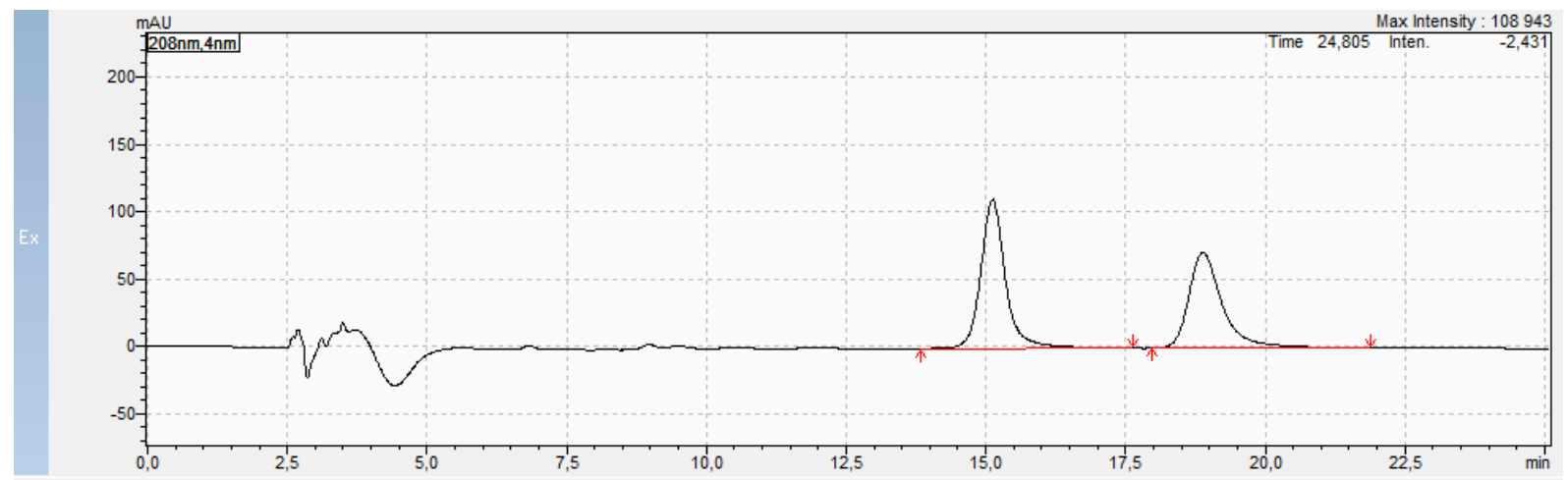

\begin{tabular}{|c|c|c|c|c|c|c|c|c|c|}
\hline Peak Table & Compound & Calibratic & & & & & & & \\
\hline Peak\# & Ret. Time & Conc. & Area & Height & Similarity Index & Mark & Peak Start & Peak End & Area\% \\
\hline 1 & 15,122 & 53,589 & 3455175 & 110675 & 0,000000 & $\mathrm{~S}$ & 13,824 & 17,643 & 53,589 \\
\hline 2 & 18,883 & 46,411 & 2992384 & 71175 & 0,000000 & S & 17,984 & 21,899 & 46,411 \\
\hline Total & & 100,000 & 6447560 & 181850 & & & & & 100,000 \\
\hline
\end{tabular}

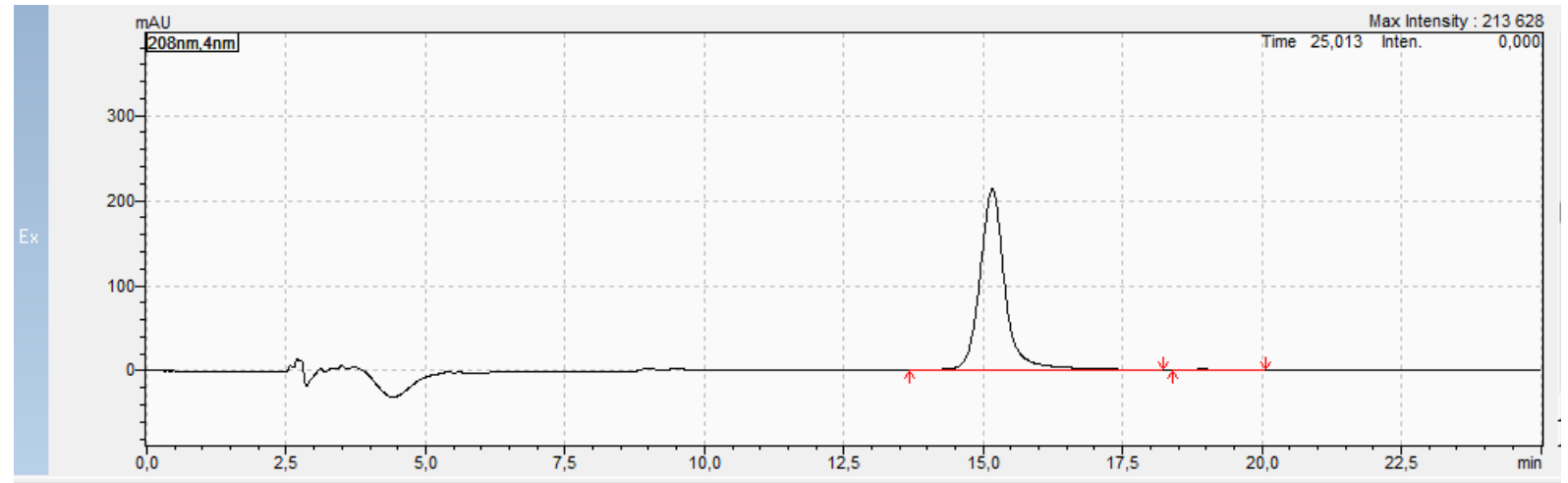

- 〈 Results View - Peok Table

\begin{tabular}{|l|l|l|l|}
\hline Peak Table Compound & Group & Calibration Curve \\
\hline
\end{tabular}

\begin{tabular}{|c|c|c|c|c|c|c|c|c|c|}
\hline Peak\# & Ret. Time & Conc. & Area & Height & Similarity Index & Mark & Peak Start & Peak End & Area\% \\
\hline 1 & 15,166 & 99,539 & 6670105 & 212689 & 0,000000 & $M$ & 13,685 & 18,229 & 99,539 \\
\hline 2 & 18,946 & 0,461 & 30918 & 918 & 0,000000 & M & 18,400 & 20,064 & 0.461 \\
\hline Total & & 100,000 & 6701023 & 213606 & & & & & 100,000 \\
\hline
\end{tabular}

for 4f: $e e=99 \%$ 


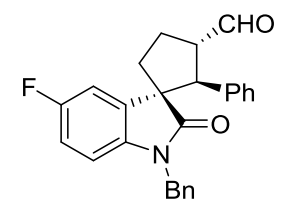

Conditions: IA column

mobile phase: heptane / $i$-PrOH $-80: 20$

$\lambda=206 \mathrm{~nm}, V=1.0 \mathrm{ml} / \mathrm{min}, t=25^{\circ} \mathrm{C}$

for 3g: $t_{\mathrm{R}}=9.1 \mathrm{~min}$ (minor), $t_{\mathrm{R}}=21.8 \mathrm{~min}$ (major).

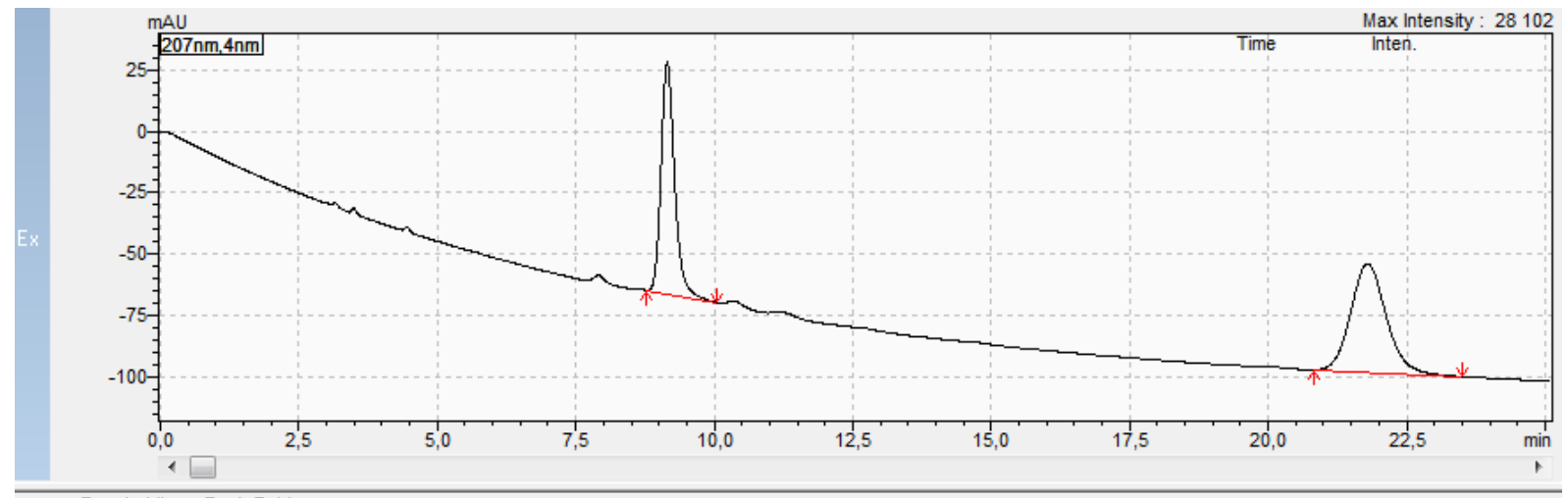

口〈Results View - Peak Table

\begin{tabular}{|l|l|l|l|}
\hline Peak Table & Compound & Group & Calibration Curve \\
\hline
\end{tabular}

\begin{tabular}{|l|r|r|r|r|r|r|r|r|r|}
\hline Peak\# & Ret. Time & \multicolumn{1}{|c|}{ Conc. } & \multicolumn{1}{c|}{ Area } & \multicolumn{1}{c|}{ Height } & Similarity Index & Mark & \multicolumn{1}{c|}{ Peak Start } & Peak End & \multicolumn{1}{c|}{ Area\% } \\
\hline 1 & 9,149 & 44,774 & 1606492 & 94157 & 0,000000 & & 8,768 & 10,037 & 44,774 \\
\hline 2 & 21,797 & 55,226 & 1981489 & 44243 & 0,000000 & & 20,821 & 23,488 & 55,226 \\
\hline Total & & 100,000 & 3587981 & 138400 & & & & & \\
\hline
\end{tabular}

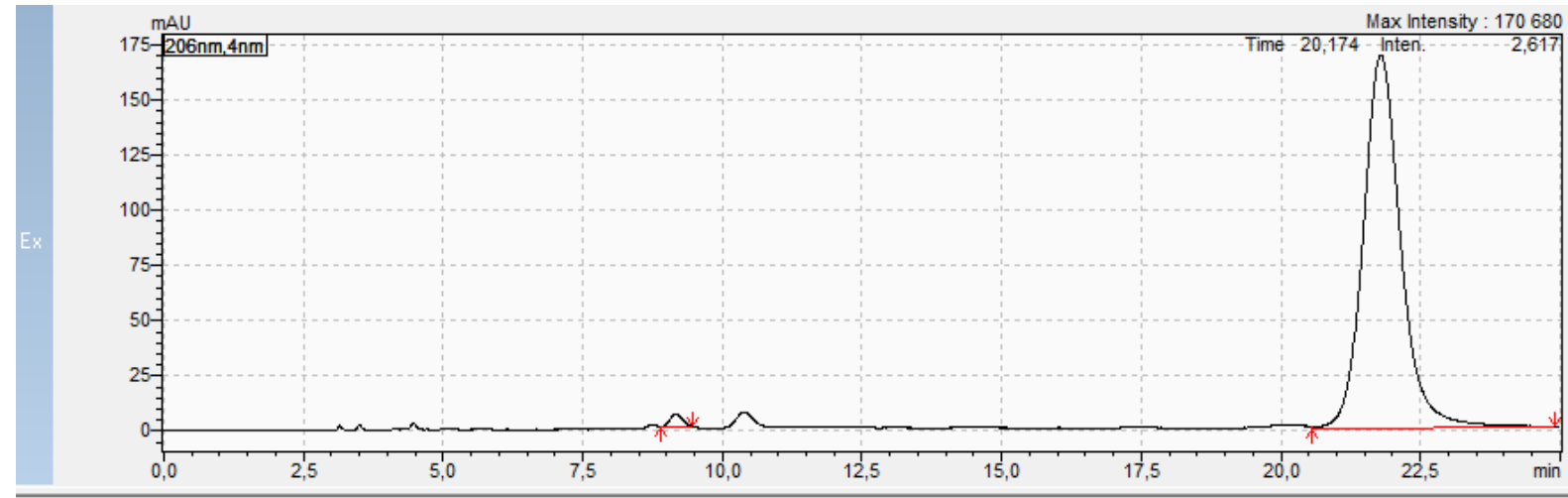

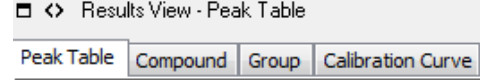

\begin{tabular}{|l|r|r|r|r|r|r|r|r|r|r|}
\hline Peak\# & Ret. Time & \multicolumn{1}{|c|}{ Conc. } & \multicolumn{1}{c|}{ Area } & Height & Similarity Index & Mark & \multicolumn{1}{c|}{ Peak Start } & Peak End & \multicolumn{1}{c|}{ Area\% } \\
\hline 1 & 9,167 & 1,172 & 93551 & 5996 & 0,000000 & M & 8,896 & 9,483 \\
\hline 2 & 21,790 & 98,828 & 7888907 & 169155 & 0,000000 & S & 20,565 & 24,917 \\
\hline Total & & 100,000 & 7982458 & 175151 & & & 98,828 \\
\hline
\end{tabular}

for 3g: $e e=98 \%$ 
<smiles>C=C[C@H]1CC[C@@]2(C(=O)N(Cc3ccccc3)c3ccc(F)cc32)C1c1ccccc1</smiles>

Conditions: IA column

mobile phase: heptane / $i$-PrOH $-80: 20$

$\lambda=207 \mathrm{~nm}, V=1.0 \mathrm{ml} / \mathrm{min}, t=25^{\circ} \mathrm{C}$

for $4 \mathrm{~g}: t_{\mathrm{R}}=10.3 \min$ (minor), $t_{\mathrm{R}}=13.8 \mathrm{~min}$ (major).

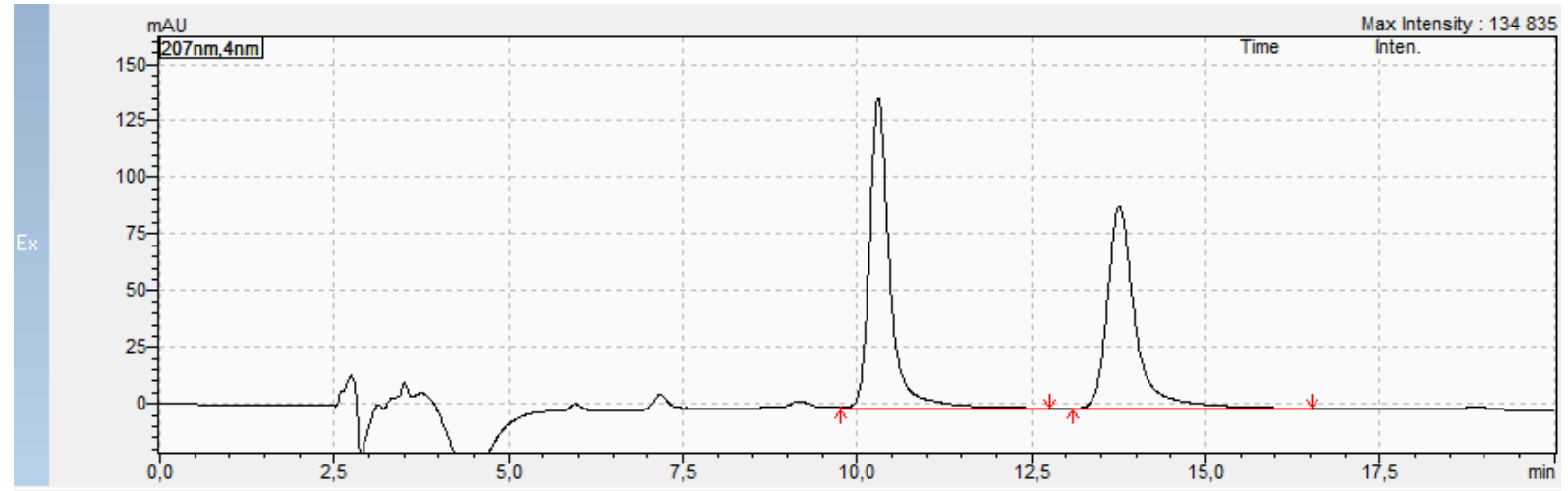

口〈 Results View - Peak Table

\begin{tabular}{|l|l|l|l|}
\hline Peak Table & Compound & Group & Calibration Curve \\
\hline
\end{tabular}

\begin{tabular}{|c|c|c|c|c|c|c|c|c|c|}
\hline Peak\# & Ret. Time & Conc. & Area & Height & Similarity Index & Mark & Peak Start & Peak End & Area\% \\
\hline $17 m m$ & 10,305 & 52,758 & 2797059 & 136858 & 0,000000 & S & 9.760 & 12,768 & 52,758 \\
\hline 2 & 13,763 & 47,242 & 2504596 & 89305 & 0,000000 & S & 13,099 & 16,523 & 47,242 \\
\hline Total & & 100,000 & 5301654 & 226163 & & & & & 100,000 \\
\hline
\end{tabular}

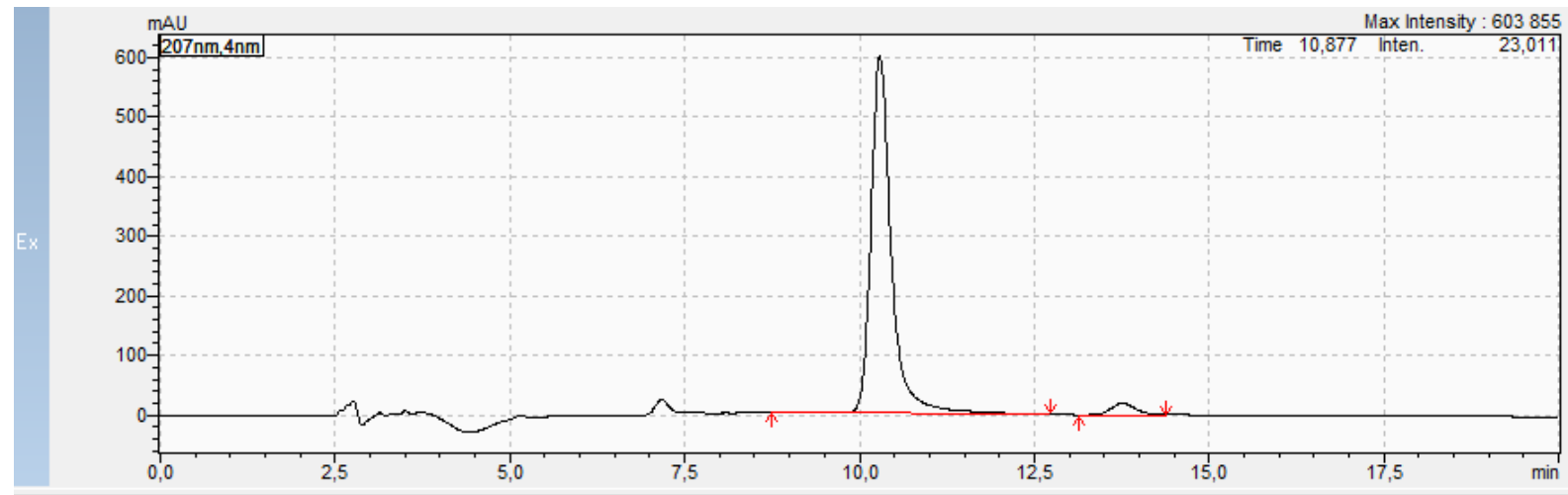

口 〈 Results View - Peak Table

\begin{tabular}{l|l|l|l|}
\hline Peak Table & Compound & Group & Calibration Curve \\
\hline
\end{tabular}

\begin{tabular}{|l|r|r|r|r|r|r|r|r|r|r|}
\hline Peak\# & Ret. Time & \multicolumn{1}{|c|}{ Conc. } & \multicolumn{1}{c|}{ Area } & \multicolumn{1}{c|}{ Height } & Similarity Index & Mark & Peak Start & \multicolumn{1}{c|}{ Peak End } & \multicolumn{1}{c|}{ Area\% } \\
\hline 1 & 10,292 & 95,969 & 12175939 & 600554 & 0,000000 & M & 8,747 & 12,736 & 95,969 \\
\hline 2 & 13,764 & 4,031 & 511463 & 19814 & 0,000000 & & 13,131 & 14,379 & 4,031 \\
\hline Total & & 100,000 & 12687402 & 620368 & & & & & \\
\hline
\end{tabular}

for 4g: $e e=90 \%$ 
<smiles>O=C[C@H]1CC[C@@]2(C(=O)N(Cc3ccccc3)c3ccc(Cl)cc32)[C@H]1c1ccccc1</smiles>

Conditions: IA column

mobile phase: heptane / $i$-PrOH $-80: 20$

$\lambda=207 \mathrm{~nm}, V=1.0 \mathrm{ml} / \mathrm{min}, t=25^{\circ} \mathrm{C}$

for $3 \mathbf{h}: t_{\mathrm{R}}=8.6 \mathrm{~min}$ (minor), $t_{\mathrm{R}}=17.3 \mathrm{~min}$ (major).

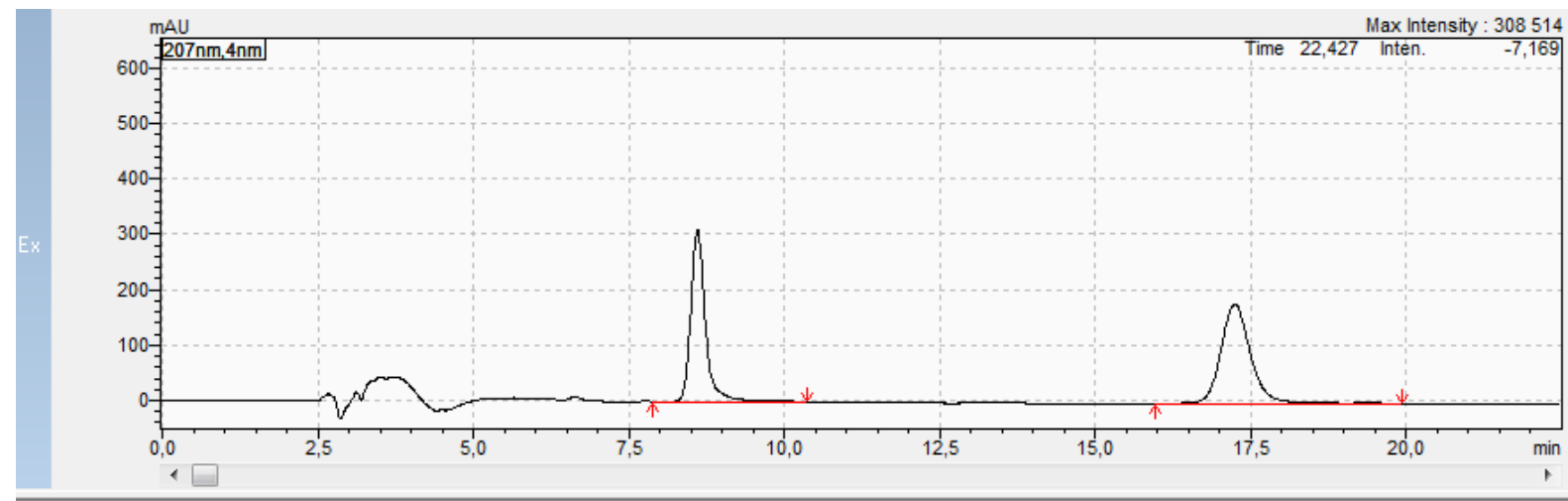

口〈 Results View - Peak Table

\begin{tabular}{l|l|l|l|}
\hline Peak Table & Compound & Group & Calibration Curve \\
\hline
\end{tabular}

\begin{tabular}{|c|c|c|c|c|c|c|c|c|c|}
\hline Peak\# & Ret. Time & Conc. & Area & Height & Similarity Index & Mark & Peak Start & Peak End & Area\% \\
\hline 1 & 8,608 & 45,501 & 5068939 & 310663 & 0,000000 & $\bar{M}$ & 7.883 & 10,368 & 45,501 \\
\hline 2 & 17,250 & 54,499 & 6071259 & 179948 & 0,000000 & M & 15.957 & 19,936 & 54,499 \\
\hline Total & & 100,000 & 11140198 & 490612 & & & & & 100,000 \\
\hline
\end{tabular}

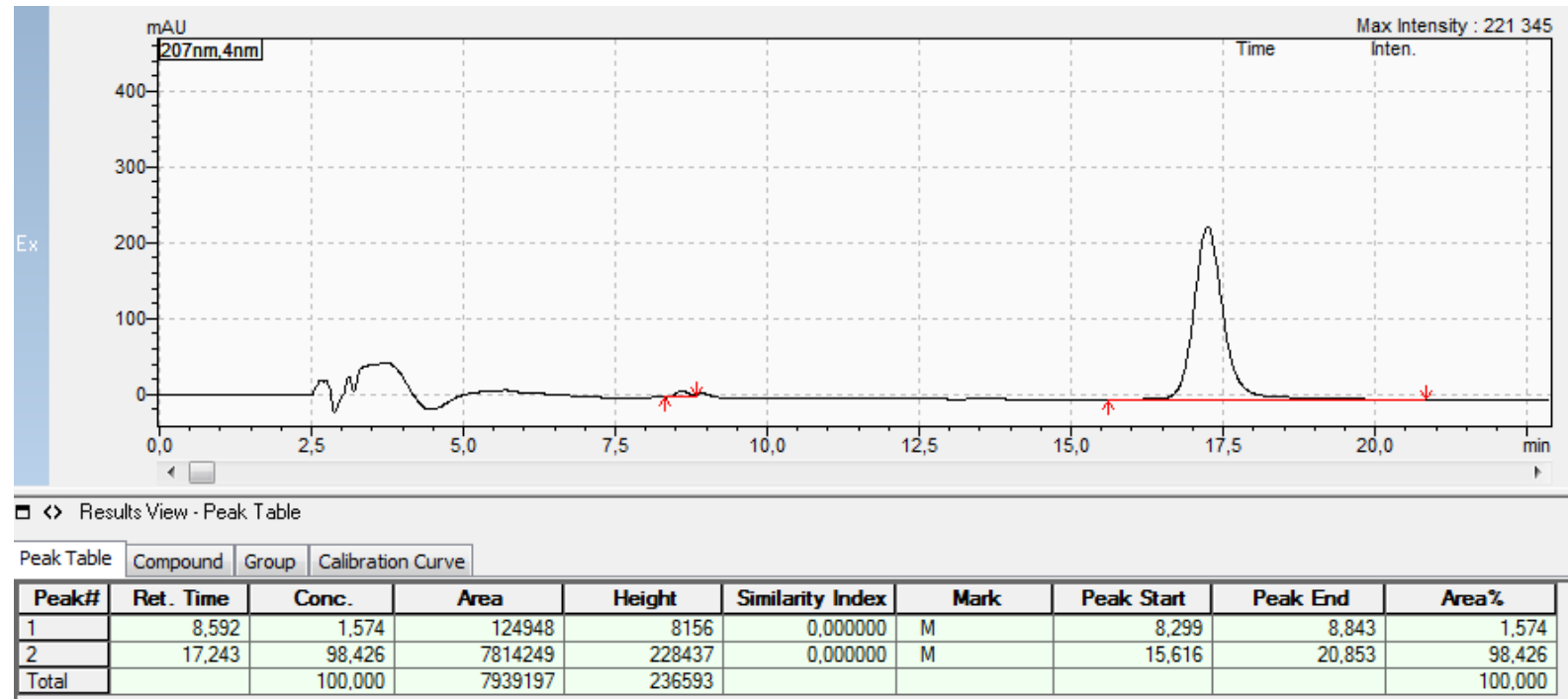

for $3 \mathbf{h}: e e=96 \%$ 
<smiles>O=C[C@@H]1CCC2(C(=O)N(Cc3ccccc3)c3ccc(Cl)cc32)[C@H]1c1ccccc1</smiles>

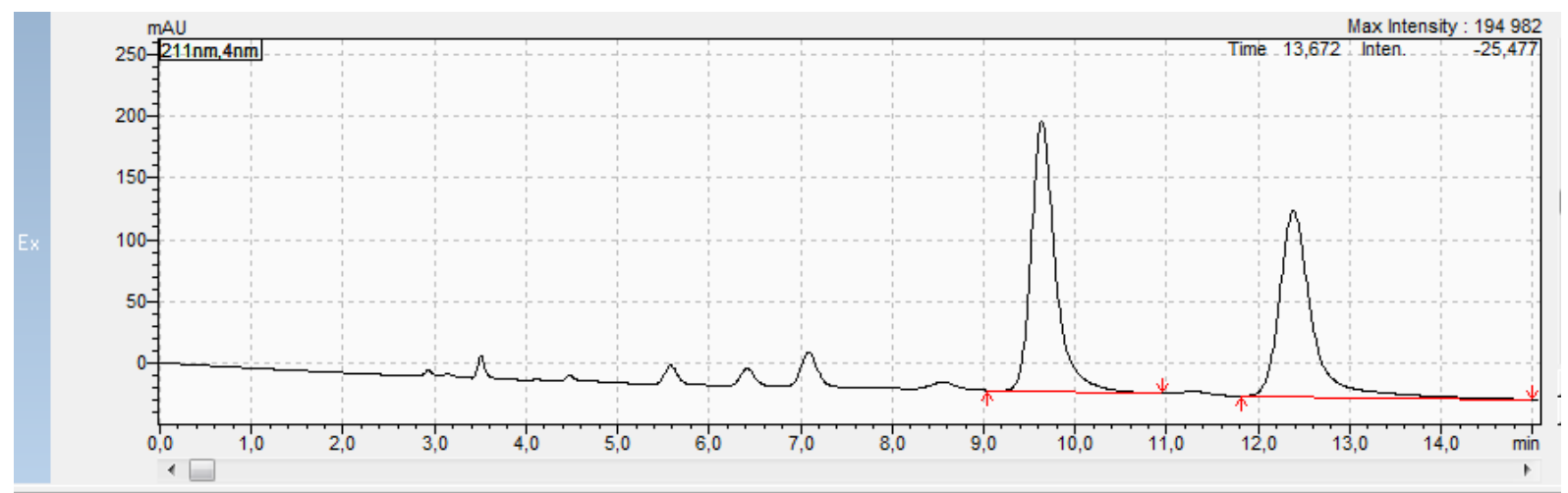

口〈 Results View - Peak Table

\begin{tabular}{|l|l|l|l|}
\hline Peak Table & Compound & Group & Calibration Curve \\
\hline
\end{tabular}

\begin{tabular}{|l|r|r|r|r|r|r|r|r|r|r|}
\hline Peak\# & Ret. Time & \multicolumn{1}{|c|}{ Conc. } & \multicolumn{1}{c|}{ Area } & Height & Similarity Index & Mark & Peak Start & Peak End & Area\% \\
\hline 1 & 9,636 & 51,973 & 4008222 & 217514 & 0,000000 & M & 9,035 & 10,944 \\
\hline 2 & 12,384 & 48,027 & 3703858 & 150181 & 0,000000 & & 51,973 \\
\hline Total & & 100,000 & 7712080 & 367695 & & & & 11,808 & 14,987 & \\
\hline
\end{tabular}

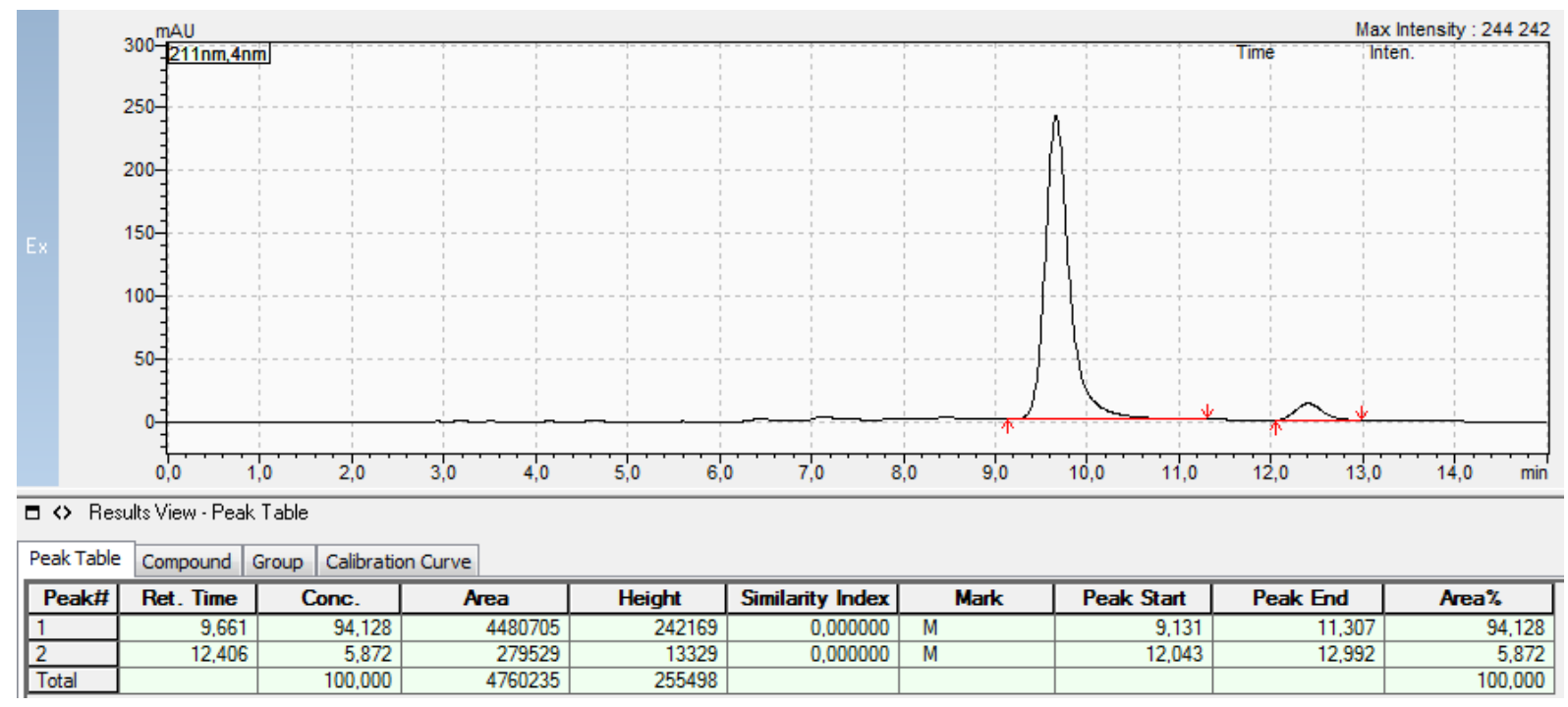

for $4 \mathrm{~h}: e e=88 \%$
Conditions: IA column

mobile phase: heptane / $i$-PrOH $-80: 20$

$\lambda=211 \mathrm{~nm}, V=1.0 \mathrm{ml} / \mathrm{min}, t=25^{\circ} \mathrm{C}$

for $4 \mathrm{~h}: t_{\mathrm{R}}=9.6 \mathrm{~min}$ (major), $t_{\mathrm{R}}=12.4 \mathrm{~min}$ (minor). 


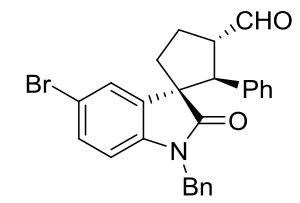

Conditions: IA column

mobile phase: heptane / $i$-PrOH $-80: 20$

$\lambda=212 \mathrm{~nm}, V=1.0 \mathrm{ml} / \mathrm{min}, t=25^{\circ} \mathrm{C}$

for 3i: $t_{\mathrm{R}}=8.7$ min (minor), $t_{\mathrm{R}}=17.8 \mathrm{~min}$ (major).

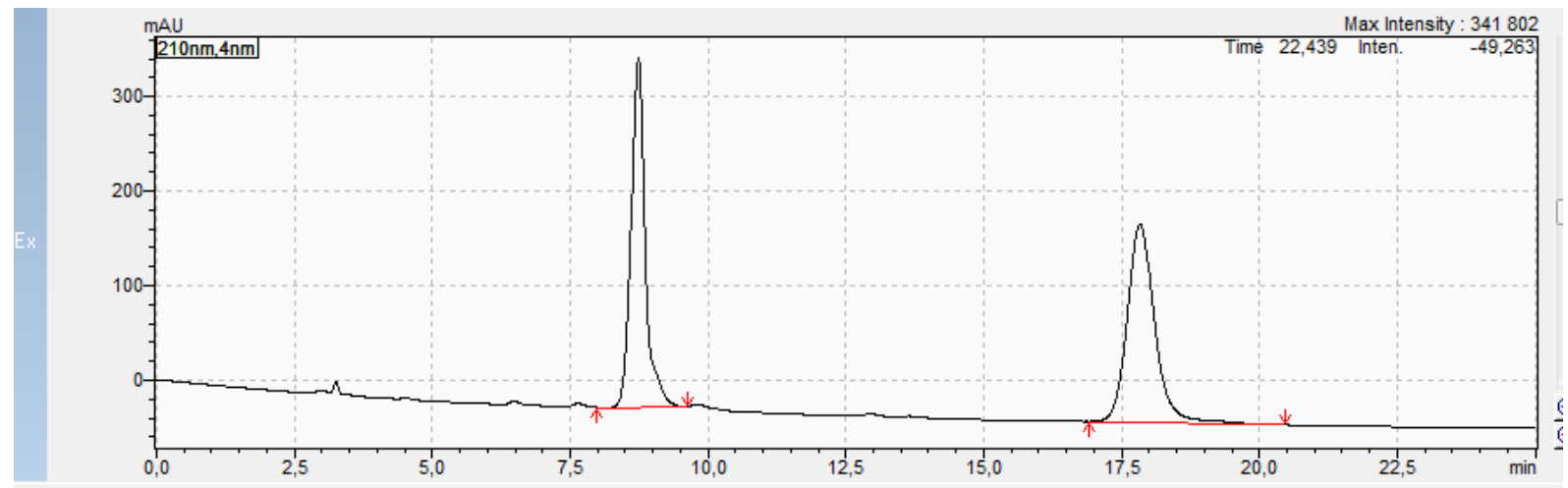

口〈R Results View - Peak Table

\begin{tabular}{l|l|l|l|}
\hline Peak Table Compound & Group & Calibration Curve \\
\hline
\end{tabular}

\begin{tabular}{|c|c|c|c|c|c|c|c|c|c|}
\hline Peak\# & Ret. Time & Conc. & Area & Height & Similarity Index & Mark & Peak Start & Peak End & Area\% \\
\hline 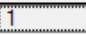 & 8,735 & 47,298 & 6507463 & 370258 & 0,000000 & M & 7,979 & 9,643 & 47,298 \\
\hline 2 & 17,839 & 52,702 & 7250889 & 209408 & 0,000000 & & 16,907 & 20,469 & 52,702 \\
\hline Total & & 100,000 & 13758352 & 579666 & & & & & 100,000 \\
\hline
\end{tabular}

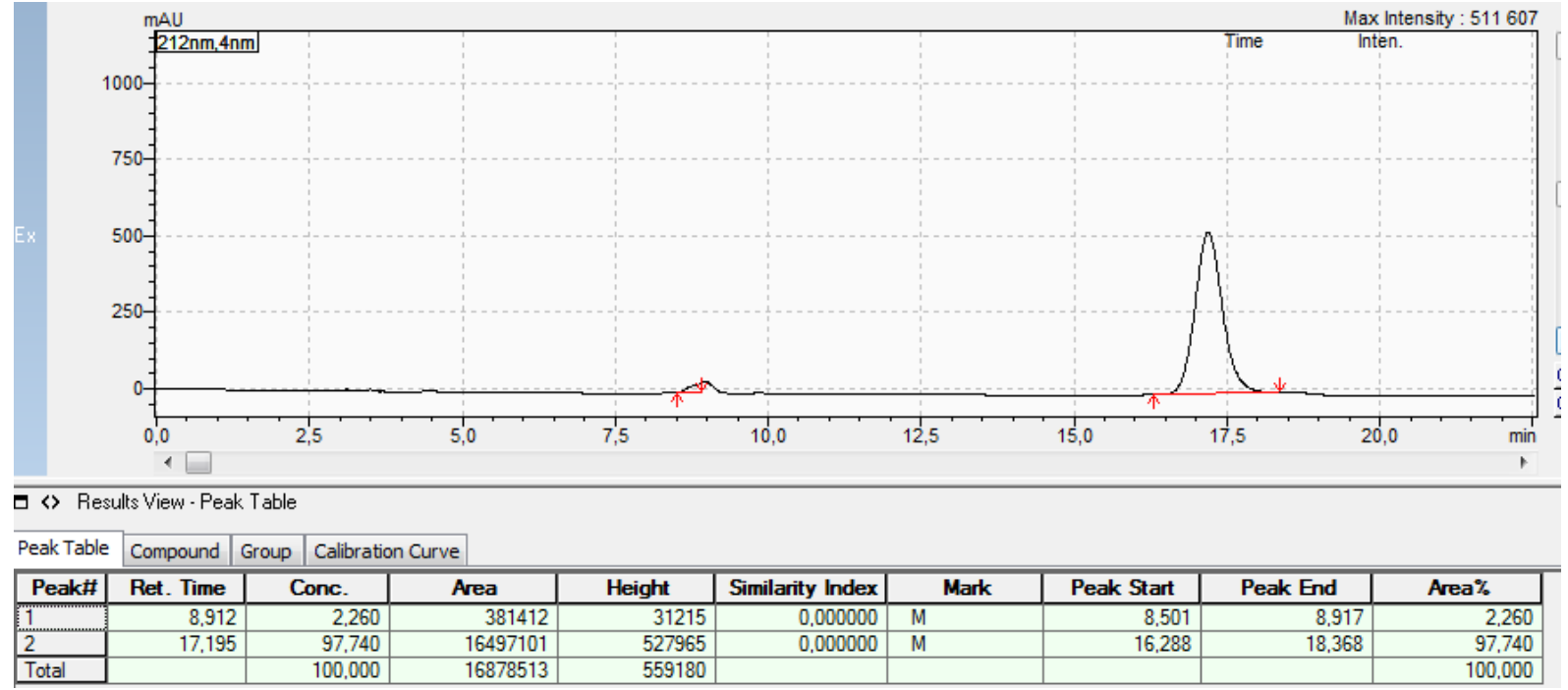

for 3i: $e e=96 \%$ 

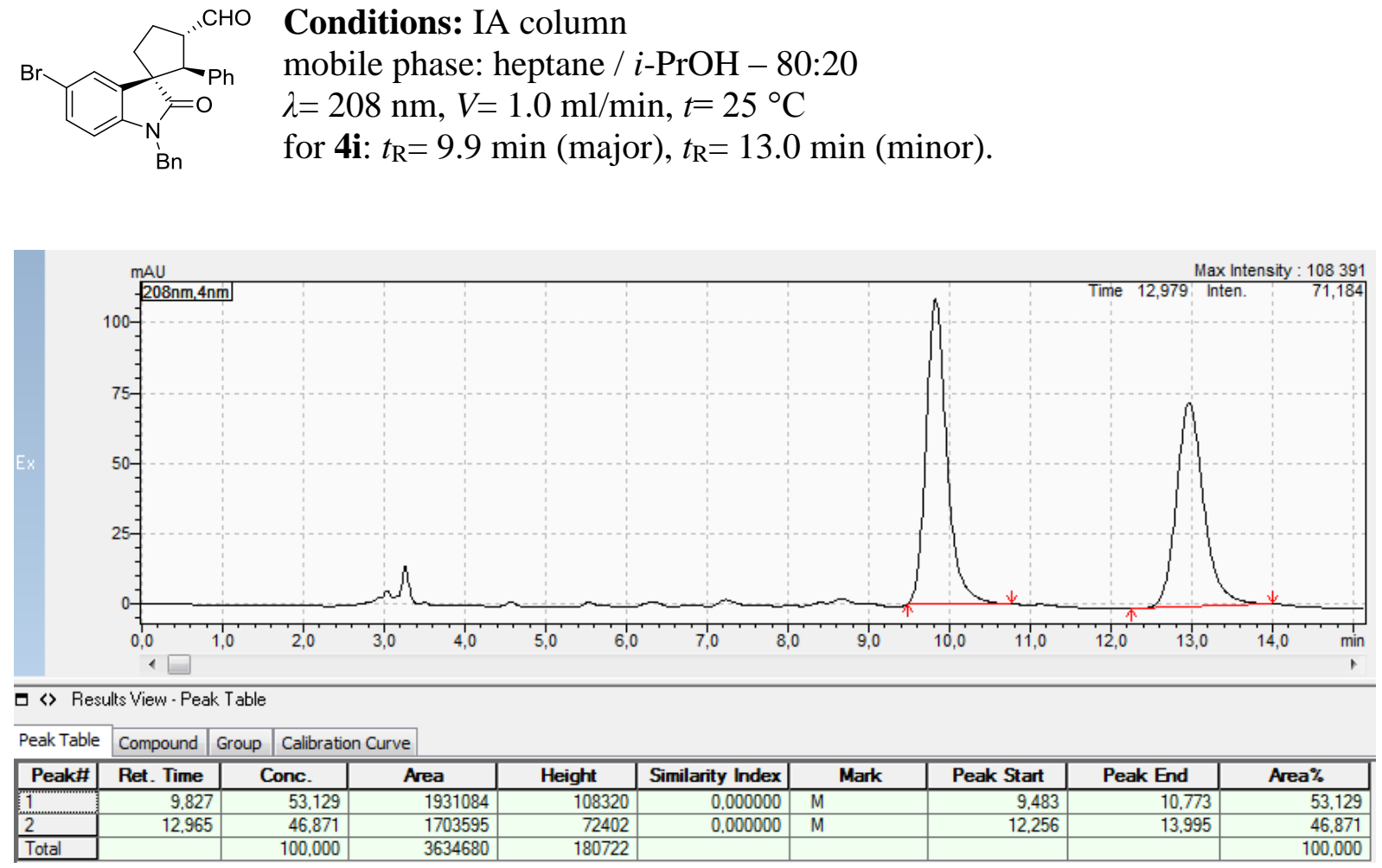

Conditions: IA column

mobile phase: heptane / $i$-PrOH $-80: 20$

$\lambda=208 \mathrm{~nm}, V=1.0 \mathrm{ml} / \mathrm{min}, t=25^{\circ} \mathrm{C}$

for 4i: $t_{\mathrm{R}}=9.9 \mathrm{~min}$ (major), $t_{\mathrm{R}}=13.0 \mathrm{~min}$ (minor).

for $4 \mathbf{i}: e e=96 \%$

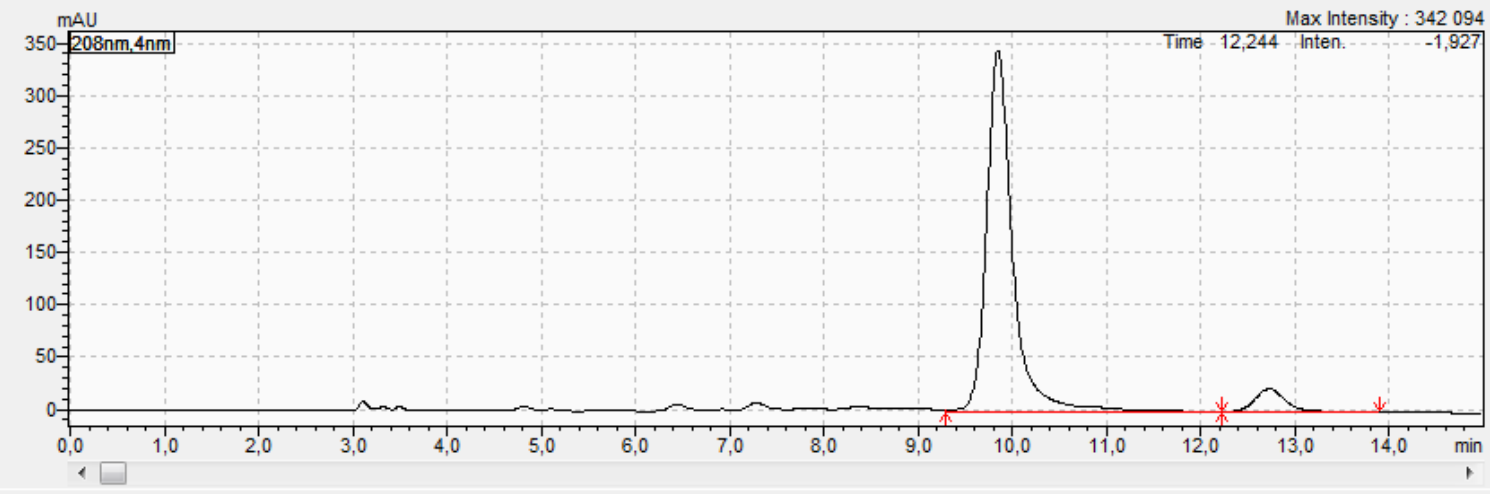

口〈 Results View - Peak Table

\begin{tabular}{|l|l|l|l|}
\hline Peak Table & Compound & Group & Calibration Curve \\
\hline
\end{tabular}

\begin{tabular}{|c|c|c|c|c|c|c|c|c|c|}
\hline Peak\# & Ret. Time & Conc. & Area & Height & Similarity Index & Mark & Peak Start & Peak End & Area\% \\
\hline $1 \quad \cdots$ & 9.850 & 93,071 & 6647837 & 343921 & 0,000000 & & 9.291 & 12,224 & 93,071 \\
\hline 2 & 12,725 & 6,929 & 494928 & 21748 & 0,000000 & & 12,224 & 13,899 & 6.929 \\
\hline Total & & 100,000 & 7142765 & 365669 & & & & & 100,000 \\
\hline
\end{tabular}


(n)

$\mathrm{Bn}$

Conditions: IA column

mobile phase: heptane / $i$-PrOH $-80: 20$

$\lambda=209 \mathrm{~nm}, V=1.0 \mathrm{ml} / \mathrm{min}, t=25^{\circ} \mathrm{C}$

for $3 \mathrm{j}$ : $t_{\mathrm{R}}=9.4 \mathrm{~min}$ (minor), $t_{\mathrm{R}}=25.6 \mathrm{~min}$ (major).

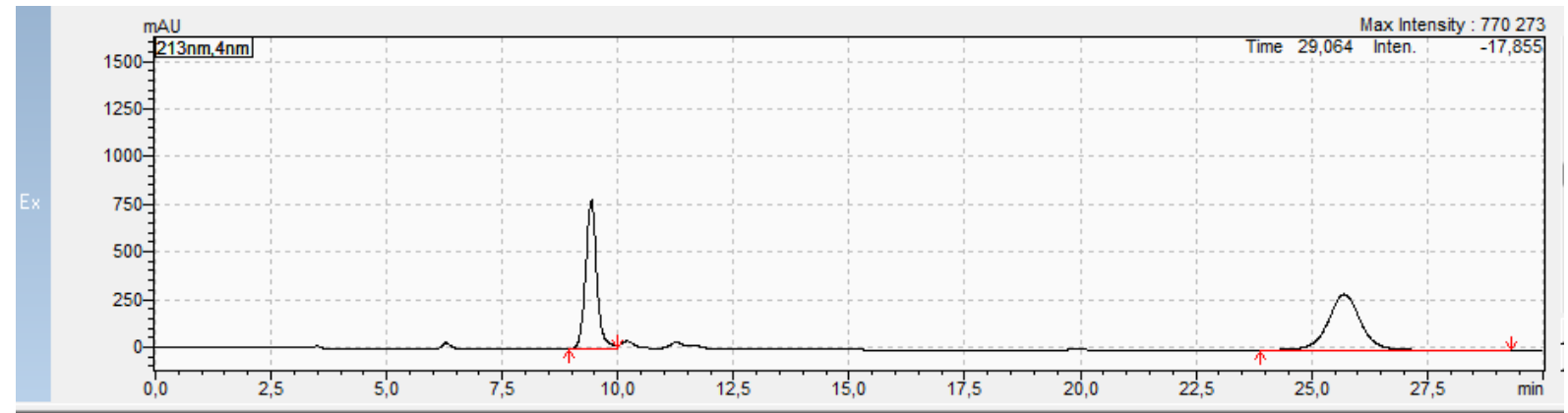

\begin{tabular}{|c|c|c|c|c|c|c|c|c|c|}
\hline Peak Table & Compound & Calibratio & & & & & & & \\
\hline Peak\# & Ret. Time & Conc. & Area & Height & Similarity Index & Mark & Peak Start & Peak End & Area\% \\
\hline 1 & 9.428 & 47.498 & 13212008 & 779993 & 0,000000 & & 8,928 & 9,973 & 47.498 \\
\hline 2 & 25,704 & 52,502 & 14603922 & 293606 & 0,000000 & & 23,893 & 29,333 & 52,502 \\
\hline Total & & 100,000 & 27815931 & 1073599 & & & & & 100,000 \\
\hline
\end{tabular}

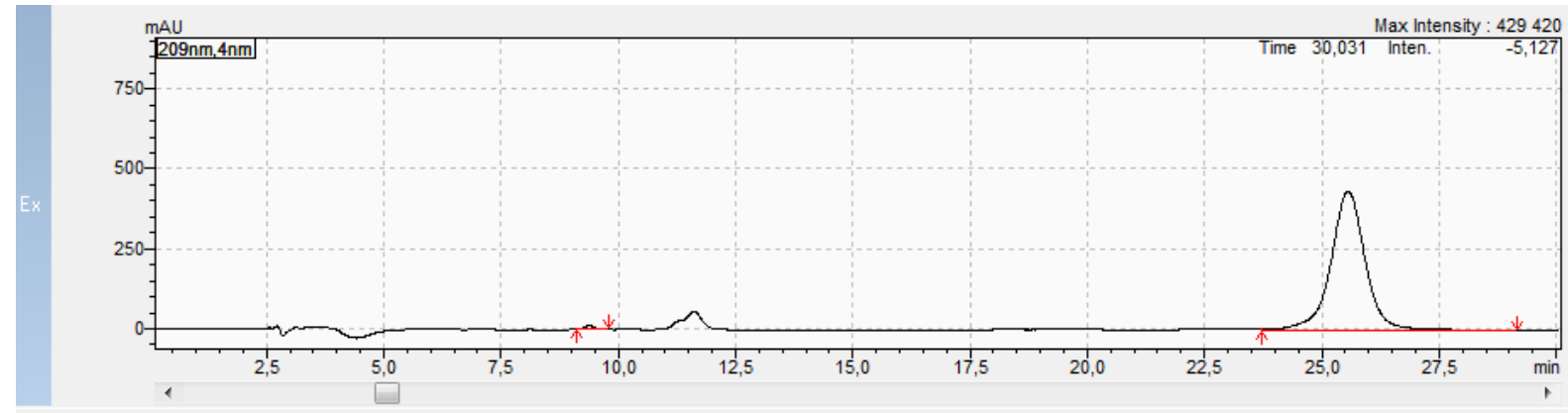

口〈 Results View - Peak Table

\begin{tabular}{l|l|l|l|}
\hline Peak Table Compound & Group & Calibration Curve \\
\hline
\end{tabular}

\begin{tabular}{|c|c|c|c|c|c|c|c|c|c|}
\hline Peak\# & Ret. Time & Conc. & Area & Height & Similarity Index & Mark & Peak Start & Peak End & Area\% \\
\hline 7 & 9,378 & 0,778 & 172506 & 11258 & 0,000000 & $M$ & 9,109 & 9,781 & 0,778 \\
\hline 2 & 25,564 & 99,222 & 22000394 & 433504 & 0,000000 & & 23,701 & 29,163 & 99.222 \\
\hline Total & & 100,000 & 22172901 & 444762 & & & & & 100,000 \\
\hline
\end{tabular}

for $\mathbf{3 j}: e e=98 \%$ 


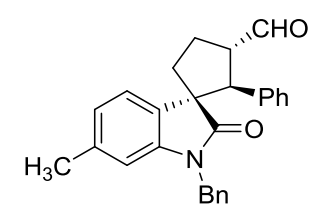

Conditions: IA column

mobile phase: heptane / $i$-PrOH $-80: 20$

$\lambda=210 \mathrm{~nm}, V=1.0 \mathrm{ml} / \mathrm{min}, t=25^{\circ} \mathrm{C}$

for 3k: $t_{\mathrm{R}}=8.6 \mathrm{~min}$ (minor), $t_{\mathrm{R}}=15.2 \mathrm{~min}$ (major).

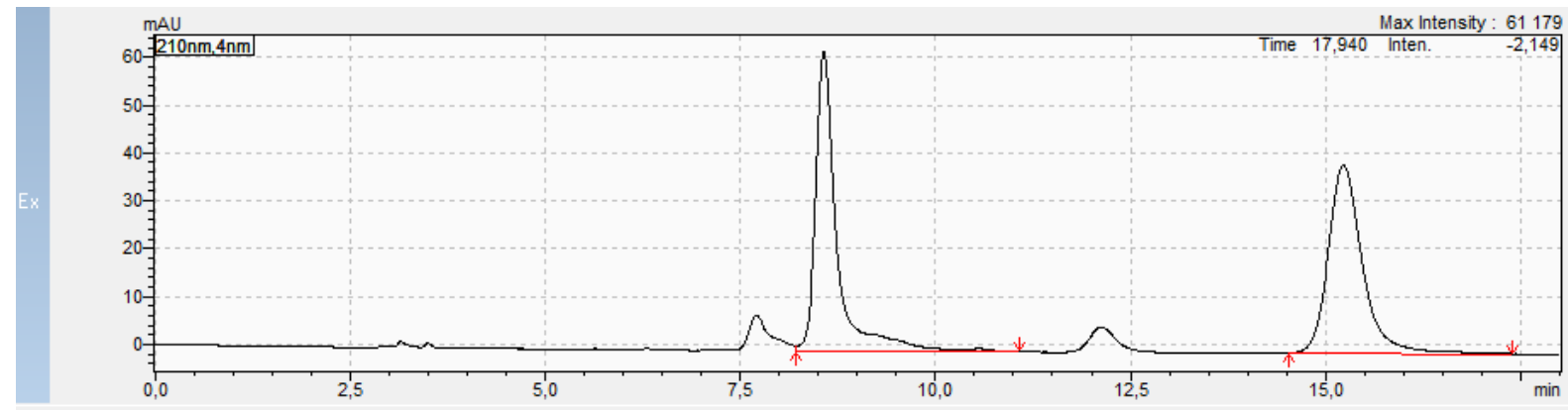

口〈 Results View - Peak Table

\begin{tabular}{|l|l|l|l|}
\hline Peak Table & Compound & Group & Calibration Curve \\
\hline
\end{tabular}

\begin{tabular}{|c|c|c|c|c|c|c|c|c|c|}
\hline Peak\# & Ret. Time & Conc. & Area & Height & Similarity Index & Mark & Peak Start & Peak End & Area $\%$ \\
\hline 1 & 8.571 & 50,083 & 1191861 & 62578 & 0,000000 & & 8,213 & 11,083 & 50,083 \\
\hline 2 & 15,233 & 49,917 & 1187897 & 39288 & 0,000000 & S & 14,539 & 17,397 & 49.917 \\
\hline Total & & 100,000 & 2379757 & 101866 & & & & & 100,000 \\
\hline
\end{tabular}

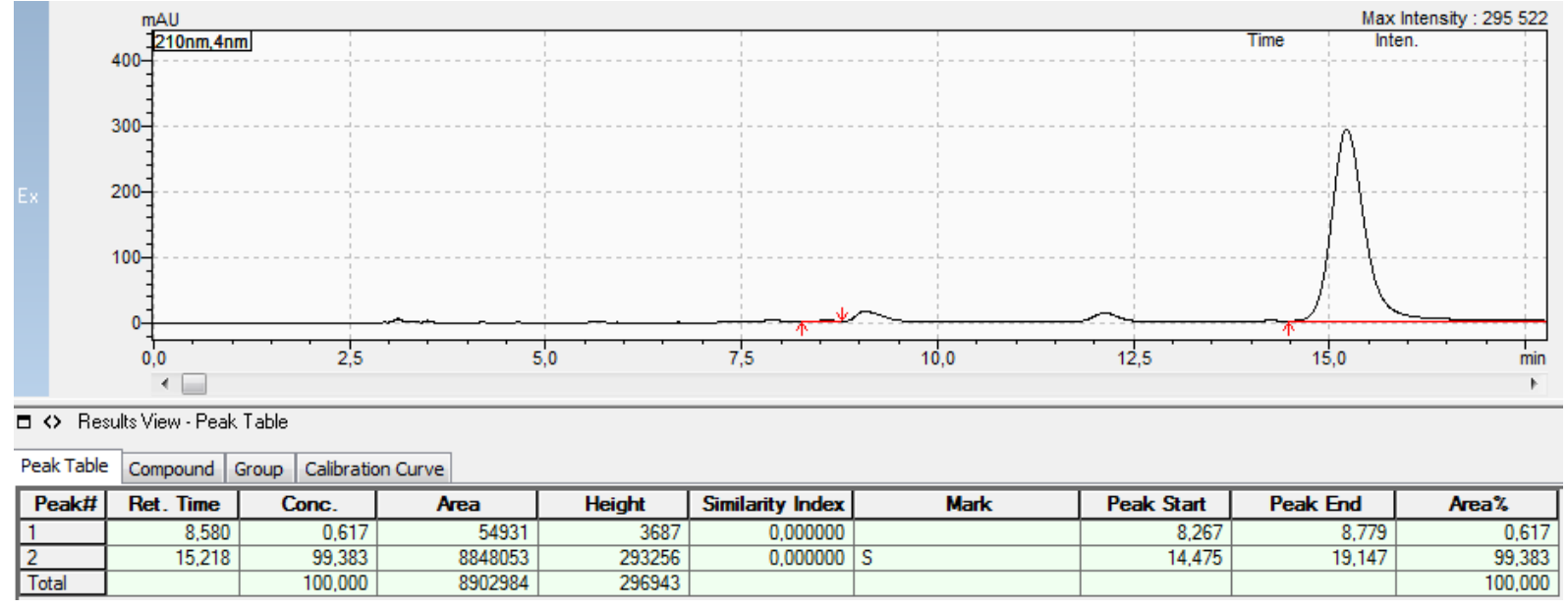

for 3k: $e e=99 \%$ 
<smiles>Cc1ccc2c(c1)CC1(C(=O)N2C(=O)c2ccccc2)C(C)CCC1C=O</smiles>

Conditions: IA column

mobile phase: heptane / $i$-PrOH $-80: 20$

$\lambda=208 \mathrm{~nm}, V=1.0 \mathrm{ml} / \mathrm{min}, t=25^{\circ} \mathrm{C}$

for $4 \mathrm{k}: t_{\mathrm{R}}=9.8 \mathrm{~min}$ (major), $t_{\mathrm{R}}=12.5 \mathrm{~min}$ (minor).
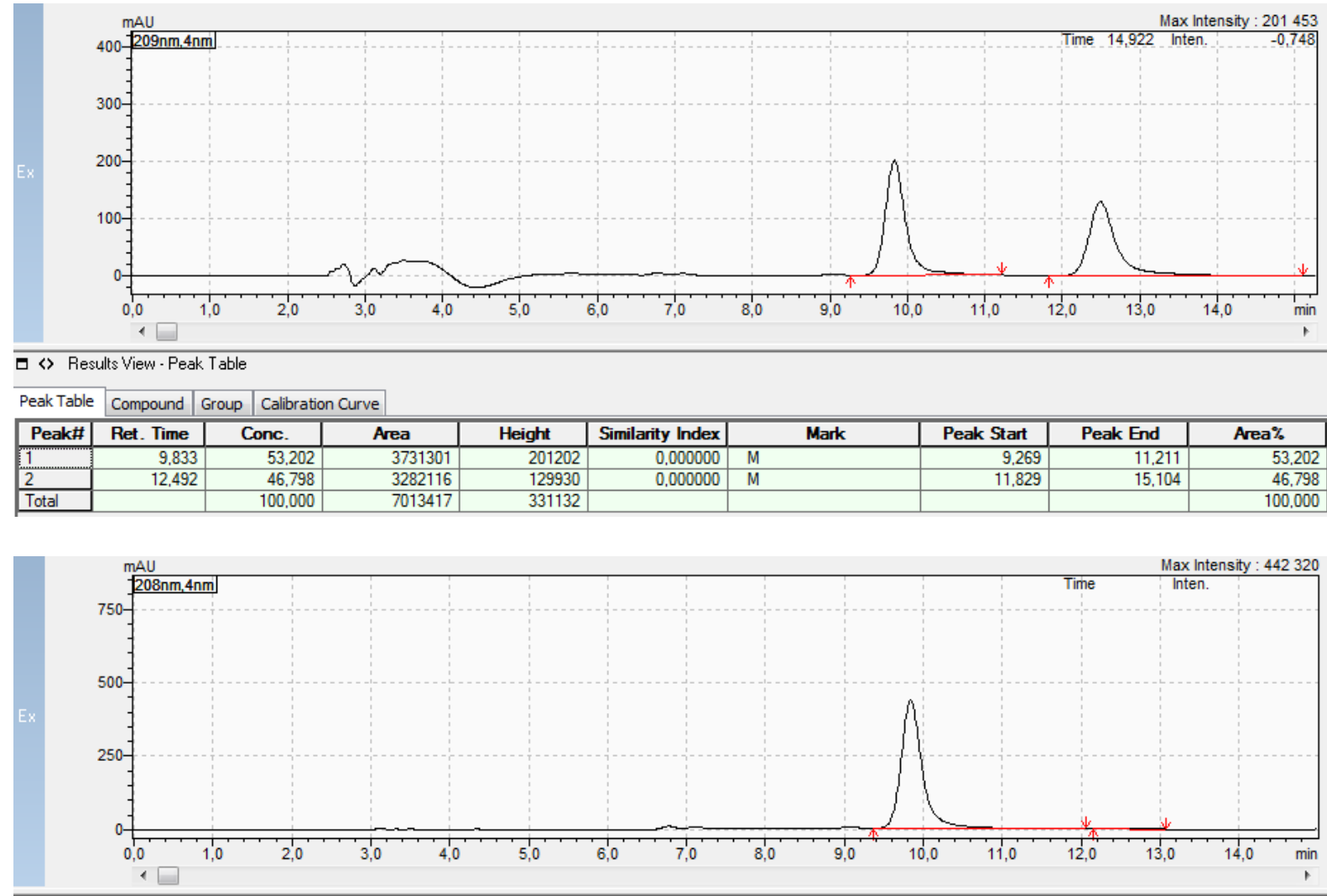

口〈R Results View - Peak Table

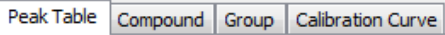

\begin{tabular}{|l|r|r|r|r|r|r|r|r|r|r|}
\hline Peak\# & Ret. Time & \multicolumn{1}{|c|}{ Conc. } & \multicolumn{1}{|c|}{ Area } & Height & Similarity Index & Mark & \multicolumn{1}{c|}{ Peak Start } & \multicolumn{1}{c|}{ Peak End } & \multicolumn{1}{c|}{ Area\% } \\
\hline 1 & 9,838 & 99,578 & 8312275 & 438817 & 0,000000 & & 9,365 & 12,064 & 99,578 \\
\hline 2 & 12,506 & 0,422 & 35187 & 1696 & 0,000000 & & 12,160 & 13,077 & 0,422 \\
\hline Total & & 100,000 & 8347462 & 440513 & & & & \\
\hline
\end{tabular}

for $4 \mathbf{k}: e e=99 \%$ 

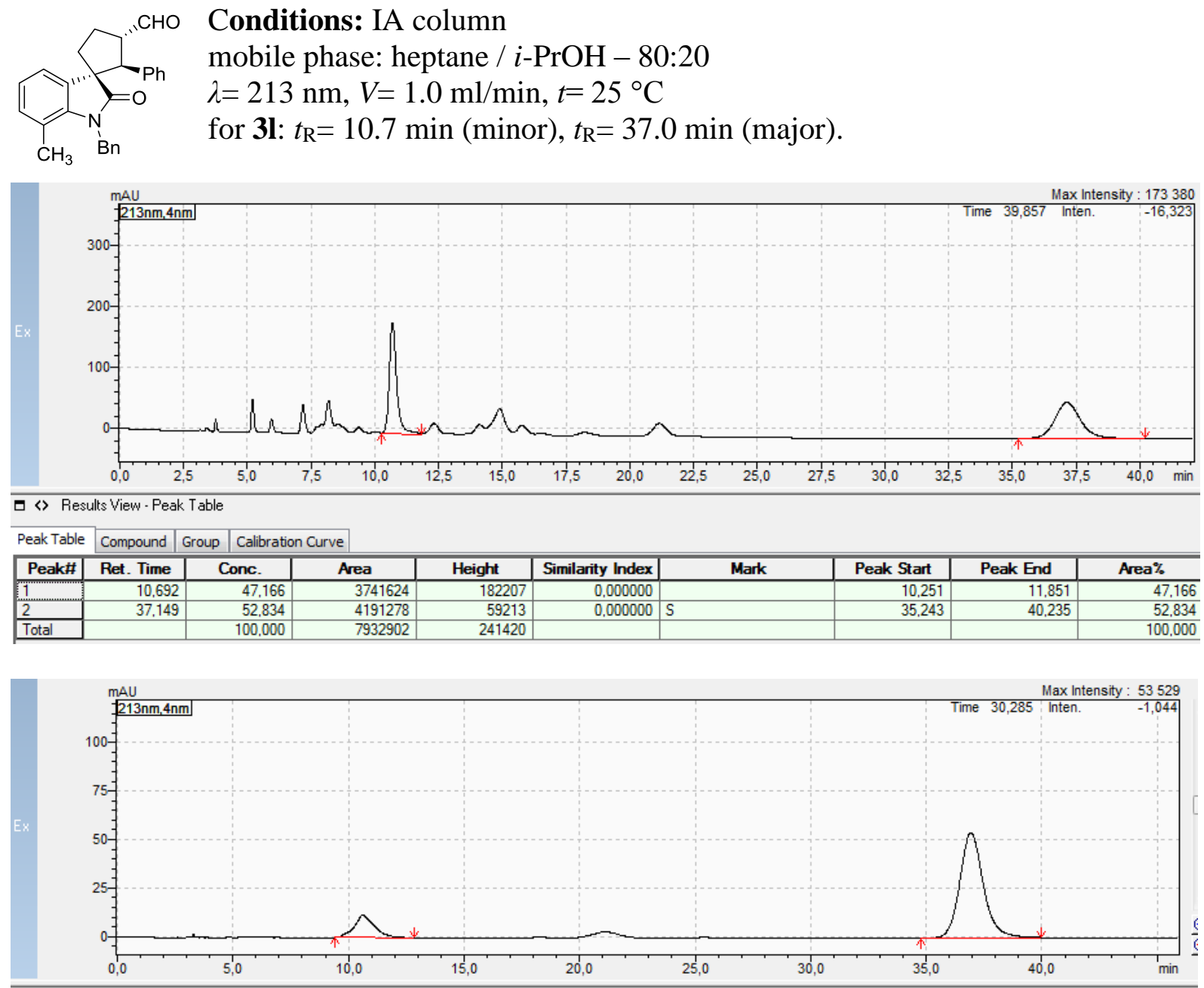

口 〈 Results View - Peak Table

\begin{tabular}{|l|l|l|l|}
\hline Peak Table & Compound & Group & Calibration Curve \\
\hline
\end{tabular}

\begin{tabular}{|c|c|c|c|c|c|c|c|c|c|}
\hline Peak\# & Ret. Time & Conc. & Area & Height & Similarity Index & Mark & Peak Start & Peak End & Area\% \\
\hline 1 & 10,606 & 15,543 & 706278 & 11613 & 0,000000 & M & 9,408 & 12,821 & 15,543 \\
\hline 2 & 36,927 & 84.457 & 3837660 & 54252 & 0,000000 & M & 34,763 & 39.968 & 84,457 \\
\hline Total & & 100,000 & 4543938 & 65865 & & & & & 100,000 \\
\hline
\end{tabular}

for 31: $e e=69 \%$ 


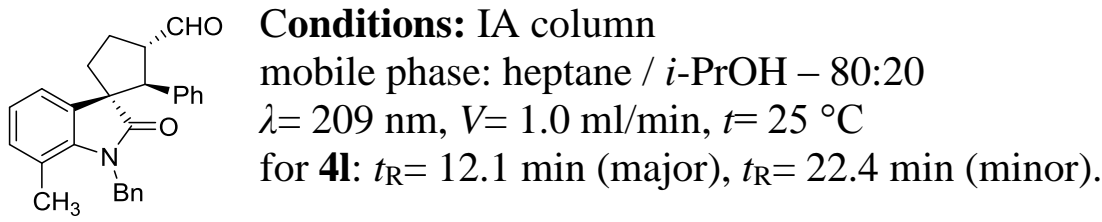

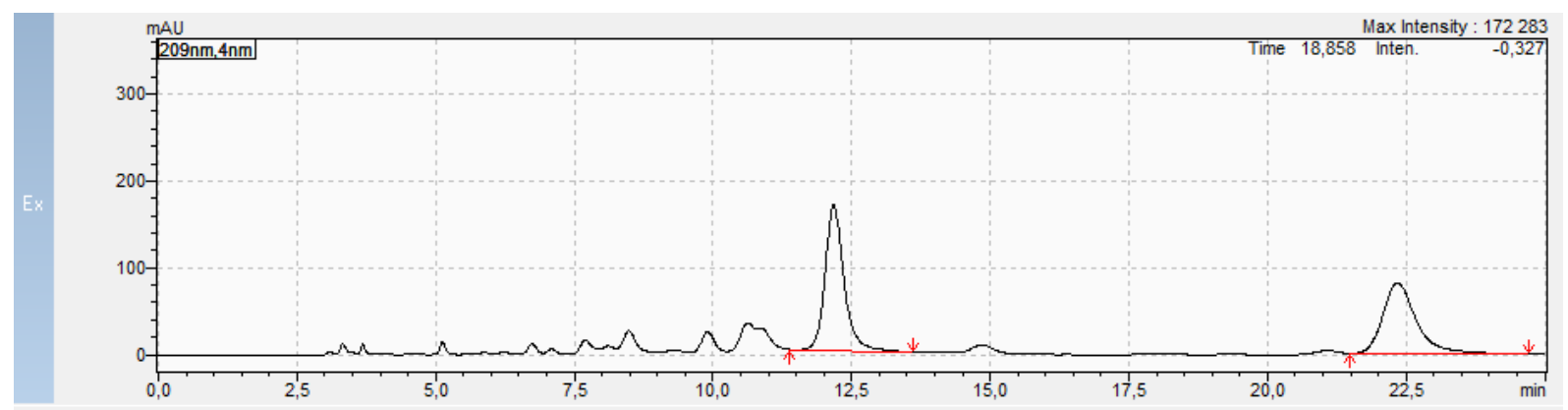

口 〈 Results View - Peak Table

\begin{tabular}{|c|c|c|c|c|c|c|c|c|c|}
\hline Peak Table & Compound & Calibratic & & & & & & & \\
\hline Peak\# & Ret. Time & Conc. & Area & Height & Similarity Index & Mark & Peak Start & Peak End & Area\% \\
\hline (1) & 12,178 & 52,910 & 3999779 & 167564 & 0,000000 & M & 11,381 & 13,600 & 52,910 \\
\hline 2 & 22,348 & 47,090 & 3559778 & 82253 & 0,000000 & & 21.493 & 24,725 & 47,090 \\
\hline Total & & 100,000 & 7559557 & 249818 & & & & & 100,000 \\
\hline
\end{tabular}

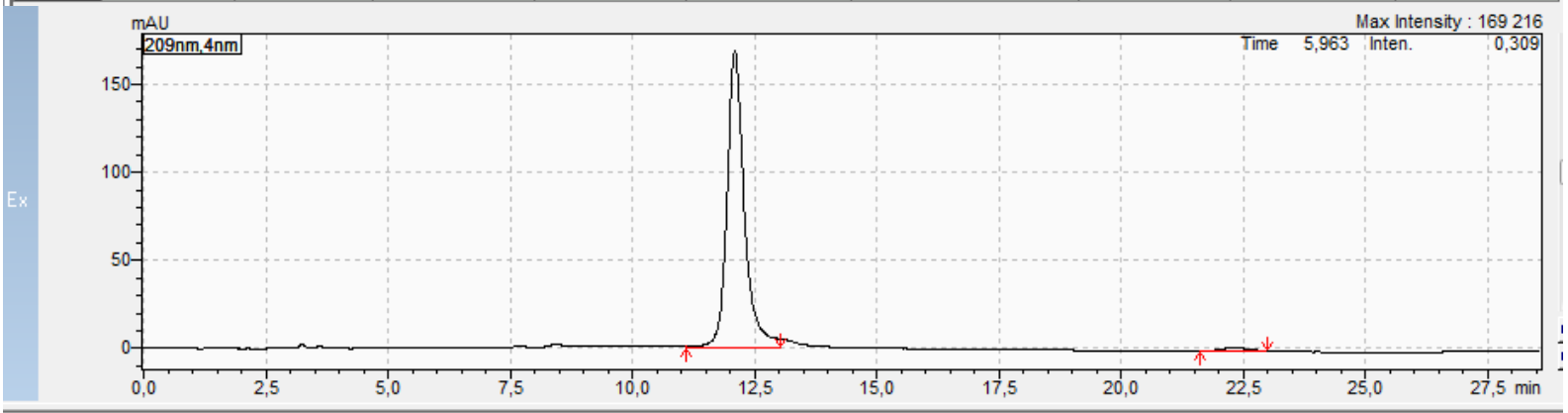

口〈 Results View - Peak Table

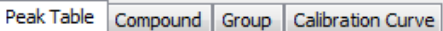

\begin{tabular}{|l|r|r|r|r|r|r|r|r|r|r|}
\hline Peak\# & Ret. Time & \multicolumn{1}{|c|}{ Conc. } & \multicolumn{1}{c|}{ Area } & \multicolumn{1}{c|}{ Height } & Similarity Index & Mark & Peak Start & Peak End & \multicolumn{1}{c|}{ Area\% } \\
\hline 1 & 12,097 & 97,970 & 4193535 & 168809 & 0,000000 & $\mathrm{M}$ & 11,115 & 13,035 & 97,970 \\
\hline 2 & 22,349 & 2,030 & 86902 & 2142 & 0,000000 & & 21,611 & 22,976 & 2,030 \\
\hline Total & & 100,000 & 4280437 & 170951 & & & & 100,000 \\
\hline
\end{tabular}

for 4l: $e e=96 \%$ 
<smiles>O=C[C@H]1CC[C@]2(C(=O)N(Cc3ccccc3)c3ccccc32)[C@H]1c1ccc2ccccc2c1</smiles>

Conditions: IA column

mobile phase: heptane / $i$-PrOH $-80: 20$

$\lambda=228 \mathrm{~nm}, V=1.0 \mathrm{ml} / \mathrm{min}, t=25^{\circ} \mathrm{C}$

for $3 \mathrm{~m}$ : $t_{\mathrm{R}}=10.4 \mathrm{~min}$ (minor), $t_{\mathrm{R}}=13.7 \mathrm{~min}$ (major).

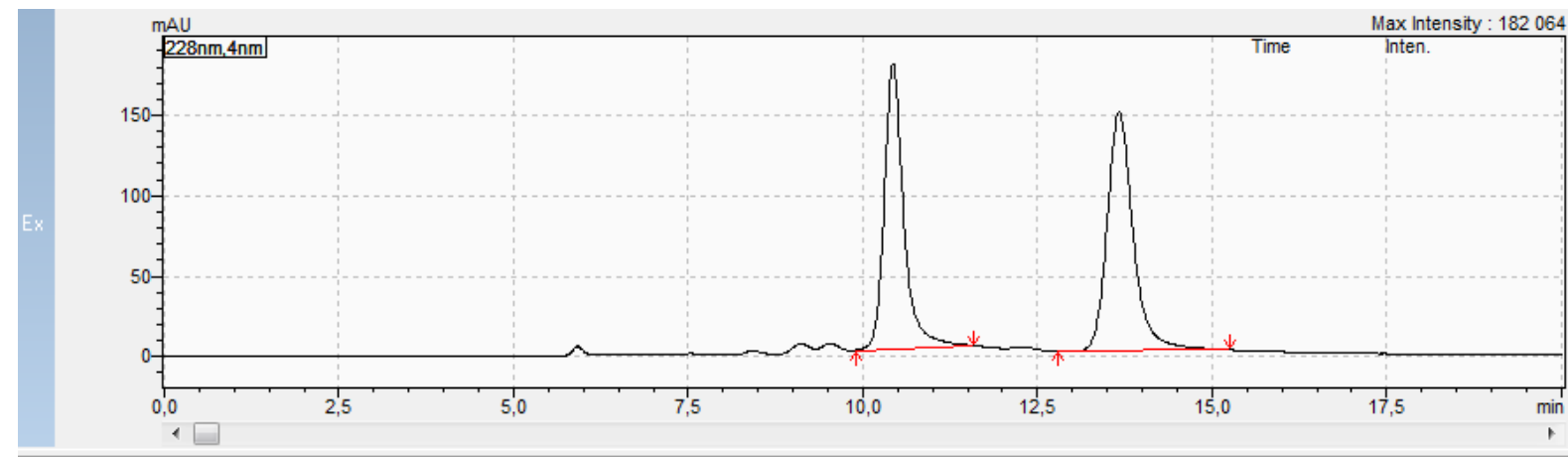

口〈 Results View - Peak Table

\begin{tabular}{|l|l|l|l|}
\hline Peak Table & Compound & Group & Calibration Curve \\
\hline
\end{tabular}

\begin{tabular}{|l|r|r|r|r|r|r|r|r|r|r|}
\hline Peak\# & Ret. Time & \multicolumn{1}{|c|}{ Conc. } & \multicolumn{1}{c|}{ Area } & \multicolumn{1}{c|}{ Height } & Similarity Index & Mark & Peak Start & Peak End & \multicolumn{1}{c|}{ Area\% } \\
\hline 1 & 10,432 & 47,125 & 3441248 & 177015 & 0,000000 & M & 9,899 & 11,595 & 47,125 \\
\hline 2 & 13,669 & 52,875 & 3861151 & 148079 & 0,000000 & M & 12,800 & 15,253 & 52,875 \\
\hline Total & & 100,000 & 7302399 & 325094 & & & & & \\
\hline
\end{tabular}

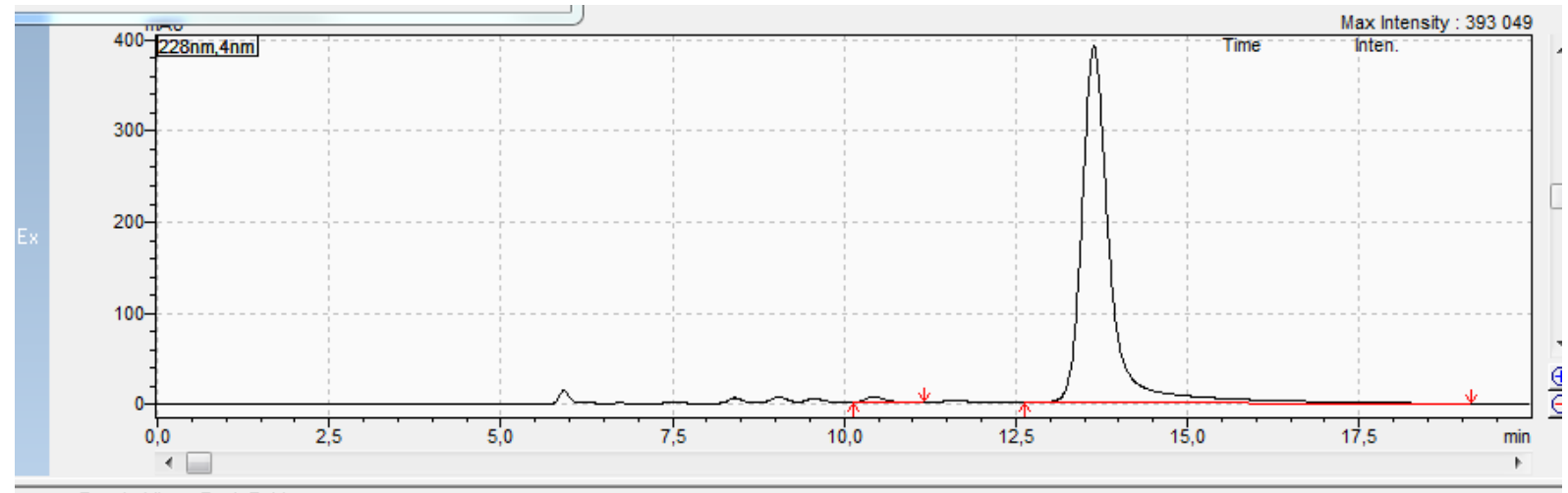

口

\begin{tabular}{|l|l|l|l|}
\hline Peak Table & Compound & Group & Calibration Curve \\
\hline
\end{tabular}

\begin{tabular}{|c|c|c|c|c|c|c|c|c|c|}
\hline Peak\# & Ret. Time & Conc. & Area & Height & Similarity Index & Mark & Peak Start & Peak End & Area\% \\
\hline 1 & 10,437 & 1,349 & 152074 & 6804 & 0,000000 & & 10,123 & 11,179 & 1,349 \\
\hline 2 & 13,638 & 98,651 & 11122914 & 390939 & 0,000000 & & 12,640 & 19,125 & 98,651 \\
\hline Total & & 100,000 & 11274989 & 397743 & & & & & 100,000 \\
\hline
\end{tabular}

for 3m: $e e=97 \%$ 


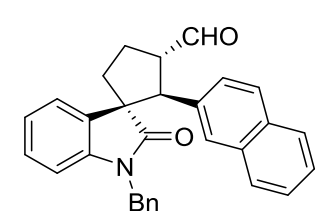

Conditions: IB column

mobile phase: heptane / $i-\mathrm{PrOH}-90: 10$

$\lambda=228 \mathrm{~nm}, V=1.0 \mathrm{ml} / \mathrm{min}, t=25^{\circ} \mathrm{C}$

for $4 \mathrm{~m}: t_{\mathrm{R}}=14.4 \mathrm{~min}$ (minor), $t_{\mathrm{R}}=18.6 \mathrm{~min}$ (major).

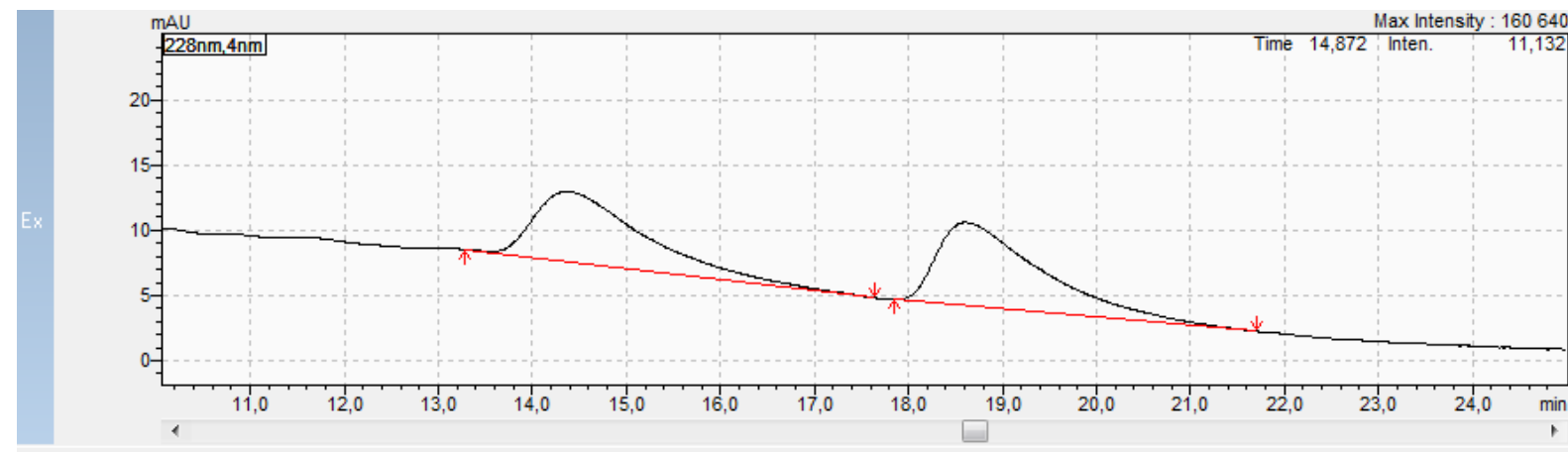

口 $〉$ Results View - Peak Table

\begin{tabular}{|l|l|l|l|}
\hline Peak Table & Compound & Group & Calibration Curve \\
\hline
\end{tabular}

\begin{tabular}{|c|c|c|c|c|c|c|c|c|c|}
\hline Peak\# & Ret. Time & Conc. & Area & Height & Similarity Index & Mark & Peak Start & Peak End & Area\% \\
\hline 1 & 14,376 & 47,016 & 449111 & 5406 & 0,000000 & $M$ & 13,280 & 17,643 & 47,016 \\
\hline 2 & 18,608 & 52,984 & 506110 & 6422 & 0,000000 & & 17,856 & 21,707 & 52,984 \\
\hline Total & & 100,000 & 955221 & 11828 & & & & & 100,000 \\
\hline
\end{tabular}

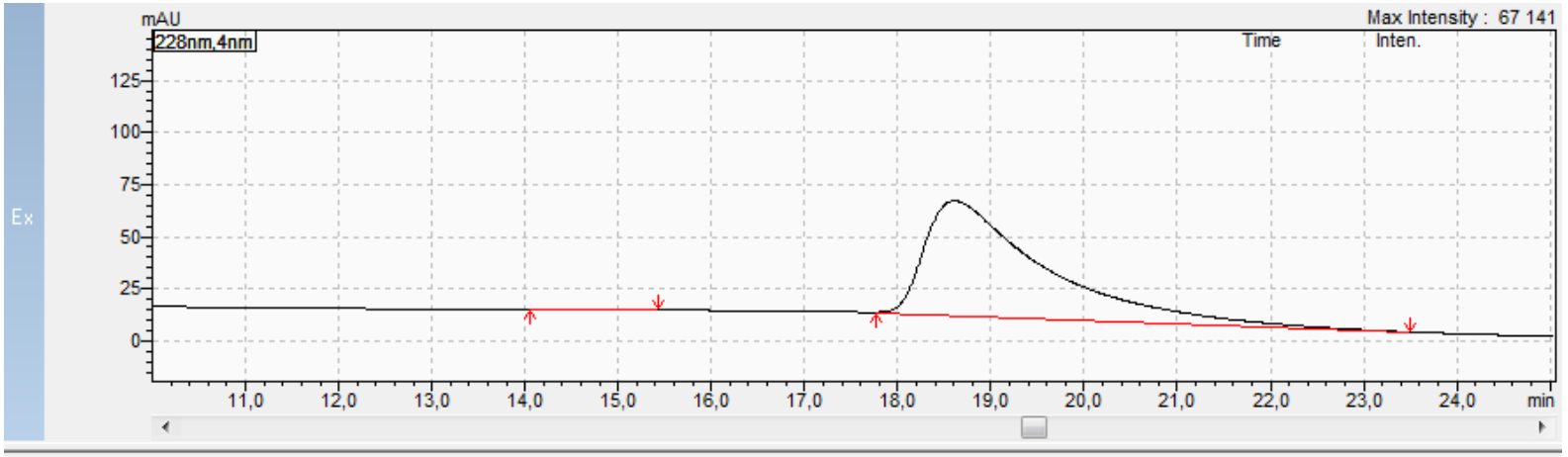

口〈 Results View - Peak Table

\begin{tabular}{|l|l|l|l|}
\hline Peak Table & Compound & Group & Calibration Curve \\
\hline
\end{tabular}

\begin{tabular}{|l|r|r|r|r|r|r|r|r|r|r|}
\hline Peak\# & Ret. Time & \multicolumn{1}{|c|}{ Conc. } & \multicolumn{1}{c|}{ Area } & \multicolumn{1}{c|}{ Height } & Similarity Index & Mark & \multicolumn{1}{c|}{ Peak Start } & \multicolumn{1}{c|}{ Peak End } & \multicolumn{1}{c|}{ Area\% } \\
\hline 1 & 14,398 & 0,222 & 10811 & 254 & 0,000000 & & 14,059 & 15,424 & 0,222 \\
\hline 2 & 18,605 & 99,778 & 4868220 & 54810 & 0,000000 & & 17,760 & 23,499 & 99,778 \\
\hline Total & & 100,000 & 4879031 & 55065 & & & & & \\
\hline
\end{tabular}

for $4 \mathbf{m}: e e=99 \%$ 
<smiles>Cc1ccc(C2[C@H](C=O)CC[C@]23C(=O)N(Cc2ccccc2)c2ccccc23)cc1</smiles>

Conditions: IA column

mobile phase: heptane / $i$-PrOH $-80: 20$

$\lambda=213 \mathrm{~nm}, V=1.0 \mathrm{ml} / \mathrm{min}, t=25^{\circ} \mathrm{C}$

for $3 \mathrm{n}$ : $t_{\mathrm{R}}=8.3 \mathrm{~min}$ (minor), $t_{\mathrm{R}}=13.5 \mathrm{~min}$ (major).

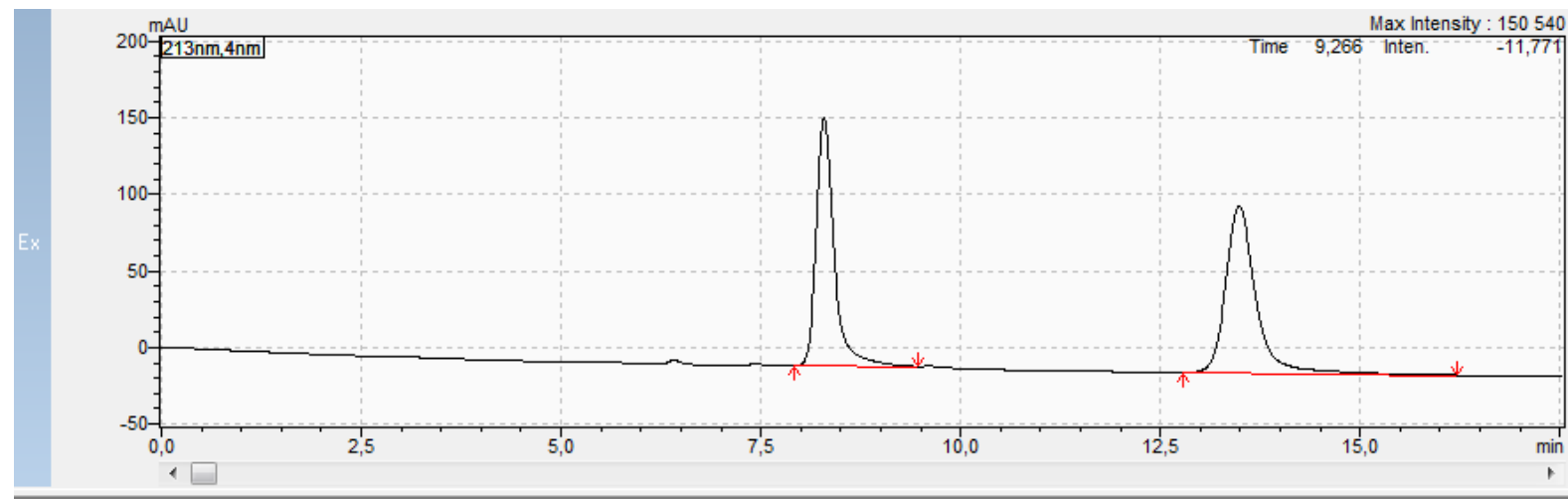

口〈Results View - Peak Table

\begin{tabular}{|l|l|l|l|}
\hline Peak Table & Compound & Group & Calibration Curve \\
\hline
\end{tabular}

\begin{tabular}{|l|r|r|r|r|r|r|r|r|r|}
\hline Peak\# & Ret. Time & \multicolumn{1}{|c|}{ Conc. } & \multicolumn{1}{c|}{ Area } & Height & Similarity Index & Mark & Peak Start & Peak End & Area\% \\
\hline 1 & 8,291 & 47,059 & 2533268 & 162802 & 0,000000 & & 7,925 & 9,472 & 47,059 \\
\hline 2 & 13,484 & 52,941 & 2849862 & 108940 & 0,000000 & & 12,789 & 16,213 & 52,941 \\
\hline Total & & 100,000 & 5383130 & 271741 & & & & & \\
\hline
\end{tabular}

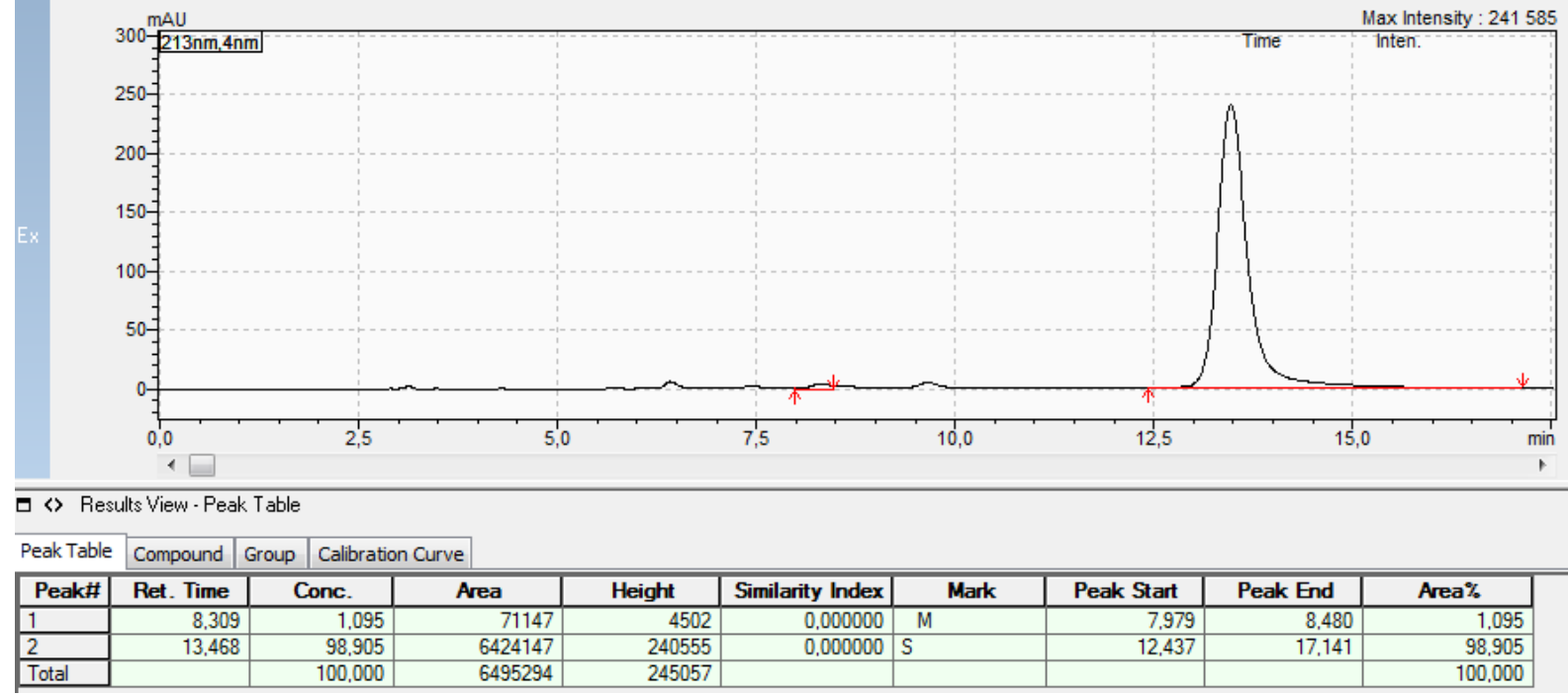

for 3n: $e e=98 \%$ 
<smiles>Cc1ccc(C2[C@H](C=O)CC[C@]23C(=O)N(Cc2ccccc2)c2ccccc23)cc1</smiles>

Conditions: IA column

mobile phase: heptane / $i$-PrOH $-80: 20$

$\lambda=208 \mathrm{~nm}, V=1.0 \mathrm{ml} / \mathrm{min}, t=25^{\circ} \mathrm{C}$

for $4 \mathrm{n}: t_{\mathrm{R}}=9.6 \mathrm{~min}$ (major), $t_{\mathrm{R}}=11.5 \mathrm{~min}$ (minor).

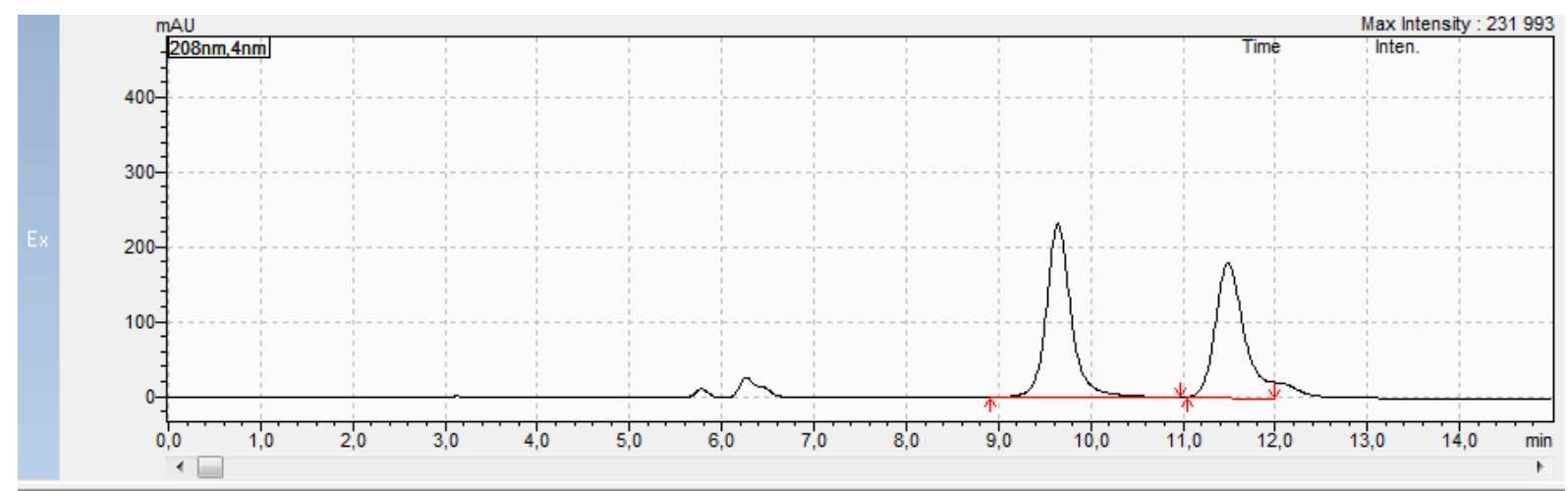

口〈Results View - Peak Table

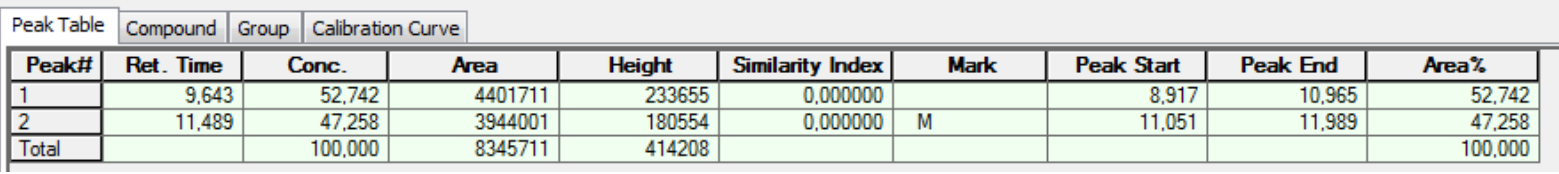

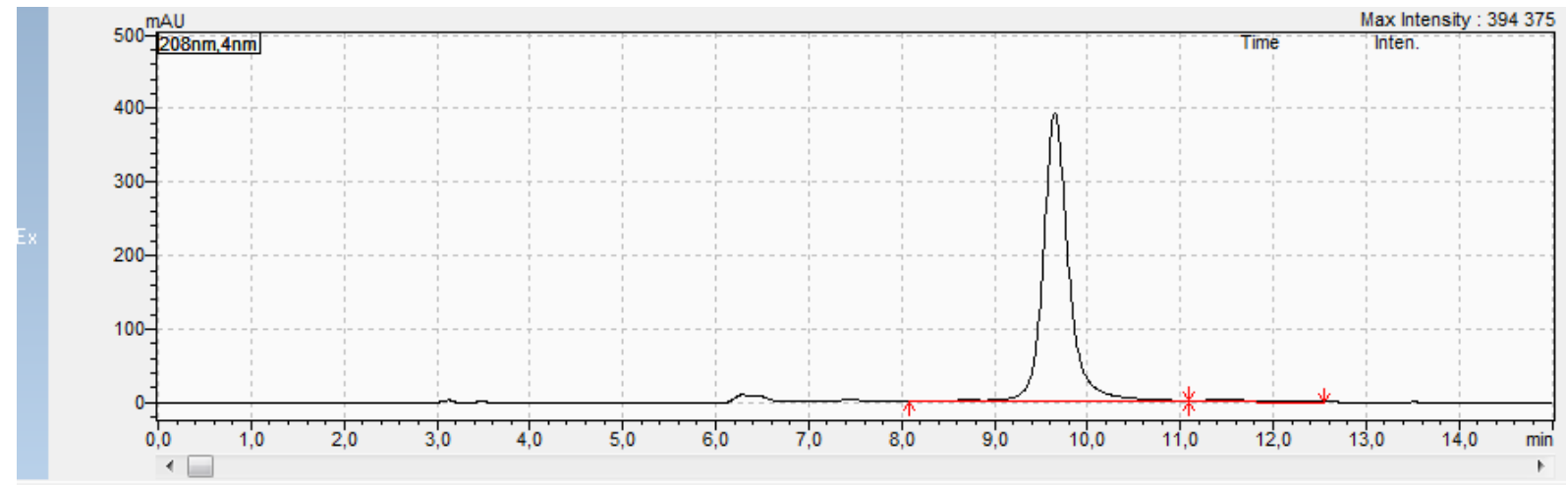

J〈R Results View - Peak Table

\begin{tabular}{l|l|l|l|}
\hline Peak Table Compound & Group & Calibration Curve \\
\hline
\end{tabular}

\begin{tabular}{|c|c|c|c|c|c|c|c|c|c|}
\hline Peak\# & Ret. Time & Conc. & Area & Height & Similarity Index & Mark & Peak Start & Peak End & Area\% \\
\hline 1 & 9.651 & 98,950 & 7640436 & 393190 & 0,000000 & $\bar{M}$ & 8.075 & 11,093 & 98,950 \\
\hline 2 & 11.494 & 1.050 & 81045 & 2841 & 0,000000 & M & 11,093 & 12,544 & 1.050 \\
\hline Total & & 100,000 & 7721482 & 396031 & & & & & 100,000 \\
\hline
\end{tabular}

for $4 \mathbf{n}: e e=98 \%$ 
<smiles>COc1ccc(C2[C@H](C=O)CC[C@]23C(=O)N(Cc2ccccc2)c2ccccc23)cc1</smiles>

Conditions: IA column

mobile phase: heptane / $i$-PrOH $-80: 20$

$\lambda=208 \mathrm{~nm}, V=1.0 \mathrm{ml} / \mathrm{min}, t=25^{\circ} \mathrm{C}$

for 3o: $t_{\mathrm{R}}=10.3 \mathrm{~min}$ (minor), $t_{\mathrm{R}}=12.6 \mathrm{~min}$ (major).

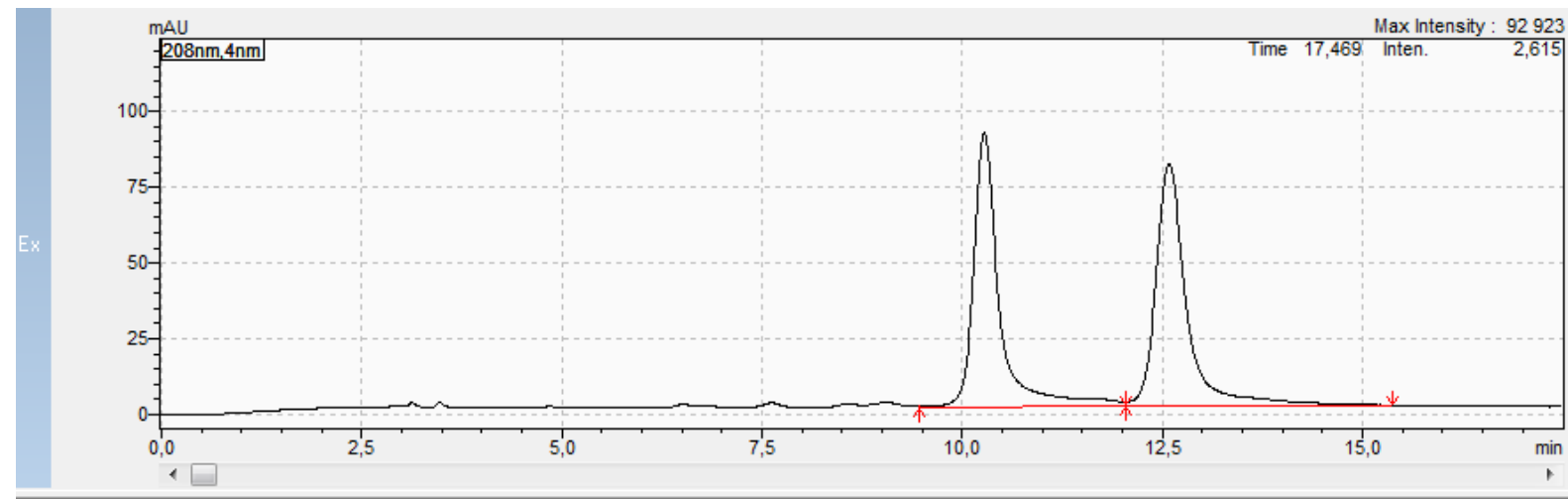

口〈R Results View - Peak Table

\begin{tabular}{l|l|l|l|}
\hline Peak Table & Compound & Group & Calibration Curve \\
\hline
\end{tabular}

\begin{tabular}{|l|r|r|r|r|r|r|r|r|r|r|}
\hline Peak\# & Ret. Time & \multicolumn{1}{|c|}{ Conc. } & \multicolumn{1}{c|}{ Area } & \multicolumn{1}{c|}{ Height } & Similarity Index & Mark & \multicolumn{1}{|c|}{ Peak Start } & Peak End & \multicolumn{1}{c|}{ Area\% } \\
\hline 1 & 10,276 & 48,496 & 2017862 & 90269 & 0,000000 & & 9,461 & 12,043 & 48,496 \\
\hline 2 & 12,582 & 51,504 & 2143047 & 80009 & 0,000000 & SV & 12,043 & 15,371 & 51,504 \\
\hline Total & & 100,000 & 4160910 & 170278 & & & & & \\
\hline
\end{tabular}

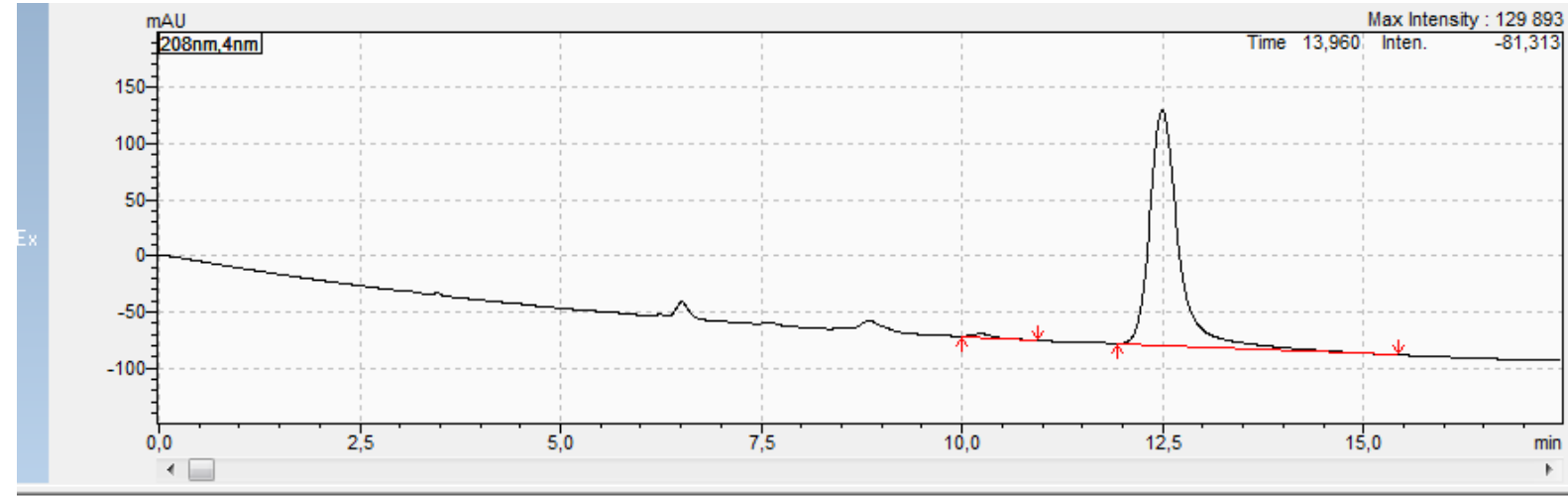

コ〈 Results View - Peak Table

\begin{tabular}{|l|l|l|l|}
\hline Peak Table & Compound & Group & Calibration Curve \\
\hline
\end{tabular}

\begin{tabular}{|l|r|r|r|r|r|r|r|r|r|r|}
\hline Peak\# & Ret. Time & \multicolumn{1}{|c|}{ Conc. } & \multicolumn{1}{c|}{ Area } & \multicolumn{1}{c|}{ Height } & Similarity Index & Mark & \multicolumn{1}{c|}{ Peak Start } & \multicolumn{1}{c|}{ Peak End } & Area\% \\
\hline 1 & 10,238 & 0,997 & 53608 & 3194 & 0,000000 & & 9,995 & 10,944 & 0,997 \\
\hline 2 & 12,494 & 99,003 & 5323333 & 209773 & 0,000000 & & 11,936 & 15,435 & \\
\hline Total & & 100,000 & 5376941 & 212967 & & & & 99,003 \\
\hline
\end{tabular}

for 3o: $e e=98 \%$ 
<smiles>COc1ccc(C2[C@H](C=O)CCC23C(=O)N(Cc2ccccc2)c2ccccc23)cc1</smiles>

Conditions: IA column

mobile phase: heptane / $i$-PrOH - 80:20

$\lambda=208 \mathrm{~nm}, V=1.0 \mathrm{ml} / \mathrm{min}, t=25^{\circ} \mathrm{C}$

for 4o: $t_{\mathrm{R}}=11.7 \mathrm{~min}$ (major), $t_{\mathrm{R}}=13.6 \mathrm{~min}$ (minor).

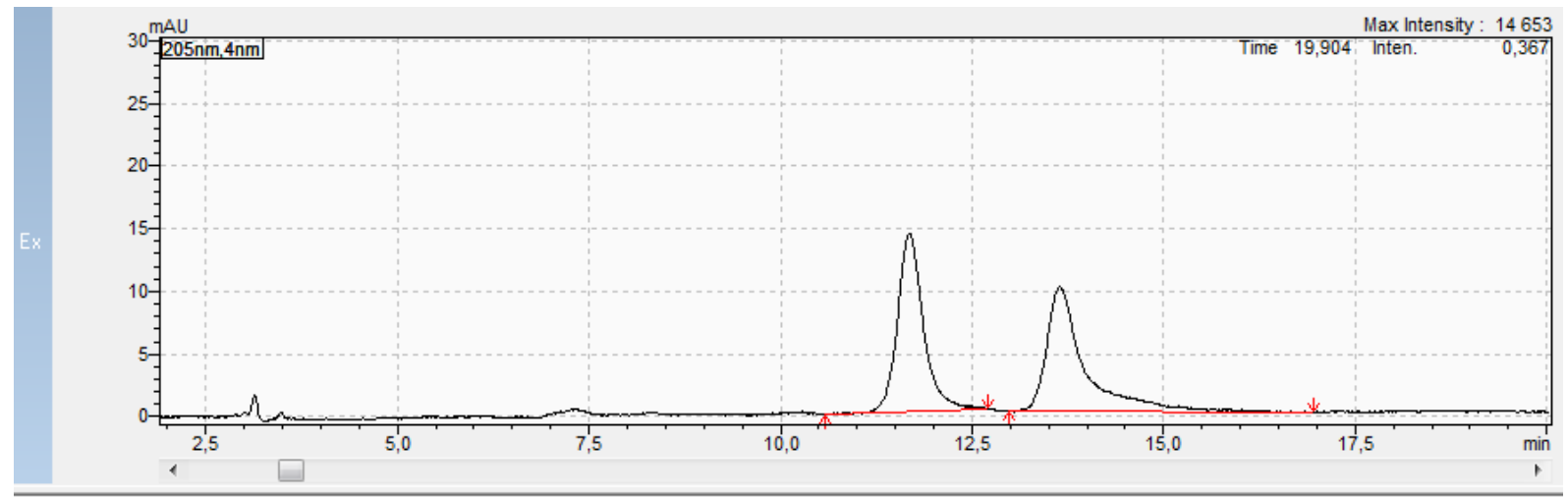

口

\begin{tabular}{|l|l|l|l|}
\hline Peak Table & Compound & Group & Calibration Curve \\
\hline
\end{tabular}

\begin{tabular}{|c|c|c|c|c|c|c|c|c|c|}
\hline Peak\# & Ret. Time & Conc. & Area & Height & Similarity Index & Mark & Peak Start & Peak End & Area\% \\
\hline 1 & 11,682 & 49,922 & 335682 & 14220 & 0,000000 & $\mathrm{M}$ & 10,592 & 12,715 & 49,922 \\
\hline 2 & 13,635 & 50,078 & 336737 & 9862 & 0,000000 & M & 12,981 & 16,949 & 50,078 \\
\hline Total & & 100,000 & 672419 & 24082 & & & & & 100,000 \\
\hline
\end{tabular}

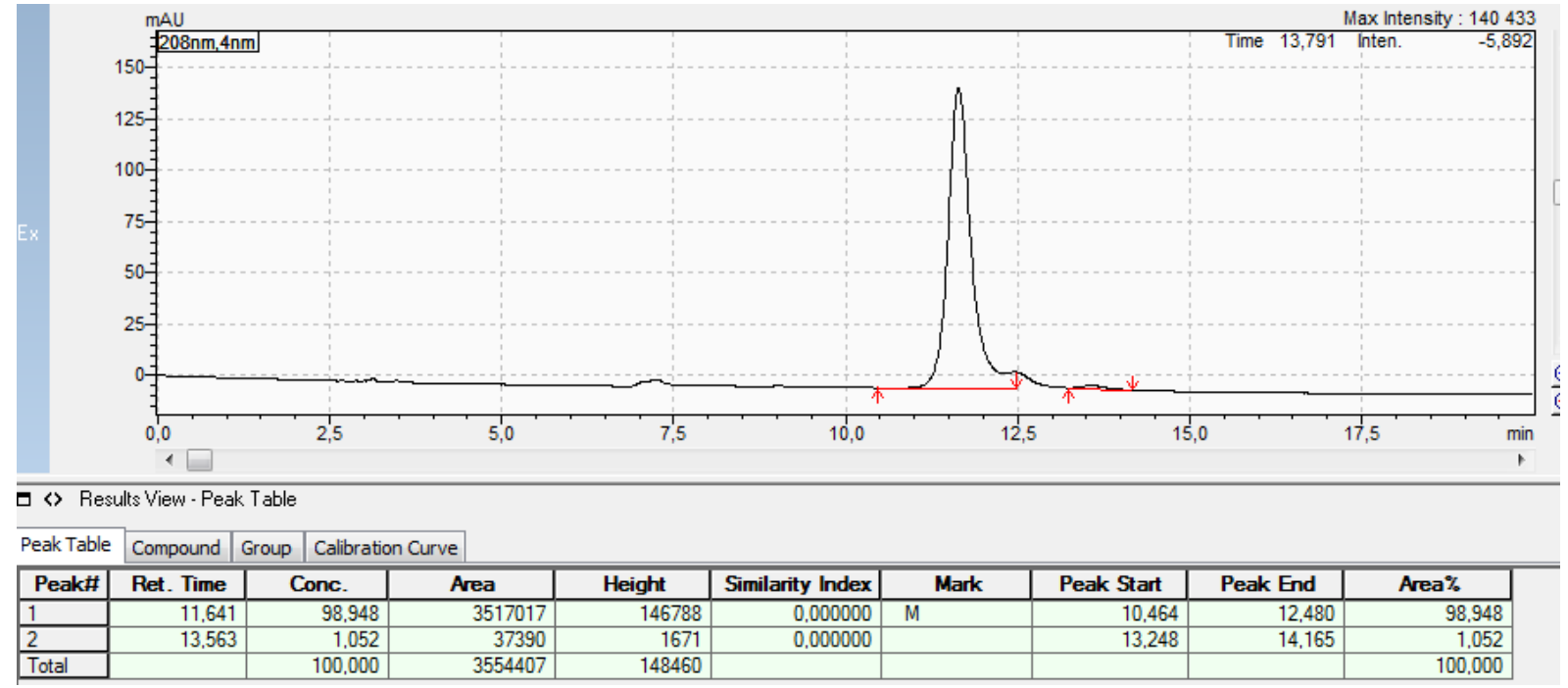

for 4o: $e e=97 \%$ 
<smiles>O=C[C@H]1CC[C@@]2(C(=O)N(Cc3ccccc3)c3ccccc32)[C@H]1c1ccc([N+](=O)[O-])cc1</smiles>

Conditions: IA column

mobile phase: heptane / $i$-PrOH $-70: 30$

$\lambda=212 \mathrm{~nm}, V=1.0 \mathrm{ml} / \mathrm{min}, t=25^{\circ} \mathrm{C}$

for 3p: $t_{\mathrm{R}}=10.0 \mathrm{~min}$ (minor), $t_{\mathrm{R}}=13.1 \mathrm{~min}$ (major).

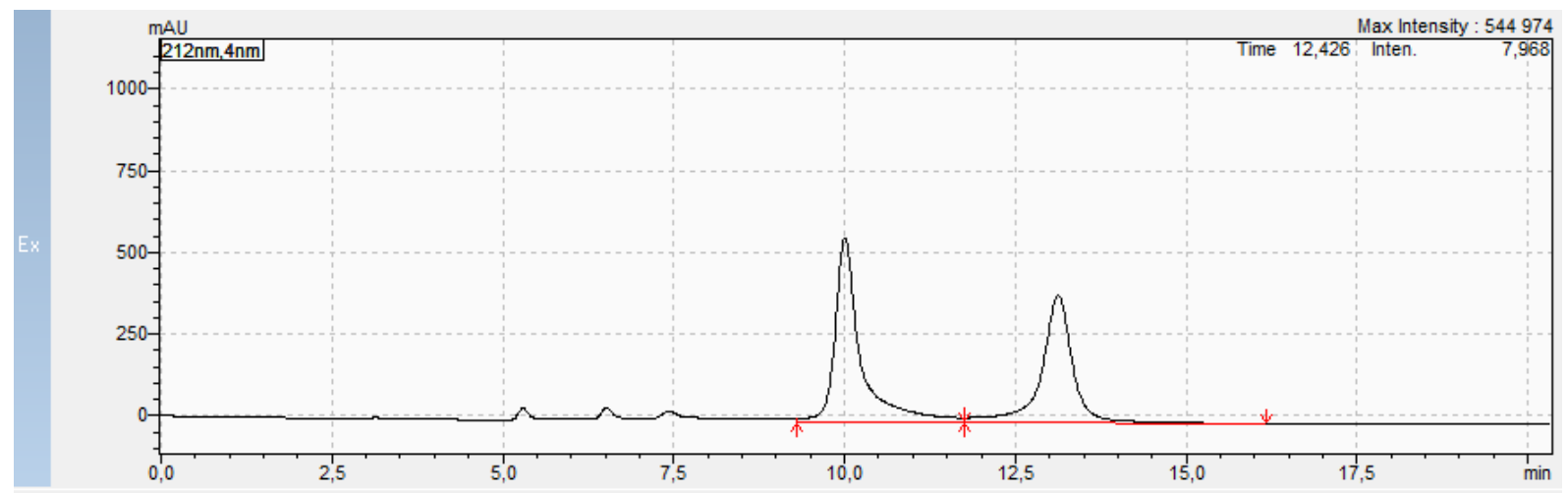

\begin{tabular}{|c|c|c|c|c|c|c|c|c|c|}
\hline Peak Table & Compound & Calibratio & & & & & & & \\
\hline Peak\# & Ret. Time & Conc. & Area & Height & Similarity Index & Mark & Peak Start & Peak End & Area\% \\
\hline 1 & 10,004 & 54,280 & 14785398 & 562415 & 0,000000 & & 9,291 & 11,744 & 54,280 \\
\hline 2 & 13,125 & 45,720 & 12453770 & 388353 & 0.000000 & V & 11,744 & 16,160 & 45,720 \\
\hline Total & & 100,000 & 27239168 & 950768 & & & & & 100,000 \\
\hline
\end{tabular}

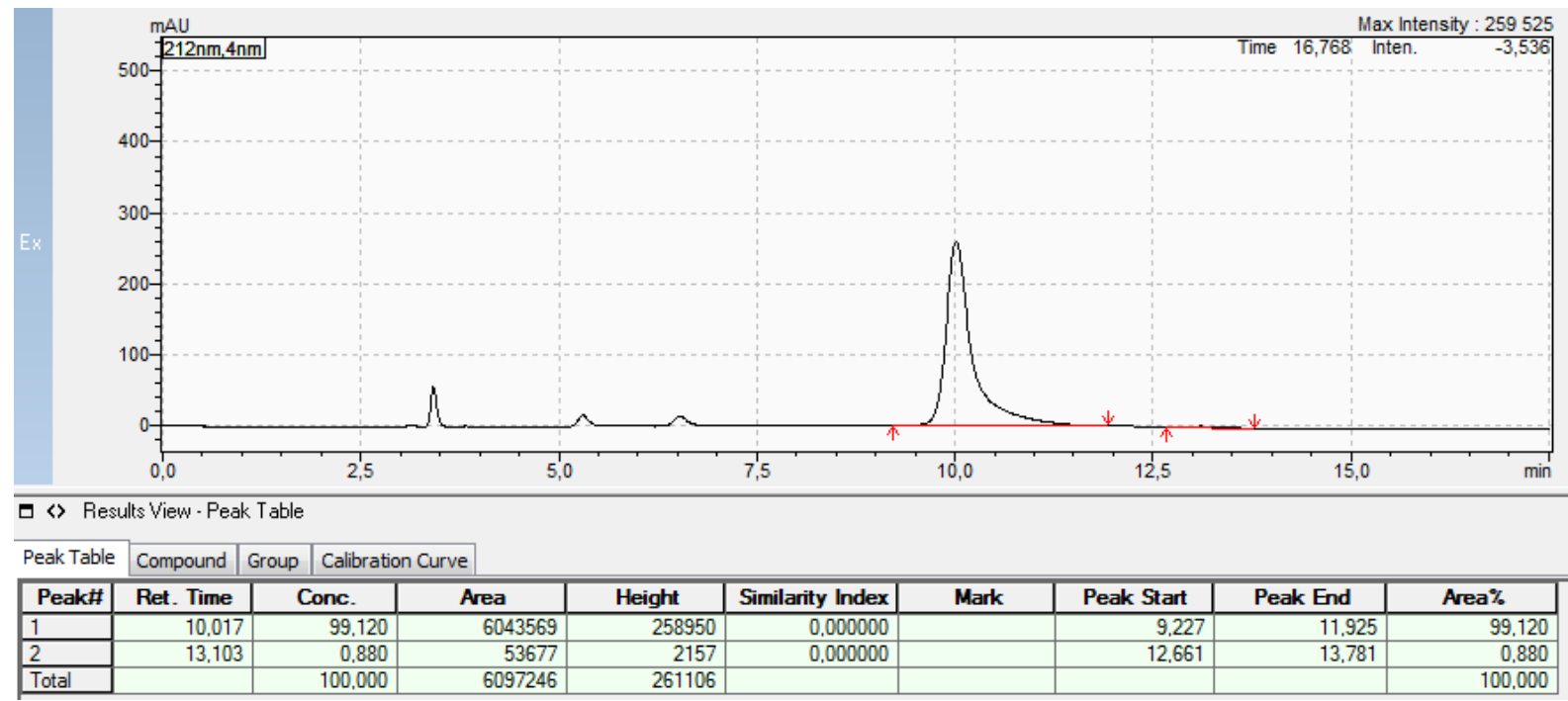

for 3p: $e e=98 \%$ 
<smiles>O=C[C@H]1CCC2(C(=O)N(Cc3ccccc3)c3ccccc32)C1c1ccc([N+](=O)[O-])cc1</smiles>

Conditions: AD column

mobile phase: heptane / $i$-PrOH $-80: 20$

$\lambda=210 \mathrm{~nm}, V=1.0 \mathrm{ml} / \mathrm{min}, t=25^{\circ} \mathrm{C}$

for $4 \mathrm{p}: t_{\mathrm{R}}=7.3 \mathrm{~min}$ (minor), $t_{\mathrm{R}}=9.7 \mathrm{~min}$ (major).

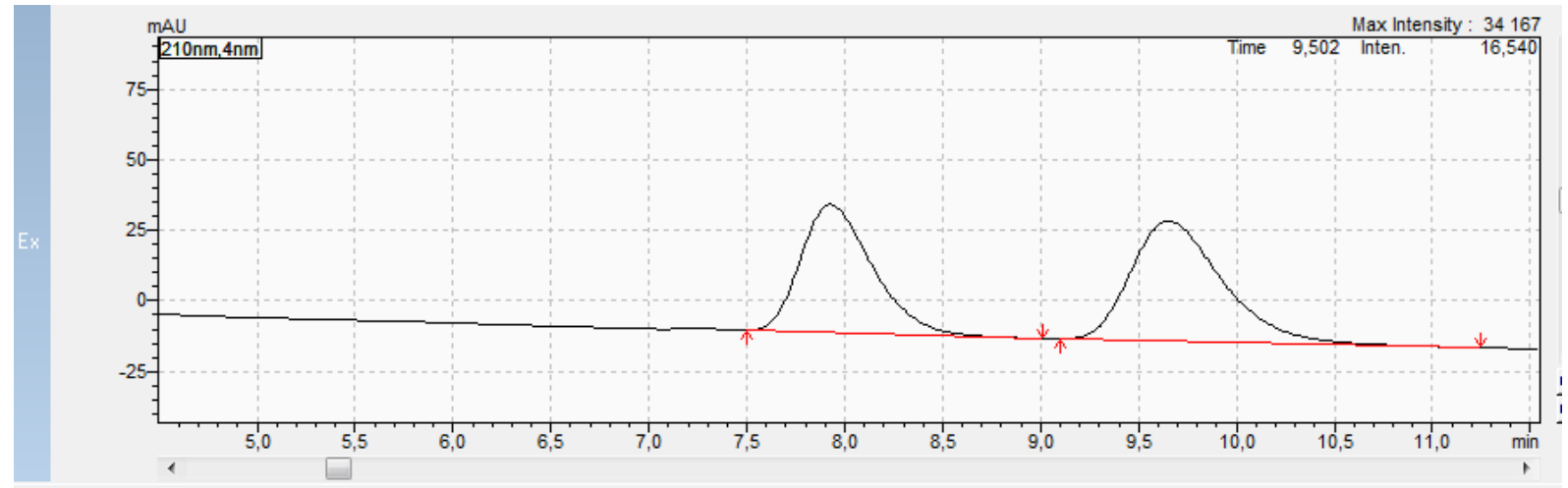

口〈 Results View - Peak Table

\begin{tabular}{|l|l|l|l|}
\hline Peak Table & Compound & Group & Calibration Curve \\
\hline
\end{tabular}

\begin{tabular}{|l|r|r|r|r|r|r|r|r|r|}
\hline Peak\# & Ret. Time & \multicolumn{1}{|c|}{ Conc. } & \multicolumn{1}{c|}{ Area } & Height & Similarity Index & Mark & \multicolumn{1}{|c|}{ Peak Start } & \multicolumn{1}{c|}{ Peak End } & \multicolumn{1}{c|}{ Area\% } \\
\hline 1 & 7,927 & 45,556 & 1162549 & 45473 & 0,000000 & & 9,499 & 9,013 & 45,556 \\
\hline 2 & 9,654 & 54,444 & 1389363 & 42614 & 0,000000 & & 9,099 & 11,243 & 54,444 \\
\hline Total & & 100,000 & 2551911 & 88087 & & & & \\
\hline
\end{tabular}

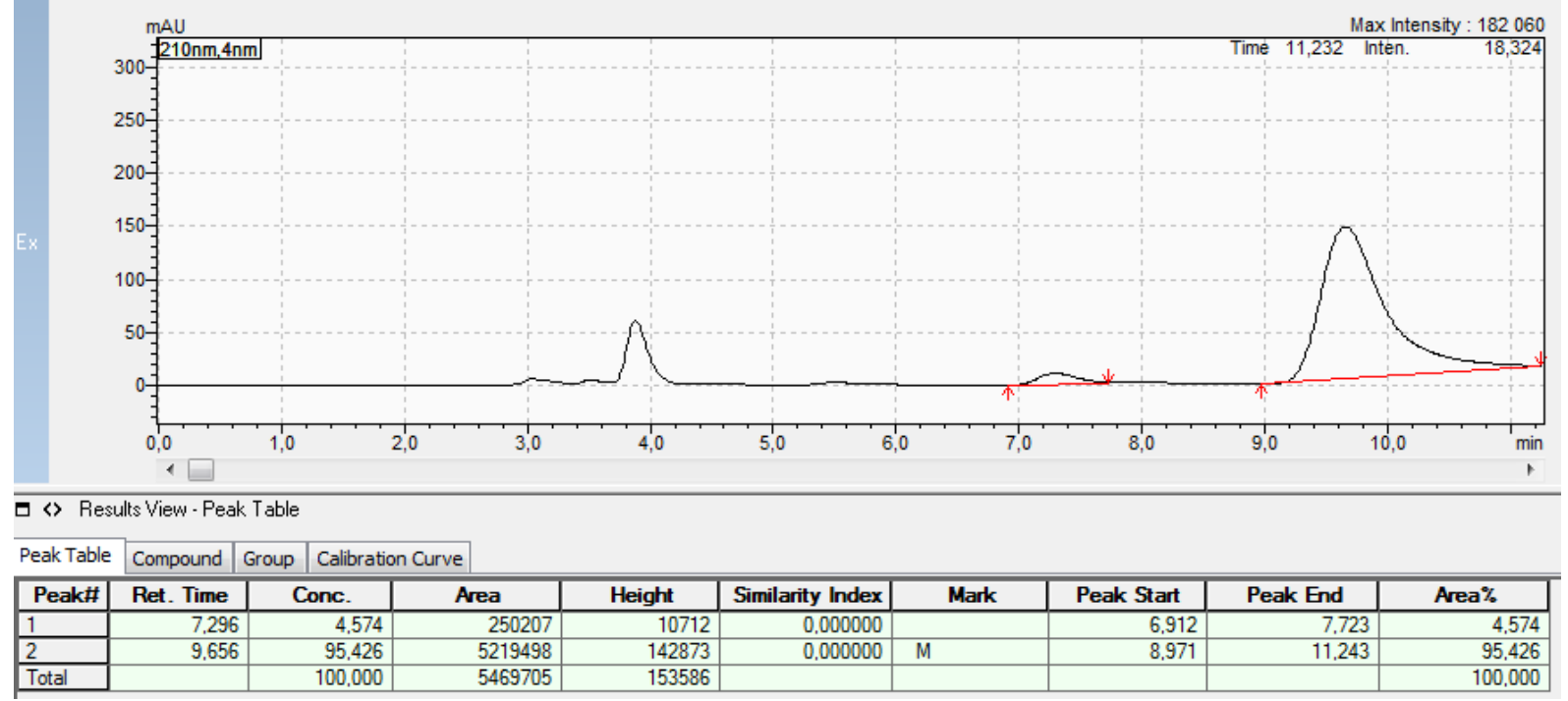

for 4p: $e e=91 \%$ 
<smiles>O=C1N(Cc2ccccc2)c2ccccc2[C@]12CC[C@H](CO)[C@H]2c1ccc(C(F)(F)F)cc1</smiles>

Conditions: IA column

mobile phase: heptane / $i$-PrOH $-80: 20$

$\lambda=217 \mathrm{~nm}, V=1.0 \mathrm{ml} / \mathrm{min}, t=25^{\circ} \mathrm{C}$

for 3q: $t_{\mathrm{R}}=9.0 \mathrm{~min}$ (major), $t_{\mathrm{R}}=9.7 \mathrm{~min}$ (minor).

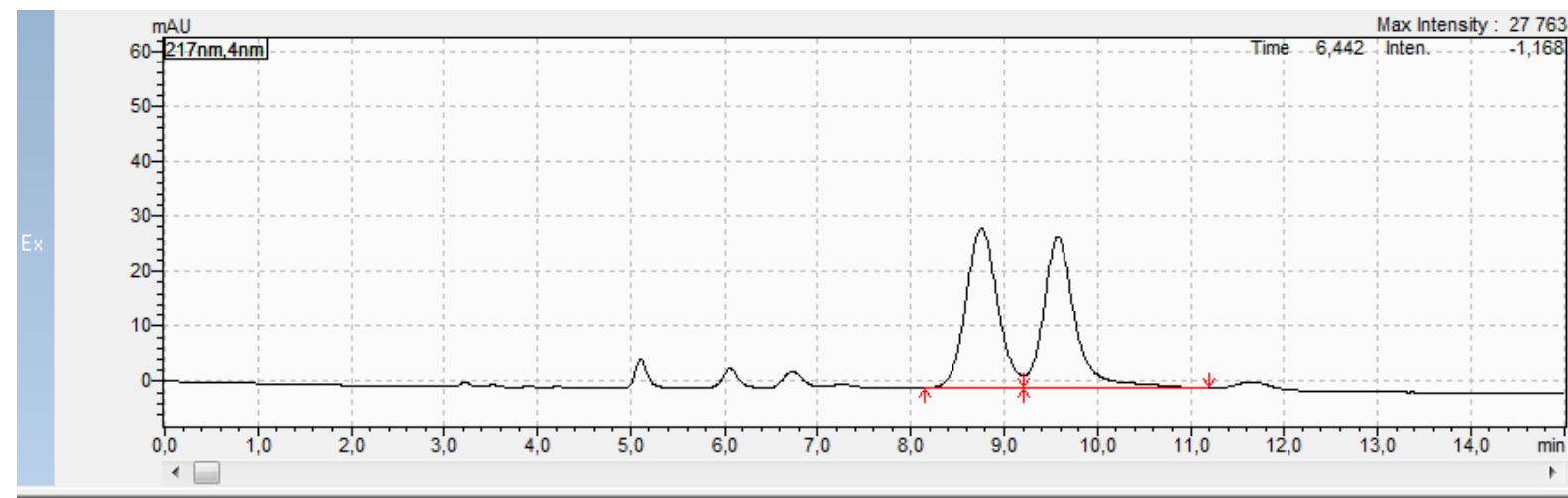

口 $\gg$ Results View - Peak Table

\begin{tabular}{|l|l|l|l|}
\hline Peak Table & Compound & Group & Calibration Curve \\
\hline
\end{tabular}

\begin{tabular}{|l|r|r|r|r|r|r|r|r|r|}
\hline Peak\# & Ret. Time & \multicolumn{1}{|c|}{ Conc. } & \multicolumn{1}{c|}{ Area } & Height & Similarity Index & Mark & \multicolumn{1}{c|}{ Peak Start } & \multicolumn{1}{c|}{ Peak End } & Area\% \\
\hline 1 & 8,758 & 50,842 & 678731 & 28931 & 0,000000 & & 8,149 & 9,205 & 50,842 \\
\hline 2 & 9,575 & 49,158 & 656245 & 27421 & 0,000000 & V & & 9,205 & 11,200 \\
\hline Total & & 100,000 & 1334976 & 56352 & & & & 49,158 \\
\hline
\end{tabular}

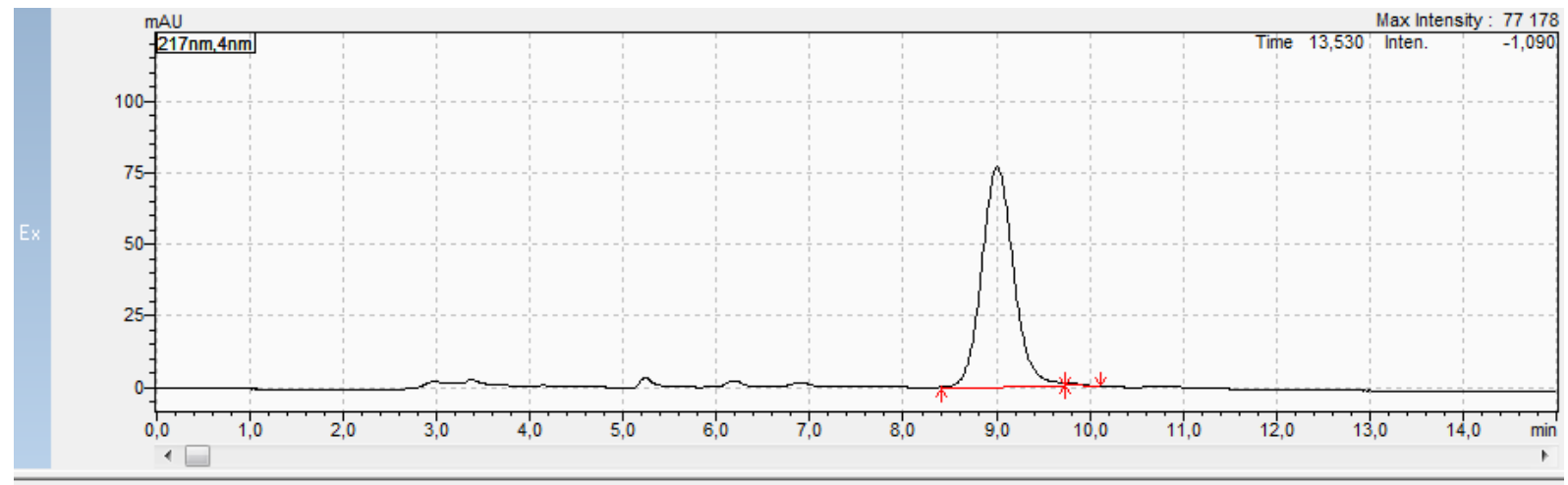

口〈 Results View - Peak Table

\begin{tabular}{|l|l|l|l|}
\hline Peak Table & Compound & Group & Calibration Curve \\
\hline
\end{tabular}

\begin{tabular}{|l|r|r|r|r|r|r|r|r|r|r|}
\hline Peak\# & Ret. Time & \multicolumn{1}{|c|}{ Conc. } & \multicolumn{1}{|c|}{ Area } & Height & Similarity Index & Mark & Peak Start & \multicolumn{1}{c|}{ Peak End } & Area\% \\
\hline 1 & 9,009 & 99,665 & 1820627 & 76886 & 0,000000 & M & 8,416 & 9,739 & 99,665 \\
\hline 2 & 9,744 & 0,335 & 6113 & 780 & 0,000000 & M & 9,739 & 10,112 \\
\hline Total & & 100,000 & 1826739 & 77666 & & & 0,335 \\
\hline
\end{tabular}

for 3q: $e e=99 \%$ 
<smiles>O=C[C@H]1CC[C@@]2(C(=O)N(Cc3ccccc3)c3ccccc32)[C@H]1c1ccc(C(F)(F)F)cc1</smiles>

Conditions: IC column

mobile phase: heptane / $i$-PrOH $-80: 20$

$\lambda=208 \mathrm{~nm}, V=1.0 \mathrm{ml} / \mathrm{min}, t=25^{\circ} \mathrm{C}$

for $\mathbf{4 q}: t_{\mathrm{R}}=9.0 \mathrm{~min}$ (major), $t_{\mathrm{R}}=10.0 \mathrm{~min}$ (minor).

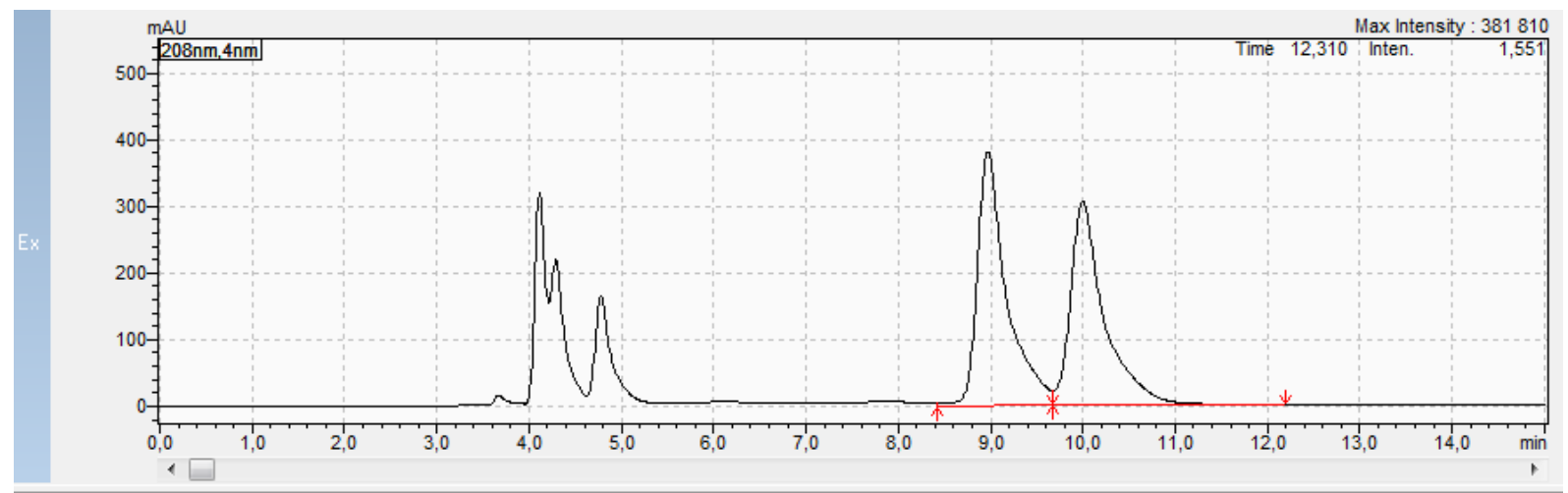

口〈 Results View - Peak Table

\begin{tabular}{|l|l|l|l|}
\hline Peak Table & Compound & Group & Calibration Curve \\
\hline
\end{tabular}

\begin{tabular}{|c|c|c|c|c|c|c|c|c|c|}
\hline Peak\# & Ret. Time & Conc. & Area & Height & Similarity Index & Mark & Peak Start & Peak End & Area\% \\
\hline 1 & 8,971 & 51,765 & 8389092 & 380717 & 0,000000 & & 8,416 & 9,675 & 51.765 \\
\hline 2 & 9,998 & 48,235 & 7816931 & 305644 & 0,000000 & SV & 9,675 & 12,192 & 48,235 \\
\hline Total & & 100,000 & 16206023 & 686361 & & & & & 100,000 \\
\hline
\end{tabular}

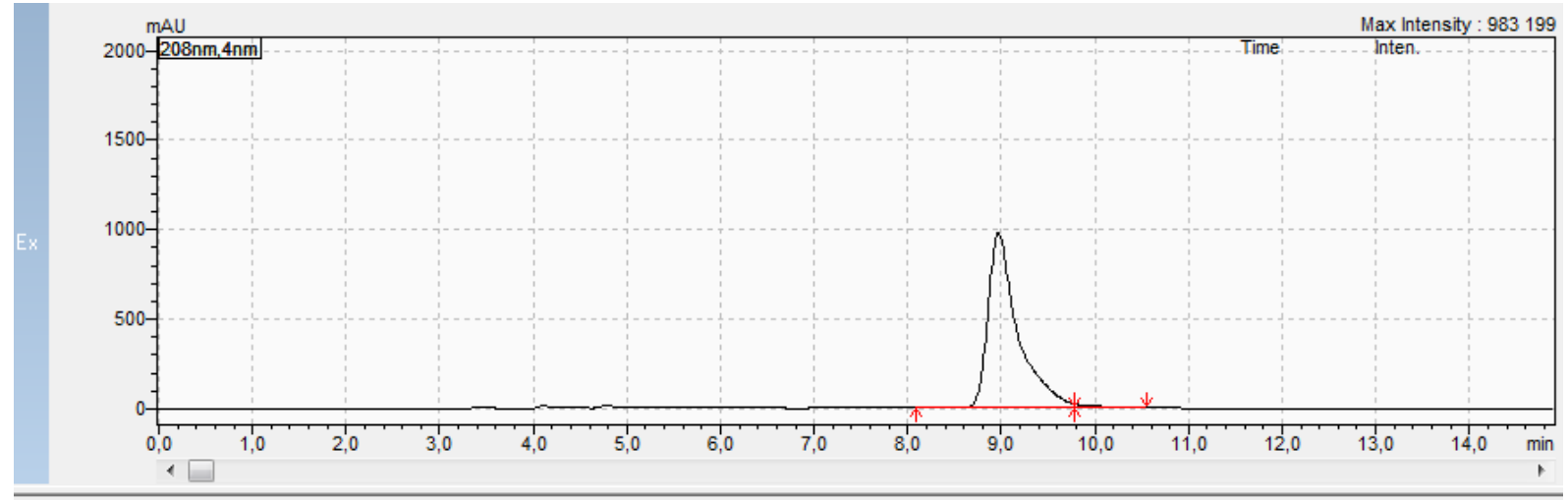

口〈R Results View - Peak Table

\begin{tabular}{|l|l|l|l|}
\hline Peak Table & Compound & Group & Calibration Curve \\
\hline
\end{tabular}

\begin{tabular}{|l|r|r|r|r|r|r|r|r|r|r|}
\hline Peak\# & Ret. Time & \multicolumn{1}{|c|}{ Conc. } & \multicolumn{1}{|c|}{ Area } & Height & Similarity Index & Mark & Peak Start & \multicolumn{1}{c|}{ Peak End } & Area\% \\
\hline 1 & 8,970 & 98,776 & 21683621 & 978440 & 0,000000 & M & 8,085 & 9,781 & 98,776 \\
\hline 2 & 9,797 & 1,224 & 268603 & 20203 & 0,000000 & M & 9,781 & 10,549 & 1,224 \\
\hline Total & & 100,000 & 21952225 & 998642 & & & & & \\
\hline
\end{tabular}

for $\mathbf{4 q}: e e=98 \%$ 
<smiles>O=C[C@H]1CC[C@]2(C(=O)N(Cc3ccccc3)c3ccccc32)[C@H]1c1ccc(F)cc1</smiles>

Conditions: IA column

mobile phase: heptane / $i$-PrOH $-80: 20$

$\lambda=218 \mathrm{~nm}, V=1.0 \mathrm{ml} / \mathrm{min}, t=25^{\circ} \mathrm{C}$

for 3r: $t_{\mathrm{R}}=9.3 \mathrm{~min}$ (minor), $t_{\mathrm{R}}=13.9 \mathrm{~min}$ (major).

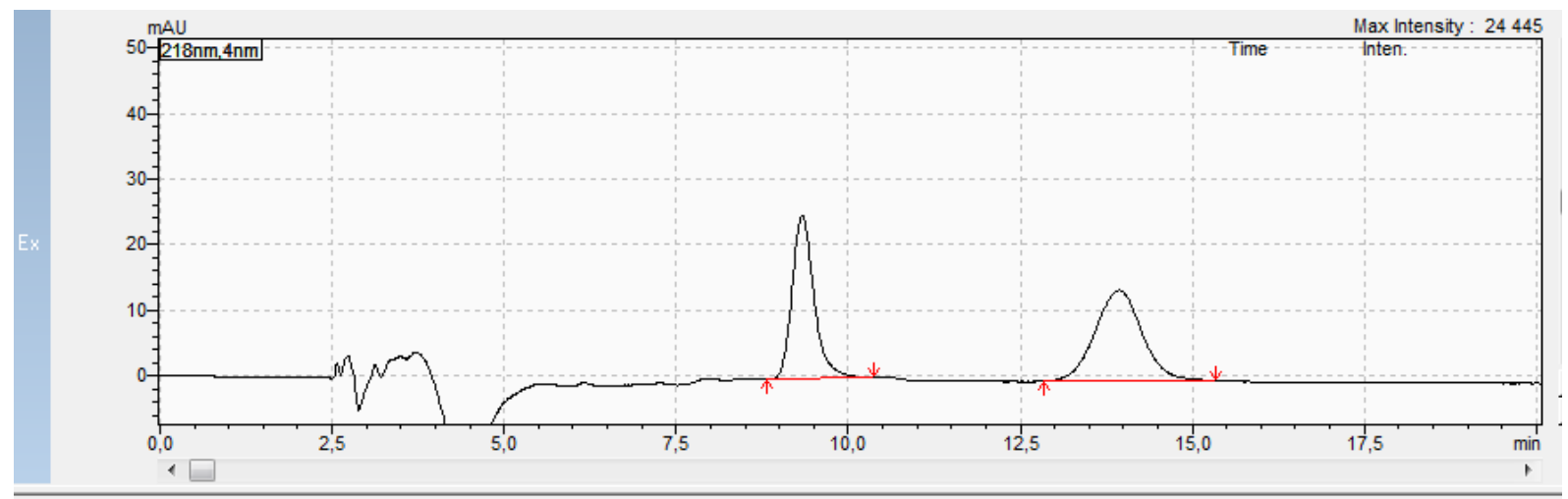

口〈 Results View - Peak Table

\begin{tabular}{|l|l|l|l|}
\hline Peak Table & Compound & Group & Calibration Curve \\
\hline
\end{tabular}

\begin{tabular}{|l|r|r|r|r|r|r|r|r|r|r|}
\hline Peak\# & Ret. Time & \multicolumn{1}{|c|}{ Conc. } & \multicolumn{1}{c|}{ Area } & Height & Similarity Index & Mark & Peak Start & Peak End & Area\% \\
\hline 1 & 9,331 & 45,807 & 555866 & 24923 & 0,000000 & & 8,821 & 10,379 & 45,807 \\
\hline 2 & 13,935 & 54,193 & 657627 & 13837 & 0,000000 & & 12,843 & 15,349 & 54,193 \\
\hline Total & & 100,000 & 1213493 & 38761 & & & & & \\
\hline
\end{tabular}

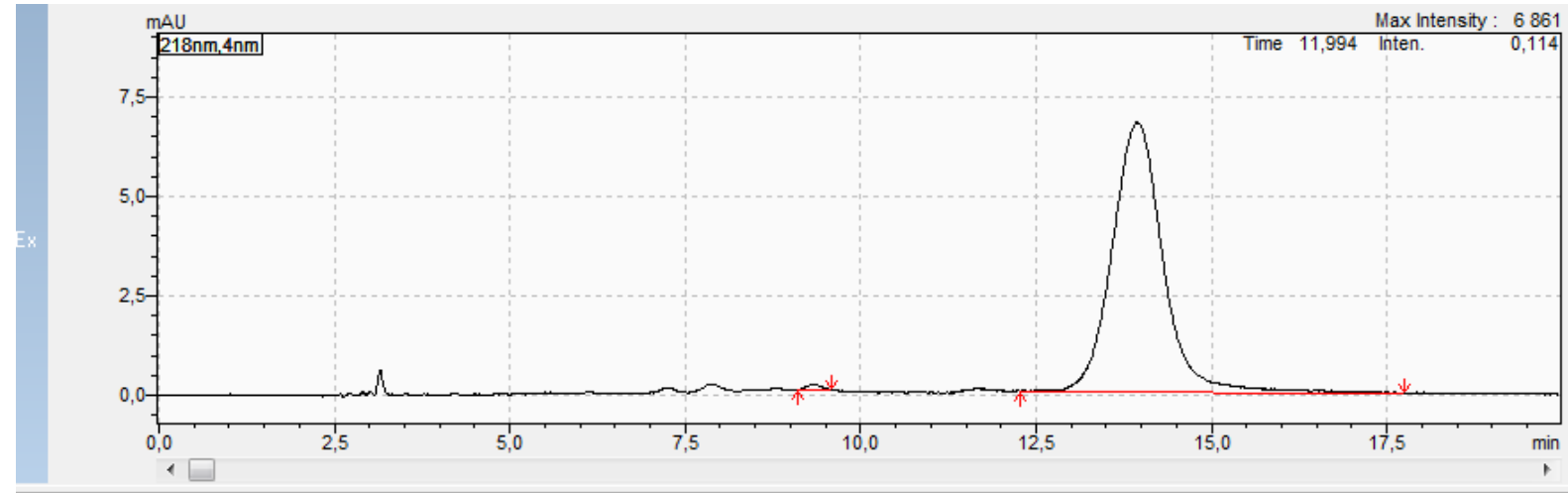

コ〈 Results View - Peak Table

\begin{tabular}{|l|l|l|l|}
\hline Peak Table & Compound & Group & Calibration Curve \\
\hline
\end{tabular}

\begin{tabular}{|l|r|r|r|r|r|r|r|r|r|}
\hline Peak\# & Ret. Time & \multicolumn{1}{|c|}{ Conc. } & \multicolumn{1}{c|}{ Area } & \multicolumn{1}{c|}{ Height } & Similarity Index & Mark & \multicolumn{1}{c|}{ Peak Start } & \multicolumn{1}{c|}{ Peak End } & \multicolumn{1}{c|}{ Area\% } \\
\hline 1 & 9,343 & 0,588 & 2011 & 133 & 0,000000 & & 9,099 & 9,579 & 0,588 \\
\hline 2 & 13,940 & 99,412 & 339814 & 6774 & 0,000000 & M & 12,277 & 17,760 & 99,412 \\
\hline Total & & 100,000 & 341825 & 6907 & & & & \\
\hline
\end{tabular}

for 3r: $e e=99 \%$ 
<smiles>O=C[C@H]1CC[C@]2(C(=O)N(Cc3ccccc3)c3ccccc32)[C@H]1c1ccc(F)cc1</smiles>

Conditions: IA column

mobile phase: heptane / $i$-PrOH $-80: 20$

$\lambda=208 \mathrm{~nm}, V=1.0 \mathrm{ml} / \mathrm{min}, t=25^{\circ} \mathrm{C}$

for 4r: $t_{\mathrm{R}}=10.4 \mathrm{~min}$ (major), $t_{\mathrm{R}}=13.5 \mathrm{~min}$ (minor).

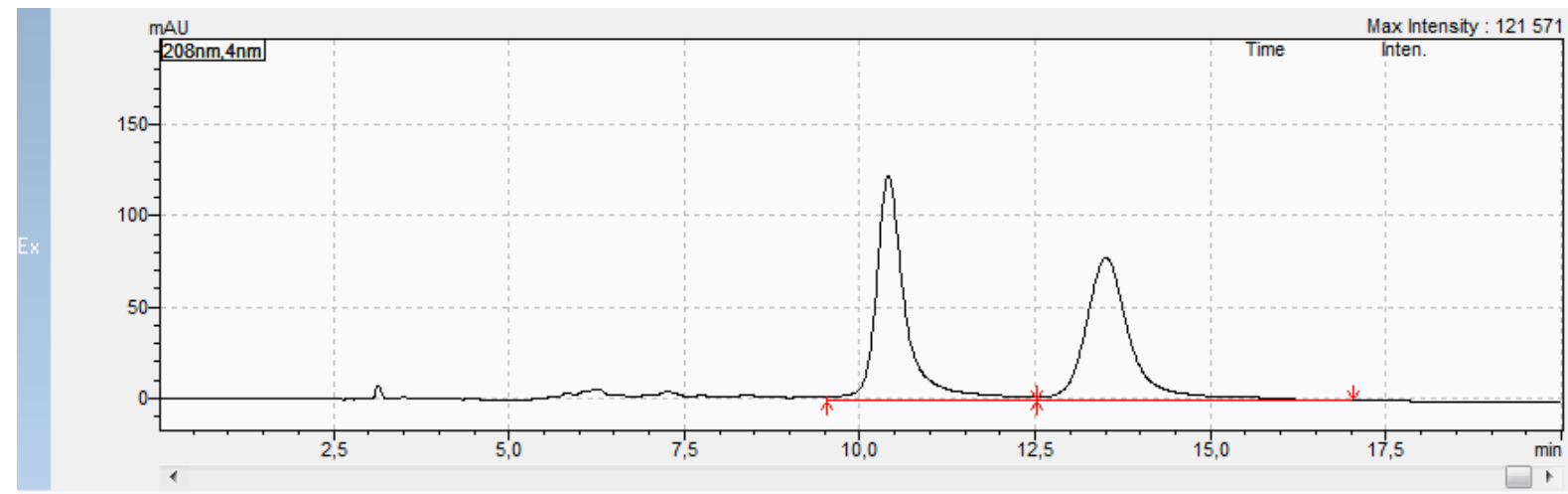

- 〈 Results View - Peak Table

\begin{tabular}{l|l|l|l|}
\hline Peak Table & Compound & Group & Calibration Curve \\
\hline
\end{tabular}

\begin{tabular}{|l|r|r|r|r|r|r|r|r|r|}
\hline Peak\#\# & Ret. Time & \multicolumn{1}{|c|}{ Conc. } & \multicolumn{1}{c|}{ Area } & \multicolumn{1}{c|}{ Height } & Similarity Index & Mark & \multicolumn{1}{c|}{ Peak Start } & Peak End & \multicolumn{1}{c|}{ Area\% } \\
\hline 1 & 10,408 & 51,054 & 3585404 & 122587 & 0,000000 & & 9,536 & 12,533 & 51,054 \\
\hline 2 & 13,511 & 48,946 & 3437408 & 78068 & 0,000000 & SV & 12,533 & 17,035 & \\
\hline Total & & 100,000 & 7022811 & 200655 & & & & 48,946 \\
\hline
\end{tabular}

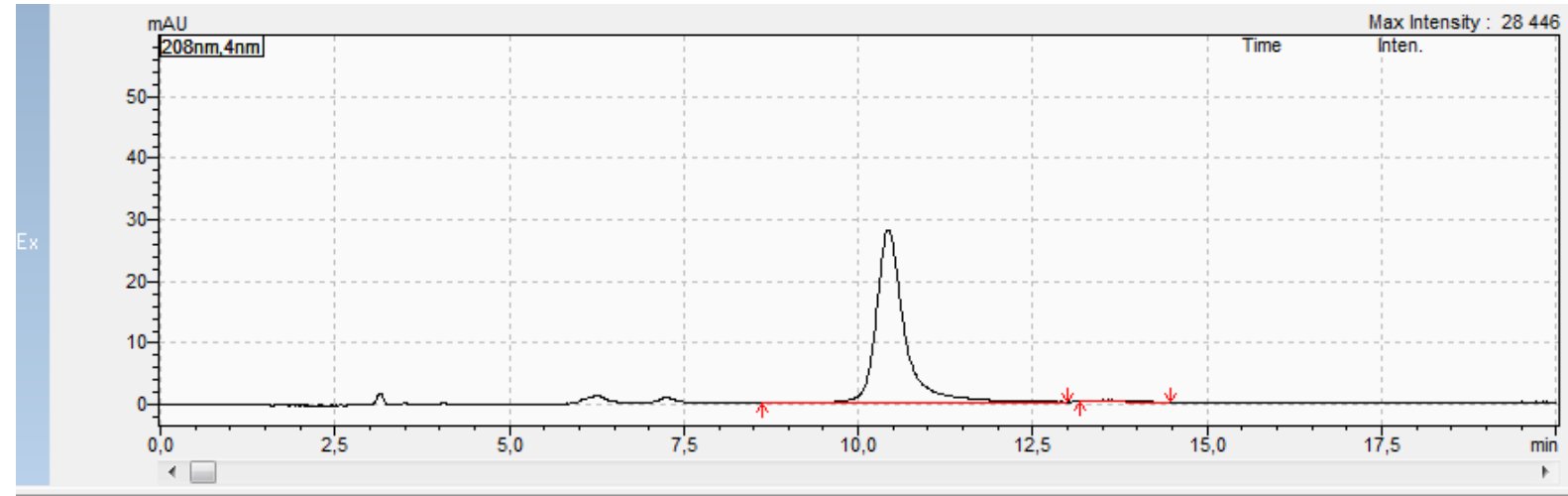

口〈Results View - Peak Table

\begin{tabular}{l|l|l|l|}
\hline Peak Table & Compound & Group & Calibration Curve \\
\hline
\end{tabular}

\begin{tabular}{|l|r|r|r|r|r|r|r|r|r|}
\hline Peak\# & Ret. Time & \multicolumn{1}{|c|}{ Conc. } & \multicolumn{1}{c|}{ Area } & \multicolumn{1}{c|}{ Height } & Similarity Index & Mark & \multicolumn{1}{c|}{ Peak Start } & Peak End & \multicolumn{1}{c|}{ Area\% } \\
\hline 1 & 10,431 & 98,612 & 773475 & 28026 & 0,000000 & M & 8,619 & 12,992 & 98,612 \\
\hline 2 & 13,507 & 1,388 & 10889 & 276 & 0,000000 & M & 13,173 & 14,464 & 1,388 \\
\hline Total & & 100,000 & 784365 & 28302 & & & & \\
\hline
\end{tabular}

for 4r: $e e=97 \%$ 
<smiles>O=C[C@H]1CC[C@]2(C(=O)N(Cc3ccccc3)c3ccccc32)C1c1ccc(Cl)cc1</smiles>

Conditions: IA column

mobile phase: heptane / $i$-PrOH $-80: 20$

$\lambda=206 \mathrm{~nm}, V=1.0 \mathrm{ml} / \mathrm{min}, t=25^{\circ} \mathrm{C}$

for 3s: $t_{\mathrm{R}}=10.2 \mathrm{~min}$ (minor), $t_{\mathrm{R}}=14.3 \mathrm{~min}$ (major).

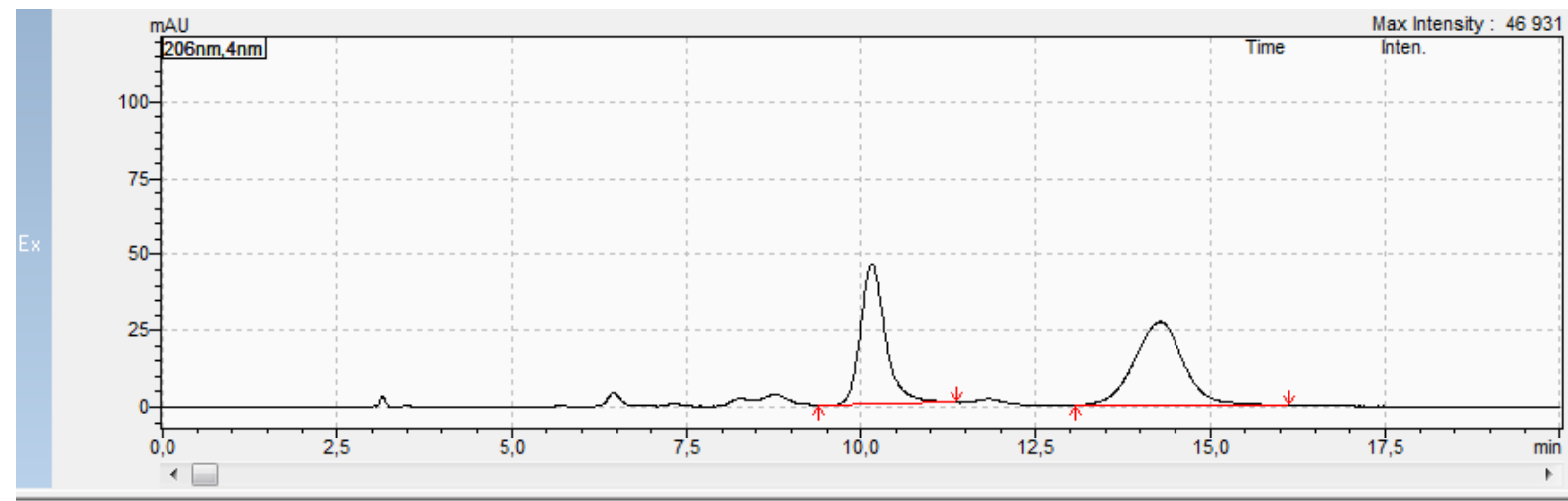

口〈R Results View - Peak Table

\begin{tabular}{l|l|l|l|}
\hline Peak Table & Compound & Group & Calibration Curve \\
\hline
\end{tabular}

\begin{tabular}{|l|r|r|r|r|r|r|r|r|r|r|}
\hline Peak\# & Ret. Time & \multicolumn{1}{|c|}{ Conc. } & \multicolumn{1}{c|}{ Area } & \multicolumn{1}{c|}{ Height } & Similarity Index & Mark & \multicolumn{1}{c|}{ Peak Start } & Peak End & \multicolumn{1}{c|}{ Area\% } \\
\hline 1 & 10,160 & 45,963 & 1129800 & 45940 & 0,000000 & M & 9,397 & 11,371 & 45,963 \\
\hline 2 & 14,276 & 54,037 & 1328246 & 27154 & 0,000000 & SV & 13,067 & 16,128 & 54,037 \\
\hline Total & & 100,000 & 2458046 & 73093 & & & & & 100,000 \\
\hline
\end{tabular}

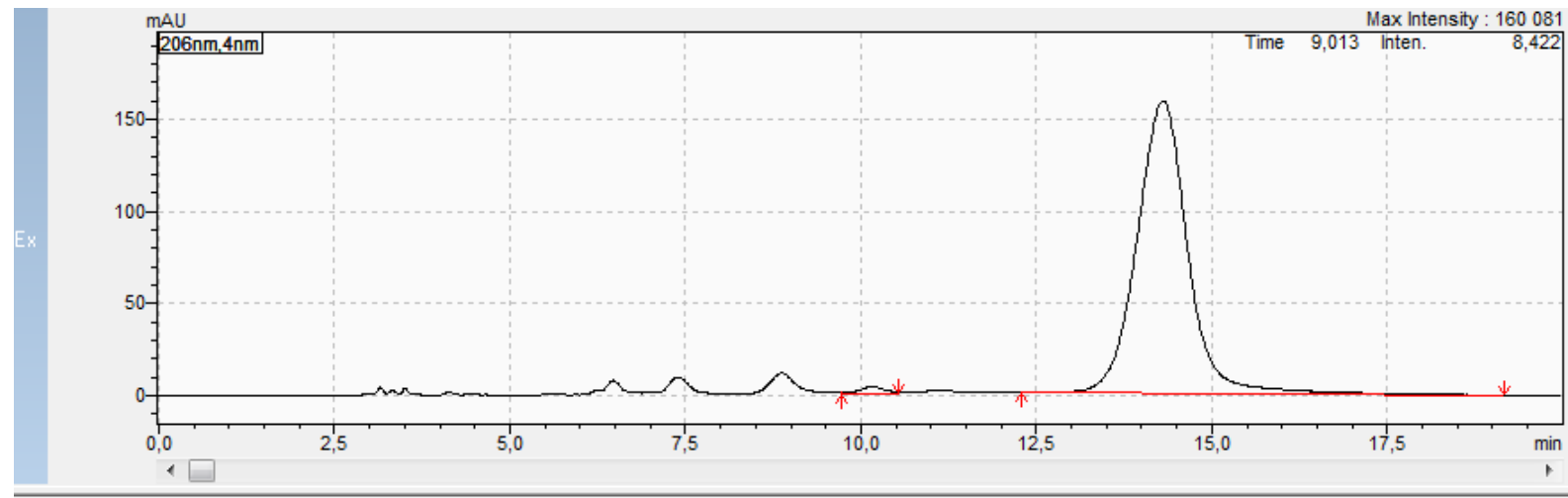

口〈 Riesults View - Peak Table

\begin{tabular}{|l|l|l|l|}
\hline Peak Table & Compound & Group & Calibration Curve \\
\hline
\end{tabular}

\begin{tabular}{|l|r|r|r|r|r|r|r|r|r|}
\hline Peak\# & Ret. Time & \multicolumn{1}{c|}{ Conc. } & \multicolumn{1}{c|}{ Area } & \multicolumn{1}{c|}{ Height } & Similarity Index & Mark & \multicolumn{1}{c|}{ Peak Start } & \multicolumn{1}{c|}{ Peak End } & \multicolumn{1}{c|}{ Area\% } \\
\hline 1 & 10,165 & 1,069 & 86631 & 3508 & 0,000000 & & 9,728 & 1,069 \\
\hline 2 & 14,308 & 98,931 & 8016912 & 158778 & 0,000000 & M & 12,277 & 19,168 & 98,931 \\
\hline Total & & 100,000 & 8103543 & 162287 & & & & & 100,000 \\
\hline
\end{tabular}

for 3s: $e e=97 \%$ 
<smiles>O=C[C@H]1CC[C@]2(C(=O)N(Cc3ccccc3)c3ccccc32)C1c1ccc(Cl)cc1</smiles>

Conditions: IA column

mobile phase: heptane / $i$-PrOH $-80: 20$

$\lambda=206 \mathrm{~nm}, V=1.0 \mathrm{ml} / \mathrm{min}, t=25^{\circ} \mathrm{C}$

for $4 \mathrm{~s}: t_{\mathrm{R}}=11.1 \mathrm{~min}$ (major), $t_{\mathrm{R}}=12.4 \mathrm{~min}$ (minor).

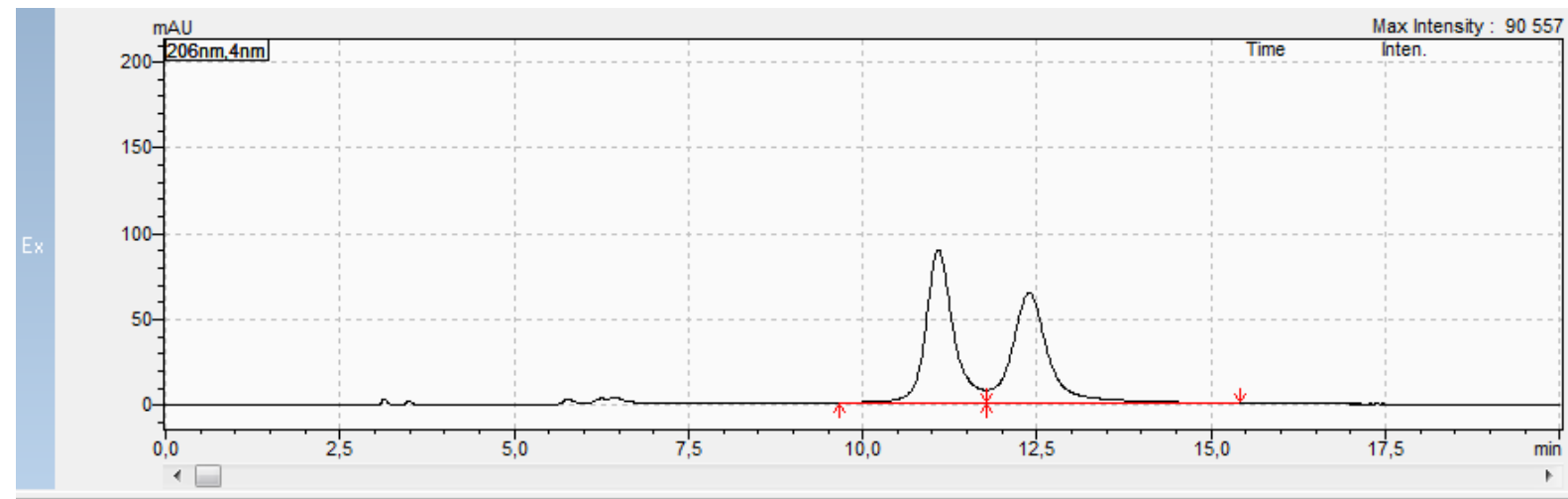

口〈 Results View - Peak Table

\begin{tabular}{|l|l|l|l|}
\hline Peak Table & Compound & Group & Calibration Curve \\
\hline
\end{tabular}

\begin{tabular}{|l|r|r|r|r|r|r|r|r|r|r|}
\hline Peak\# & Ret. Time & \multicolumn{1}{|c|}{ Conc. } & \multicolumn{1}{c|}{ Area } & Height & Similarity Index & Mark & Peak Start & Peak End & Area\% \\
\hline 1 & 11,083 & 51,500 & 2503160 & 89309 & 0,000000 & & 9,664 & 11,787 & 51,500 \\
\hline 2 & 12,389 & 48,500 & 2357336 & 64320 & 0,000000 & SV & 11,787 & 15,413 \\
\hline Total & & 100,000 & 4860496 & 153629 & & & & 48,500 \\
\hline
\end{tabular}

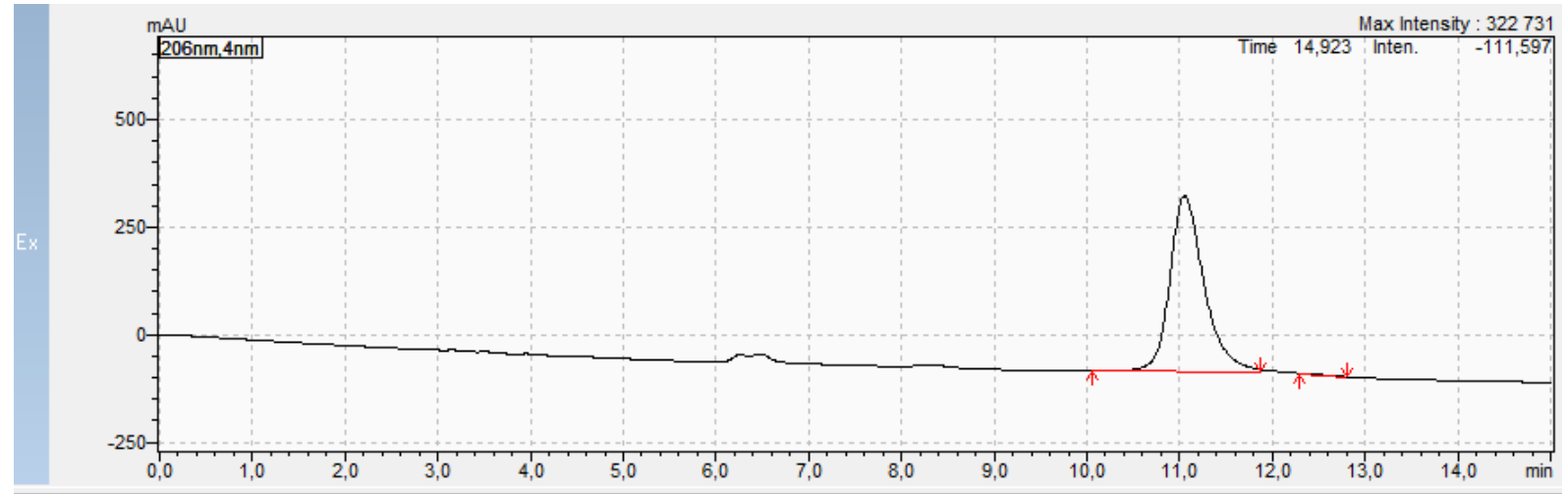

口〈R Results View - Peak Table

\begin{tabular}{|l|l|l|}
\hline Peak Table Compound & Group & Calibration Curve \\
\hline
\end{tabular}

\begin{tabular}{|l|r|r|r|r|r|r|r|r|r|}
\hline Peak\# & Ret. Time & \multicolumn{1}{|c|}{ Conc. } & \multicolumn{1}{|c|}{ Area } & \multicolumn{1}{c|}{ Height } & Similarity Index & Mark & Peak Start & \multicolumn{1}{c|}{ Peak End } & \multicolumn{1}{c|}{ Area\% } \\
\hline 1 & 11,054 & 99,985 & 10466194 & 408036 & 0,000000 & M & 10,059 & 11,872 & 99,985 \\
\hline 2 & 12,304 & 0,015 & 1605 & 82 & 0,000000 & M & 12,288 & 12,800 & 0,015 \\
\hline Total & & 100,000 & 10467799 & 408119 & & & & & \\
\hline
\end{tabular}

for 4s: $e e=99 \%$ 
<smiles>O=C[C@H]1CC[C@]2(C(=O)N(Cc3ccccc3)c3ccccc32)[C@H]1c1ccc(Br)cc1</smiles>

Conditions: IA column

mobile phase: heptane / $i$-PrOH $-80: 20$

$\lambda=206 \mathrm{~nm}, V=1.0 \mathrm{ml} / \mathrm{min}, t=25^{\circ} \mathrm{C}$

for 3t: $t_{\mathrm{R}}=11.0 \mathrm{~min}$ (minor), $t_{\mathrm{R}}=14.3 \mathrm{~min}$ (major).

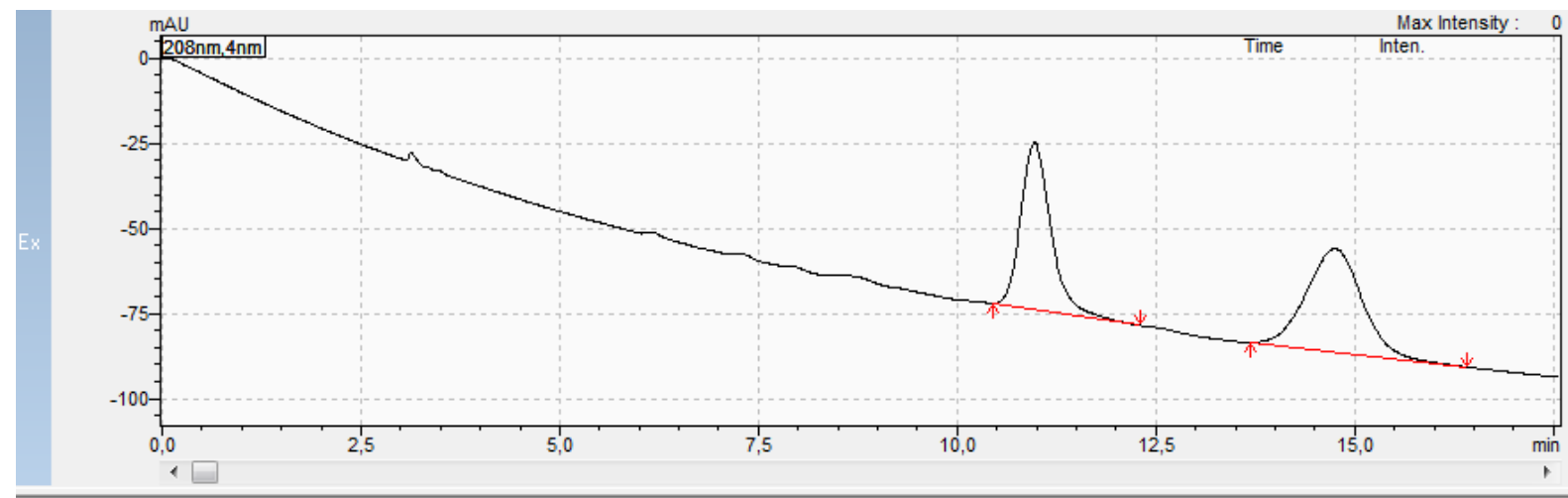

〈 Results View - Peak Table

\begin{tabular}{|l|l|l|l|}
\hline Peak Table & Compound & Group & Calibration Curve \\
\hline
\end{tabular}

\begin{tabular}{|l|r|r|r|r|r|r|r|r|r|}
\hline Peak\# & Ret. Time & \multicolumn{1}{|c|}{ Conc. } & \multicolumn{1}{c|}{ Area } & Height & Similarity Index & Mark & \multicolumn{1}{|c|}{ Peak Start } & Peak End & \multicolumn{1}{c|}{ Area\% } \\
\hline 1 & 10,972 & 46,595 & 1299137 & 49148 & 0,000000 & & 10,453 & 12,309 & 46,595 \\
\hline 2 & 14,745 & 53,405 & 1489012 & 30336 & 0,000000 & & 13,685 & 16,416 \\
\hline Total & & 100,000 & 2788149 & 79484 & & & & & \\
\hline
\end{tabular}

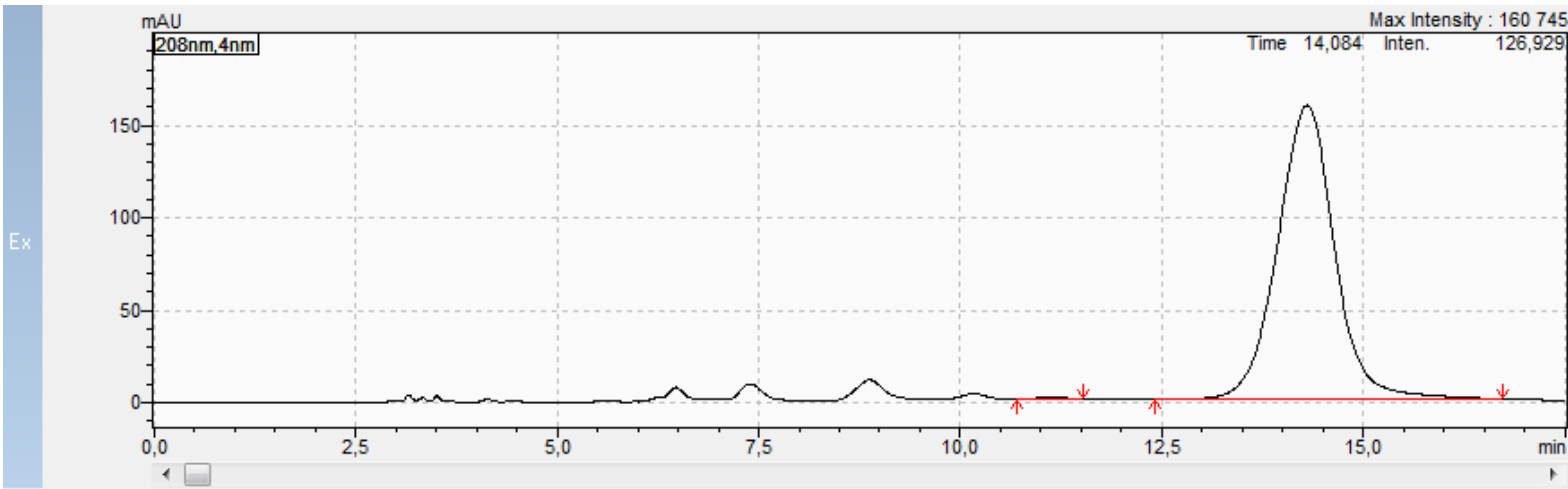

口〈 Results View - Peak Table

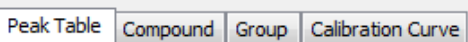

\begin{tabular}{|l|r|r|r|r|r|r|r|r|r|r|}
\hline Peak\# & Ret. Time & \multicolumn{1}{c|}{ Conc. } & \multicolumn{1}{c|}{ Area } & \multicolumn{1}{c|}{ Height } & Similarity Index & Mark & Peak Start & \multicolumn{1}{c|}{ Peak End } & \multicolumn{1}{c|}{ Area\% } \\
\hline 1 & 11,144 & 0,391 & 30707 & 1474 & 0,000000 & M & 10,699 & 11,531 & 0,391 \\
\hline 2 & 14,307 & 99,609 & 7817143 & 158746 & 0,000000 & M & 12,405 & 16,736 \\
\hline Total & & 100,000 & 7847851 & 160221 & & & 99,609 \\
\hline
\end{tabular}

for 3t: $e e=99 \%$ 
<smiles>O=C[C@H]1CC[C@]2(C(=O)N(Cc3ccccc3)c3ccccc32)[C@H]1c1ccc(Br)cc1</smiles>

Conditions: IB column

mobile phase: heptane / $i$-PrOH $-80: 20$

$\lambda=207 \mathrm{~nm}, V=1.0 \mathrm{ml} / \mathrm{min}, t=25^{\circ} \mathrm{C}$

for $4 \mathrm{t}: t_{\mathrm{R}}=8.3 \mathrm{~min}$ (minor), $t_{\mathrm{R}}=9.7 \mathrm{~min}$ (major).

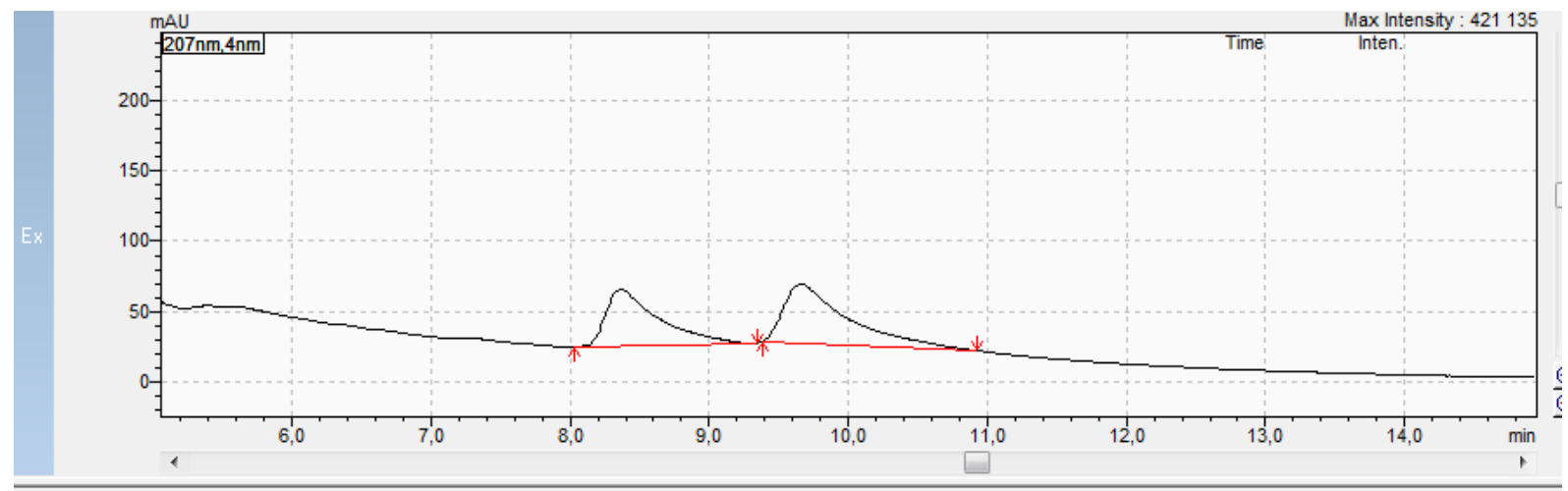

口〈 Results View - Peak Table

\begin{tabular}{l|l|l|l|}
\hline Peak Table & Compound & Group & Calibration Curve \\
\hline
\end{tabular}

\begin{tabular}{|l|r|r|r|r|r|r|r|r|r|r|}
\hline Peak\# & Ret. Time & \multicolumn{1}{|c|}{ Conc. } & \multicolumn{1}{|c|}{ Area } & \multicolumn{1}{c|}{ Height } & Similarity Index & Mark & Peak Start & \multicolumn{1}{c|}{ Peak End } & \multicolumn{1}{c|}{ Area\% } \\
\hline 1 & 8,364 & 43,616 & 1024690 & 39789 & 0,000000 & M & 8,032 & 9,344 \\
\hline 2 & 9,658 & 56,384 & 1324657 & 41428 & 0,000000 & M & 43,616 \\
\hline Total & & 100,000 & 2349346 & 81218 & & & 9,387 & 10,923 & 56,384 \\
\hline
\end{tabular}

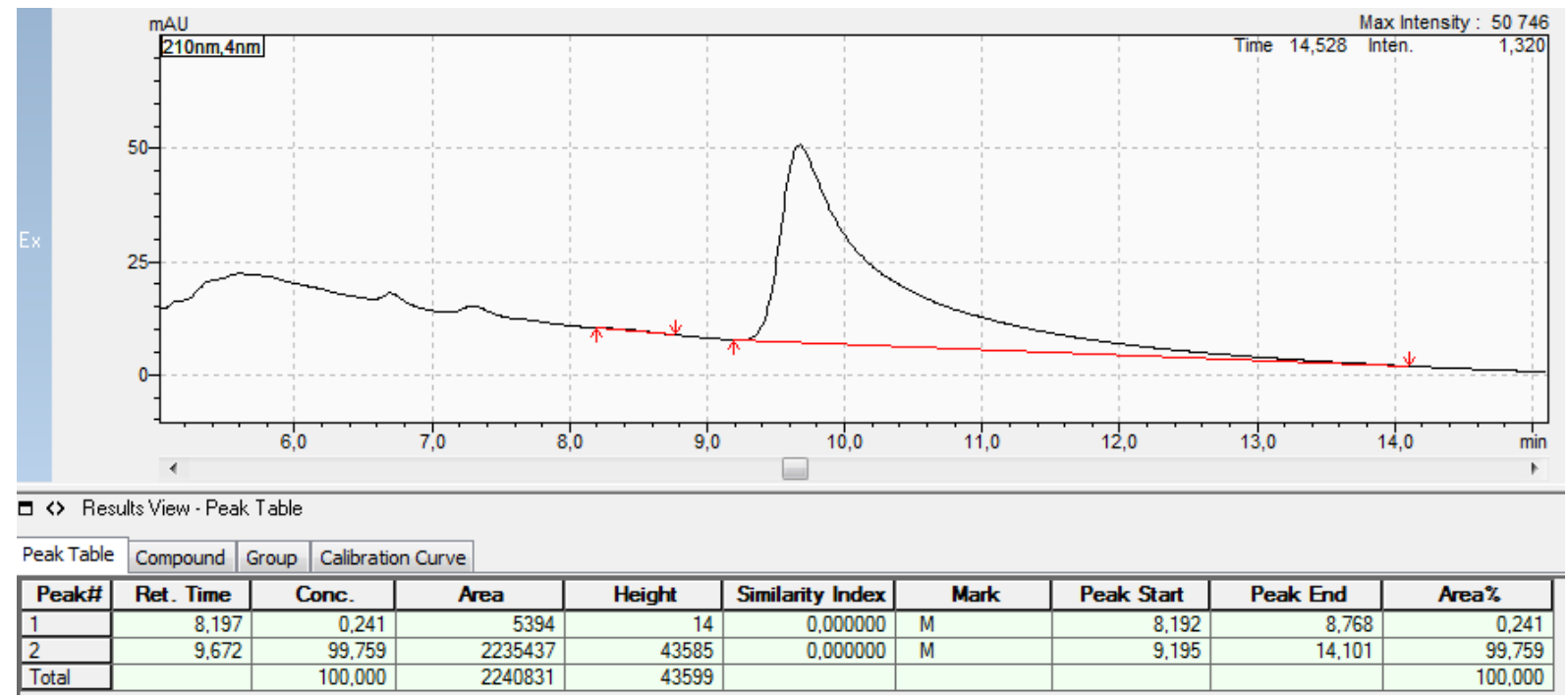

for $\mathbf{4 t}: e e=99 \%$ 


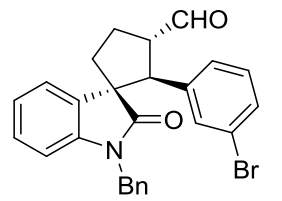

Conditions: IA column

mobile phase: heptane / $i$-PrOH $-80: 20$

$\lambda=214 \mathrm{~nm}, V=1.0 \mathrm{ml} / \mathrm{min}, t=25^{\circ} \mathrm{C}$

for $3 \mathrm{u}: t_{\mathrm{R}}=8.5 \mathrm{~min}$ (minor), $t_{\mathrm{R}}=20.7 \mathrm{~min}$ (major).

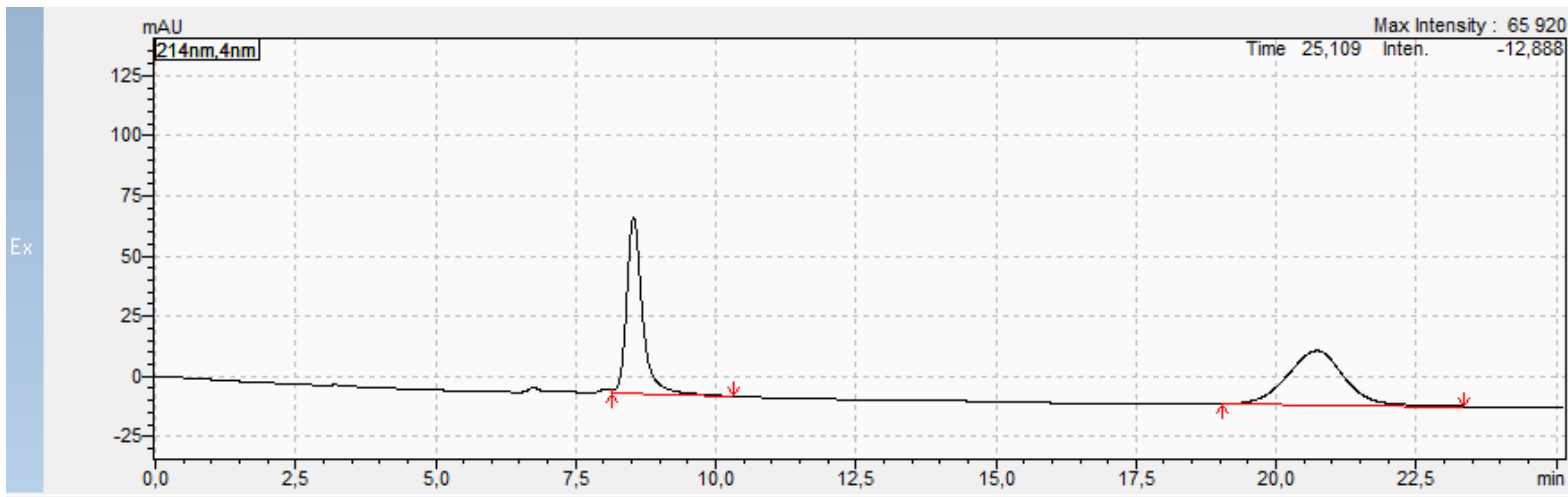

- $<$ Results View - Peak Table

\begin{tabular}{|l|l|l|l|}
\hline Peak Table Compound & Group & Calibration Curve \\
\hline
\end{tabular}

\begin{tabular}{|l|r|r|r|r|r|r|r|r|r|}
\hline Peak\# & Ret. Time & \multicolumn{1}{|c|}{ Conc. } & \multicolumn{1}{c|}{ Area } & Height & Similarity Index & Mark & Peak Start & \multicolumn{1}{c|}{ Peak End } & \multicolumn{1}{c|}{ Area\% } \\
\hline 1 & 8,538 & 47,938 & 1467608 & 73317 & 0,000000 & & 8,160 & 10,315 & 47,938 \\
\hline 2 & 20,731 & 52,062 & 1593861 & 22591 & 0,000000 & M & 19,040 & 23,360 \\
\hline Total & & 100,000 & 3061469 & 95909 & & & 52,062 \\
\hline
\end{tabular}

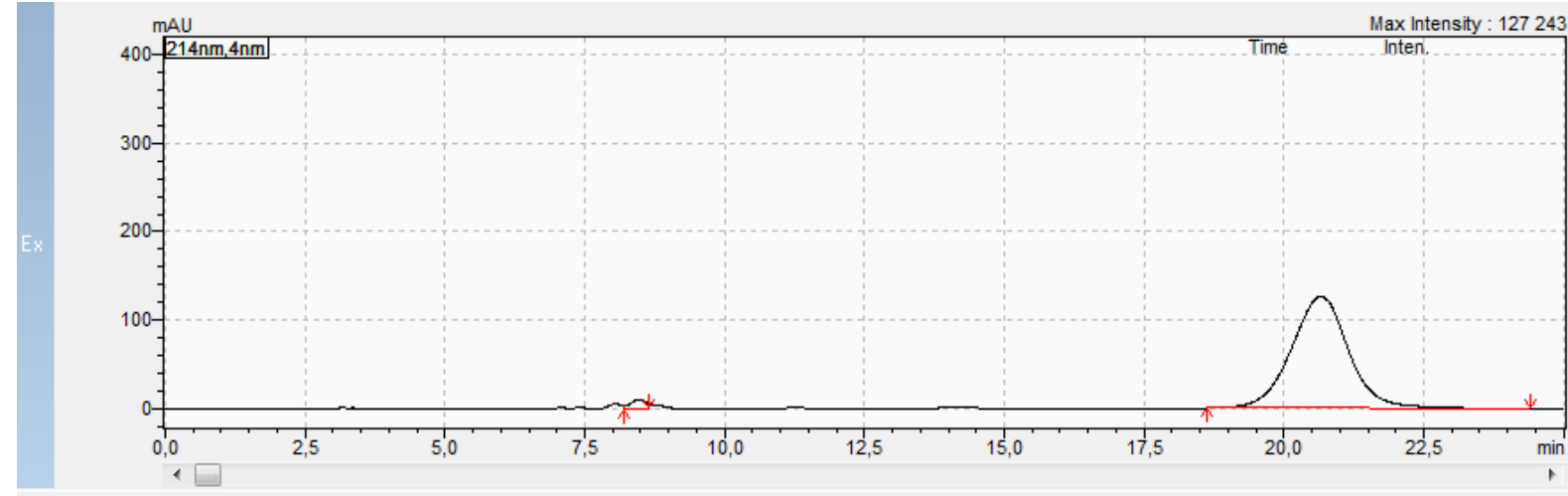

- $く$ Results View - Peak Table

\begin{tabular}{l|l|l|l|}
\hline Peak Table Compound & Group & Calibration Curve \\
\hline
\end{tabular}

\begin{tabular}{|c|c|c|c|c|c|c|c|c|c|}
\hline Peak\# & Ret. Time & Conc. & Area & Height & Similarity Index & Mark & Peak Start & Peak End & Area\% \\
\hline 1 & 8.464 & 2,419 & 223771 & 11396 & 0,000000 & $M$ & 8,192 & 8,651 & 2,419 \\
\hline 2 & 20,663 & 97,581 & 9026475 & 126778 & 0,000000 & & 18,635 & 24,405 & 97,581 \\
\hline Total & & 100,000 & 9250246 & 138174 & & & & & 100,000 \\
\hline
\end{tabular}

for $3 \mathbf{u}: e e=95 \%$ 

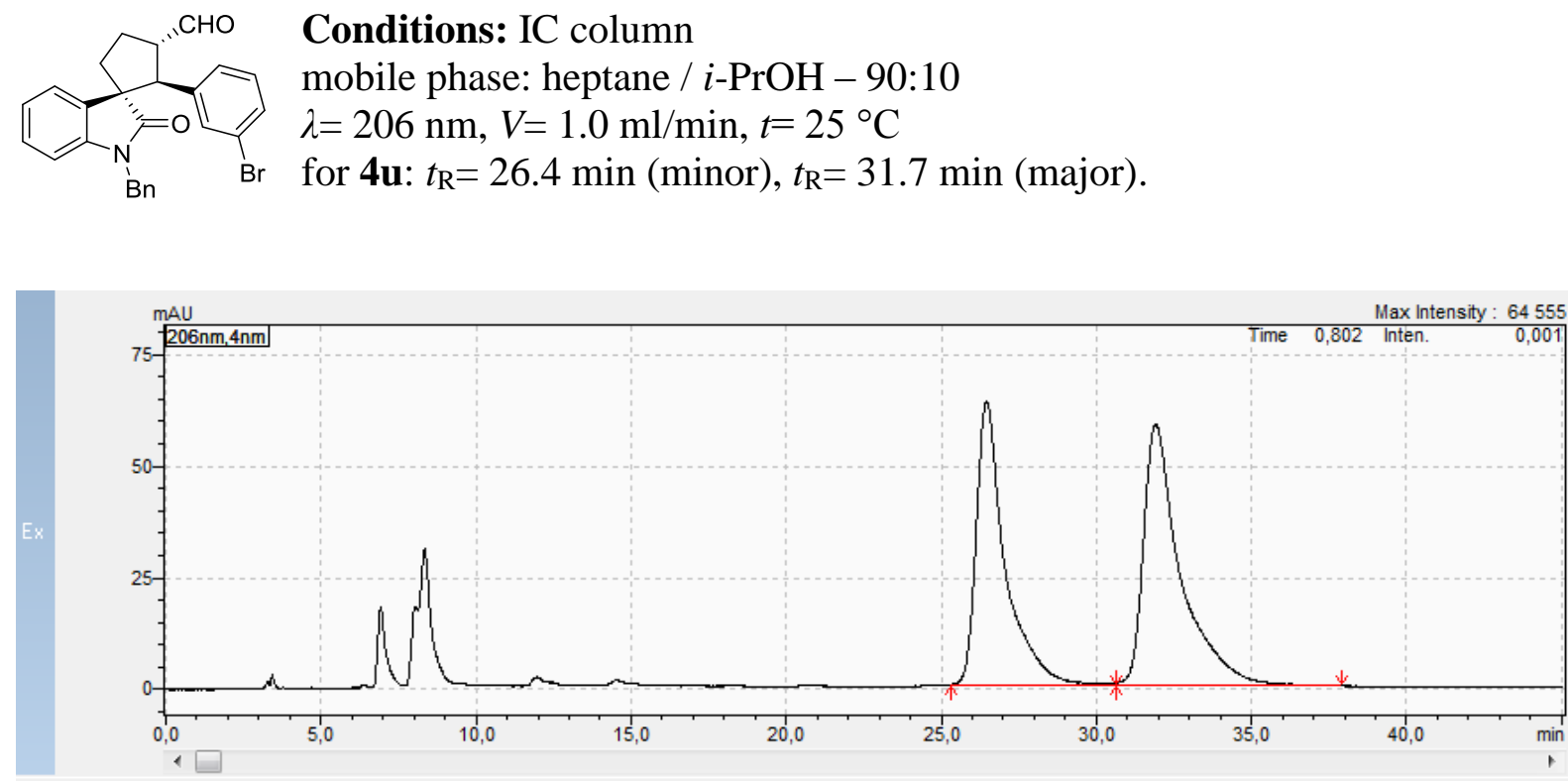

\begin{tabular}{|c|c|c|c|c|c|c|c|c|c|}
\hline Peak Table & Compound & Calibratio & & & & & & & \\
\hline Peak\# & Ret. Time & Conc. & Area & Height & Similarity Index & Mark & Peak Start & Peak End & Area\% \\
\hline 1 & 26,467 & 46,009 & 4163666 & 63733 & 0,000000 & $S$ & 25,291 & 30,624 & 46,009 \\
\hline 2 & 31,907 & 53,991 & 4885992 & 58552 & 0,000000 & SV & 30,624 & 37,941 & 53,991 \\
\hline Total & & 100,000 & 9049658 & 122285 & & & & & 100,000 \\
\hline
\end{tabular}

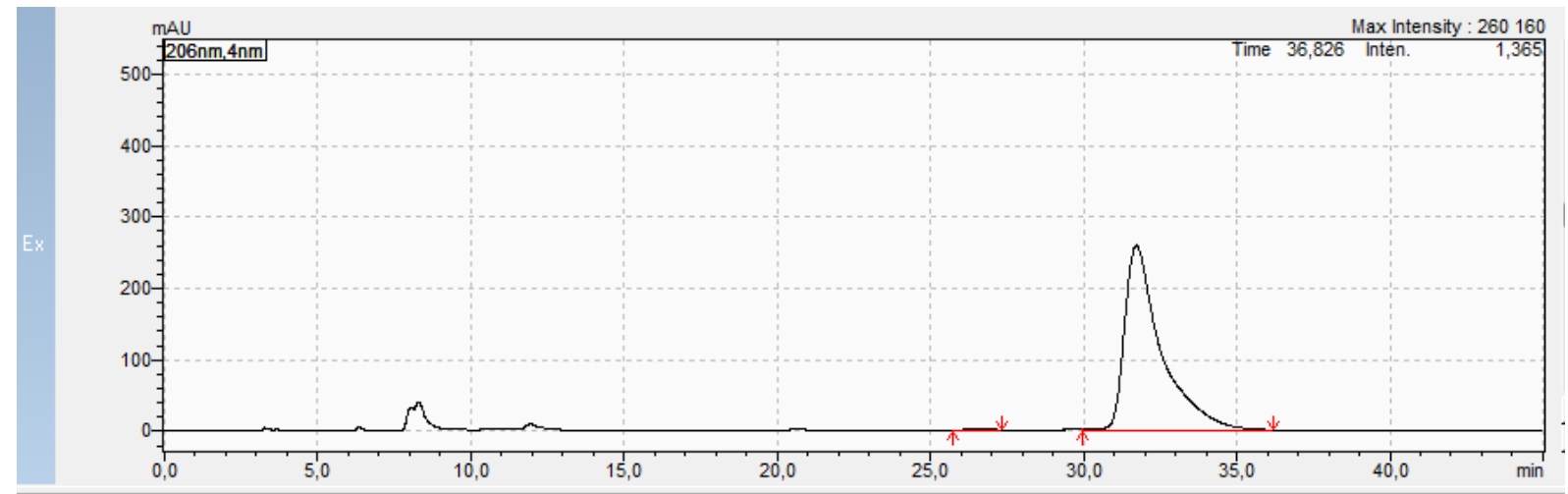

口〈R Results View - Peak Table

\begin{tabular}{|l|l|l|l|}
\hline Peak Table & Compound & Group & Calibration Curve \\
\hline
\end{tabular}

\begin{tabular}{|l|r|r|r|r|r|r|r|r|r|r|r|}
\hline Peak\# & Ret. Time & \multicolumn{1}{|c|}{ Conc. } & \multicolumn{1}{|c|}{ Area } & \multicolumn{1}{c|}{ Height } & Similarity Index & Mark & Peak Start & \multicolumn{1}{c|}{ Peak End } & Area\% \\
\hline 1 & 26,437 & 0,407 & 87597 & 1958 & 0,000000 & M & 25,728 & 27,328 & 0,407 \\
\hline 2 & 31,722 & 99,593 & 21421190 & 258416 & 0,000000 & S & 29,973 & 36,224 & 99,593 \\
\hline Total & & 100,000 & 21508787 & 260375 & & & & \\
\hline
\end{tabular}

for $4 \mathbf{u}: e e=99 \%$ 
(N)

Conditions: IA column

mobile phase: heptane / $i$ - $\mathrm{PrOH}-80: 20$

$\lambda=213 \mathrm{~nm}, V=1.0 \mathrm{ml} / \mathrm{min}, t=25^{\circ} \mathrm{C}$

for 3v: $t_{\mathrm{R}}=8.8 \mathrm{~min}$ (minor), $t_{\mathrm{R}}=18.0 \mathrm{~min}$ (major).

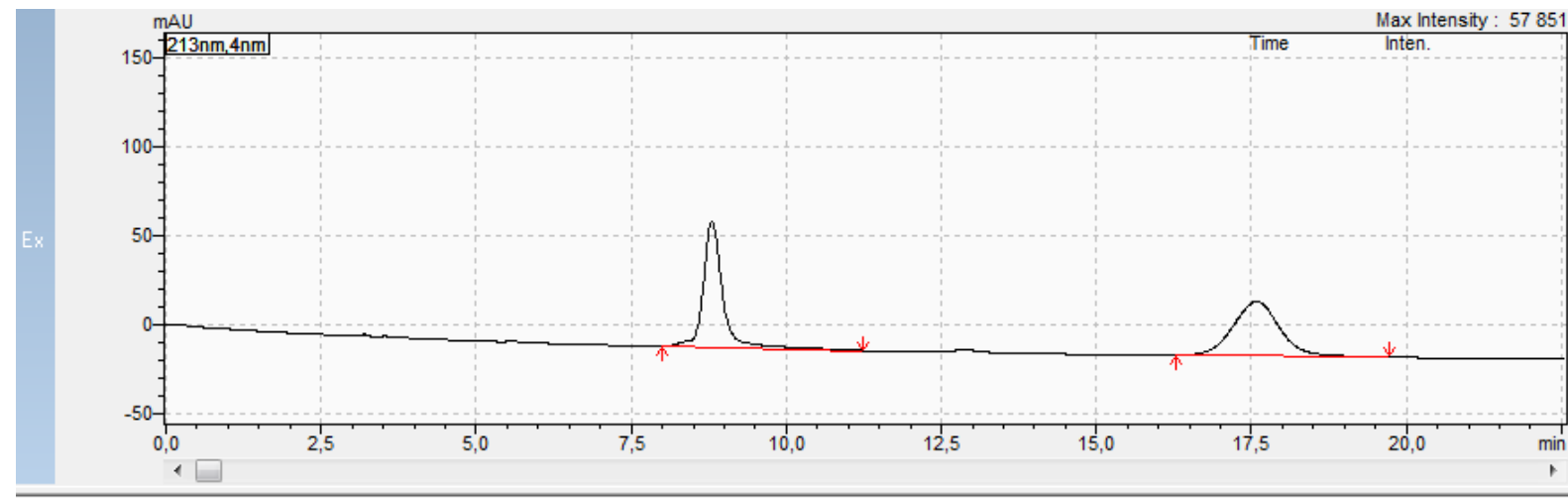

口

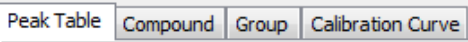

\begin{tabular}{|l|r|r|r|r|r|r|r|r|r|}
\hline Peak\# & Ret. Time & \multicolumn{1}{c|}{ Conc. } & \multicolumn{1}{c|}{ Area } & Height & Similarity Index & Mark & Peak Start & \multicolumn{1}{c|}{ Peak End } & \multicolumn{1}{c|}{ Area\% } \\
\hline 1 & 8,804 & 49,421 & 1571475 & 71115 & 0,000000 & & 7,989 & 11,232 & 49,421 \\
\hline 2 & 17,585 & 50,579 & 1608323 & 30689 & 0,000000 & & 16,288 & 19,733 & 50,579 \\
\hline Total & & 100,000 & 3179798 & 101804 & & & & & \\
\hline
\end{tabular}

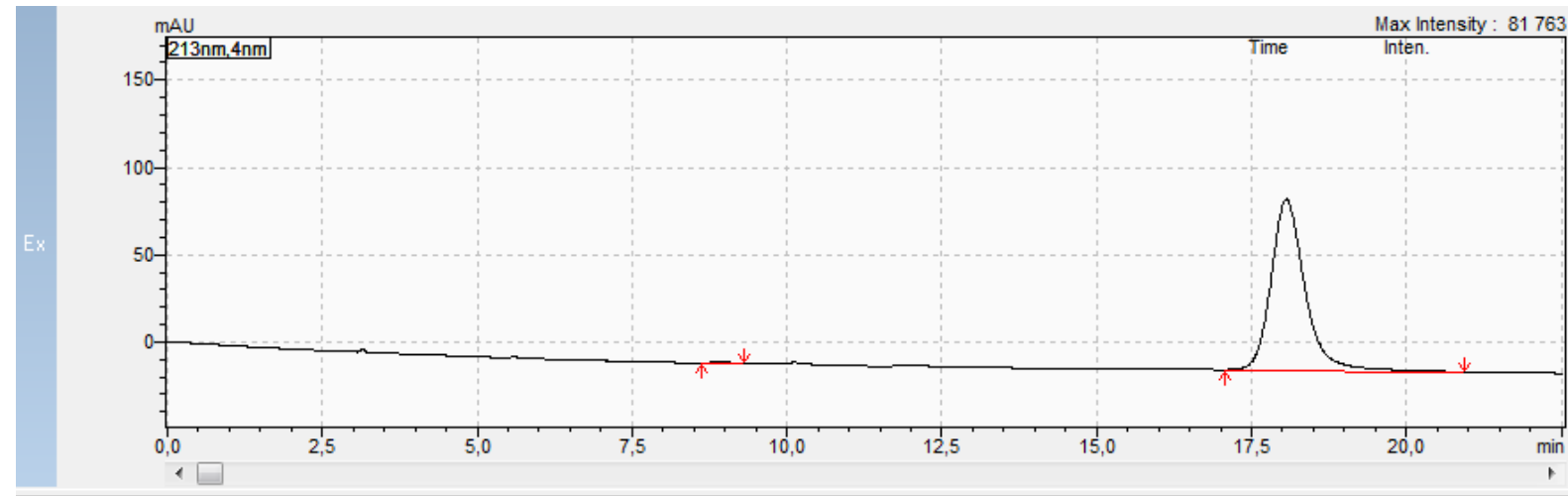

口〈 Results View - Peak Table

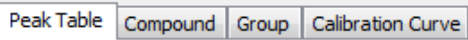

\begin{tabular}{|l|r|r|r|r|r|r|r|r|r|r|}
\hline Peak\# & Ret. Time & \multicolumn{1}{|c|}{ Conc. } & \multicolumn{1}{c|}{ Area } & \multicolumn{1}{c|}{ Height } & Similarity Index & Mark & \multicolumn{1}{c|}{ Peak Start } & \multicolumn{1}{c|}{ Peak End } & \multicolumn{1}{c|}{ Area\% } \\
\hline 1 & 8,906 & 0,537 & 20105 & 1145 & 0,000000 & & 8,629 & 9,301 & 0,537 \\
\hline 2 & 18,063 & 99,463 & 3723724 & 97920 & 0,000000 & & 17,056 & 20,928 \\
\hline Total & & 100,000 & 3743829 & 99065 & & & & 99,463 \\
\hline
\end{tabular}

for 3v: $e e=99 \%$ 
<smiles></smiles>

Conditions: IC column

mobile phase: heptane / $i$-PrOH - 80:20

$\lambda=204 \mathrm{~nm}, V=1.0 \mathrm{ml} / \mathrm{min}, t=25^{\circ} \mathrm{C}$

for $4 \mathrm{v}: t_{\mathrm{R}}=36.5 \mathrm{~min}$ (minor), $t_{\mathrm{R}}=40.8 \mathrm{~min}$ (major).

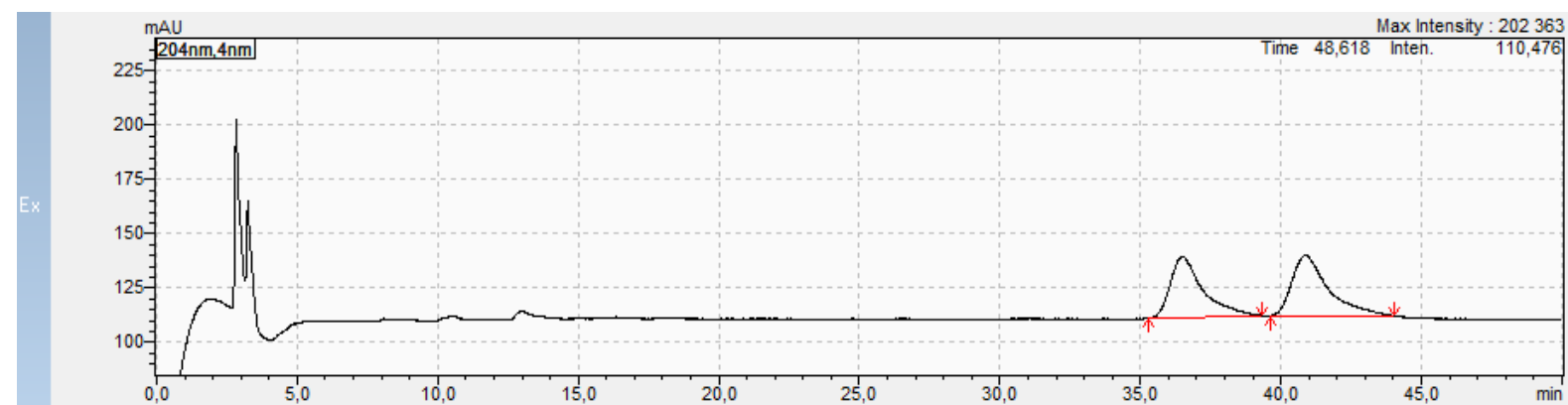

口〈 Results View - Peak Table

\begin{tabular}{l|l|l|l|}
\hline Peak Table Compound & Group & Calibration Curve \\
\hline
\end{tabular}

\begin{tabular}{|l|r|r|r|r|r|r|r|r|r|}
\hline Peak\# & Ret. Time & \multicolumn{1}{|c|}{ Conc. } & \multicolumn{1}{c|}{ Area } & \multicolumn{1}{c|}{ Height } & Similarity Index & Mark & Peak Start & \multicolumn{1}{c|}{ Peak End } & \multicolumn{1}{c|}{ Area\% } \\
\hline 1 & 36,483 & 47,401 & 2303865 & 28101 & 0,000000 & SV & 35,307 & 39,349 & 47,401 \\
\hline 2 & 40,832 & 52,599 & 2556495 & 27977 & 0,000000 & & & 39,616 & 44,021 \\
\hline Total & & 100,000 & 4860361 & 56077 & & & & 52,599 \\
\hline
\end{tabular}

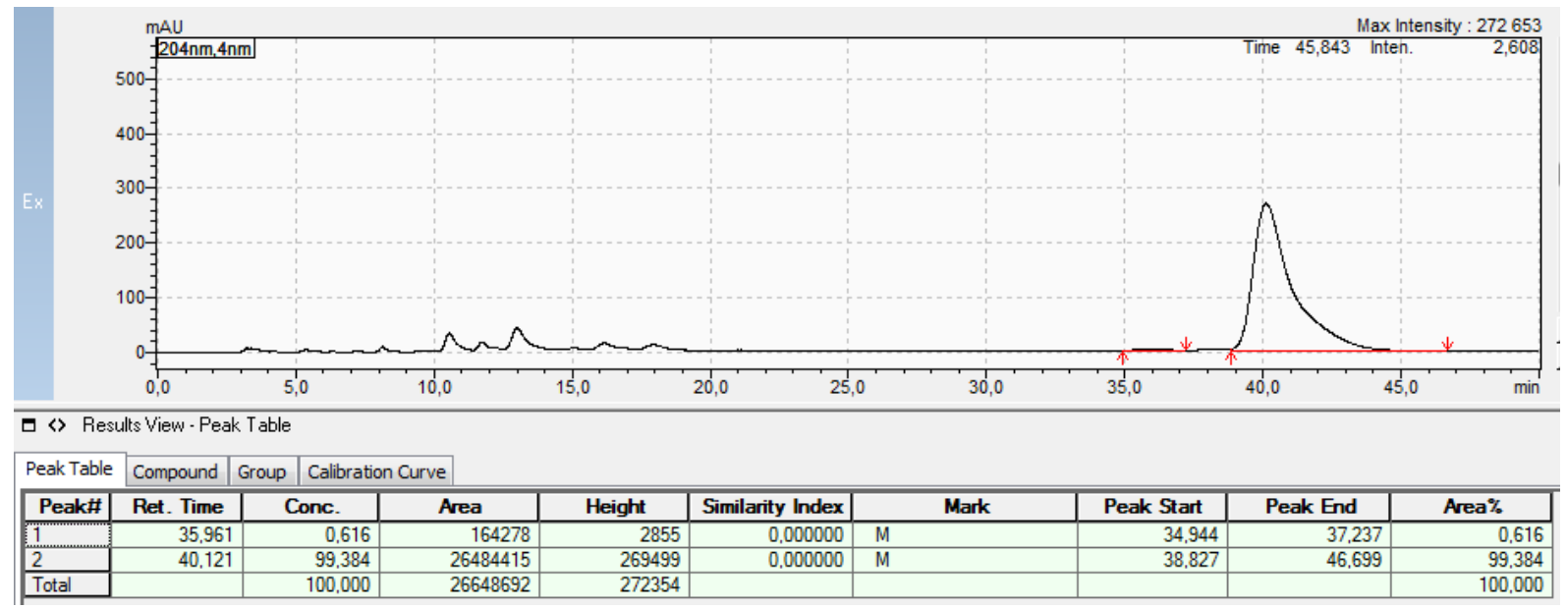

for $\mathbf{4 v}: e e=99 \%$ 
<smiles>O=C[C@H]1CC[C@]2(C(=O)N(Cc3ccccc3)c3ccccc32)C1c1ccco1</smiles>

Conditions: IA column

mobile phase: heptane / $i$-PrOH $-80: 20$

$\lambda=217 \mathrm{~nm}, V=1.0 \mathrm{ml} / \mathrm{min}, t=25^{\circ} \mathrm{C}$

for $3 \mathbf{w}: t_{\mathrm{R}}=13.0 \mathrm{~min}$ (minor), $t_{\mathrm{R}}=18.4 \mathrm{~min}$ (major).

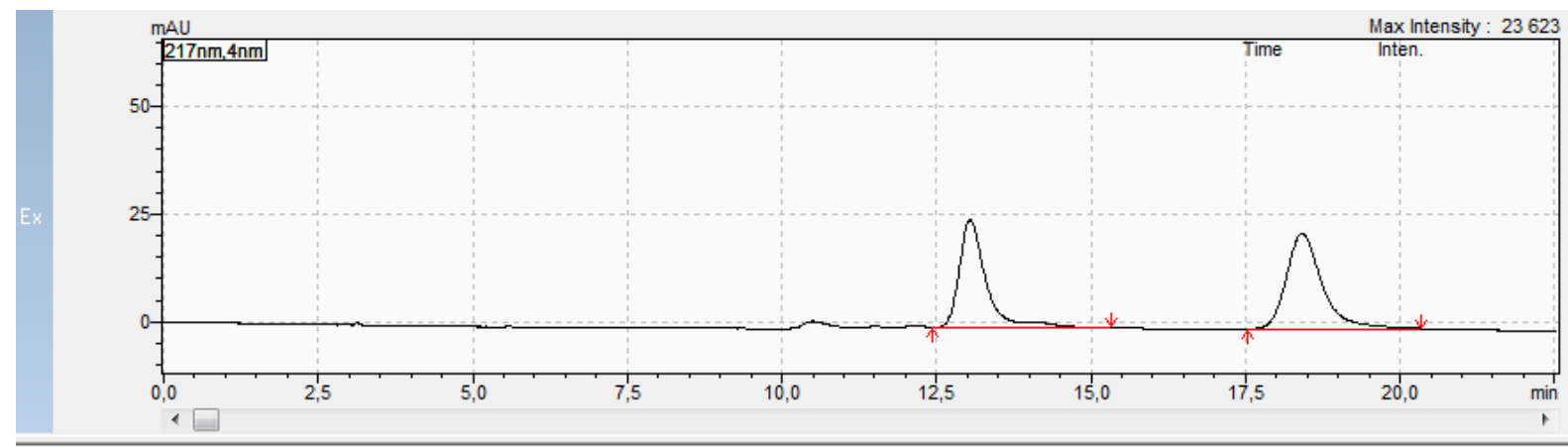

口〈R Results View - Peak Table

\begin{tabular}{l|l|l|l|}
\hline Peak Table & Compound & Group & Calibration Curve \\
\hline
\end{tabular}

\begin{tabular}{|l|r|r|r|r|r|r|r|r|r|r|}
\hline Peak\# & Ret. Time & \multicolumn{1}{|c|}{ Conc. } & \multicolumn{1}{|c|}{ Area } & \multicolumn{1}{c|}{ Height } & Similarity Index & Mark & Peak Start & Peak End & \multicolumn{1}{c|}{ Area\% } \\
\hline 1 & 13,047 & 46,032 & 759745 & 24930 & 0,000000 & M & 12,427 & 15,328 & 46,032 \\
\hline 2 & 18,418 & 53,968 & 890721 & 22247 & 0,000000 & & 17,536 & 20,341 & 53,968 \\
\hline Total & & 100,000 & 1650466 & 47178 & & & & \\
\hline
\end{tabular}

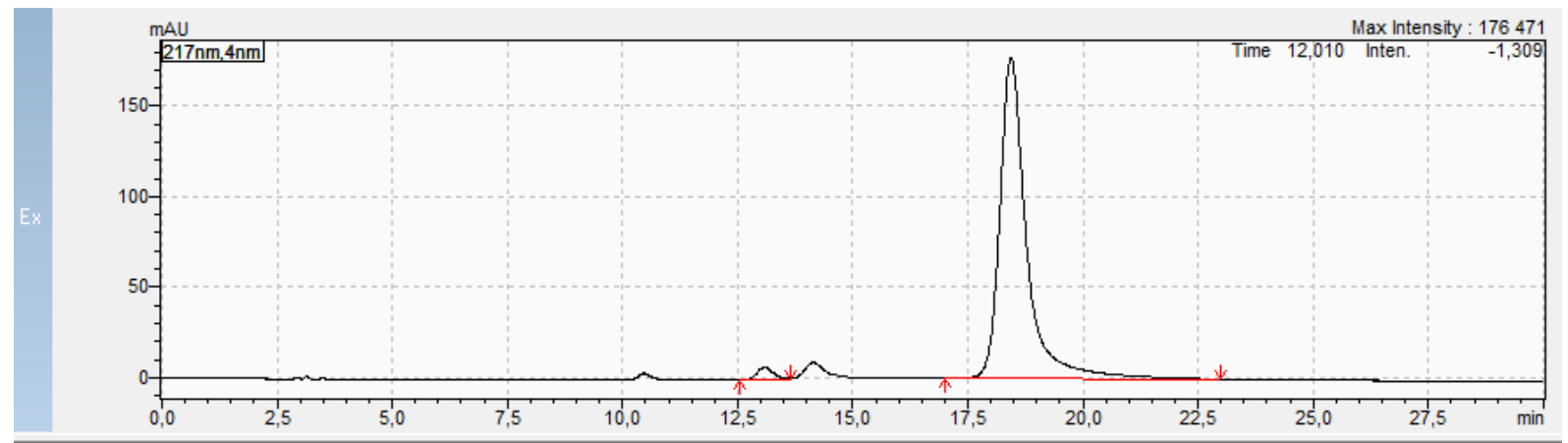

口〈R Results View - Peak Table

\begin{tabular}{|l|l|l|l|}
\hline Peak Table & Compound & Group & Calibration Curve \\
\hline
\end{tabular}

\begin{tabular}{|c|c|c|c|c|c|c|c|c|c|}
\hline Peak\# & Ret. Time & Conc. & Area & Height & Similarity Index & Mark & Peak Start & Peak End & Area\% \\
\hline 1 & 13,087 & 2,356 & 172855 & 6817 & 0,000000 & & 12,544 & 13,632 & 2,356 \\
\hline 2 & 18,439 & 97,644 & 7163406 & 177041 & 0,000000 & & 17,003 & 22,987 & 97,644 \\
\hline Total & & 100,000 & 7336260 & 183858 & & & & & 100,000 \\
\hline
\end{tabular}

for 3w: $e e=95 \%$ 
<smiles>O=C[C@H]1CCC2(C(=O)N(Cc3ccccc3)c3ccccc32)[C@H]1c1ccco1</smiles>

Conditions: IA column

mobile phase: heptane / $i$-PrOH $-80: 20$

$\lambda=206 \mathrm{~nm}, V=1.0 \mathrm{ml} / \mathrm{min}, t=25^{\circ} \mathrm{C}$

for $4 \mathrm{w}: t_{\mathrm{R}}=14.6 \mathrm{~min}$ (major), $t_{\mathrm{R}}=15.7 \mathrm{~min}$ (minor).

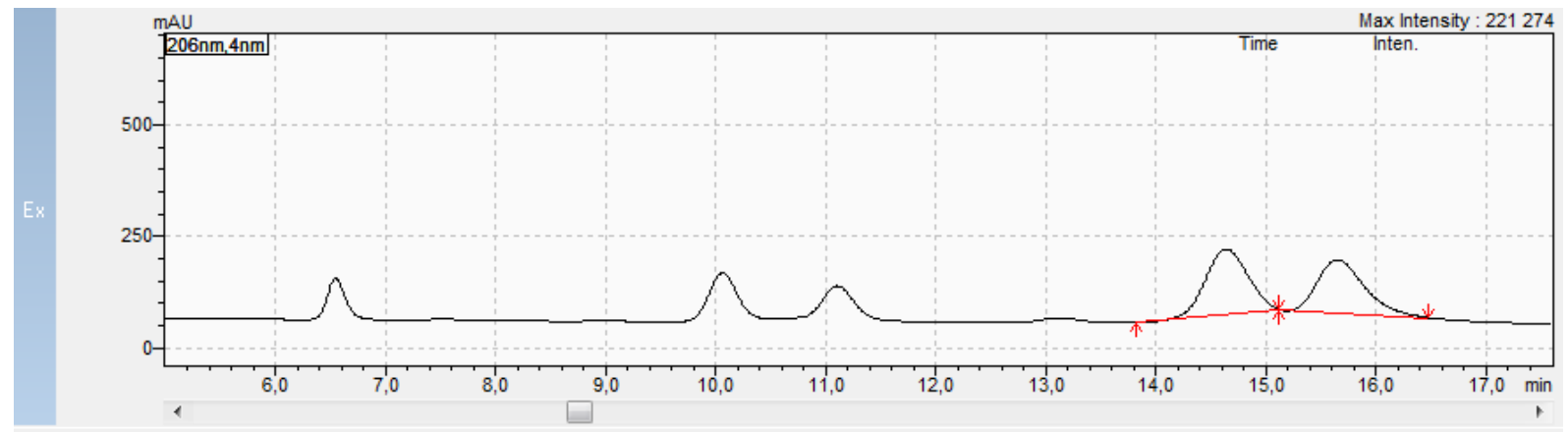

口〈 Results View - Peak Table

\begin{tabular}{|c|c|c|c|c|c|c|c|c|c|}
\hline Peak Table & Compound & Calibratic & & & & & & & \\
\hline Peak\# & Ret. Time & Conc. & Area & Height & Similarity Index & Mark & Peak Start & Peak End & Area\% \\
\hline 1 & 14,639 & 52,675 & 3848292 & 145895 & 0,000000 & $\mathrm{M}$ & 13,813 & 15,115 & 52,675 \\
\hline 2 & 15,646 & 47,325 & 3457501 & 118171 & 0,000000 & M & 15,115 & 16,480 & 47,325 \\
\hline Total & & 100,000 & 7305794 & 264066 & & & & & 100,000 \\
\hline
\end{tabular}

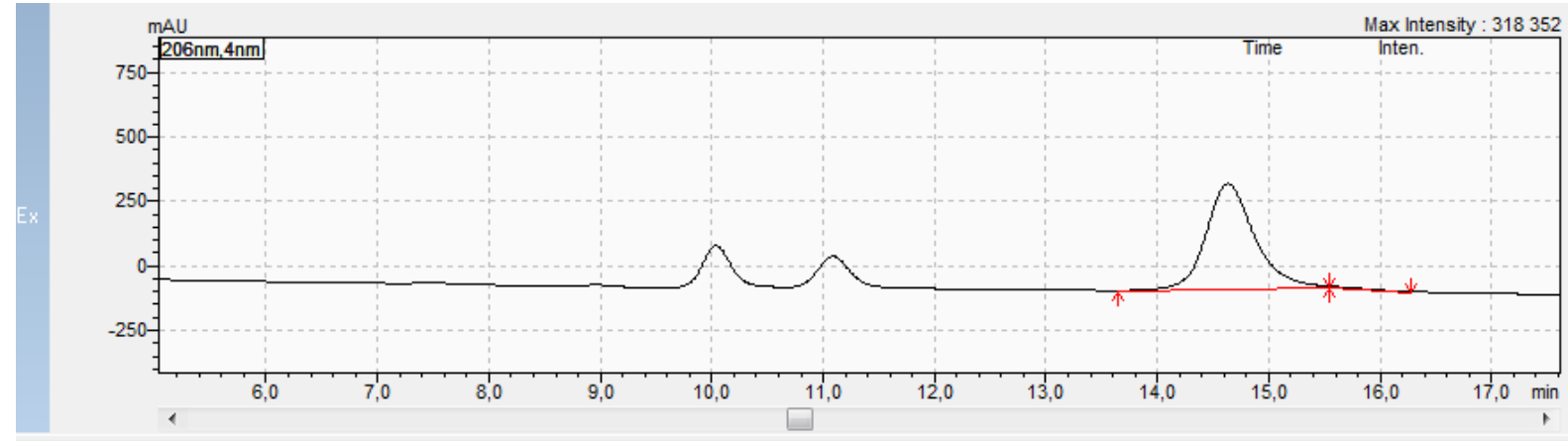

口〈 Results View - Peak Table

\begin{tabular}{l|l|l|l|}
\hline Peak Table & Compound & Group & Calibration Curve \\
\hline
\end{tabular}

\begin{tabular}{|c|c|c|c|c|c|c|c|c|c|}
\hline Peak\# & Ret. Time & Conc. & Area & Height & Similarity Index & Mark & Peak Start & Peak End & Area\% \\
\hline 1 & 14,634 & 98,951 & 12500895 & 408779 & 0,000000 & $M$ & 13,643 & 15,541 & 98,951 \\
\hline 2 & 15,557 & 1.049 & 132555 & 4961 & 0,000000 & M & 15,541 & 16,277 & 1,049 \\
\hline Total & & 100,000 & 12633449 & 413740 & & & & & 100,000 \\
\hline
\end{tabular}

for $4 \mathbf{w}: e e=98 \%$ 
<smiles>O=C[C@H]1CC[C@]2(C(=O)N(Cc3ccccc3)c3ccccc32)C1c1cccs1</smiles>

Conditions: IA column

mobile phase: heptane / $i$-PrOH $-80: 20$

$\lambda=213 \mathrm{~nm}, V=1.0 \mathrm{ml} / \mathrm{min}, t=25^{\circ} \mathrm{C}$

for $3 \mathbf{x}: t_{\mathrm{R}}=13.4 \mathrm{~min}$ (major), $t_{\mathrm{R}}=25.4 \mathrm{~min}$ (minor).

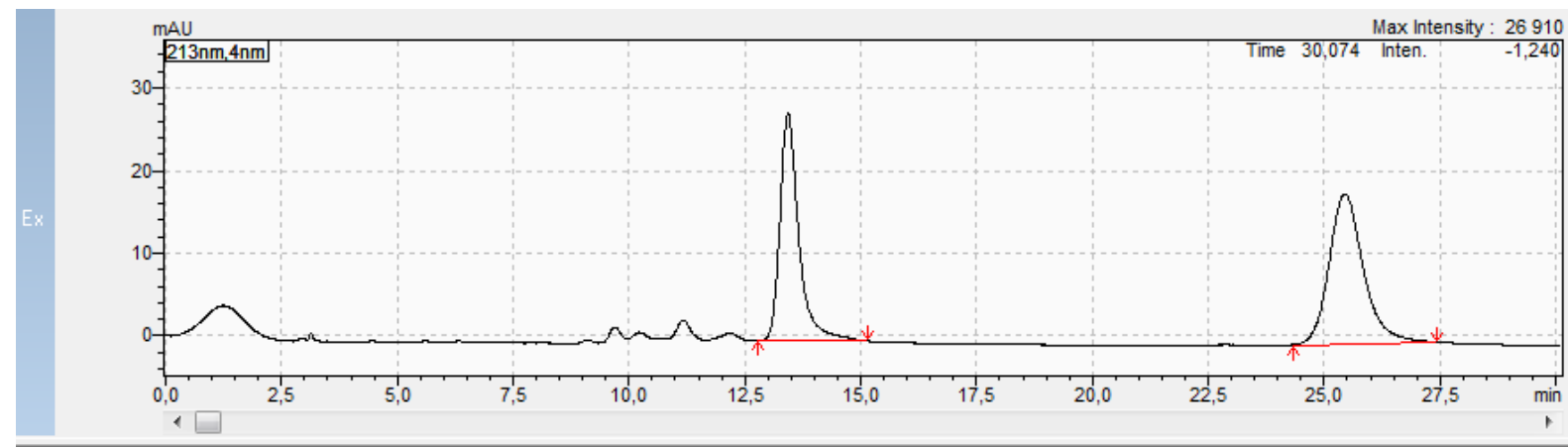

口 $〉$ Results View - Peak Table

\begin{tabular}{|l|l|l|l|}
\hline Peak Table & Compound & Group & Calibration Curve \\
\hline
\end{tabular}

\begin{tabular}{|l|r|r|r|r|r|r|r|r|r|}
\hline Peak\# & Ret. Time & \multicolumn{1}{|c|}{ Conc. } & \multicolumn{1}{|c|}{ Area } & Height & Similarity Index & \multicolumn{1}{|c|}{ Mark } & \multicolumn{1}{l|}{ Peak Start } & \multicolumn{1}{c|}{ Peak End } & \multicolumn{1}{c|}{ Area\% } \\
\hline 1 & 13,430 & 45,539 & 781408 & 27488 & 0,000000 & & 12,789 & 15,168 & 45,539 \\
\hline 2 & 25,444 & 54,461 & 934501 & 18158 & 0,000000 & & 24,331 & 27,424 & 54,461 \\
\hline Total & & 100,000 & 1715910 & 45646 & & & & & \\
\hline
\end{tabular}

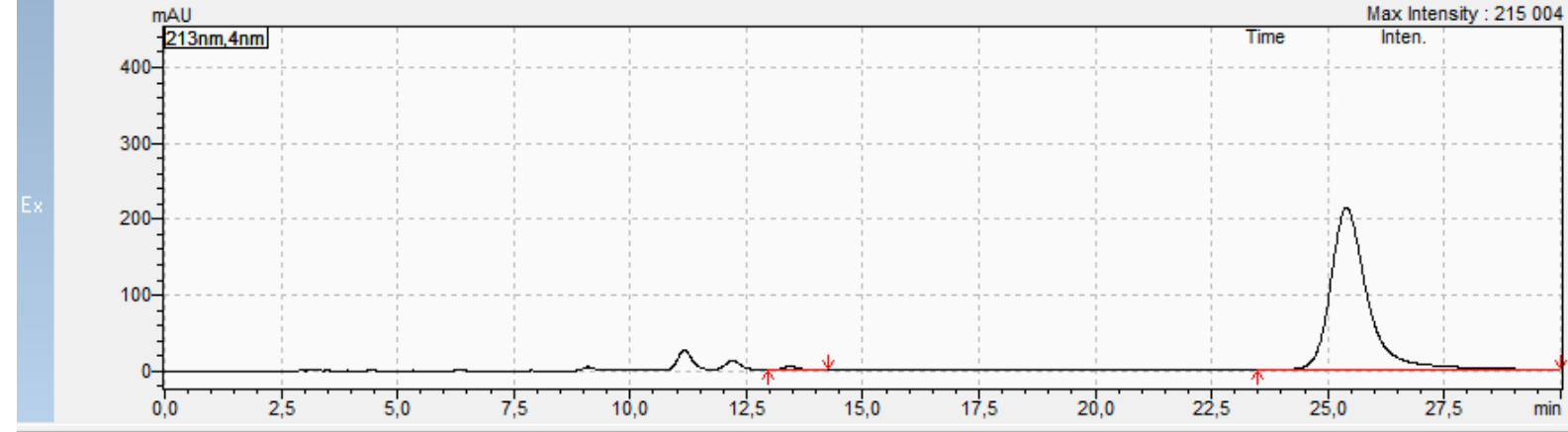

口〈 Results View - Peak Table

\begin{tabular}{|l|l|l|l|}
\hline Peak Table & Compound & Group & Calibration Curve \\
\hline
\end{tabular}

\begin{tabular}{|l|r|r|r|r|r|r|r|r|r|}
\hline Peak\# & Ret. Time & \multicolumn{1}{|c|}{ Conc. } & \multicolumn{1}{|c|}{ Area } & Height & Similarity Index & Mark & Peak Start & \multicolumn{1}{c|}{ Peak End } & \multicolumn{1}{c|}{ Area\% } \\
\hline 1 & 13,426 & 1,015 & 120260 & 4634 & 0,000000 & & 12,971 & 14,272 & 1,015 \\
\hline 2 & 25,398 & 98,985 & 11724888 & 213164 & 0,000000 & V & 23,488 & 29,995 & 98,985 \\
\hline Total & & 100,000 & 11845148 & 217798 & & & & & \\
\hline
\end{tabular}

for 3x: $e e=98 \%$ 
<smiles>O=C[C@H]1CCC2(C(=O)N(Cc3ccccc3)c3ccccc32)[C@H]1c1cccs1</smiles>

Conditions: IA column

mobile phase: heptane / $i$-PrOH $-80: 20$

$\lambda=207 \mathrm{~nm}, V=1.0 \mathrm{ml} / \mathrm{min}, t=25^{\circ} \mathrm{C}$

for $4 \mathrm{x}$ : $t_{\mathrm{R}}=14.4 \mathrm{~min}$ (major), $t_{\mathrm{R}}=16.6 \mathrm{~min}$ (minor).

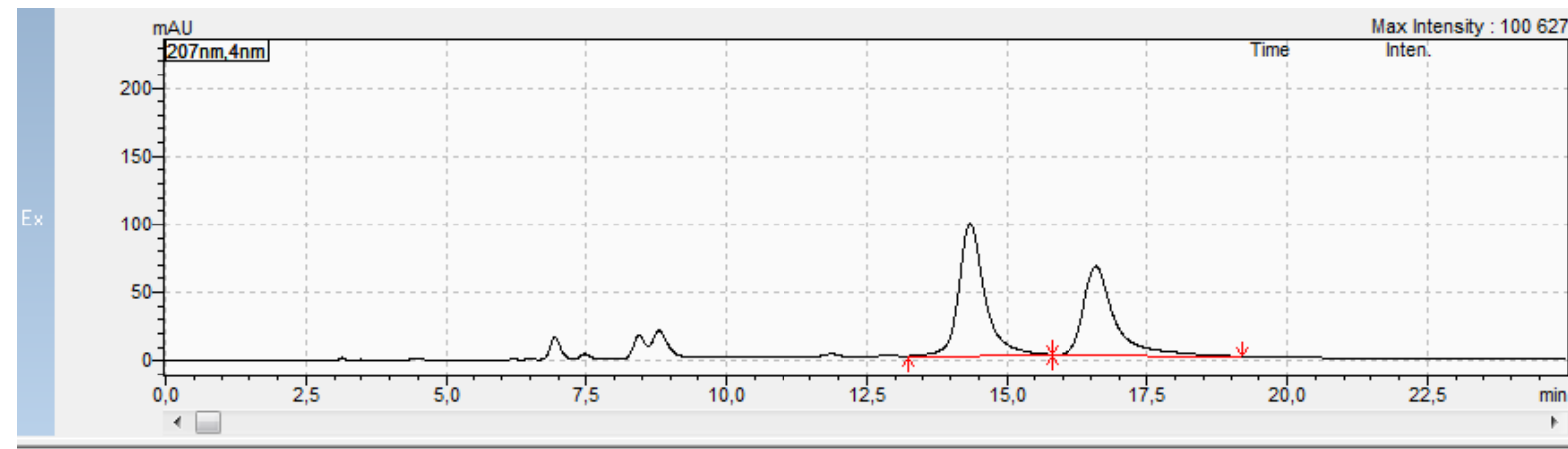

口〈 Results View - Peak Table

\begin{tabular}{|l|l|l|l|}
\hline Peak Table & Compound & Group & Calibration Curve \\
\hline
\end{tabular}

\begin{tabular}{|l|r|r|r|r|r|r|r|r|r|}
\hline Peak\# & Ret. Time & \multicolumn{1}{|c|}{ Conc. } & \multicolumn{1}{c|}{ Area } & \multicolumn{1}{c|}{ Height } & Similarity Index & \multicolumn{1}{|c|}{ Mark } & Peak Start & Peak End & Area\% \\
\hline 1 & 14,352 & 55,541 & 3102338 & 96724 & 0,000000 & M & 13,237 & 15,819 \\
\hline 2 & 16,588 & 44,459 & 2483304 & 64901 & 0,000000 & M & 55,541 \\
\hline Total & & 100,000 & 5585642 & 161625 & & & 15,819 & 19,189 \\
\hline
\end{tabular}

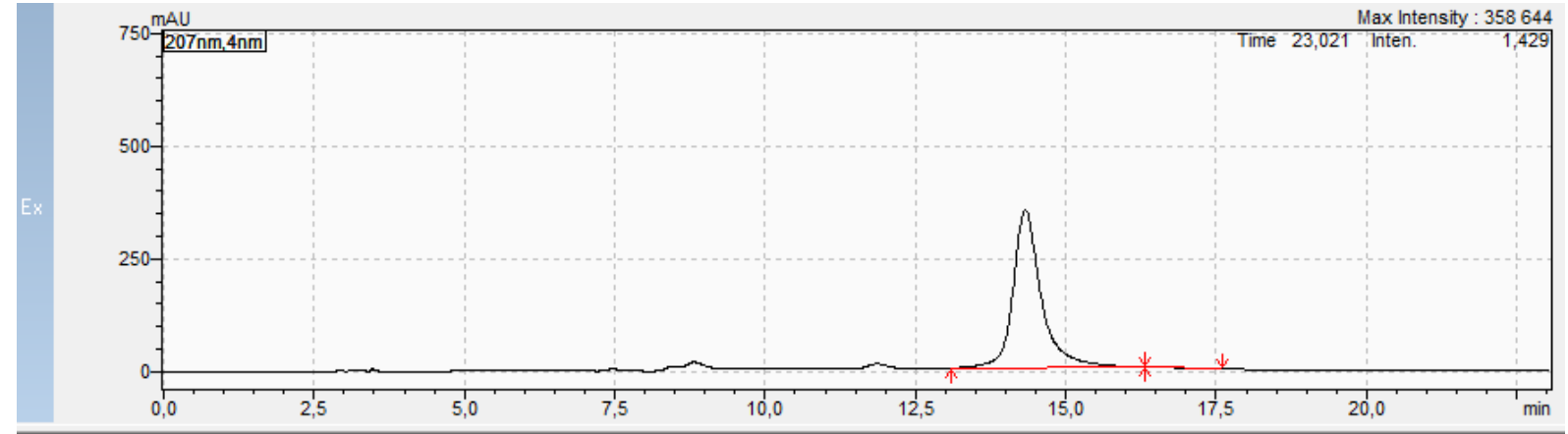

(

\begin{tabular}{|l|l|l|l|}
\hline Peak Table Compound & Group & Calibration Curve \\
\hline
\end{tabular}

\begin{tabular}{|l|r|r|r|r|r|r|r|r|r|r|}
\hline Peak\# & Ret. Time & \multicolumn{1}{|c|}{ Conc. } & \multicolumn{1}{|c|}{ Area } & \multicolumn{1}{c|}{ Height } & Similarity Index & Mark & Peak Start & Peak End & Area\% \\
\hline 1 & 14,325 & 99,877 & 11557667 & 350154 & 0,000000 & M & 13,088 & 16,309 & 99,877 \\
\hline 2 & 16,547 & 0,123 & 14274 & 1433 & 0,000000 & M & 16,309 & 17,600 & 0,123 \\
\hline Total & & 100,000 & 11571941 & 351588 & & & & & 100,000 \\
\hline
\end{tabular}

for $4 \mathbf{x}: e e=99 \%$ 
$\underbrace{\mathrm{CHO}}_{\mathrm{Bn}}$

Conditions: IA column

mobile phase: heptane / $i$-PrOH $-80: 20$

$\lambda=214 \mathrm{~nm}, V=1.0 \mathrm{ml} / \mathrm{min}, t=25^{\circ} \mathrm{C}$

for $3 \mathbf{y}: t_{\mathrm{R}}=14.4 \mathrm{~min}$ (major), $t_{\mathrm{R}}=15.5 \mathrm{~min}$ (minor).

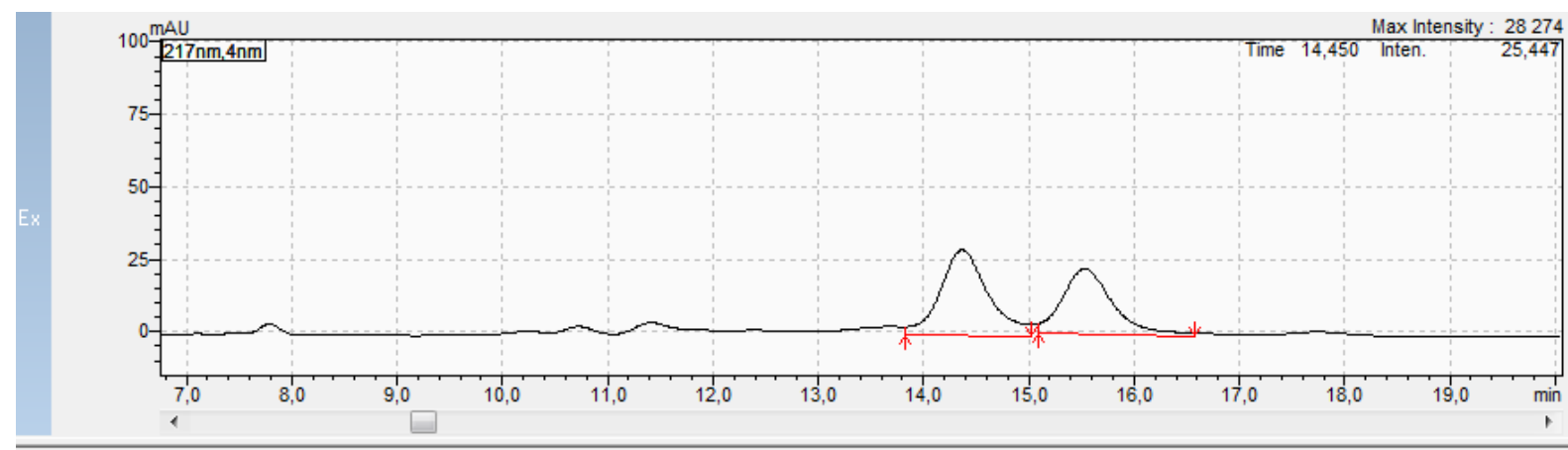

口

\begin{tabular}{|l|l|l|l|}
\hline Peak Table & Compound & Group & Calibration Curve \\
\hline
\end{tabular}

\begin{tabular}{|l|r|r|r|r|r|r|r|r|r|r|}
\hline Peak\# & Ret. Time & \multicolumn{1}{c|}{ Conc. } & \multicolumn{1}{c|}{ Area } & Height & Similarity Index & Mark & Peak Start & Peak End & \multicolumn{1}{c|}{ Area\% } \\
\hline 1 & 14,367 & 56,174 & 942069 & 29647 & 0,000000 & & 13,824 & 15,019 & 56,174 \\
\hline 2 & 15,533 & 43,826 & 734984 & 22283 & 0,000000 & M & 15,093 & 16,576 \\
\hline Total & & 100,000 & 1677053 & 51929 & & & 43,826 \\
\hline
\end{tabular}

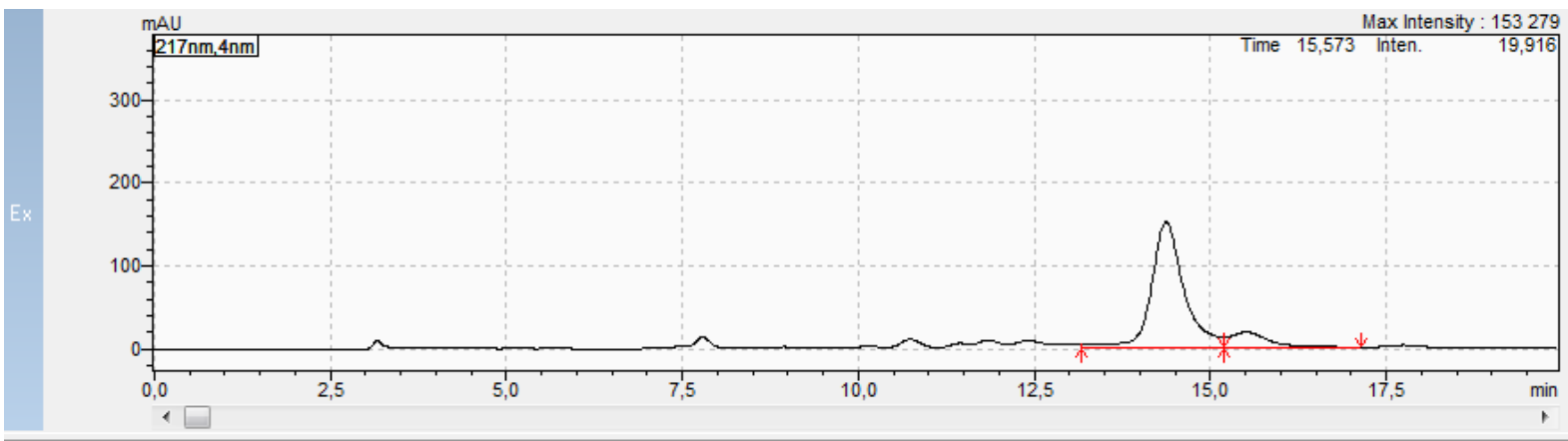

口〈Results View - Peak Table

\begin{tabular}{|l|l|l|l|}
\hline Peak Table & Compound & Group & Calibration Curve \\
\hline
\end{tabular}

\begin{tabular}{|l|r|r|r|r|r|r|r|r|r|}
\hline Peak\# & Ret. Time & \multicolumn{1}{|c|}{ Conc. } & \multicolumn{1}{c|}{ Area } & \multicolumn{1}{c|}{ Height } & Similarity Index & Mark & Peak Start & Peak End & Area\% \\
\hline 1 & 14,371 & 85,469 & 5043191 & 152631 & 0,000000 & & 13,173 & 15,189 \\
\hline 2 & 15,519 & 14,531 & 857444 & 19742 & 0,000000 & V & 85,469 \\
\hline Total & & 100,000 & 5900636 & 172373 & & & & 15,189 & 17,131 \\
\hline
\end{tabular}

for $3 \mathbf{y}: e e=71 \%$ 
$\overbrace{\mathrm{Bn}}^{\mathrm{CHO}}$

Conditions: IA column

mobile phase: heptane / $i$-PrOH $-80: 20$

$\lambda=207 \mathrm{~nm}, V=1.0 \mathrm{ml} / \mathrm{min}, t=25^{\circ} \mathrm{C}$

for $4 \mathrm{y}: t_{\mathrm{R}}=11.4 \mathrm{~min}$ (minor), $t_{\mathrm{R}}=13.7 \mathrm{~min}$ (major).

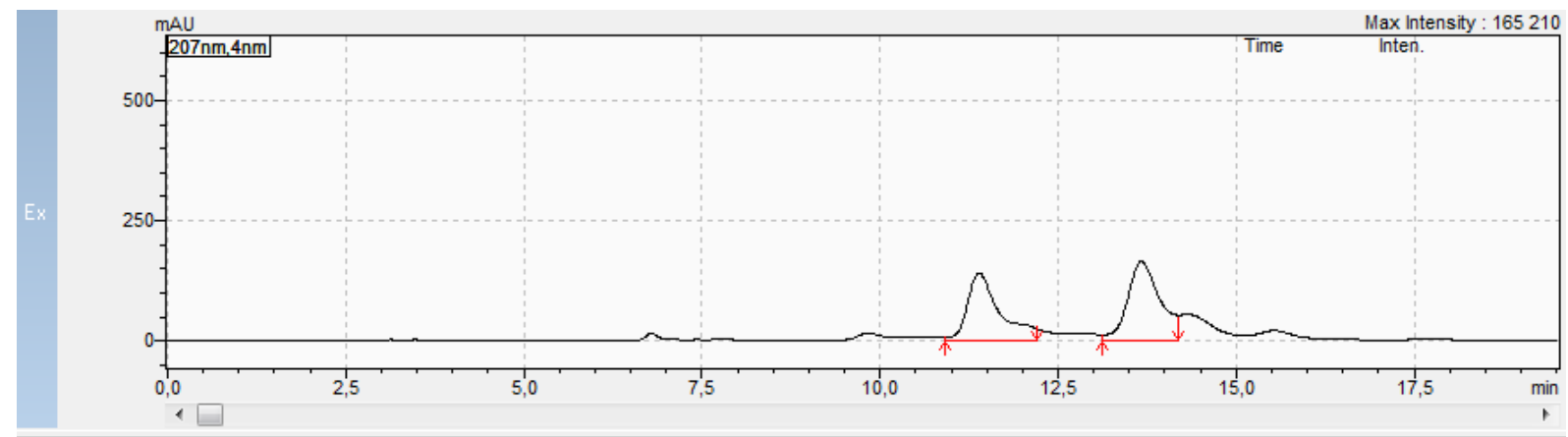

口〈 Results View - Peak Table

\begin{tabular}{|l|l|l|l|}
\hline Peak Table & Compound & Group & Calibration Curve \\
\hline
\end{tabular}

\begin{tabular}{|l|r|r|r|r|r|r|r|r|r|}
\hline Peak\# & Ret. Time & \multicolumn{1}{|c|}{ Conc. } & \multicolumn{1}{c|}{ Area } & \multicolumn{1}{c|}{ Height } & Similarity Index & Mark & Peak Start & \multicolumn{1}{c|}{ Peak End } & \multicolumn{1}{c|}{ Area\% } \\
\hline 1 & 11,391 & 46,139 & 4507592 & 141385 & 0,000000 & M & 10,923 & 12,203 & 46,139 \\
\hline 2 & 13,670 & 53,861 & 5261997 & 165096 & 0,000000 & & 13,120 & 14,187 & 53,861 \\
\hline Total & & 100,000 & 9769589 & 306480 & & & & & 100,000 \\
\hline
\end{tabular}

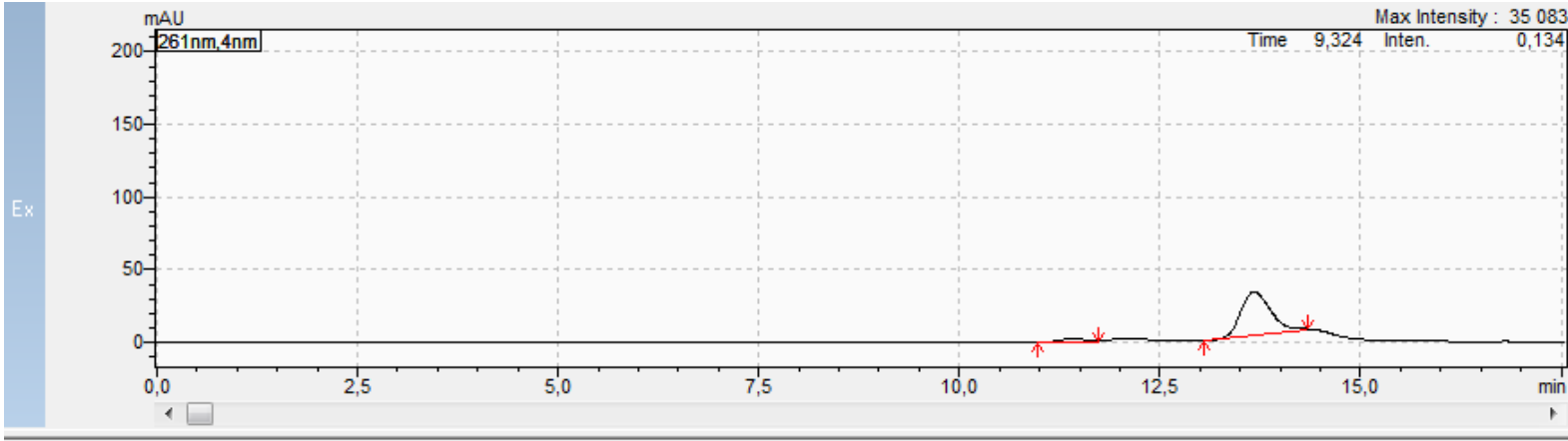

口

\begin{tabular}{l|l|l|l|}
\hline Peak Table & Compound & Group & Calibration Curve \\
\hline
\end{tabular}

\begin{tabular}{|c|c|c|c|c|c|c|c|c|c|}
\hline Peak\# & Ret. Time & Conc. & Area & Height & Similarity Index & Mark & Peak Start & Peak End & Area\% \\
\hline 1 & 11.409 & 6.996 & 55679 & 2331 & 0,000000 & & 10,976 & 11,733 & 6.996 \\
\hline 2 & 13,676 & 93,004 & 740235 & 29702 & 0,000000 & M & 13,045 & 14,347 & 93,004 \\
\hline Total & & 100,000 & 795914 & 32033 & & & & & 100,000 \\
\hline
\end{tabular}

for $\mathbf{4 y}: e e=86 \%$ 

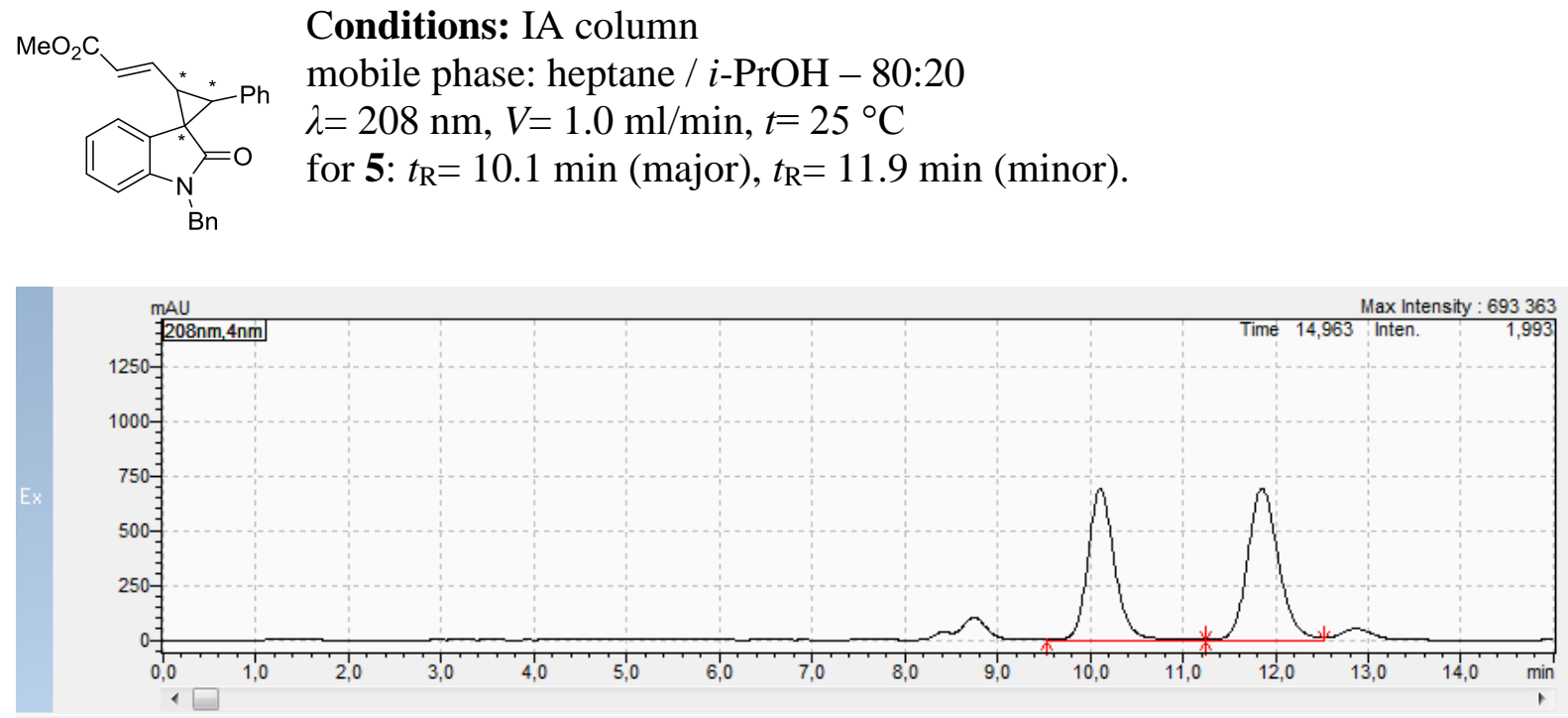

口〈 Results View - Peak Table

\begin{tabular}{|l|l|l|l|}
\hline Peak Table & Compound & Group & Calibration Curve \\
\hline
\end{tabular}

\begin{tabular}{|l|r|r|r|r|r|r|r|r|r|r|}
\hline Peak\# & Ret. Time & \multicolumn{1}{|c|}{ Conc. } & \multicolumn{1}{c|}{ Area } & \multicolumn{1}{c|}{ Height } & Similarity Index & Mark & \multicolumn{1}{|c|}{ Peak Start } & \multicolumn{1}{c|}{ Peak End } & Area\% \\
\hline 1 & 10,109 & 46,154 & 13674213 & 690565 & 0,000000 & & 9,525 & 11,253 & 46,154 \\
\hline 2 & 11,854 & 53,846 & 15952955 & 693943 & 0,000000 & V & 11,253 & 12,523 \\
\hline Total & & 100,000 & 29627167 & 1384508 & & & & \\
\hline
\end{tabular}

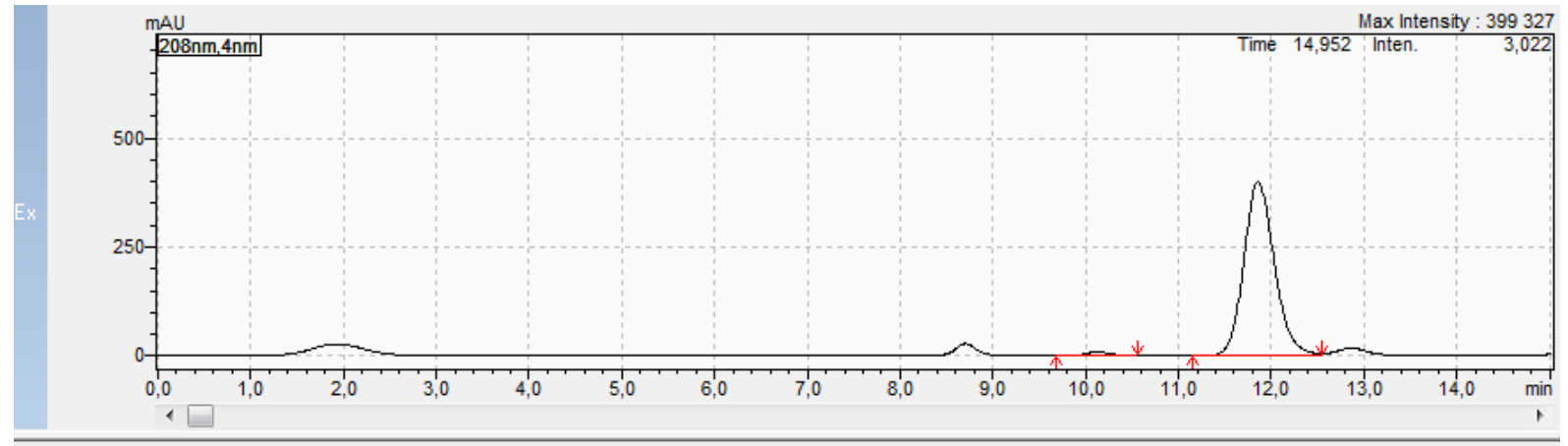

口〈 Results View - Peak Table

\begin{tabular}{|l|l|l|l|}
\hline Peak Table Compound & Group & Calibration Curve \\
\hline
\end{tabular}

\begin{tabular}{|c|c|c|c|c|c|c|c|c|c|}
\hline Peak\# & Ret. Time & Conc. & Area & Height & Similarity Index & Mark & Peak Start & Peak End & Area\% \\
\hline 1 & 10,123 & 1,804 & 168974 & 8752 & 0,000000 & & 9.685 & 10,560 & 1,804 \\
\hline 2 & 11,859 & 98,196 & 9198048 & 398864 & 0,000000 & & 11,147 & 12,555 & 98,196 \\
\hline Total & & 100,000 & 9367023 & 407615 & & & & & 100,000 \\
\hline
\end{tabular}

for 5: $e e=96 \%$ 


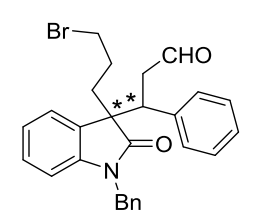

Conditions: IA column

mobile phase: heptane / $i$-PrOH $-80: 20$

$\lambda=206 \mathrm{~nm}, V=1.0 \mathrm{ml} / \mathrm{min}, t=25^{\circ} \mathrm{C}$

for 6: $t_{\mathrm{R}}=12.9 \mathrm{~min}$ (minor), $t_{\mathrm{R}}=18.8 \mathrm{~min}$ (major).

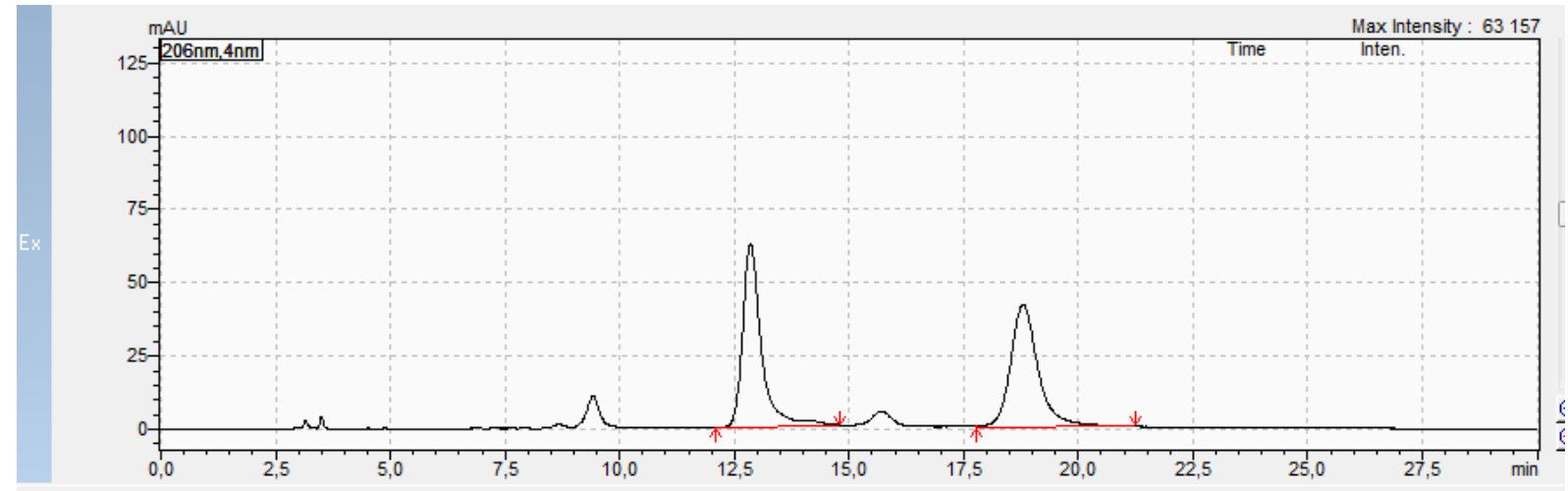

- 〈 Results View - Peak Table

\begin{tabular}{l|l|l|l|}
\hline Peak Table Compound & Group & Calibration Curve \\
\hline
\end{tabular}

\begin{tabular}{|c|c|c|c|c|c|c|c|c|c|}
\hline Peak\# & Ret. Time & Conc. & Area & Height & Similarity Index & Mark & Peak Start & Peak End & Area\% \\
\hline $17 m \cdots \cdots$ & 12,851 & 50,447 & 1780733 & 62297 & 0,000000 & $\bar{S}$ & 12,107 & 14,795 & 50,447 \\
\hline 2 & 18,793 & 49,553 & 1749169 & 41813 & 0,000000 & S & 17,781 & 21,248 & 49,553 \\
\hline Total & & 100,000 & 3529902 & 104111 & & & & & 100,000 \\
\hline
\end{tabular}

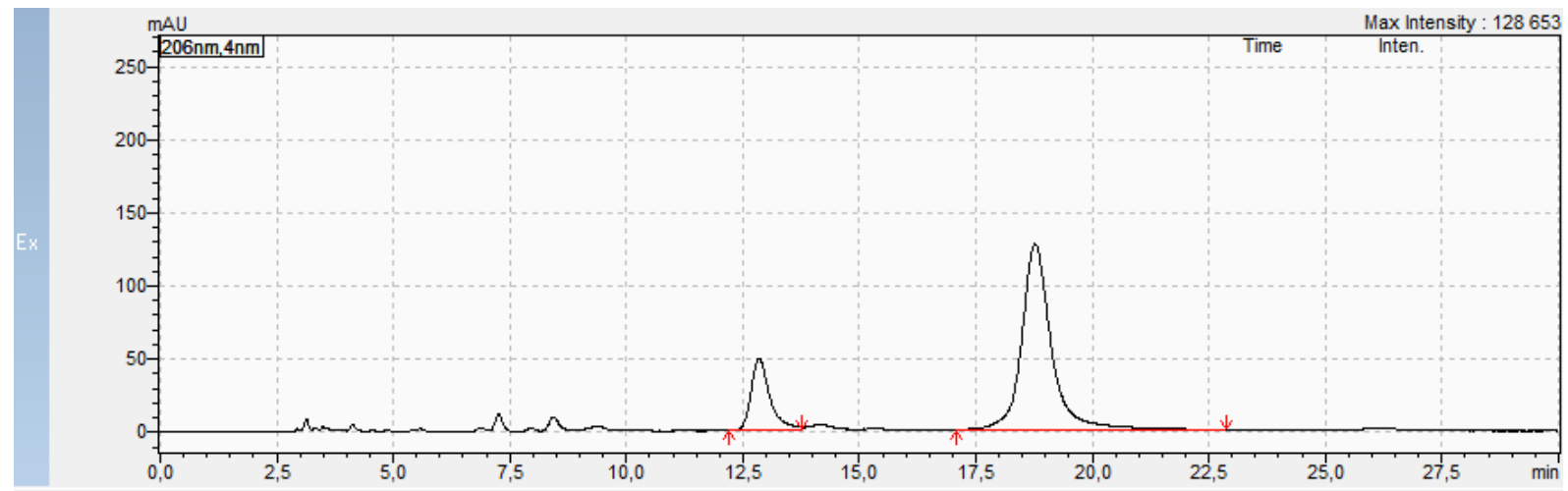

口〈R Results View - Peak Table

\begin{tabular}{|l|l|l|l|}
\hline Peak Table & Compound & Group & Calibration Curve \\
\hline
\end{tabular}

\begin{tabular}{|c|c|c|c|c|c|c|c|c|c|}
\hline Peak\# & Ret. Time & Conc. & Area & Height & Similarity Index & Mark & Peak Start & Peak End & Area\% \\
\hline 1 & 12,847 & 19,827 & 1400660 & 49155 & 0,000000 & & 12,181 & 13,771 & 19,827 \\
\hline 2 & 18,775 & 80,173 & 5663836 & 126878 & 0,000000 & SV & 17,088 & 22,869 & 80,173 \\
\hline Total & & 100,000 & 7064496 & 176033 & & & & & 100,000 \\
\hline
\end{tabular}

for 6: $e e=60 \%$ 
<smiles>O=CC/C(=C\CCCBr)C(=O)N(Cc1ccccc1)c1ccccc1</smiles>

Conditions: IA column

mobile phase: heptane / $i$-PrOH - 80:20

$\lambda=204 \mathrm{~nm}, V=1.0 \mathrm{ml} / \mathrm{min}, t=25^{\circ} \mathrm{C}$

for 7: $t_{\mathrm{R}}=15.3 \mathrm{~min}$ (major), $t_{\mathrm{R}}=31.6 \mathrm{~min}$ (minor).

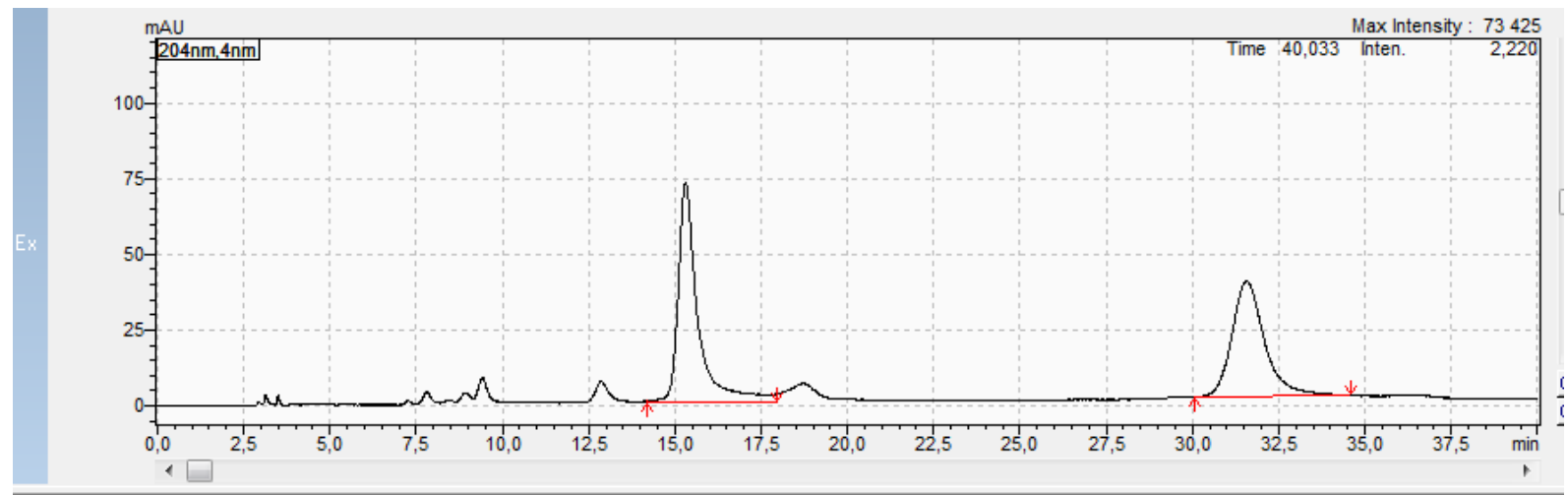

- 〈 Results View - Peak Table

\begin{tabular}{|l|l|l|l|}
\hline Peak Table & Compound & Group & Calibration Curve \\
\hline
\end{tabular}

\begin{tabular}{|l|r|r|r|r|r|r|r|r|r|r|}
\hline Peak\# & Ret. Time & \multicolumn{1}{|c|}{ Conc. } & \multicolumn{1}{c|}{ Area } & \multicolumn{1}{c|}{ Height } & Similarity Index & Mark & Peak Start & Peak End & Area\% \\
\hline 1 & 15,308 & 53,678 & 2906296 & 72341 & 0,000000 & S & 14,208 & 17,963 \\
\hline 2 & 31,574 & 46,322 & 2507981 & 38067 & 0,000000 & M & 53,678 \\
\hline Total & & 100,000 & 5414276 & 110407 & & & & 30,048 & 34,581 & \\
\hline
\end{tabular}

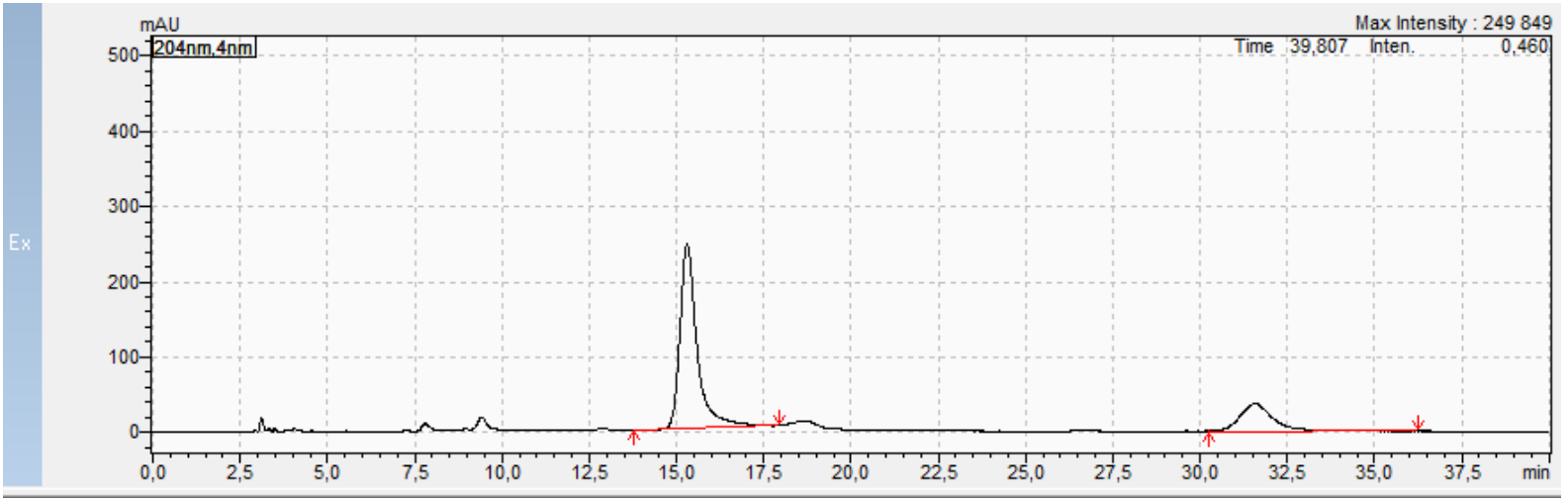

口 〈 Results View - Peak Table

\begin{tabular}{|l|l|l|l|}
\hline Peak Table & Compound & Group & Calibration Curve \\
\hline
\end{tabular}

\begin{tabular}{|l|r|r|r|r|r|r|r|r|r|}
\hline Peak\# & Ret. Time & \multicolumn{1}{|c|}{ Conc. } & \multicolumn{1}{c|}{ Area } & \multicolumn{1}{c|}{ Height } & Similarity Index & Mark & Peak Start & Peak End & \multicolumn{1}{c|}{ Area\% } \\
\hline 1 & 15,305 & 77,485 & 8502893 & 245294 & 0,000000 & M & 13,781 & 17,941 \\
\hline 2 & 31,581 & 22,515 & 2470763 & 36550 & 0,000000 & S & 77,485 \\
\hline Total & & 100,000 & 10973656 & 281844 & & & 30,272 & 36,245 & 22,515 \\
\hline
\end{tabular}

for $7: e e=55 \%$ 


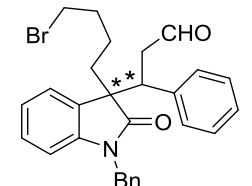

$\mathrm{Bn}$

Conditions: IA column

mobile phase: heptane / $i$-PrOH $-80: 20$

$\lambda=209 \mathrm{~nm}, V=1.0 \mathrm{ml} / \mathrm{min}, t=25^{\circ} \mathrm{C}$

for 8: $t_{\mathrm{R}}=12.8 \mathrm{~min}$ (minor), $t_{\mathrm{R}}=20.5 \mathrm{~min}$ (major).

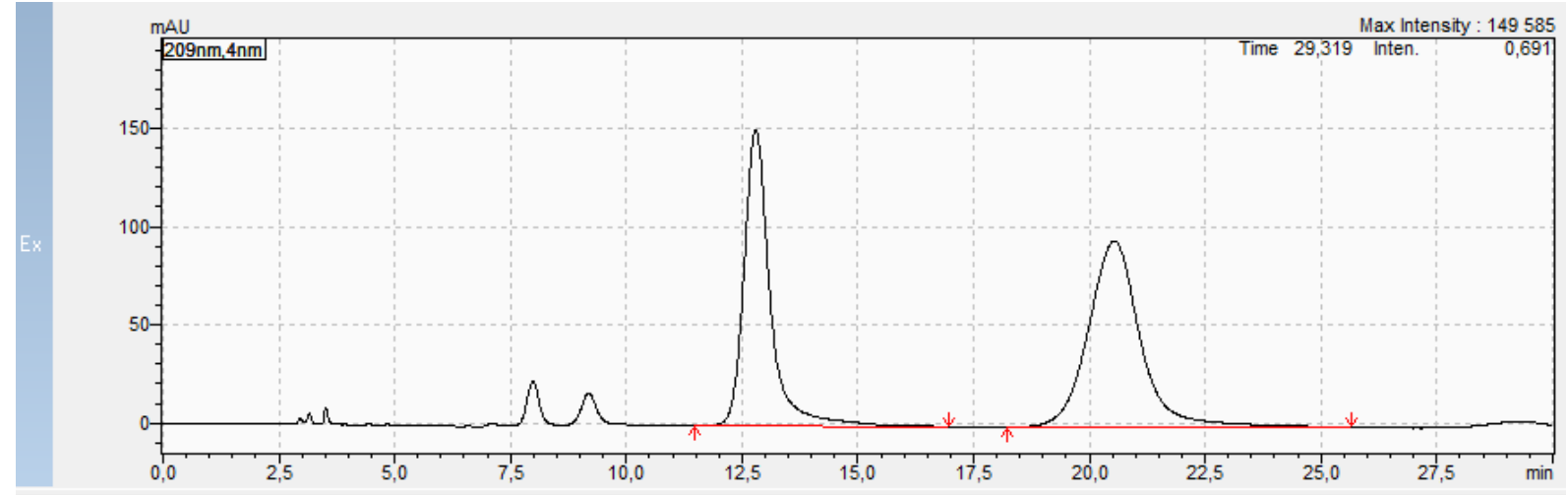

口〈R Results View - Peak Table

\begin{tabular}{|l|l|l|l|}
\hline Peak Table & Compound & Group & Calibration Curve \\
\hline
\end{tabular}

\begin{tabular}{|l|r|r|r|r|r|r|r|r|r|r|}
\hline Peak\# & Ret. Time & \multicolumn{1}{|c|}{ Conc. } & \multicolumn{1}{c|}{ Area } & \multicolumn{1}{c|}{ Height } & Similarity Index & Mark & Peak Start & \multicolumn{1}{c|}{ Peak End } & \multicolumn{1}{c|}{ Area\% } \\
\hline 1 & 12,797 & 44,518 & 5949775 & 150884 & 0,000000 & M & 11,477 & 16,971 & 44,518 \\
\hline 2 & 20,539 & 55,482 & 7415042 & 94953 & 0,000000 & S & 18,208 & 25,653 \\
\hline Total & & 100,000 & 13364817 & 245837 & & & 55,482 \\
\hline
\end{tabular}

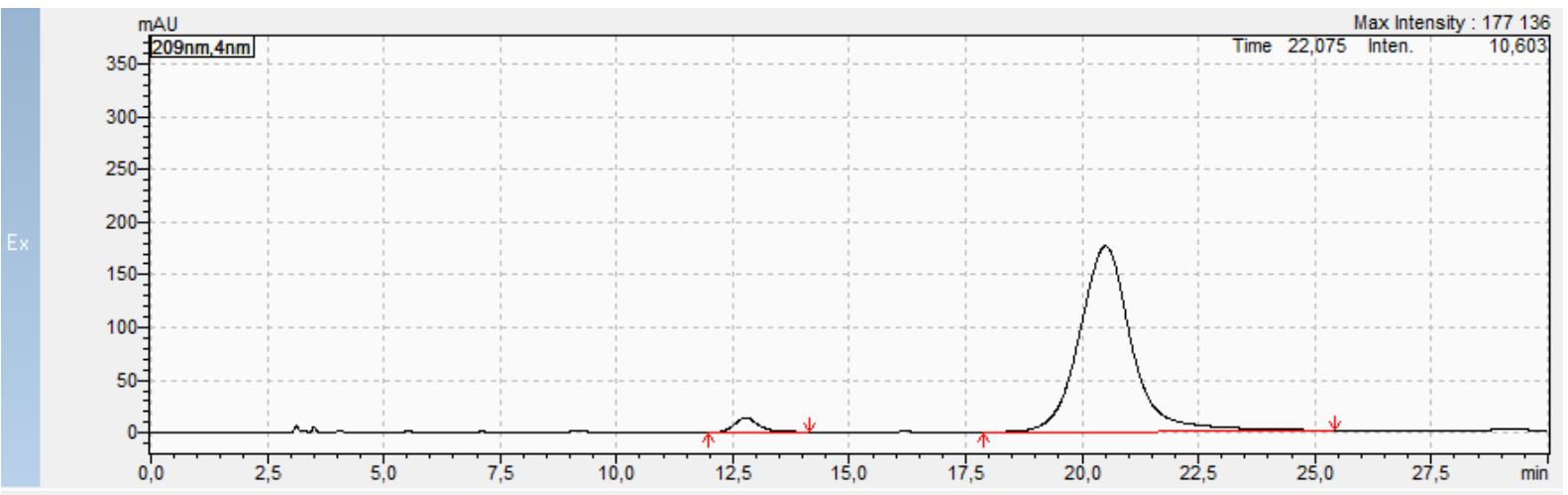

口〈 Results View - Peak Table

\begin{tabular}{|l|l|l|l|}
\hline Peak Table & Compound & Group & Calibration Curve \\
\hline
\end{tabular}

\begin{tabular}{|l|r|r|r|r|r|r|r|r|r|r|}
\hline Peak\# & Ret. Time & \multicolumn{1}{|c|}{ Conc. } & \multicolumn{1}{c|}{ Area } & \multicolumn{1}{c|}{ Height } & Similarity Index & Mark & Peak Start & \multicolumn{1}{c|}{ Peak End } & \multicolumn{1}{c|}{ Area\% } \\
\hline 1 & 12,779 & 3,531 & 501090 & 13691 & 0,000000 & & 11,979 & 14,155 & 3,531 \\
\hline 2 & 20,502 & 96,469 & 13690213 & 175936 & 0,000000 & & 17,877 & 25,440 & 96,469 \\
\hline Total & & 100,000 & 14191303 & 189627 & & & & & \\
\hline
\end{tabular}

for 8: $e e=93 \%$ 


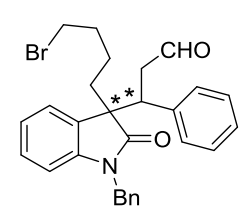

Conditions: IA column

mobile phase: heptane / $i$-PrOH $-80: 20$

$\lambda=214 \mathrm{~nm}, V=1.0 \mathrm{ml} / \mathrm{min}, t=25^{\circ} \mathrm{C}$

for 9: $t_{\mathrm{R}}=15.8 \mathrm{~min}$ (minor), $t_{\mathrm{R}}=38.0 \mathrm{~min}$ (major).

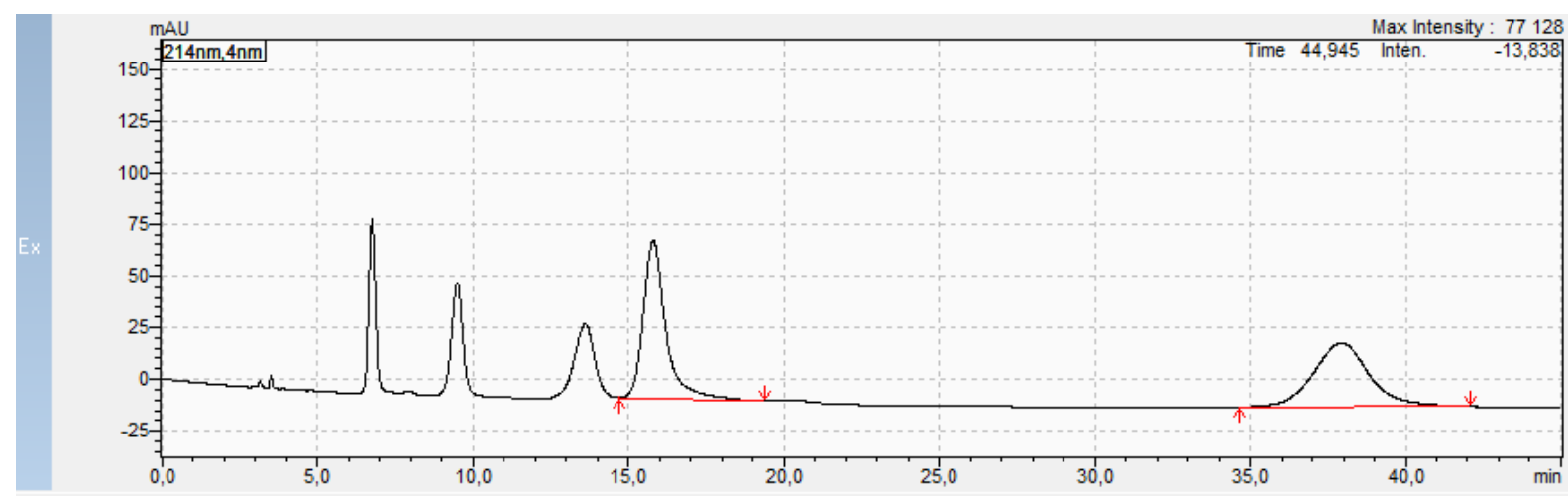

口〈 Results View - Peak Table

\begin{tabular}{|l|l|l|l|}
\hline Peak Table & Compound & Group & Calibration Curve \\
\hline
\end{tabular}

\begin{tabular}{|l|r|r|r|r|r|r|r|r|r|}
\hline Peak\# & Ret. Time & \multicolumn{1}{|c|}{ Conc. } & \multicolumn{1}{c|}{ Area } & Height & Similarity Index & Mark & Peak Start & \multicolumn{1}{c|}{ Peak End } & \multicolumn{1}{c|}{ Area\% } \\
\hline 1 & 15,794 & 50,646 & 3973799 & 76899 & 0,000000 & & 14,688 & 19,403 & 50,646 \\
\hline 2 & 37,954 & 49,354 & 3872458 & 30853 & 0,000000 & S & 34,667 & 42,101 & 49,354 \\
\hline Total & & 100,000 & 7846257 & 107753 & & & & & \\
\hline
\end{tabular}

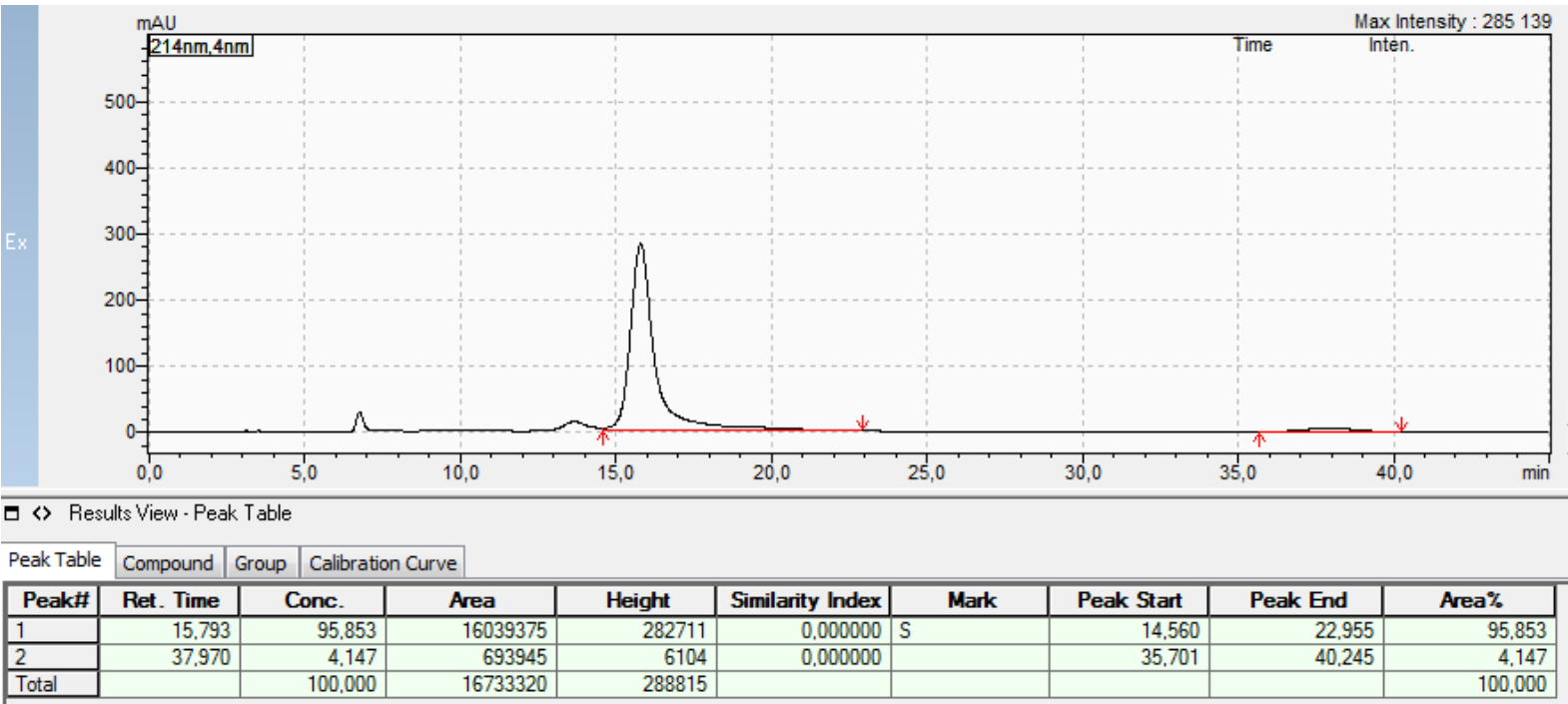

for 9: $e e=92 \%$ 


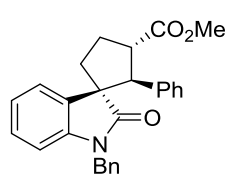

Conditions: IA column

mobile phase: heptane / $i$-PrOH $-80: 20$

$\lambda=209 \mathrm{~nm}, V=1.0 \mathrm{ml} / \mathrm{min}, t=25^{\circ} \mathrm{C}$

for 11a: $t_{\mathrm{R}}=10.2 \mathrm{~min}$ (major), $t_{\mathrm{R}}=11.3 \mathrm{~min}$ (minor).

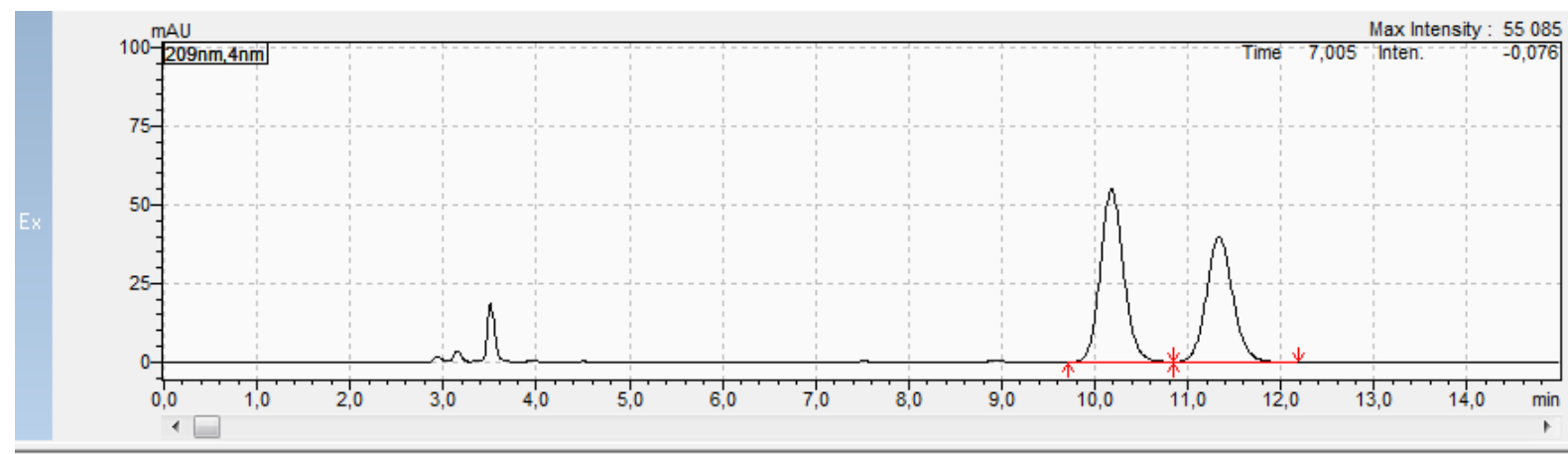

口〈R Results View - Peak Table

\begin{tabular}{l|l|l|l|}
\hline Peak Table & Compound & Group & Calibration Curve \\
\hline
\end{tabular}

\begin{tabular}{|l|r|r|r|r|r|r|r|r|r|}
\hline Peak\# & Ret. Time & \multicolumn{1}{|c|}{ Conc. } & \multicolumn{1}{c|}{ Area } & Height & Similarity Index & Mark & Peak Start & \multicolumn{1}{c|}{ Peak End } & \multicolumn{1}{c|}{ Area\% } \\
\hline 1 & 10,178 & 55,012 & 969154 & 55223 & 0,000000 & V & 9,707 & 10,837 & 55,012 \\
\hline 2 & 11,334 & 44,988 & 792555 & 40032 & 0,000000 & SV & 10,837 & 12,192 & 44,988 \\
\hline Total & & 100,000 & 1761709 & 95255 & & & & 100,000 \\
\hline
\end{tabular}

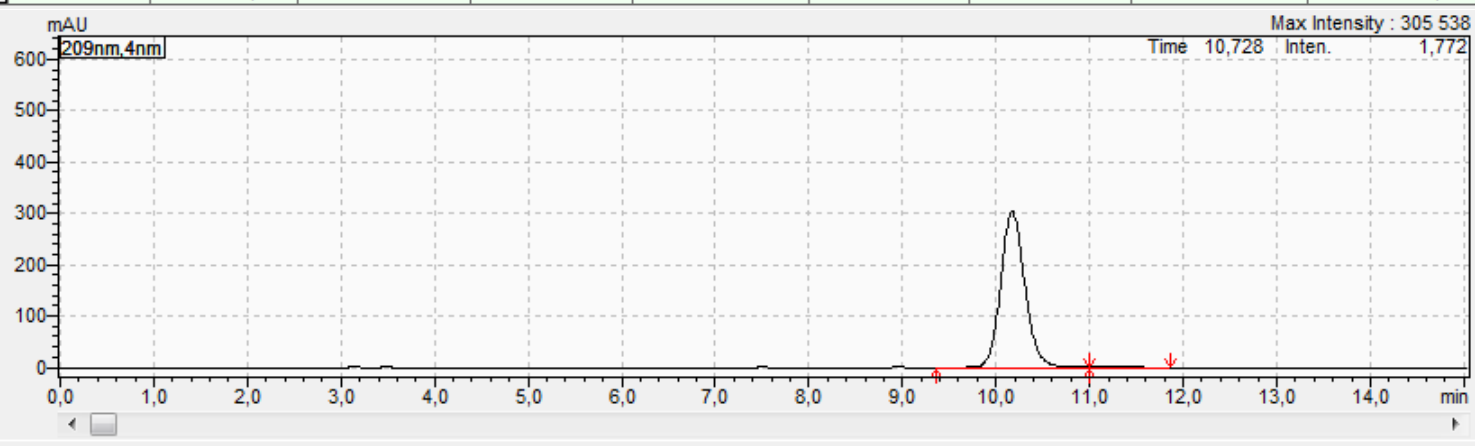

口〈R Results View - Peak Table

\begin{tabular}{l|l|l|l}
\hline Peak Table Compound & Group & Calibration Curve
\end{tabular}

\begin{tabular}{|c|c|c|c|c|c|c|c|c|c|}
\hline Peak\# & Ret. Time & Conc. & Area & Height & Similarity Index & Mark & Peak Start & Peak End & Area\% \\
\hline 1 & 10,173 & 98,928 & 5493153 & 305938 & 0,000000 & & 9.355 & 10,997 & 98,928 \\
\hline 2 & 11,329 & 1.072 & 59545 & 2642 & 0,000000 & V & 10,997 & 11,872 & 1,072 \\
\hline Total & & 100,000 & 5552698 & 308580 & & & & & 100,000 \\
\hline
\end{tabular}

for 11a: $e e=98 \%$ 
<smiles>CC(=O)[C@H]1CC[C@]2(C(=O)Nc3ccccc32)[C@H]1c1ccccc1</smiles>

Conditions: IA column

mobile phase: heptane / $i$-PrOH $-80: 20$

$\lambda=209 \mathrm{~nm}, V=1.0 \mathrm{ml} / \mathrm{min}, t=25^{\circ} \mathrm{C}$

for 11d': $t_{\mathrm{R}}=5.0 \mathrm{~min}$ (major), $t_{\mathrm{R}}=6.6 \mathrm{~min}$ (minor).

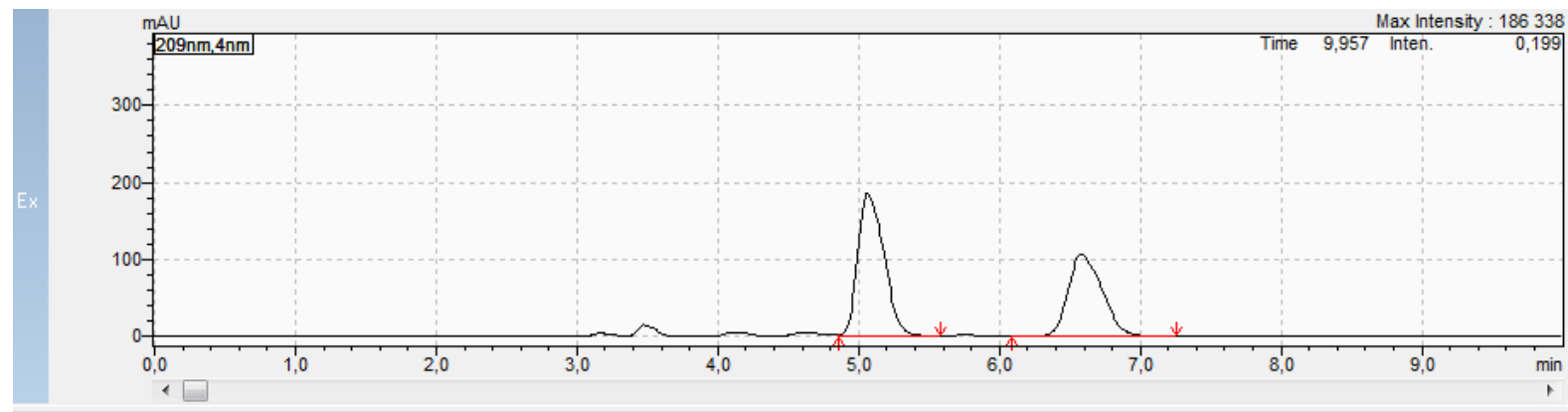

- 〈 Results View - Peak Table

\begin{tabular}{l|l|l|l|}
\hline Peak Table & Compound & Group & Calibration Curve \\
\hline
\end{tabular}

\begin{tabular}{|l|r|r|r|r|r|r|r|r|r|}
\hline Peak\# & Ret. Time & \multicolumn{1}{|c|}{ Conc. } & \multicolumn{1}{c|}{ Area } & Height & Similarity Index & Mark & Peak Start & Peak End & \multicolumn{1}{c|}{ Area\% } \\
\hline 1 & 5,063 & 57,436 & 2407740 & 186227 & 0,000000 & & 5,853 & 5,579 \\
\hline 2 & 6.584 & 42,564 & 1784305 & 106082 & 0,000000 & & 6,091 & 7,253 \\
\hline Total & & 100,000 & 4192045 & 292309 & & & & 42,564 \\
\hline
\end{tabular}

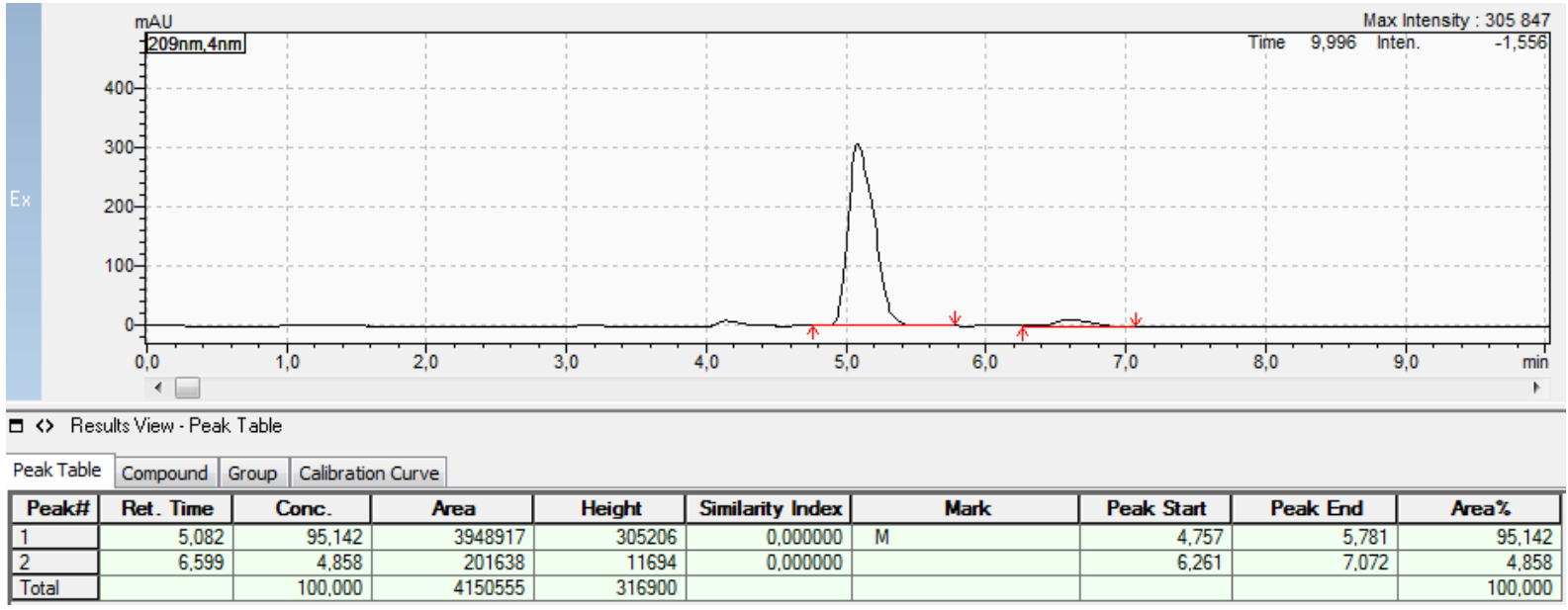

for 11d': ee $=90 \%$ 
Conditions: IA column

mobile phase: heptane / $i$-PrOH $-80: 20$

$\lambda=208 \mathrm{~nm}, V=1.0 \mathrm{ml} / \mathrm{min}, t=25^{\circ} \mathrm{C}$

for 12a: $t_{\mathrm{R}}=8.9 \mathrm{~min}$ (major), $t_{\mathrm{R}}=13.2 \mathrm{~min}$ (minor).

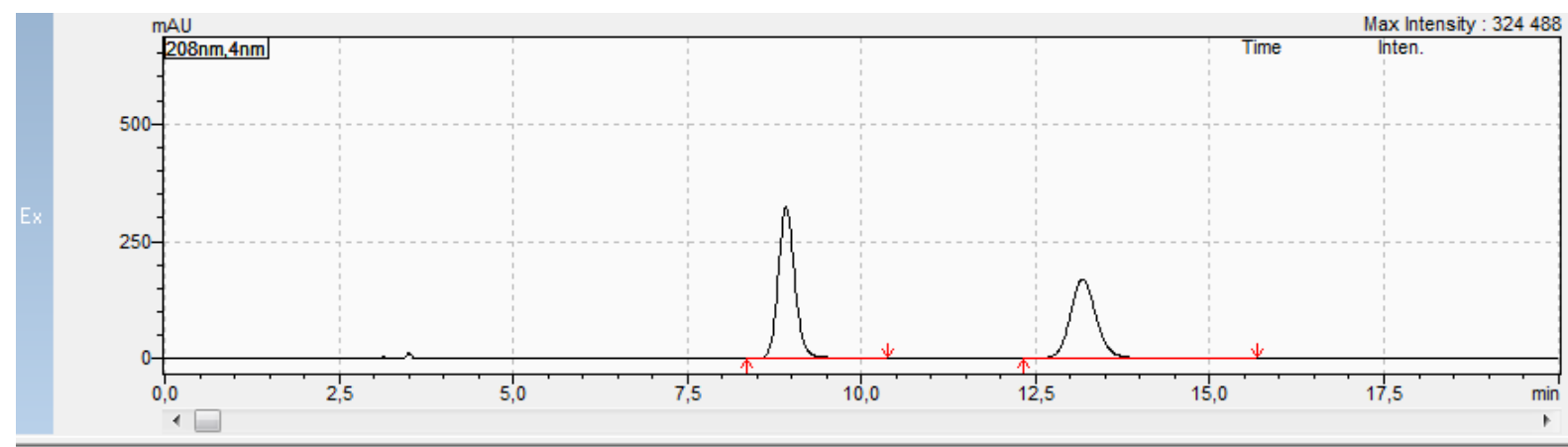

口 \ Results View - Peak Table

\begin{tabular}{|l|l|l|l|}
\hline Peak Table & Compound & Group & Calibration Curve \\
\hline
\end{tabular}

\begin{tabular}{|l|r|r|r|r|r|r|r|r|r|r|}
\hline Peak\# & Ret. Time & \multicolumn{1}{|c|}{ Conc. } & \multicolumn{1}{c|}{ Area } & \multicolumn{1}{c|}{ Height } & Similarity Index & Mark & Peak Start & Peak End & \multicolumn{1}{c|}{ Area\% } \\
\hline 1 & 8,917 & 55,031 & 5367880 & 323577 & 0,000000 & S & 8,363 & 10,389 & 55,031 \\
\hline 2 & 13,175 & 44,969 & 4386388 & 169104 & 0,000000 & SV & 12,320 & 15,680 & 44,969 \\
\hline Total & & 100,000 & 9754268 & 492681 & & & & & 100,000 \\
\hline
\end{tabular}

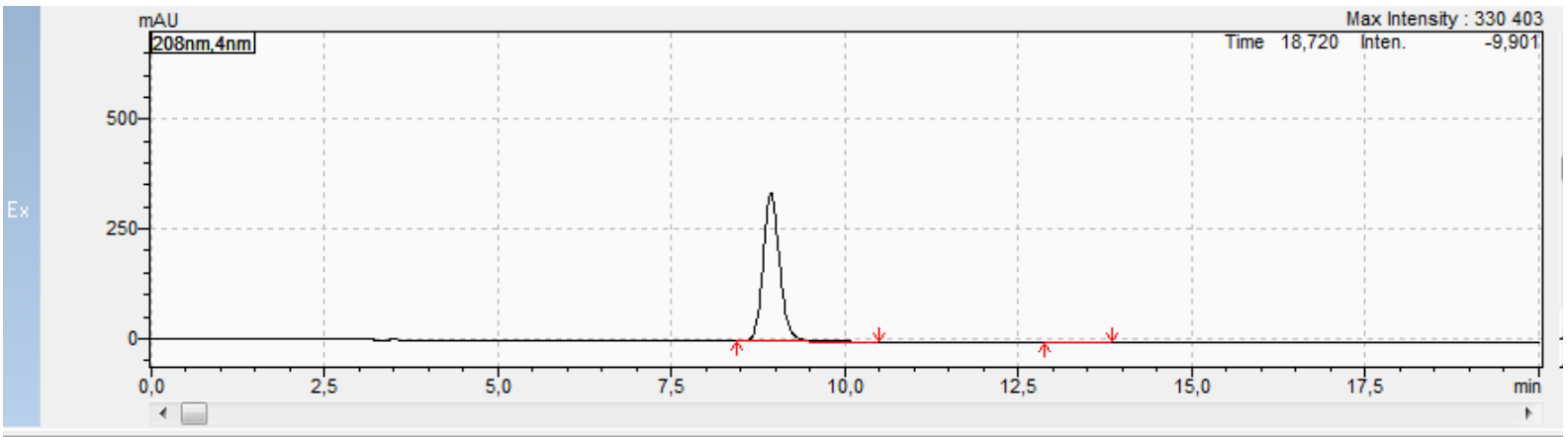

口 〈 Results View - Peak Table

\begin{tabular}{|l|l|l|l|}
\hline Peak Table & Compound & Group & Calibration Curve \\
\hline
\end{tabular}

\begin{tabular}{|l|r|r|r|r|r|r|r|r|r|}
\hline Peak\# & Ret. Time & \multicolumn{1}{|c|}{ Conc. } & \multicolumn{1}{c|}{ Area } & \multicolumn{1}{c|}{ Height } & Similarity Index & Mark & \multicolumn{1}{c|}{ Peak Start } & \multicolumn{1}{c|}{ Peak End } & \multicolumn{1}{c|}{ Area\% } \\
\hline 1 & 8,930 & 99,425 & 5625410 & 336537 & 0,000000 & & 8,427 & 10,496 & 99,425 \\
\hline 2 & 13,205 & 0,575 & 32545 & 1413 & 0,000000 & & 12,864 & 13,856 & 0,575 \\
\hline Total & & 100,000 & 5657955 & 337950 & & & & & \\
\hline
\end{tabular}

for 12a: $e e=99 \%$ 


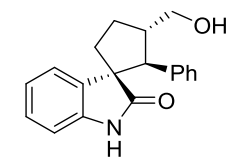

Conditions: IA column

mobile phase: heptane / $i$-PrOH $-80: 20$

$\lambda=208 \mathrm{~nm}, V=1.0 \mathrm{ml} / \mathrm{min}, t=25^{\circ} \mathrm{C}$

for 12d $\mathrm{d}^{\prime}: t_{\mathrm{R}}=5.3 \mathrm{~min}$ (major), $t_{\mathrm{R}}=6.1 \mathrm{~min}$ (minor).

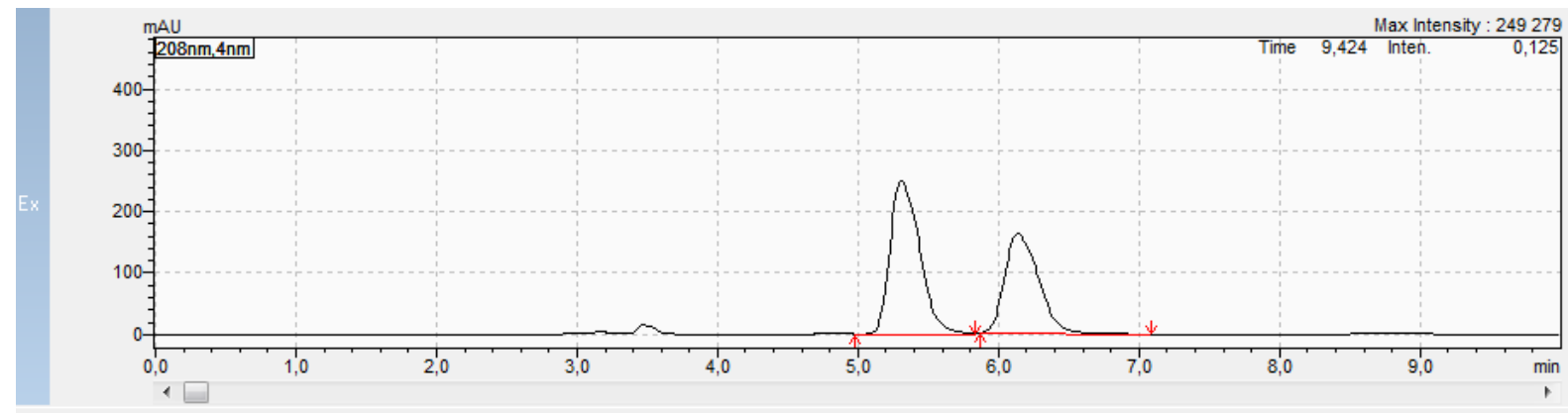

口〈 Results View - Peak Table

\begin{tabular}{l|l|l|l|}
\hline Peak Table & Compound & Group & Calibration Curve \\
\hline
\end{tabular}

\begin{tabular}{|c|c|c|c|c|c|c|c|c|c|}
\hline Peak\# & Ret. Time & Conc. & Area & Height & Similarity Index & Mark & Peak Start & Peak End & Area\% \\
\hline 1 & 5,308 & 57,768 & 3698026 & 249387 & 0,000000 & & 4,981 & 5,835 & 57,768 \\
\hline 2 & 6.139 & 42,232 & 2703474 & 161627 & 0,000000 & M & 5,867 & 7,083 & 42,232 \\
\hline Total & & 100,000 & 6401500 & 411013 & & & & & 100,000 \\
\hline
\end{tabular}

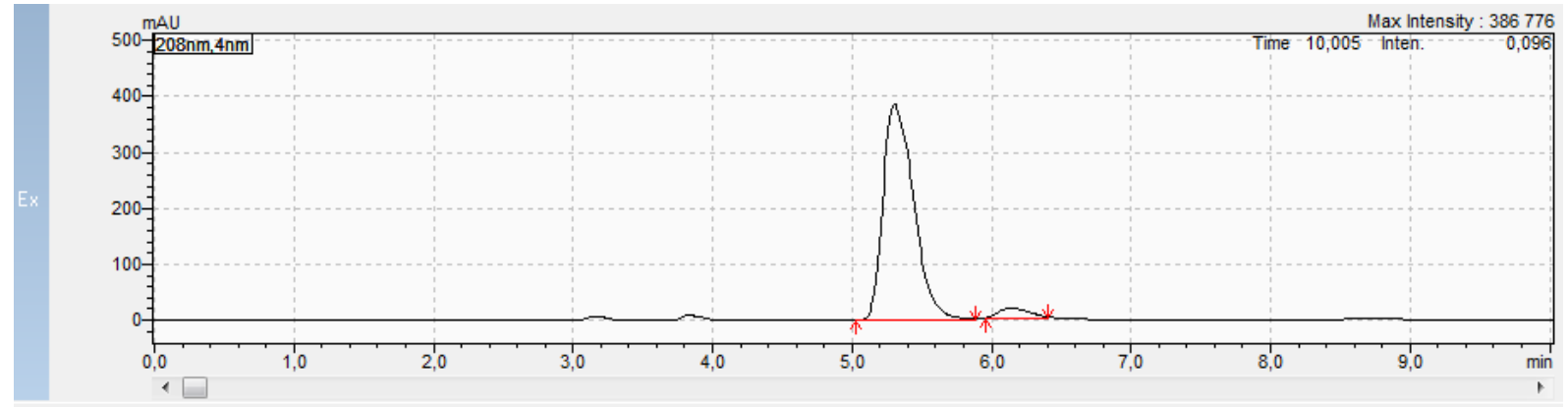

口〈 Results View - Peak Table

\begin{tabular}{l|l|l|l|}
\hline Peak Table Compound & Group & Calibration Curve \\
\hline
\end{tabular}

\begin{tabular}{|c|c|c|c|c|c|c|c|c|c|}
\hline Peak\# & Ret. Time & Conc. & Area & Height & Similarity Index & Mark & Peak Start & Peak End & Area\% \\
\hline 1 & 5,303 & 95,001 & 5934454 & 386537 & 0,000000 & & 5,024 & 5,888 & 95,001 \\
\hline 2 & 6.137 & 4.999 & 312295 & 19424 & 0,000000 & M & 5,963 & 6.400 & 4.999 \\
\hline Total & & 100,000 & 6246749 & 405961 & & & & & 100,000 \\
\hline
\end{tabular}

for 12d': ee $=90 \%$ 


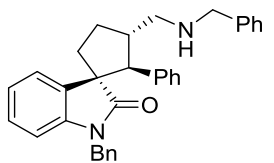

Conditions: IA column

mobile phase: heptane / $i$-PrOH $-50: 50$

$\lambda=256 \mathrm{~nm}, V=1.0 \mathrm{ml} / \mathrm{min}, t=25^{\circ} \mathrm{C}$

for 13a: $t_{\mathrm{R}}=6.6 \mathrm{~min}$ (major), $t_{\mathrm{R}}=7.5 \mathrm{~min}$ (minor).

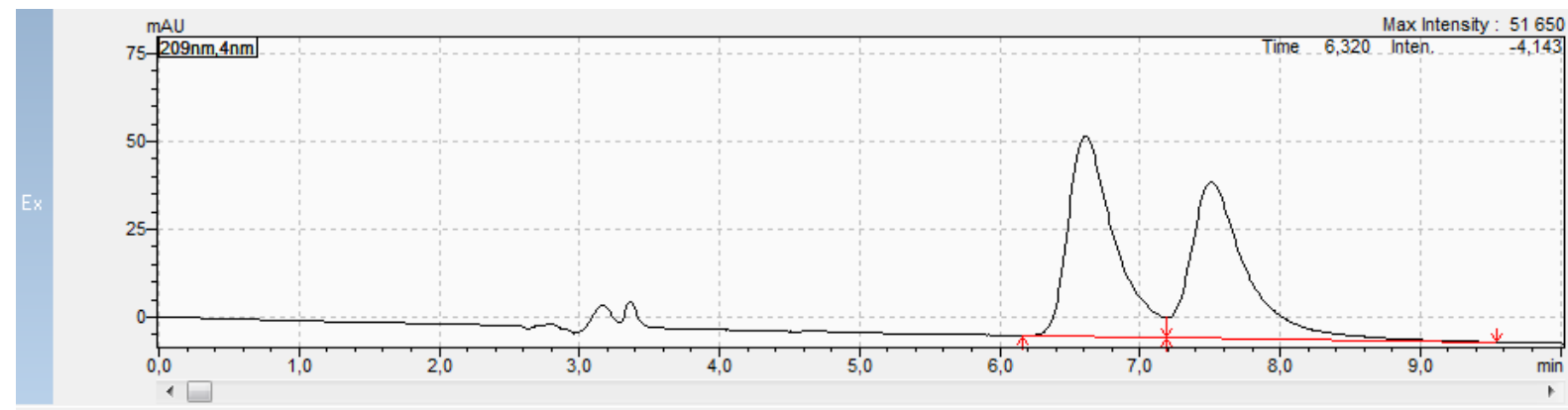

口 $\gg$ Results View - Peak Table

\begin{tabular}{|l|l|l|l|}
\hline Peak Table & Compound & Group & Calibration Curve \\
\hline
\end{tabular}

\begin{tabular}{|c|c|c|c|c|c|c|c|c|c|}
\hline Peak\# & Ret. Time & Conc. & Area & Height & Similarity Index & Mark & Peak Start & Peak End & Area\% \\
\hline 1 & 6.610 & 51.739 & 1320686 & 57199 & 0,000000 & & 6.165 & 7.189 & 51.739 \\
\hline 2 & 7,510 & 48,261 & 1231902 & 44530 & 0,000000 & V & 7,189 & 9,547 & 48,261 \\
\hline Total & & 100,000 & 2552588 & 101728 & & & & & 100,000 \\
\hline
\end{tabular}

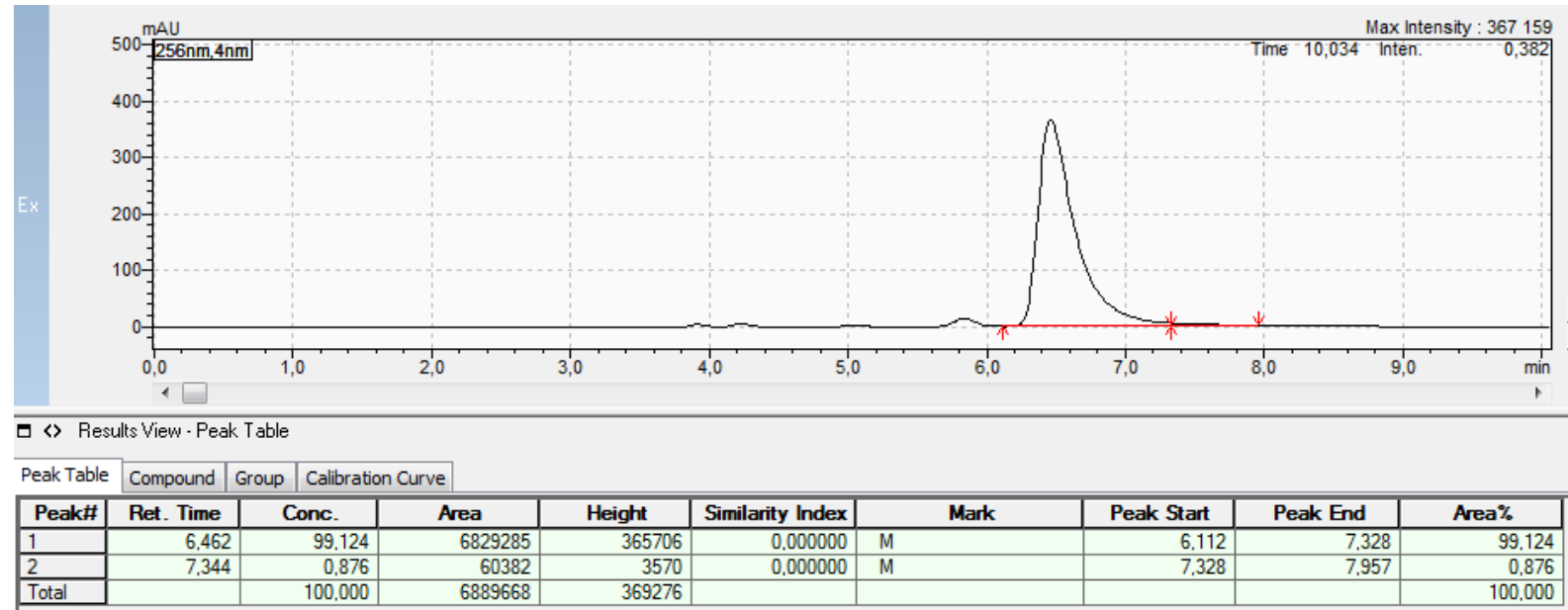

for 13a: $e e=98 \%$ 
Conditions: IA column

mobile phase: heptane / $i$-PrOH $-50: 50$

$\lambda=258 \mathrm{~nm}, V=1.0 \mathrm{ml} / \mathrm{min}, t=25{ }^{\circ} \mathrm{C}$

for 13a': $t_{\mathrm{R}}=8.4 \mathrm{~min}$ (minor), $t_{\mathrm{R}}=11.6 \mathrm{~min}$ (major).

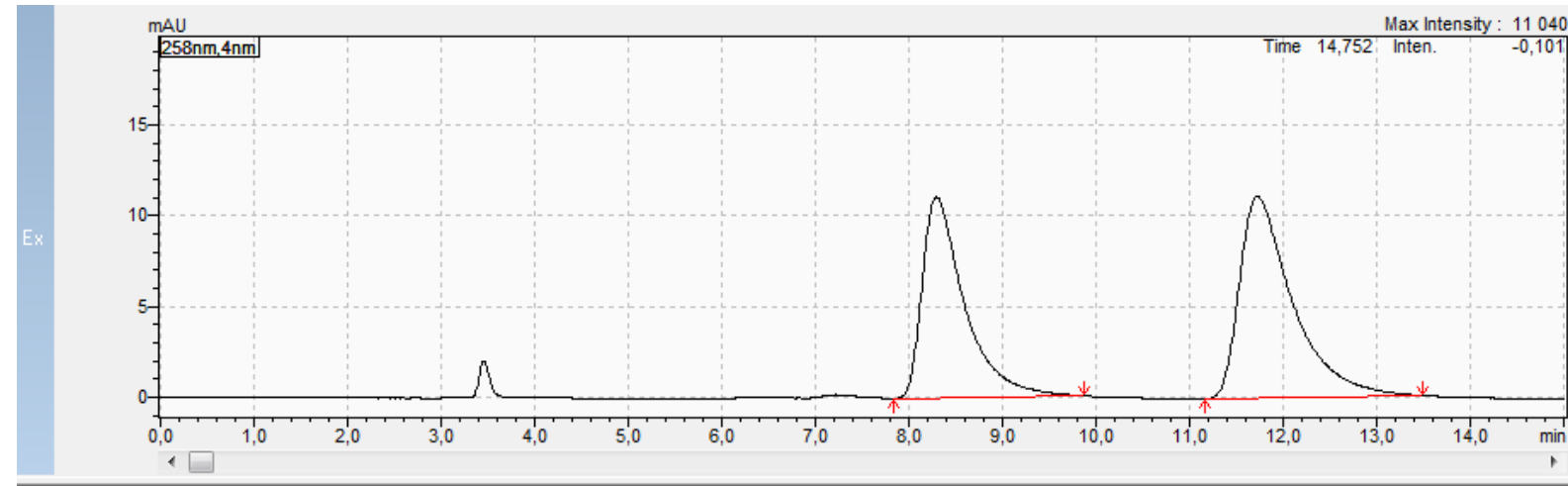

口 〈 Results View - Peak Table

\begin{tabular}{|l|l|l|l|}
\hline Peak Table & Compound & Group & Calibration Curve \\
\hline
\end{tabular}

\begin{tabular}{|l|r|r|r|r|r|r|r|r|r|}
\hline Peak\# & Ret. Time & \multicolumn{1}{|c|}{ Conc. } & \multicolumn{1}{c|}{ Area } & Height & Similarity Index & Mark & Peak Start & \multicolumn{1}{c|}{ Peak End } & \multicolumn{1}{c|}{ Area\% } \\
\hline 1 & 8,298 & 44,295 & 348616 & 11064 & 0,000000 & & 7,829 & 9,877 & 44,295 \\
\hline 2 & 11,728 & 55,705 & 438412 & 11093 & 0,000000 & & 11,157 & 13,493 & 55,705 \\
\hline Total & & 100,000 & 787028 & 22157 & & & & \\
\hline
\end{tabular}

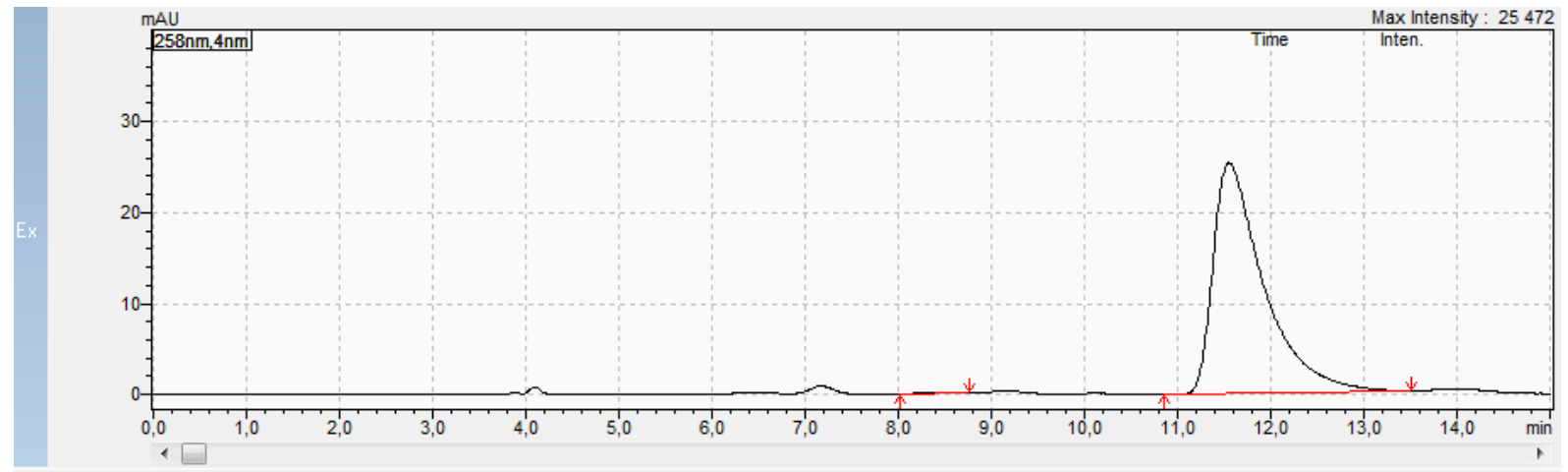

口〈 Results View - Peak Table

\begin{tabular}{|l|l|l|l|}
\hline Peak Table & Compound & Group & Calibration Curve \\
\hline
\end{tabular}

\begin{tabular}{|l|r|r|r|r|r|r|r|r|r|r|}
\hline Peak\# & Ret. Time & \multicolumn{1}{c|}{ Conc. } & \multicolumn{1}{c|}{ Area } & \multicolumn{1}{c|}{ Height } & Similarity Index & Mark & \multicolumn{1}{c|}{ Peak Start } & \multicolumn{1}{c|}{ Peak End } & \multicolumn{1}{c|}{ Area\% } \\
\hline 1 & 8,413 & 0,361 & 3453 & 136 & 0,000000 & M & 8,011 & 8,757 & 0,361 \\
\hline 2 & 11,549 & 99,639 & 954243 & 25316 & 0,000000 & $M$ & 10,848 & 13,504 & 99,639 \\
\hline Total & & 100,000 & 957696 & 25452 & & & & & \\
\hline
\end{tabular}

for 13a': $e e=99 \%$ 
Conditions: IA column

mobile phase: heptane / $i$-PrOH $-50: 50$

$\lambda=256 \mathrm{~nm}, V=1.0 \mathrm{ml} / \mathrm{min}, t=25{ }^{\circ} \mathrm{C}$

for 13d': $t_{\mathrm{R}}=5.1 \mathrm{~min}$ (major), $t_{\mathrm{R}}=6.5 \mathrm{~min}$ (minor).

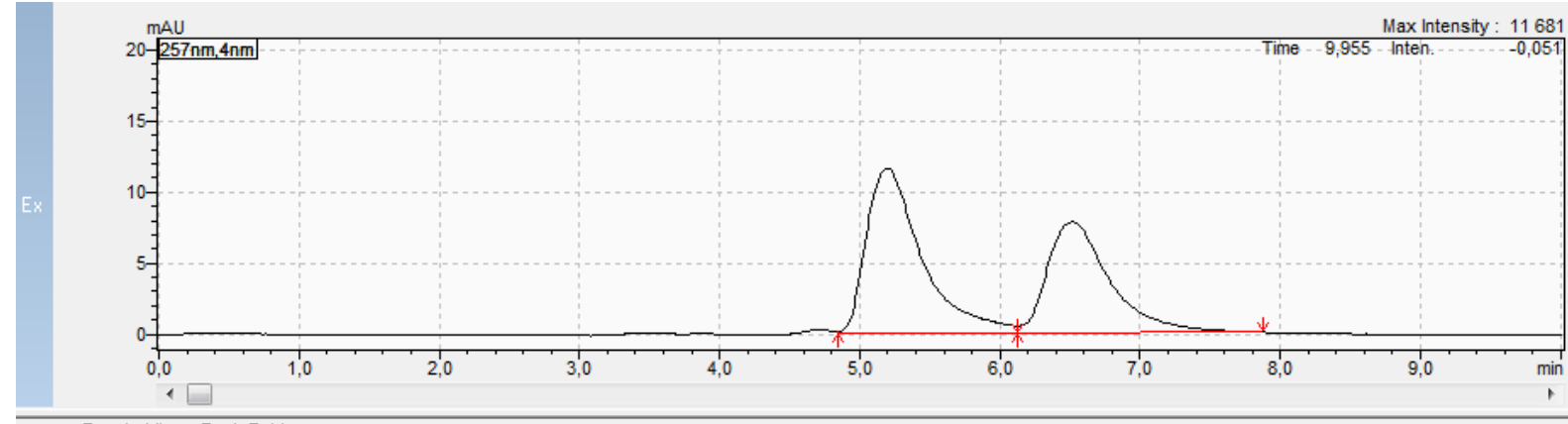

口 〈 Results View - Peak Table

Peak Table Compound Group Calibration Curve

\begin{tabular}{|l|r|r|r|r|r|r|r|r|r|}
\hline Peak\# & Ret. Time & \multicolumn{1}{|c|}{ Conc. } & \multicolumn{1}{c|}{ Area } & Height & Similarity Index & Mark & Peak Start & Peak End & \multicolumn{1}{c|}{ Area\% } \\
\hline 1 & 5,195 & 56,988 & 323112 & 11655 & 0,000000 & & 4,843 & 6,123 & 56,988 \\
\hline 2 & 6,512 & 43,012 & 243870 & 7835 & 0,000000 & V & & 6,123 & \\
\hline Total & & 100,000 & 566981 & 19490 & & & & 43,883 & \\
\hline
\end{tabular}

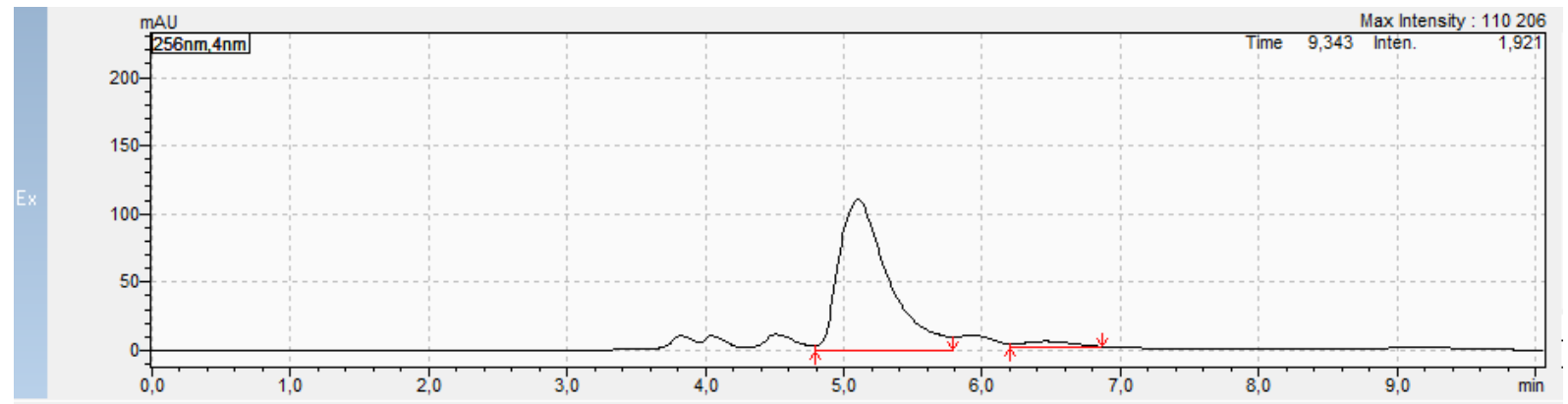

口〈 Results View - Peak Table

\begin{tabular}{l|l|l|l|}
\hline Peak Table & Compound & Group & Calibration Curve \\
\hline
\end{tabular}

\begin{tabular}{|c|c|c|c|c|c|c|c|c|c|}
\hline Peak\# & Ret. Time & Conc. & Area & Height & Similarity Index & Mark & Peak Start & Peak End & Area\% \\
\hline 1 & 5,103 & 95,360 & 2766681 & 109948 & 0,000000 & & 4,789 & 5,792 & 95,360 \\
\hline 2 & 6.456 & 4,640 & 134635 & 4889 & 0,000000 & M & 6.197 & 6.869 & 4.640 \\
\hline Total & & 100,000 & 2901316 & 114837 & & & & & 100,000 \\
\hline
\end{tabular}

for $13 d^{\prime}:$ e $e=90 \%$ 


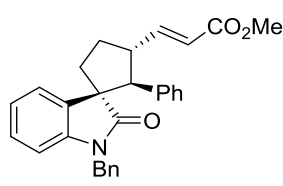

Conditions: IA column

mobile phase: heptane / $i$-PrOH $-80: 20$

$\lambda=208 \mathrm{~nm}, V=1.0 \mathrm{ml} / \mathrm{min}, t=25^{\circ} \mathrm{C}$

for 14a: $t_{\mathrm{R}}=11.2 \mathrm{~min}$ (major), $t_{\mathrm{R}}=15.3 \mathrm{~min}$ (minor).

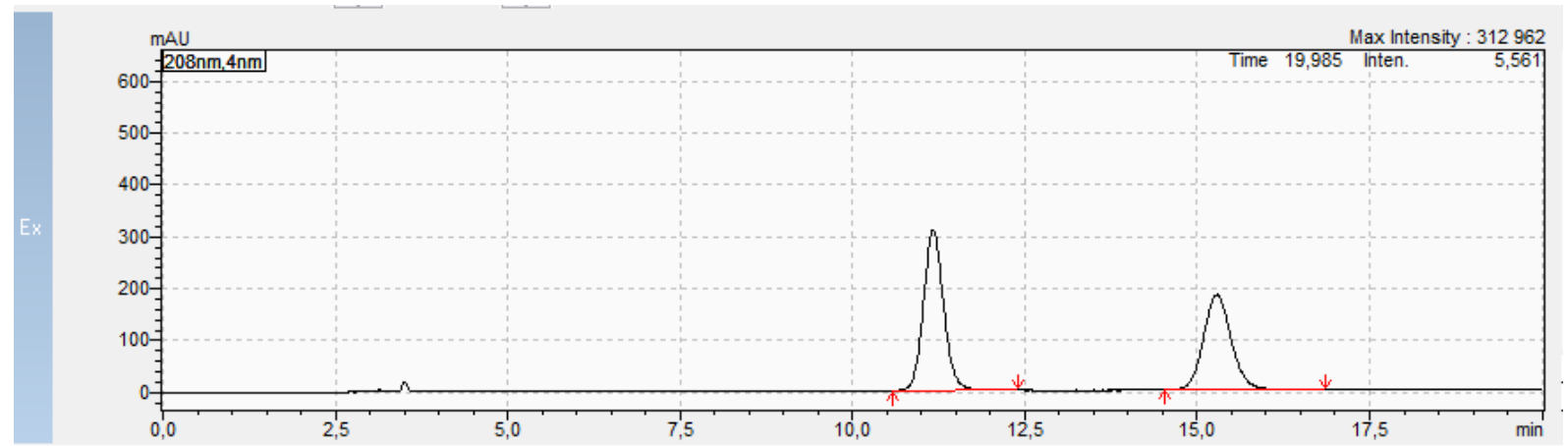

口〈 Results View - Peak Table

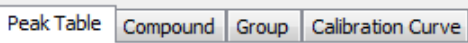

\begin{tabular}{|l|r|r|r|r|r|r|r|r|r|r|}
\hline Peak\# & Ret. Time & \multicolumn{1}{|c|}{ Conc. } & \multicolumn{1}{c|}{ Area } & \multicolumn{1}{c|}{ Height } & Similarity Index & Mark & Peak Start & Peak End & \multicolumn{1}{c|}{ Area\% } \\
\hline 1 & 11,171 & 54,852 & 6300561 & 308964 & 0,000000 & & 10,592 & 12,405 & 54,852 \\
\hline 2 & 15,285 & 45,148 & 5185873 & 183898 & 0,000000 & S & 14,539 & 16,853 & 45,148 \\
\hline Total & & 100,000 & 11486434 & 492862 & & & & \\
\hline
\end{tabular}

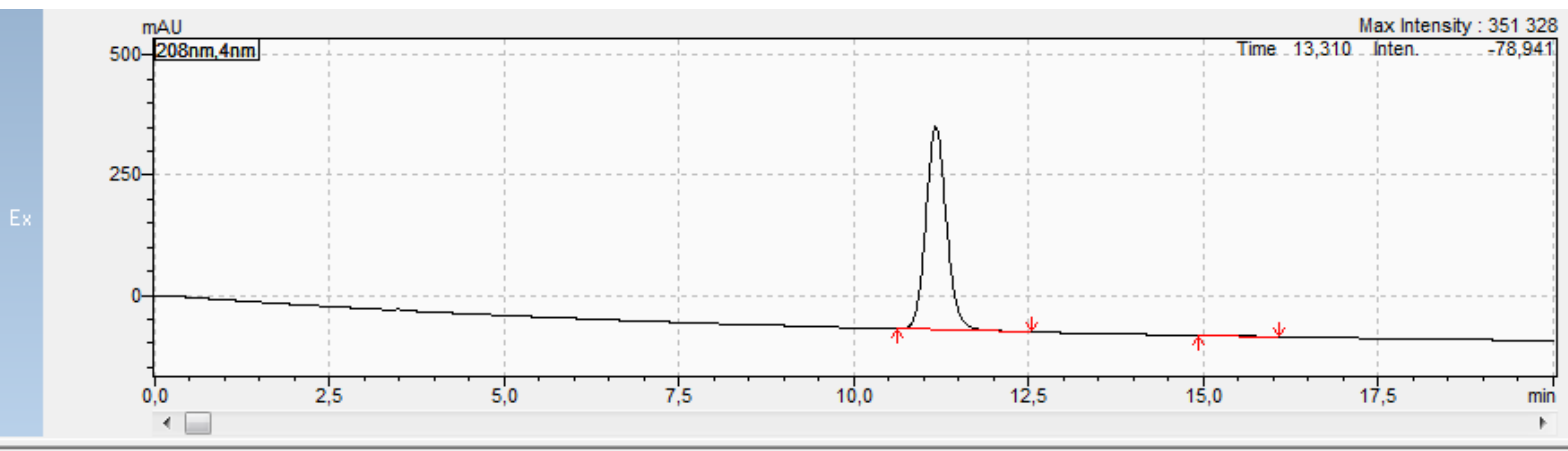

口〈Results View - Peak Table

\begin{tabular}{|l|l|l|l|}
\hline Peak Table & Compound & Group & Calibration Curve \\
\hline
\end{tabular}

\begin{tabular}{|l|r|r|r|r|r|r|r|r|r|r|}
\hline Peak\# & Ret. Time & \multicolumn{1}{|c|}{ Conc. } & \multicolumn{1}{c|}{ Area } & \multicolumn{1}{c|}{ Height } & Similarity Index & Mark & \multicolumn{1}{c|}{ Peak Start } & \multicolumn{1}{c|}{ Peak End } & \multicolumn{1}{c|}{ Area\% } \\
\hline 1 & 11,169 & 99,372 & 8697478 & 422895 & 0,000000 & & 10,624 & 12,544 & 99,372 \\
\hline 2 & 15,296 & 0,628 & 54972 & 2087 & 0,000000 & & 14,923 & 16,075 & 0,628 \\
\hline Total & & 100,000 & 8752450 & 424982 & & & & 100,000 \\
\hline
\end{tabular}

for 14a: $e e=99 \%$ 


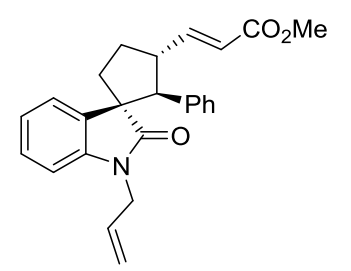

Conditions: IA column

mobile phase: heptane / $i$-PrOH $-90: 10$

$\lambda=211 \mathrm{~nm}, V=1.0 \mathrm{ml} / \mathrm{min}, t=25^{\circ} \mathrm{C}$

for 14b: $t_{\mathrm{R}}=9.8 \mathrm{~min}$ (major), $t_{\mathrm{R}}=11.6 \mathrm{~min}$ (minor).

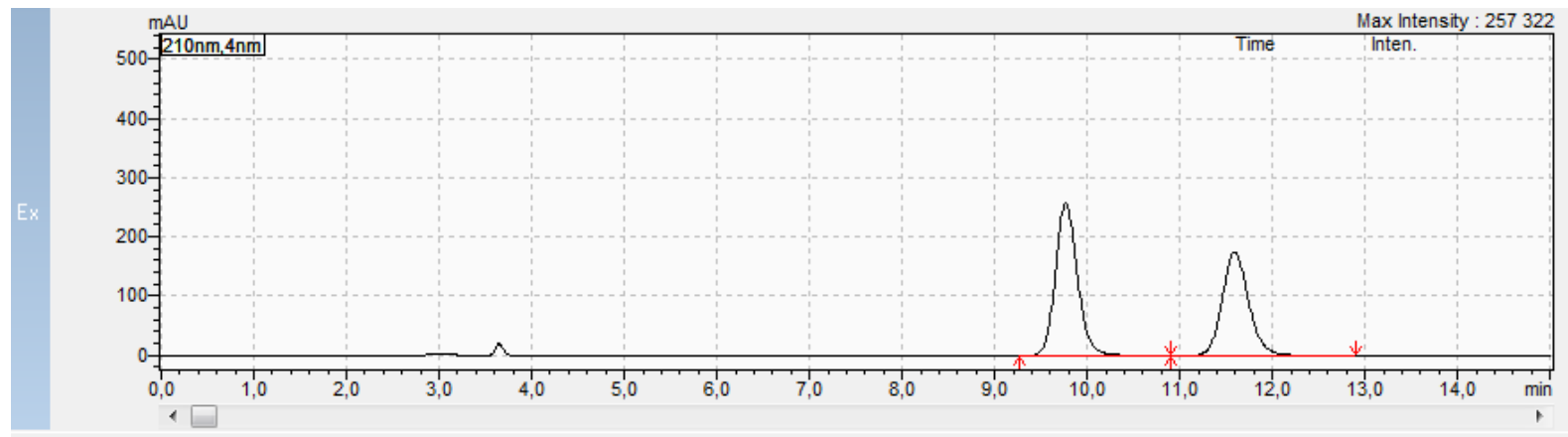

口〈 Results View - Peak Table

\begin{tabular}{|l|l|l|}
\hline Peak Table Compound & Group & Calibration Curve \\
\hline
\end{tabular}

\begin{tabular}{|l|r|r|r|r|r|r|r|r|r|}
\hline Peak\# & Ret. Time & \multicolumn{1}{c|}{ Conc. } & \multicolumn{1}{c|}{ Area } & Height & Similarity Index & Mark & \multicolumn{1}{c|}{ Peak Start } & \multicolumn{1}{c|}{ Peak End } & \multicolumn{1}{c|}{ Area\% } \\
\hline 1 & 9,768 & 55,098 & 4302319 & 258532 & 0,000000 & & 9,269 & 10,891 & 55,098 \\
\hline 2 & 11,592 & 44,902 & 3506181 & 175007 & 0,000000 & V & 10,891 & 12,907 \\
\hline Total & & 100,000 & 7808500 & 433539 & & & 44,902 \\
\hline
\end{tabular}

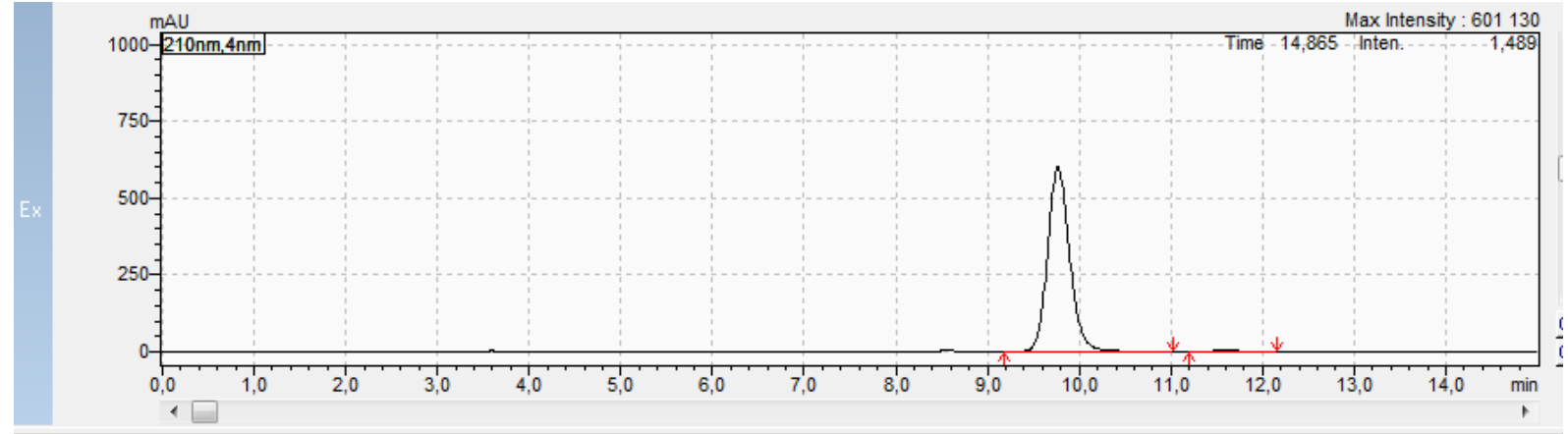

口〈 Results View - Peak Table

\begin{tabular}{|l|l|l|l|}
\hline Peak Table & Compound & Group & Calibration Curve \\
\hline
\end{tabular}

\begin{tabular}{|c|c|c|c|c|c|c|c|c|c|}
\hline Peak\# & Ret. Time & Conc. & Area & Height & Similarity Index & Mark & Peak Start & Peak End & Area\% \\
\hline 1 & 9,768 & 99,311 & 10025761 & 600546 & 0,000000 & & 9.173 & 11.019 & 99,311 \\
\hline 2 & 11,602 & 0.689 & 69595 & 3593 & 0,000000 & & 11,189 & 12,149 & 0,689 \\
\hline Total & & 100,000 & 10095356 & 604139 & & & & & 100,000 \\
\hline
\end{tabular}

for 14b: $e e=99 \%$ 


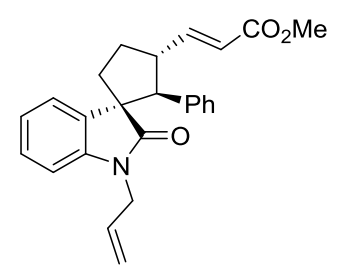

Conditions: IA column

mobile phase: heptane / $i$-PrOH $-90: 10$

$\lambda=211 \mathrm{~nm}, V=1.0 \mathrm{ml} / \mathrm{min}, t=25^{\circ} \mathrm{C}$

for $14 \mathbf{b}^{\prime}: t_{\mathrm{R}}=7.3 \min$ (minor), $t_{\mathrm{R}}=8.0 \mathrm{~min}$ (major).

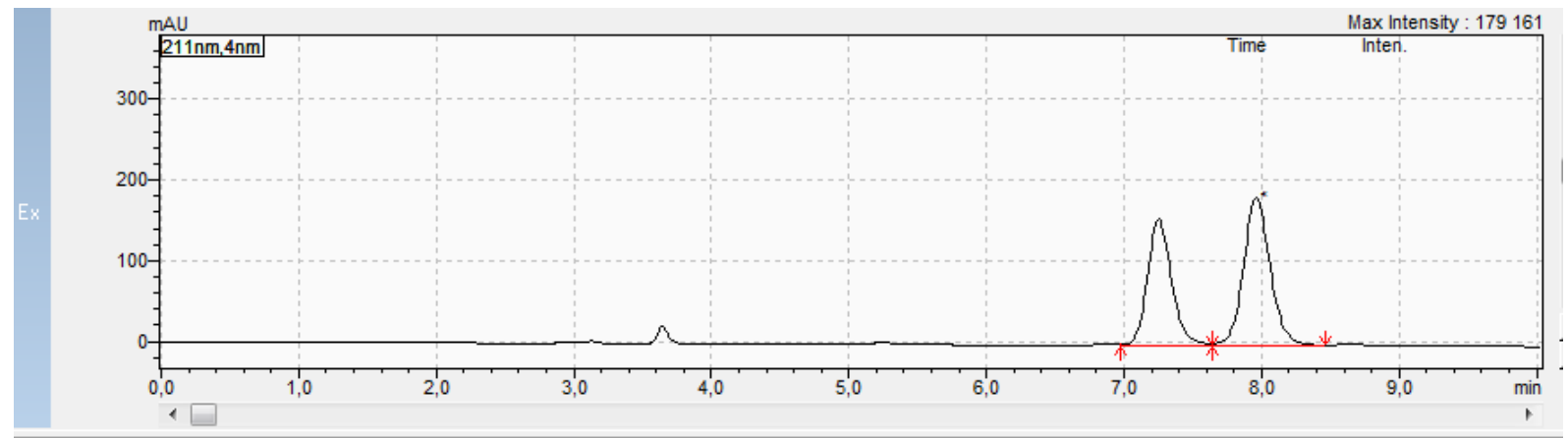

口〈 Results View - Peak Table

\begin{tabular}{|l|l|l|l|}
\hline Peak Table & Compound & Group & Calibration Curve \\
\hline
\end{tabular}

\begin{tabular}{|c|c|c|c|c|c|c|c|c|c|}
\hline Peak\# & Ret. Time & Conc. & Area & Height & Similarity Index & Mark & Peak Start & Peak End & Area\% \\
\hline $11 \ldots$ & 7.252 & 43,268 & 1914139 & 156299 & 0,000000 & & 6.976 & 7,637 & 43,268 \\
\hline 2 & 7,961 & 56,732 & 2509751 & 184513 & 0,000000 & V & 7.637 & 8.459 & 56.732 \\
\hline Total & & 100,000 & 4423890 & 340812 & & & & & 100,000 \\
\hline
\end{tabular}

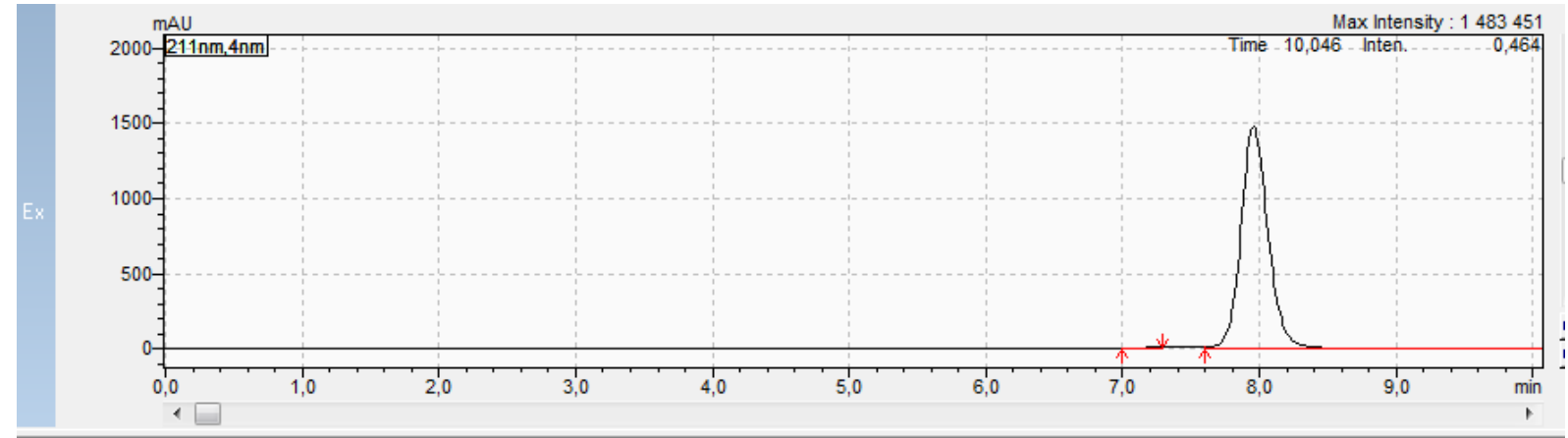

口〈 Results View - Peak Table

\begin{tabular}{l|l|l|l|}
\hline Peak Table & Compound & Group & Calibration Curve \\
\hline
\end{tabular}

\begin{tabular}{|l|r|r|r|r|r|r|r|r|r|}
\hline Peak\# & Ret. Time & \multicolumn{1}{|c|}{ Conc. } & \multicolumn{1}{c|}{ Area } & Height & Similarity Index & Mark & Peak Start & \multicolumn{1}{c|}{ Peak End } & \multicolumn{1}{c|}{ Area\% } \\
\hline 1 & 7,253 & 0,584 & 120966 & 13408 & 0,000000 & M & 6,997 & 7,296 & 0,584 \\
\hline 2 & 7,957 & 99,416 & 20598107 & 1483242 & 0,000000 & & 7,605 & 10,325 & 99,416 \\
\hline Total & & 100,000 & 20719073 & 1496650 & & & & & \\
\hline
\end{tabular}

for $14 \mathbf{b}^{\prime}: e e=99 \%$ 
<smiles>CCCC(C=CC(C)=O)C1(O)c2ccccc2C(=O)N1C</smiles>
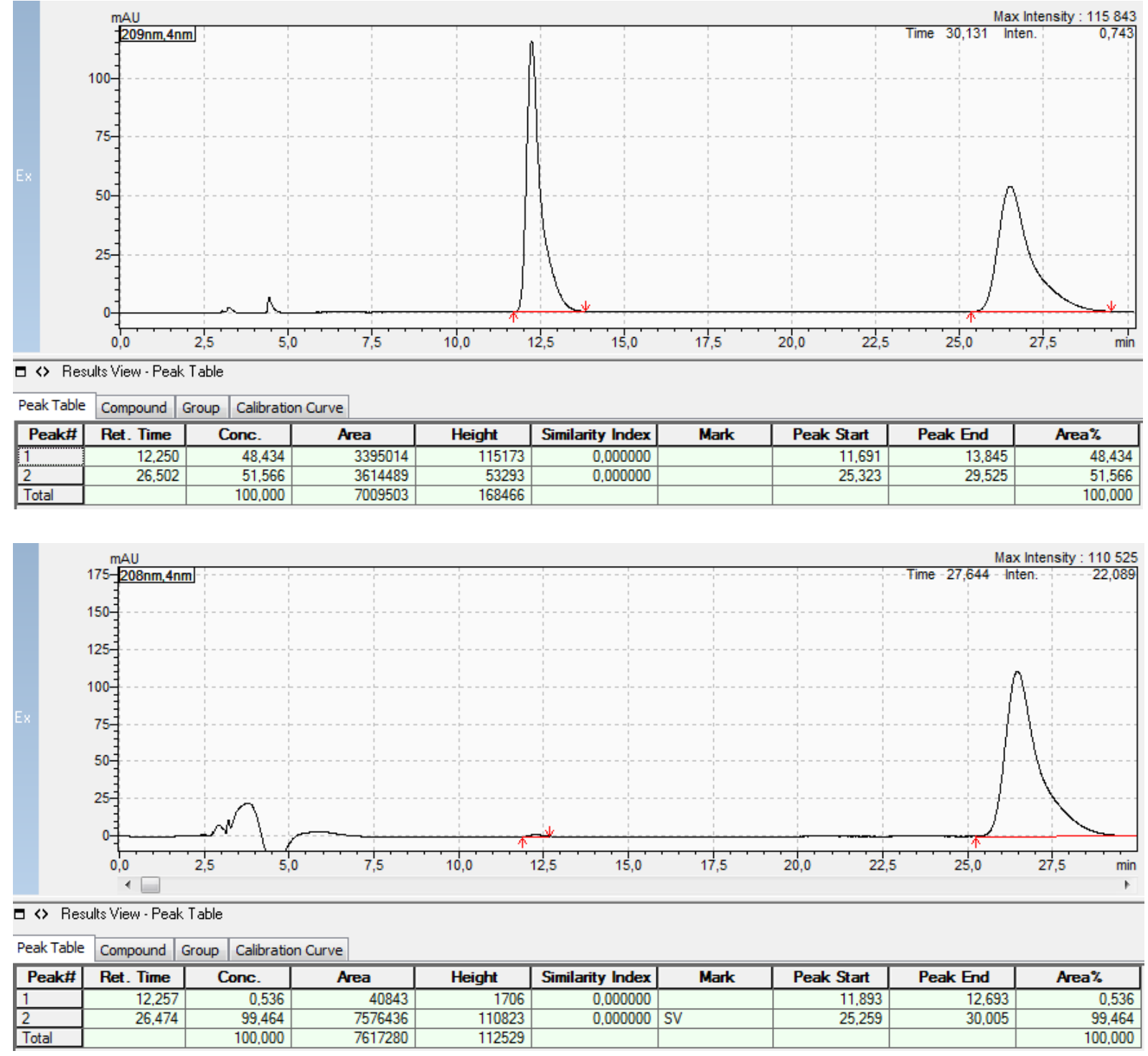

for 14c: $e e=99 \%$ mobile phase: heptane / $i$-PrOH $-80: 20$

$\lambda=209 \mathrm{~nm}, V=1.0 \mathrm{ml} / \mathrm{min}, t=25^{\circ} \mathrm{C}$

for 14c: $t_{\mathrm{R}}=12.3 \mathrm{~min}$ (major), $t_{\mathrm{R}}=26.5 \mathrm{~min}$ (minor).
Conditions: IC column 


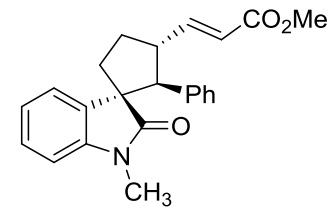

Conditions: IC column

mobile phase: heptane / $i$-PrOH $-80: 20$

$\lambda=258 \mathrm{~nm}, V=1.0 \mathrm{ml} / \mathrm{min}, t=25^{\circ} \mathrm{C}$

for $14 \mathbf{c}^{\prime}: t_{\mathrm{R}}=9.4 \mathrm{~min}$ (major), $t_{\mathrm{R}}=11.7 \mathrm{~min}$ (minor).

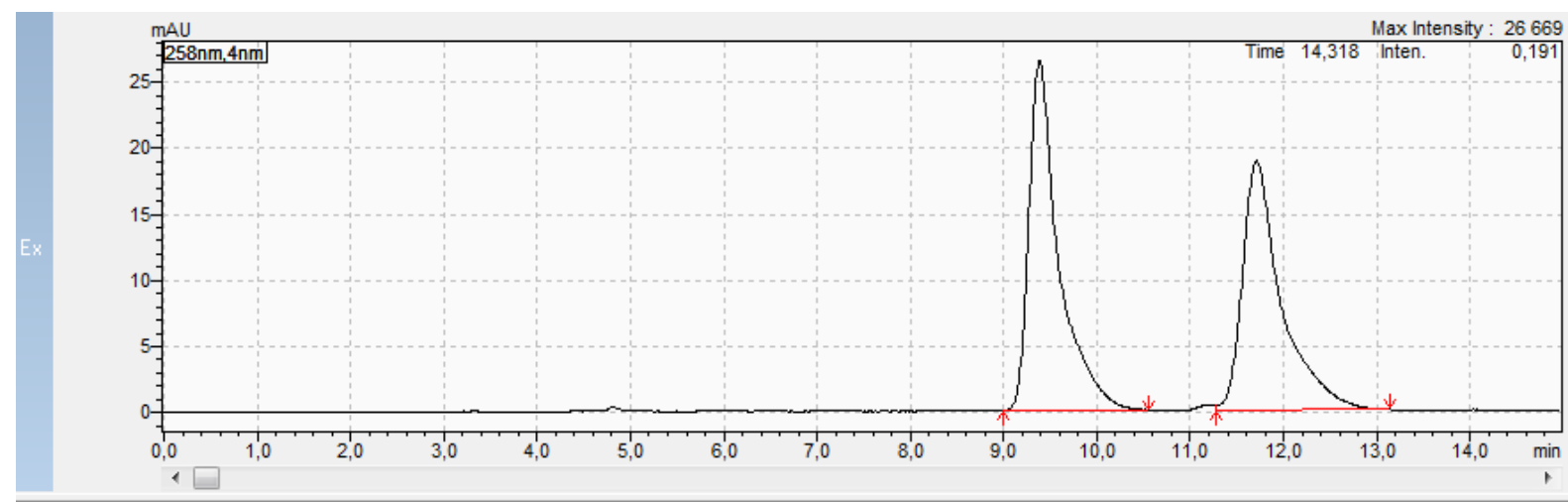

口 $\gg$ Results View - Peak Table

\begin{tabular}{|l|l|l|l|}
\hline Peak Table Compound & Group & Calibration Curve \\
\hline
\end{tabular}

\begin{tabular}{|c|c|c|c|c|c|c|c|c|c|}
\hline Peak\# & Ret. Time & Conc. & Area & Height & Similarity Index & Mark & Peak Start & Peak End & Area\% \\
\hline $11 m-m$ & 9.384 & 51,912 & 588445 & 26507 & 0,000000 & & 8,992 & 10,549 & 51.912 \\
\hline 2 & 11.710 & 48,088 & 545102 & 18900 & 0,000000 & & 11,285 & 13,131 & 48,088 \\
\hline Total & & 100,000 & 1133547 & 45406 & & & & & 100,000 \\
\hline
\end{tabular}

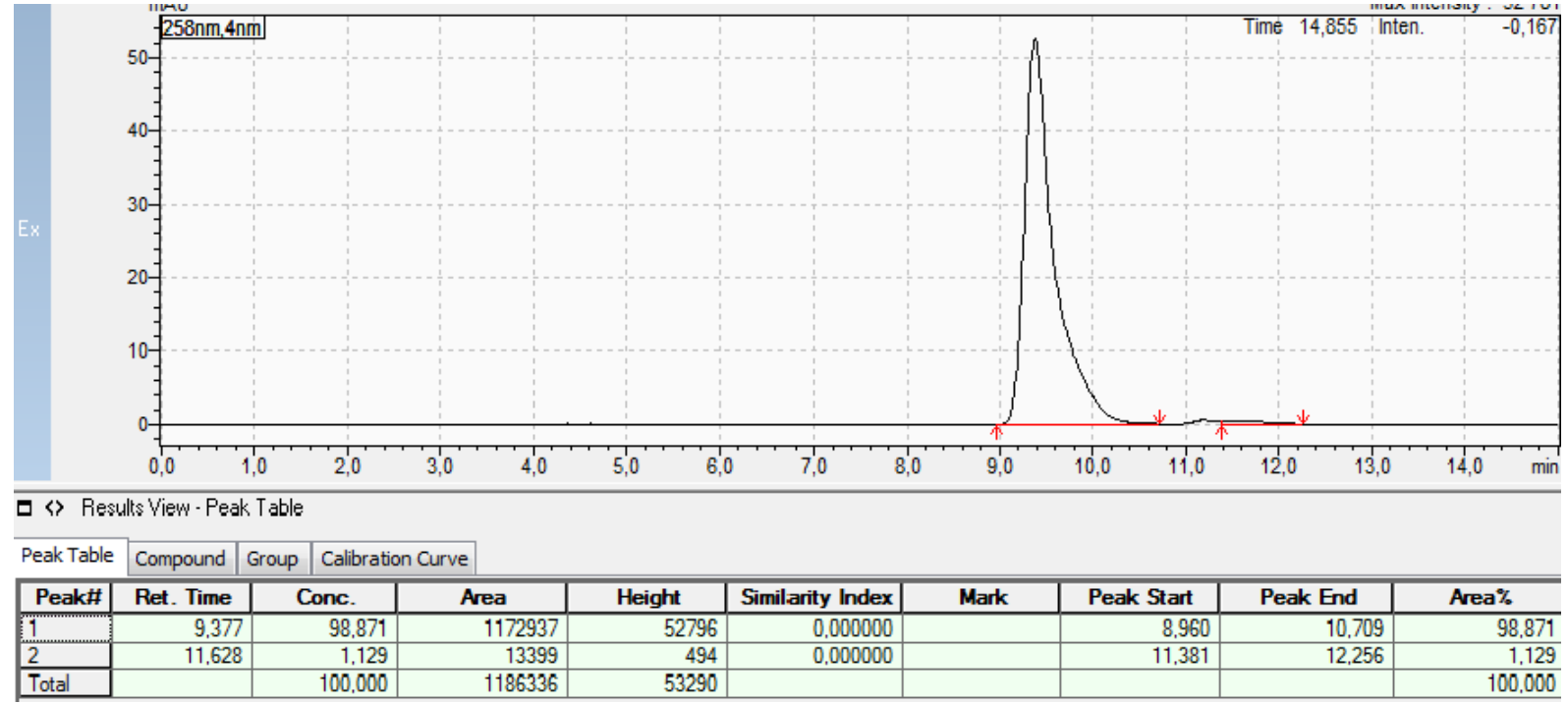

for $14 \mathbf{c}^{\prime}: e e=98 \%$ 


\section{References}

(1) Sheldrick, G. M. SHELXT - Integrated Space-Group and Crystal-Structure Determination. Acta Crystallogr. Sect. A Found. Crystallogr. 2015, 71, 3-8.

(2) Sheldrick, G. M. Crystal Structure Refinement with SHELXL. Acta Crystallogr. Sect. C Struct. Chem. 2015, 71, 3-8.

(3) Frisch, M. J. G. W. Trucks, H. B. Schlegel, G. E. Scuseria, M. A. Robb, J. R. Cheeseman, G. Scalmani, V. Barone, G. A. Petersson, H. Nakatsuji, X. Li, X. Caricato, A. V. Marenich, J. Bloino, B. G. Janesko, R. Gomperts, B. Mennucci, H. P. Hratchian, J. V. Ortiz, A. F. Izmaylov, J. L Sonnenberg, D. Williams-Young, F. Ding, F. Lipparini, F. Egidi, J. Goings, B. Peng, A. T. Petrone, T. Henderson, D. Ranasinghe, V. G. Zakrzewski, J. Gao, N. Rega, G. Zheng, W. Liang, M. Hada, M. Ehara, K. Toyota, R. Fukuda, J. Hasegawa, M. Ishida, T. Nakajima, Y. Honda, O. Kitao, H. Nakai, T. Vreven, K. Throssell, J. A. Montgomery, J. E. Peralta, F. Ogliaro, M. J. Bearpark, J. J. Heyd, E. N. Brothers, K. N. Kudin, V. N. Staroverov, T. A. Keith, R. Kobayashi, J. Normand, K. Raghavachari, A. P. Rendell, J. C. Burant, S. S. Iyengar, J. Tomasi, M. Cossi, J. M. Millam, M. Klene, C. Adamo, R. Cammi, J. W. Ochterski, R. L. Martin, K. Morokuma, O. Farkas, J. B. Foresman, D. J. Fox, Gaussian 16, Revision A.03, Gaussian, Inc., Wallingford CT, 2016.

(4) Zhao, Y.; Truhlar, D. G. The M06 Suite of Density Functionals for Main Group Thermochemistry, Thermochemical Kinetics, Noncovalent Interactions, Excited States, and Transition Elements: Two New Functionals and Systematic Testing of Four M06-Class Functionals and 12 Other Function. Theor. Chem. Acc. 2008, 120, 215-241.

(5) Weigend, F. Accurate Coulomb-Fitting Basis Sets for H to Rn. Phys. Chem. Chem. Phys. 2006, 8, 1057-1065.

(6) Marenich, A. V.; Cramer, C. J.; Truhlar, D. G. Universal Solvation Model Based on Solute Electron Density and on a Continuum Model of the Solvent Defined by the Bulk Dielectric Constant and Atomic Surface Tensions. J. Phys. Chem. B 2009, 113, 6378-6396.

(7) Determination of Minimum Inhibitory Concentrations (MICs) of Antibacterial Agents by Broth Dilution. Clin. Microbiol. Infect. 2003, 9, 1-7.

(8) a) EUCAST DEFINITIVE DOCUMENT E.DEF 7.3.1. Method for the determination of broth dilution minimum inhibitory concentrations of antifungal agents for yeasts. 2017, 1-21. b) EUCAST DEFINITIVE DOCUMENT E.DEF 9.3.1. Method for the determination of broth dilution minimum inhibitory concentrations of antifungal agents for conidia forming moulds. 2017, 1-23. 